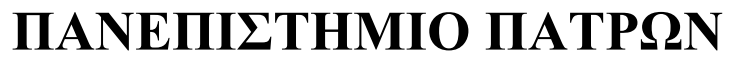

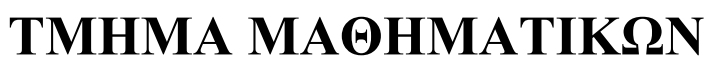

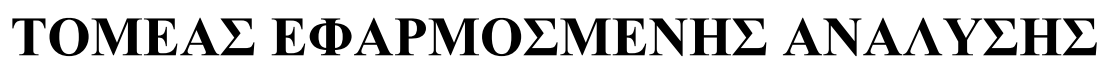

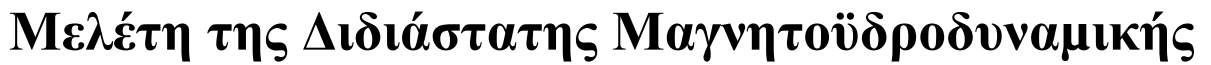

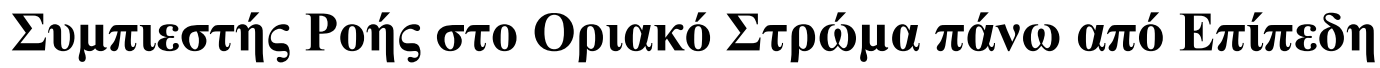

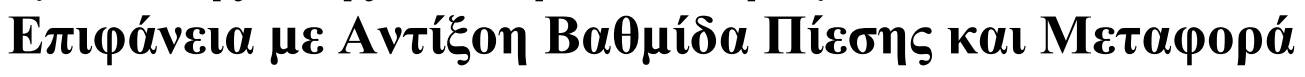

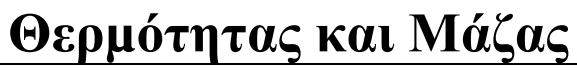

\section{$\Delta$ I $\Delta$ AKTOPIKH $\Delta$ IATPIBH}

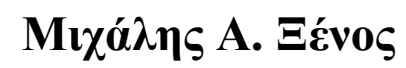

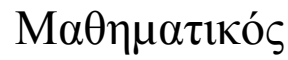



The speculations of today may be the standard methods, or the anathemata, of tomorrow.

W.C. Reynolds and T. Cebeci

$\Sigma \tau \eta \Phi \omega \tau \varepsilon เ v \eta ́$ 


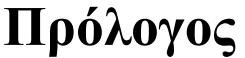

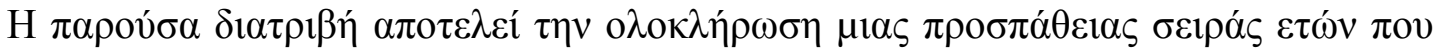

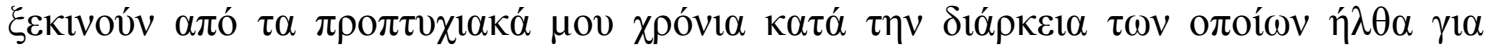

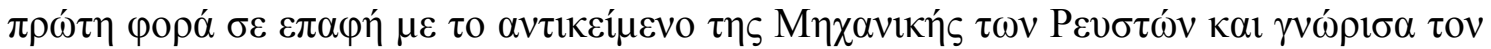

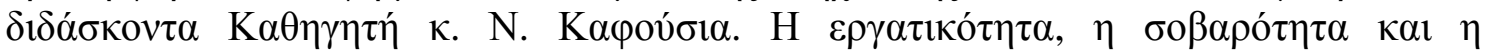

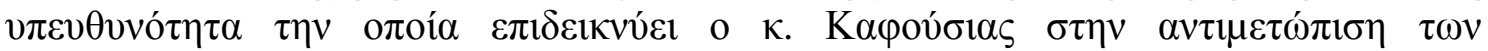

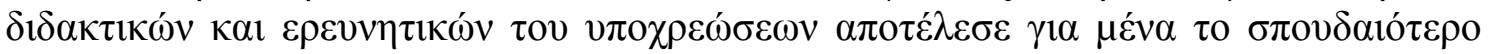

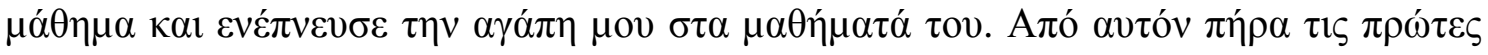

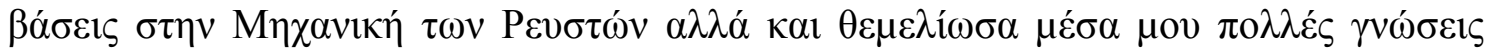

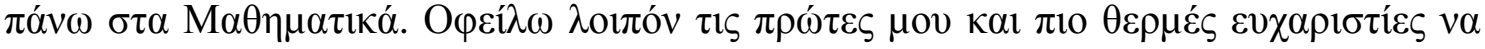

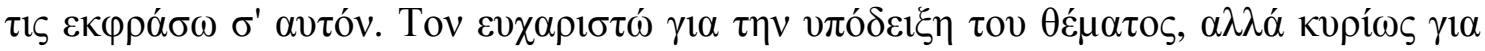

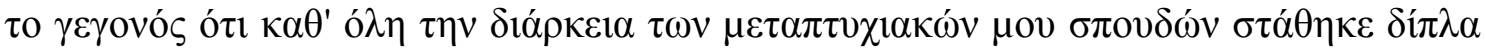

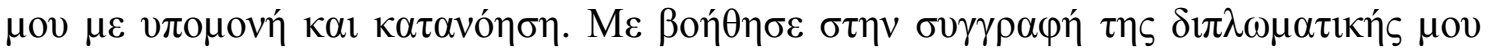

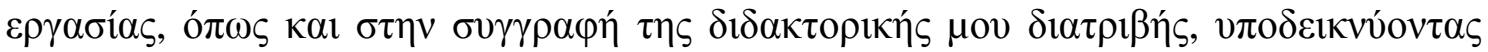

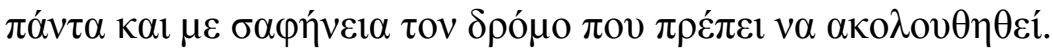

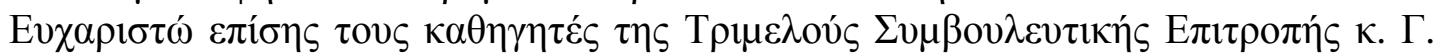

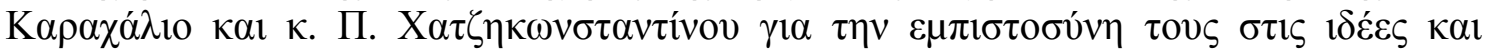

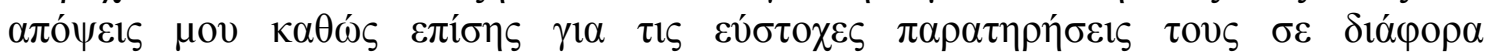

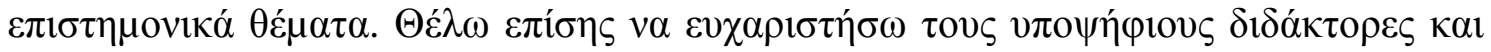

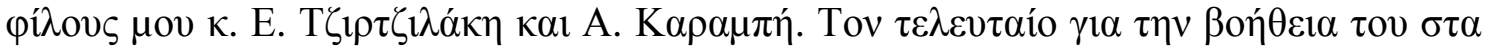

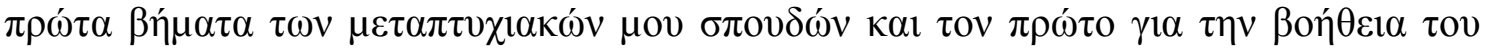

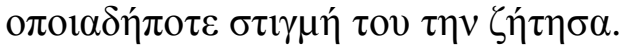

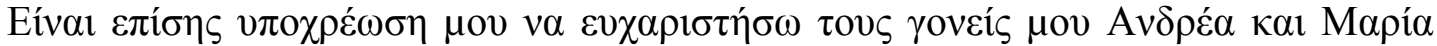

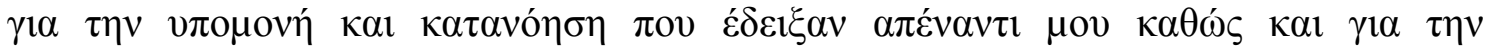

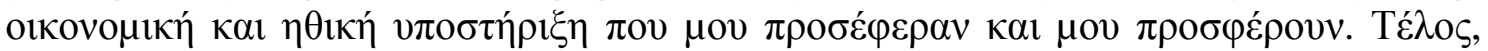

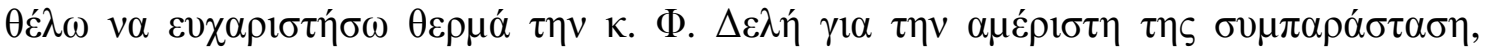

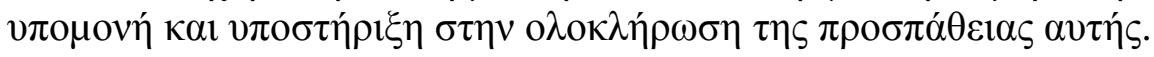

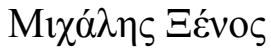

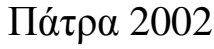




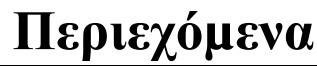

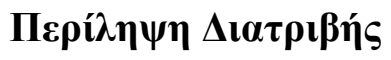

КЕФАААIO I

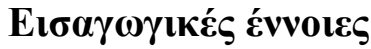

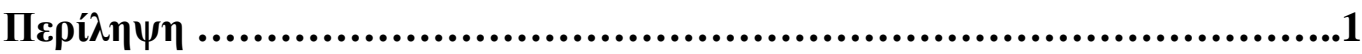

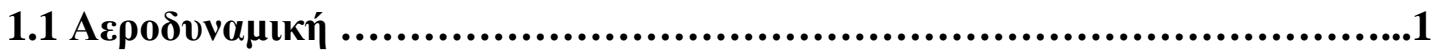

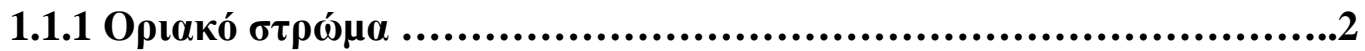

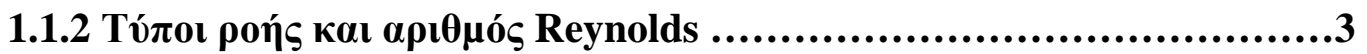

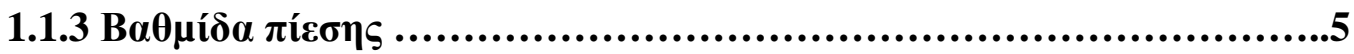

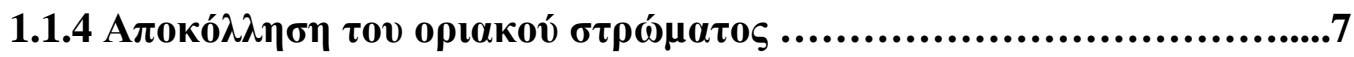

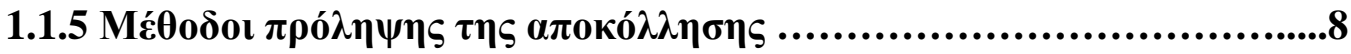

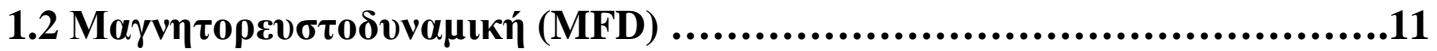

\section{КЕФАААIO II}

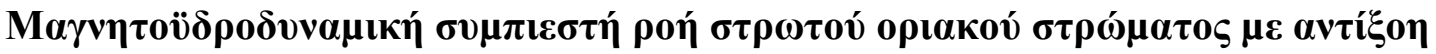

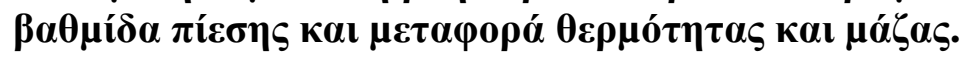

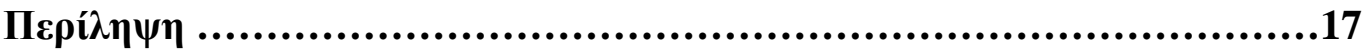

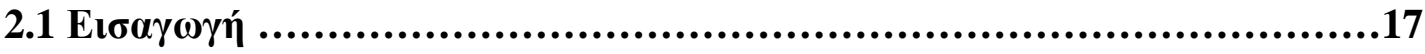

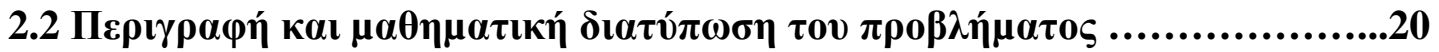

2.3 А

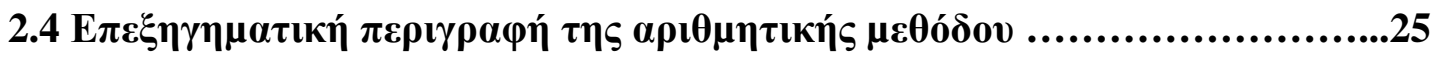

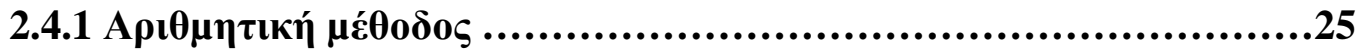

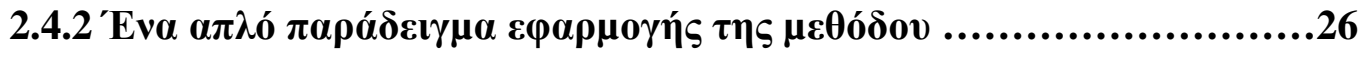

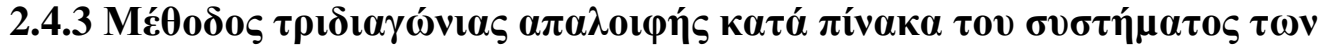
$\varepsilon \xi \iota \sigma \omega ́ \sigma \varepsilon \omega v$........................................................29

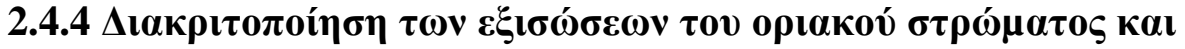

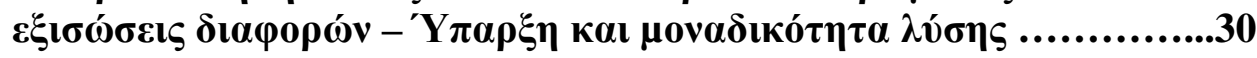

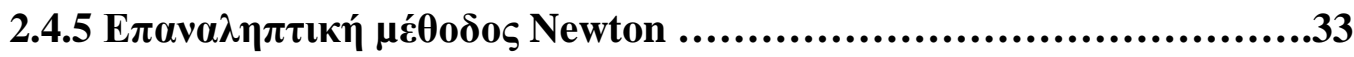

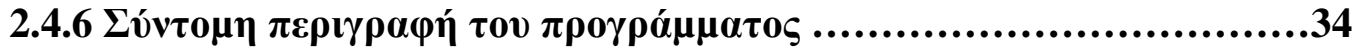




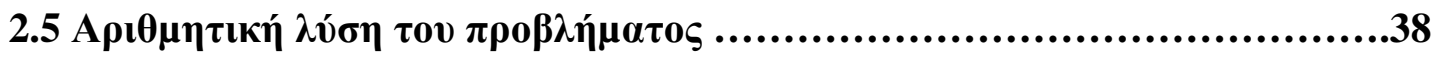

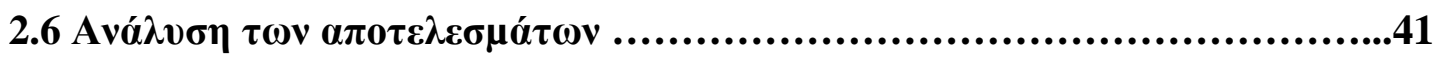

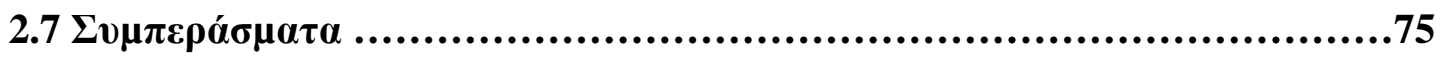

\section{КЕФАААIO III}

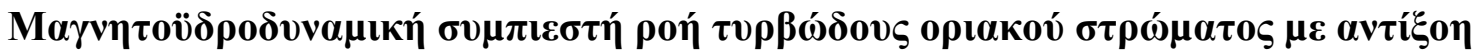

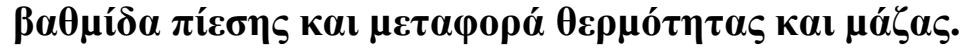

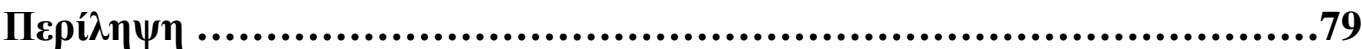

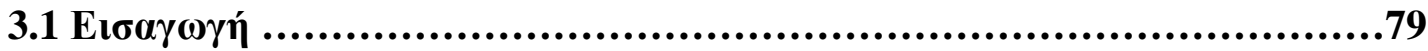

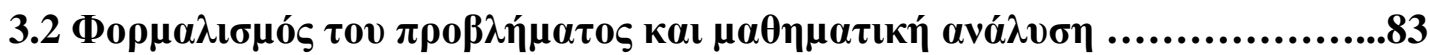

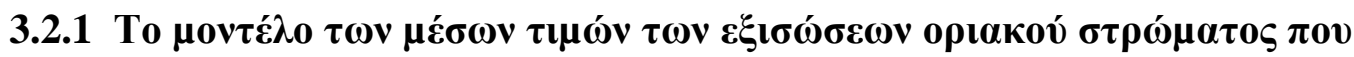
$\pi \rho o ́ \tau \varepsilon เ v \varepsilon$ o Reynolds ...................................................83

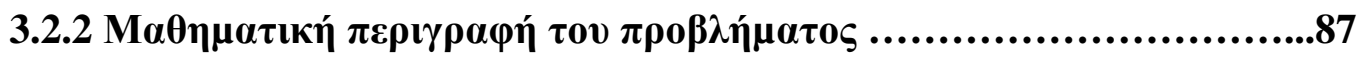

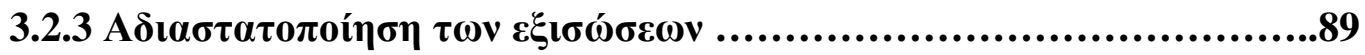

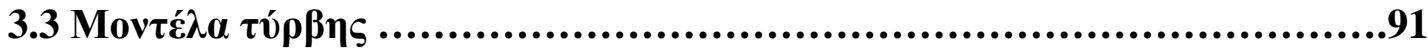

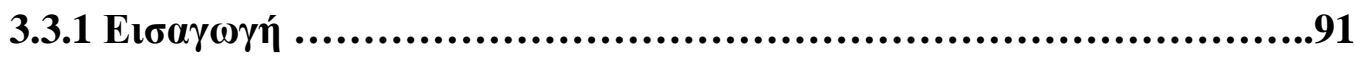

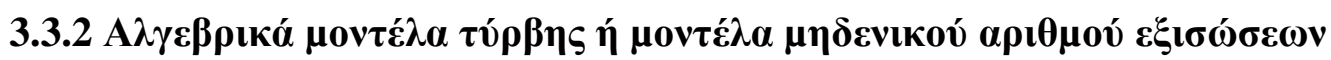

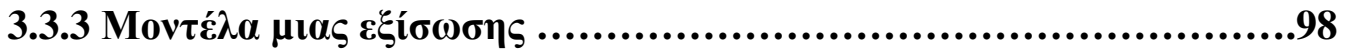

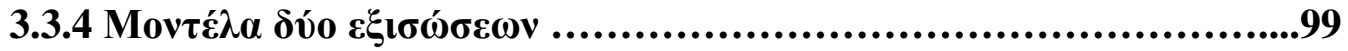

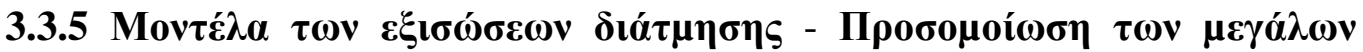

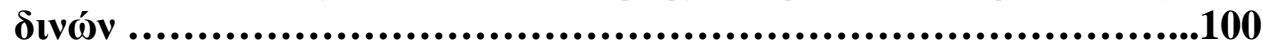

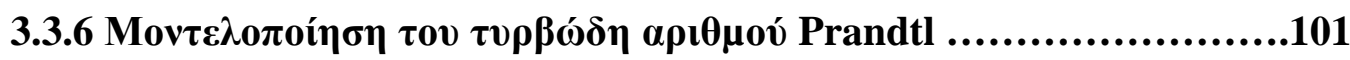

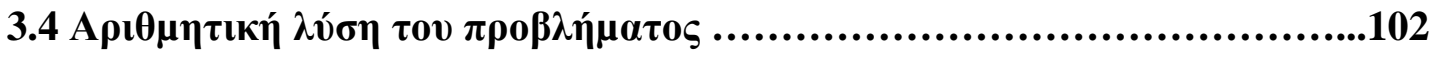

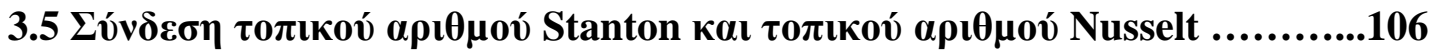

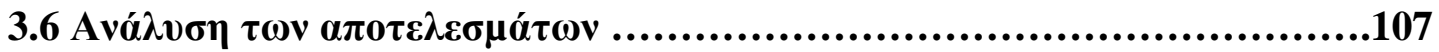

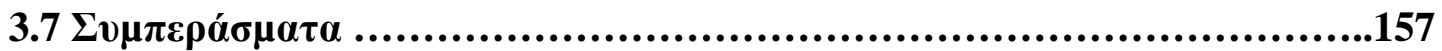

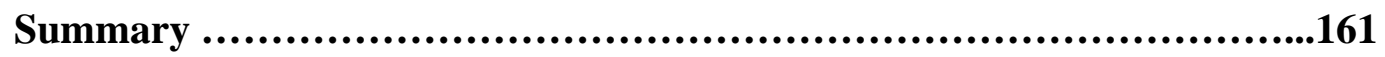

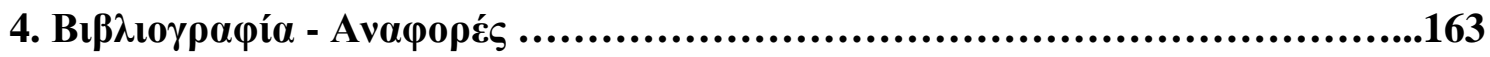




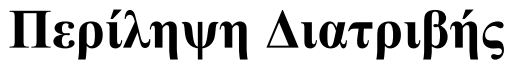

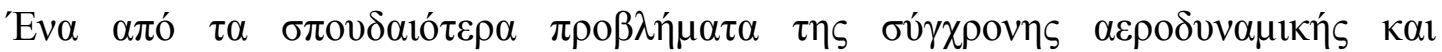

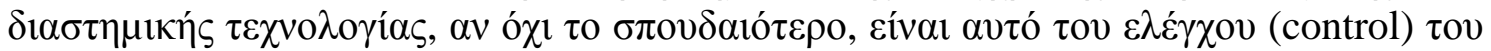

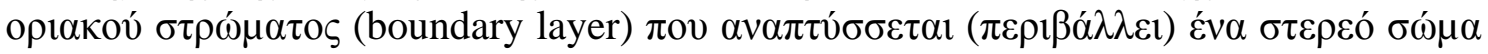

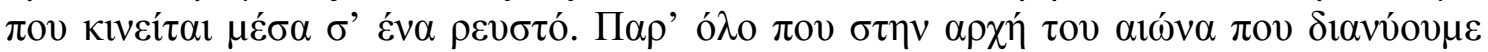

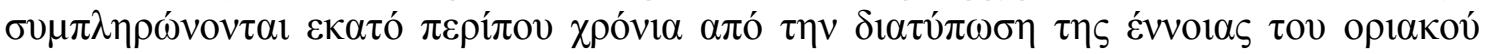

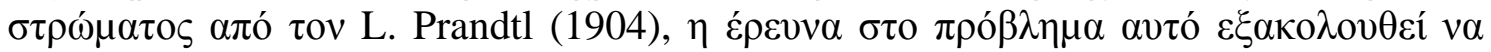

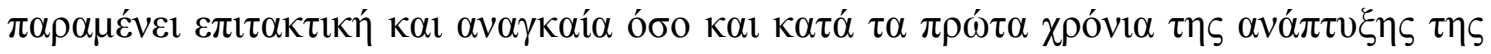

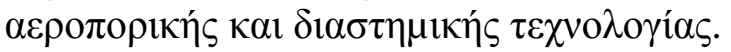

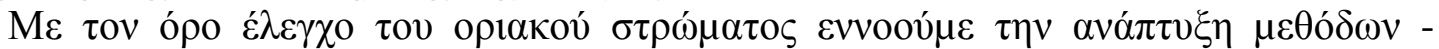

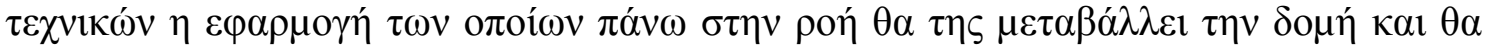

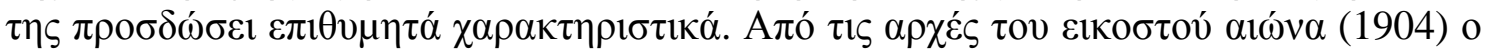

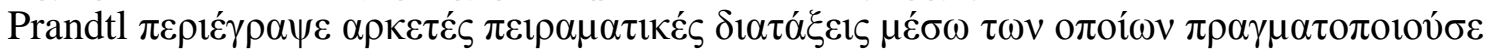

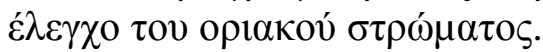

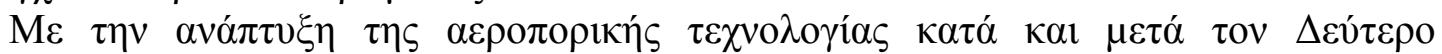

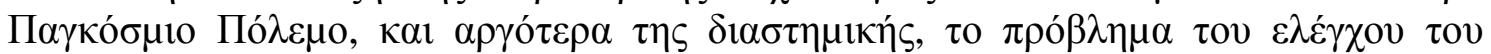

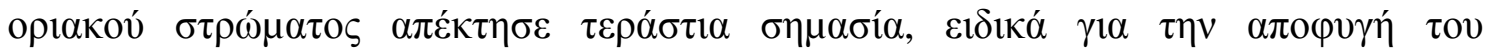

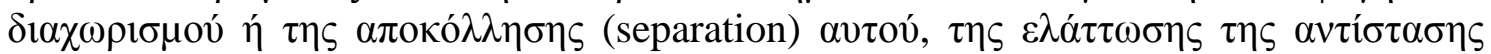

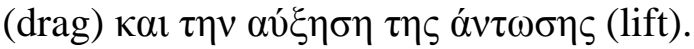

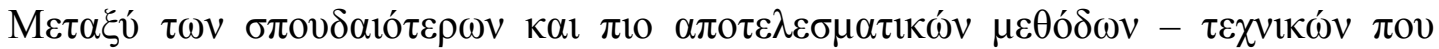

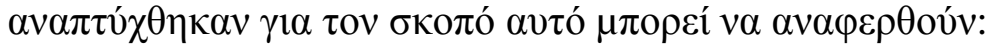

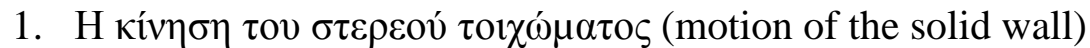

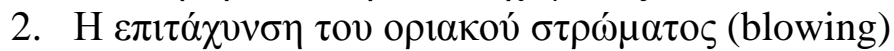

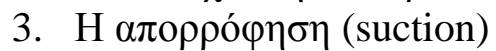

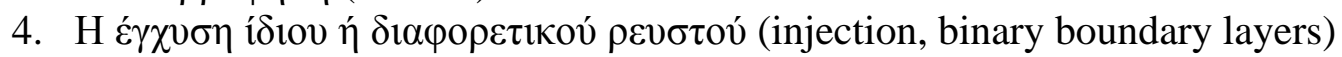

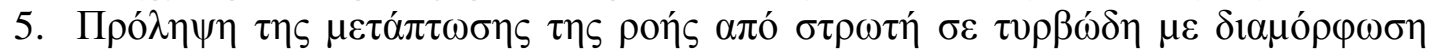

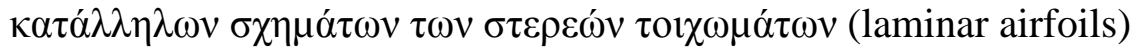

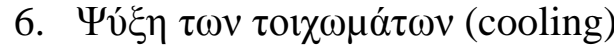

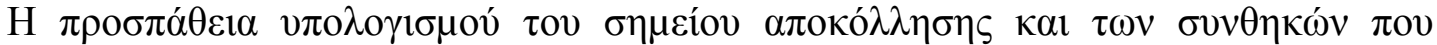

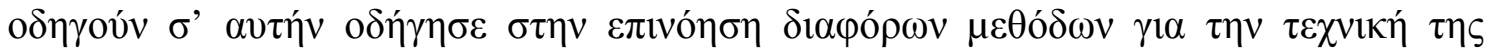

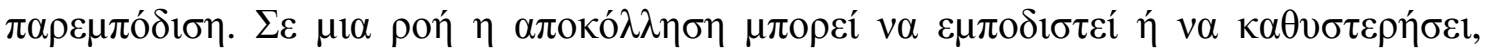

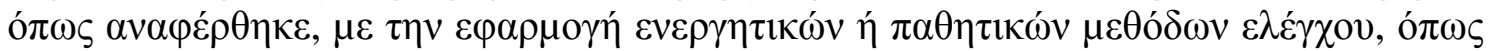

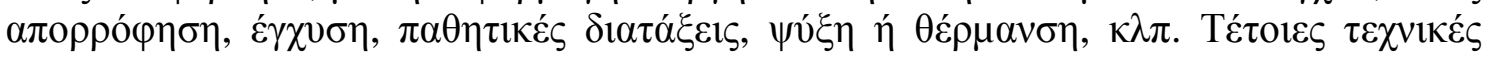

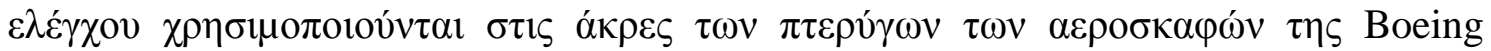

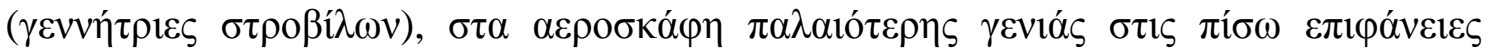

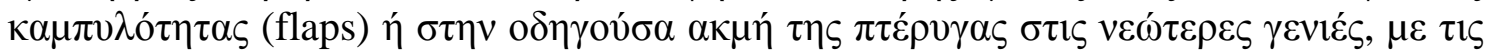

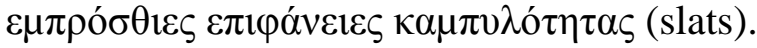

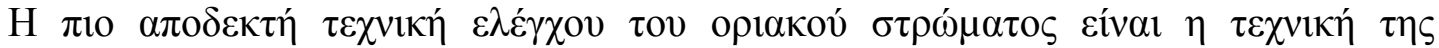

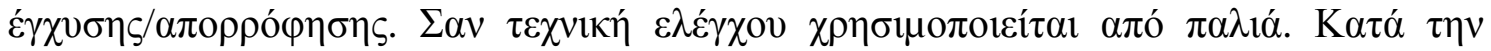

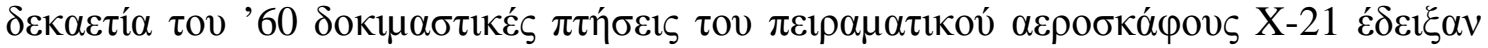

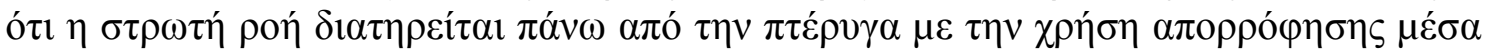

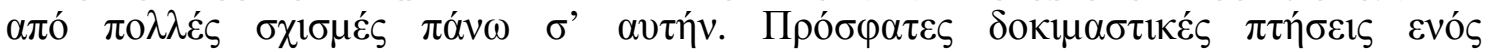

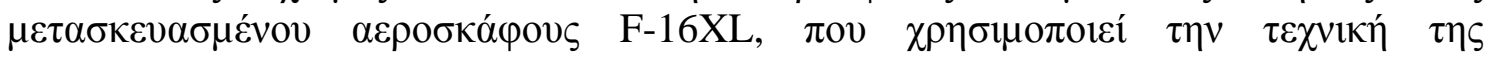

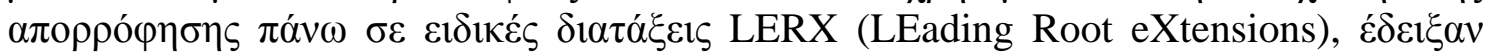

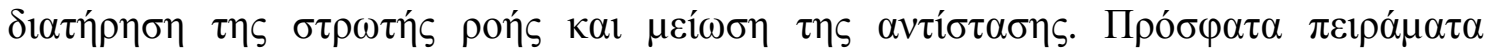

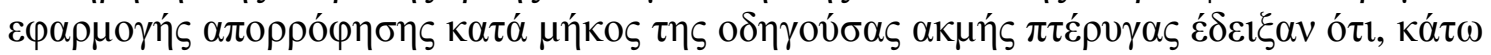




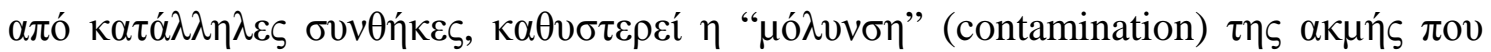

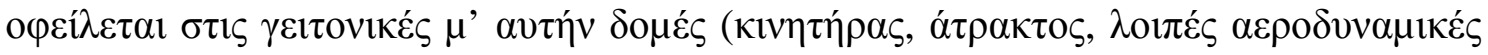
$\delta 1 \alpha \tau \alpha \dot{\xi \varepsilon \varepsilon \varsigma) ~ \pi o v ~ \sigma o \mu \mu \varepsilon \tau \varepsilon ́ \chi 0 v v ~ \sigma \tau \eta v ~ \rho o \eta ́ . ~ П o \lambda \lambda o i ́ ~ \varepsilon i ́ v \alpha r ~ \alpha v \tau o i ́ ~ \pi o v ~ \varepsilon ́ \chi o v v ~ \pi \rho o \tau \varepsilon i ́ v \varepsilon เ ~}$

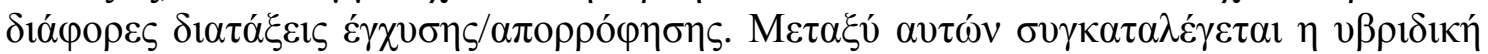

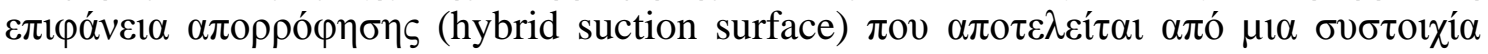

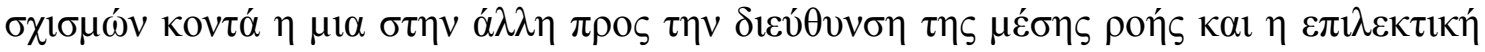

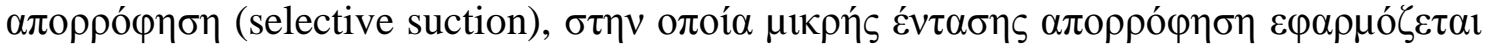

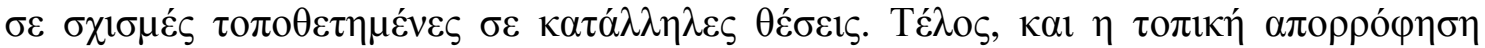

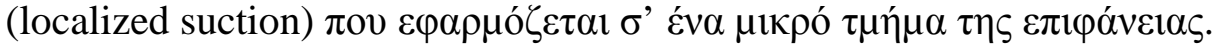

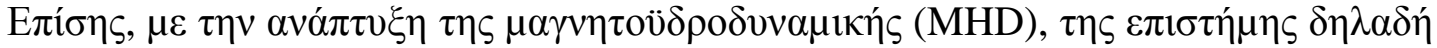

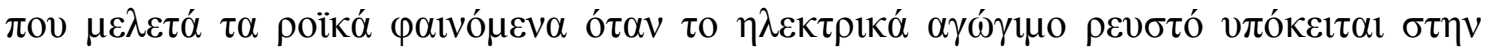

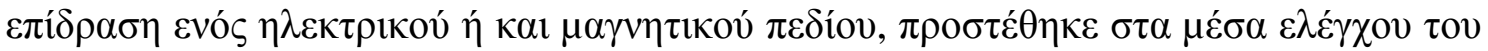

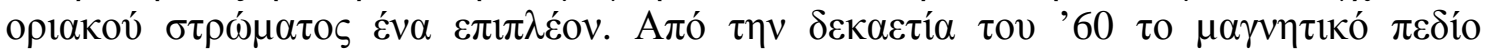

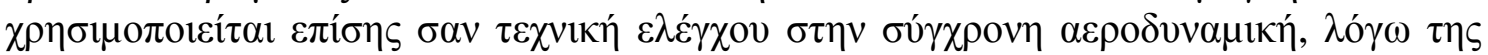

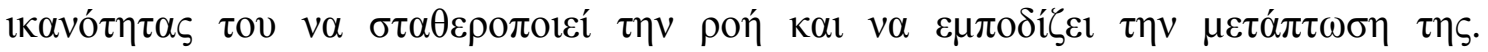

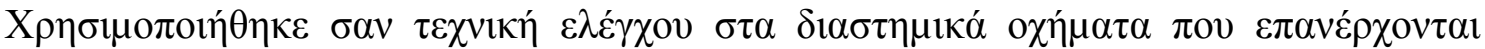

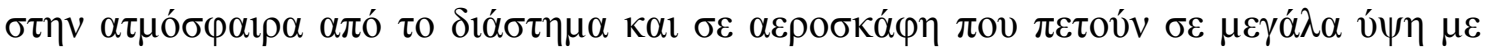

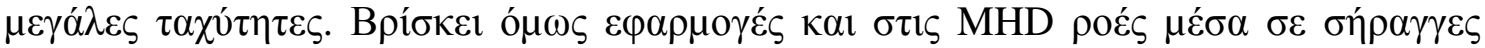

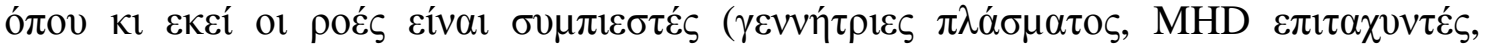

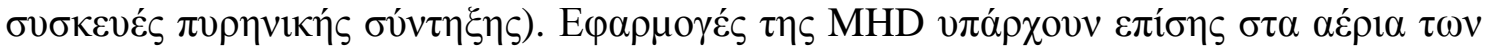

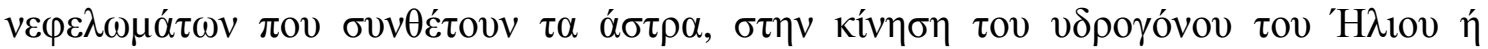

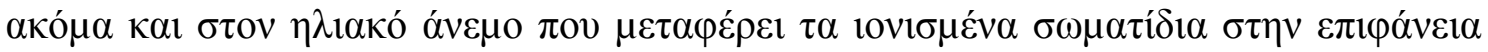
$\tau \eta \varsigma \Gamma \eta s^{2}$

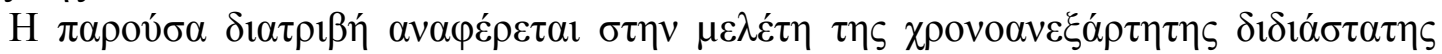

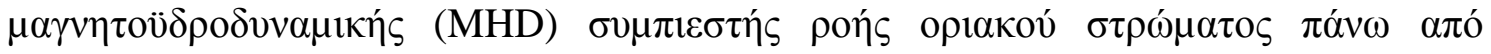

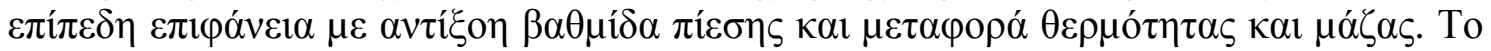

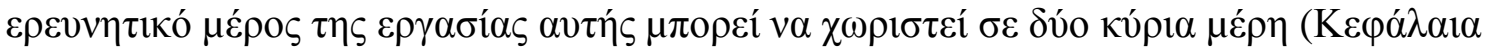
II $\kappa \alpha$ III).

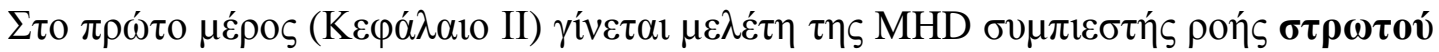

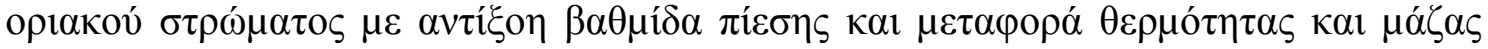

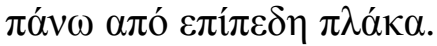

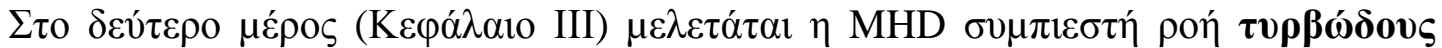

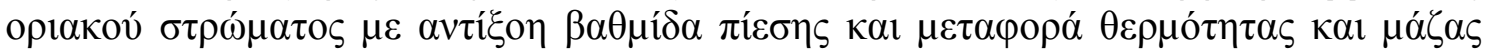
$\pi \alpha ́ v \omega \alpha \pi$ $\varepsilon \pi i ́ \pi \varepsilon \delta \eta \pi \lambda \alpha ́ \kappa \alpha$.

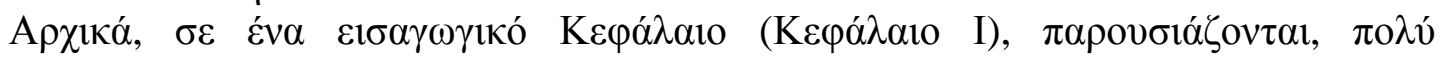

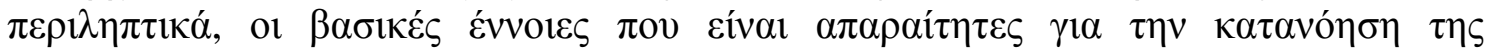

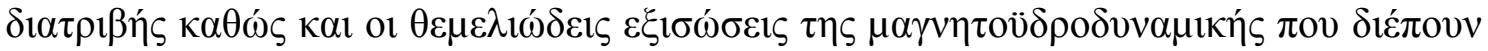

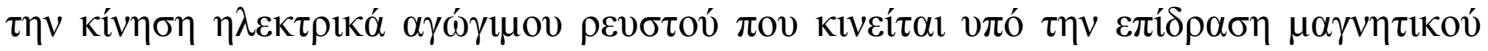
$\pi \varepsilon \delta$ íov.

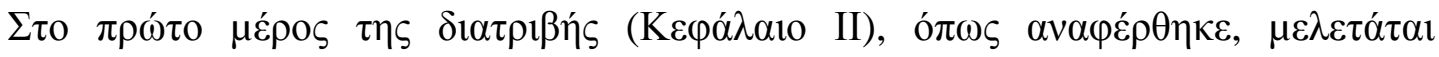

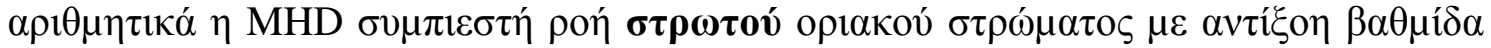

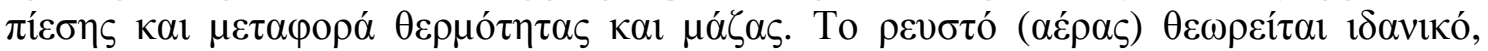

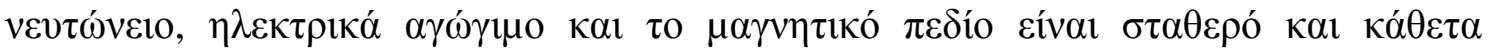

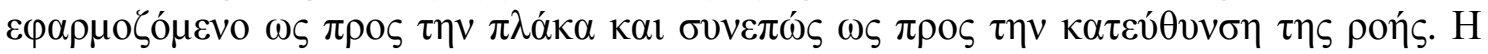

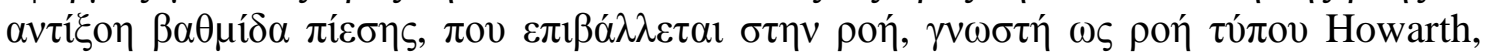

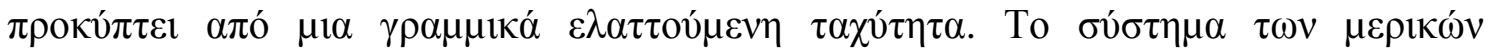

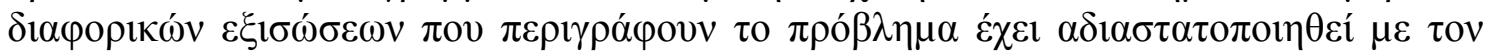

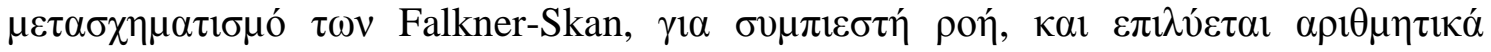

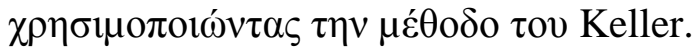




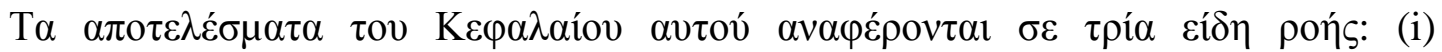

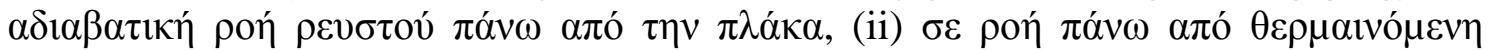

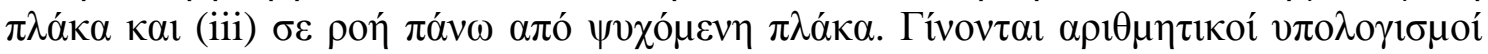

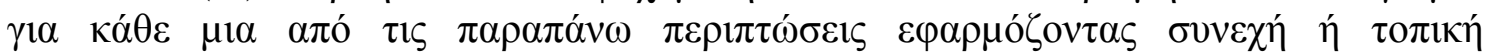

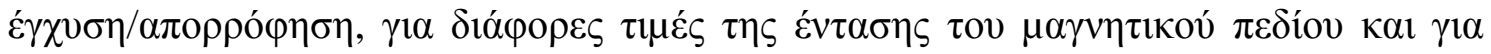

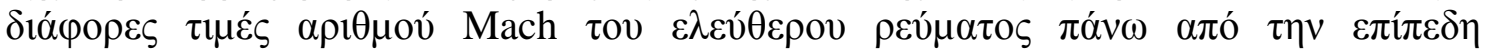

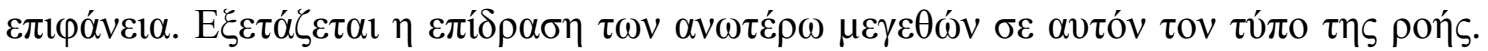

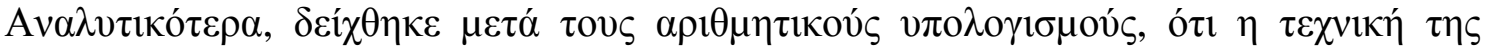

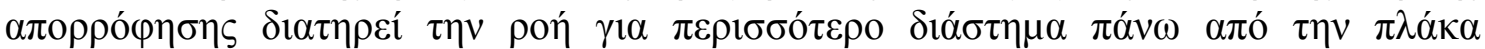

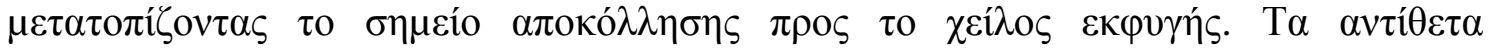

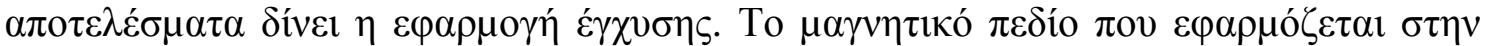

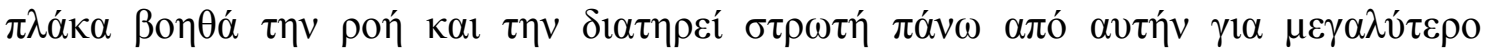

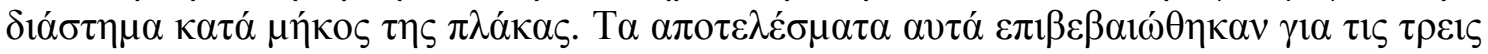

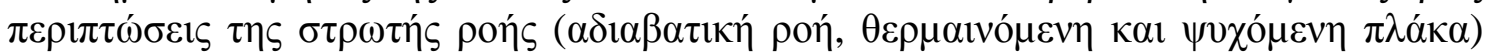

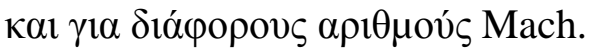

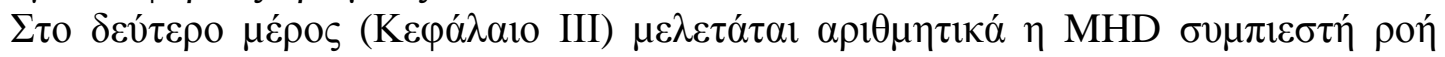

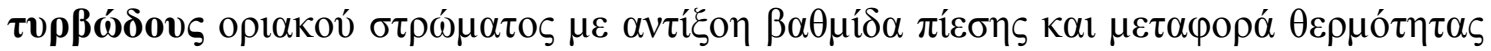

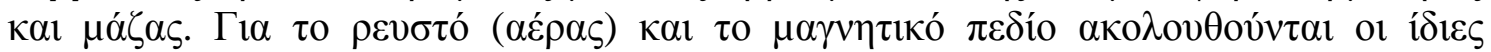

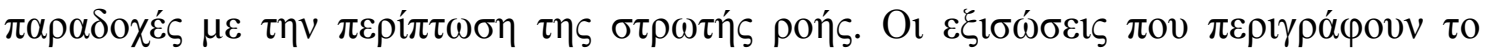

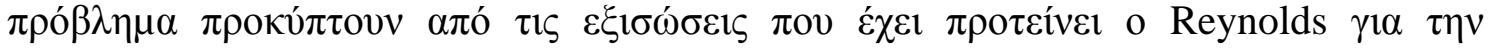

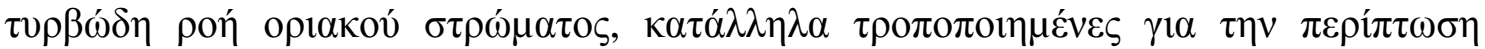

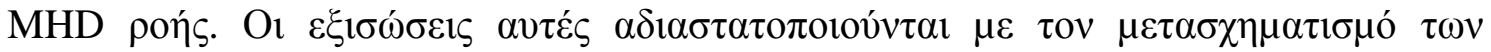

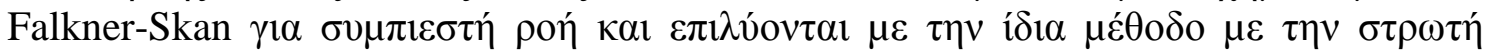

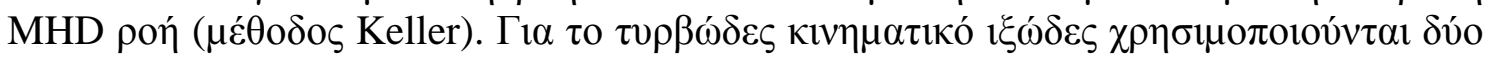

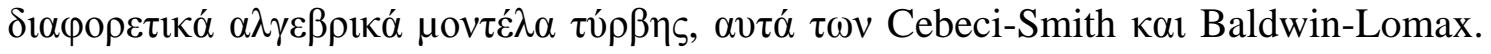

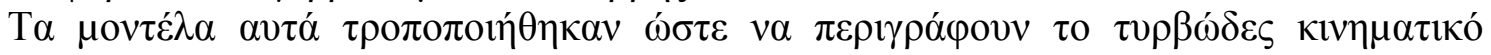

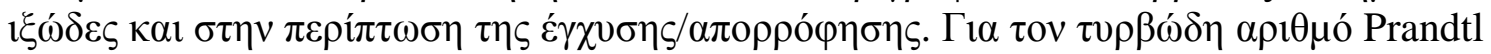

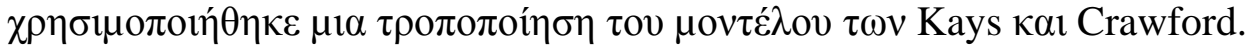

A

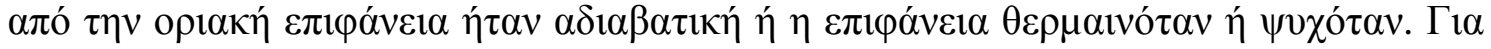

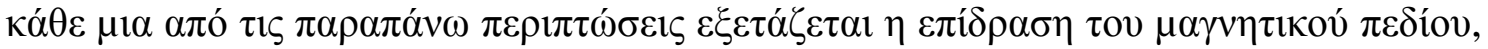

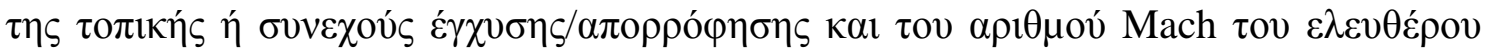

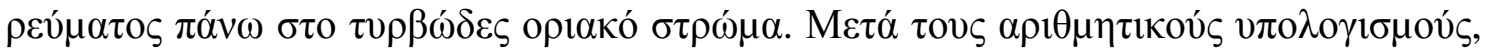

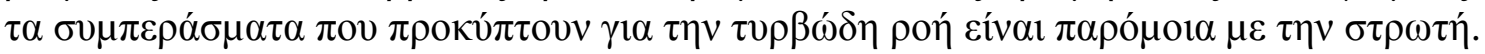

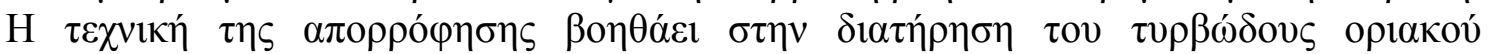

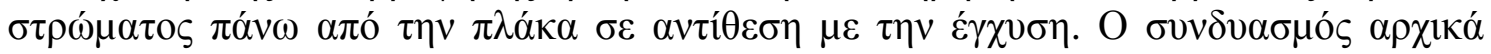

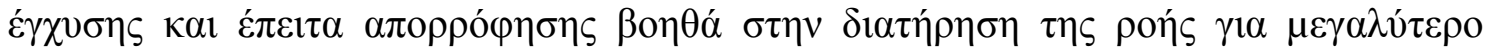

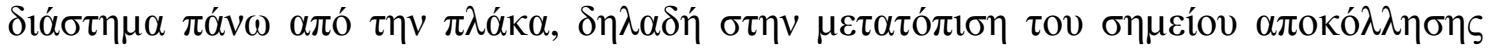

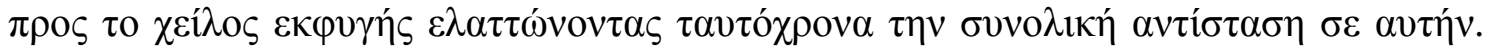

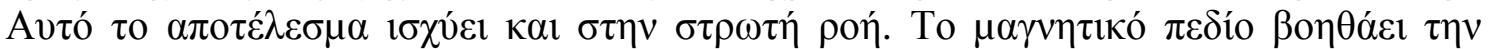

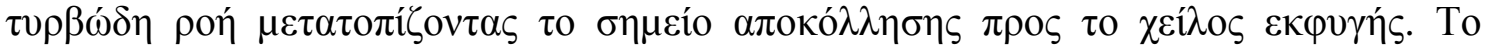

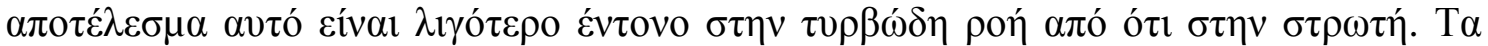

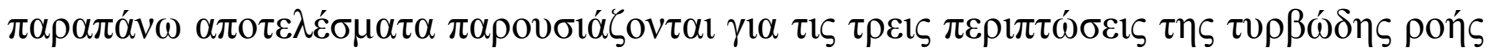

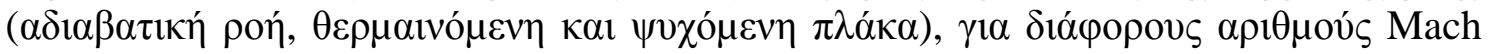

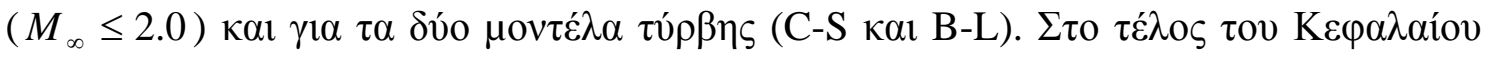

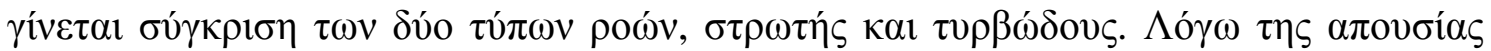

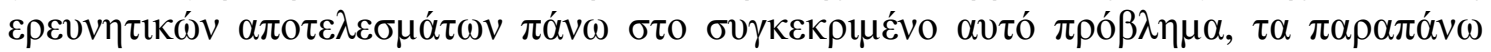

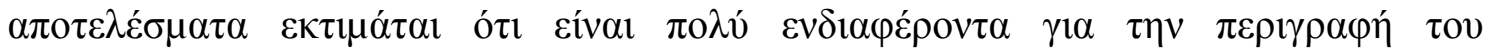

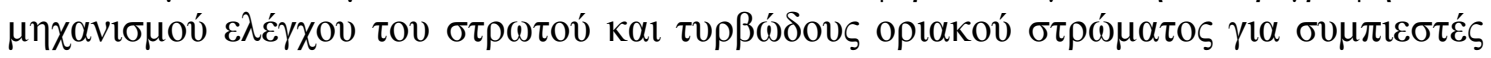
ров́s. 



\title{
КЕФААAIO I
}

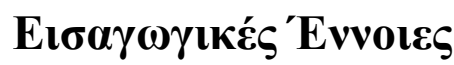

\section{Пєрí $\eta \psi \eta$}

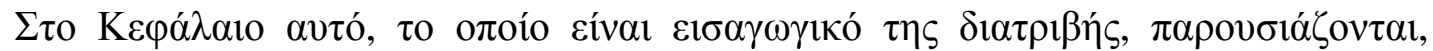

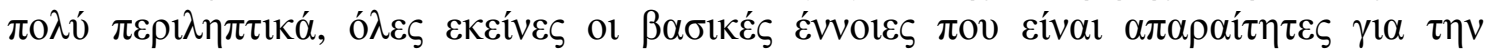

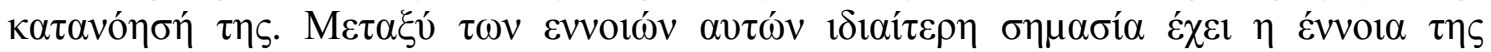

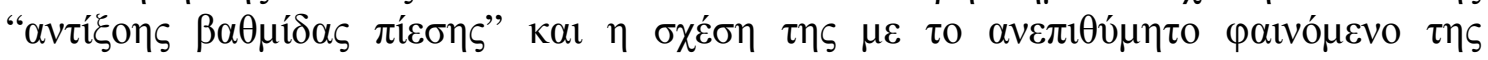

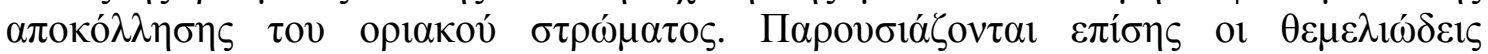

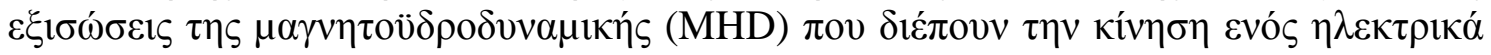

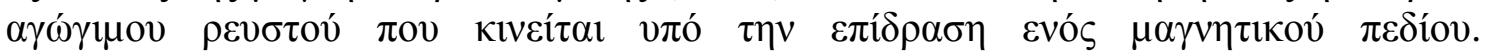

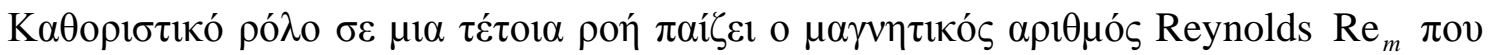

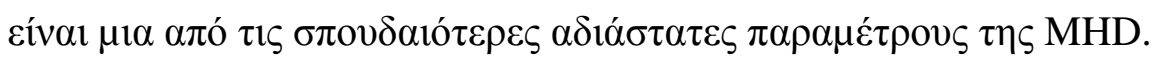

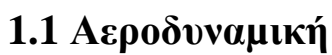

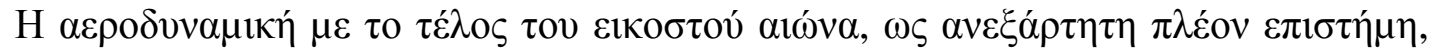

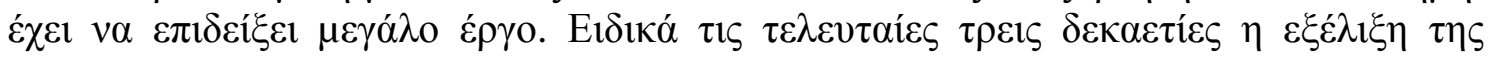

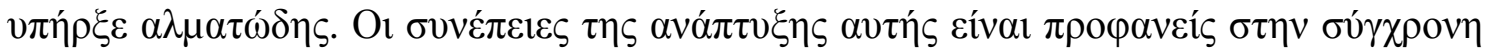

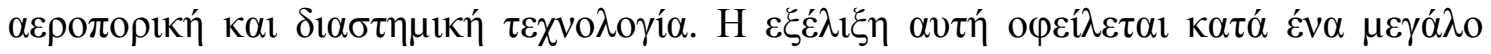

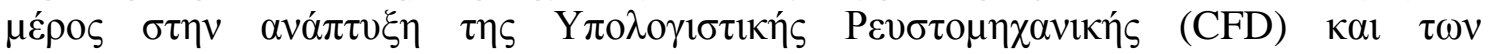

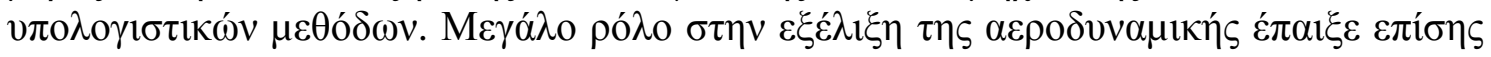

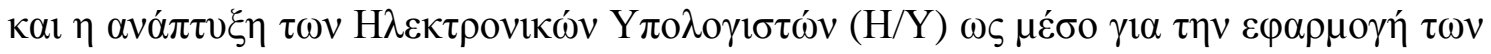

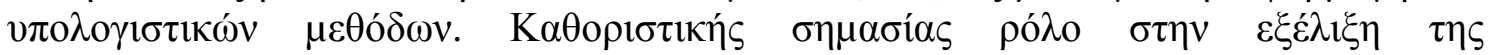

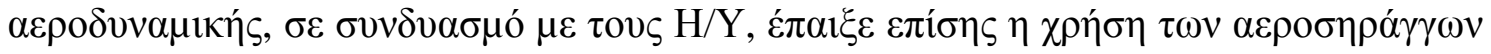

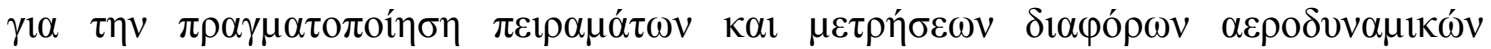

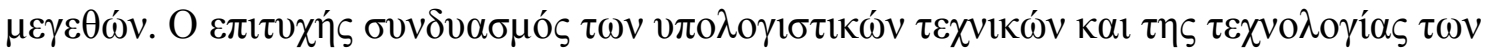

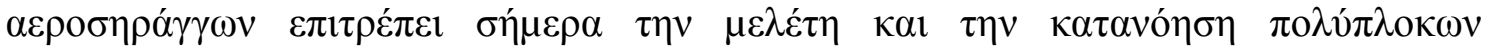

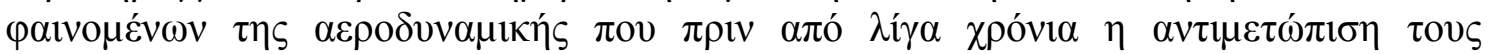

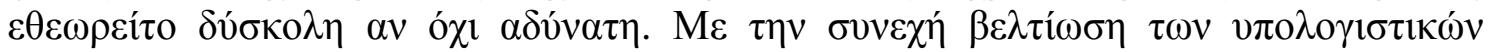

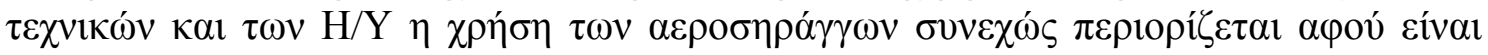

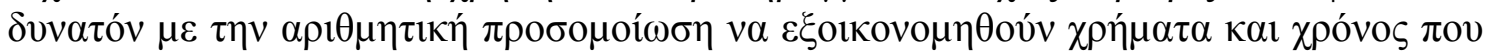

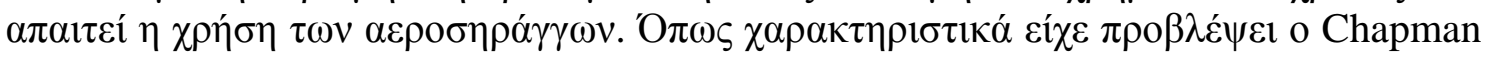

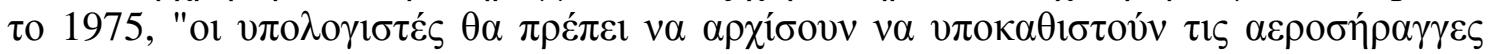

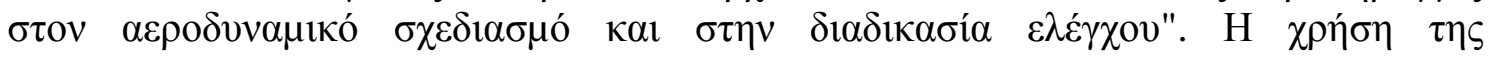

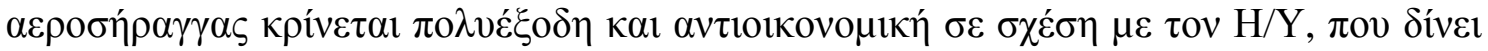

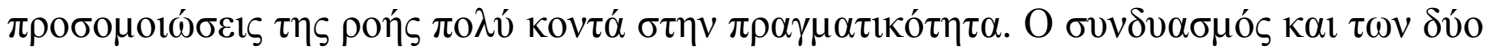

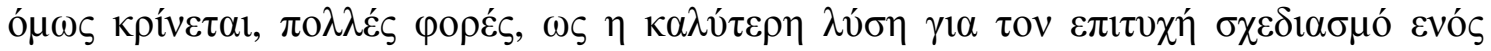

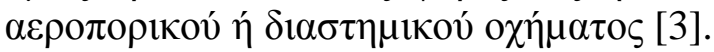

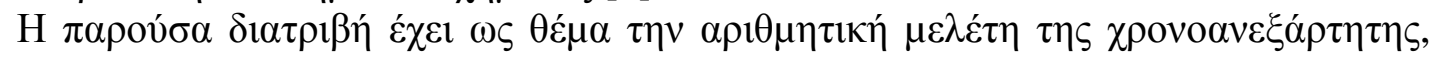

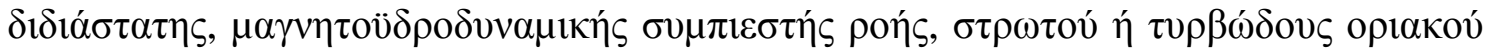

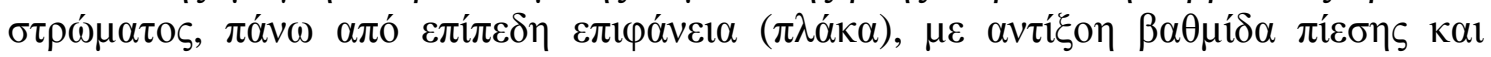

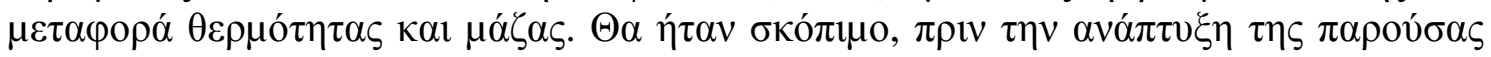

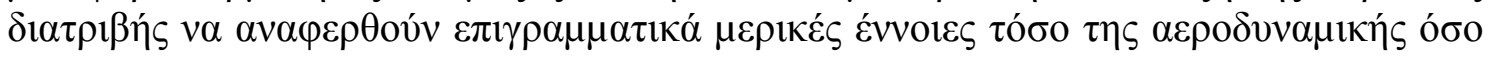

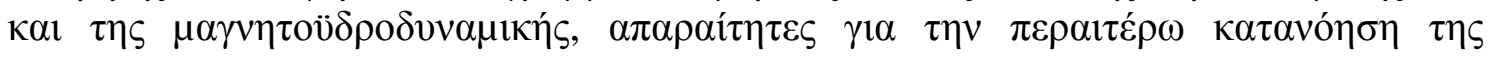




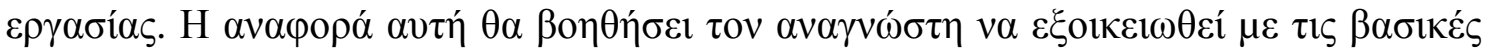

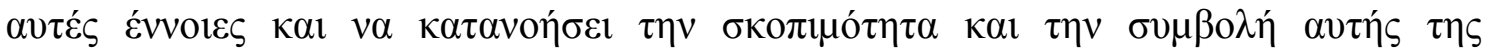

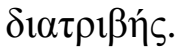

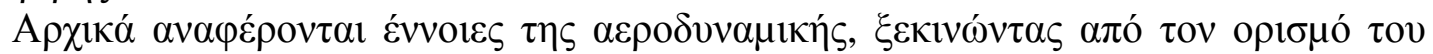

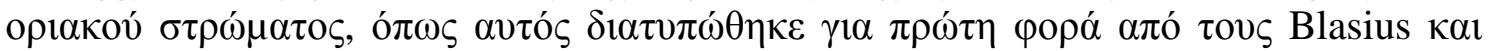

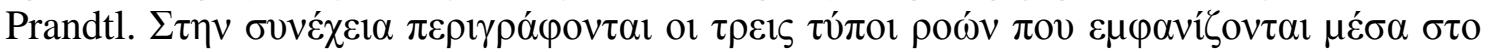

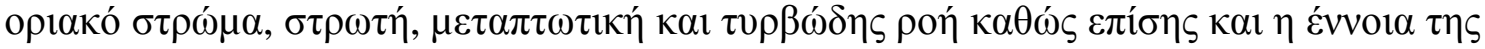

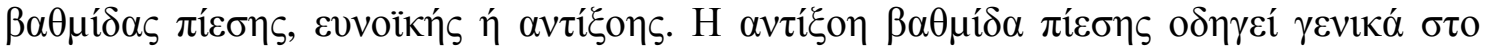

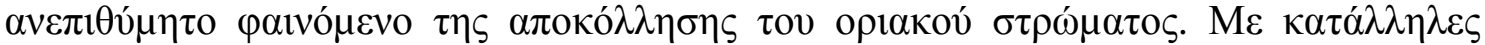

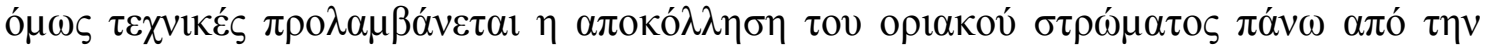

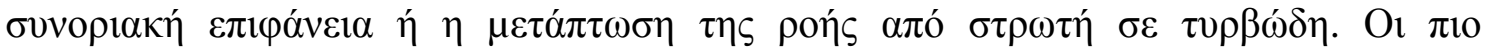

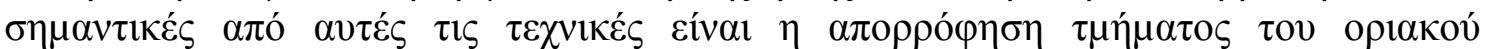

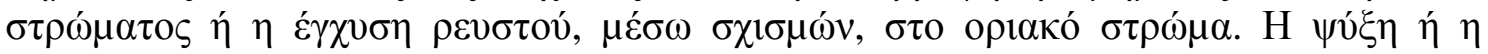

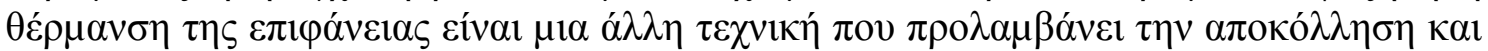

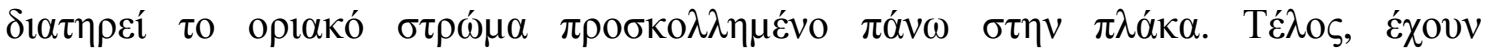

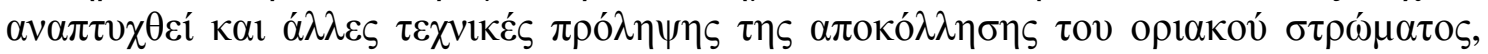

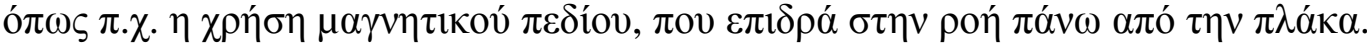

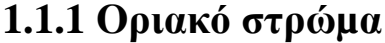

H

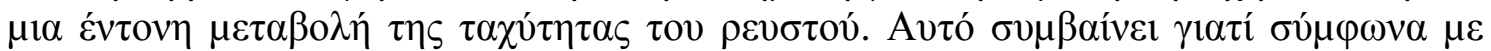

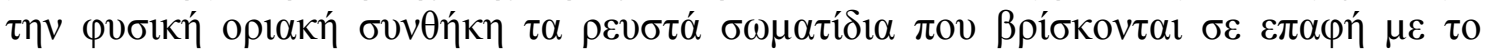

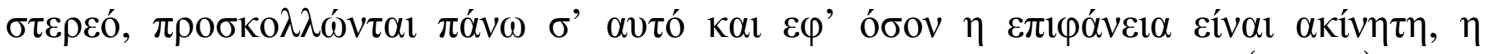

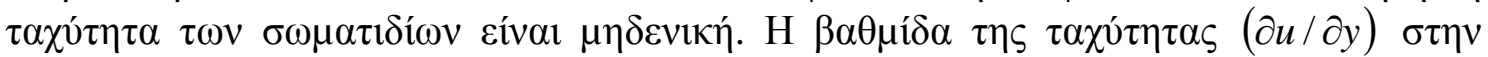

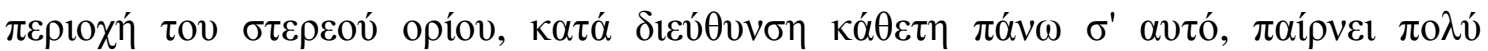

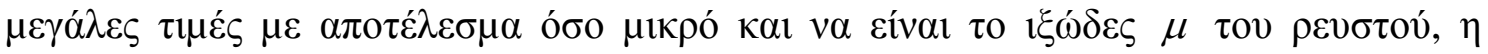

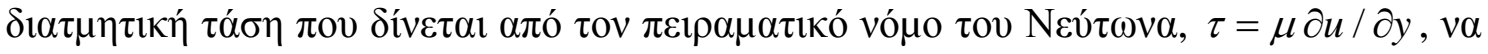

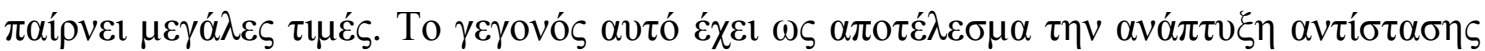

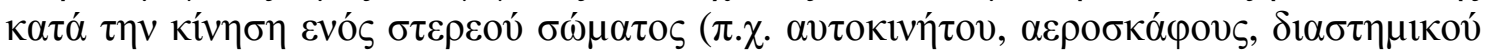

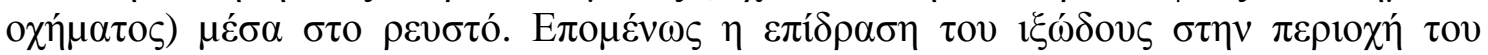

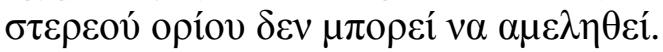

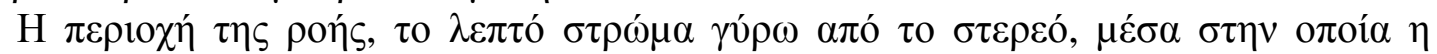

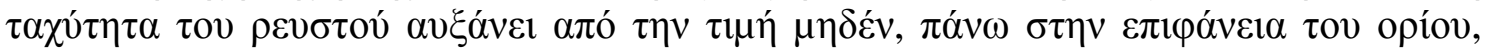

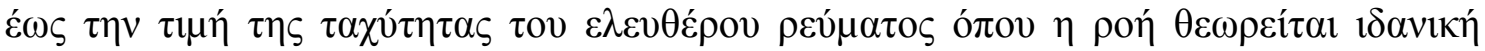

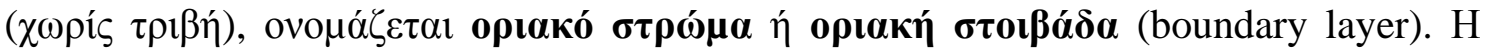

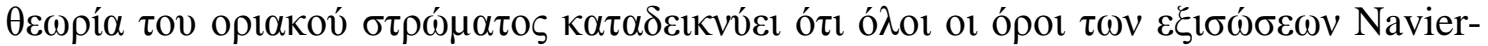

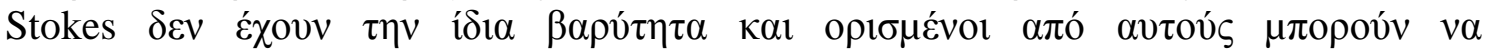

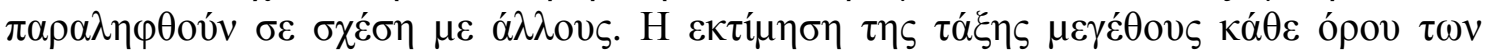

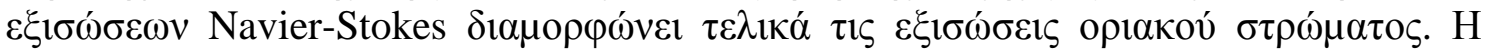

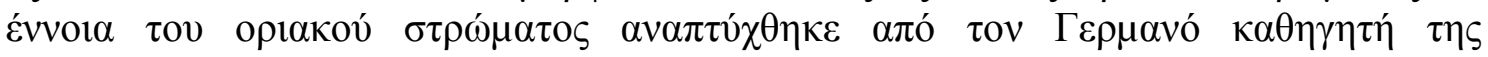

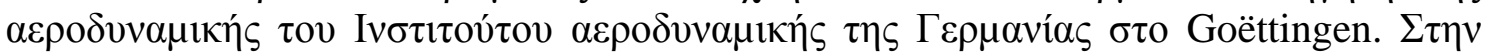

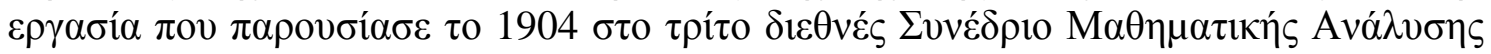

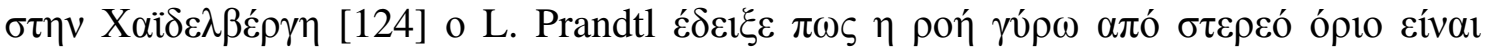

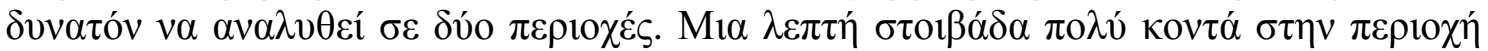

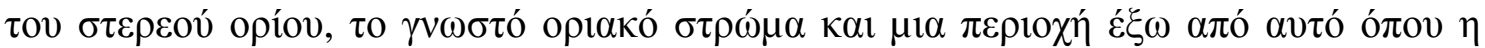

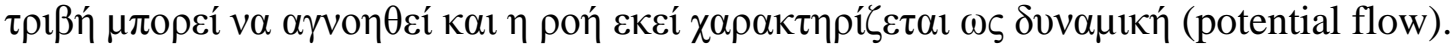




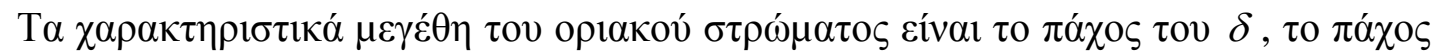

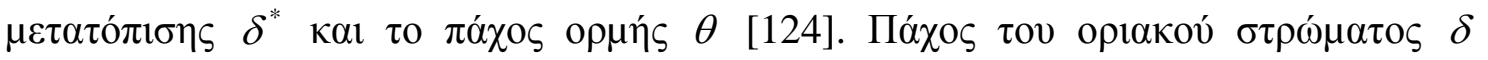

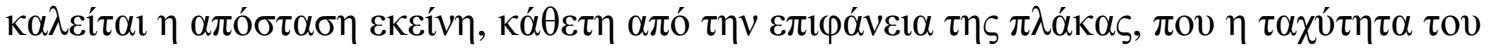

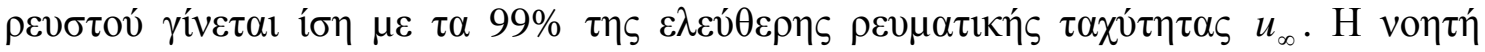

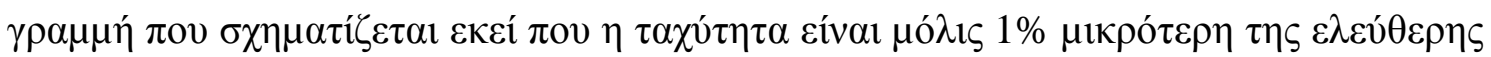

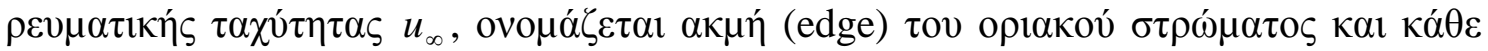

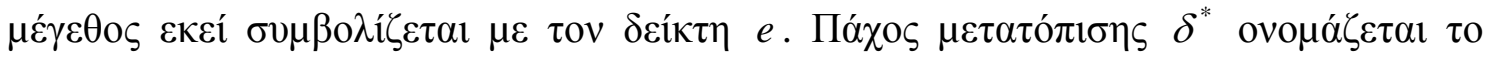

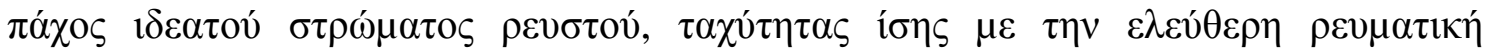

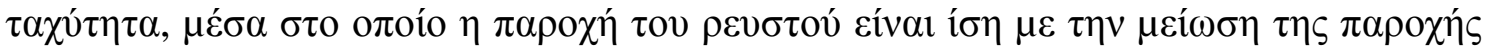

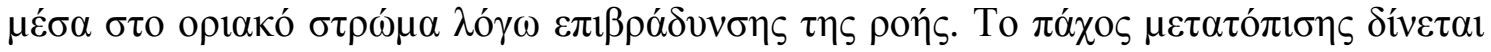

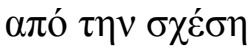

$$
\delta^{*}=\int_{0}^{\infty}\left(1-\frac{\rho u}{\rho_{e} u_{e}}\right) d y .
$$

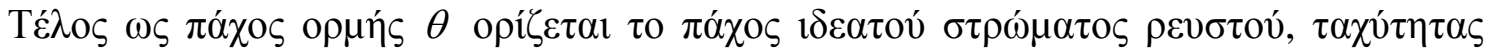

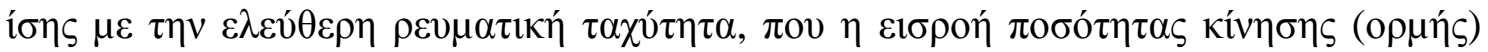

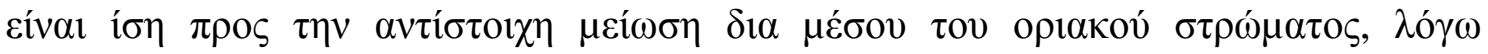

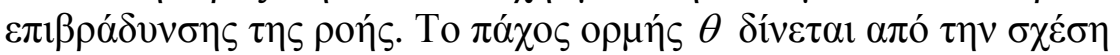

$$
\theta=\int_{0}^{\infty} \frac{\rho u}{\rho_{e} u_{e}}\left(1-\frac{u}{u_{e}}\right) d y .
$$

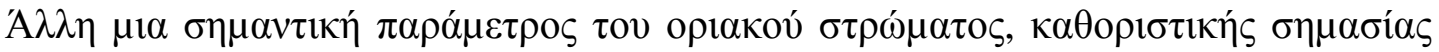

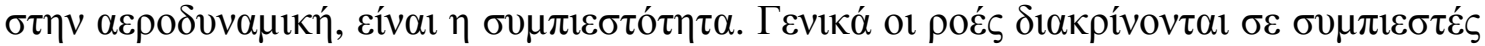

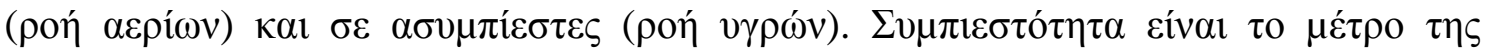

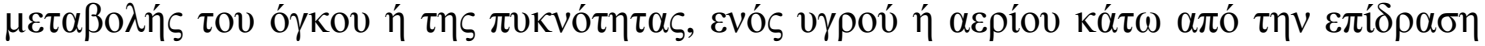
$\tau \omega v \varepsilon \xi \omega \tau \varepsilon \rho ı \kappa \omega ́ v ~ \delta v v \alpha ́ \mu \varepsilon \omega v ~[124]$.

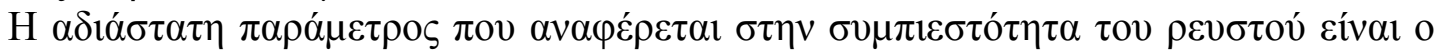

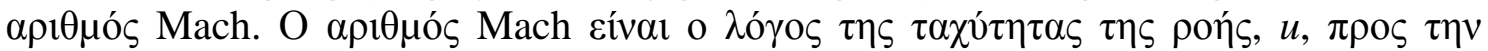

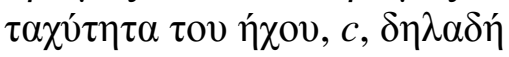

$$
M=\frac{u}{c} .
$$

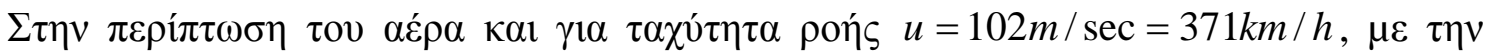

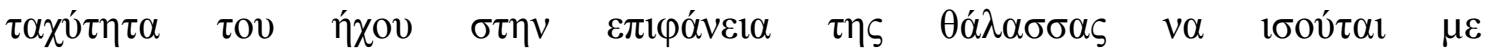

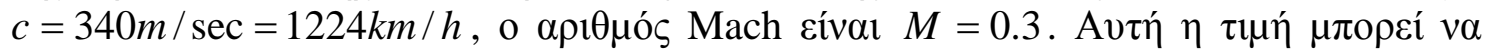

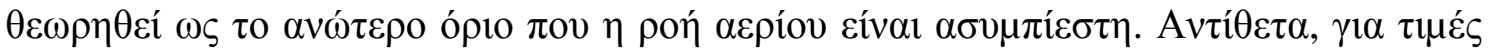

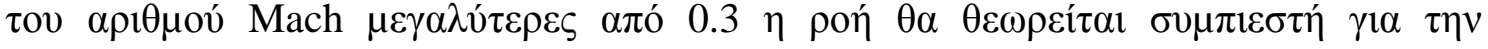

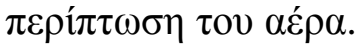

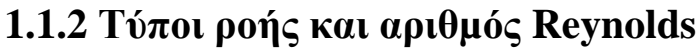

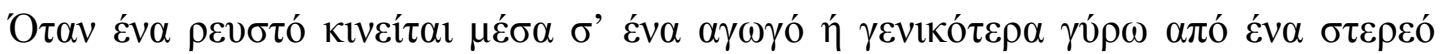

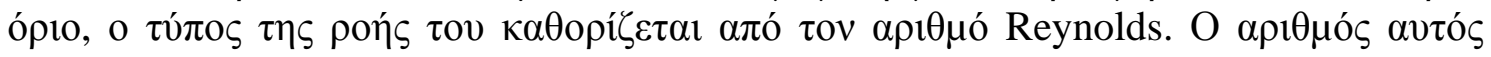




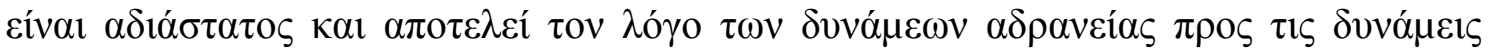

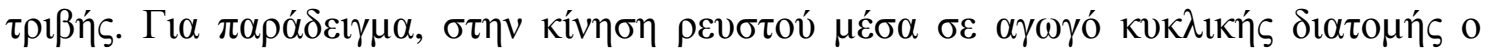

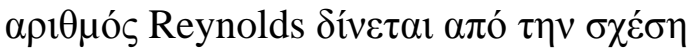

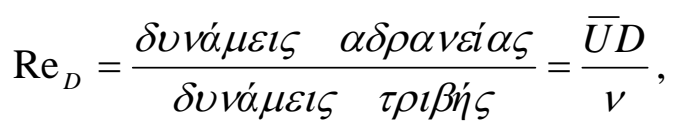

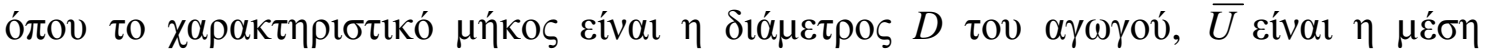

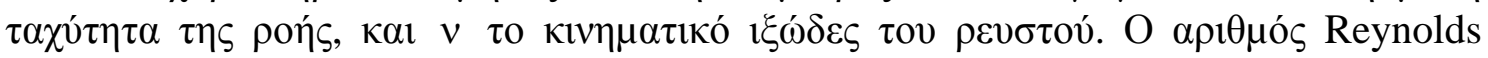

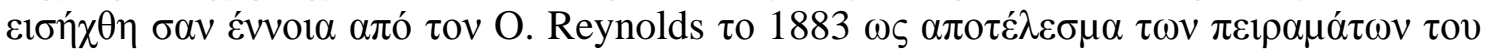

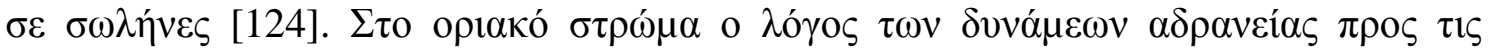

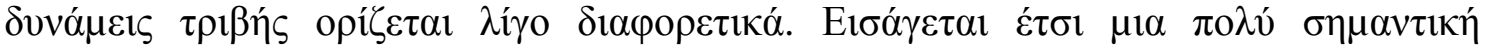

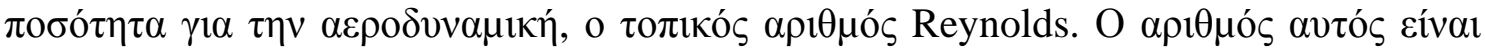

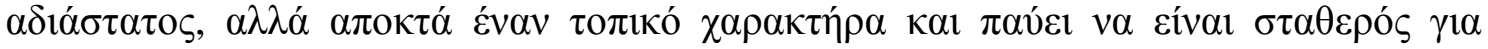

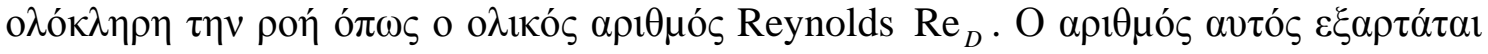

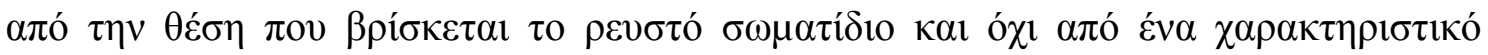

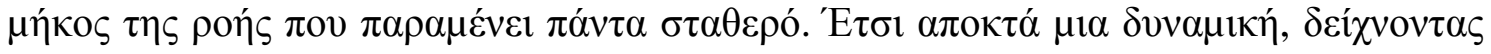

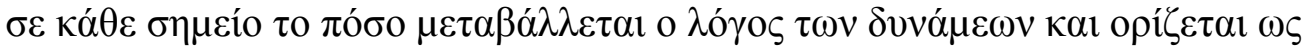

$$
\operatorname{Re}_{x}=\frac{u_{e} x}{v_{e}}
$$

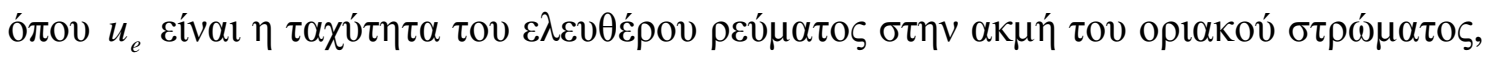

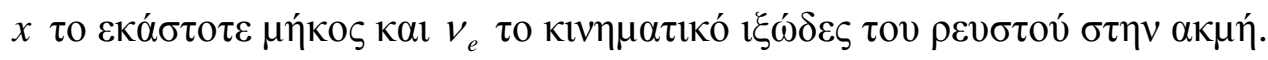

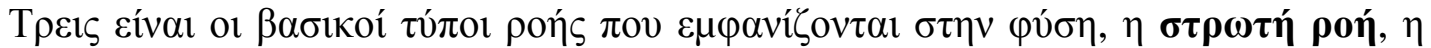

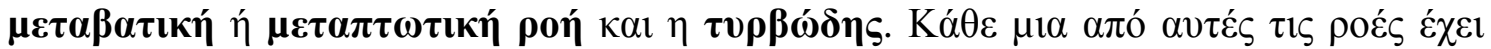

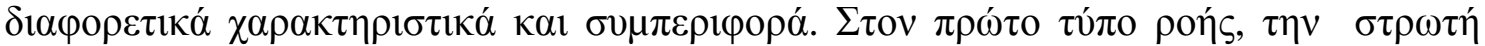

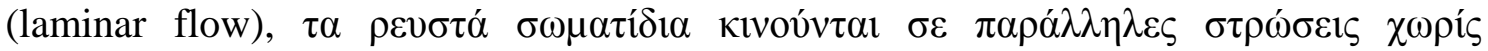

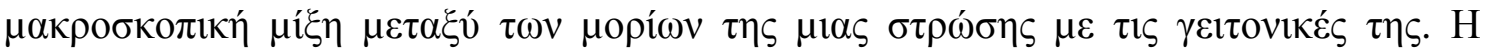

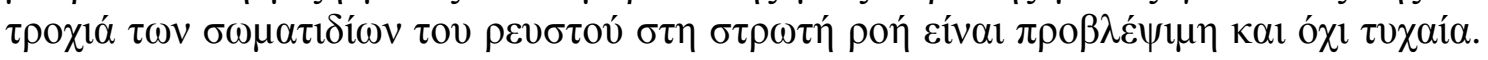

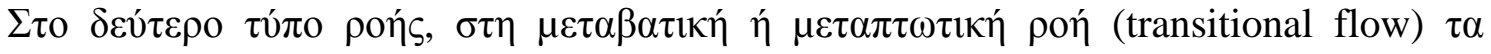

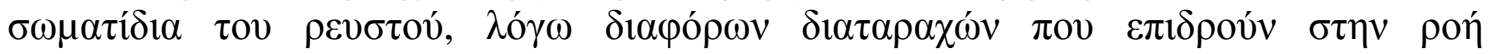

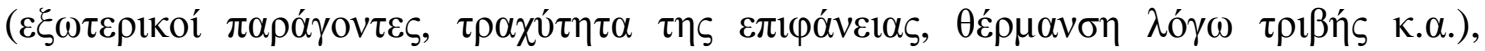

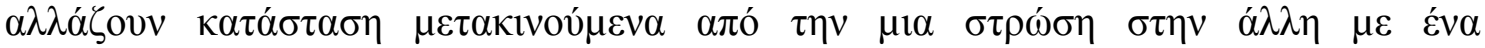

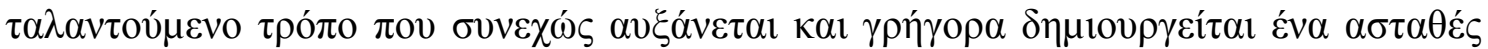

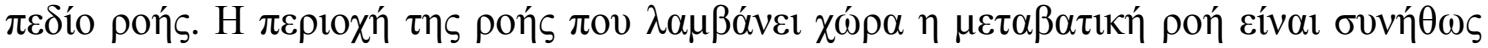

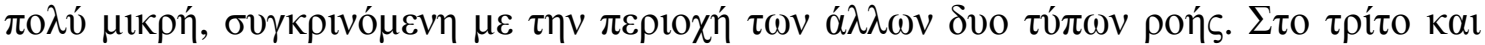

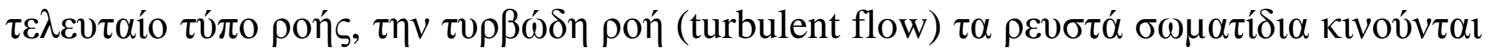

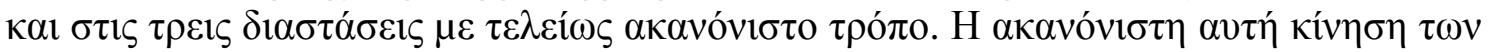

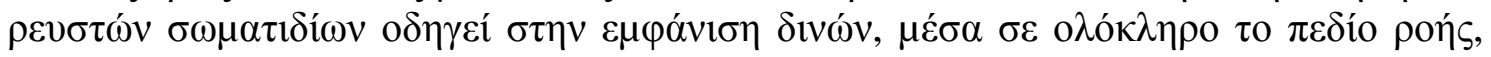

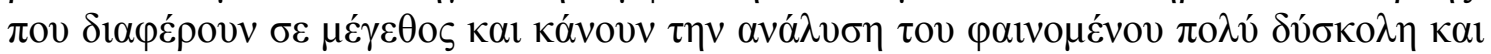

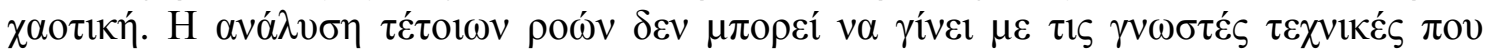

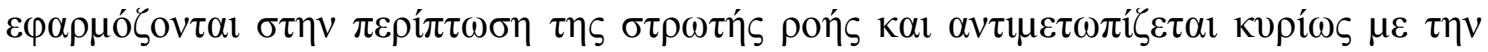

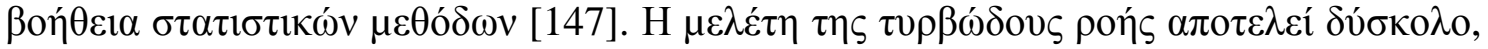

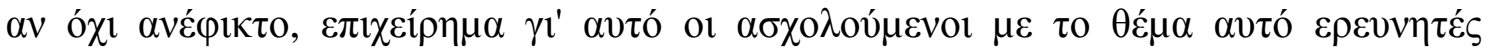

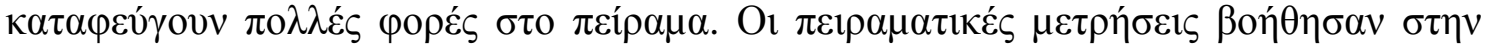

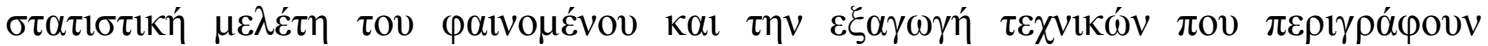

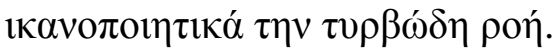




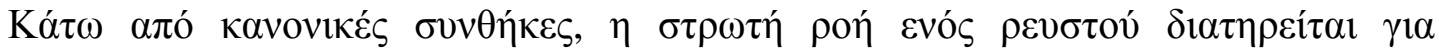

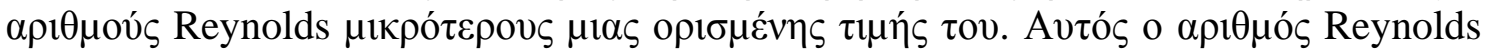

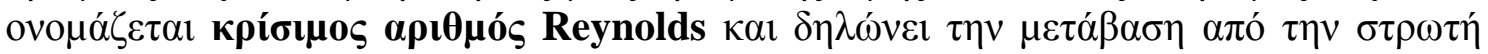

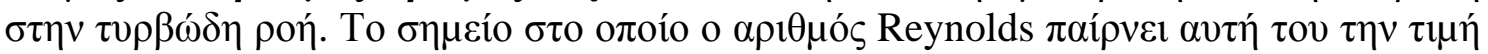

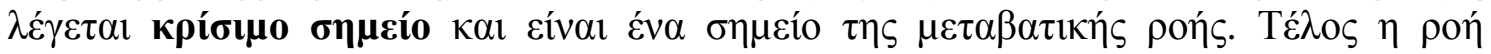

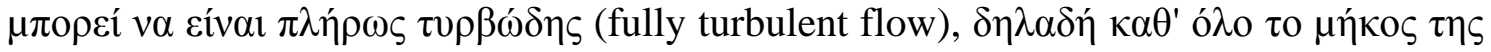

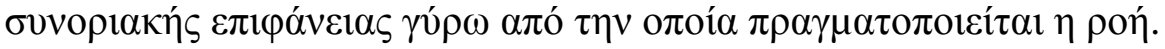

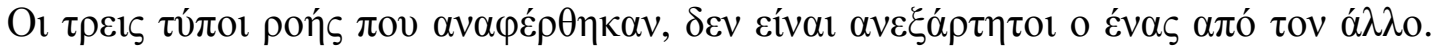

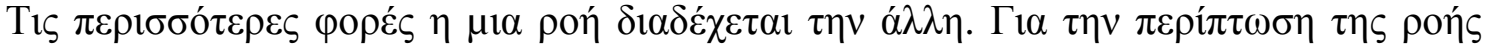
$\pi \alpha ́ v \omega$ a

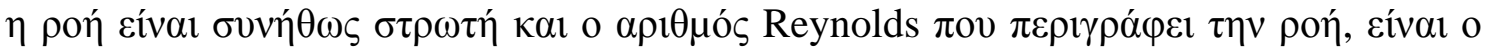

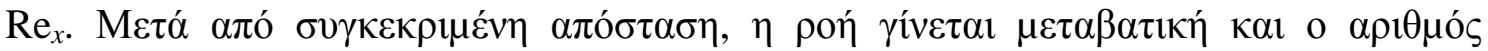

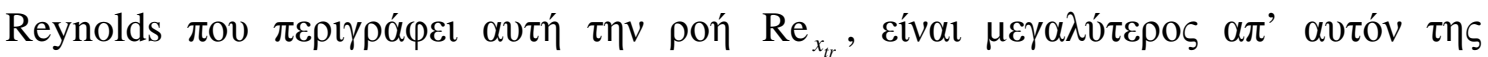

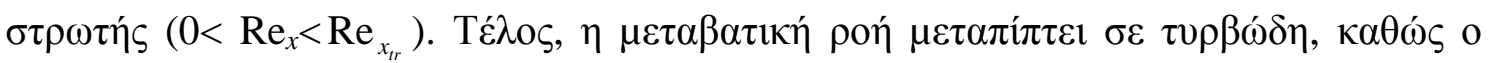

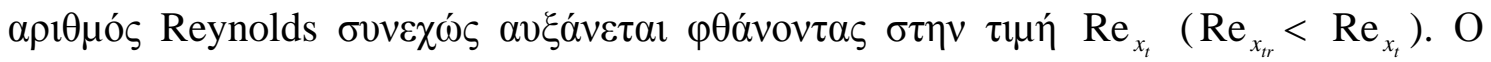

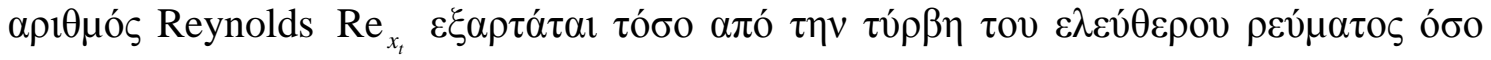

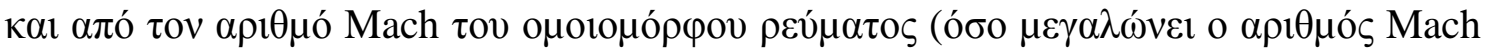

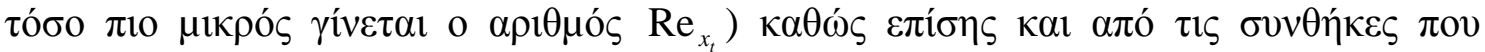

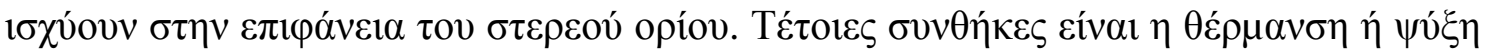

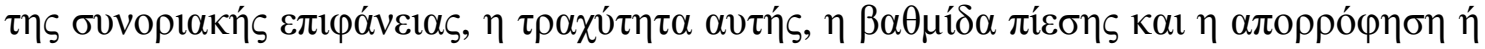

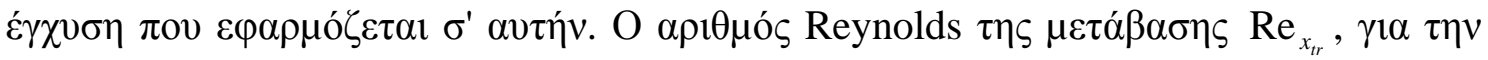

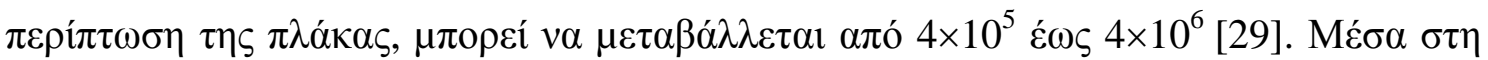

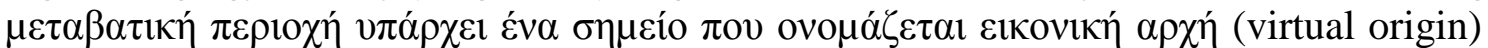

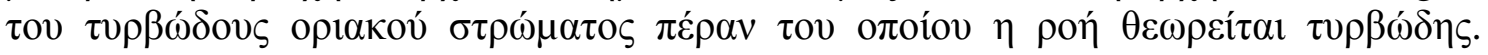

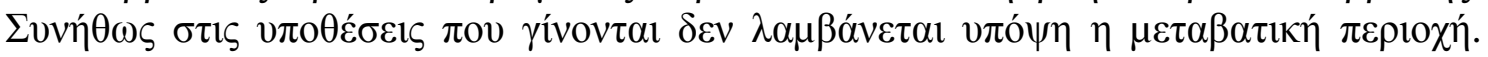

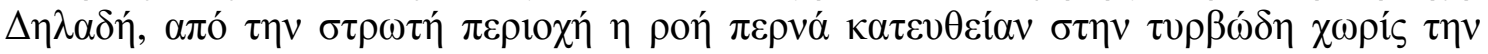

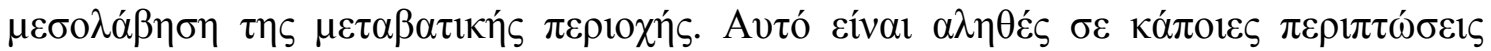

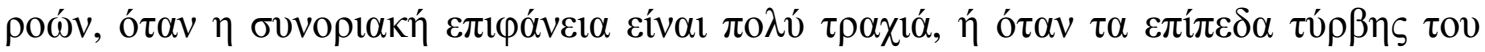

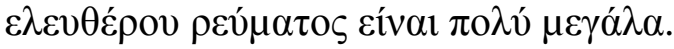

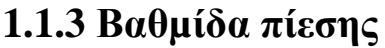

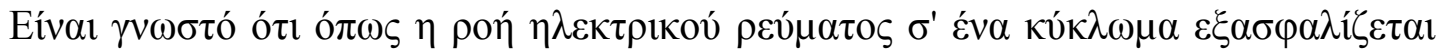

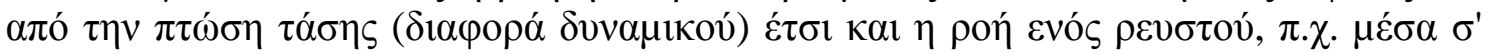

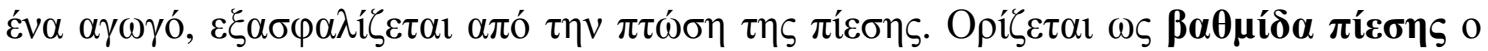

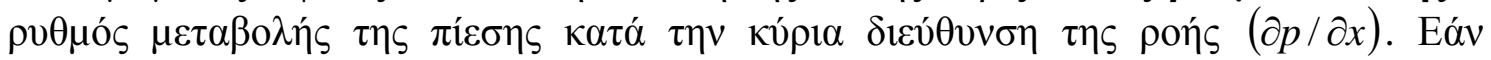

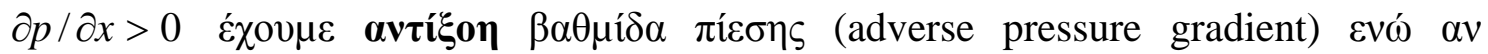

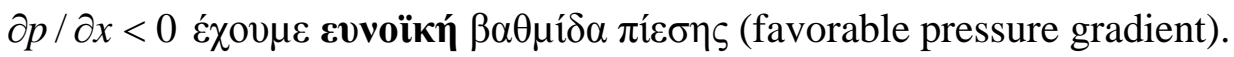

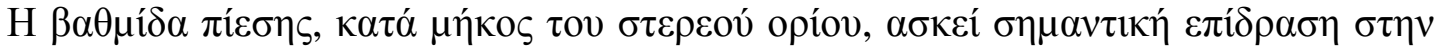

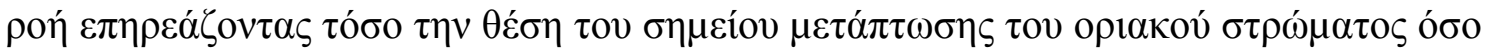

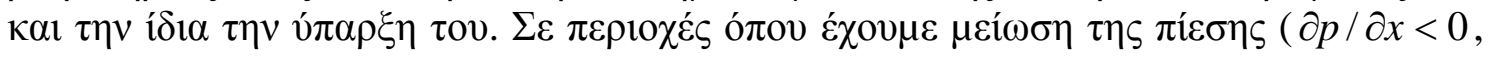

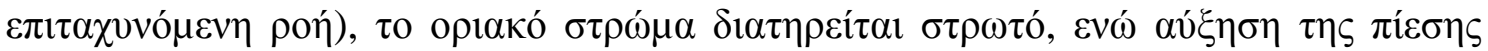

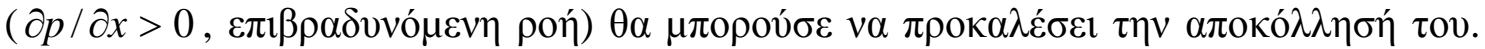

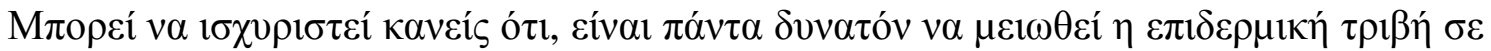

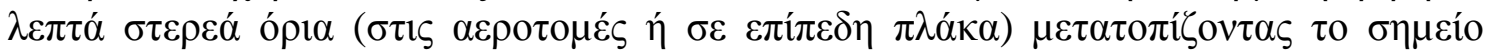

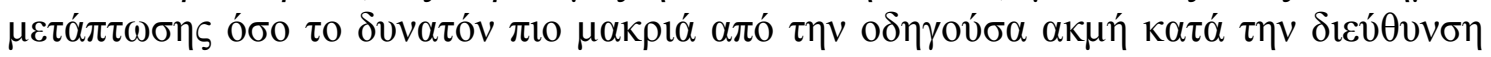




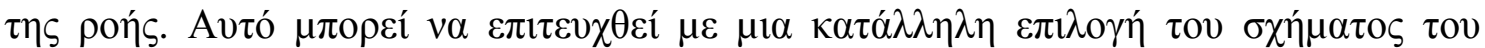

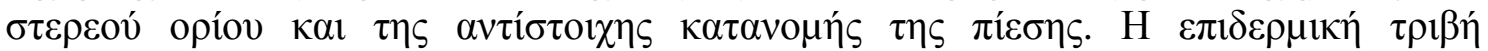

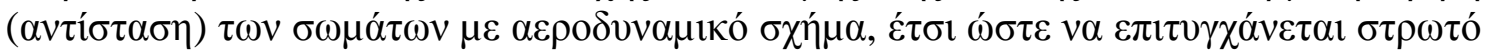

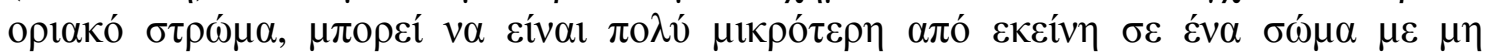

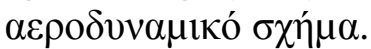

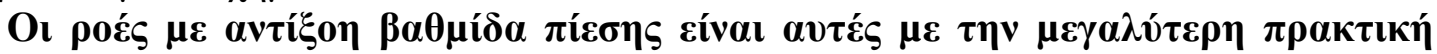

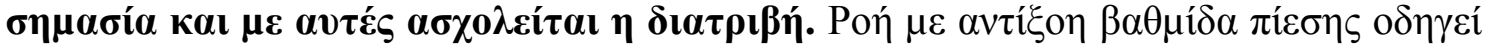

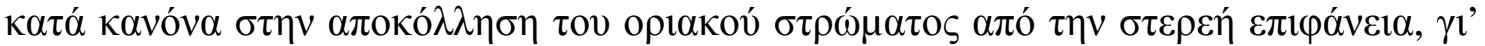

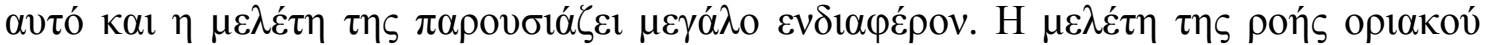

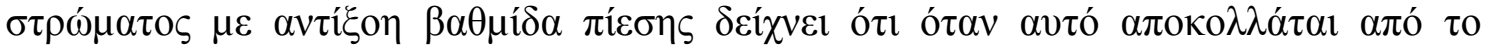

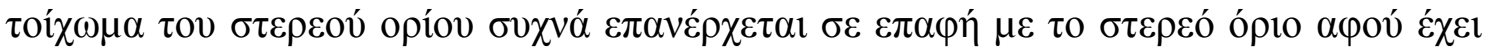

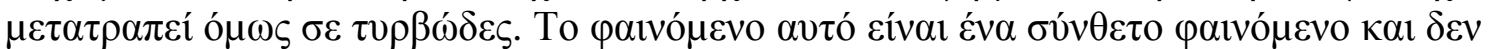

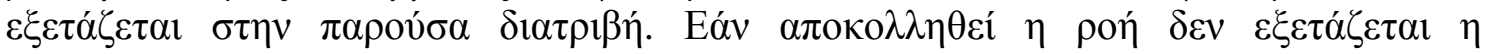

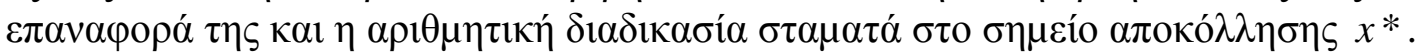

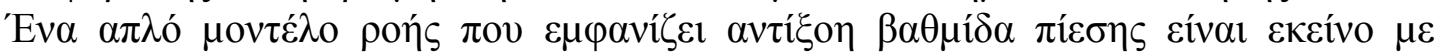

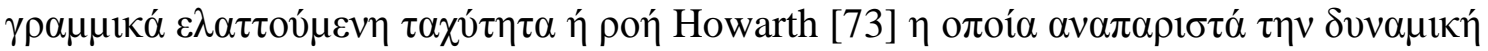

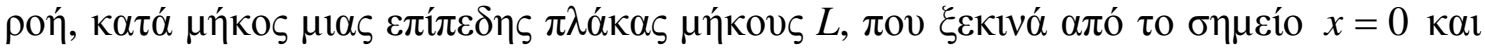

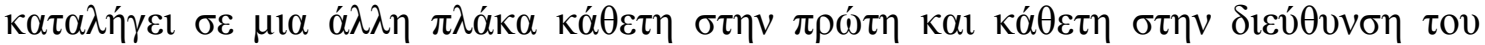

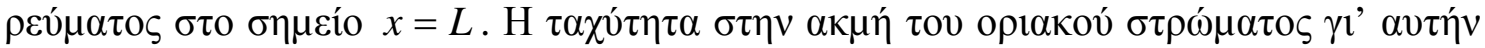

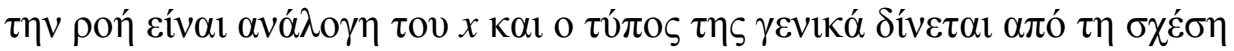

$$
u_{e}=u_{\infty}\left(1-\frac{x}{L}\right)
$$

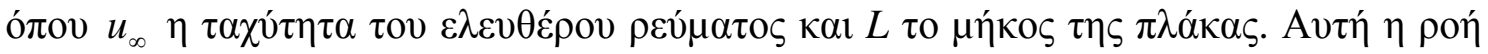

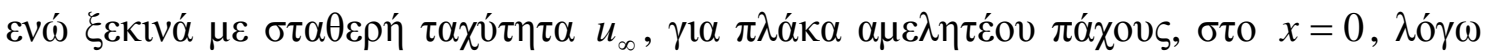

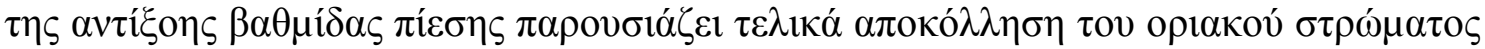

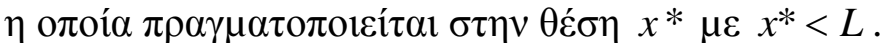

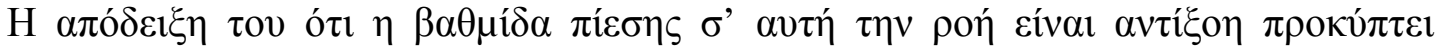

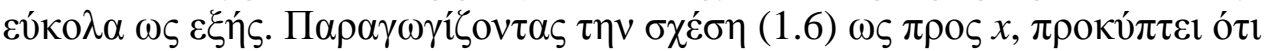

$$
\frac{d u_{e}}{d x}=-\frac{u_{\infty}}{L} .
$$

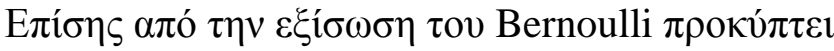

$$
\frac{d p}{d x}=-\rho_{e} u_{e} \frac{d u_{e}}{d x} .
$$

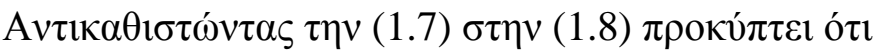

$$
\frac{d p}{d x}=\frac{\rho_{e} u_{e} u_{\infty}}{L}>0 .
$$

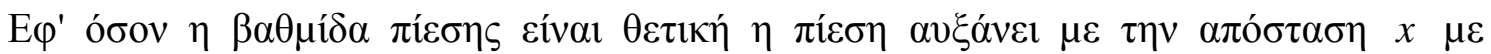

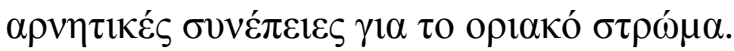

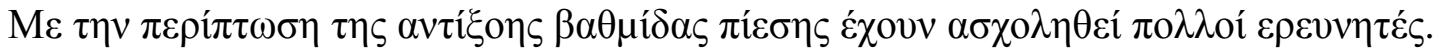

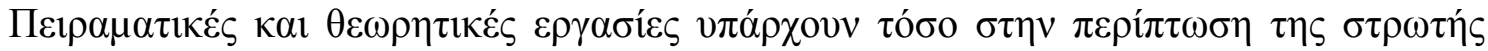




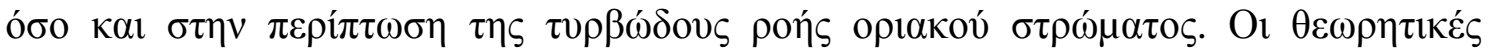

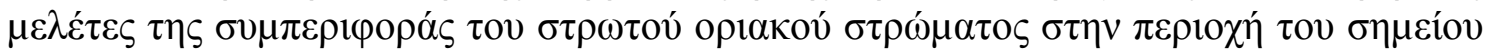

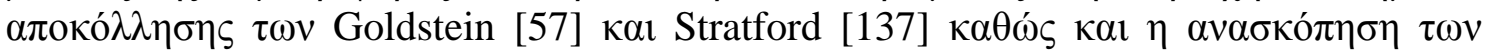

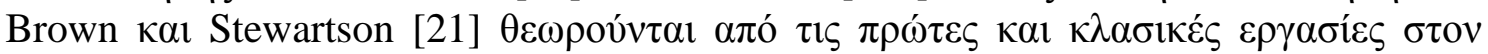

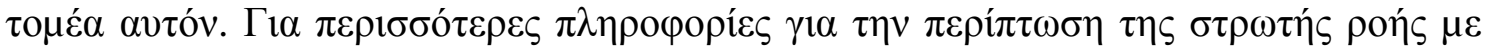

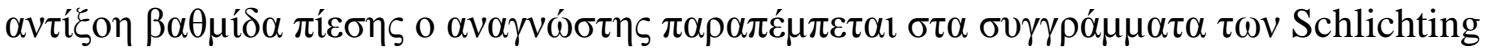

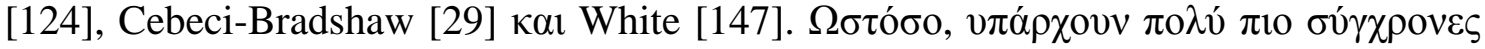

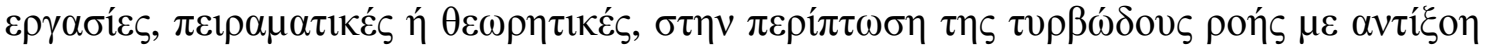

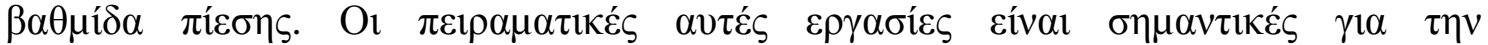

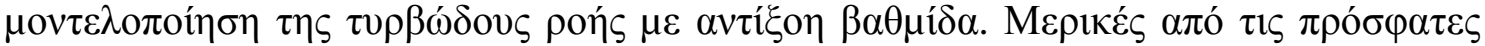

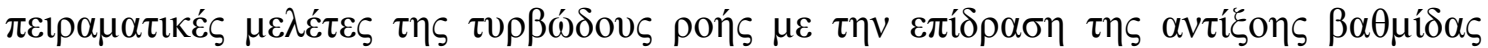

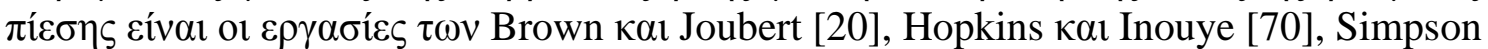

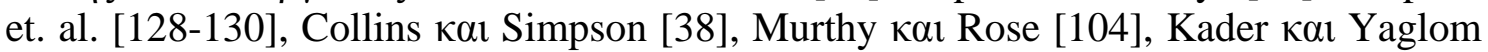

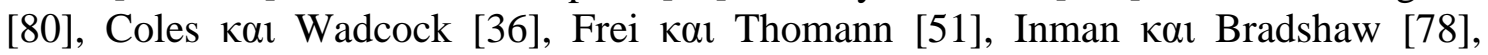

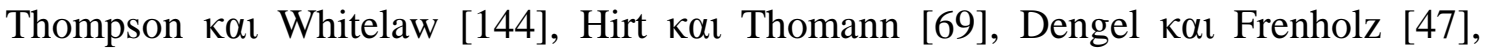

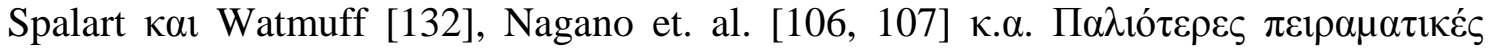

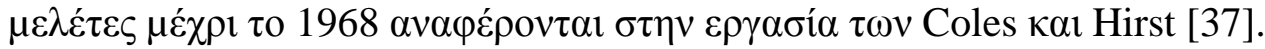

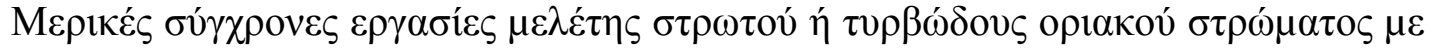

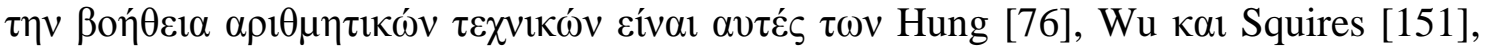

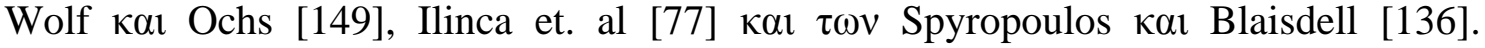

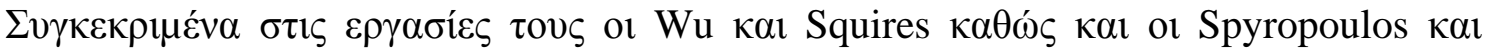

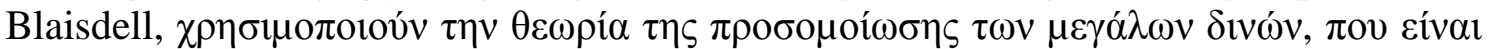

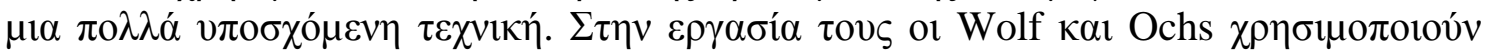

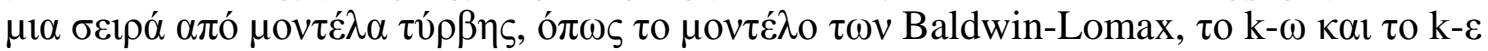

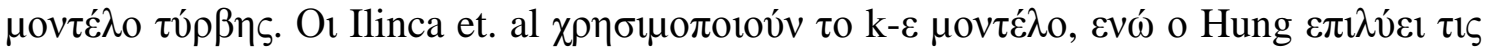

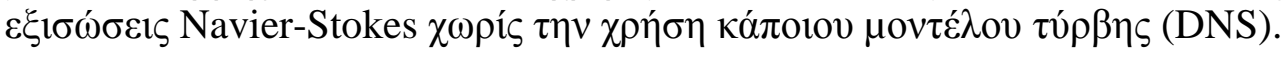

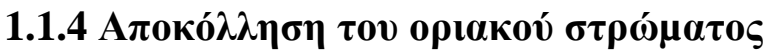

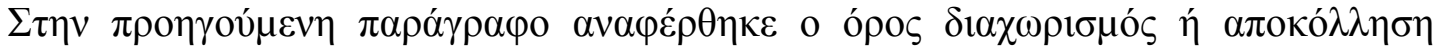

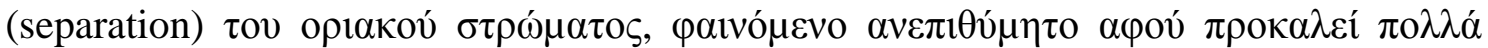

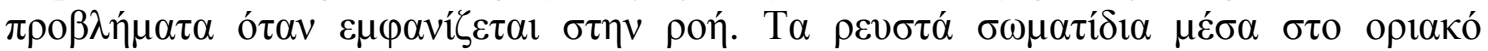

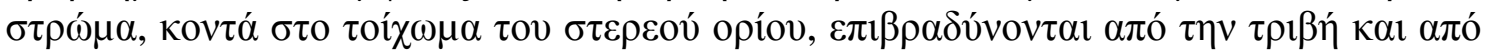

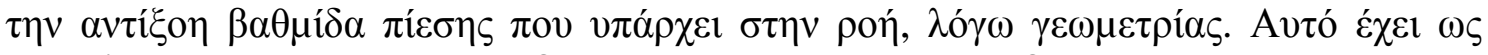

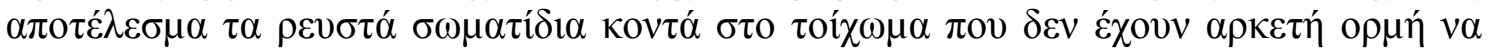

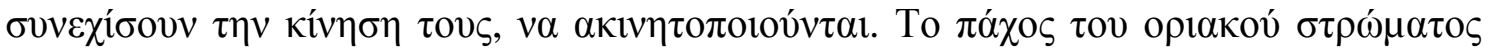

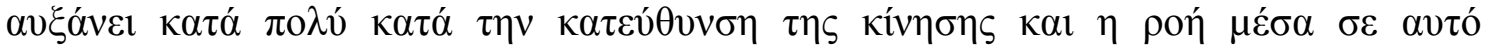

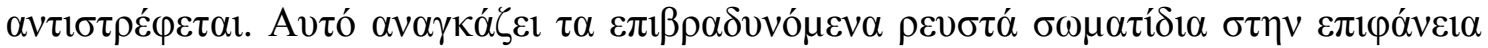

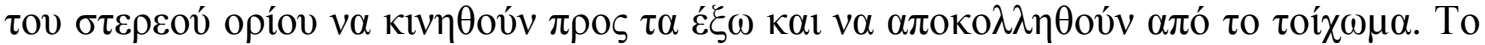

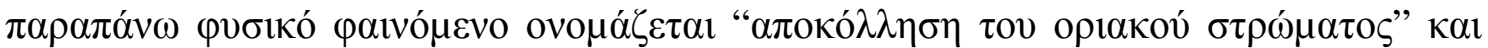

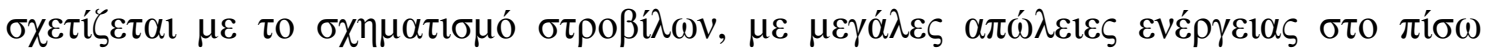

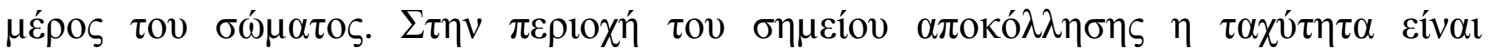

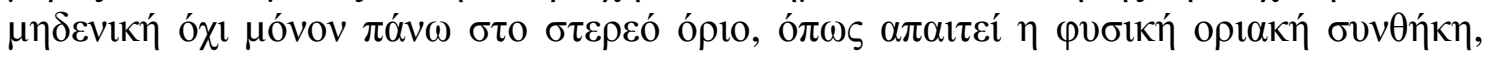

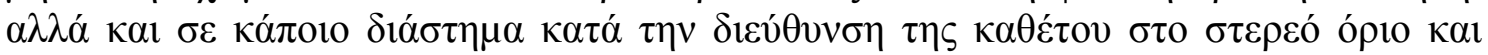

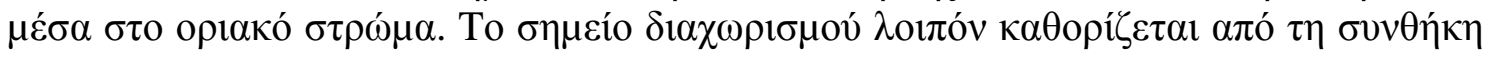

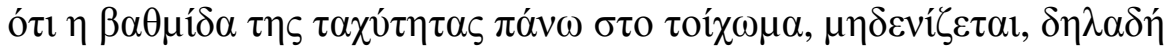




$$
\left(\frac{\partial u}{\partial y}\right)_{w}=\left(\frac{\partial u}{\partial y}\right)_{\tau o i \chi \omega \mu \alpha}=0
$$

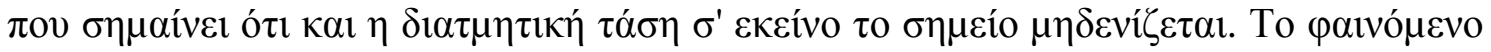

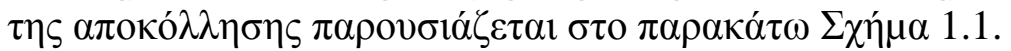

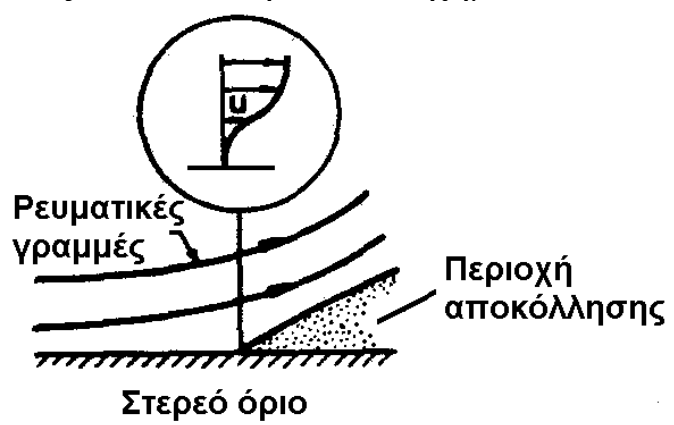

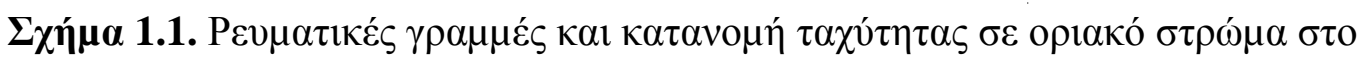

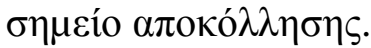

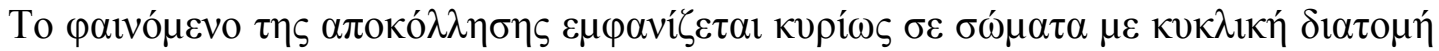

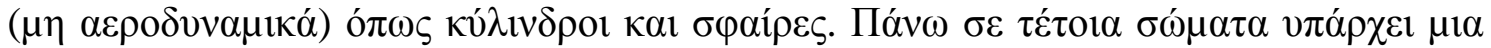

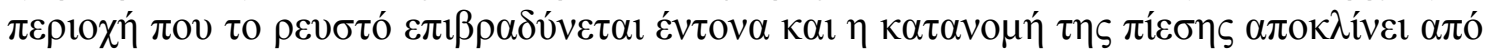

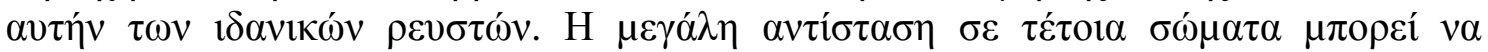

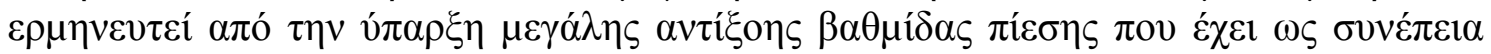

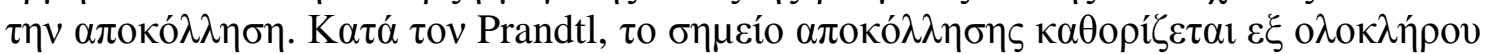

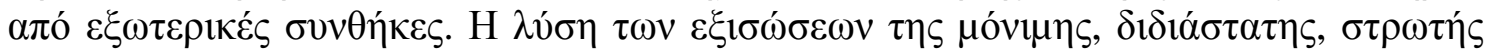

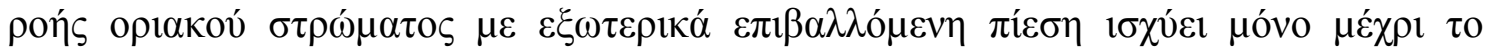

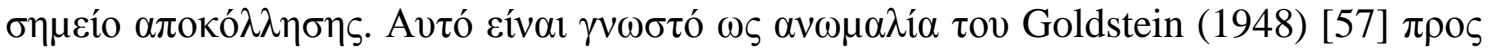

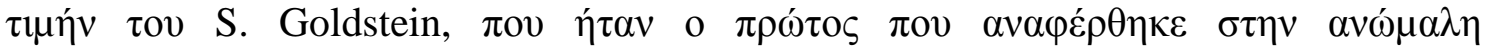

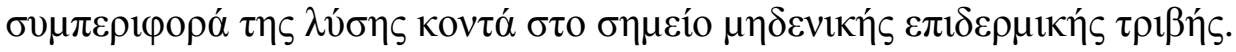

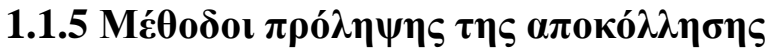

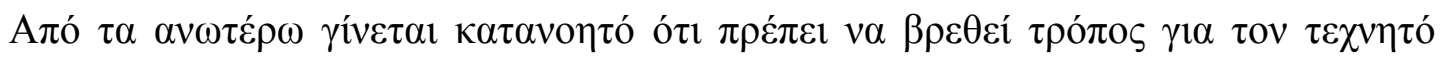

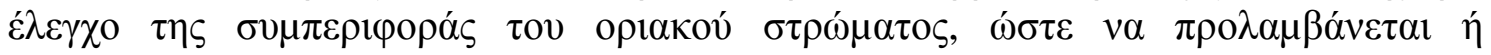

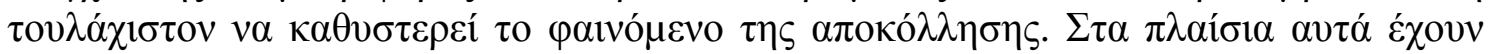

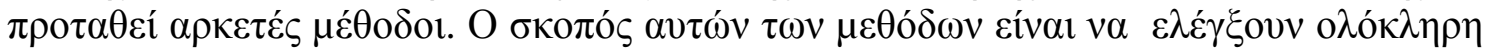

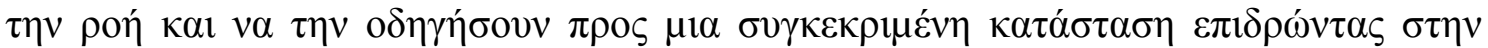

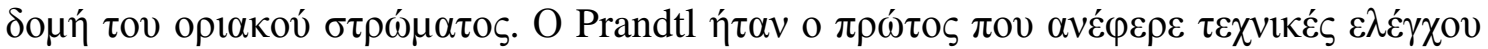

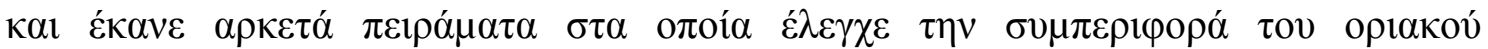

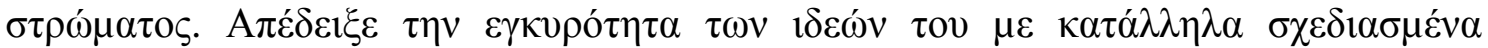

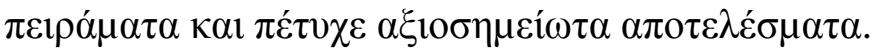

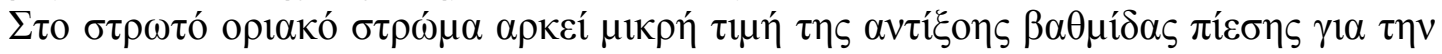

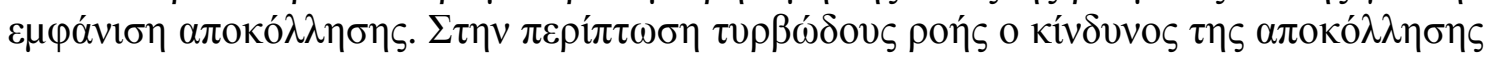

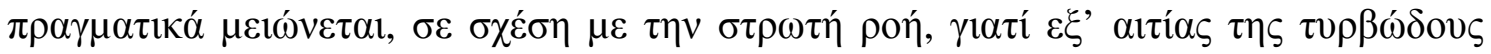

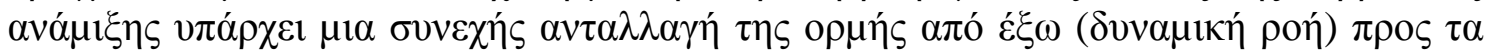
$\mu \varepsilon ́ \sigma \alpha$ (

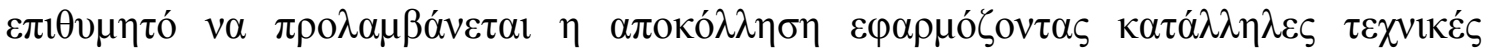

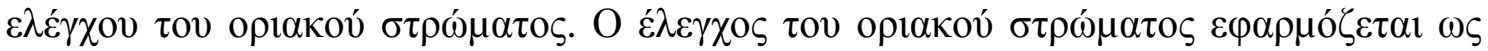

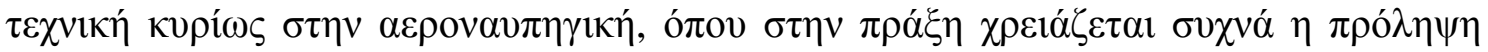




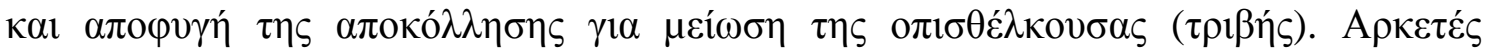

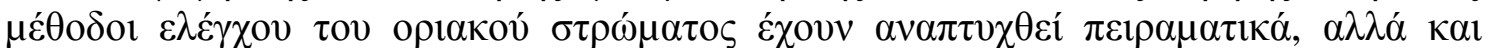

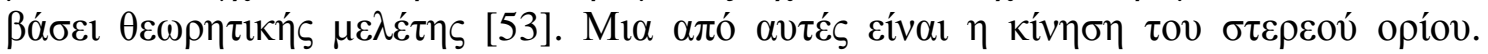

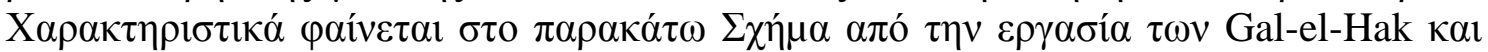

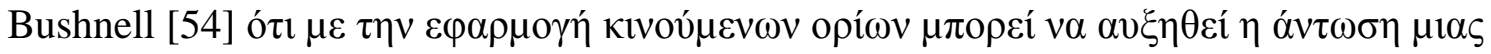

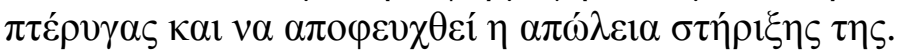

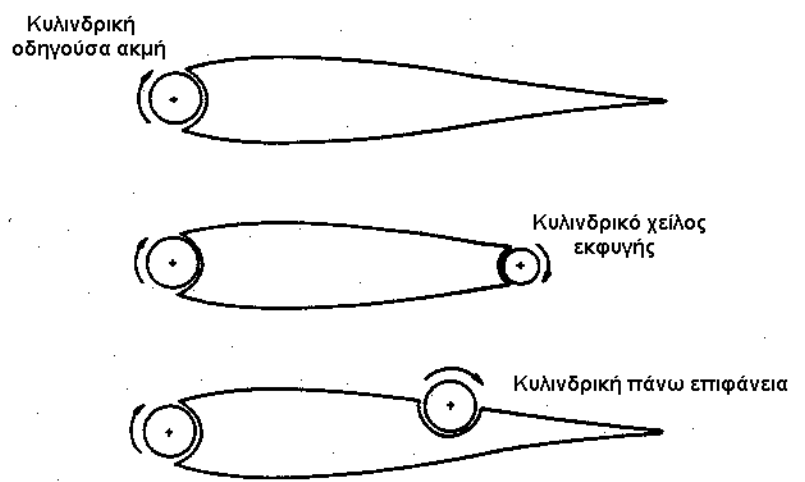

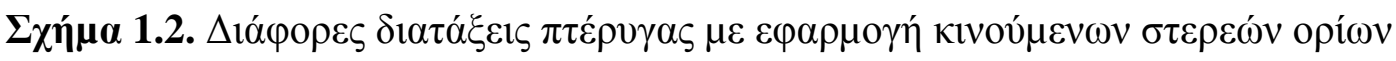

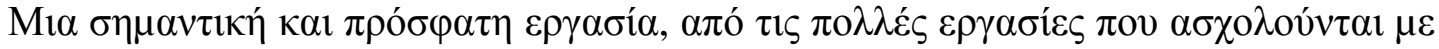

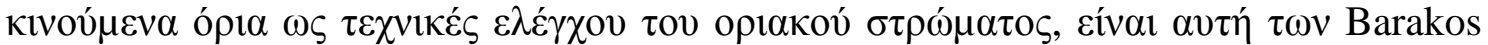

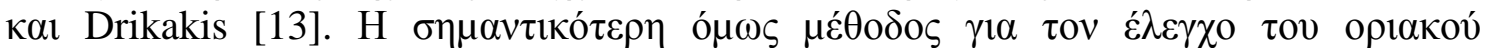

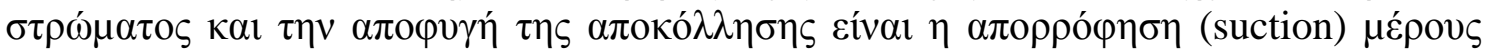

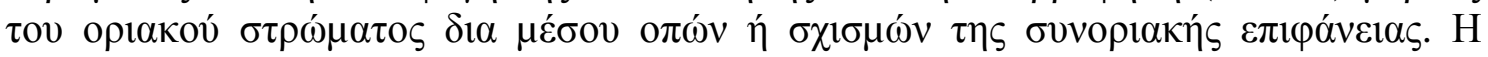

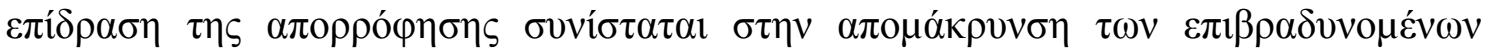

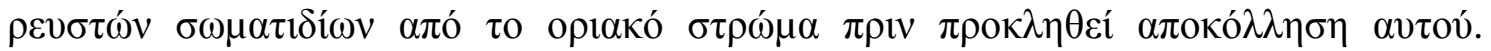

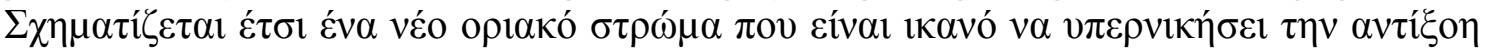

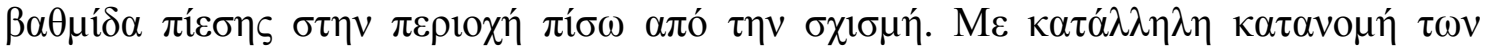

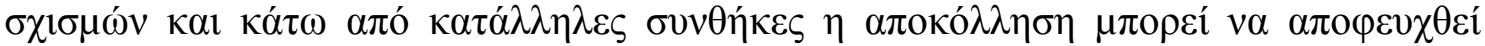

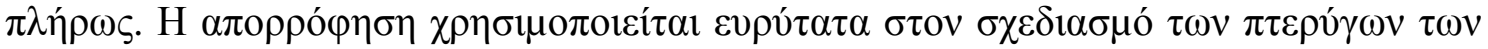

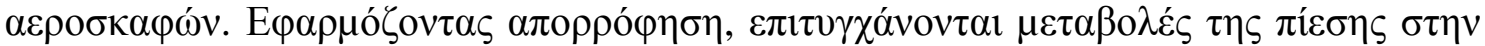

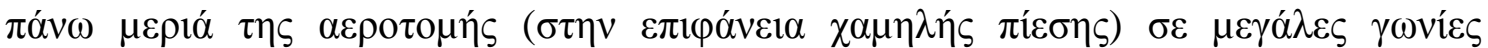

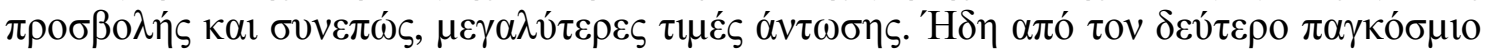

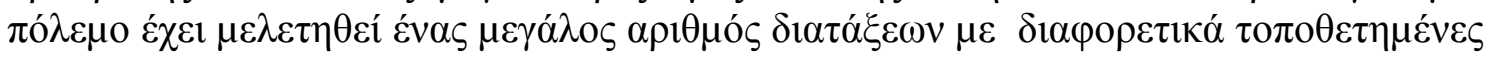

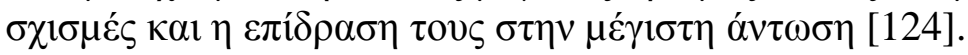

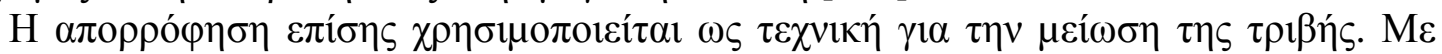

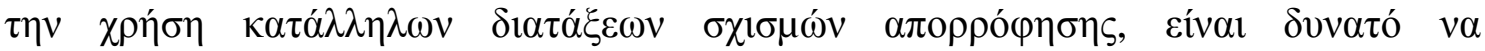

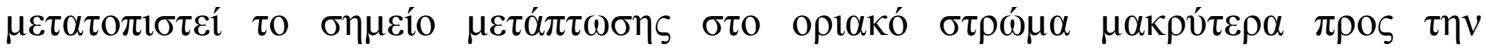

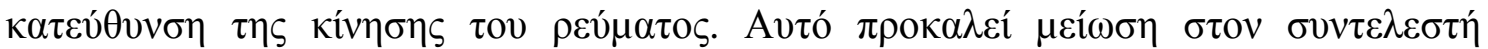

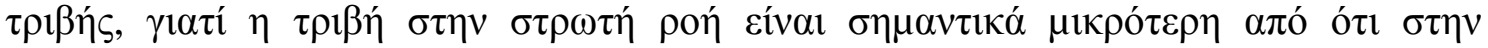
$\tau \cup \rho \beta \omega ́ \delta \eta$.

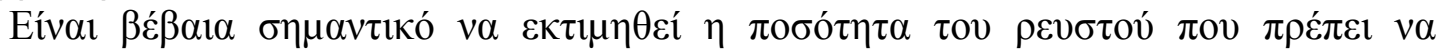

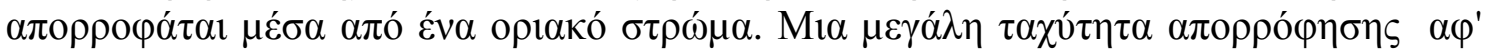

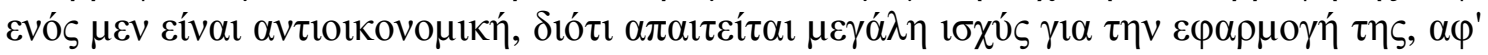

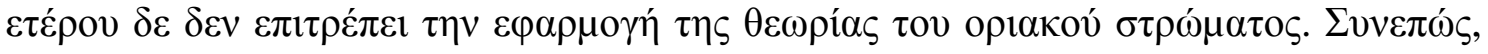

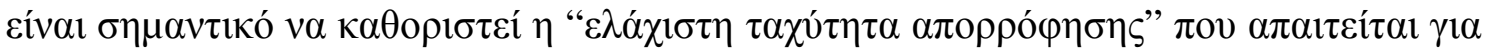

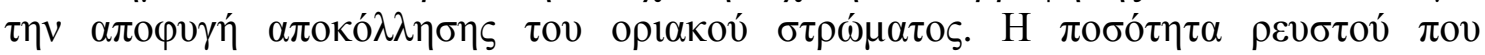

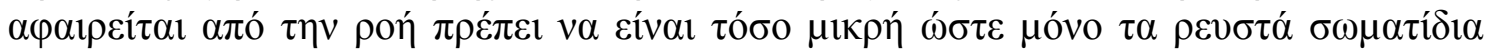

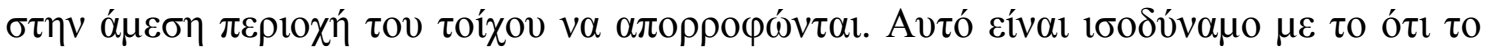




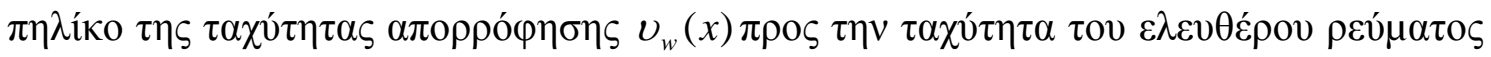

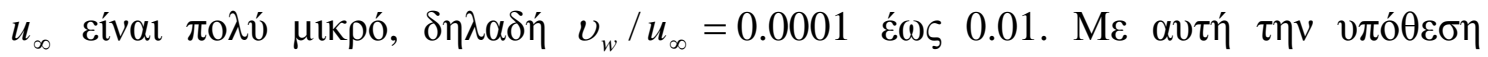

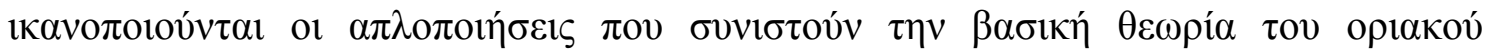

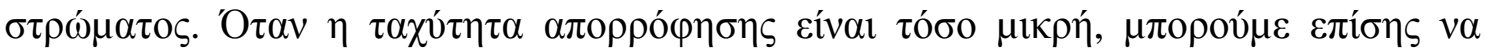

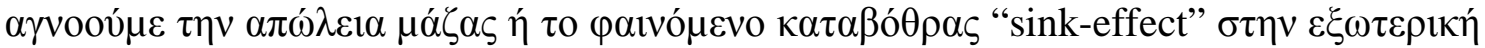

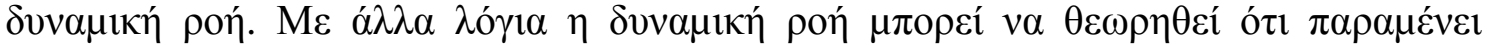

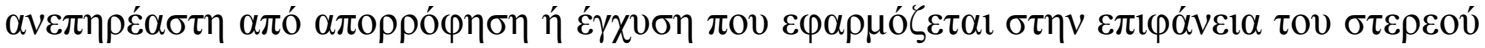

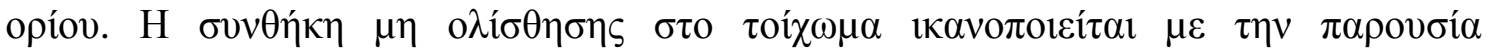

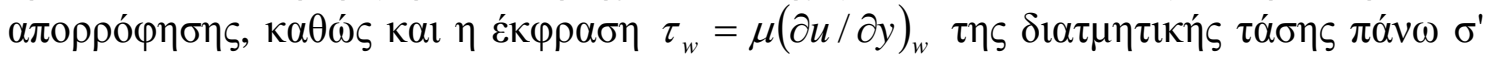

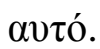

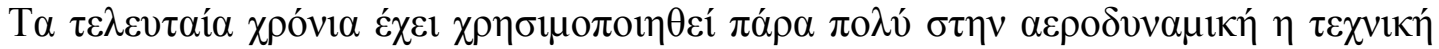

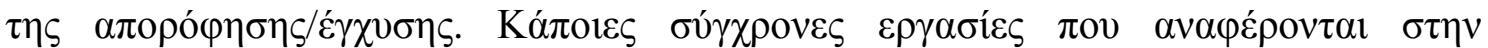

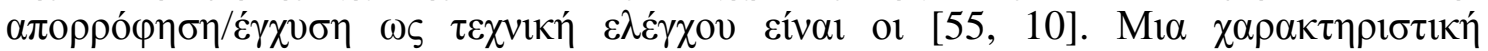

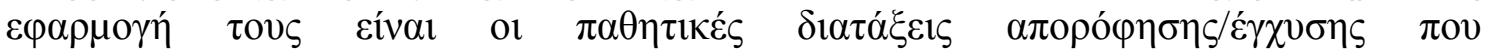

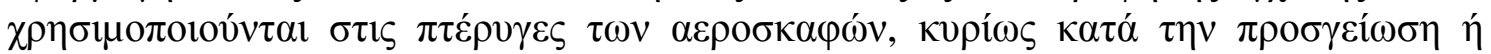

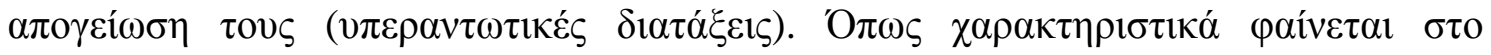

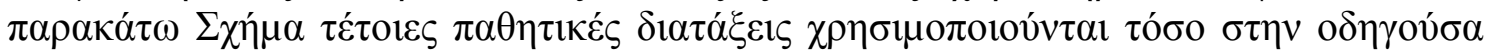

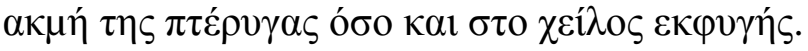
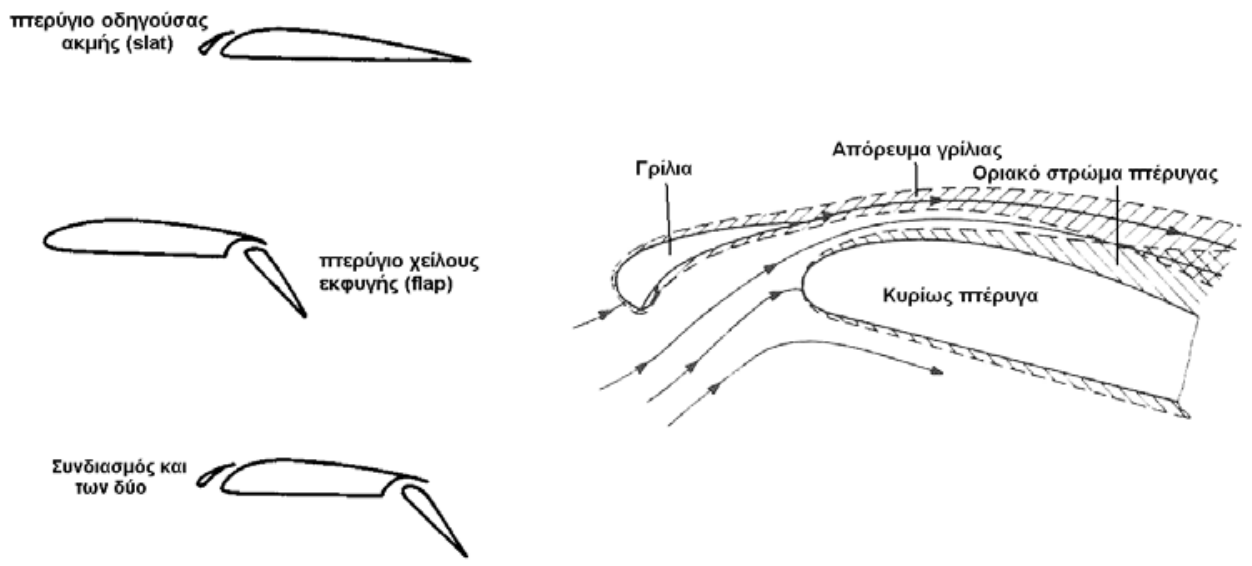

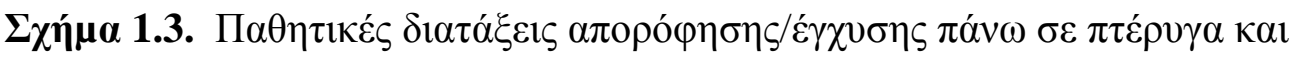

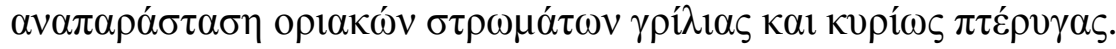

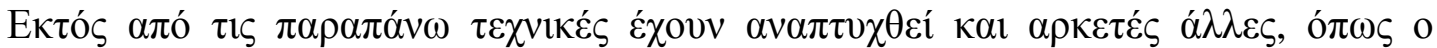

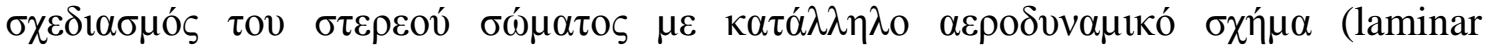

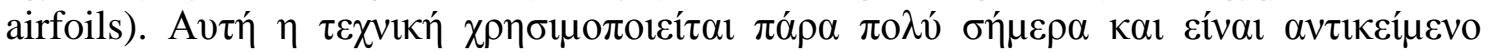

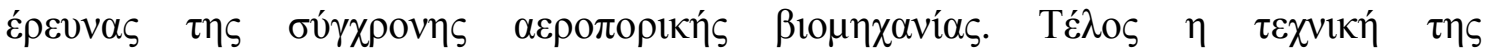

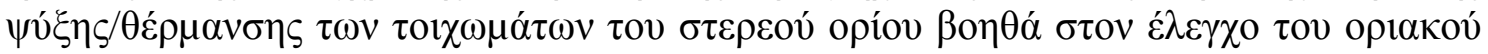

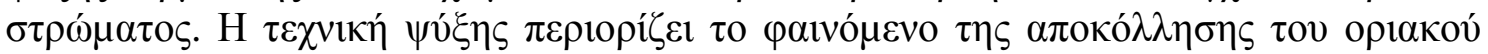

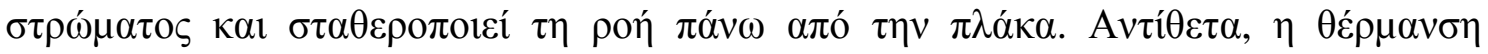
$\alpha \pi$

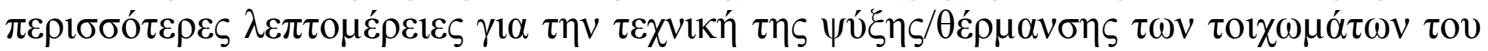

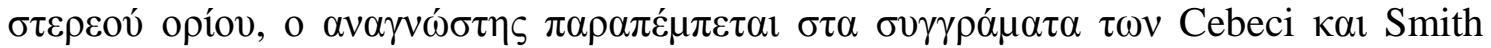

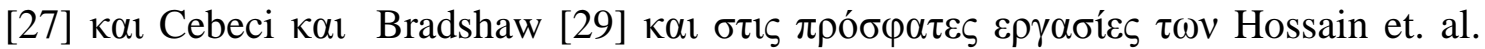
[72] кaı Acharya et. al. [1]. 


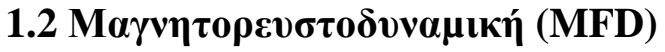

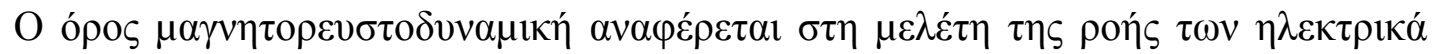

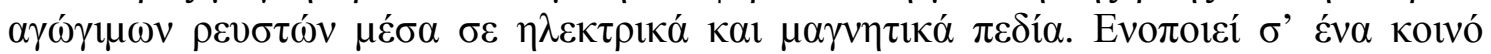

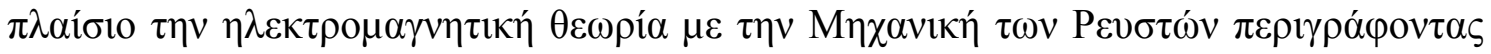

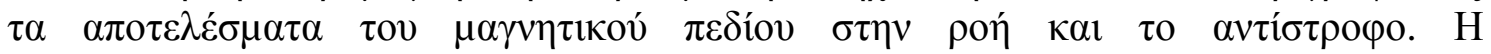

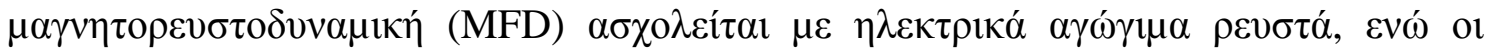

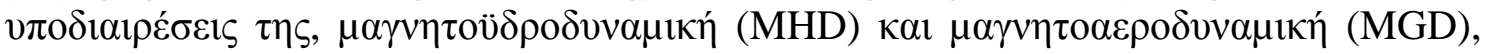

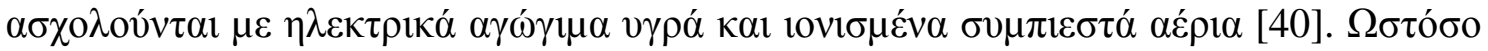

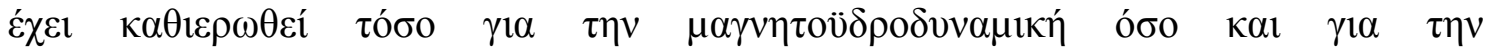

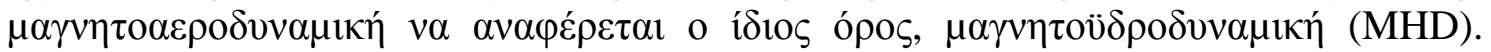

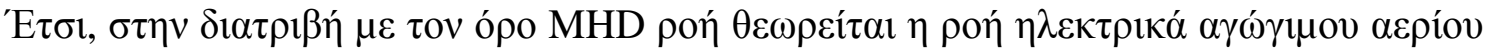

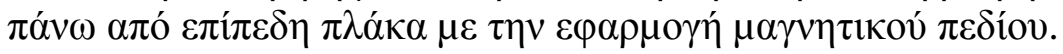

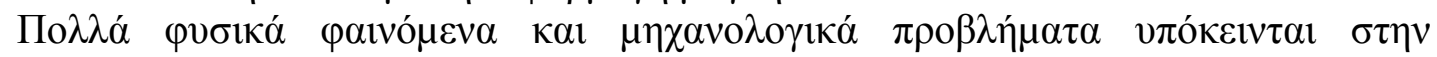

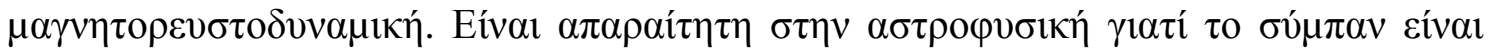

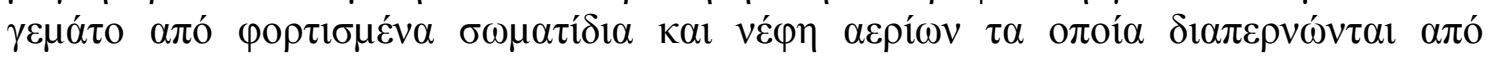

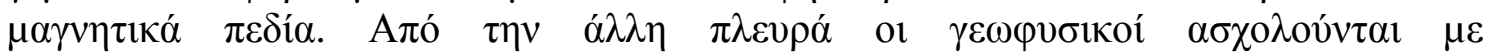

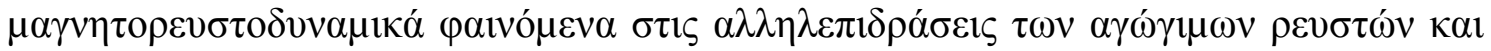

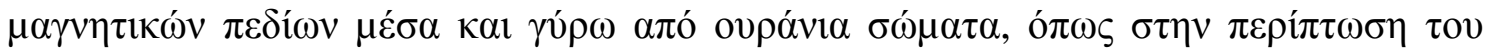

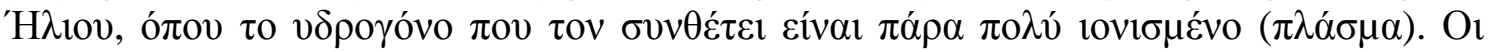

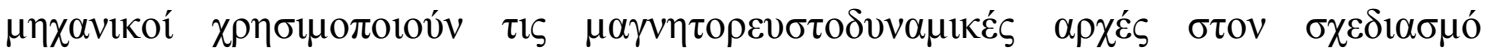

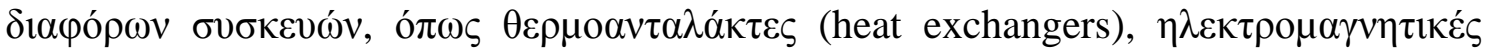

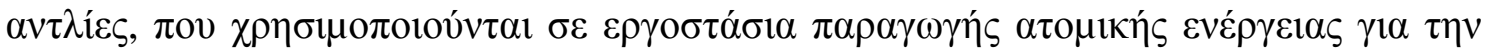

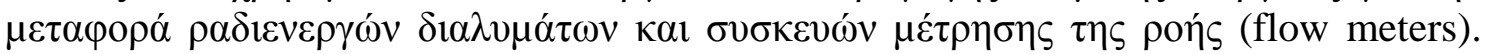

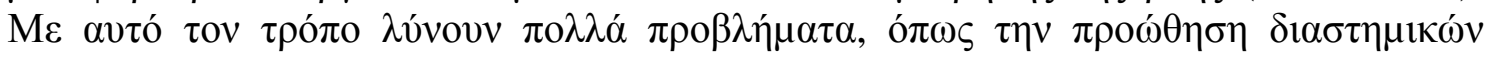

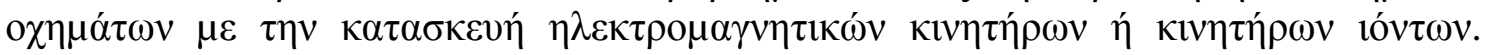

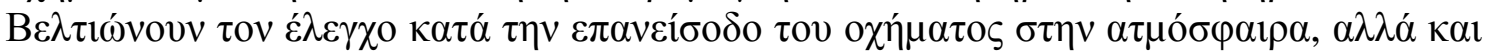

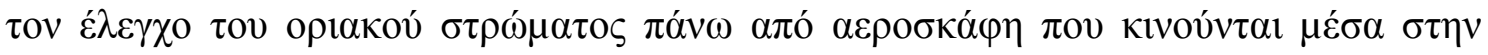

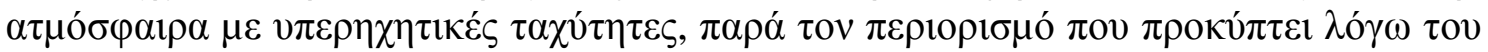

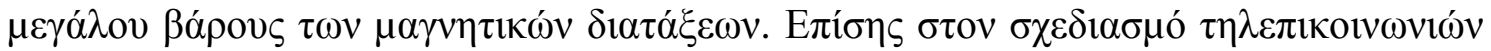

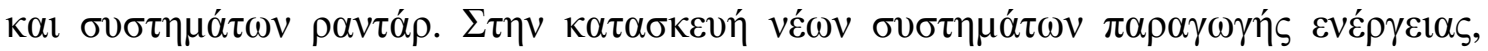

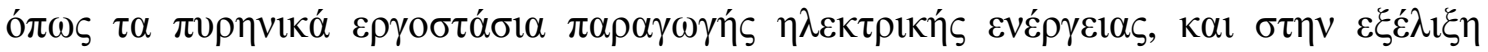

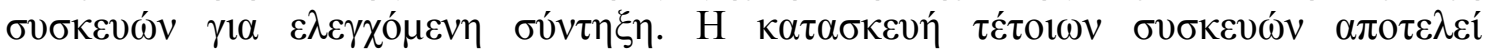

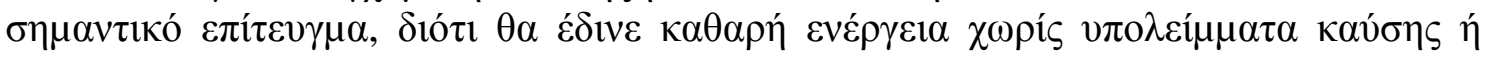

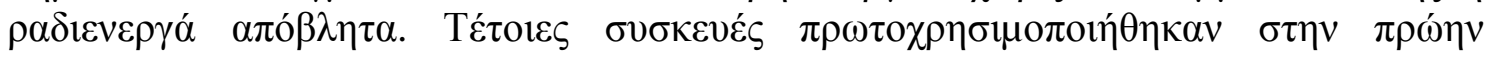

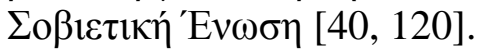

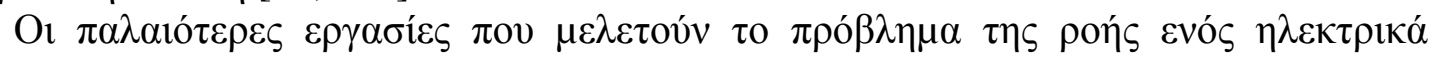

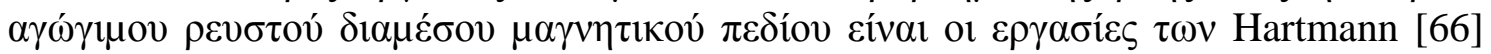

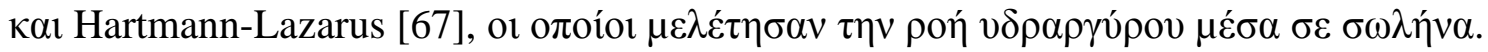

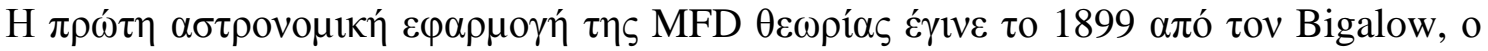

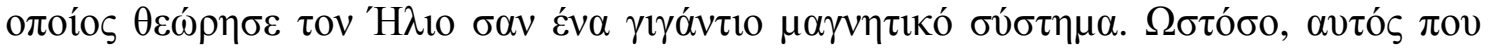

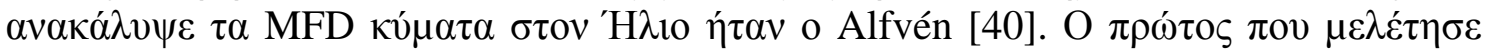

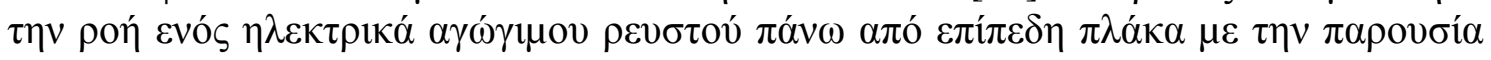

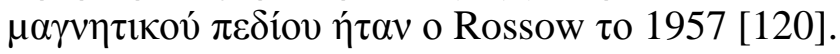

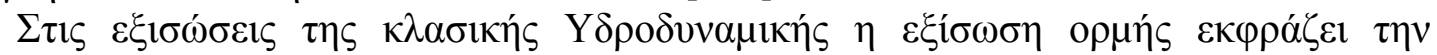

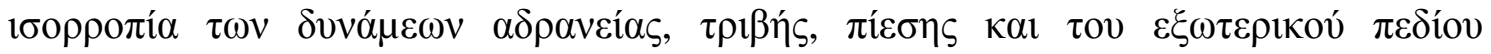

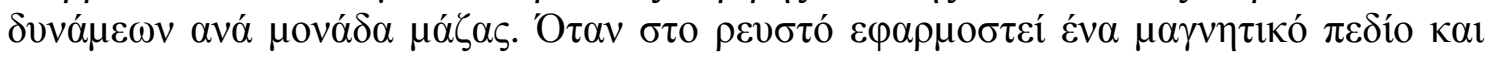

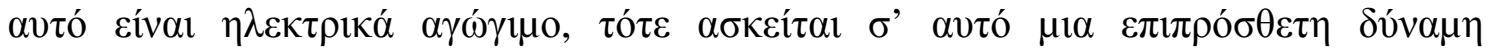

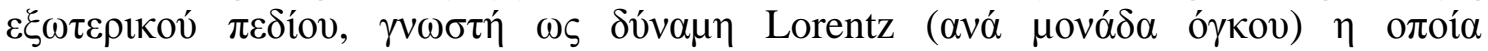
$\varepsilon \kappa \varphi \rho \alpha ́ \zeta \varepsilon \tau \alpha \iota \omega \varsigma$ 


$$
\vec{F}_{L}=\rho_{e} \vec{E}+\vec{J} \times \vec{B}
$$

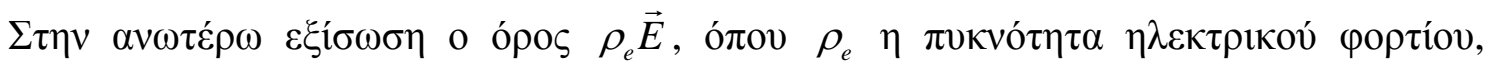

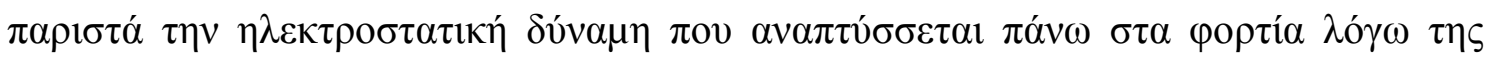

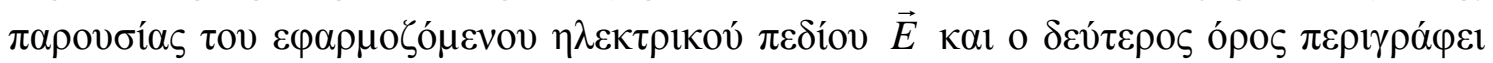

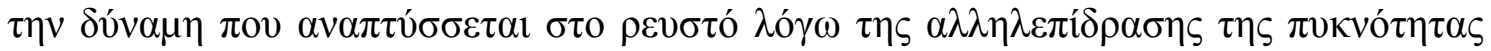

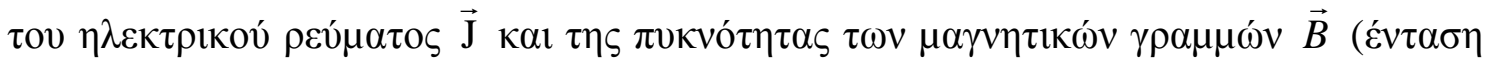

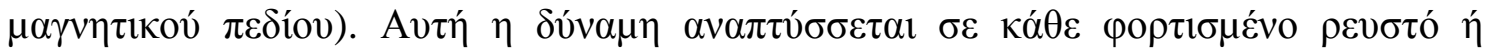

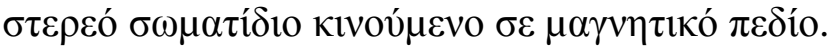

A

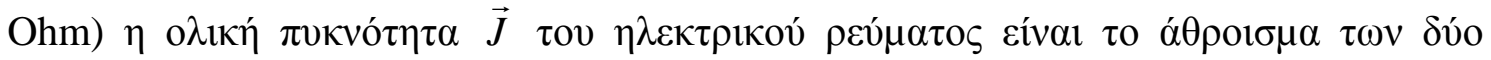
$\rho \varepsilon v \mu \alpha ́ \tau \omega v$

$$
\vec{J}=\vec{J}_{v}+\vec{J}_{c}
$$

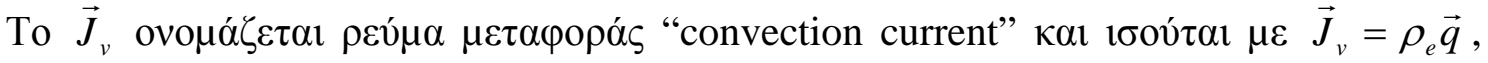

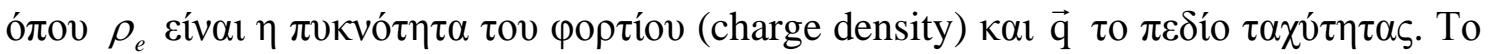

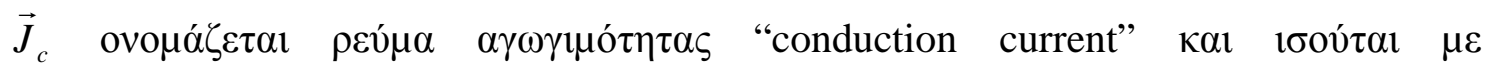

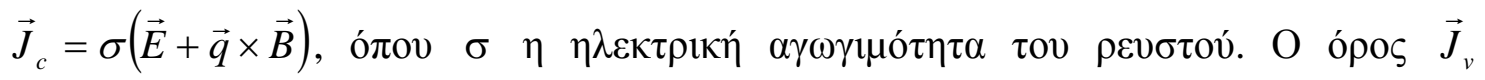

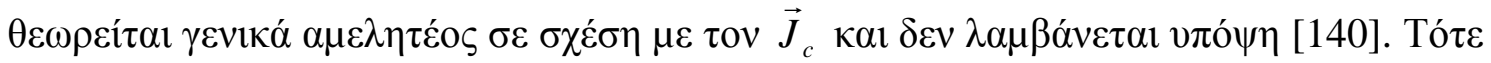

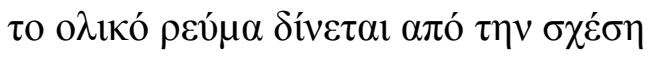

$$
\vec{J}=\vec{J}_{c}=\sigma(\vec{E}+\vec{q} \times \vec{B})
$$

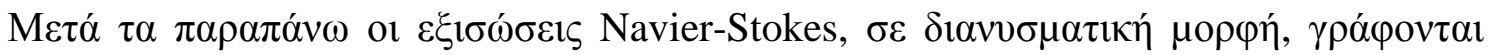
$\tau \omega ́ \rho \alpha$

$$
\frac{D \vec{q}}{D t}=-\frac{1}{\rho} \vec{\nabla} p+\frac{1}{\rho} \nabla^{2}(\mu \vec{q})+\frac{\vec{E}}{\rho}+\frac{1}{\rho} \vec{J} \times \vec{B},
$$

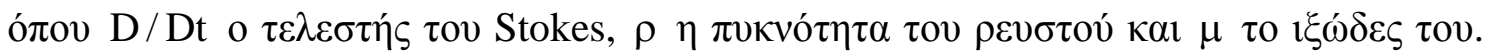

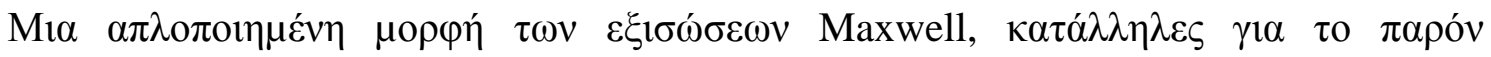

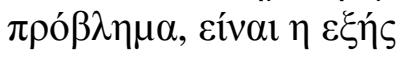

$$
\operatorname{div} \vec{B}=0, \quad \operatorname{div} \vec{J}=0, \quad \vec{B}=\mu_{0} \vec{H}, \quad \operatorname{curl} \vec{H}=4 \pi \vec{J},
$$

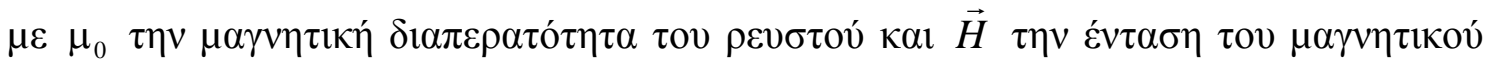
$\pi \varepsilon \delta i ́ o v$.

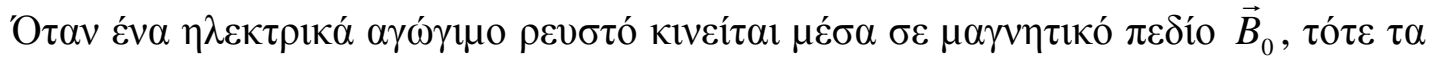

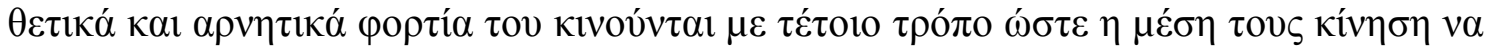

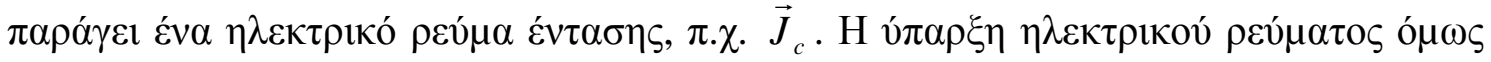

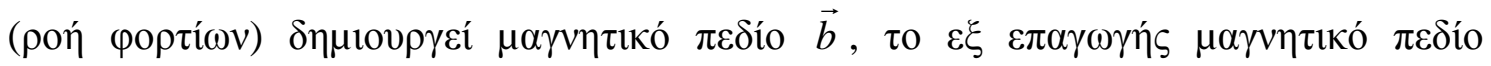




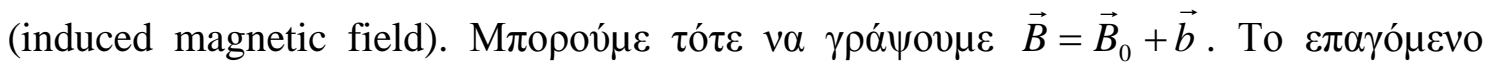

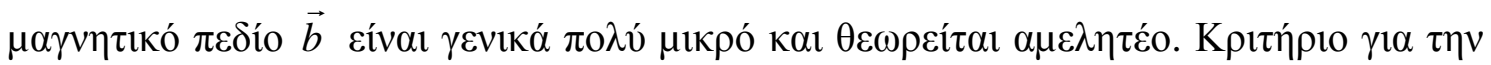

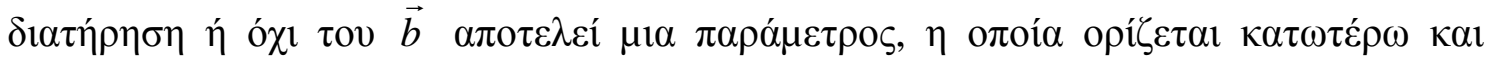

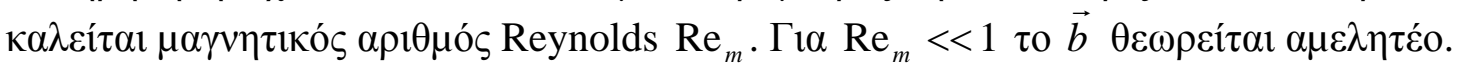

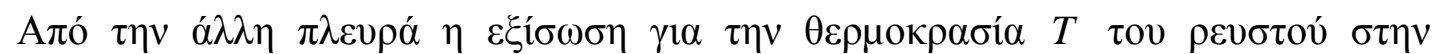

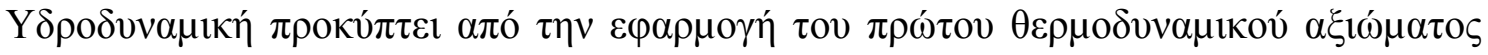

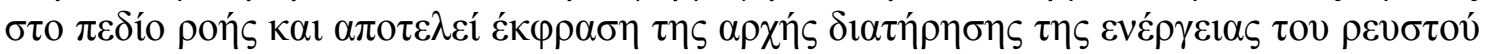

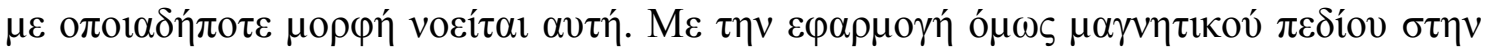

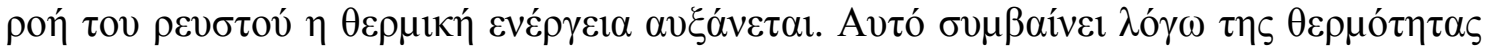

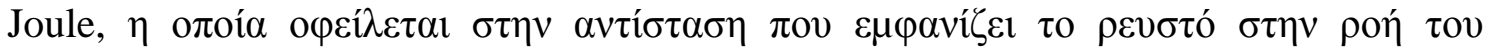

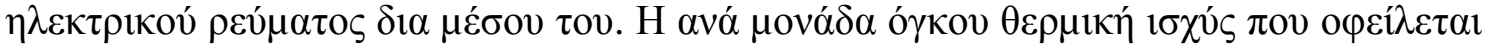

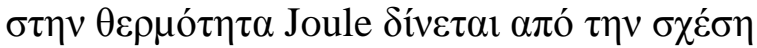

$$
\frac{\vec{J}_{c} \cdot \vec{J}_{c}}{\sigma}=\sigma(\vec{E}+\vec{q} \times \vec{B})^{2}
$$

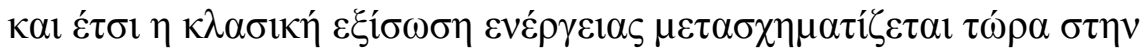

$$
\rho c_{p} \frac{D T}{D t}=\nabla^{2}(k T)+\frac{D p}{D t}+\frac{\vec{J}_{c} \cdot \vec{J}_{c}}{\sigma}+\mu \Phi,
$$

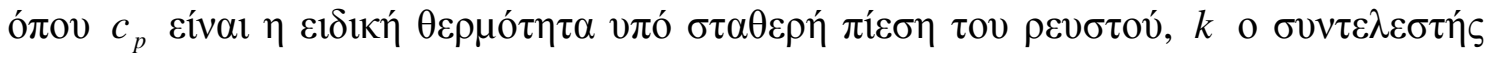

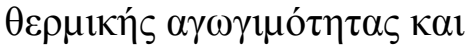

$$
\begin{gathered}
\Phi=2\left[\left(\frac{\partial u}{\partial x}\right)^{2}+\left(\frac{\partial v}{\partial y}\right)^{2}+\left(\frac{\partial w}{\partial z}\right)^{2}\right]+\left(\frac{\partial v}{\partial x}+\frac{\partial u}{\partial y}\right)^{2}+ \\
\left.+\frac{\partial w}{\partial y}+\frac{\partial v}{\partial z}\right)^{2}+\left(\frac{\partial u}{\partial z}+\frac{\partial w}{\partial x}\right)^{2} \\
-\frac{2}{3}\left(\frac{\partial u}{\partial x}+\frac{\partial v}{\partial y}+\frac{\partial w}{\partial z}\right)^{2}
\end{gathered}
$$

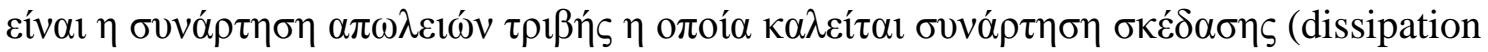

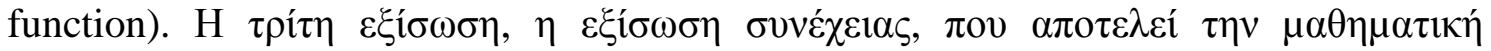

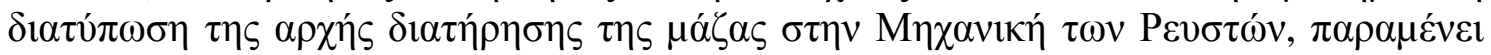

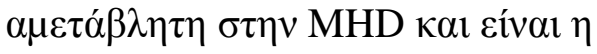

$$
\frac{\partial \rho}{\partial t}+\vec{\nabla} \cdot(\rho \vec{q})=0
$$

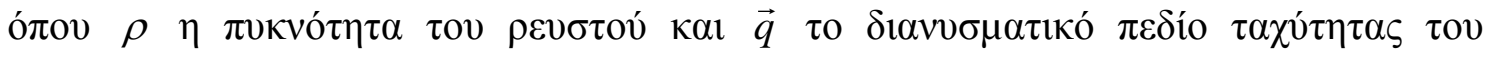

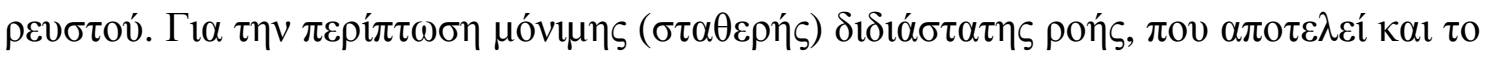

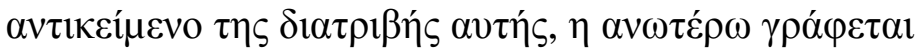

$$
\frac{\partial}{\partial x}(\rho u)+\frac{\partial}{\partial y}(\rho v)=0
$$




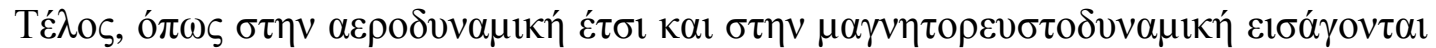

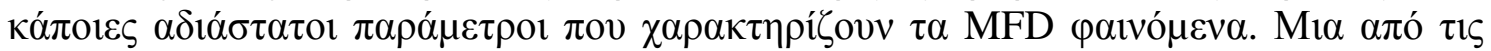

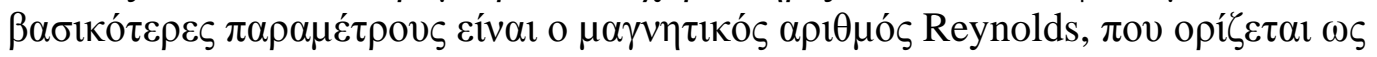

$$
\operatorname{Re}_{m}=\frac{u_{\infty} L}{\frac{1}{\mu_{0} \sigma}},
$$

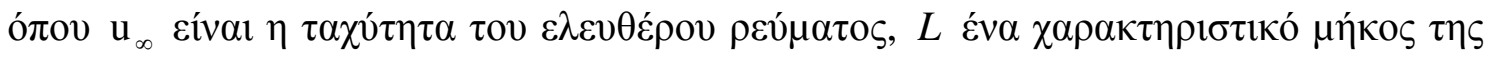

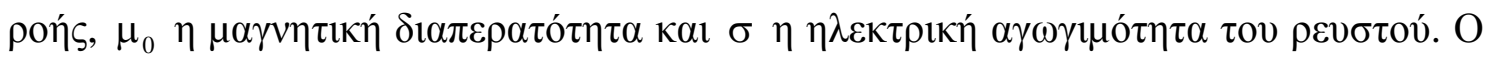

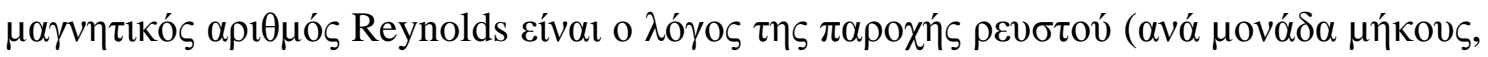

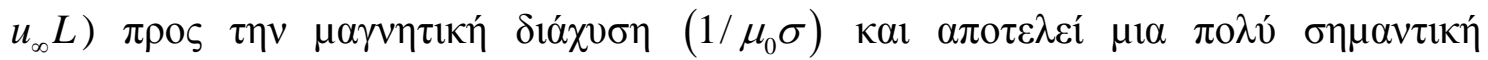

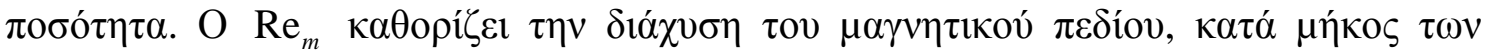

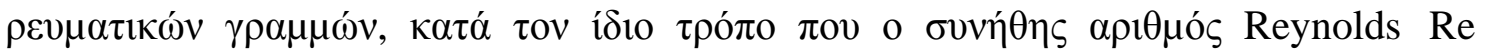

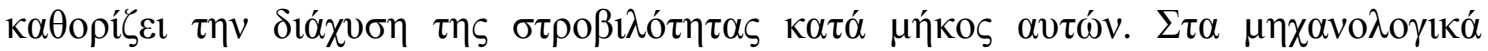

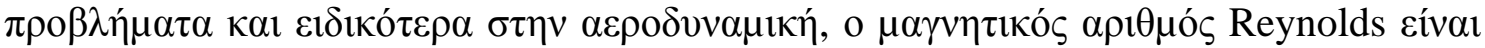

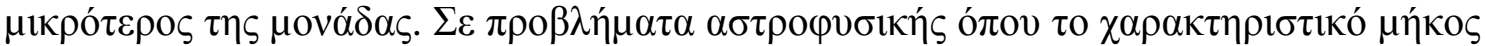

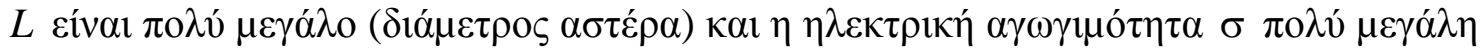

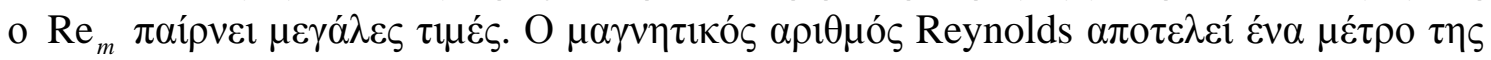

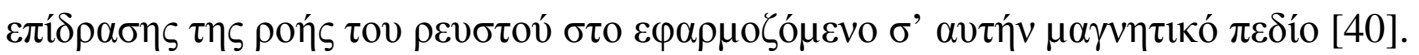

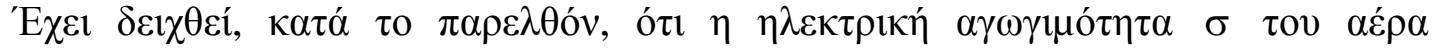

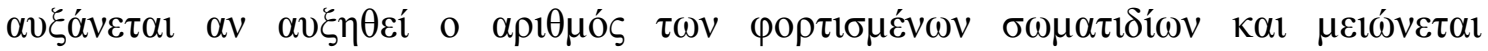

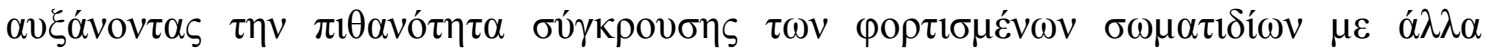

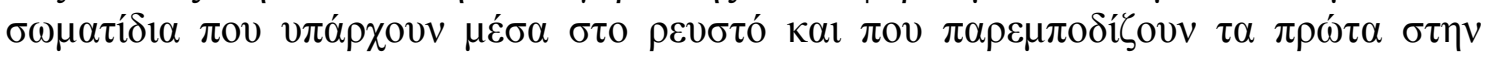

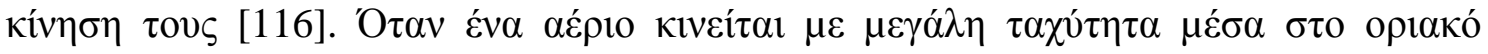

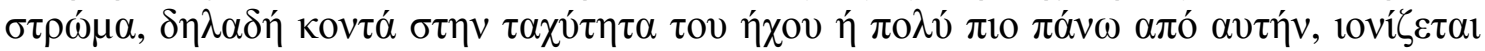

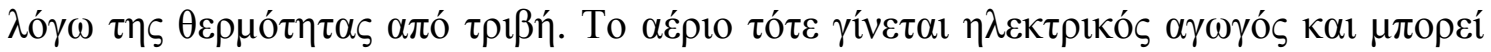

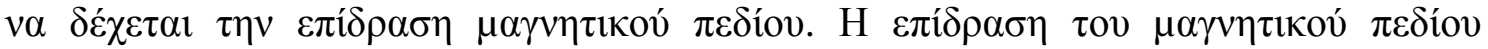

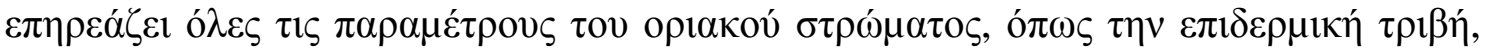

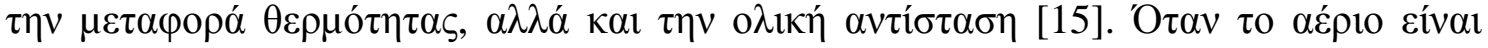

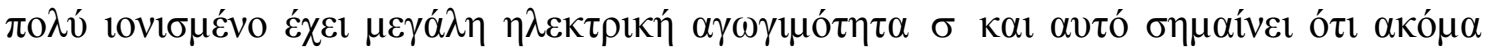

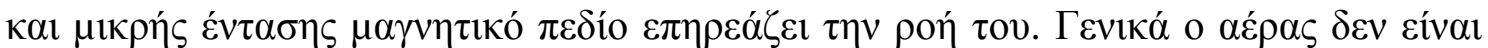

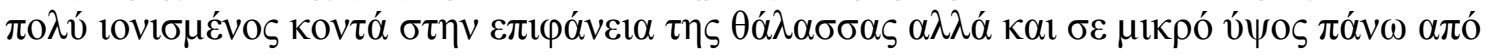

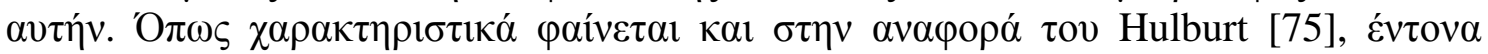

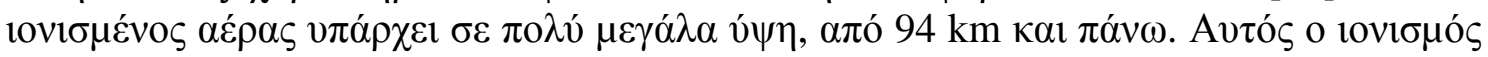

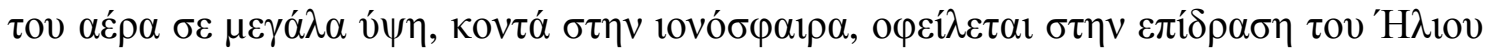

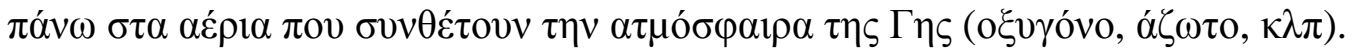

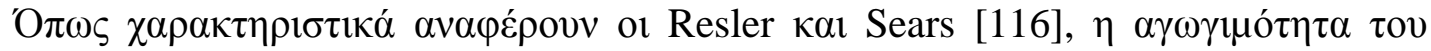

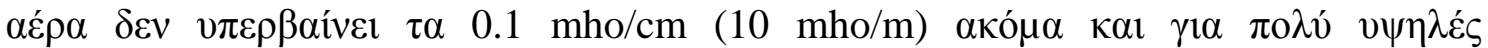

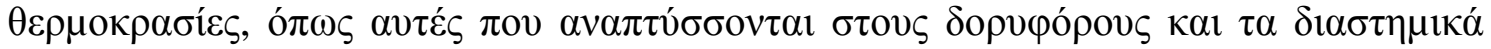

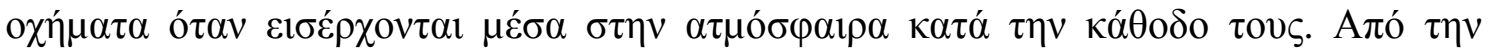

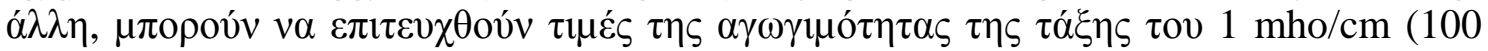

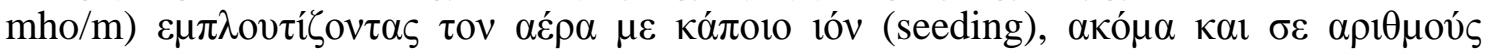

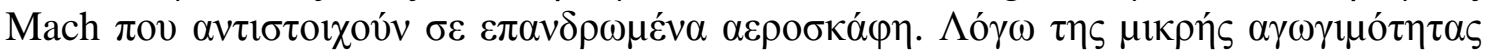

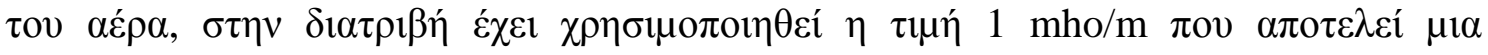

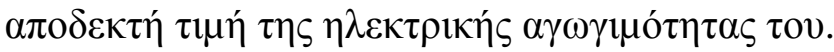

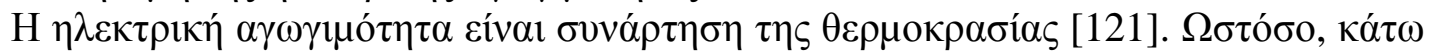

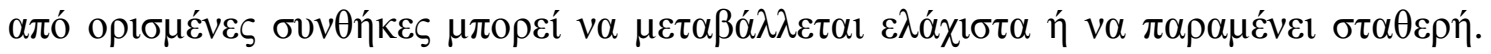




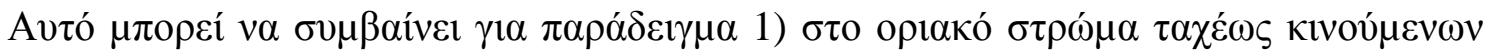

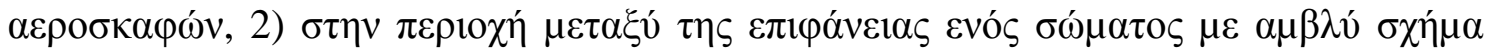

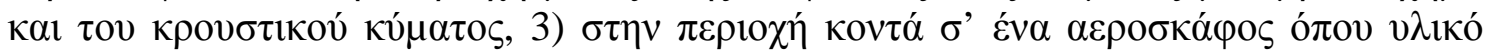

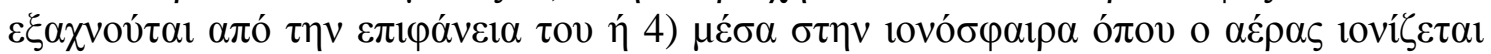
$\lambda$

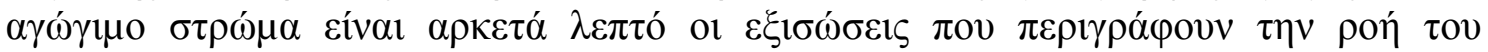

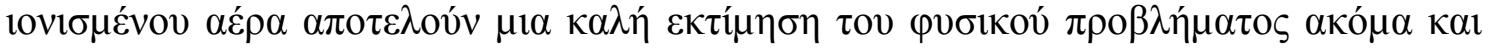
$\alpha \nu \tau o \sigma \pi \alpha \rho \alpha \mu \varepsilon ́ v \varepsilon l \sigma \tau \alpha \theta \varepsilon \rho o ́$ [120].

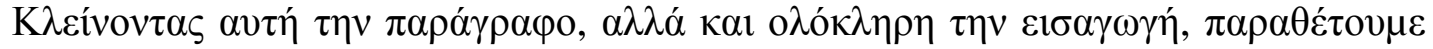

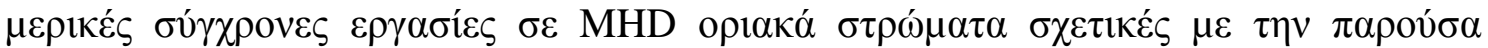

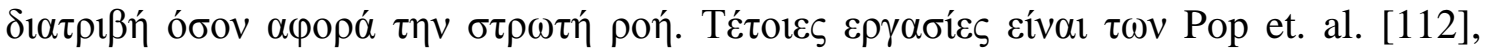

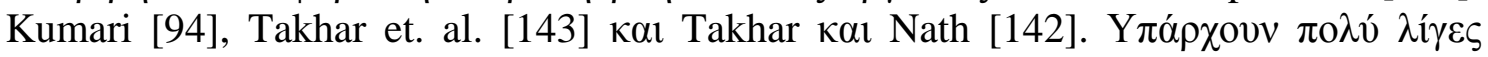

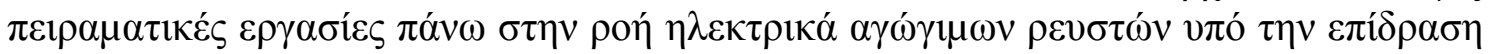

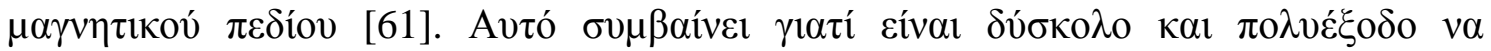

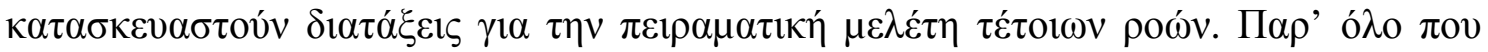

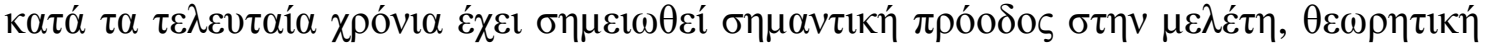

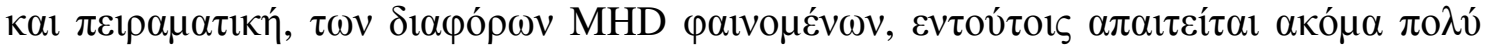

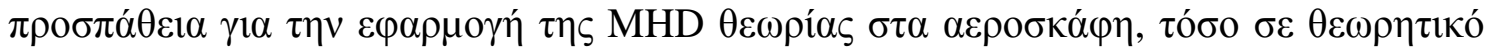

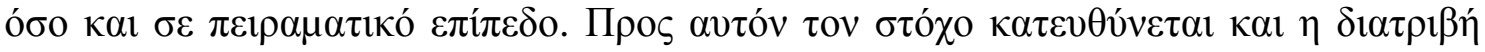
$\alpha v \tau \eta ́$. 


\title{
КЕФАААIO II
}

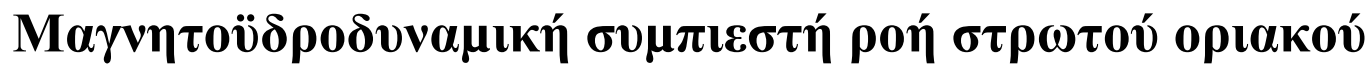

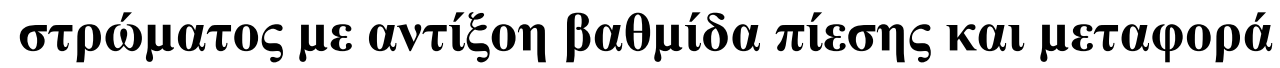

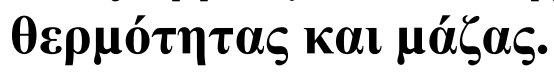

\begin{abstract}
Пєрí $\eta \psi \eta$

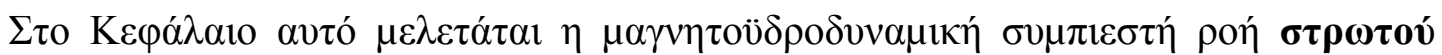

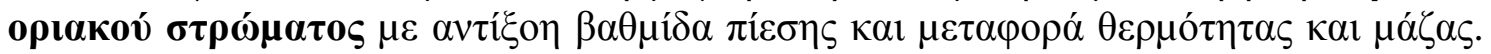

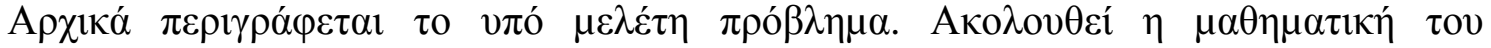

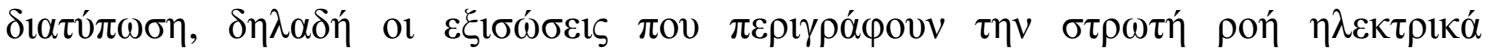

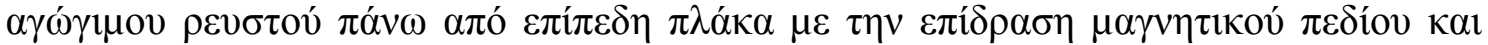

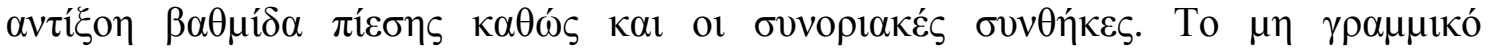

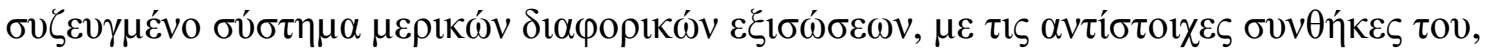

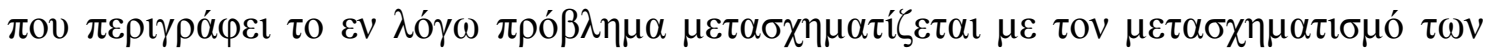

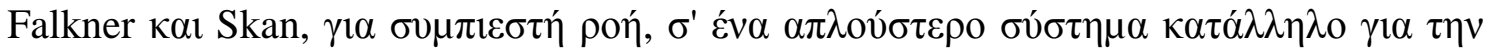

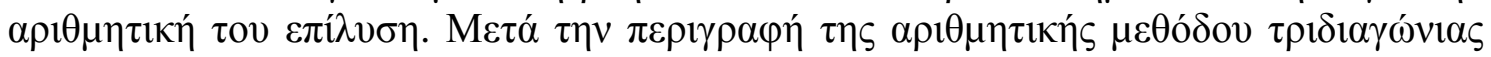

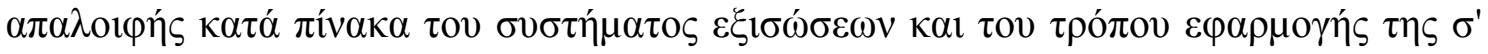

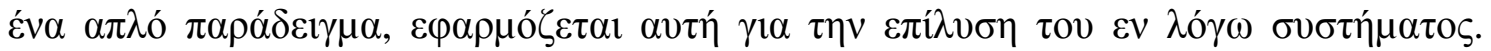

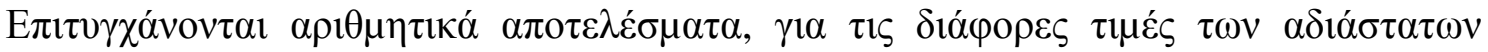

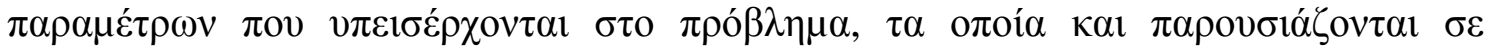

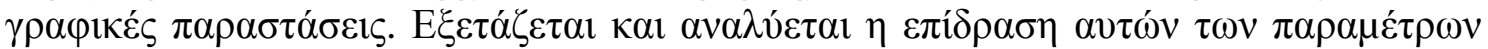

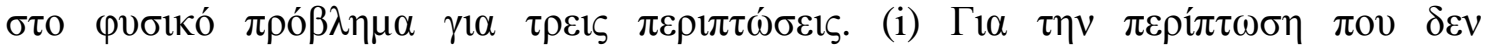

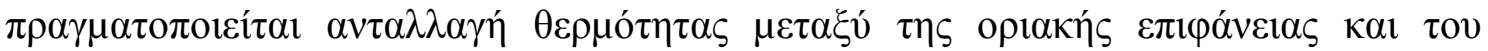

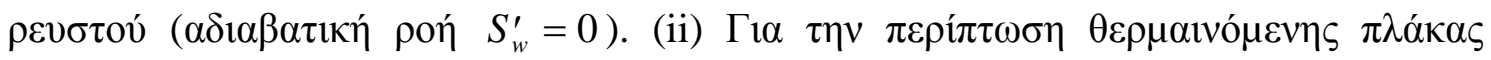

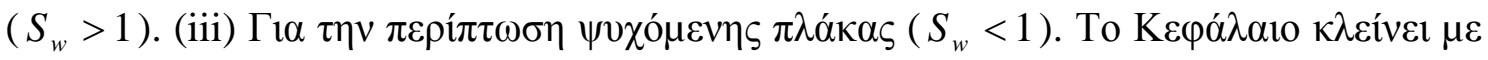

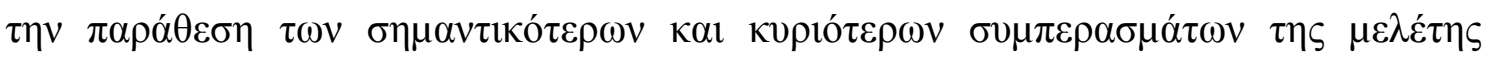

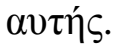

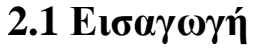

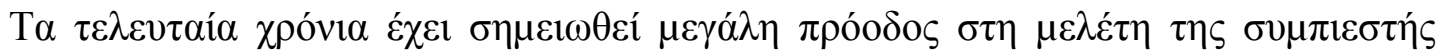

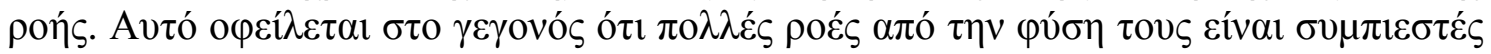

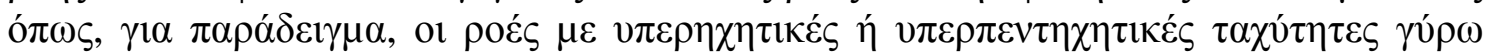

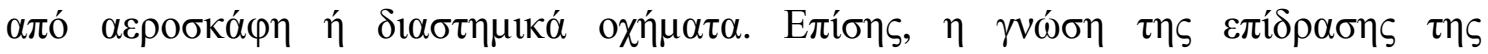

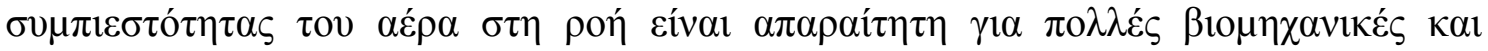

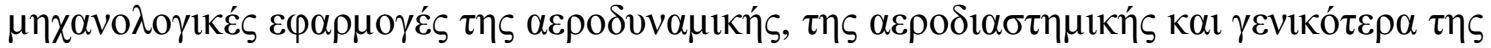

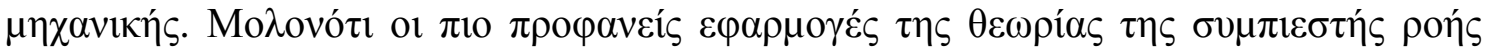

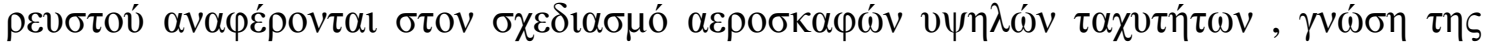

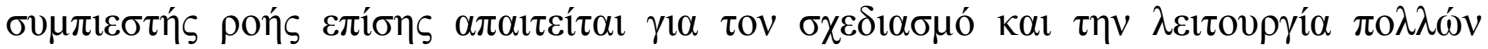

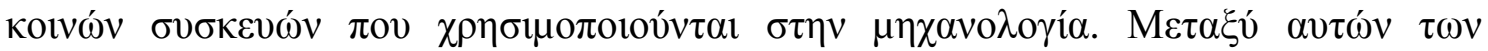

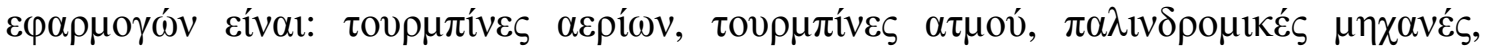

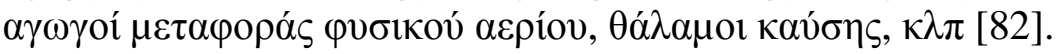

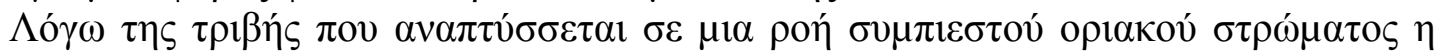

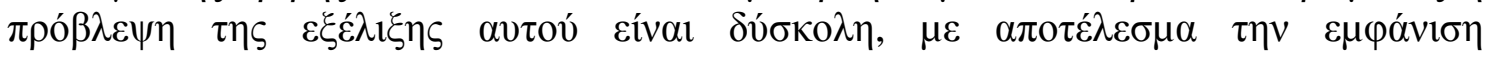




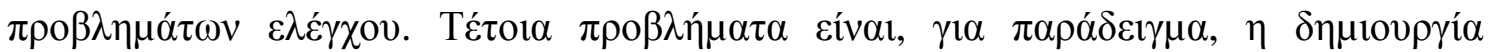

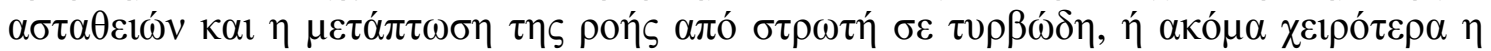

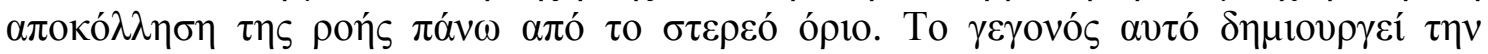

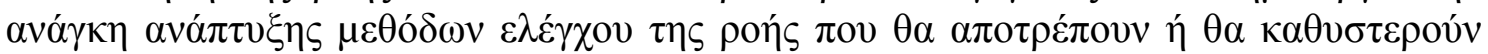

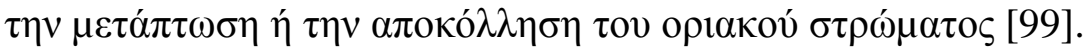

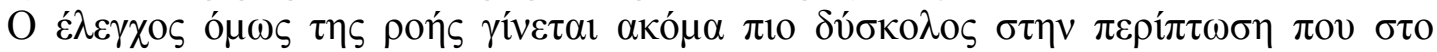

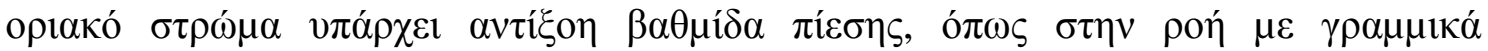

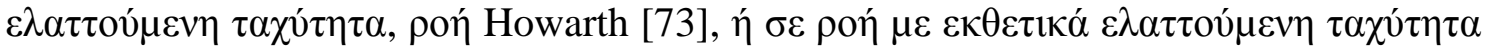

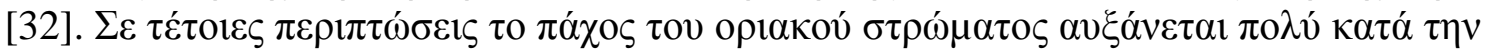

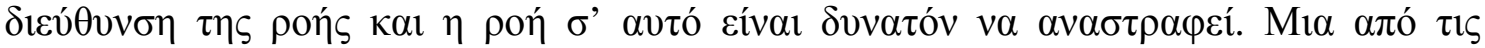

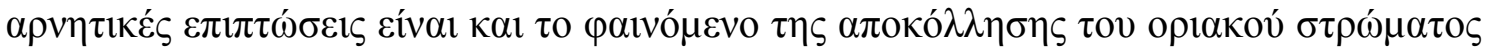

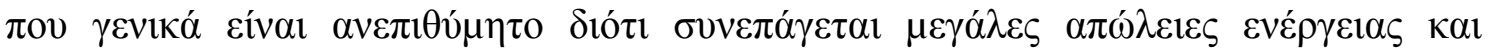

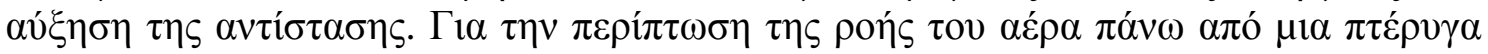

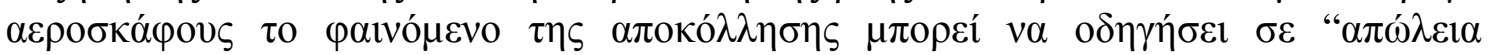

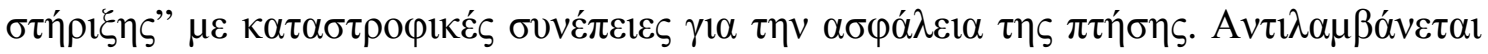

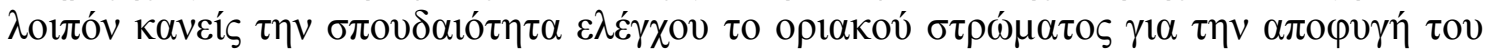

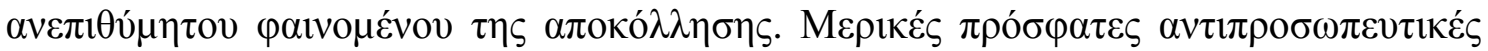

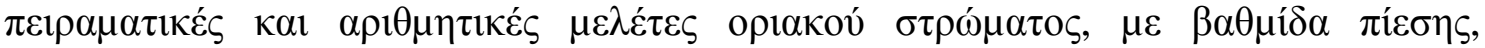

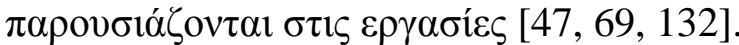

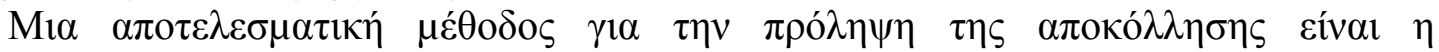

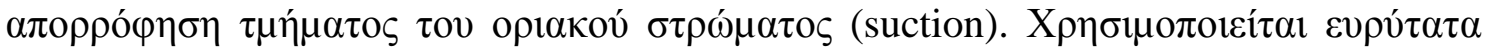

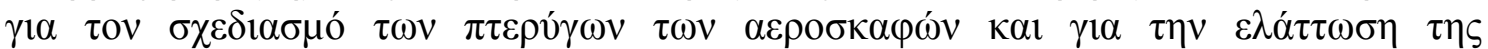

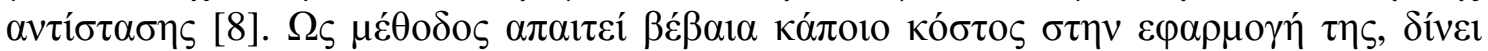

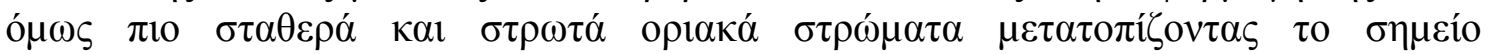

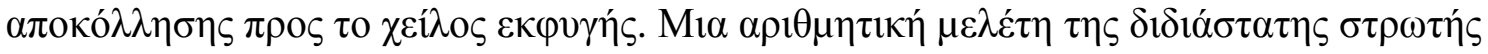

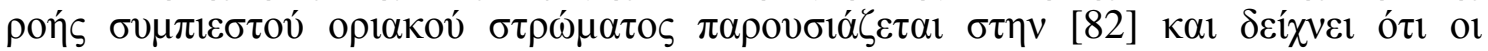

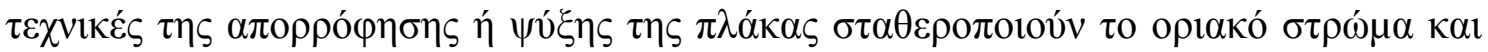

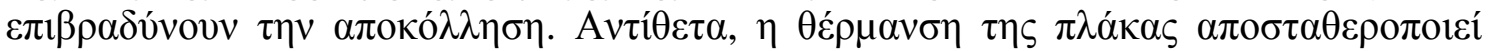

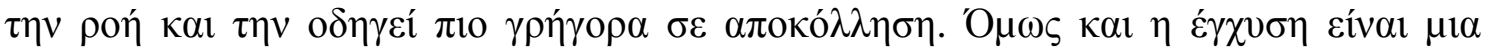

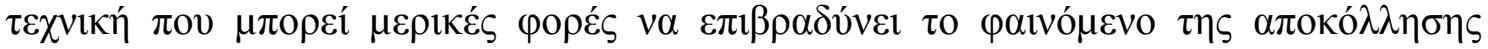

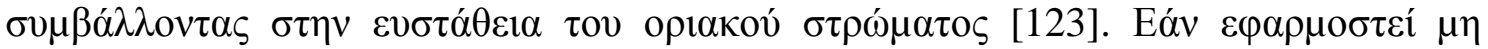

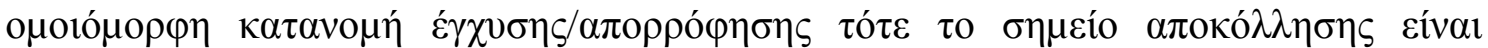

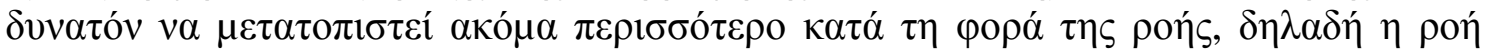

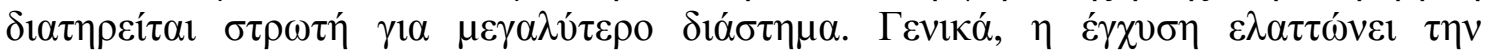

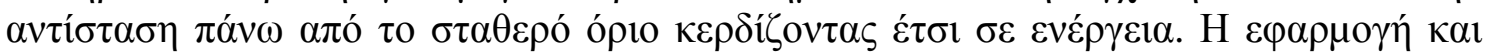

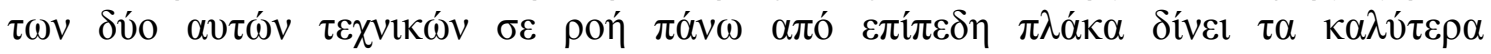

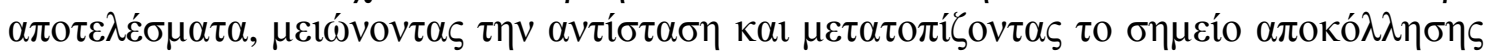

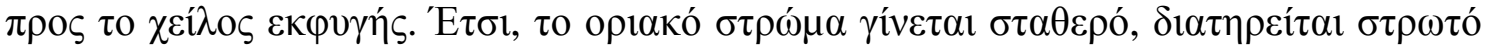

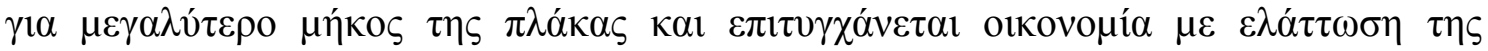
$\alpha \nu \tau i ́ \sigma \tau \alpha \sigma \eta \varsigma$.

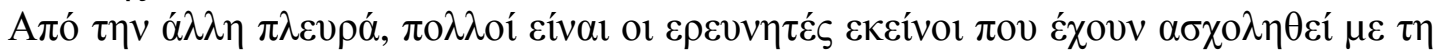

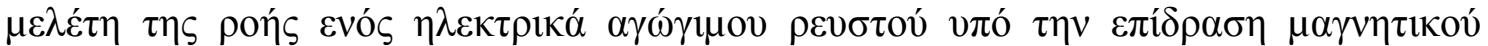

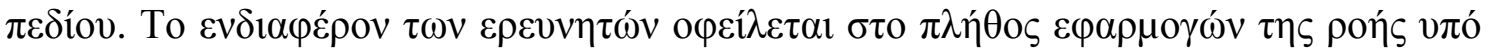

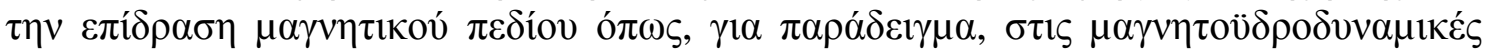

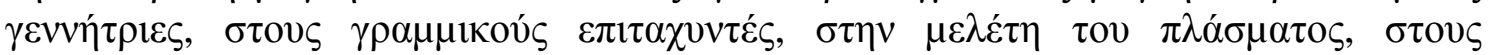

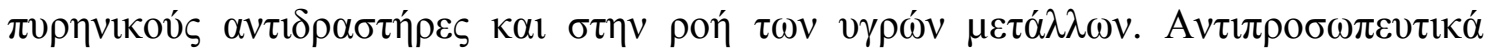

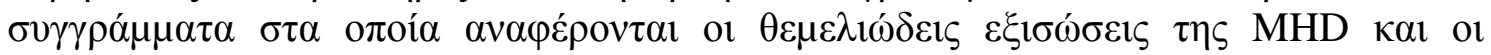

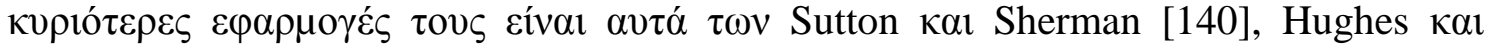

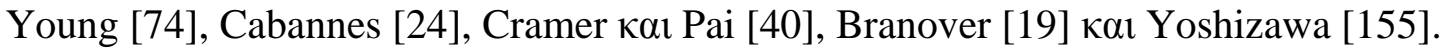




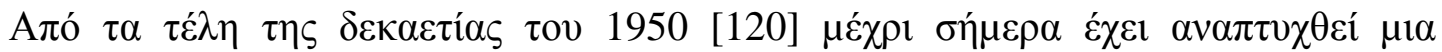

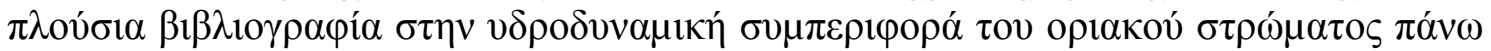

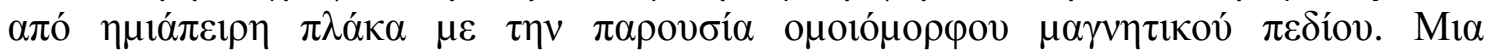

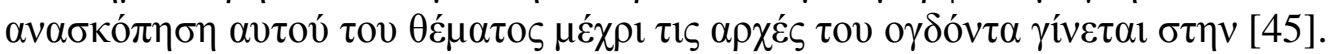

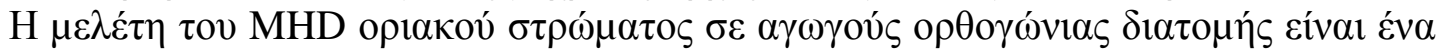

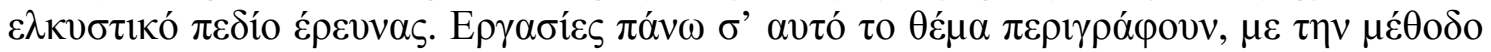

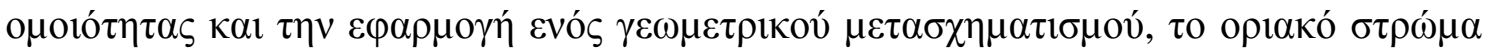

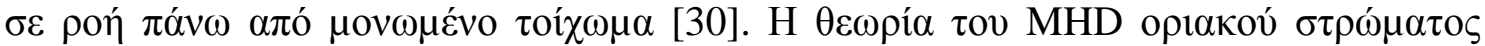

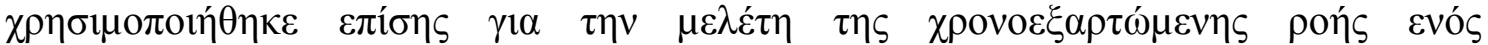

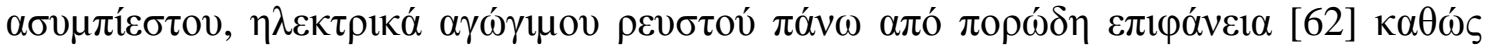

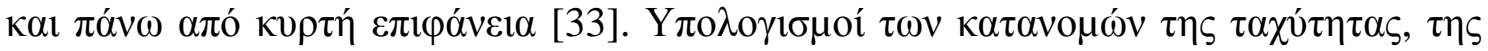

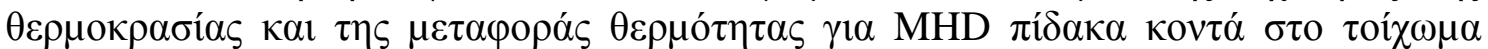

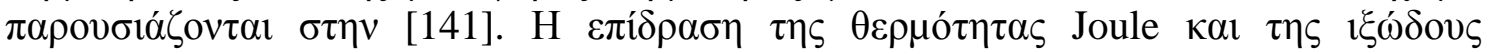

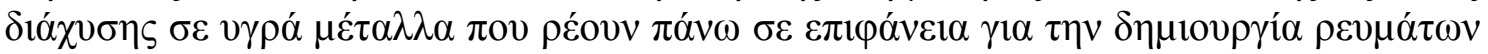

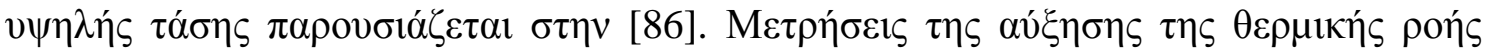

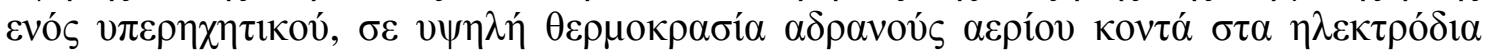

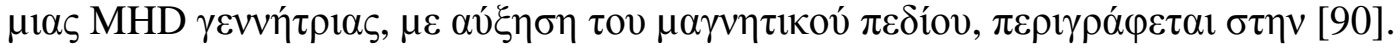

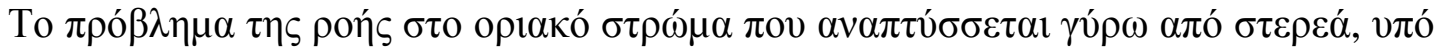

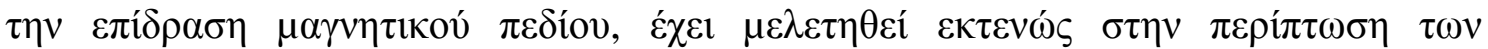

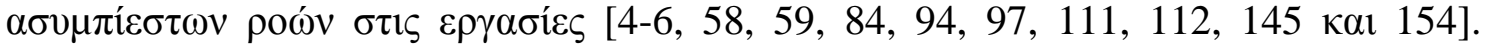

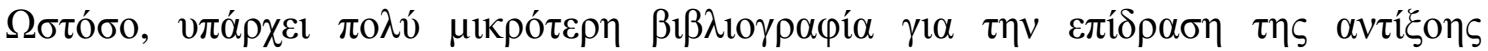

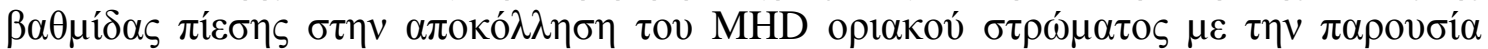

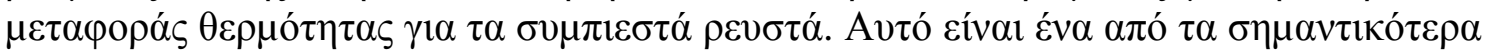

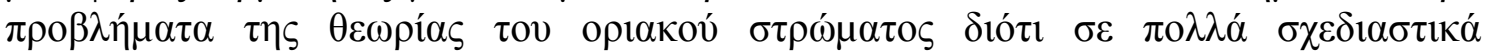

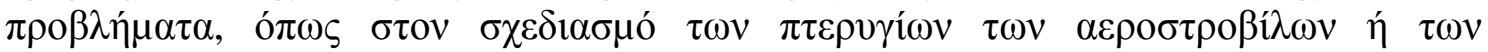

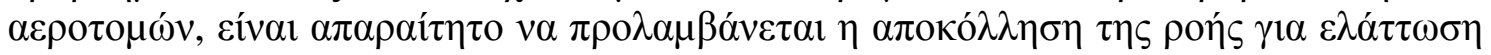

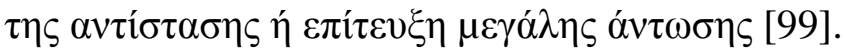

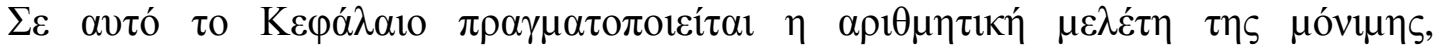

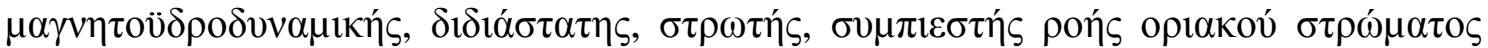

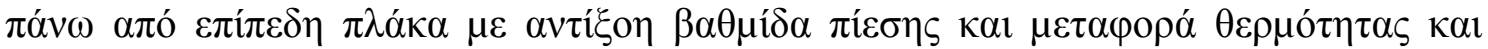
$\mu \alpha ́ \zeta \alpha \varsigma$.

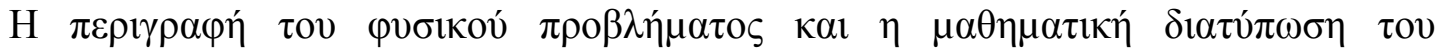

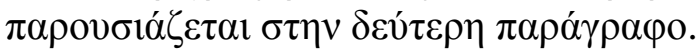

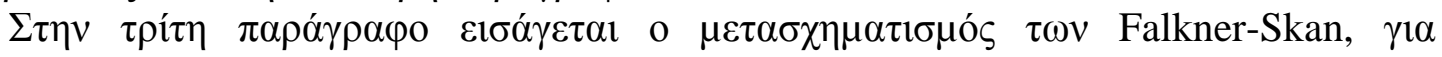

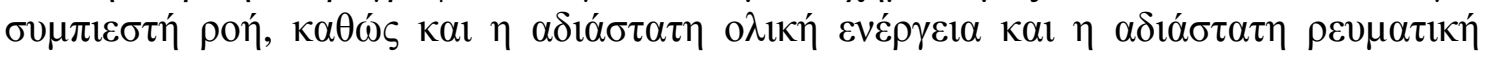

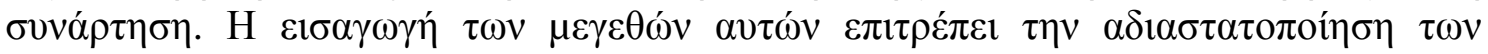

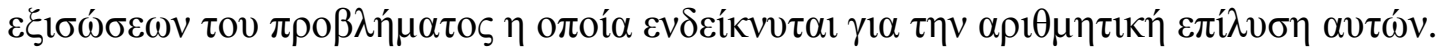

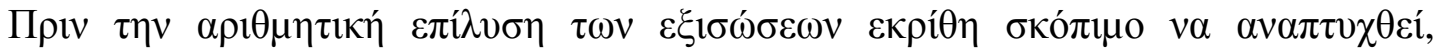

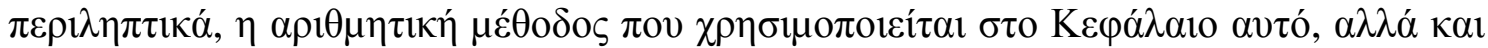

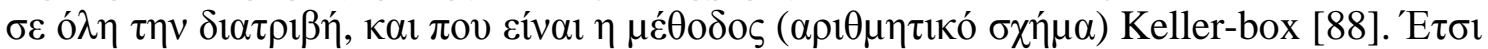

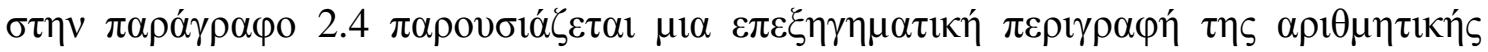

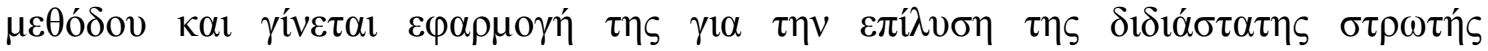

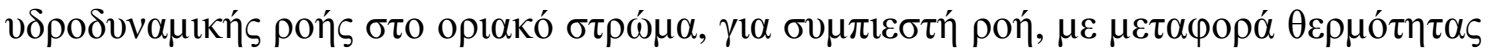

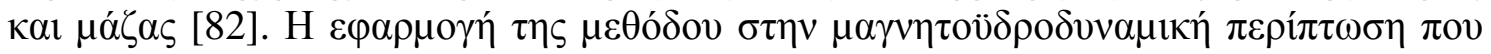

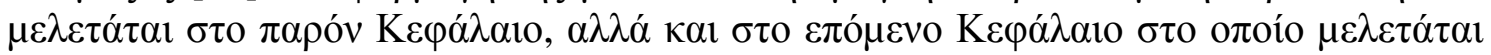

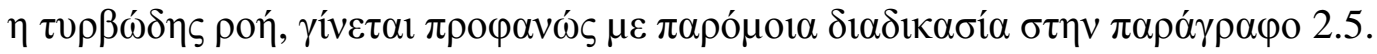

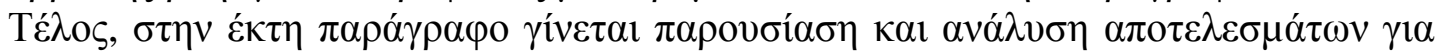

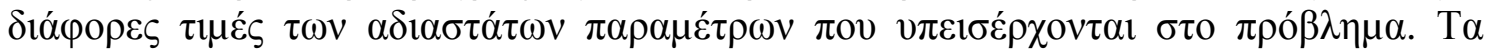

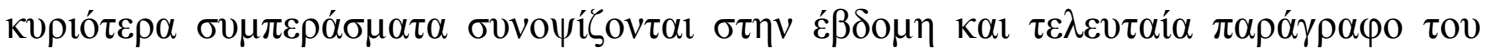

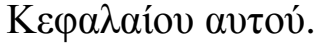




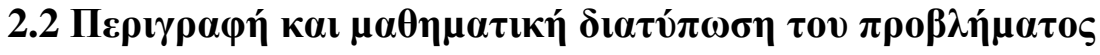

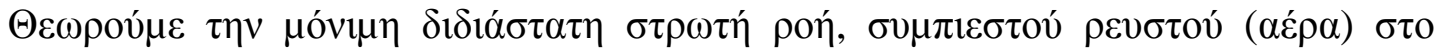

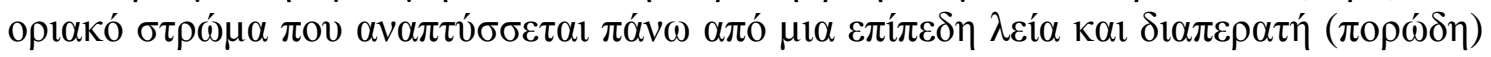

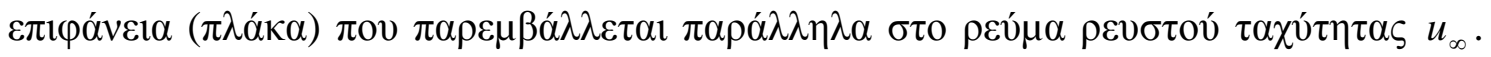

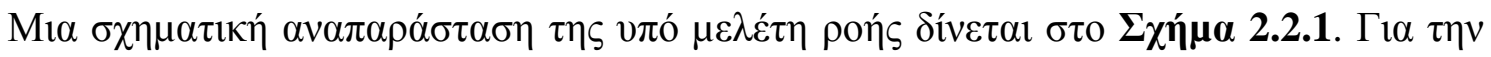

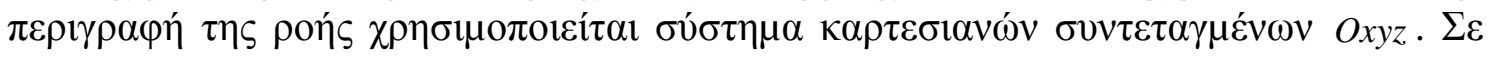

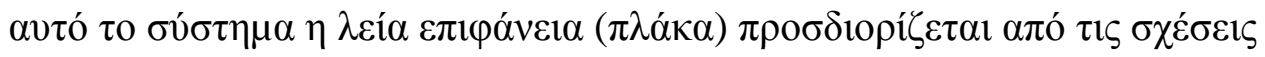

$$
y=0, \quad 0 \leq x \leq L, \quad-\infty<z<+\infty,
$$

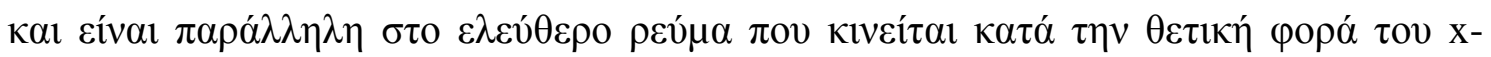

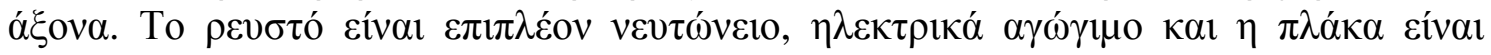

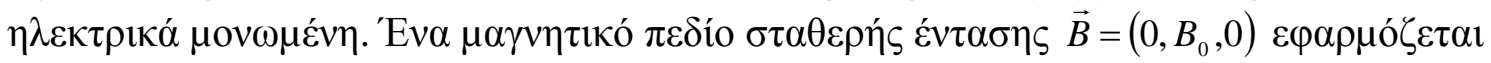

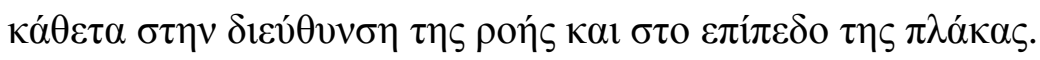

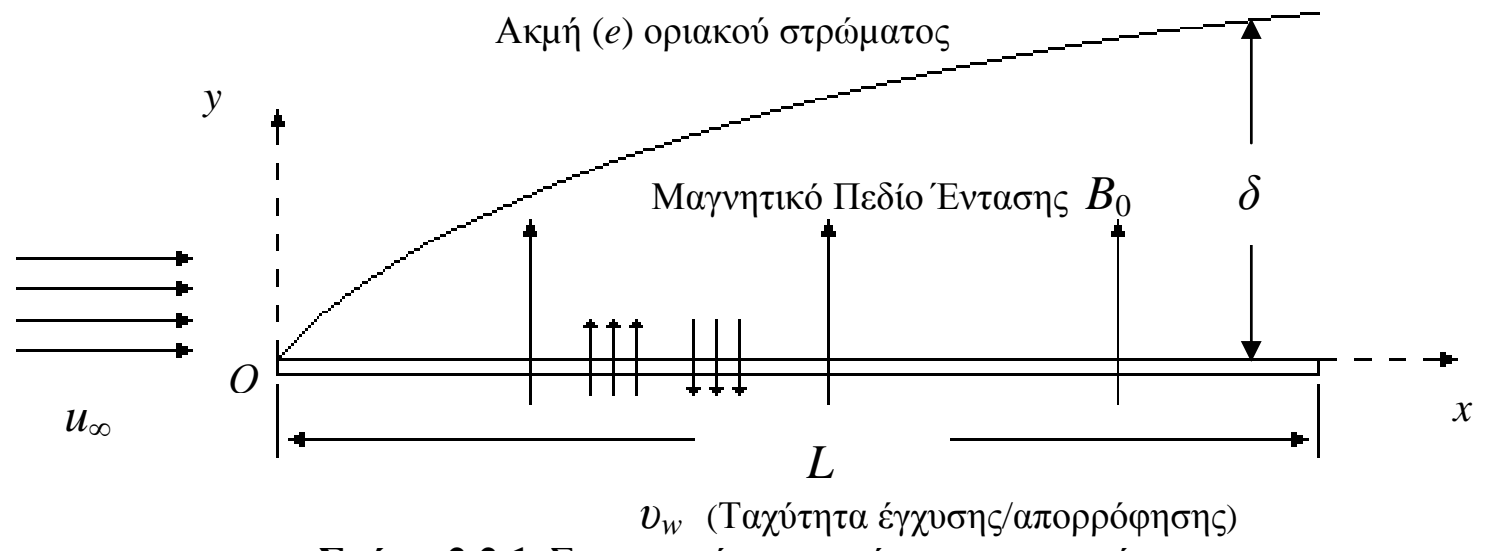

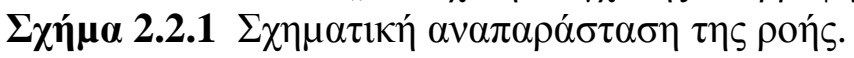

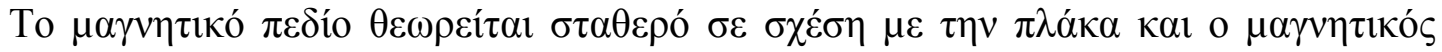

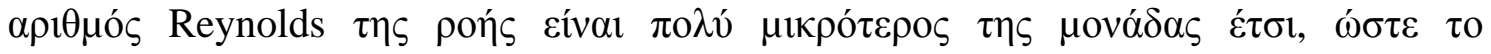

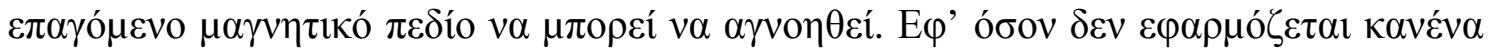

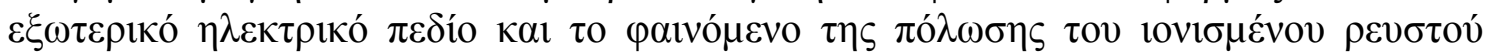

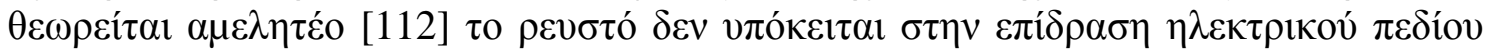
$(\vec{E}=\overrightarrow{0})$.

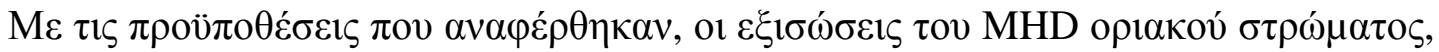

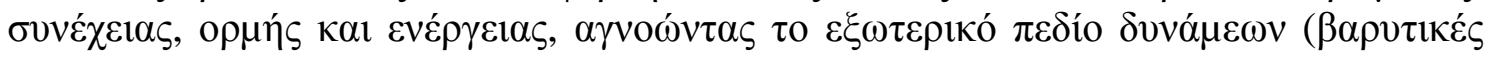

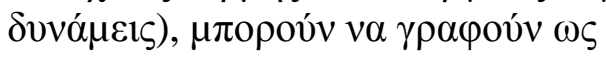

$$
\begin{array}{cc}
\frac{\partial}{\partial x}(\rho u)+\frac{\partial}{\partial y}(\rho v)=0, & (\sigma v v \varepsilon ́ \chi \varepsilon 1 \alpha \varsigma) \\
\rho u \frac{\partial u}{\partial x}+\rho v \frac{\partial u}{\partial y}=-\frac{d p}{d x}+\frac{\partial}{\partial y}\left(\mu \frac{\partial u}{\partial y}\right)+\left(\vec{J}_{c} \times \vec{B}\right)_{x}, & \text { (о } \mu \mu \eta ́ \varsigma \varsigma)
\end{array}
$$




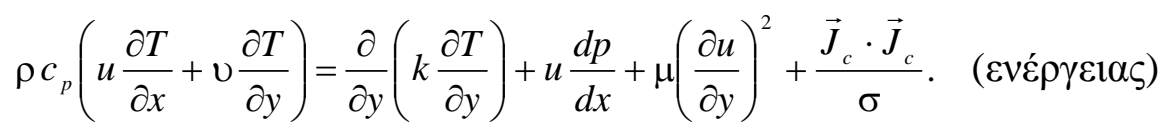

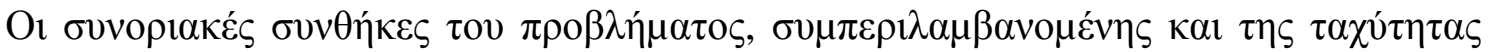

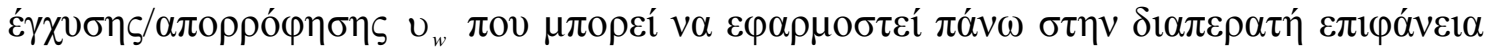
(wall), síval

$$
\begin{aligned}
& y=0: u=0, v=\mathrm{v}_{w}(x), T=T_{w}(x), \\
& y \rightarrow \delta: u=u_{e}(x), T=T_{e}(x) .
\end{aligned}
$$

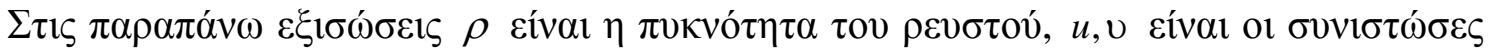

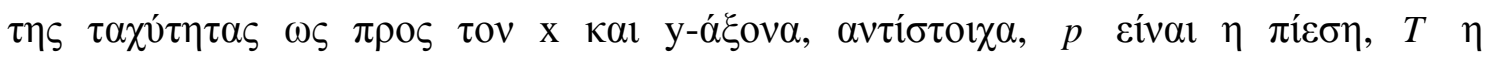

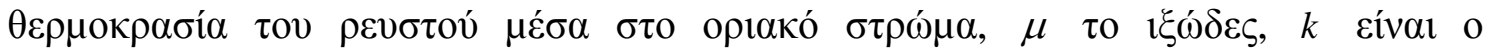

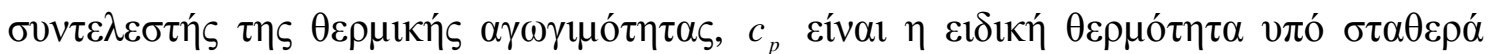

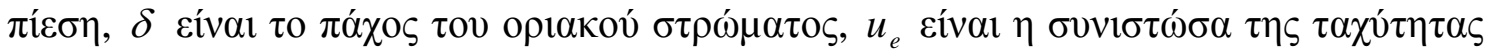

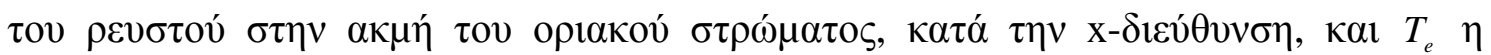

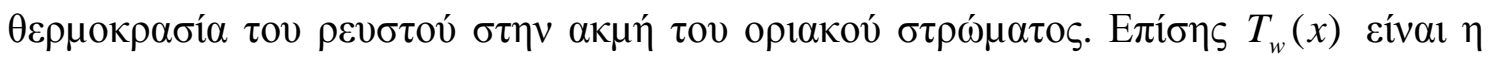

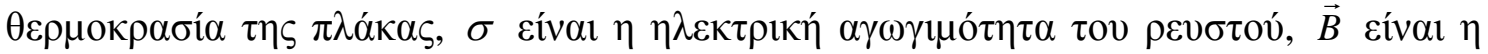

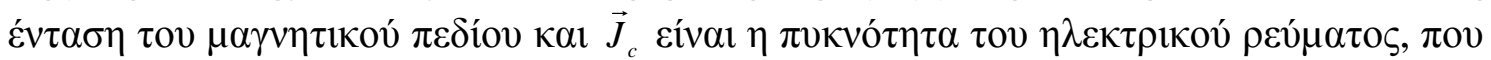

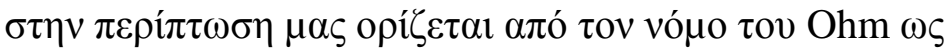

$$
\vec{J}_{c}=\sigma(\vec{q} \times \vec{B}), \quad(\vec{E}=\overrightarrow{0})
$$

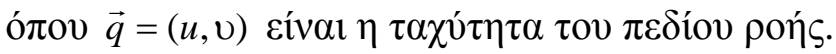

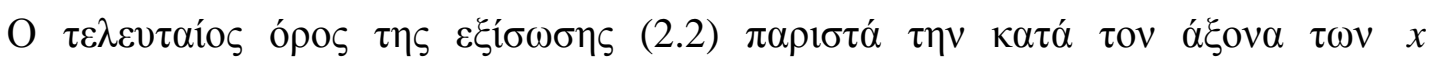

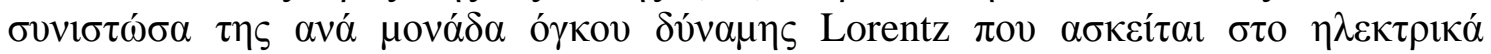

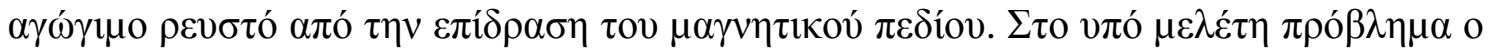

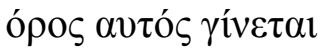

$$
\left(\vec{J}_{c} \times \vec{B}\right)_{x}=-\sigma u B_{0}^{2} .
$$

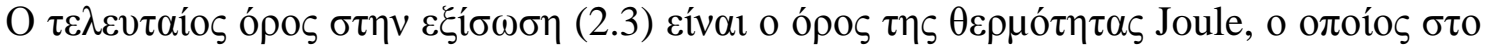

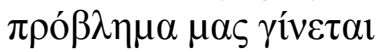

$$
\frac{\vec{J}_{c} \cdot \vec{J}_{c}}{\sigma}=\sigma u^{2} B_{0}^{2} .
$$

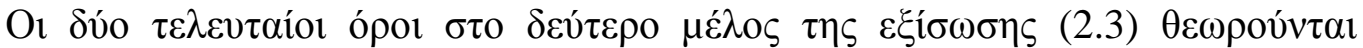

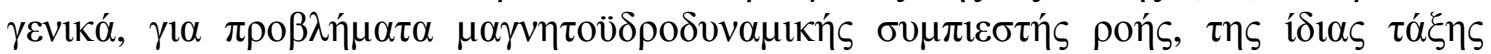

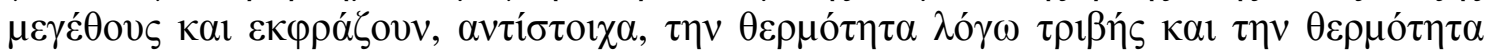
Joule $[40,74,140]$.

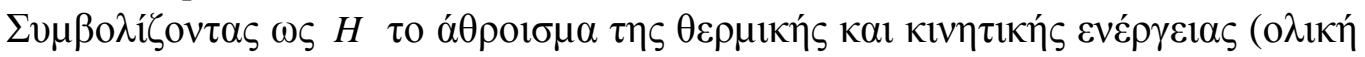

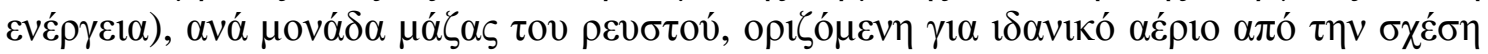




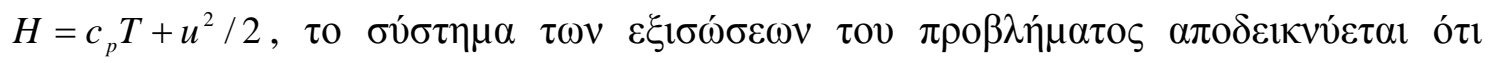

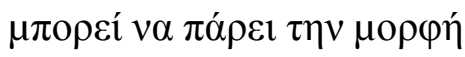

$$
\begin{gathered}
\frac{\partial}{\partial x}(\rho u)+\frac{\partial}{\partial y}(\rho \cup)=0 \\
\rho u \frac{\partial u}{\partial x}+\rho \cup \frac{\partial u}{\partial y}=-\frac{d p}{d x}+\frac{\partial}{\partial y}\left(\mu \frac{\partial u}{\partial y}\right)-\sigma B_{0}^{2} u, \\
\rho u \frac{\partial H}{\partial x}+\rho \cup \frac{\partial H}{\partial y}=\frac{\partial}{\partial y}\left[\frac{\mu}{\operatorname{Pr}} \frac{\partial H}{\partial y}+\mu\left(1-\frac{1}{\operatorname{Pr}}\right) u \frac{\partial u}{\partial y}\right] .
\end{gathered}
$$

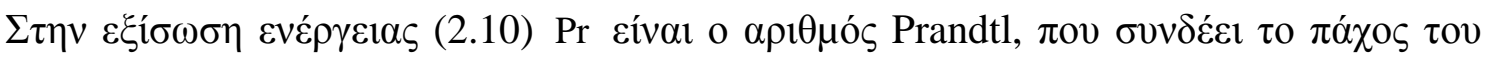

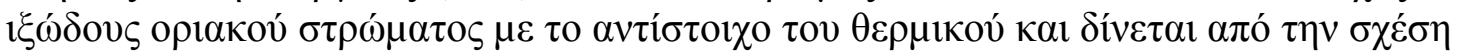

$$
\operatorname{Pr}=\frac{\mu c_{P}}{k}
$$

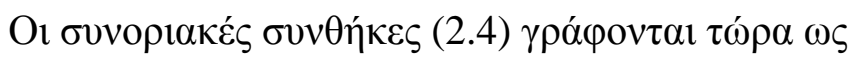

$$
\begin{aligned}
& y=0: \quad u=0, \quad v=v_{w}(x), \quad H=H_{w}(x), \\
& y \rightarrow \delta: \quad u=u_{e}(x), \quad H=H_{e}(x),
\end{aligned}
$$

о́ $\operatorname{tov} H_{w}(x)=c_{p} T_{w}(x) \kappa \alpha \imath H_{e}(x)=c_{p} T_{e}(x)+\frac{1}{2} u_{e}^{2}(x)$.

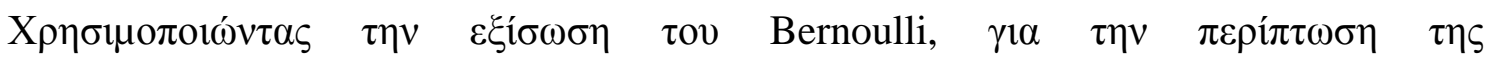

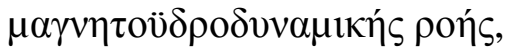

$$
-\frac{d p}{d x}=\rho_{e} u_{e} \frac{d u_{e}}{d x}+\sigma B_{0}^{2} u_{e}
$$

ó

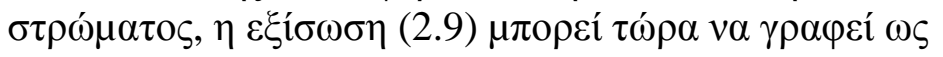

$$
\rho u \frac{\partial u}{\partial x}+\rho v \frac{\partial u}{\partial y}=\rho_{e} u_{e} \frac{d u_{e}}{d x}+\sigma B_{0}^{2} u_{e}+\frac{\partial}{\partial y}\left(\mu \frac{\partial u}{\partial y}\right)-\sigma B_{0}^{2} u .
$$

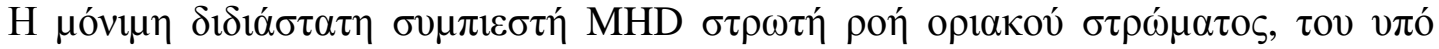

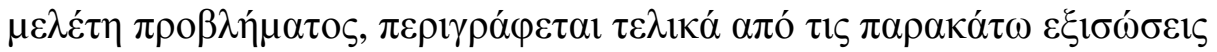

$$
\frac{\partial}{\partial x}(\rho u)+\frac{\partial}{\partial y}(\rho v)=0
$$




$$
\begin{gathered}
\rho u \frac{\partial u}{\partial x}+\rho v \frac{\partial u}{\partial y}=\rho_{e} u_{e} \frac{d u_{e}}{d x}+\frac{\partial}{\partial y}\left(\mu \frac{\partial u}{\partial y}\right)-\sigma B_{0}^{2}\left(u-u_{e}\right), \\
\rho u \frac{\partial H}{\partial x}+\rho v \frac{\partial H}{\partial y}=\frac{\partial}{\partial y}\left[\frac{\mu}{\operatorname{Pr}} \frac{\partial H}{\partial y}+\mu\left(1-\frac{1}{\operatorname{Pr}}\right) u \frac{\partial u}{\partial y}\right],
\end{gathered}
$$

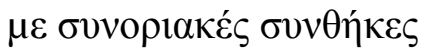

$$
\begin{aligned}
& y=0: \quad u=0, \quad v=v_{w}(x), \quad H=H_{w}(x), \\
& y \rightarrow \delta: \quad u=u_{e}(x), \quad H=H_{e}(x) .
\end{aligned}
$$

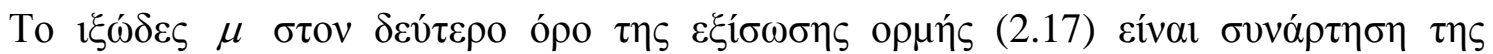

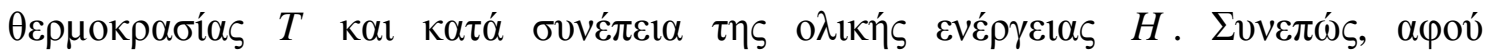

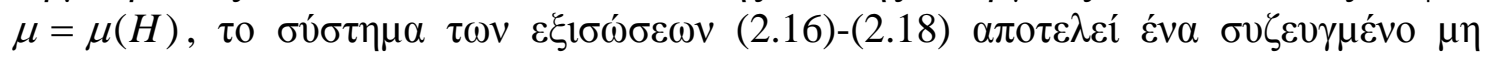

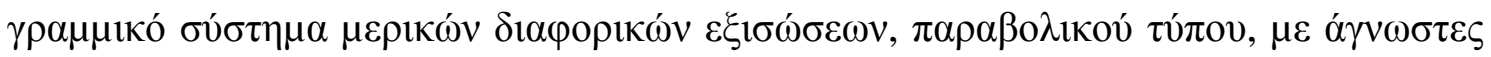

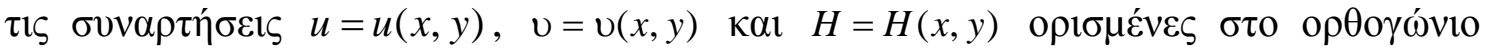

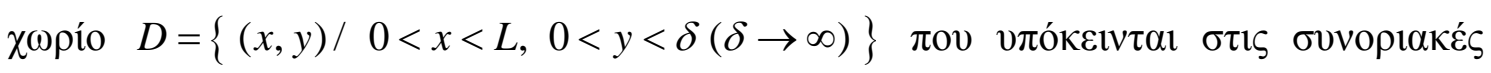

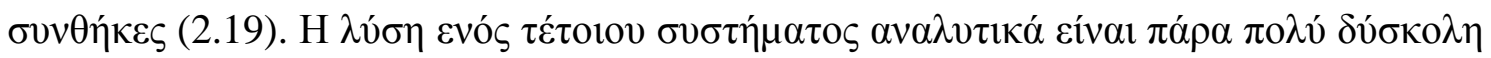

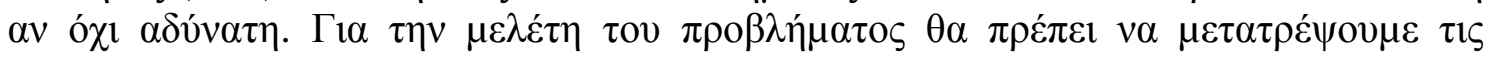

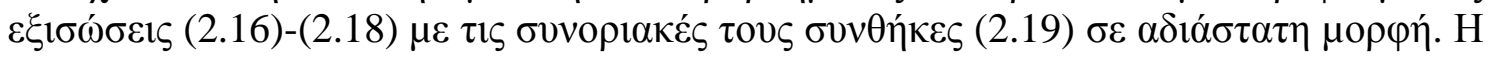

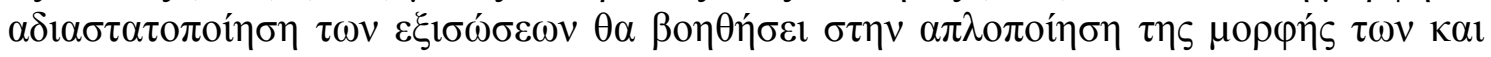

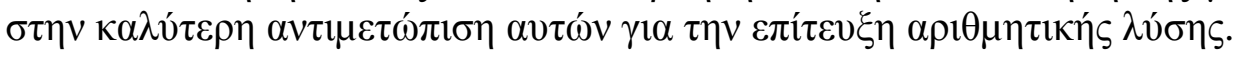

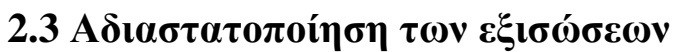

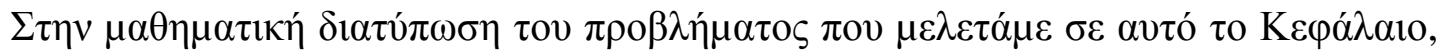

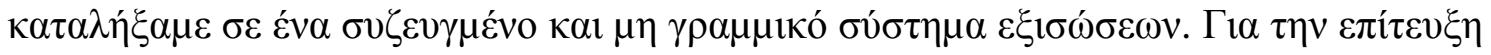

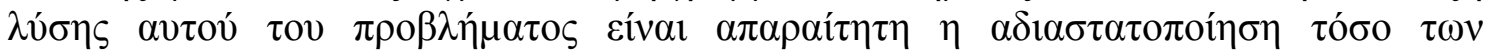

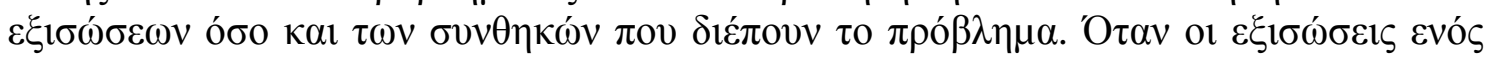

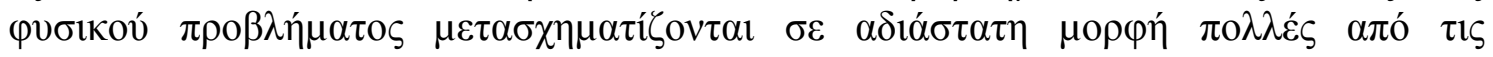

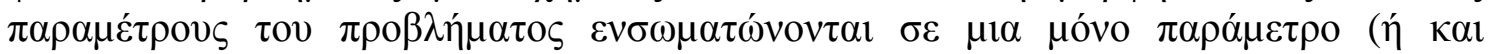

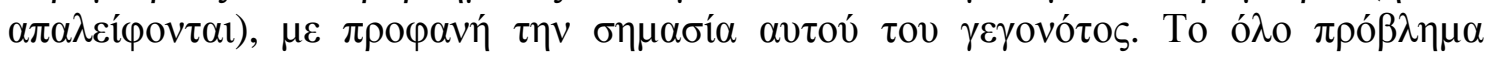

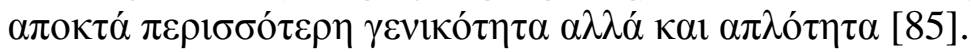

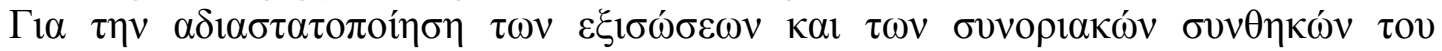

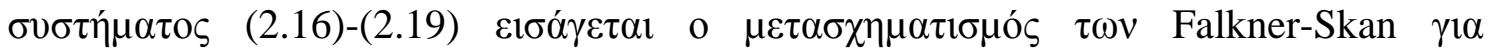

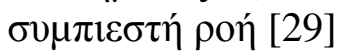

$$
\eta(x, y)=\int_{0}^{y}\left(\frac{u_{e}(x)}{x v_{e}(x)}\right)^{1 / 2} \frac{\rho(x, y)}{\rho_{e}(x)} d y, \quad \psi(x, y)=\left(\rho_{e} \mu_{e} u_{e} x\right)^{1 / 2} f(x, \eta),
$$

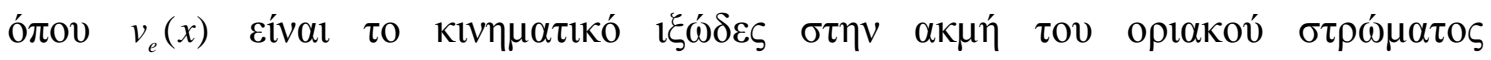

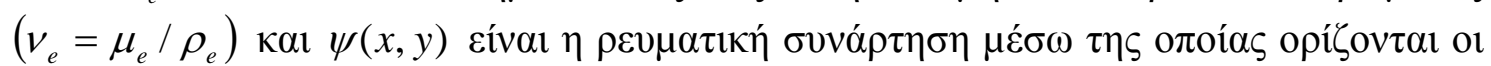

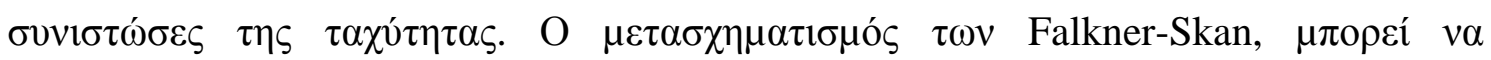




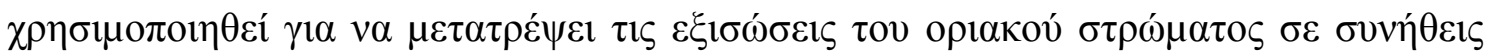

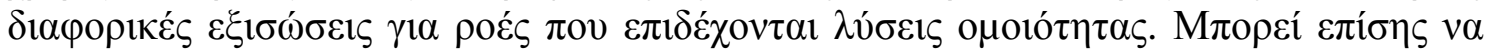

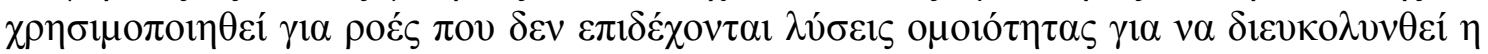

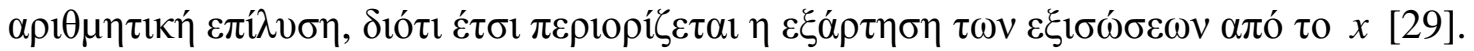

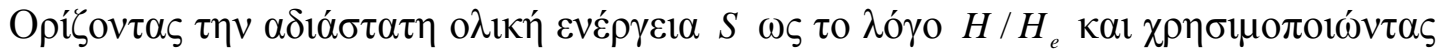

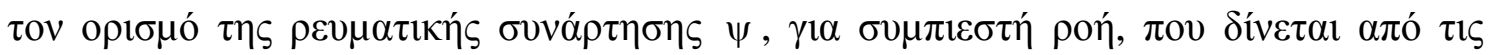
$\sigma \chi \varepsilon \dot{\sigma \varepsilon l \varsigma}$

$$
\rho u=\frac{\partial \psi}{\partial y}, \quad \rho v=-\frac{\partial \psi}{\partial x},
$$

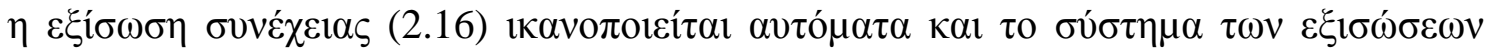

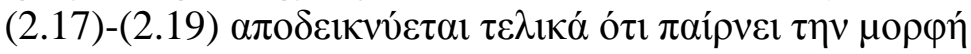

$$
\begin{gathered}
\left(b f^{\prime \prime}\right)^{\prime}+m_{1} f f^{\prime \prime}+m_{2}\left[c-\left(f^{\prime}\right)^{2}\right]=x\left[m_{3}\left(f^{\prime}-1\right)+f^{\prime} \frac{\partial f^{\prime}}{\partial x}-f^{\prime \prime} \frac{\partial f}{\partial x}\right], \\
\left(e S^{\prime}+d f^{\prime} f^{\prime \prime}\right)^{\prime}+m_{1} f S^{\prime}=x\left(f^{\prime} \frac{\partial S}{\partial x}-S^{\prime} \frac{\partial f}{\partial x}\right), \\
\eta=0: f^{\prime}=0, f_{w}=f(x, 0)=-\frac{1}{\left(u_{e} \mu_{e} \rho_{e} x\right)^{1 / 2}} \int_{0}^{x} \rho_{w} v_{w}(x) d x, S=S_{w}(x) \\
\eta=\eta_{e}: \quad f^{\prime}=1, \quad S=1,
\end{gathered}
$$

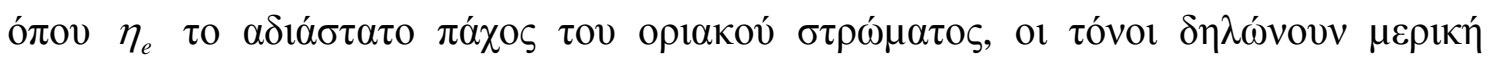

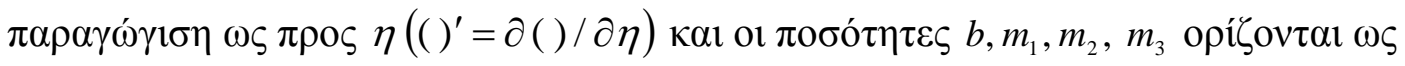

$$
\begin{gathered}
b=C, \quad C=\frac{\rho \mu}{\rho_{e} \mu_{e}}, \quad c=\frac{\rho_{e}}{\rho}, \quad d=\frac{C u_{e}^{2}}{H_{e}}\left(1-\frac{1}{\operatorname{Pr}}\right), \\
e=\frac{b}{\operatorname{Pr}}, \quad S=\frac{H}{H_{e}}, \\
m_{1}=\frac{1}{2}\left[1+m_{2}+\frac{x}{\rho_{e} \mu_{e}} \frac{d}{d x}\left(\rho_{e} \mu_{e}\right)\right], \quad m_{2}=\frac{x}{u_{e}} \frac{d u_{e}}{d x}, \quad R_{x}=\frac{u_{e} x}{v_{e}}, \\
m_{3}=\frac{m_{0} c}{\rho_{e} u_{e}}, \quad m_{0}=\sigma B_{0}^{2} .
\end{gathered}
$$

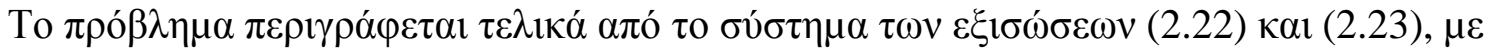

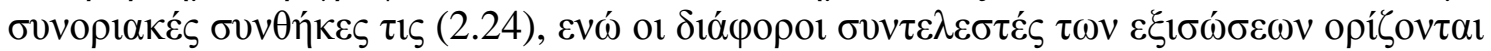
$\sigma \tau \eta v(2.25)$.

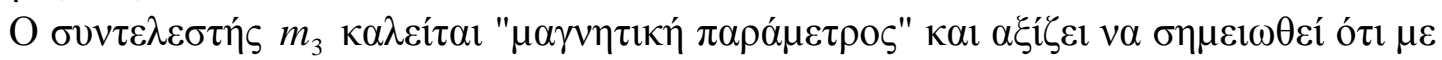
$\tau \eta \nu$ a

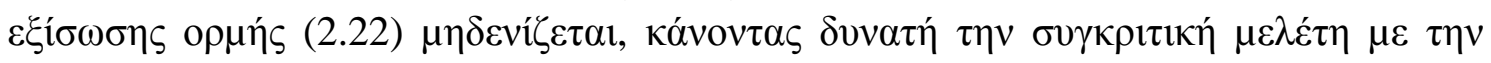




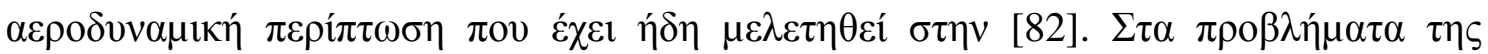

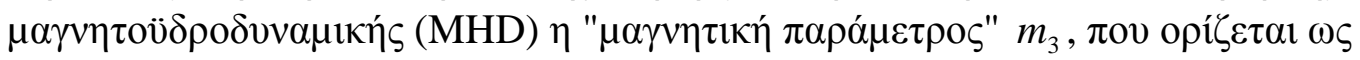

$$
m_{3}=\frac{m_{0} c}{\rho_{e} u_{e}}, \text { ó } \pi \mathrm{ov} c=\frac{\rho_{e}}{\rho} \kappa \alpha \imath m_{0}=\sigma B_{0}^{2},
$$

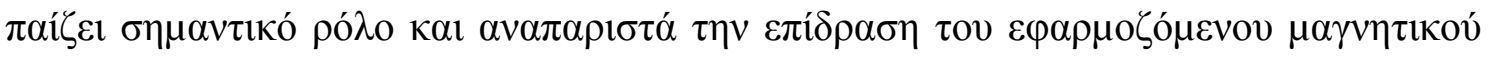

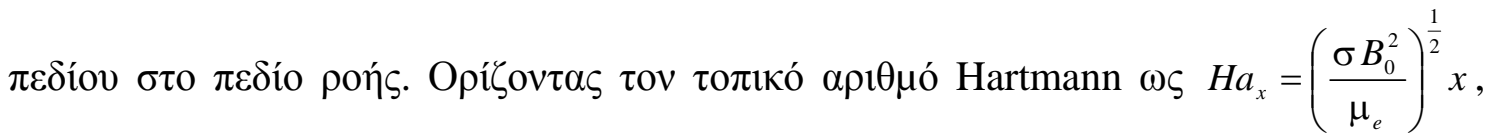

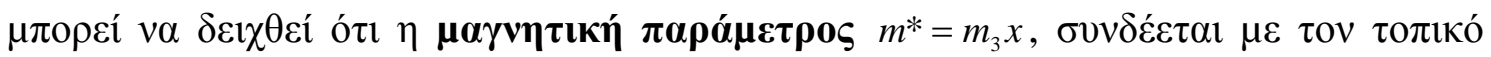

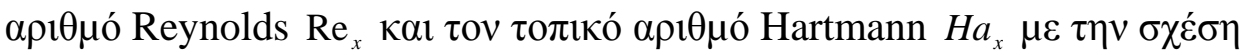

$$
m^{*}=m_{3} x=c \frac{H a_{x}^{2}}{\operatorname{Re}_{x}},
$$

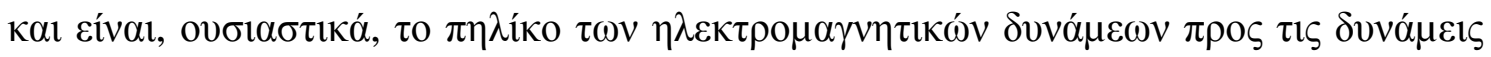

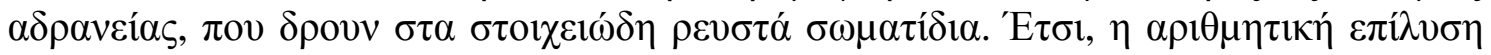

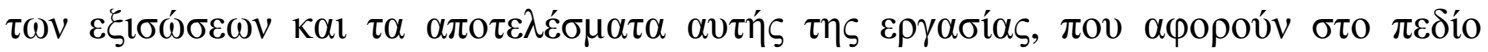

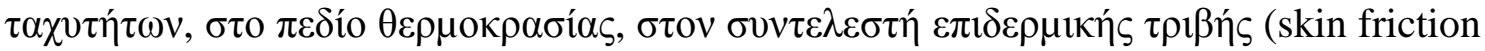

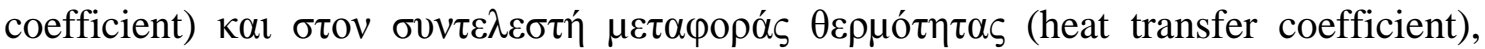

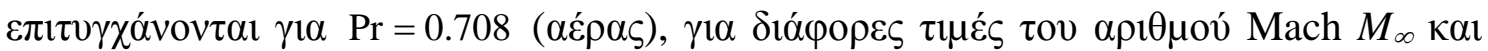

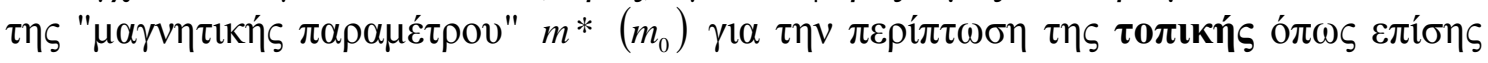

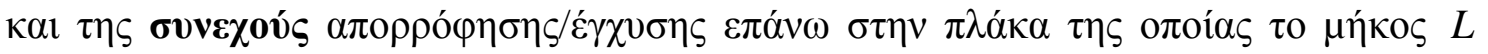
$\lambda \alpha \mu \beta \alpha ́ v \varepsilon \tau \alpha \imath$ íбo $\mu \varepsilon 8 \mathrm{~m}$.

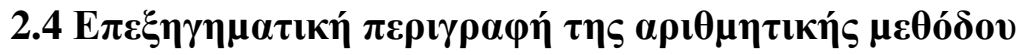

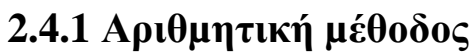

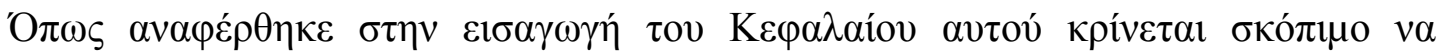

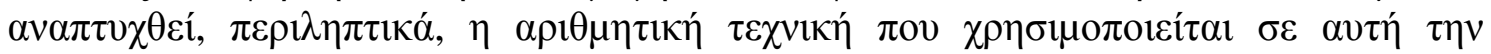

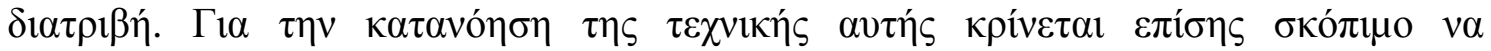

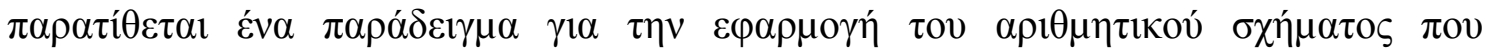

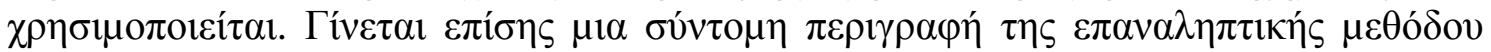

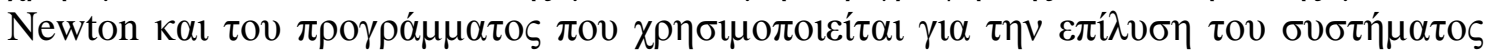

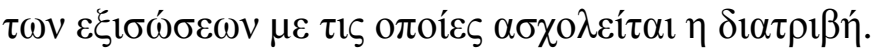

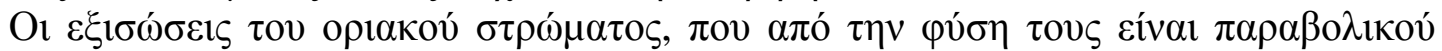

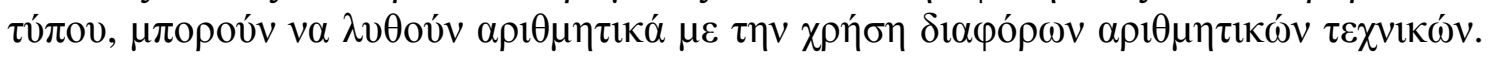

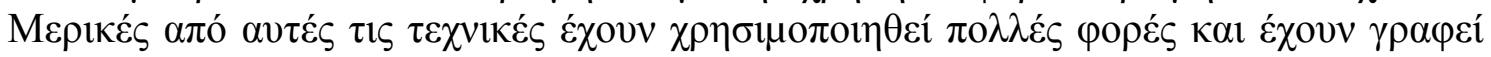

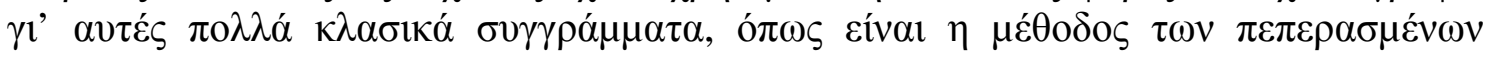

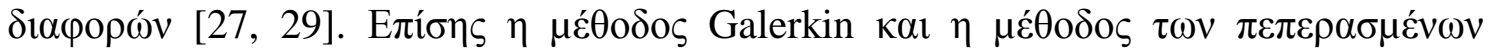

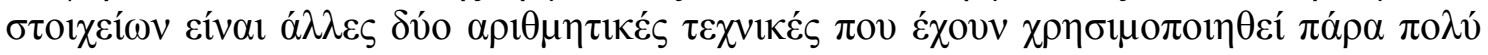

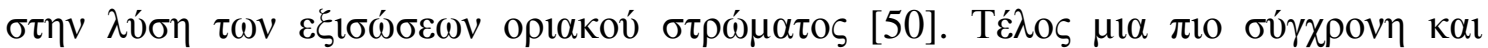

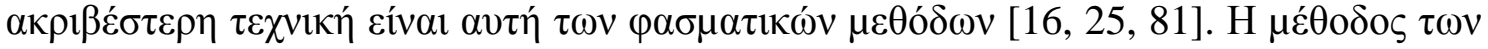

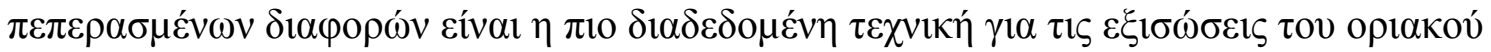




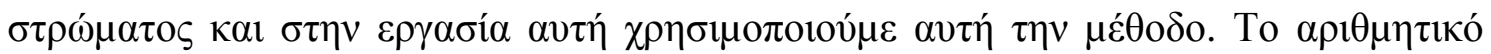

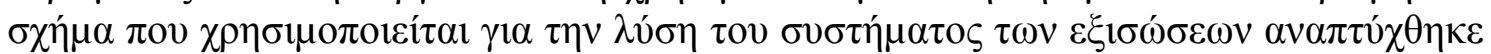

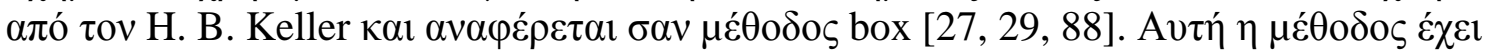

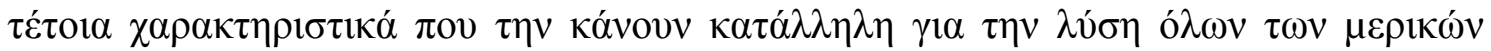

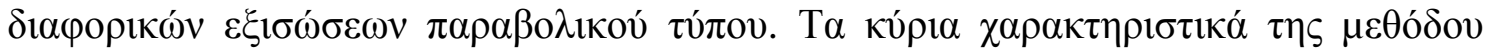
Eíval:

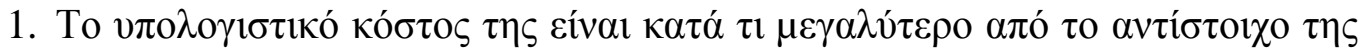
$\mu \varepsilon \theta$ ódov Crank-Nicolson [85].

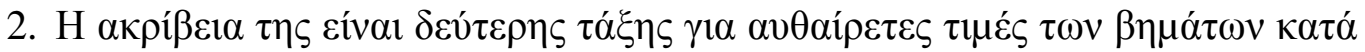

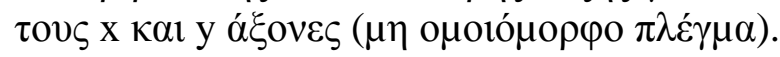

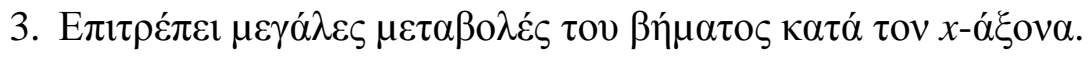

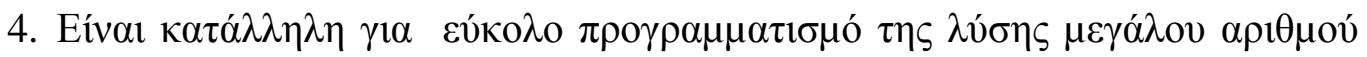
$\sigma v \zeta \varepsilon v \gamma \mu \varepsilon \dot{v} \omega v \varepsilon \xi \xi \sigma \omega ́ \sigma \varepsilon \omega v$.

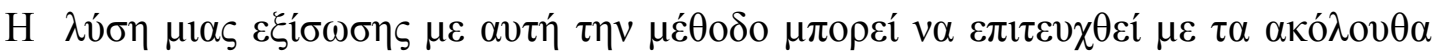
$\tau \varepsilon ́ \sigma \sigma \varepsilon \rho \alpha \beta \eta \dot{\mu} \mu \tau \alpha$ :

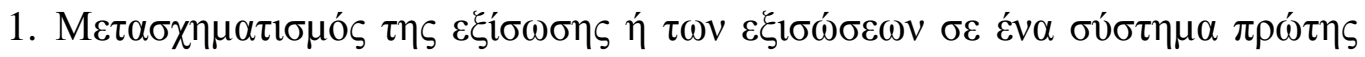
$\tau \alpha \dot{\xi \eta \eta s . ~}$

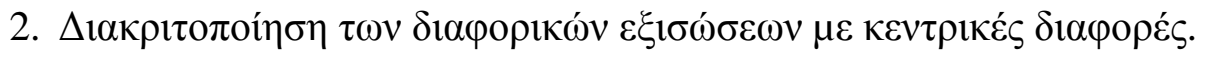

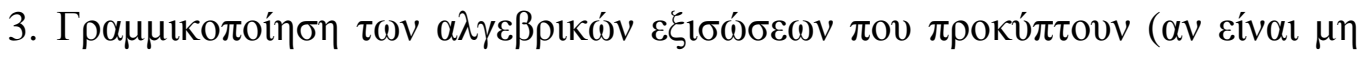

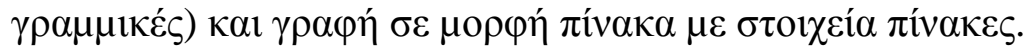

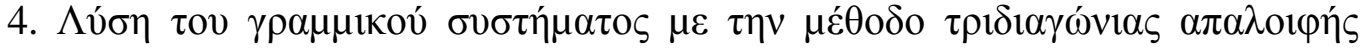

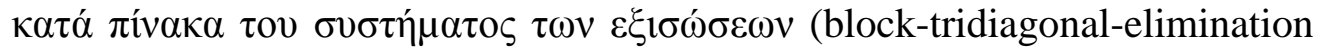
method).

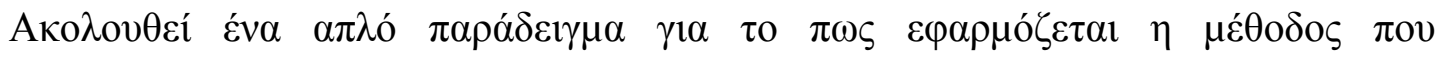

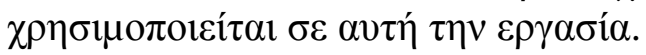

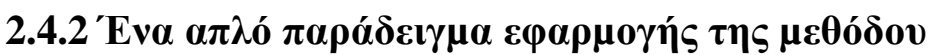

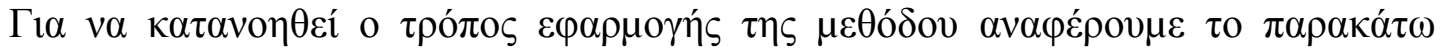

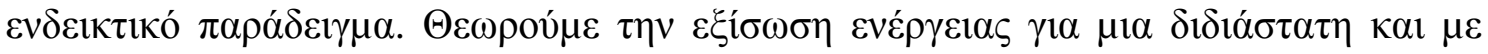

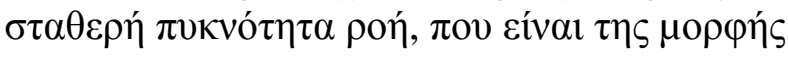

$$
u \frac{\partial T}{\partial x}=\frac{v}{\operatorname{Pr}} \frac{\partial^{2} T}{\partial y^{2}}
$$

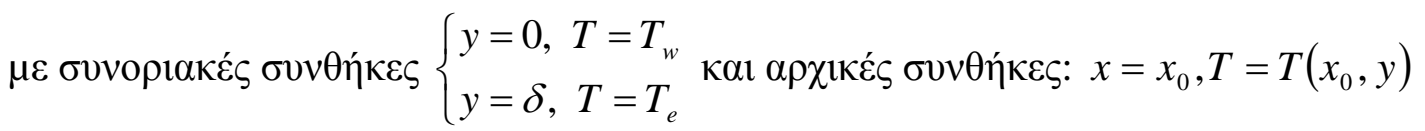




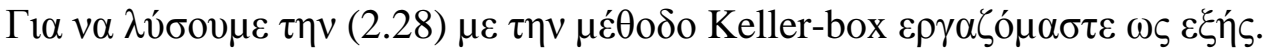

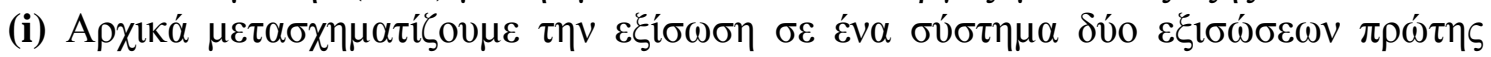
$\tau \alpha \dot{\xi} \eta \varsigma$ $\theta \dot{\varepsilon} \tau 0 v \tau \alpha \varsigma$

$$
T^{\prime}=p \quad\left(()^{\prime}=\frac{\partial()}{\partial y}\right)
$$

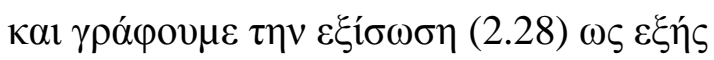

$$
p^{\prime}=\frac{\operatorname{Pr}}{v} u \frac{\partial T}{\partial x}
$$

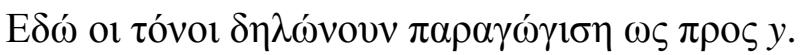

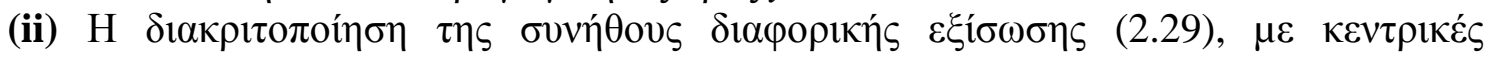

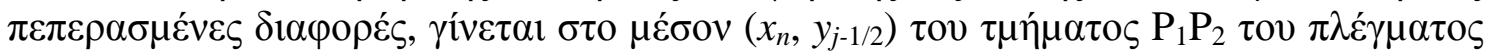

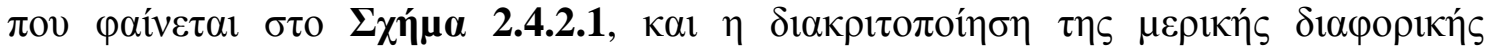

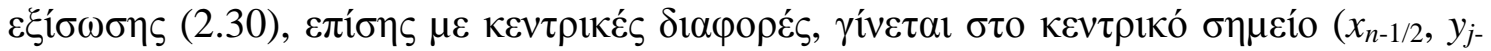

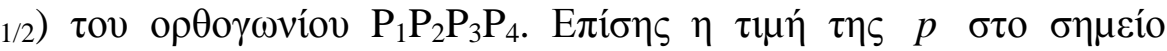
$(n, j-1 / 2)$

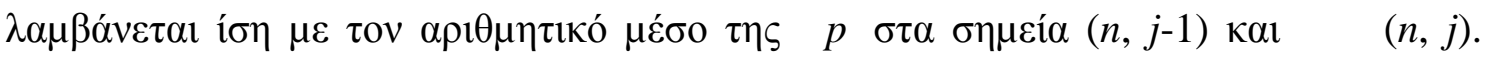

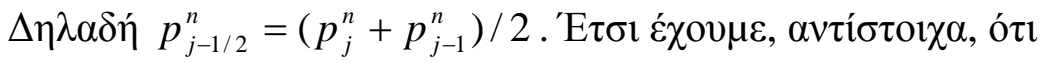

$$
\begin{gathered}
\frac{T_{j}^{n}-T_{j-1}^{n}}{2 h_{j} / 2}=\frac{p_{j}^{n}+p_{j-1}^{n}}{2}=p_{j-1 / 2}^{n}, \\
p_{j-1 / 2}^{\prime n-1 / 2}=\frac{p_{j-1 / 2}^{\prime n}+p_{j-1 / 2}^{\prime n-1}}{2}=\frac{1}{2}\left(\frac{p_{j}^{n}-p_{j-1}^{n}}{h_{j}}+\frac{p_{j}^{n-1}-p_{j-1}^{n-1}}{h_{j}}\right) \\
=\frac{\operatorname{Pr}}{v} u_{j-1 / 2}^{n-1 / 2} \frac{T_{j-1 / 2}^{n}-T_{j-1 / 2}^{n-1}}{k_{n}} .
\end{gathered}
$$

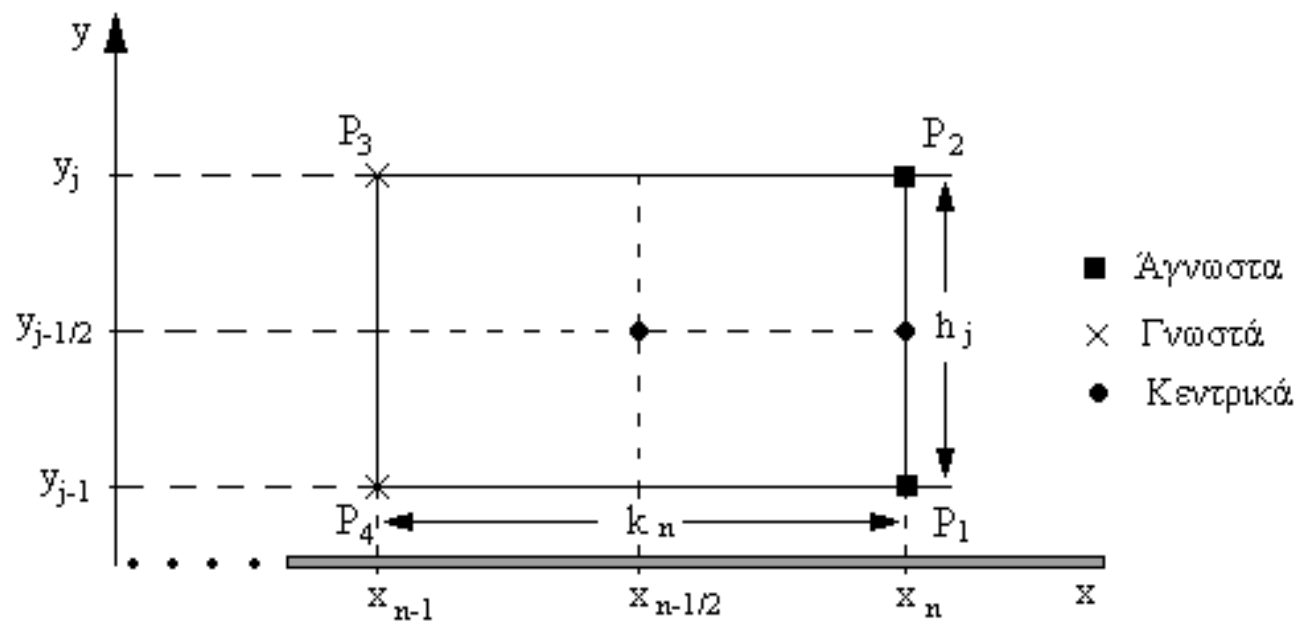

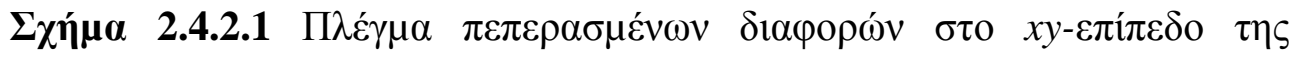

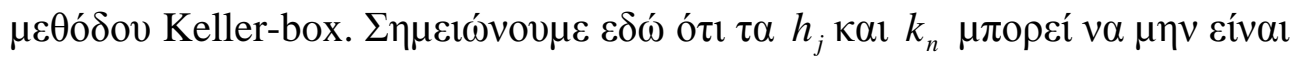

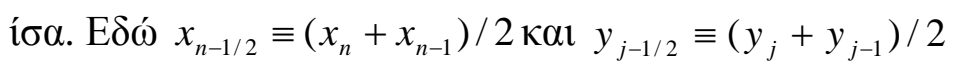




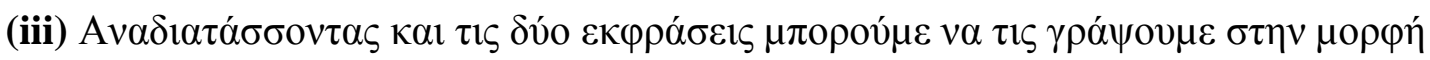

$$
\begin{aligned}
& T_{j}^{n}-T_{j-1}^{n}-\frac{h_{j}}{2}\left(p_{j}^{n}+p_{j-1}^{n}\right)=0 \\
& \left(s_{1}\right)_{j} p_{j}^{n}+\left(s_{2}\right)_{j} p_{j-1}^{n}+\left(s_{3}\right)_{j}\left(T_{j}^{n}+T_{j-1}^{n}\right)=R_{j-1 / 2}^{n-1}
\end{aligned}
$$

о́

$$
\begin{aligned}
& \left(s_{1}\right)_{j}=1,\left(s_{2}\right)_{j}=-1,\left(s_{3}\right)_{j}=-\frac{\lambda_{j}}{2} \\
& R_{j-1 / 2}^{n-1}=-\lambda_{j} T_{j-1 / 2}^{n-1}+p_{j-1}^{n-1}-p_{j}^{n-1} \kappa \alpha \iota \\
& \lambda_{j}=\frac{2 \operatorname{Pr}}{v} u_{j-1 / 2}^{n-1 / 2} \frac{h_{j}}{k_{n}}
\end{aligned}
$$

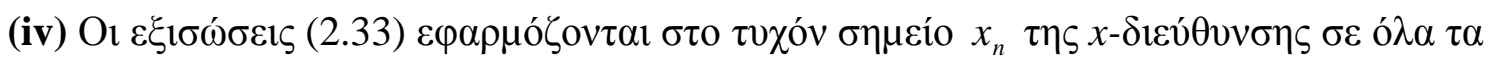

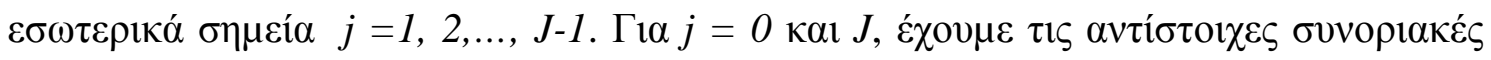

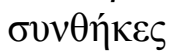

$$
T_{0}=T_{w}, T_{J}=T_{e}
$$

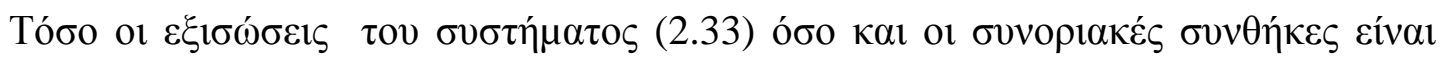

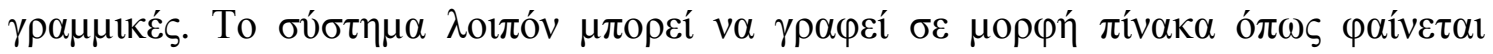

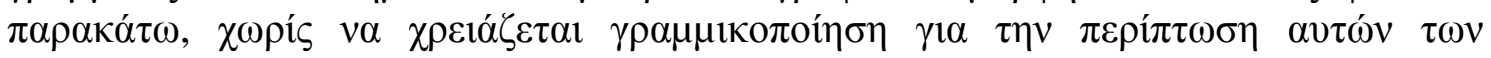
$\varepsilon \xi 1 \sigma \omega ́ \sigma \varepsilon \omega \nu$

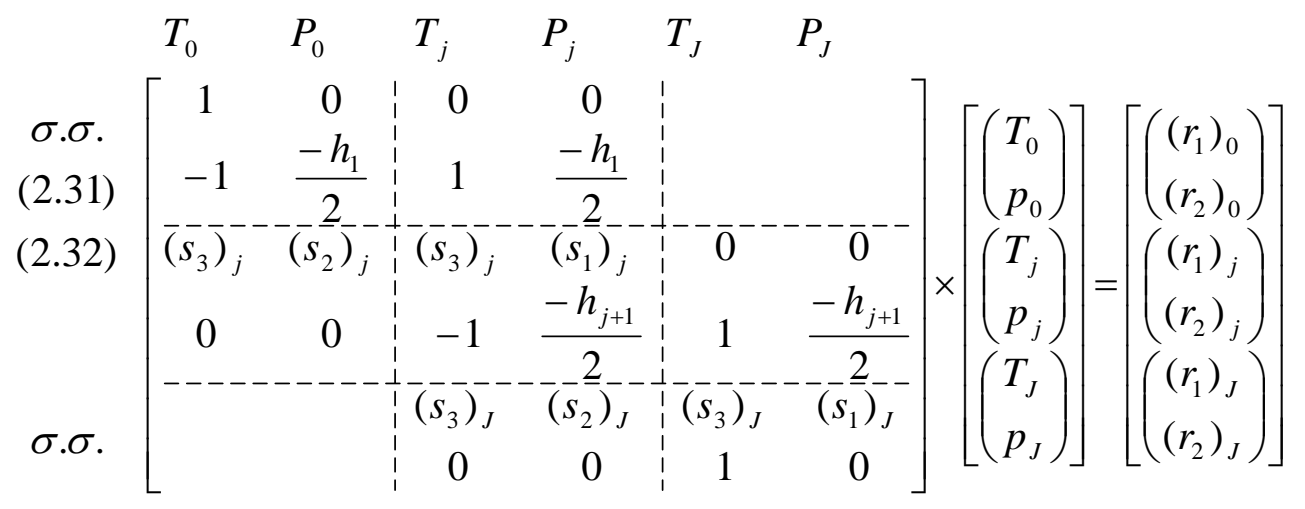

о́тоv

$$
\begin{aligned}
& \left(\mathrm{r}_{1}\right)_{0}=\mathrm{T}_{\mathrm{w}}, \quad\left(\mathrm{r}_{1}\right)_{\mathrm{j}}=\mathrm{R}_{\mathrm{j}-1 / 2}^{\mathrm{n}-1}, 1 \leq \mathrm{j} \leq \mathrm{J}, \\
& \left(\mathrm{r}_{2}\right)_{\mathrm{j}}=0,0 \leq \mathrm{j} \leq \mathrm{J}-1,\left(\mathrm{r}_{2}\right)_{\mathrm{J}}=\mathrm{T}_{\mathrm{e}}
\end{aligned}
$$

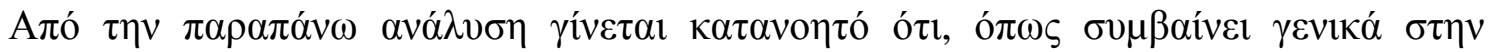

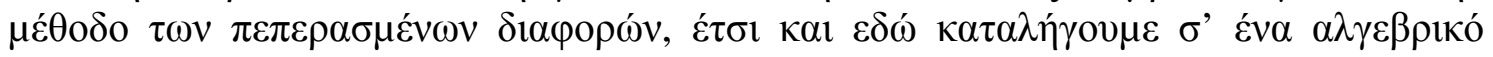

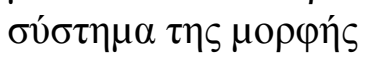

$$
\boldsymbol{A} \delta=\mathbf{r}
$$

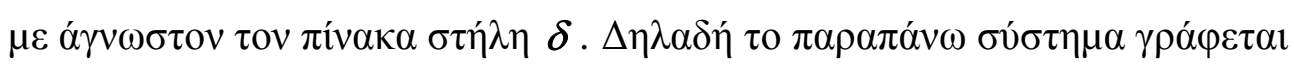




$$
\boldsymbol{A}=\left[\begin{array}{ccccccc}
A_{0} & C_{0} & & & & \\
B_{1} & A_{1} & C_{1} & & & \\
& & & & & \\
& & B_{j} & A_{j} & C_{j} & \\
& & & B_{J-1} & A_{J-1} & C_{J-1} \\
& & & & B_{J} & A_{J}
\end{array}\right], \quad \boldsymbol{\delta}=\left[\begin{array}{c}
\delta_{0} \\
\delta_{1} \\
\vdots \\
\delta_{j} \\
\vdots \\
\delta_{J}
\end{array}\right], \quad \boldsymbol{r}=\left[\begin{array}{c}
r_{0} \\
r_{1} \\
\vdots \\
r_{j} \\
\vdots \\
r_{J}
\end{array}\right] .
$$

O $\pi \mathrm{ov} \quad \boldsymbol{\delta}_{j} \equiv\left[\begin{array}{l}T_{j} \\ p_{j}\end{array}\right], \boldsymbol{r}_{j} \equiv\left[\begin{array}{l}\left(r_{1}\right)_{j} \\ \left(r_{2}\right)_{j}\end{array}\right]$

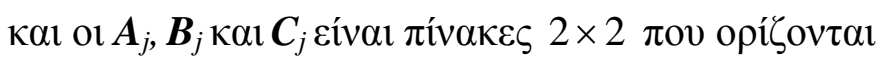

$$
\begin{aligned}
& \boldsymbol{A}_{0} \equiv\left[\begin{array}{cc}
1 & 0 \\
-1 & \frac{-h_{1}}{2}
\end{array}\right], \quad \boldsymbol{A}_{j} \equiv\left[\begin{array}{cc}
\left(s_{3}\right)_{j} & \left(s_{1}\right)_{j} \\
-1 & \frac{-h_{j+1}}{2}
\end{array}\right], \quad 1 \leq j \leq J-1, \\
& \boldsymbol{A}_{J} \equiv\left[\begin{array}{cc}
\left(s_{3}\right)_{J} & \left(s_{1}\right)_{J} \\
1 & 0
\end{array}\right], \boldsymbol{B}_{j} \equiv\left[\begin{array}{cc}
\left(\mathrm{s}_{3}\right)_{\mathrm{j}} & \left(\mathrm{s}_{2}\right)_{\mathrm{j}} \\
0 & 0
\end{array}\right], \quad 1 \leq j \leq J, \\
& \boldsymbol{C}_{j} \equiv\left[\begin{array}{cc}
0 & 0 \\
1 & \frac{-h_{j+1}}{2}
\end{array}\right], \quad 0 \leq j \leq J-1 .
\end{aligned}
$$

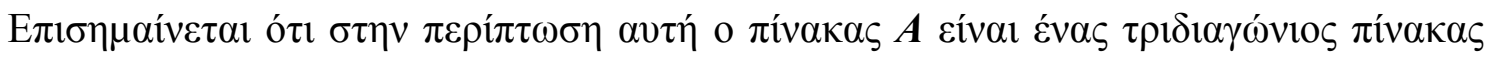

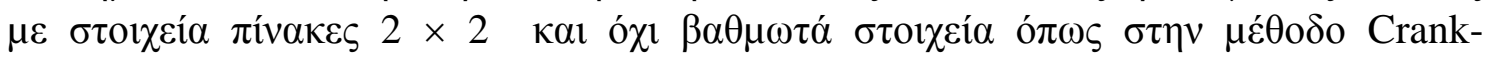

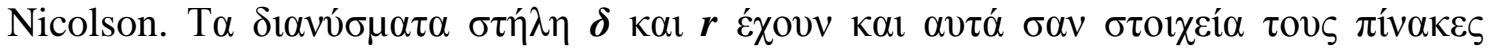

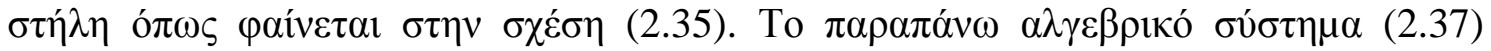

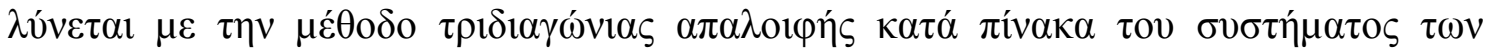

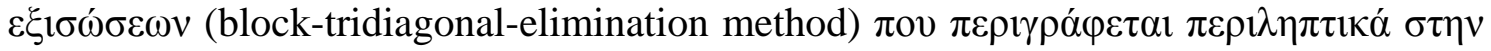
$\pi \alpha \rho \alpha \kappa \alpha ́ \tau \omega ~ \pi \alpha \rho \alpha ́ \gamma \rho \alpha \rho \circ$.

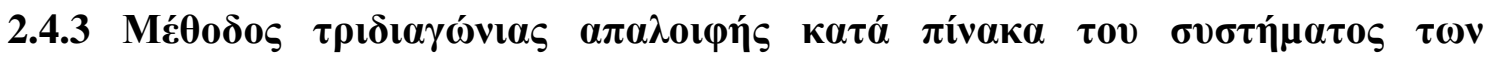 $\varepsilon \xi\llcorner\sigma \omega ́ \sigma \varepsilon \omega v$ (Block-tridiagonal-elimination method)}

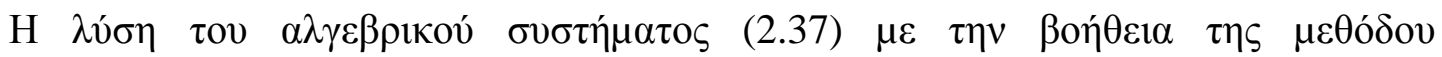

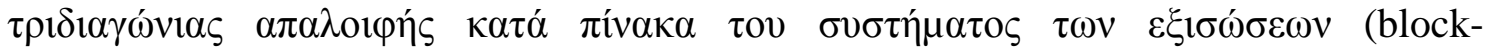

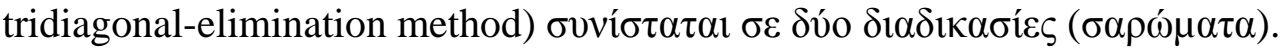

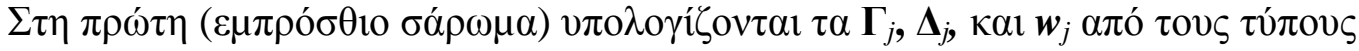

$$
\begin{aligned}
\Delta_{0} & =A_{0}, \\
\Gamma_{j} \Delta_{j-1} & =B_{j}, \\
\Delta_{j} & =A_{j}-\Gamma_{j} C_{j-1} \\
w_{0} & =r_{0} \\
w_{j} & =r_{j}-\Gamma_{j} w_{j-1}, \quad 1 \leq j \leq J .
\end{aligned}
$$




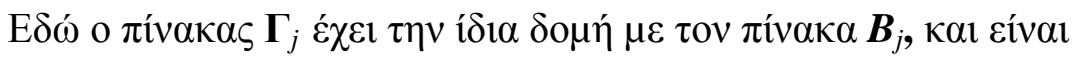

$$
\Gamma_{j} \equiv\left[\begin{array}{cc}
\left(\gamma_{11}\right)_{j} & \left(\gamma_{12}\right)_{j} \\
0 & 0
\end{array}\right] \text {. }
$$

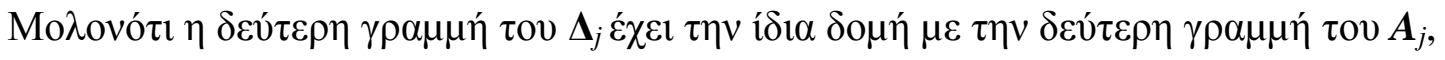

$$
\Delta_{j} \equiv\left[\begin{array}{cc}
\left(a_{11}\right)_{j} & \left(a_{12}\right)_{j} \\
-1 & \frac{-h_{j+1}}{2}
\end{array}\right]
$$

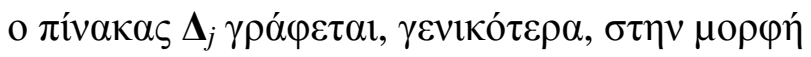

$$
\Delta_{j} \equiv\left[\begin{array}{ll}
\left(a_{11}\right)_{j} & \left(a_{12}\right)_{j} \\
\left(a_{21}\right)_{j} & \left(a_{22}\right)_{j}
\end{array}\right]
$$

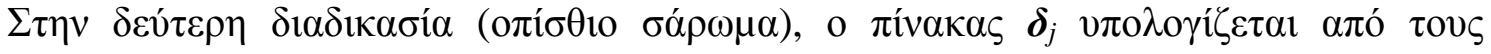

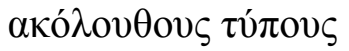

$$
\begin{aligned}
& \Delta_{J} \delta_{J}=w_{J}, \\
& \Delta_{j} \delta_{j}=w_{j}-C_{j} \delta_{j+1}, \quad j=J-1, J-2, \ldots, 0
\end{aligned}
$$

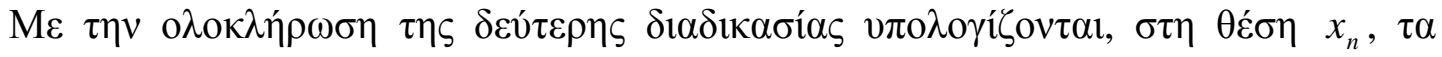

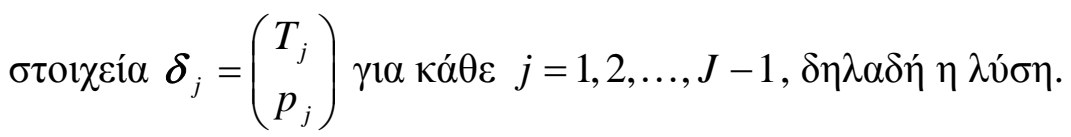

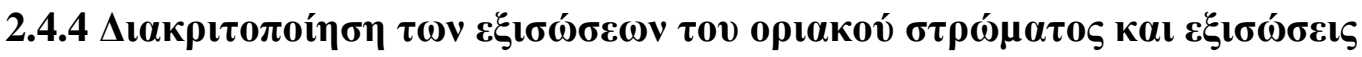

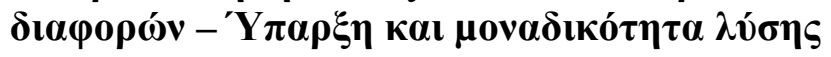

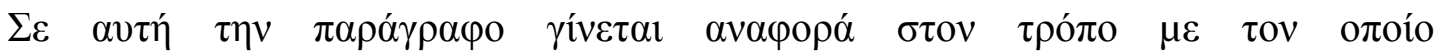

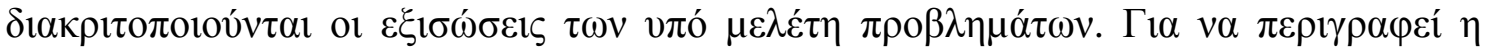

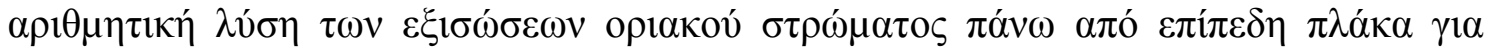

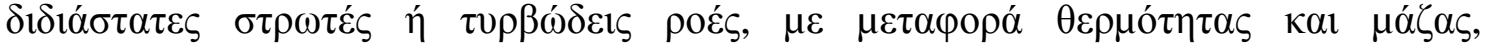

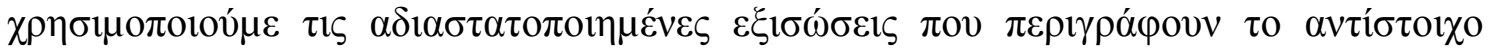

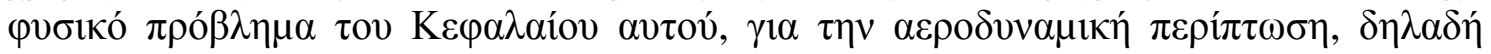

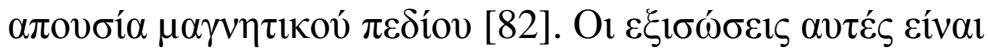

$$
\begin{gathered}
\left(b f^{\prime \prime}\right)^{\prime}+m_{1} f f^{\prime \prime}+m_{2}\left[c-\left(f^{\prime}\right)^{2}\right]=x\left(f^{\prime} \frac{\partial f^{\prime}}{\partial x}-f^{\prime \prime} \frac{\partial f}{\partial x}\right) \\
\left(e S^{\prime}+d f^{\prime} f^{\prime \prime}\right)^{\prime}+m_{1} f S^{\prime}=x\left(f^{\prime} \frac{\partial S}{\partial x}-S^{\prime} \frac{\partial f}{\partial x}\right)
\end{gathered}
$$

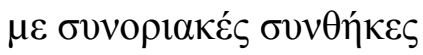




$$
\begin{aligned}
& \eta=0: f^{\prime}=0, f_{w}=f(0, x), S=S_{w}(x) \eta S^{\prime}=S_{w}^{\prime}(x), \\
& \eta=\eta_{e}: \quad f^{\prime}=1, \quad S=1 .
\end{aligned}
$$

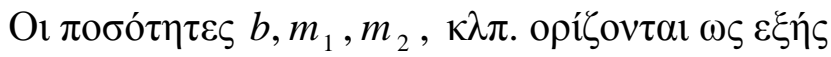

$$
\begin{gathered}
b=C, \quad C=\frac{\rho \mu}{\rho_{e} \mu_{e}}, \quad c=\frac{\rho_{e}}{\rho}, \quad d=\frac{C u_{e}^{2}}{H_{e}}\left(1-\frac{1}{\operatorname{Pr}}\right), \\
e=\frac{b}{\operatorname{Pr}}, S=\frac{H}{H_{e}}, \\
m_{1}=\frac{1}{2}\left[1+m_{2}+\frac{x}{\rho_{e} \mu_{e}} \frac{d}{d x}\left(\rho_{e} \mu_{e}\right)\right], m_{2}=\frac{x}{u_{e}} \frac{d u_{e}}{d x}, R_{x}=\frac{u_{e} x}{v_{e}} .
\end{gathered}
$$

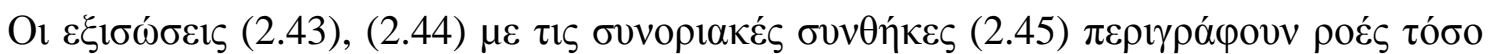

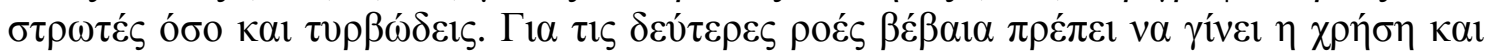

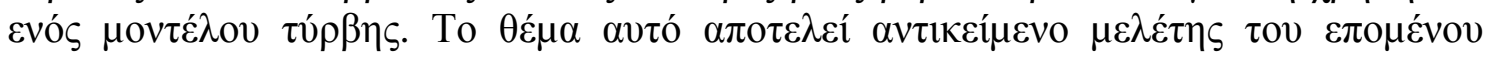

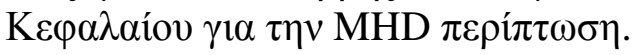

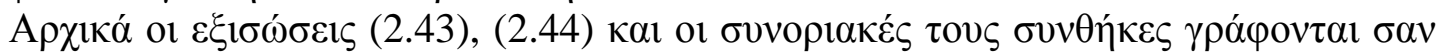

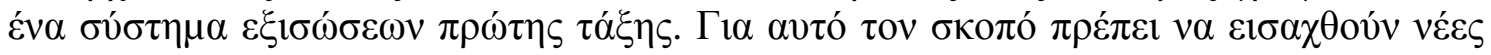

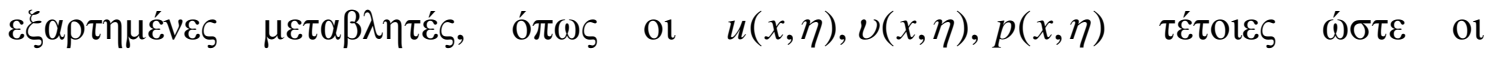

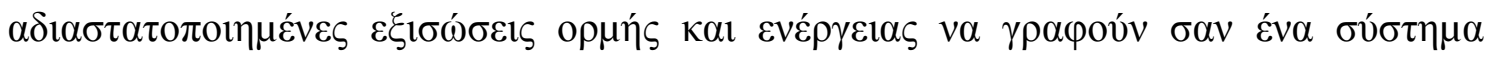

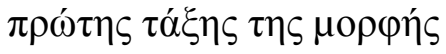

$$
\begin{gathered}
f^{\prime}=u, \\
u^{\prime}=v, \\
g^{\prime}=p, \\
(b v)^{\prime}+m_{1} f v+m_{2}\left[c-u^{2}\right]=x\left(u \frac{\partial u}{\partial x}-v \frac{\partial f}{\partial x}\right), \\
(e p+d u v)^{\prime}+m_{1} f p=x\left(u \frac{\partial g}{\partial x}-p \frac{\partial f}{\partial x}\right),
\end{gathered}
$$

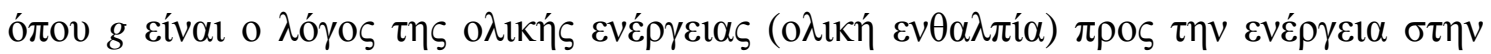

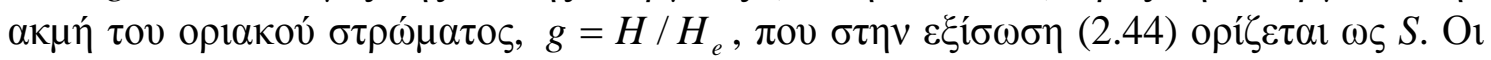

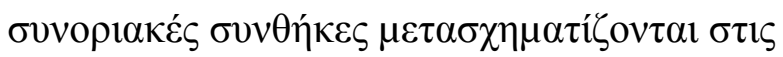

$$
\begin{aligned}
& f(x, 0)=f_{w}(x), \quad u(x, 0)=0, \quad g(x, 0)=g_{w}(x) \text { ฑं } p(x, 0)=p_{w}(x) \\
& u\left(x, \eta_{e}\right)=1, \quad g\left(x, \eta_{e}\right)=1
\end{aligned}
$$




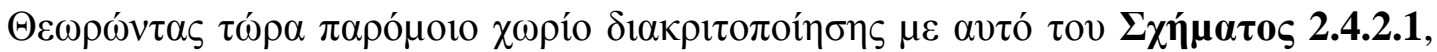

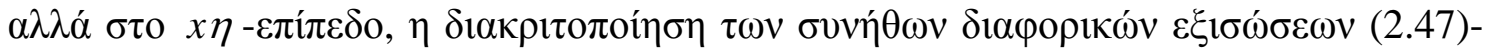

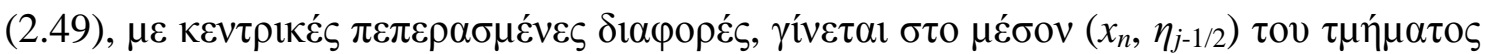

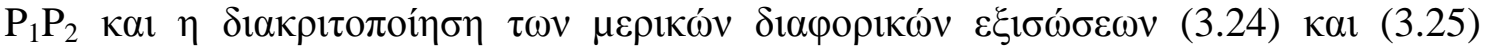

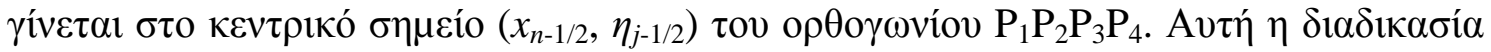

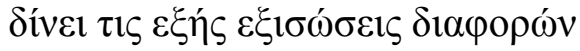

$$
\begin{gathered}
h_{j}^{-1}\left(f_{j}^{n}-f_{j-1}^{n}\right)=u_{j-1 / 2}^{n}, \\
h_{j}^{-1}\left(u_{j}^{n}-u_{j-1}^{n}\right)=v_{j-1 / 2}^{n}, \\
h_{j}^{-1}\left(g_{j}^{n}-g_{j-1}^{n}\right)=p_{j-1 / 2}^{n}, \\
h_{j}^{-1}\left(b_{j}^{n} v_{j}^{n}-b_{j-1}^{n} v_{j-1}^{n}\right)+\left(m_{1}^{n}+a_{n}\right)(f v)_{j-1 / 2}^{n}-\left(m_{2}^{n}+a_{n}\right)\left(u^{2}\right)_{j-1 / 2}^{n} \\
+a_{n}\left(v_{j-1 / 2}^{n-1} f_{j-1 / 2}^{n}-f_{j-1 / 2}^{n-1} v_{j-1 / 2}^{n}\right)=R_{j-1 / 2}^{n-1}, \\
h_{j}^{-1}\left(e_{j}^{n} p_{j}^{n}-e_{j-1}^{n} p_{j-1}^{n}\right)+h_{j}^{-1}\left(d_{j}^{n} u_{j}^{n} v_{j}^{n}-d_{j-1}^{n} u_{j-1}^{n} v_{j-1}^{n}\right)+\left(m_{1}^{n}+a_{n}\right)(f p)_{j-1 / 2}^{n} \\
-a_{n}\left[(u g)_{j-1 / 2}^{n}+u_{j-1 / 2}^{n-1} g_{j-1 / 2}^{n}-g_{j-1 / 2}^{n-1} u_{j-1 / 2}^{n}+f_{j-1 / 2}^{n-1} p_{j-1 / 2}^{n}-p_{j-1 / 2}^{n-1} f_{j-1 / 2}^{n}\right]=T_{j-1 / 2}^{n-1},
\end{gathered}
$$

ó $\pi 0 v$

$$
\begin{gathered}
a_{n}=\frac{x^{n-1 / 2}}{k_{n}} \\
R_{j-1 / 2}^{n-1}=-L_{j-1 / 2}^{n-1}+a_{n}\left[(f v)_{j-1 / 2}^{n-1}-\left(u^{2}\right)_{j-1 / 2}^{n-1}\right]-m_{2}^{n} c_{j-1 / 2}^{n}, \\
L_{j-1 / 2}^{n-1}=\left\{h_{j}^{-1}\left(b_{j} v_{j}-b_{j-1} v_{j-1}\right)+m_{1}(f v)_{j-1 / 2}+m_{2}\left[c_{j-1 / 2}-\left(u^{2}\right)_{j-1 / 2}\right]\right\}^{n-1}, \\
T_{j-1 / 2}^{n-1}=-M_{j-1 / 2}^{n-1}+a_{n}\left[(f p)_{j-1 / 2}^{n-1}-(u g)_{j-1 / 2}^{n-1}\right], \\
M_{j-1 / 2}^{n-1}=\left[h_{j}^{-1}\left(e_{j} p_{j}-e_{j-1} p_{j-1}\right)+h_{j}^{-1}\left(d_{j} u_{j} v_{j}-d_{j-1} u_{j-1} v_{j-1}\right)+m_{1}(f p)_{j-1 / 2}\right]^{n-1} .
\end{gathered}
$$

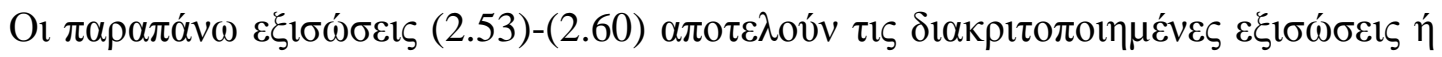

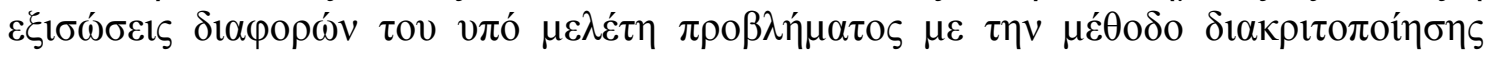
Keller-box.

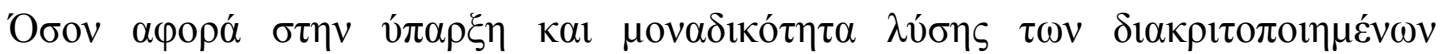

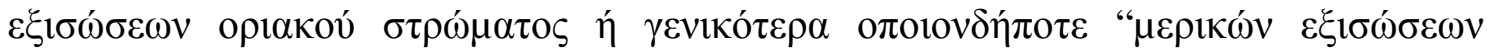

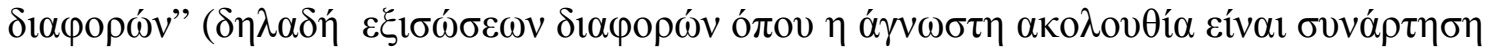

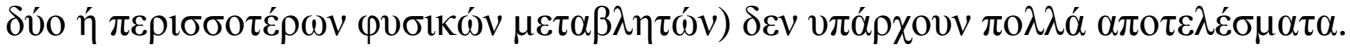

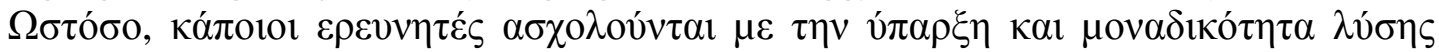

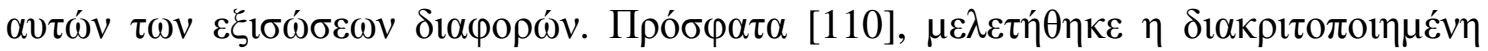

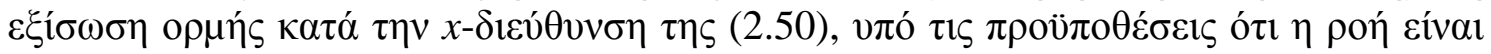

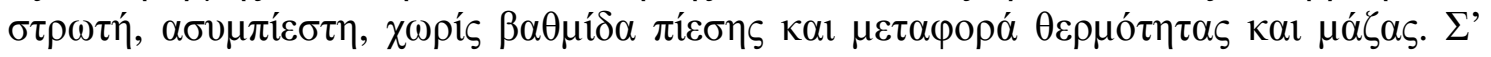

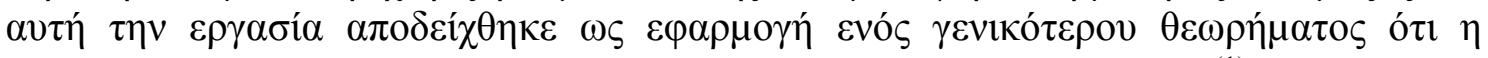

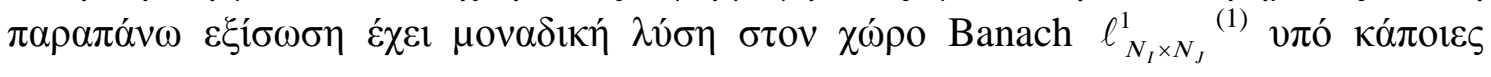

(1) $\ell_{N_{I} \times N_{J}}^{1}=\left\{f(i, j): N_{I} \times N_{J} \rightarrow R / \sum_{i=1}^{I} \sum_{j=1}^{J}|f(i, j)|<+\infty\right\}$ 


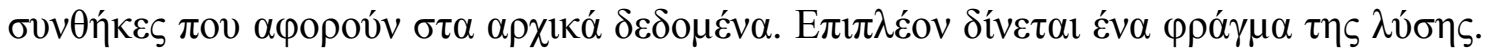

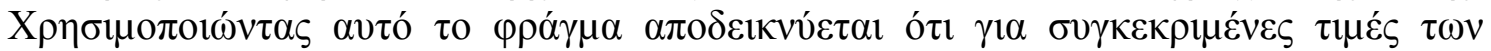

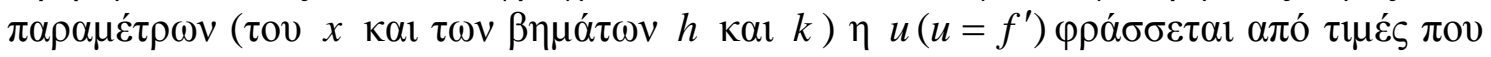

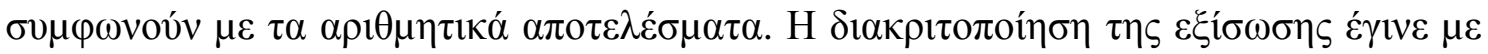

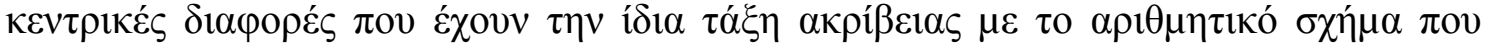

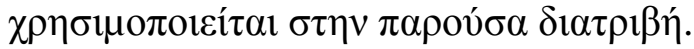

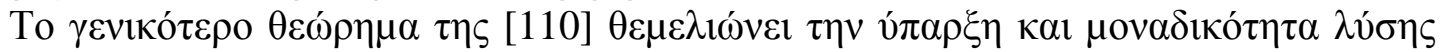

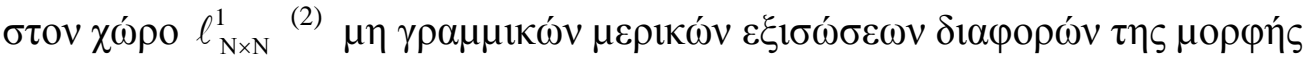

$$
\begin{aligned}
& \sum_{n=1}^{N} a_{n}(i, j) f\left(i-\sigma_{n 1}, j-\tau_{n 1}\right)+\sum_{m=1}^{M} b_{m}(i, j) f\left(i+\sigma_{m 2}, j+\tau_{m 2}\right)+ \\
& \sum_{k=1}^{K} c_{k}(i, j) f\left(i-\sigma_{k 3}, j+\tau_{k 3}\right)+\sum_{l=1}^{\Lambda} d_{l}(i, j) f\left(i+\sigma_{l 4}, j-\tau_{l 4}\right)=p(i, j)+ \\
& \sum_{s=2}^{\infty} g_{s}(i, j)[f(i+\sigma, j+\tau)]^{s}+\sum_{t=1}^{T} q_{t}(i, j) f\left(i+\sigma_{t 5}, j+\tau_{t 5}\right) f\left(i+\sigma_{t 6}, j+\tau_{t 6}\right),
\end{aligned}
$$

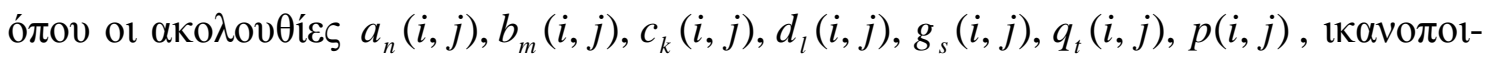

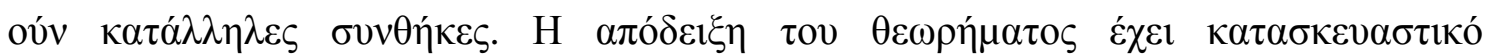

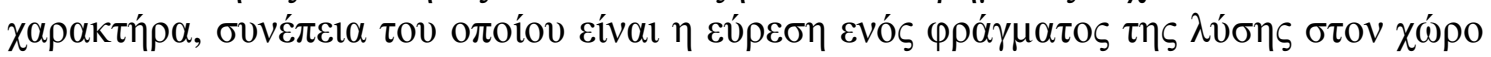

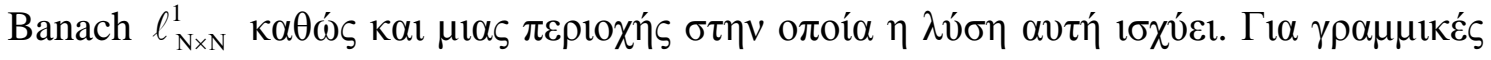

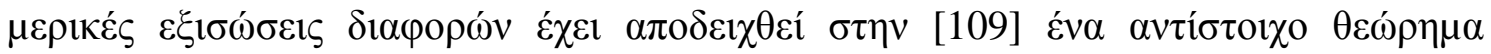

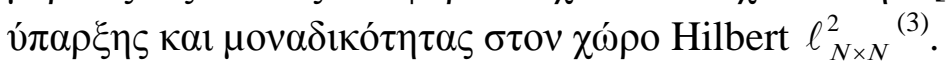

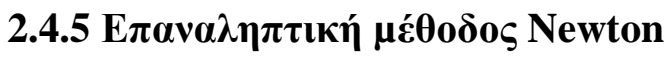

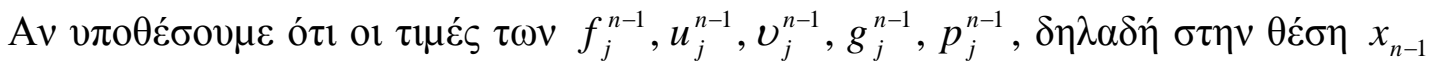

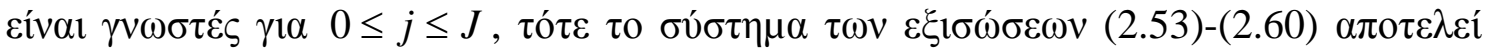

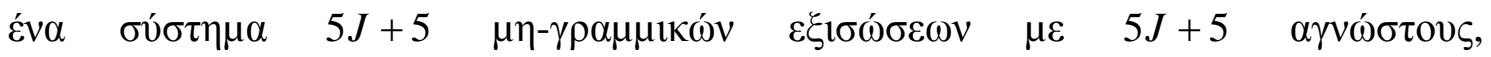

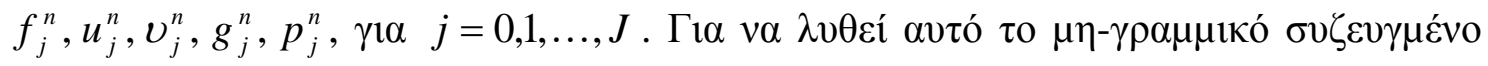

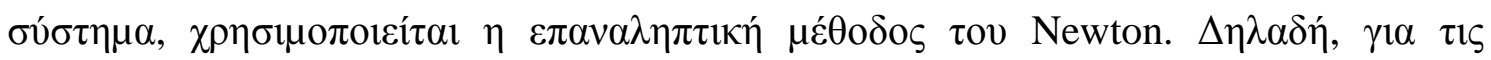

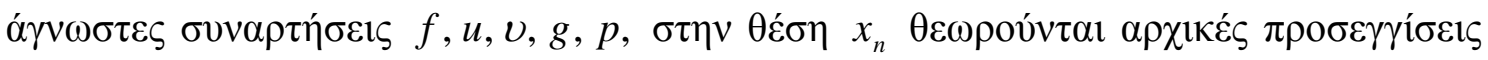

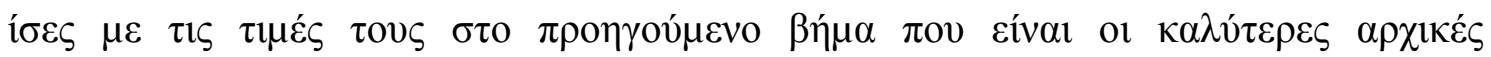

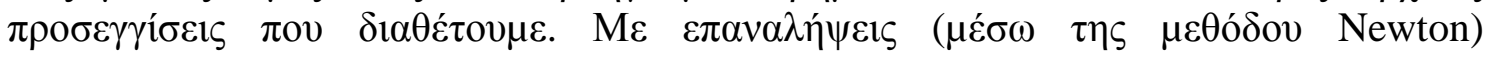

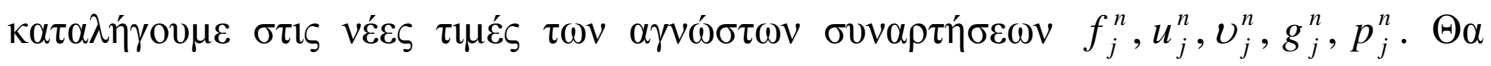

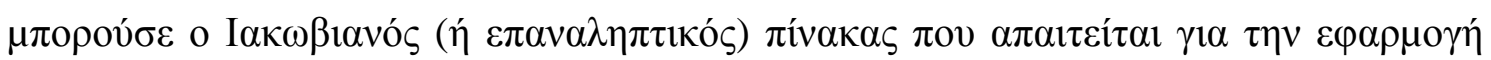

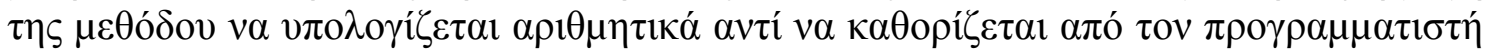

$\begin{aligned} & \text { (2) } \ell_{N \times N}^{1}=\left\{f(i, j): N \times N \rightarrow C / \sum_{i=1}^{\infty} \sum_{j=1}^{\infty}|f(i, j)|<+\infty\right\} \\ & \text { (3) } \ell_{N \times N}^{2}=\left\{f(i, j): N \times N \rightarrow C / \sum_{i=1}^{\infty} \sum_{j=1}^{\infty}|f(i, j)|^{2}<+\infty\right\}\end{aligned}$ 


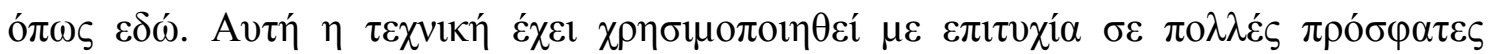

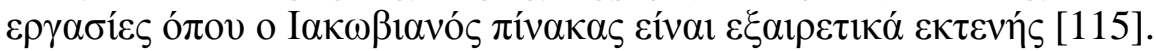

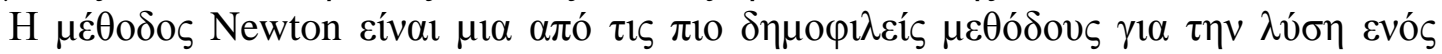

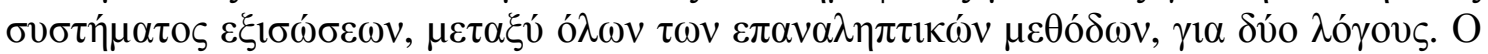

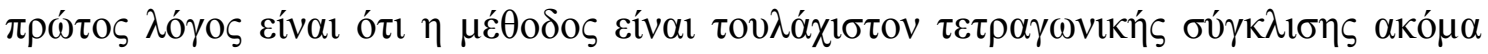

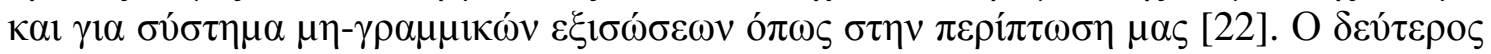

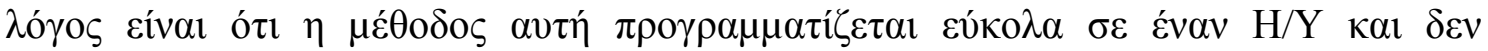

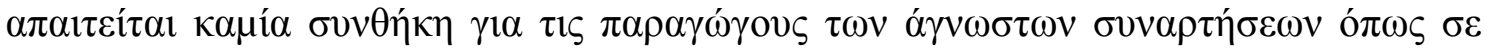

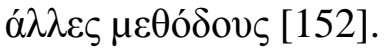

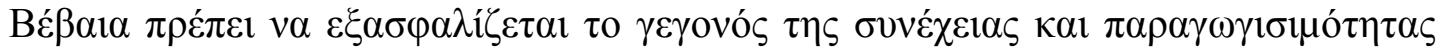

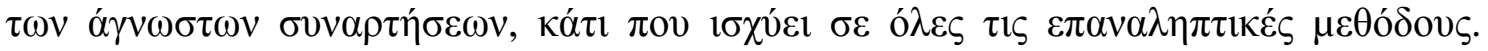

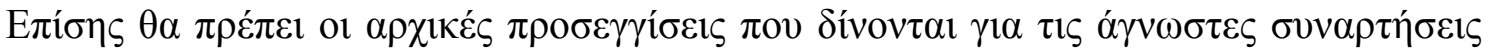

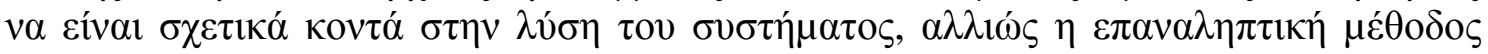

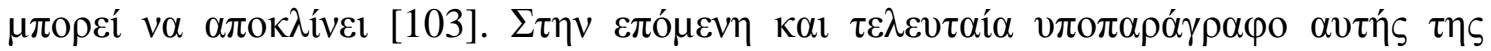

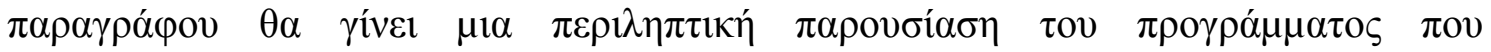

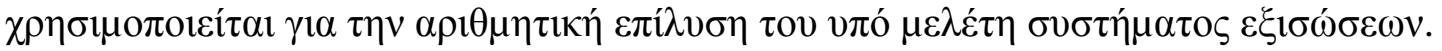

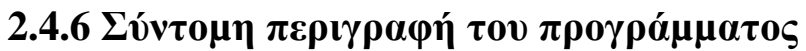

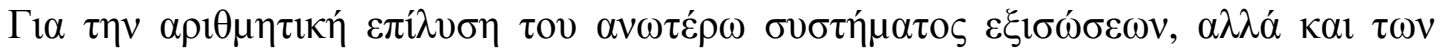

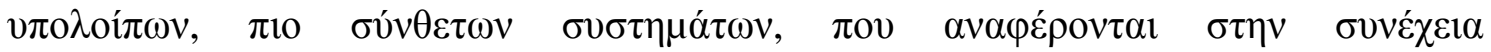

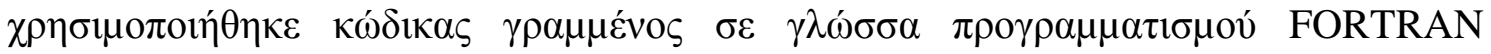

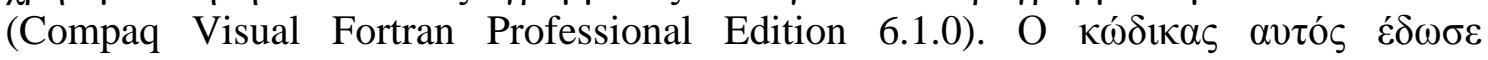

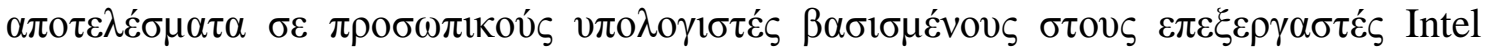

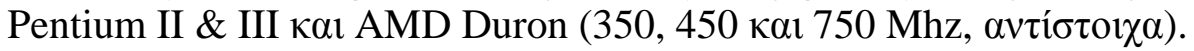

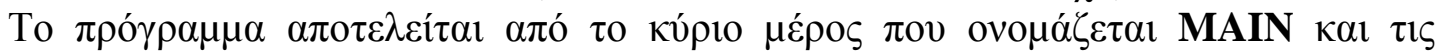

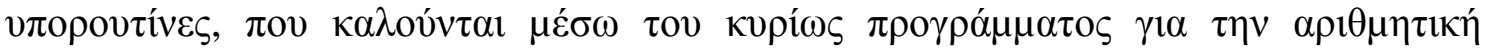

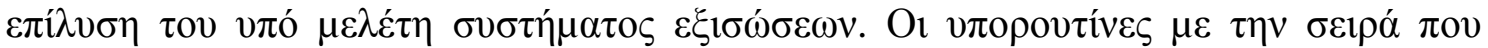

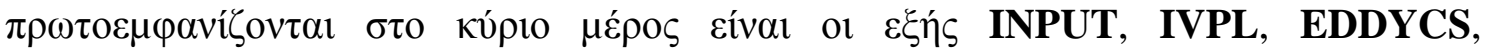

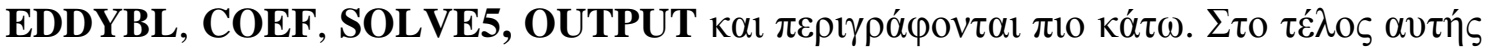

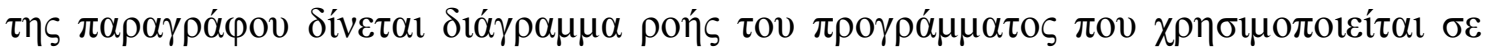
$\alpha v \tau \eta \dot{~} \tau \eta \nu \delta 1 \alpha \tau \rho ß \beta \dot{~}$

\section{MAIN}

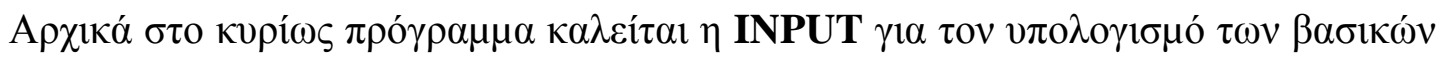

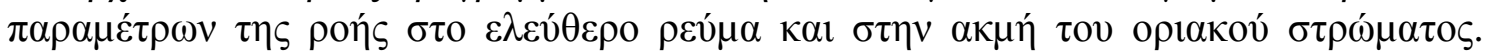

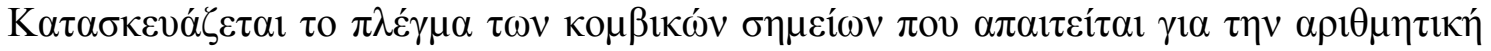

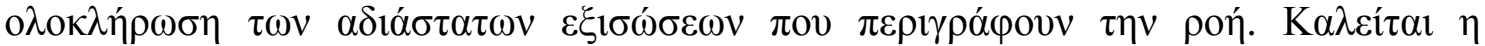

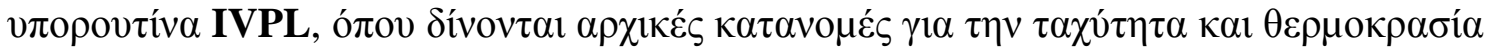

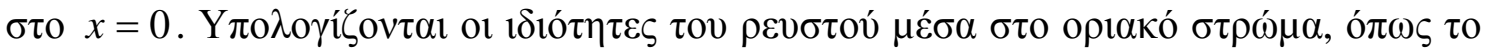

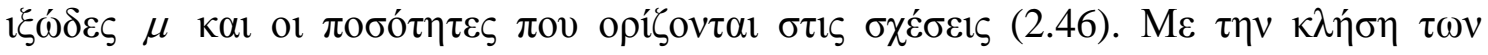

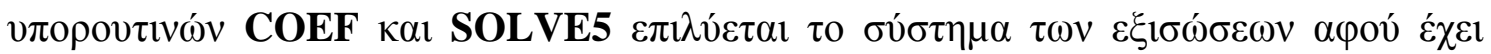

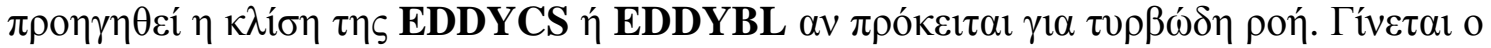

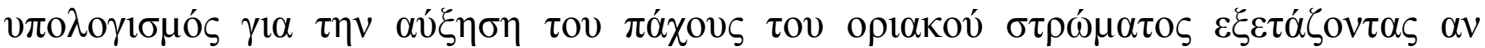

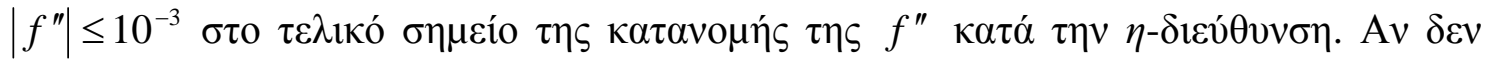

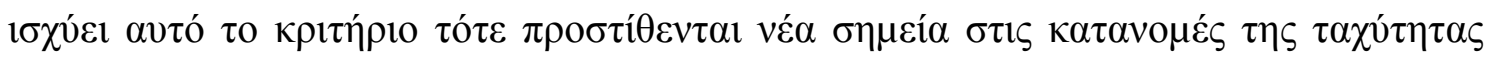

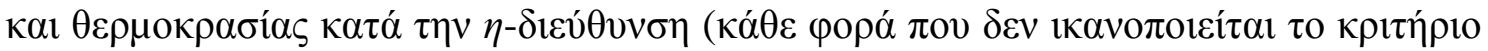

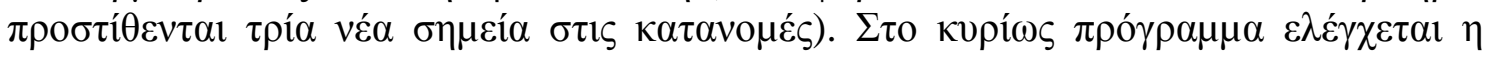




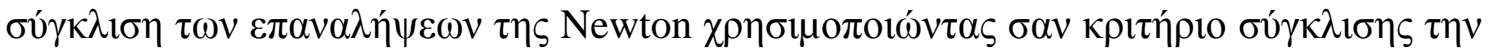

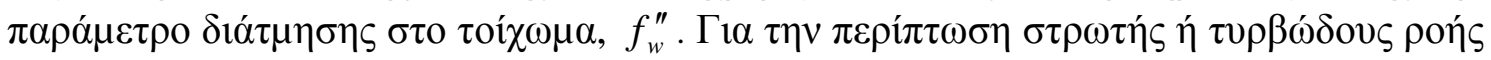

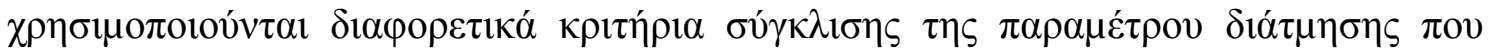

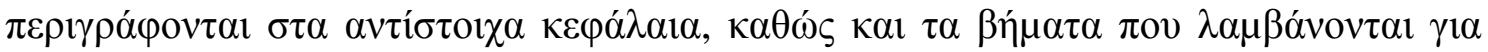

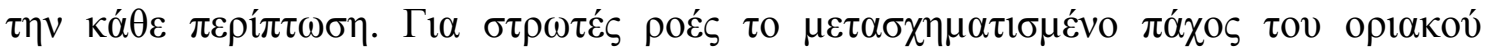

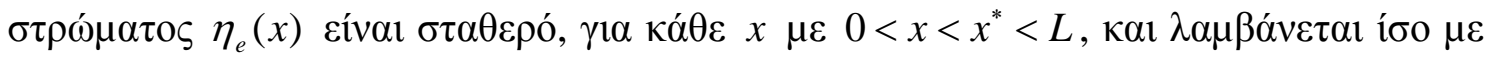

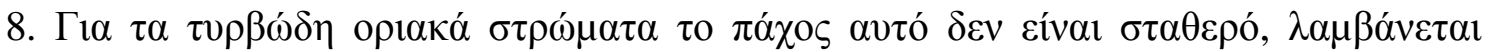

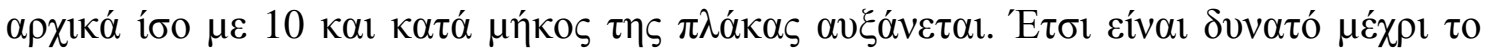

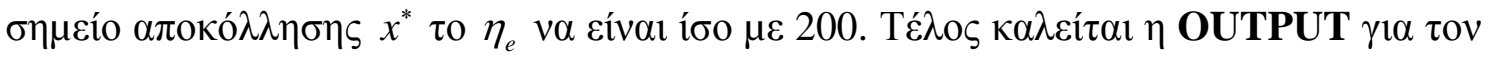

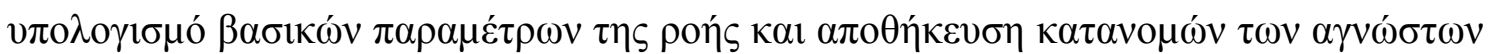
$\sigma v v \alpha \rho \tau \eta \dot{\sigma \varepsilon} \omega \nu$ ( $\tau \alpha \chi v ́ \tau \eta \tau \alpha, \theta \varepsilon \rho \mu о \kappa \rho \alpha \sigma i ́ \alpha, \kappa \lambda \pi$.).

\section{INPUT}

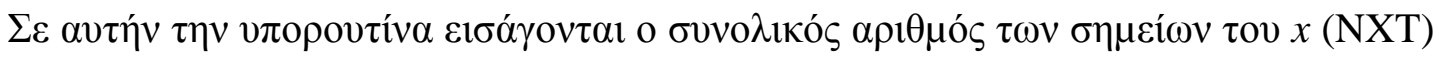

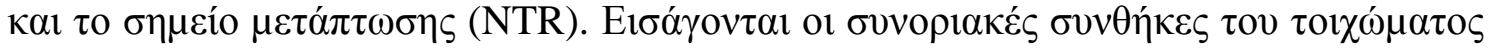

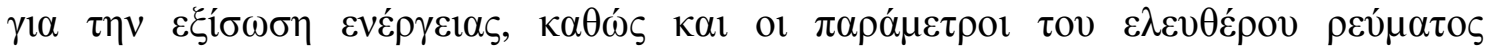

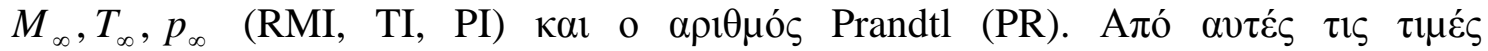

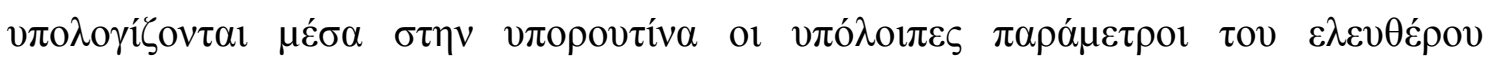

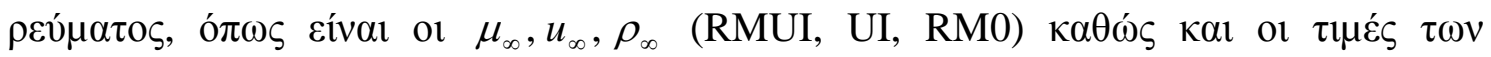

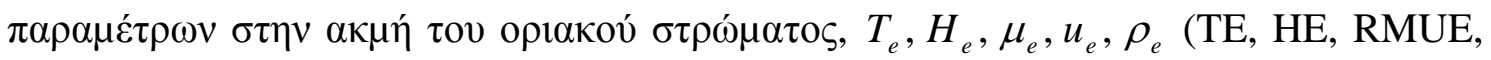

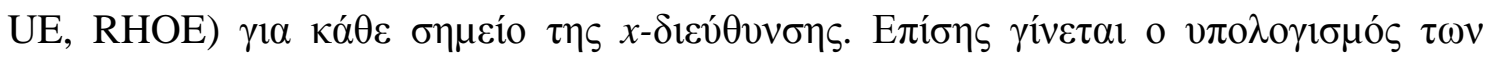

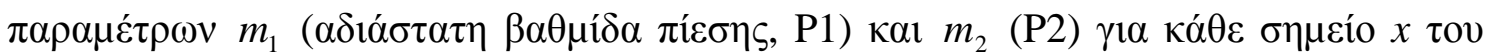

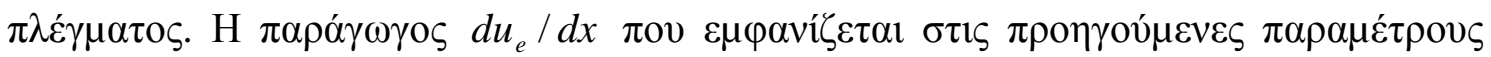

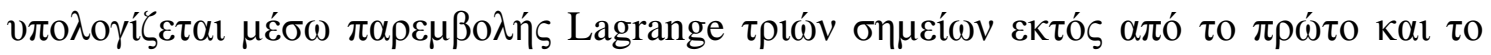
$\tau \varepsilon \lambda \varepsilon v \tau \alpha$ ío [29].

\section{IVPL}

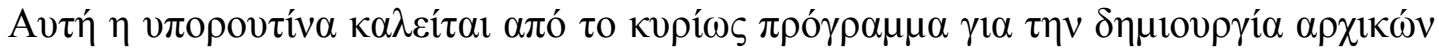

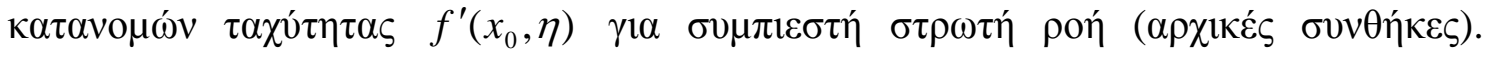

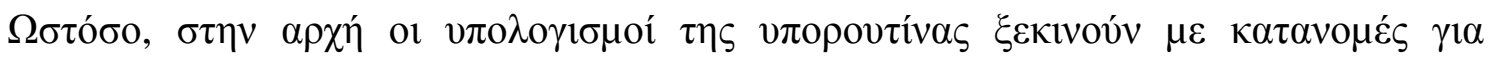

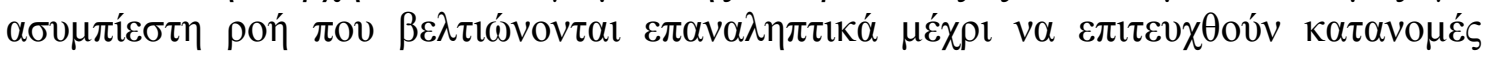

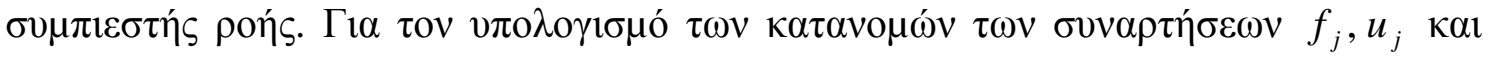

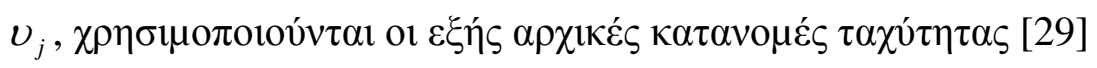

$$
\begin{aligned}
& f_{j}=\frac{\eta_{e}}{4}\left(\frac{\eta_{j}}{\eta_{e}}\right)^{2}\left[3-\frac{1}{2}\left(\frac{\eta_{j}}{\eta_{e}}\right)^{2}\right], \\
& u_{j}=\frac{1}{2} \frac{\eta_{j}}{\eta_{e}}\left[3-\left(\frac{\eta_{j}}{\eta_{e}}\right)^{2}\right], \\
& v_{j}=\frac{3}{2} \frac{1}{\eta_{e}}\left[1-\left(\frac{\eta_{j}}{\eta_{e}}\right)^{2}\right] .
\end{aligned}
$$

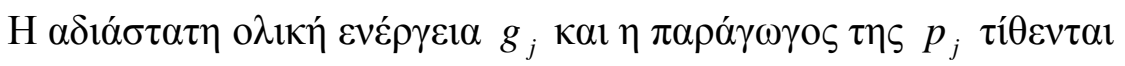




$$
\begin{gathered}
g_{j}=\frac{\eta_{j}}{\eta_{e}}, \\
p_{j}=\frac{1}{\eta_{e}} .
\end{gathered}
$$

\section{EDDYCS $\kappa \alpha 1$ EDDYBL}

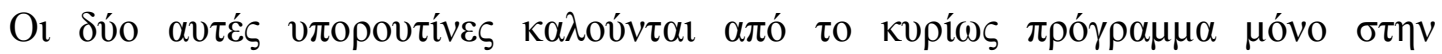

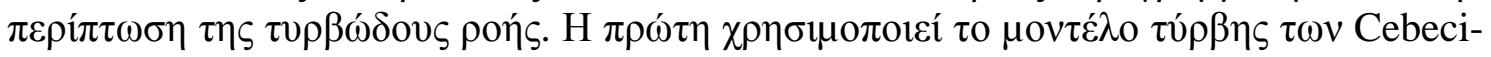

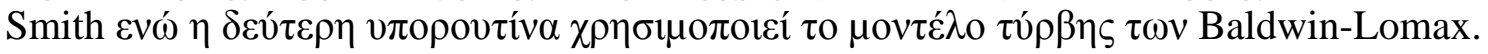

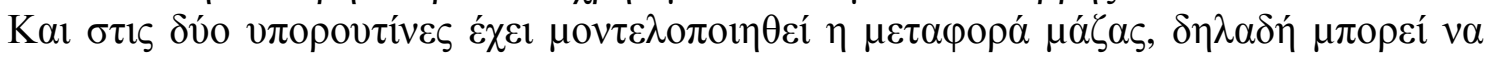

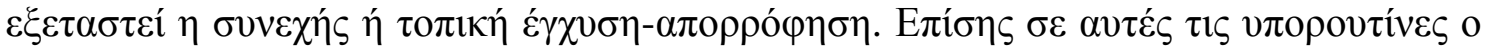

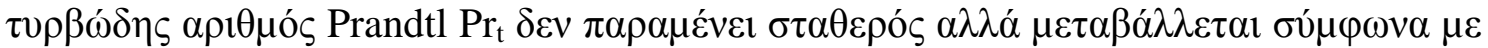

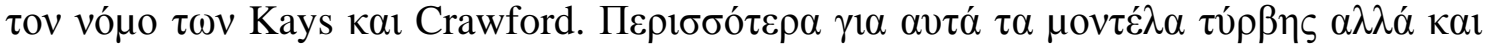

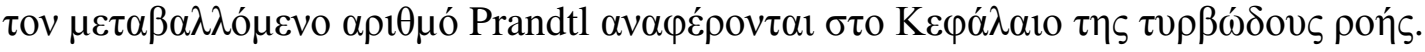

\section{COEF}

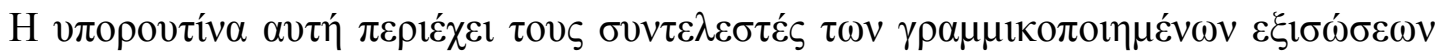

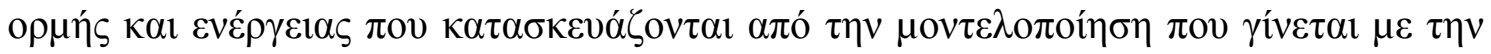

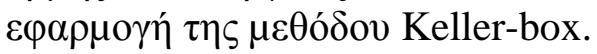

\section{SOLVE5}

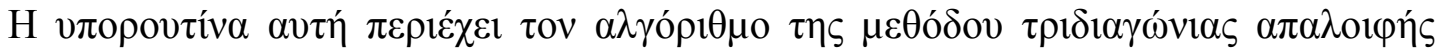

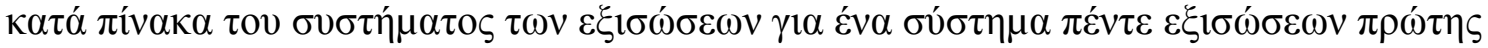

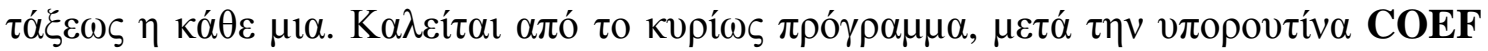

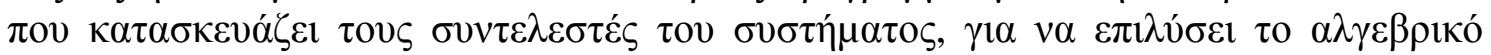

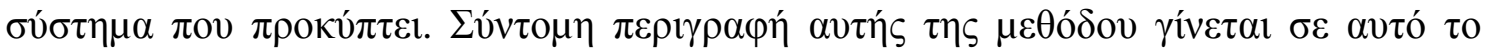

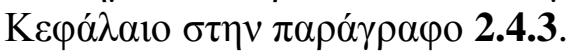

\section{OUTPUT}

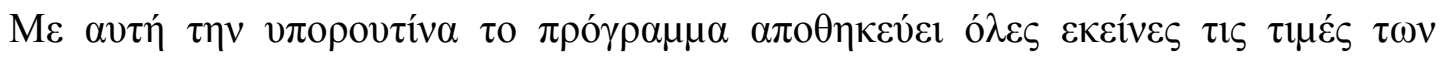

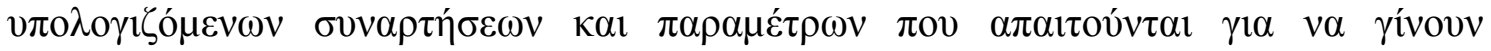

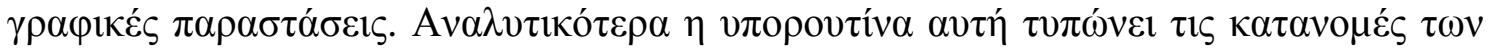

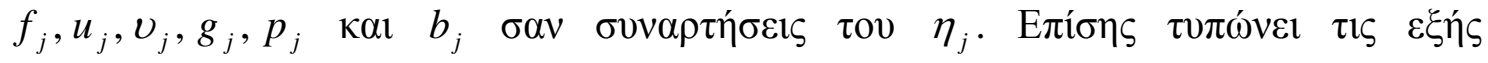

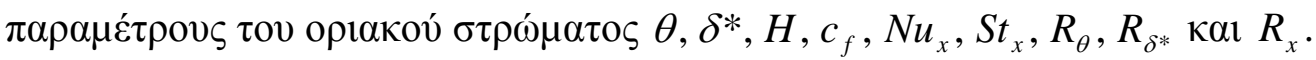




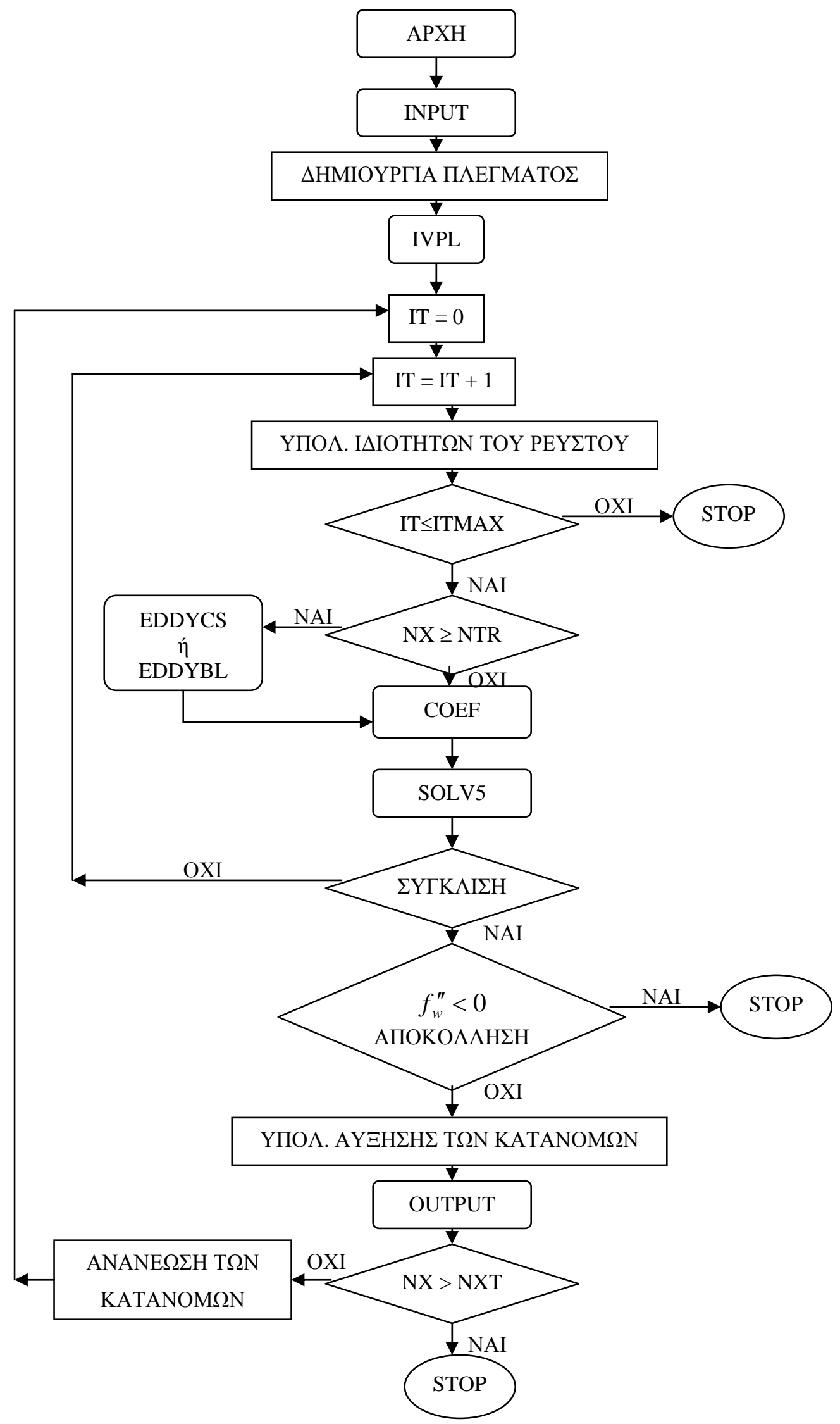

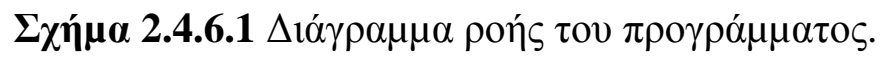




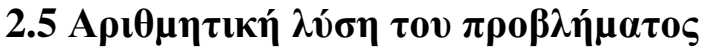

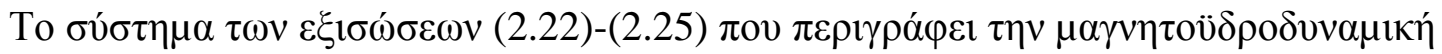

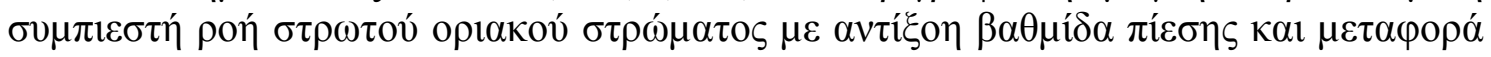

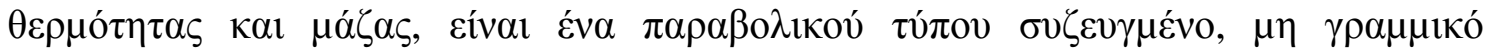

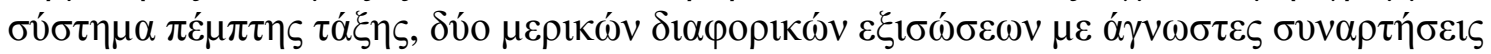

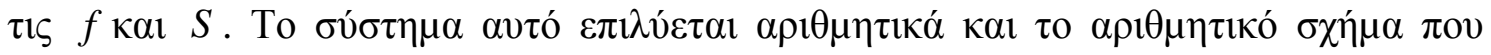

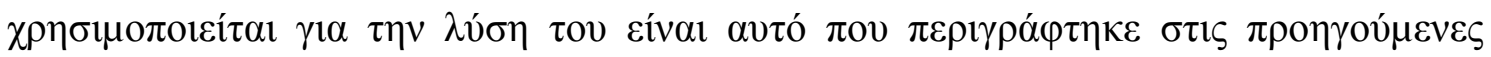

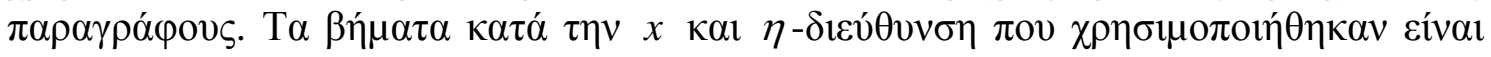

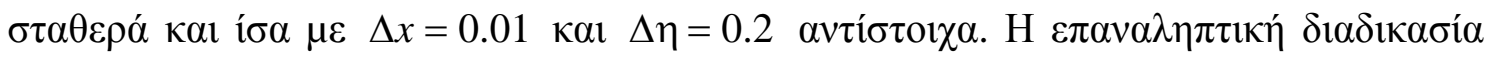

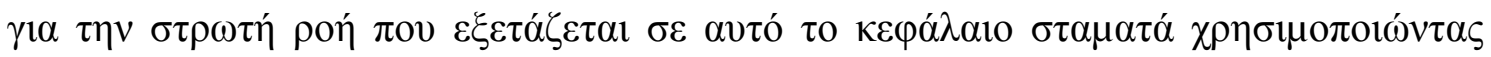

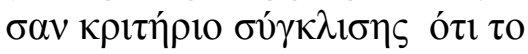

$$
\left|\delta f_{w}^{\prime \prime}\right|<10^{-5}
$$

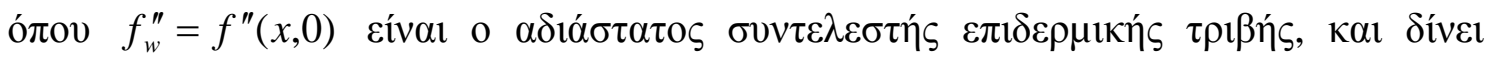

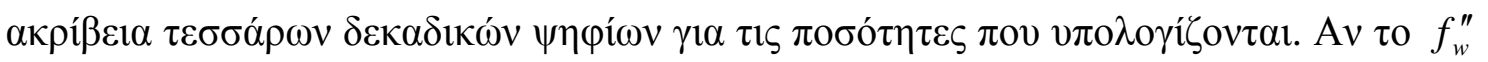

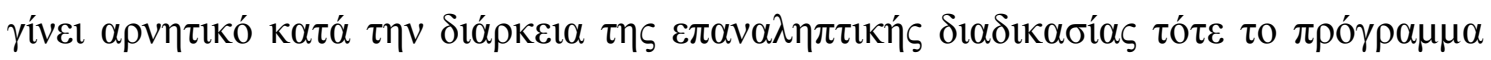
$\sigma \tau \alpha \mu \alpha \tau \alpha ́$.

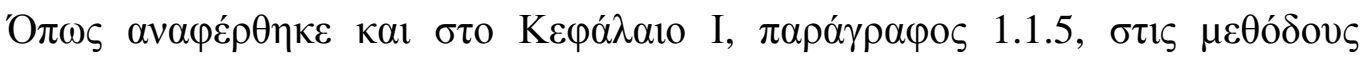

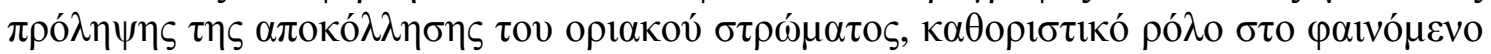

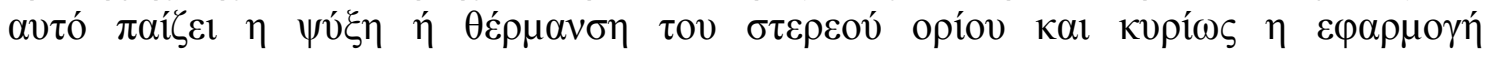

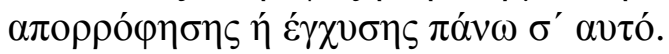

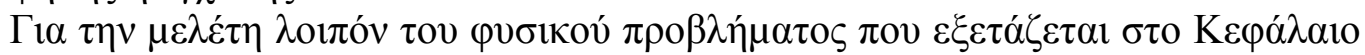

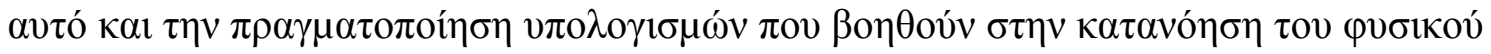

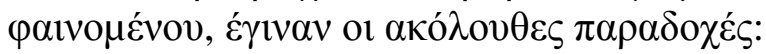

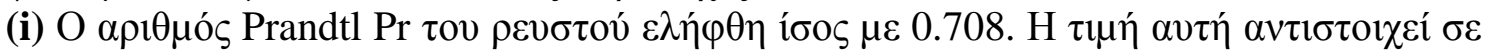

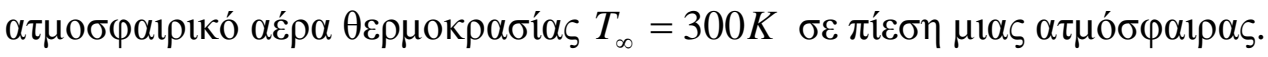

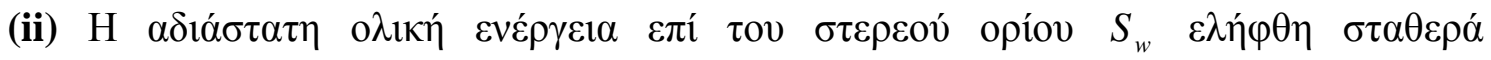

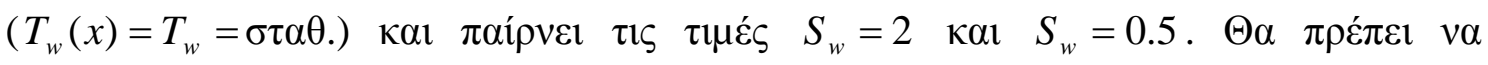

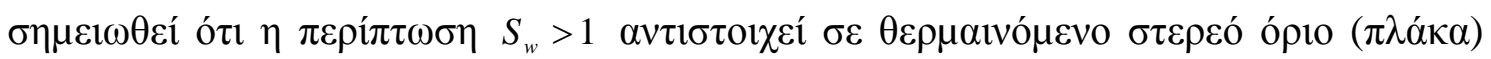

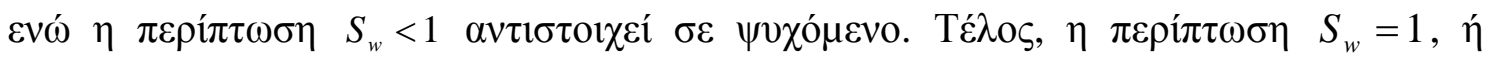

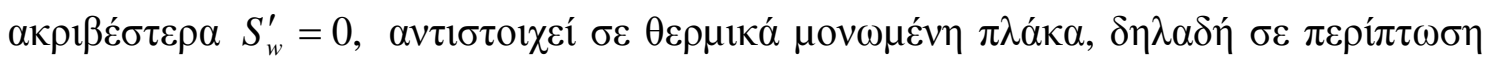

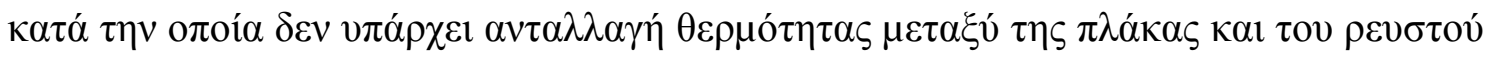
( $\alpha \delta \iota \alpha \beta \alpha \tau \iota \kappa \eta ́)$.

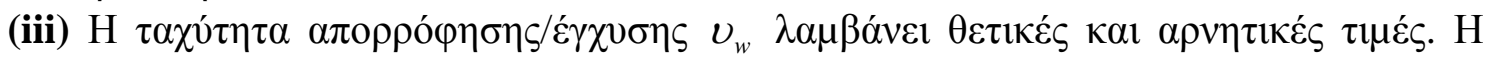

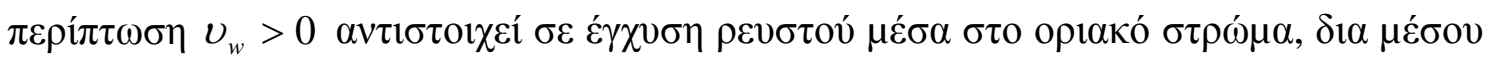

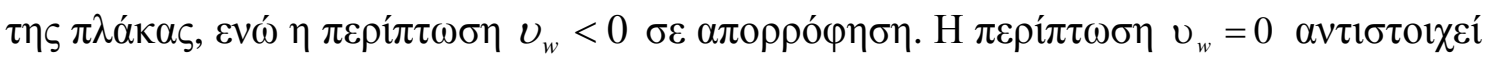

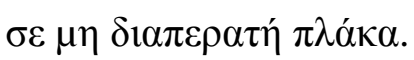

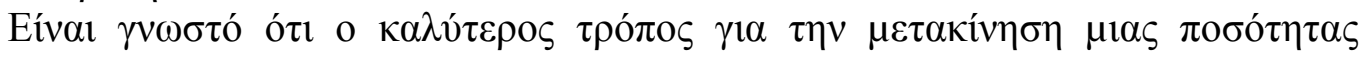

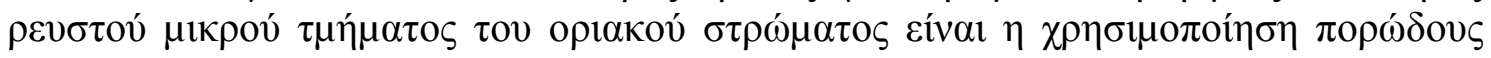

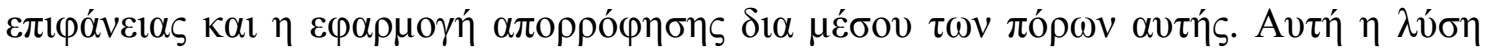

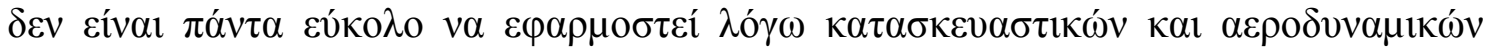

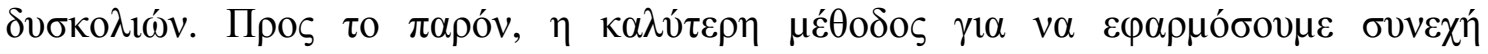
$\alpha \pi$

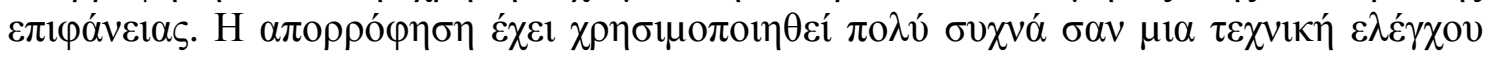




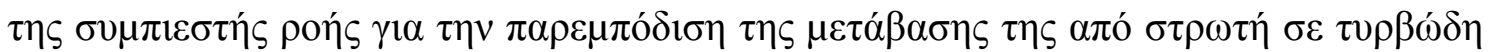

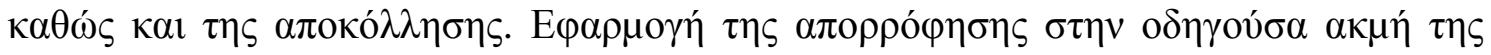

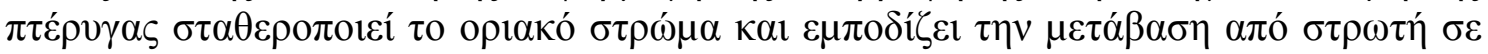

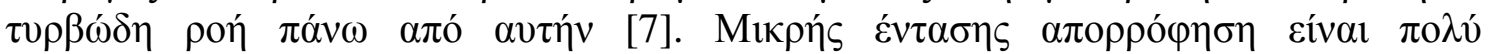

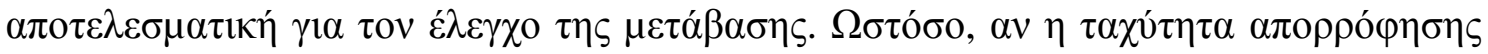

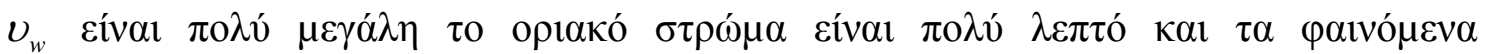

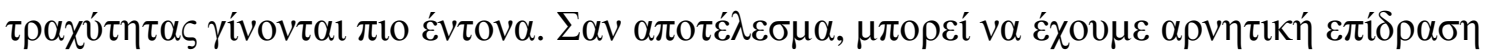

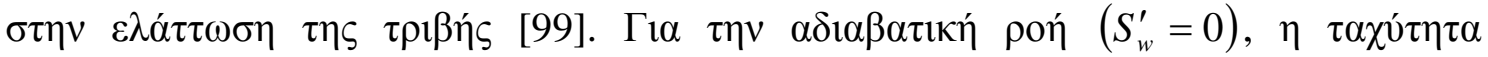

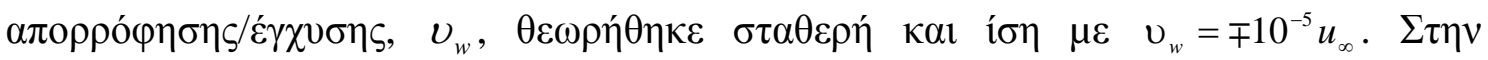

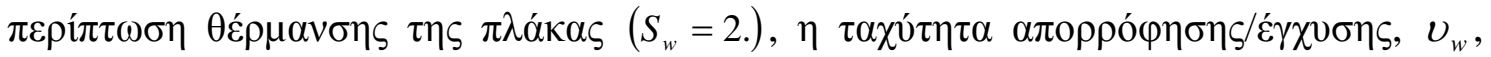

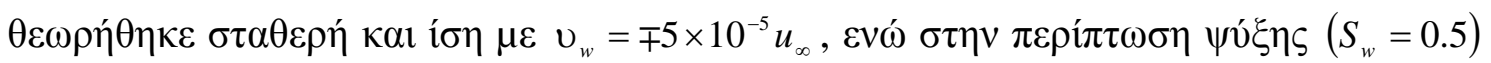

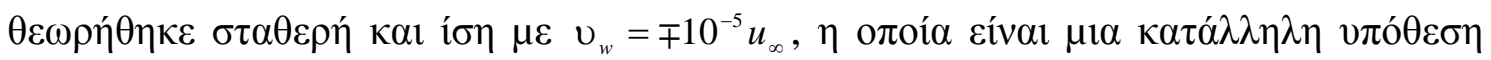

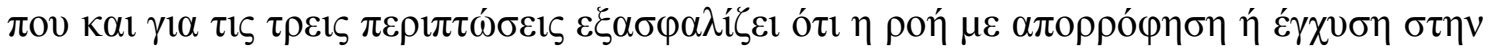

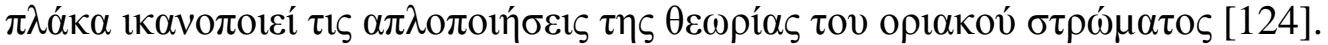

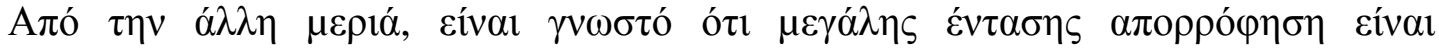

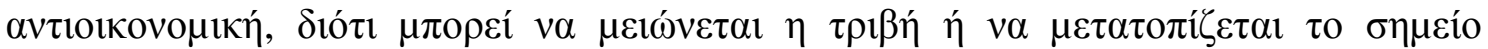

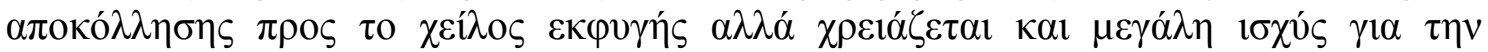

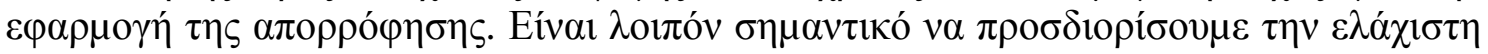

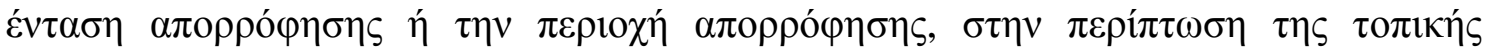

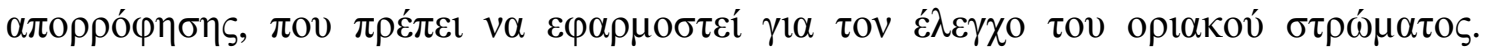

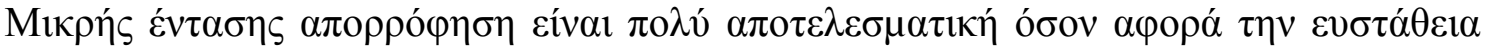

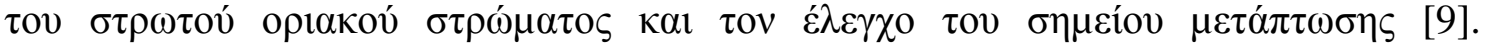

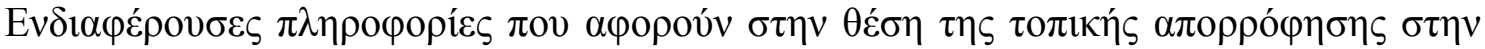

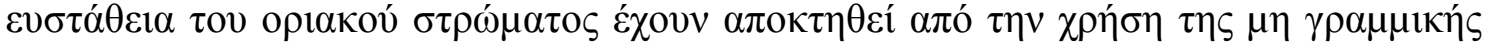

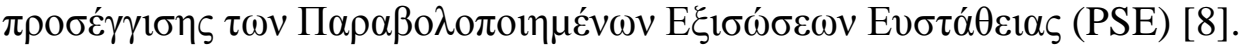

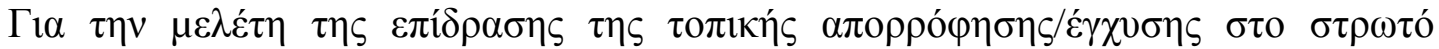

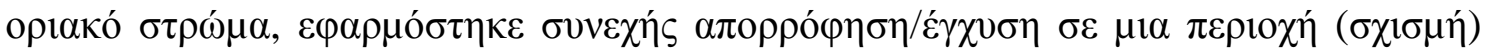

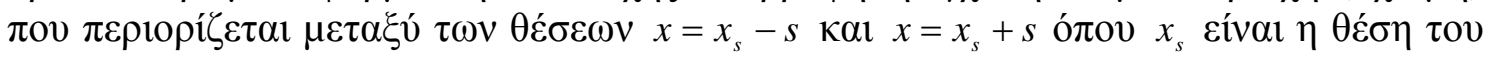

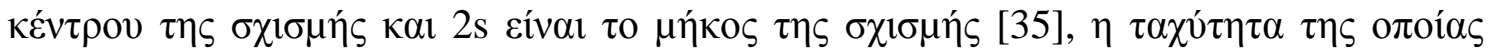

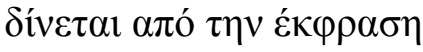

$$
\mathrm{v}_{w}(x)=\mp A_{s} e^{-\left(x-x_{s}\right)^{2} / s^{2}},
$$

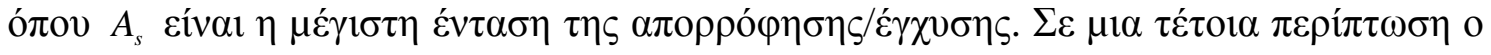

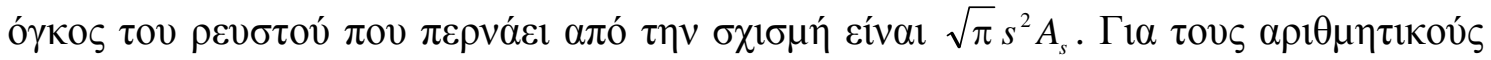

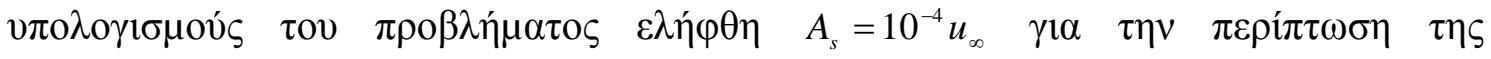

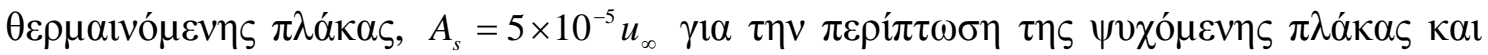

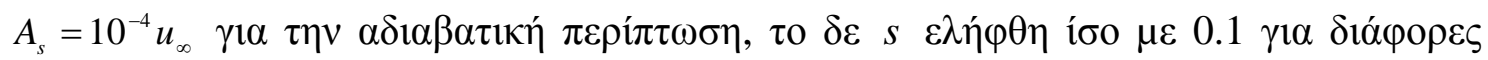
$\tau \mu \dot{\varepsilon} \varsigma \tau \mathrm{ov} x_{s}$.

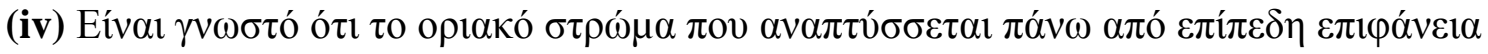

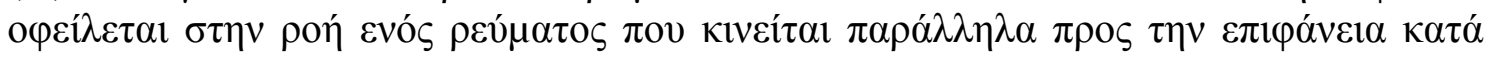

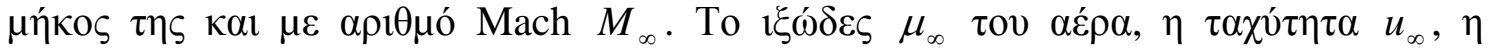

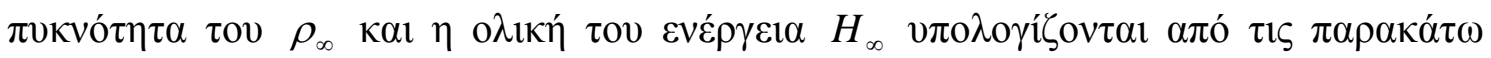
$\sigma \chi \varepsilon \dot{\varepsilon \varepsilon 1 \varsigma}$ 


$$
\begin{aligned}
\mu_{\infty} & =1.45 \times 10^{-6} T_{\infty}^{3 / 2} /\left(T_{\infty}+110.33\right), \quad \text { (Nó } \cos \tau \text { ov Sutherland) } \\
u_{\infty} & =20.04 M_{\infty} \sqrt{T_{\infty}} \\
\rho_{\infty} & =p_{\infty} / 287 T_{\infty} \\
H_{\infty} & =1005.7 T_{\infty}+\frac{1}{2} u_{\infty}^{2}, \quad\left(c_{p}=1005.7\right)
\end{aligned}
$$

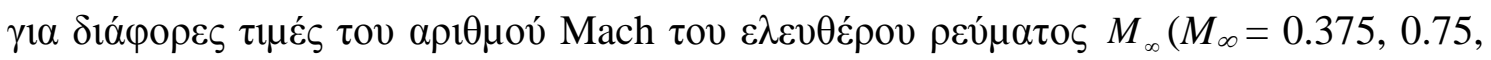

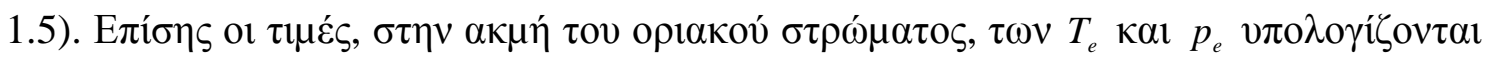
$\alpha \pi$ ó $\tau 0 \cup \varsigma \tau u ́ \pi 0 \cup \varsigma$ [29]

$$
T_{e}=T_{\infty}\left\{1-\frac{\gamma-1}{2} M_{\infty}^{2}\left[\left(\frac{u_{e}}{u_{\infty}}\right)^{2}-1\right]\right\}, \quad p_{e}=p_{\infty}\left(\frac{T_{e}}{T_{\infty}}\right)^{\gamma /(\gamma-1)},
$$

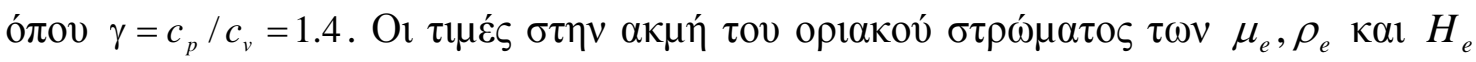

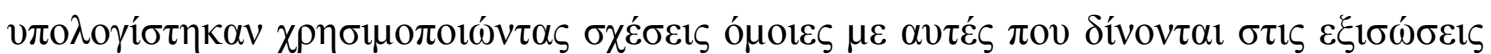

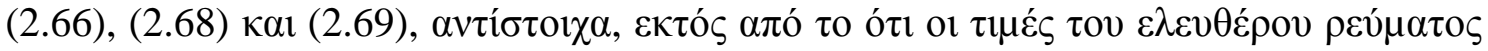

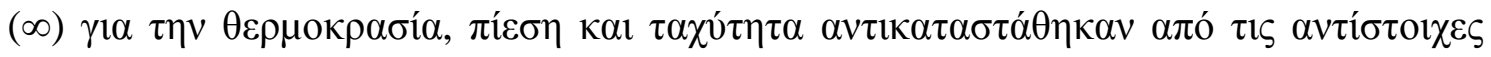
$\tau \mu \dot{\varepsilon} \varsigma \tau \eta \zeta \alpha \kappa \mu \eta ́ \varsigma(e)$.

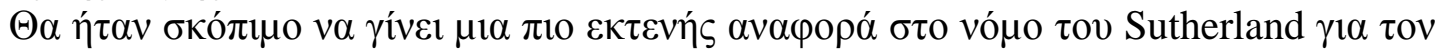

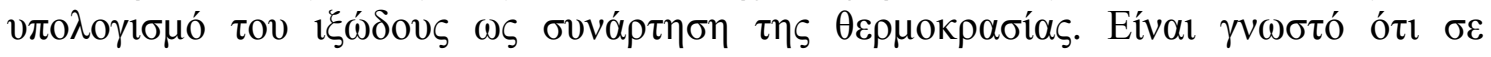

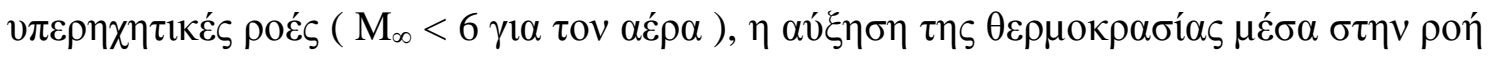

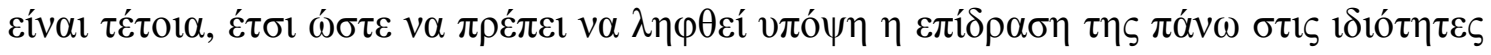

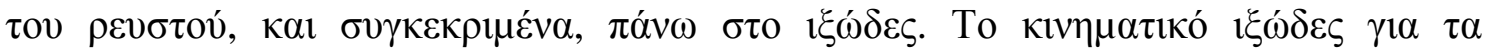

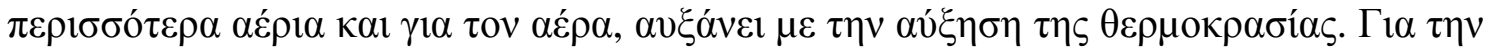

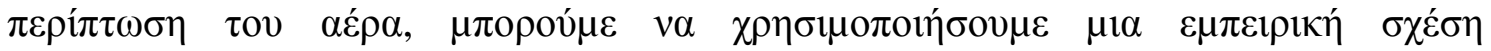

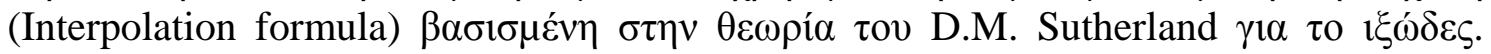

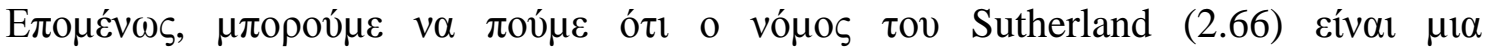

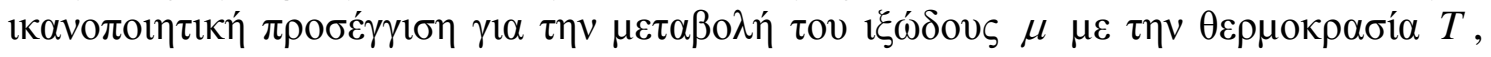

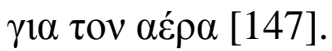

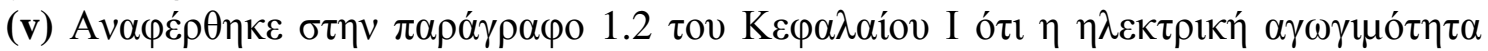

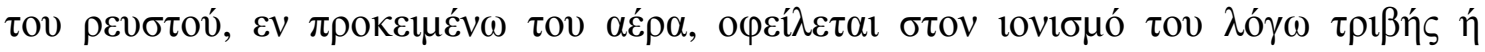

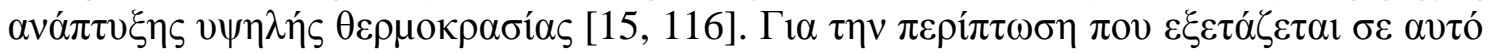

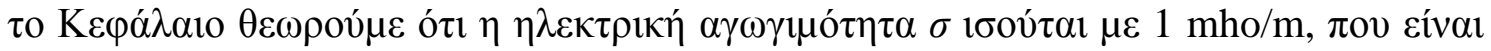

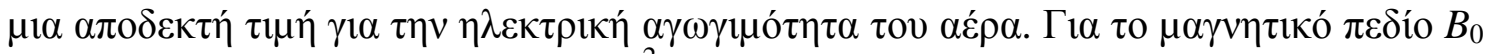

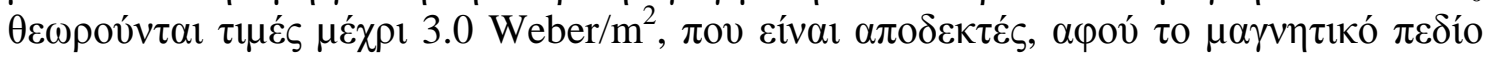

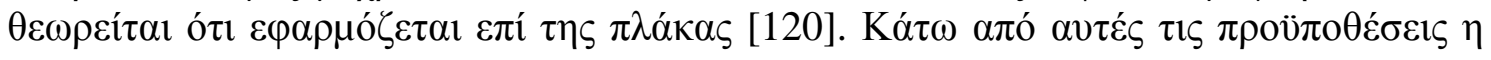

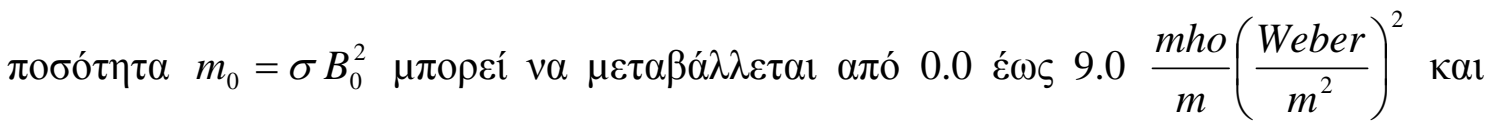

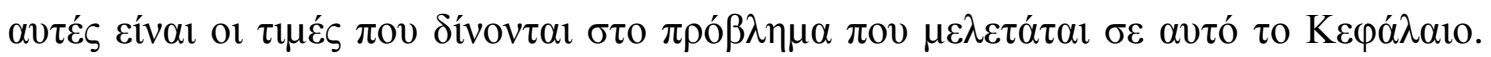

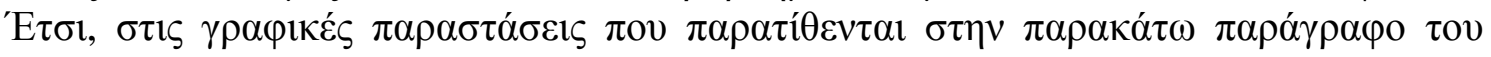

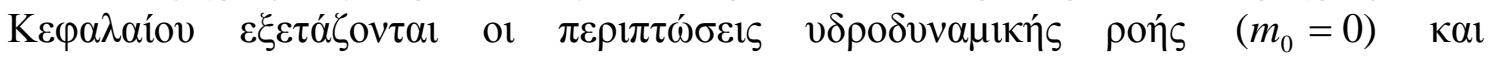

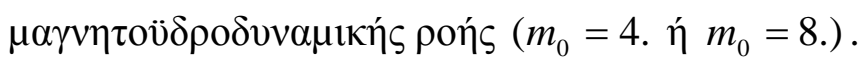

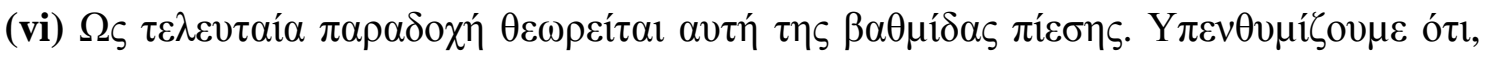

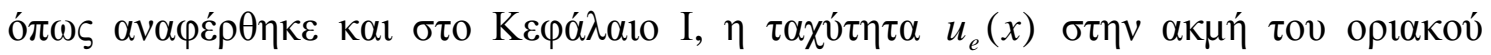




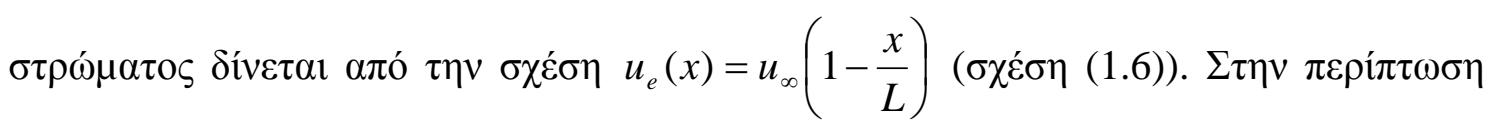

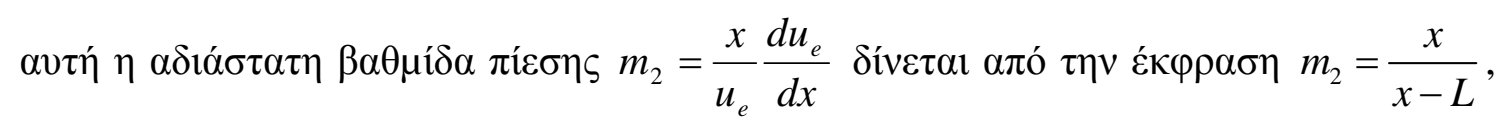

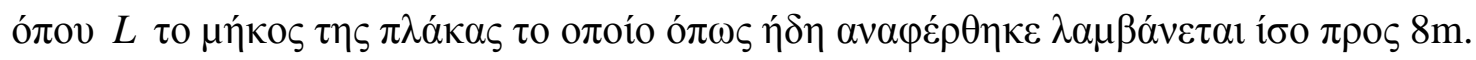

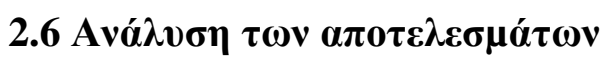

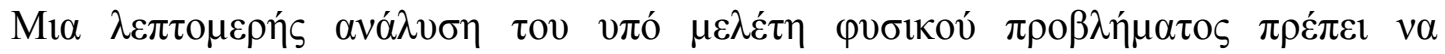

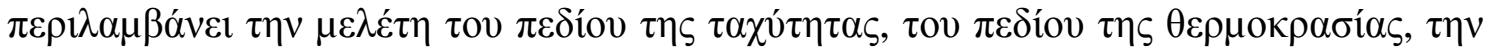

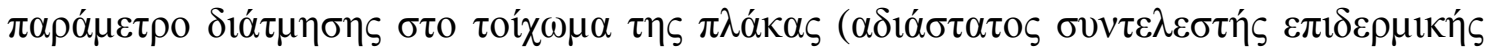

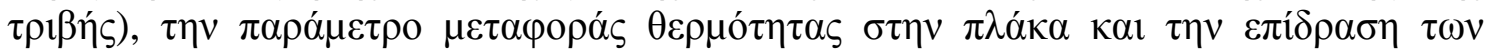

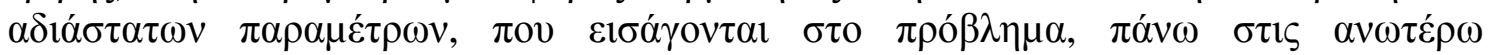

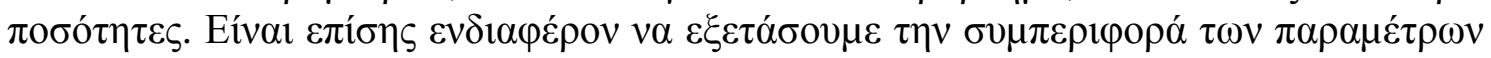

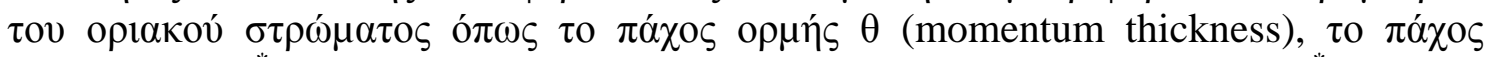

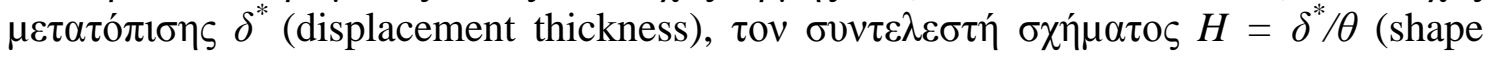
factor), $\kappa \lambda \pi$.

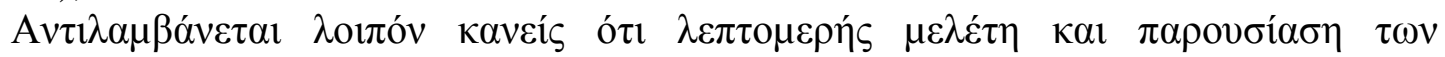

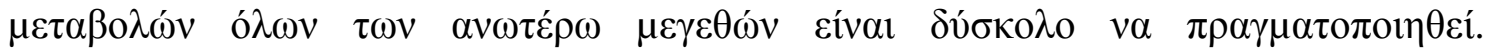

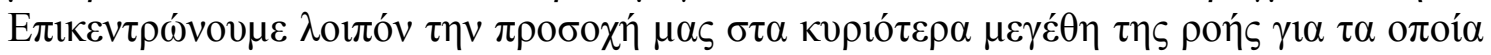

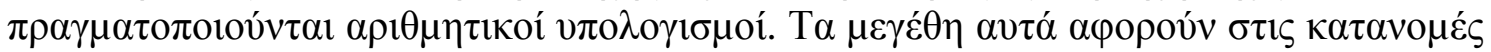

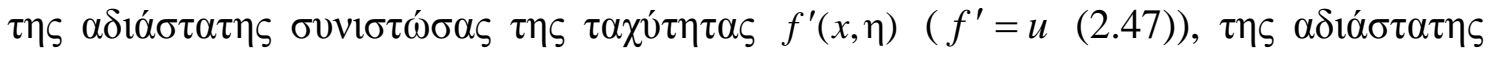

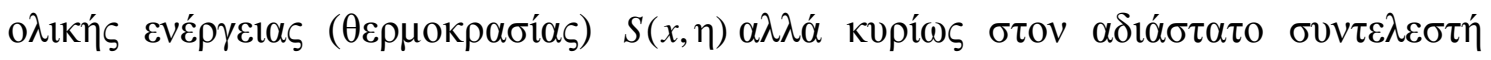

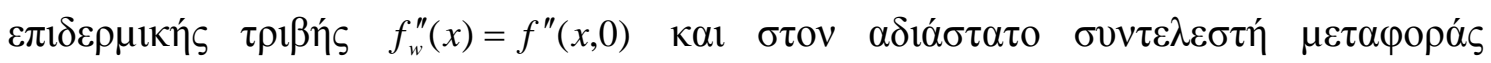

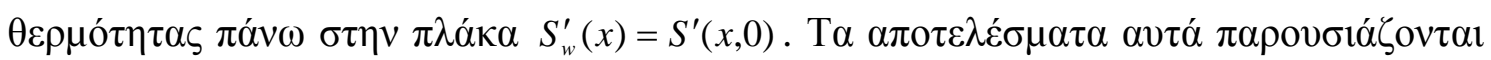

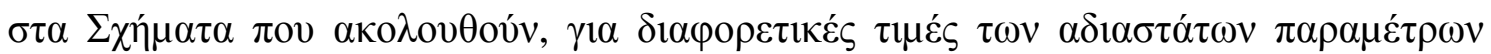

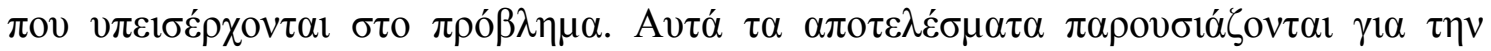

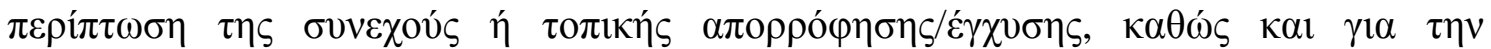

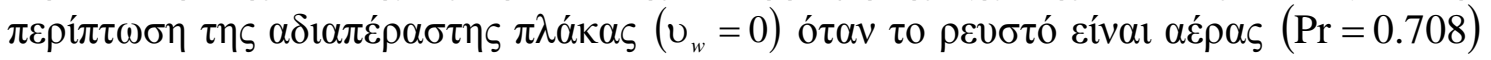

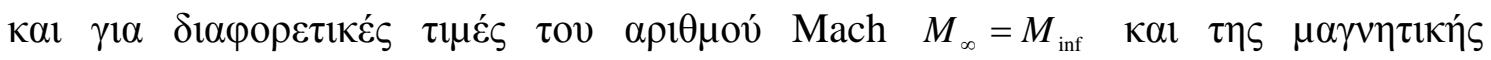

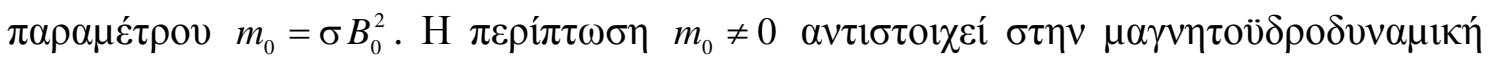


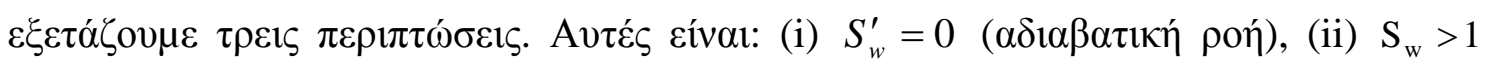

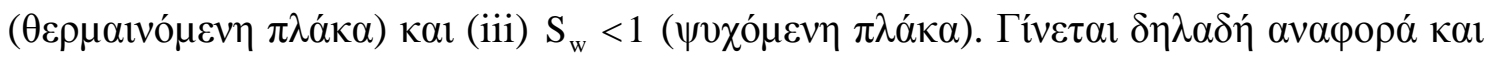

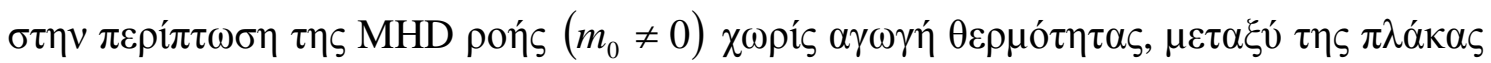

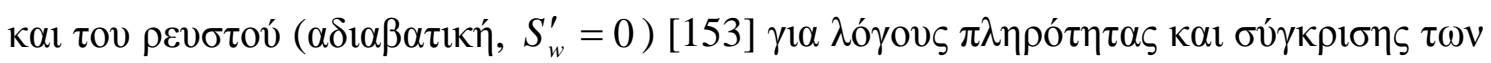

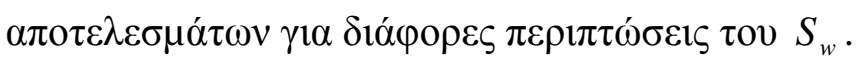

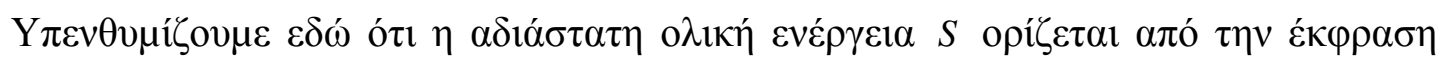

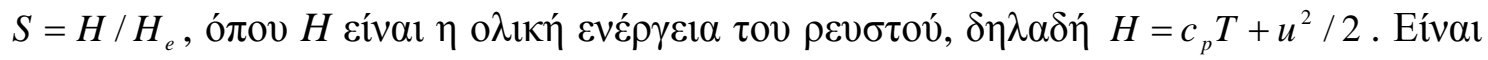

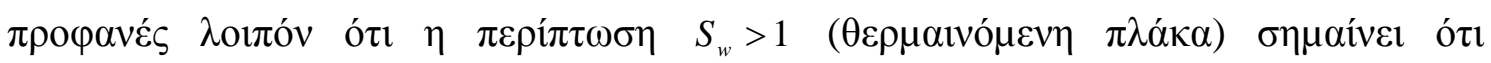

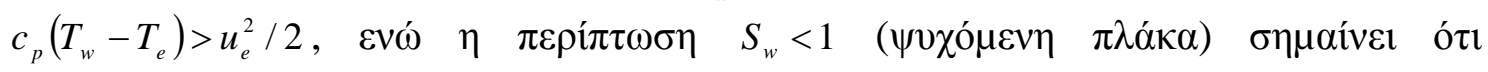

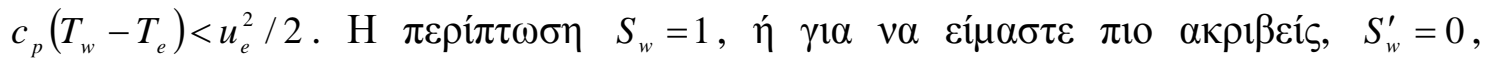

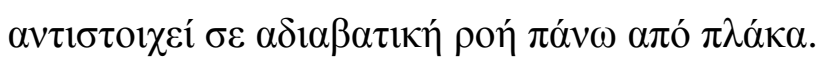




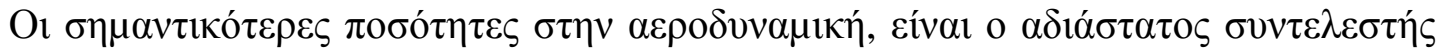

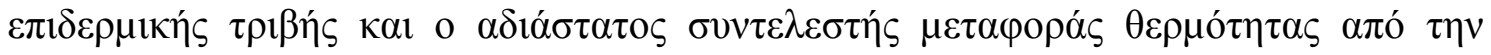

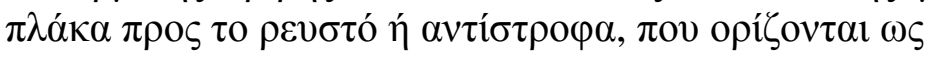

$$
\begin{gathered}
C_{f_{x}}=\frac{\tau_{w}}{\frac{1}{2} \rho_{e} u_{e}^{2}}, \text { ó } \pi \mathrm{ov} \tau_{w}=\left[\mu \frac{\partial u}{\partial y}\right]_{y=0}, \\
N u_{x}=\frac{\dot{q}_{w} x}{k\left(T_{w}-T_{e}\right)}, \text { ó } \pi \mathrm{ov} \quad \dot{q}_{w}=-\left[k \frac{\partial T}{\partial y}\right]_{y=0},
\end{gathered}
$$

$\alpha v \tau i ́ \sigma \tau o \imath \chi \alpha$.

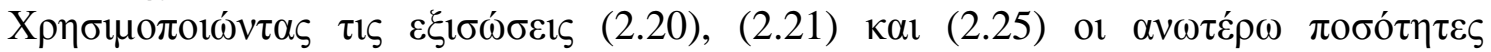
$\mu \varepsilon \tau \alpha \sigma \chi \eta \mu \alpha \tau i \zeta o v \tau \alpha l \sigma \tau \iota \varsigma$

$$
\begin{gathered}
C_{f_{x}}=\frac{2 C_{w}}{\sqrt{R_{x}}} f_{w}^{\prime \prime}, \\
N u_{x}=-\frac{C_{w} \sqrt{R_{x}}}{1-S_{w}} S_{w}^{\prime} \quad\left(\mathrm{S}_{\mathrm{w}} \neq 1\right),
\end{gathered}
$$

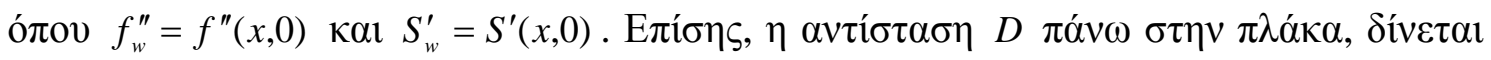
$\alpha \pi$ ó $\tau \eta \nu \sigma \chi \varepsilon ́ \sigma \eta$

$$
D=\int_{0}^{b} \int_{0}^{x^{*}}\left[\mu \frac{\partial u}{\partial y}\right]_{y=0} d x d z
$$

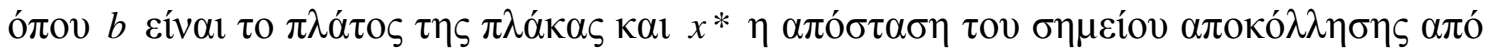

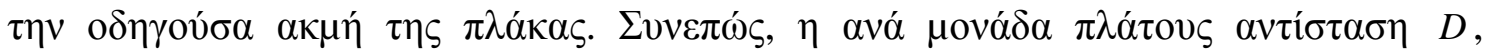

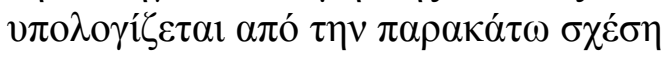

$$
D=\int_{0}^{x^{*}} \frac{C_{w} f_{w}^{\prime \prime}(x, 0)}{\sqrt{\operatorname{Re}_{x}}} \rho_{e}(x) u_{e}^{2}(x) d x
$$

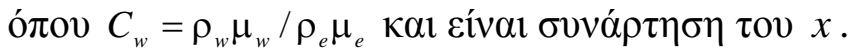

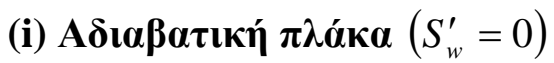

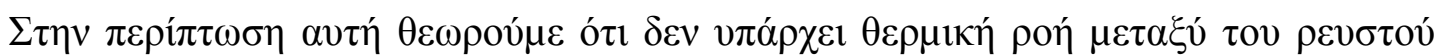

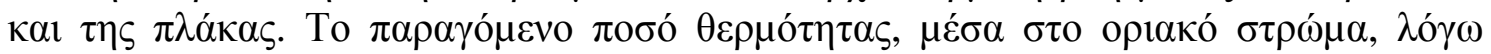

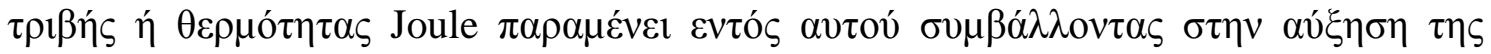

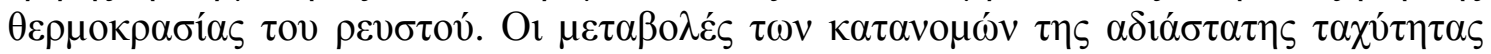

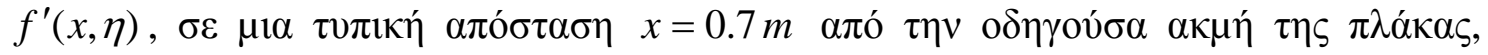

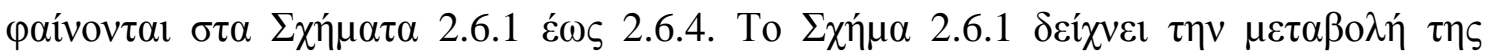

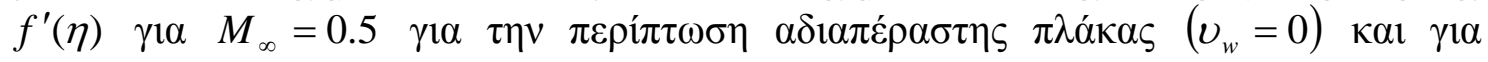

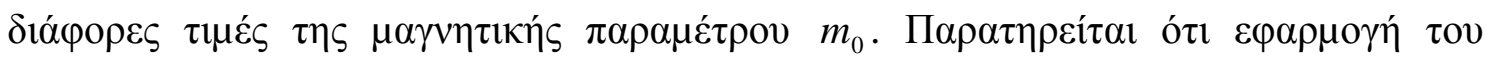




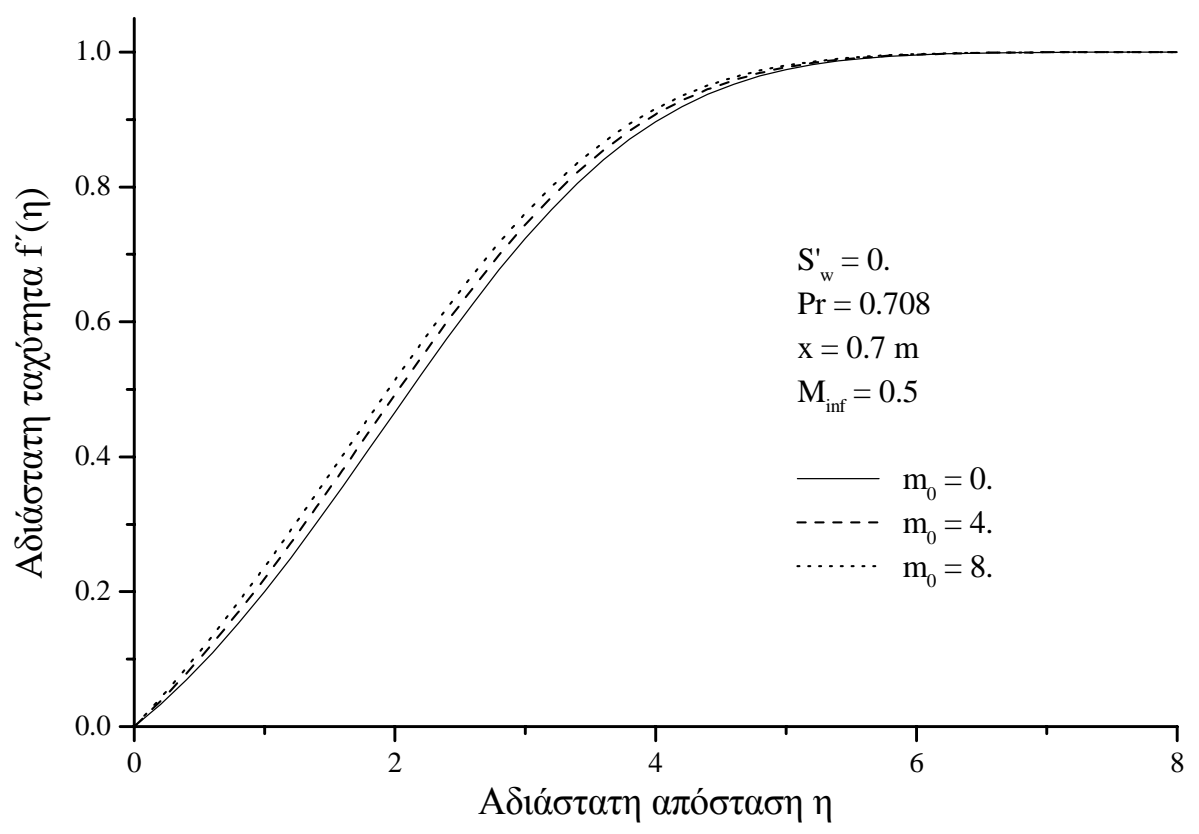

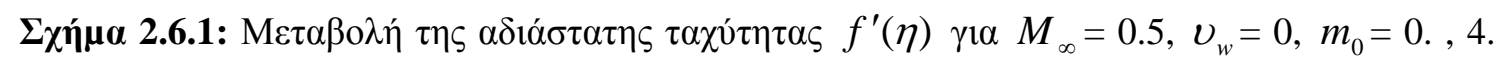
каl 8.

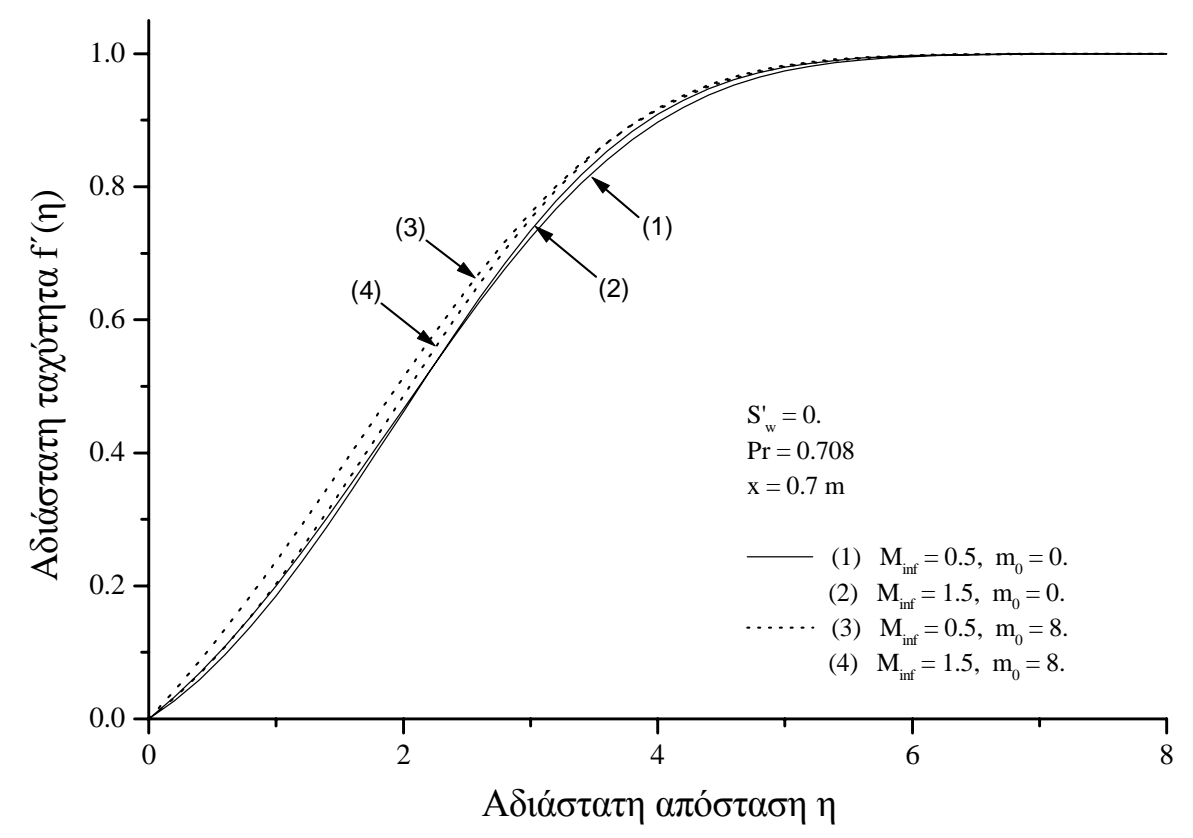

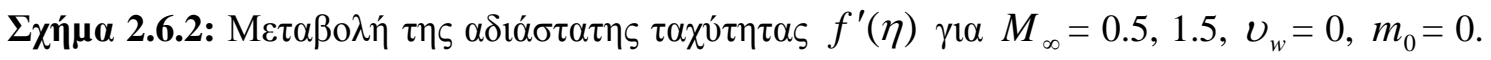
ка1 8. 


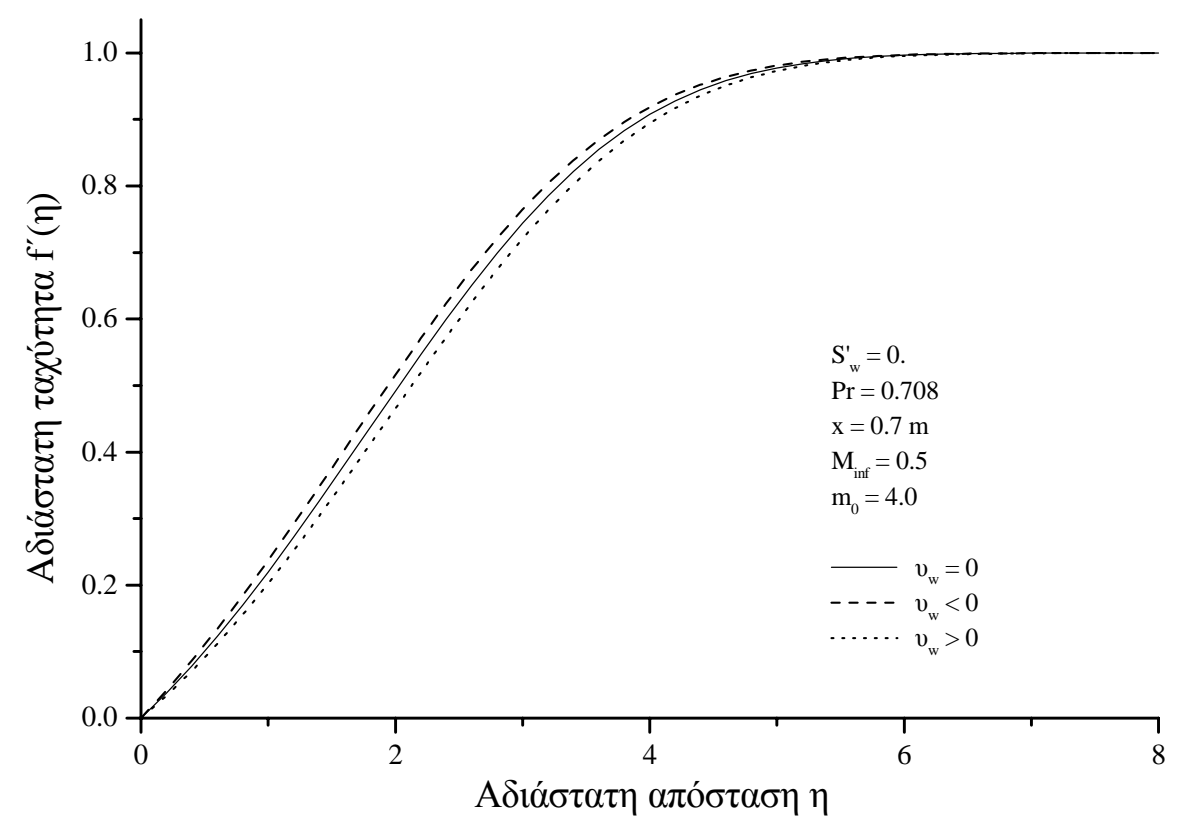

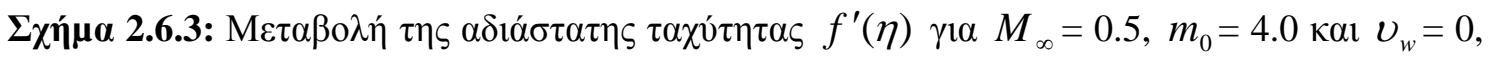
$<0,>0$.

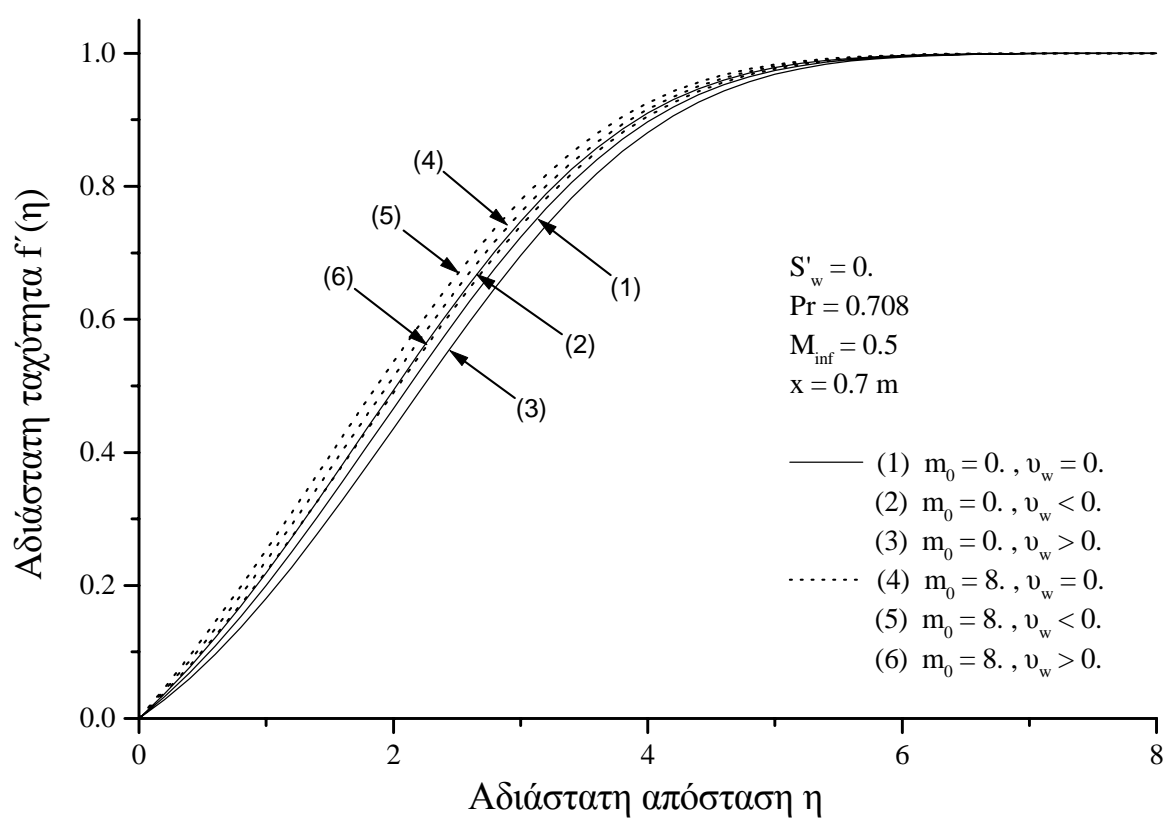

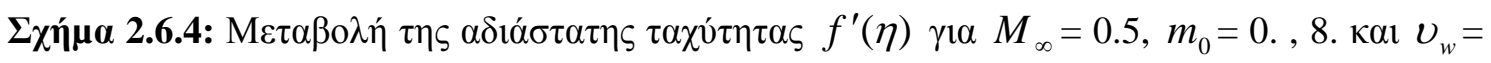
$0,<0,>0$. 


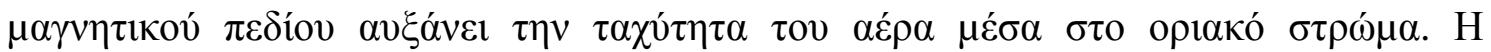

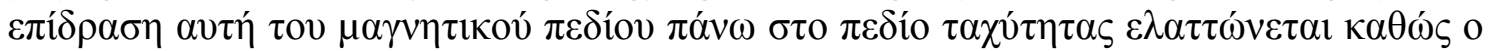

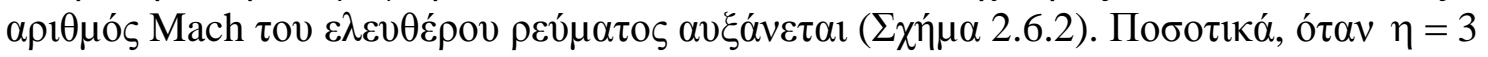

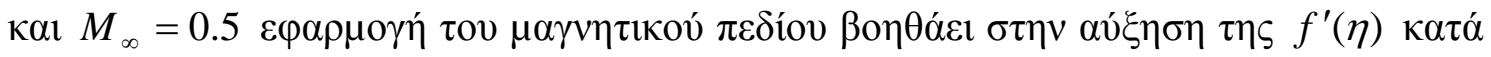

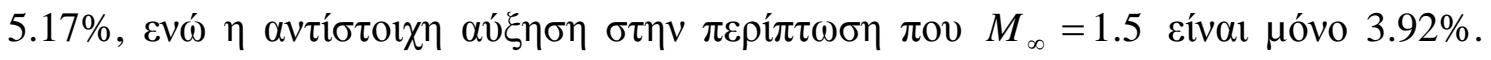

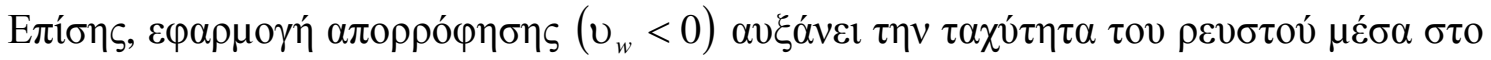

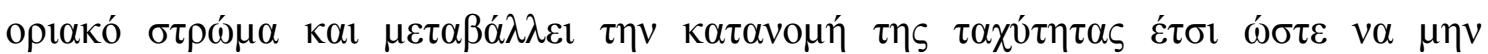

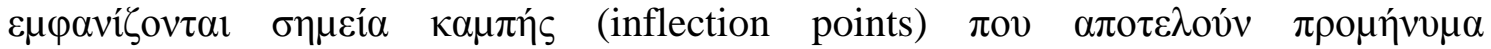

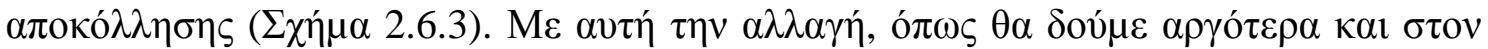

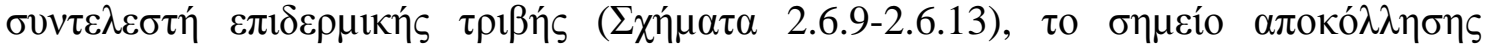

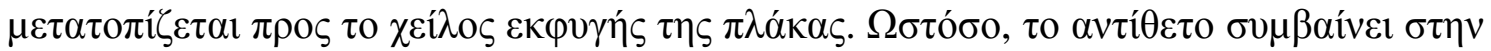
$\pi \varepsilon \rho i ́ \pi \tau \omega \sigma \eta \tau \eta \varsigma ~ \varepsilon ́ \gamma \chi v \sigma \eta \varsigma\left(v_{w}>0\right)$.

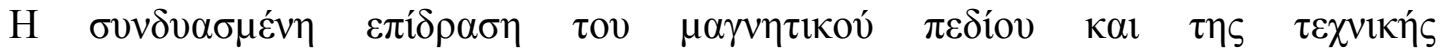

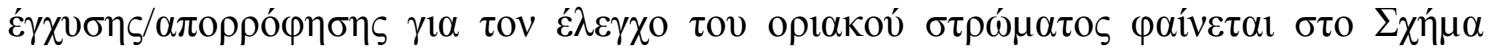

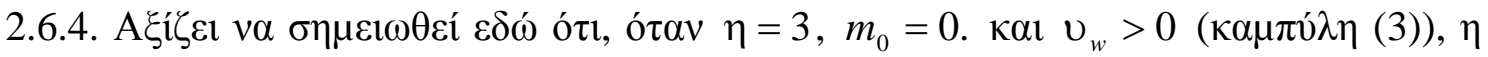

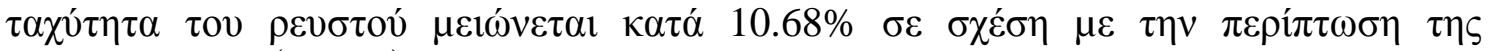

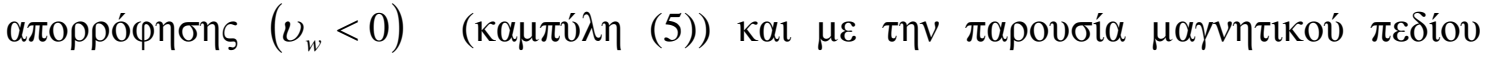
$\left(m_{0}=8\right.$.).

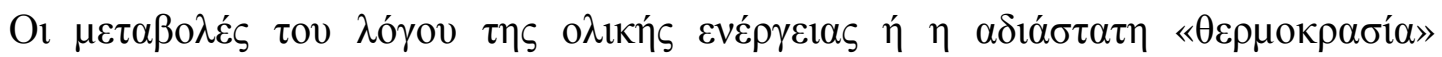

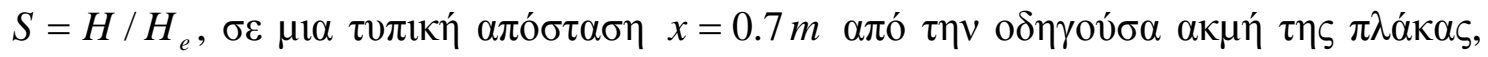

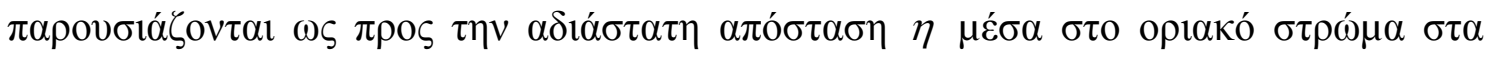

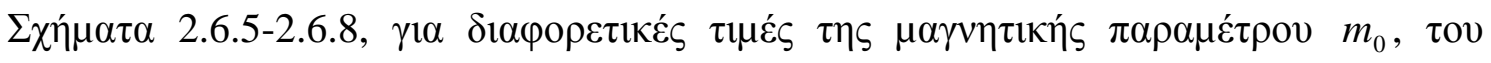

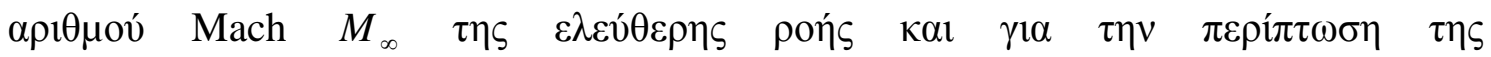

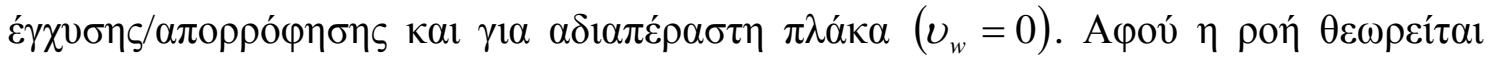

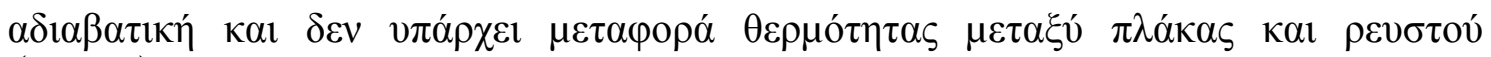

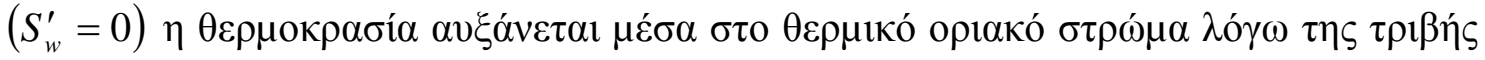

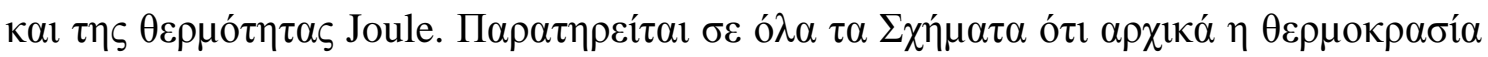

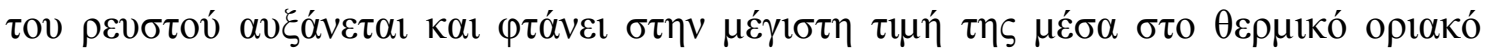

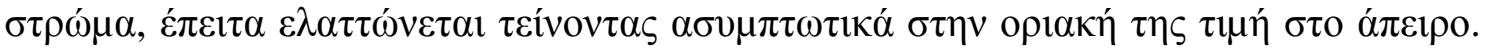

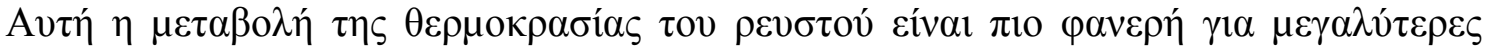

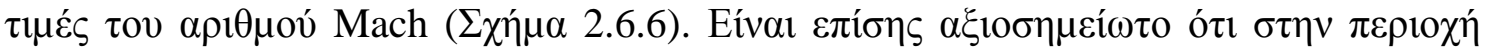

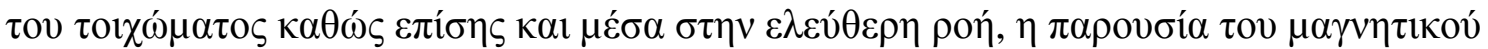

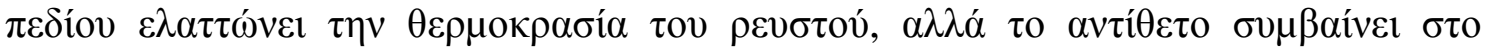

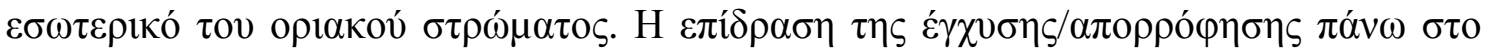

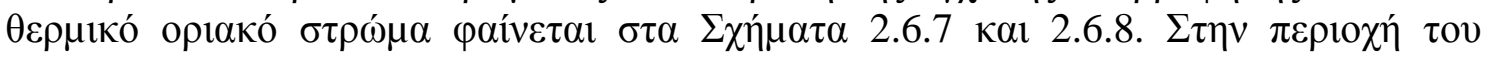

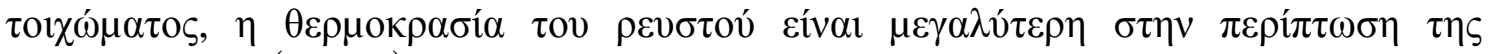

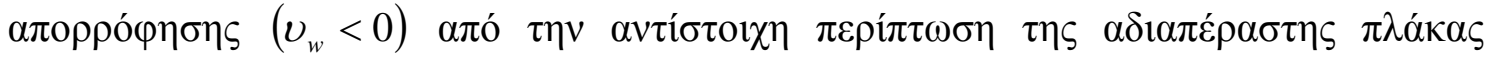

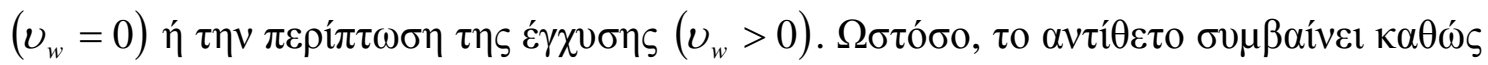

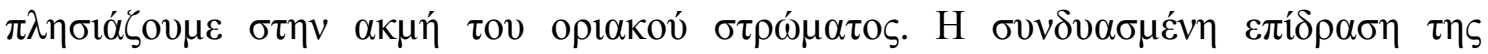

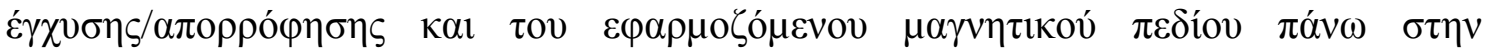

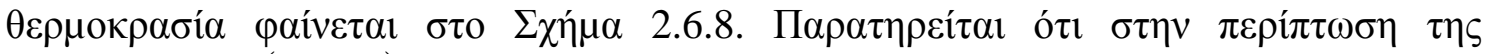

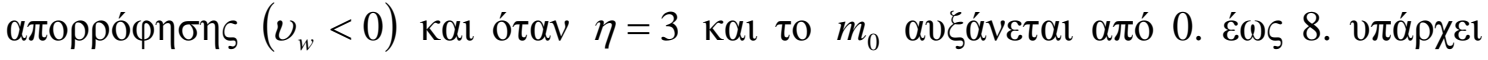

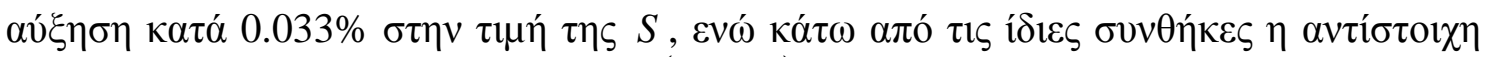

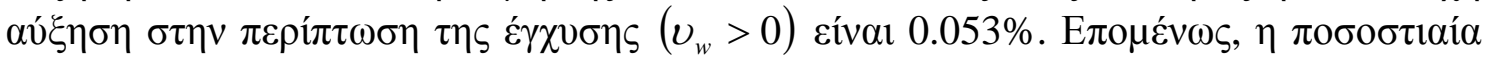

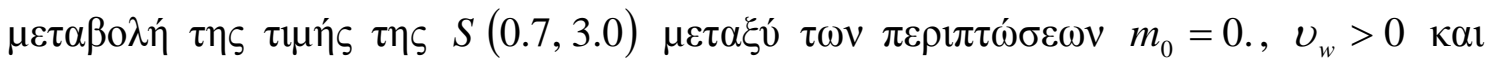




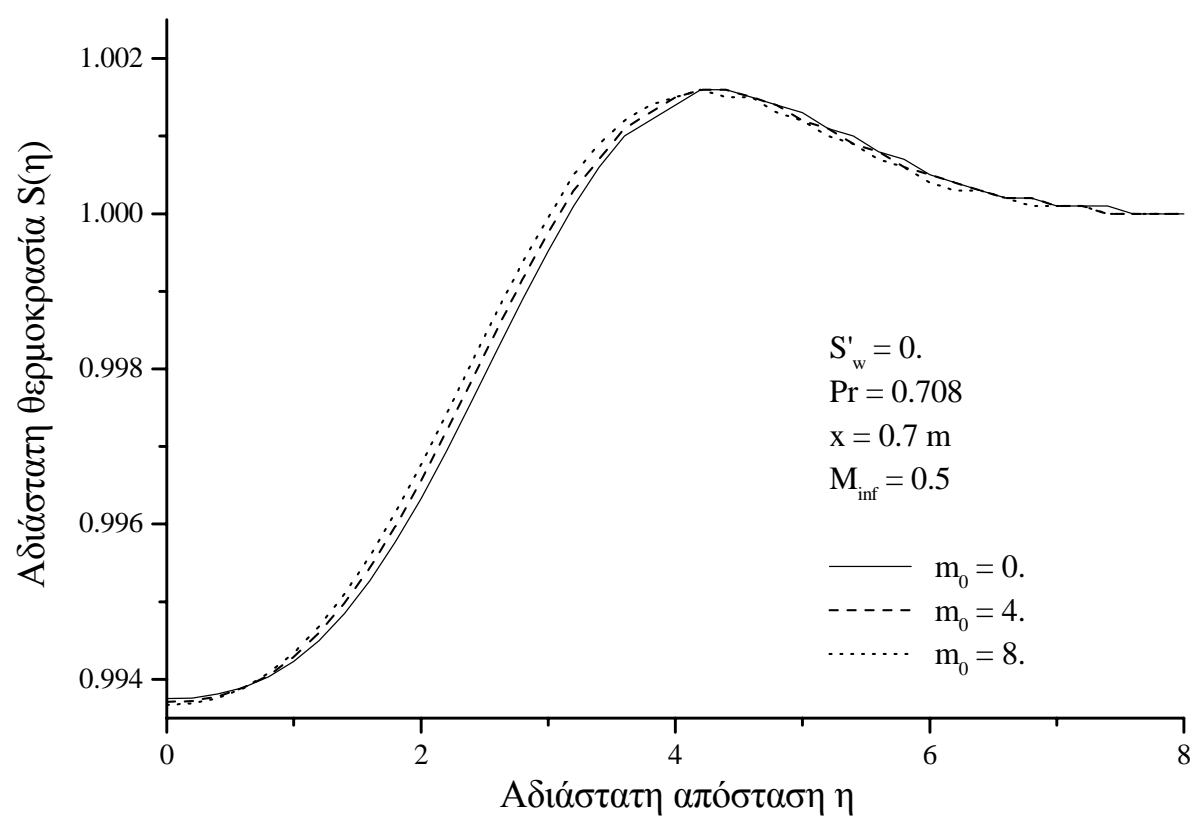

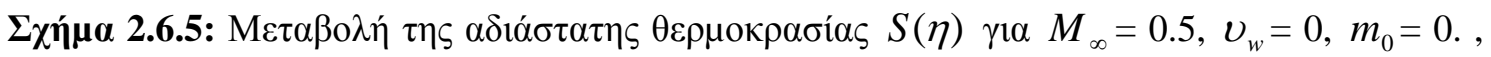
4. $\mathrm{kal} 8$.

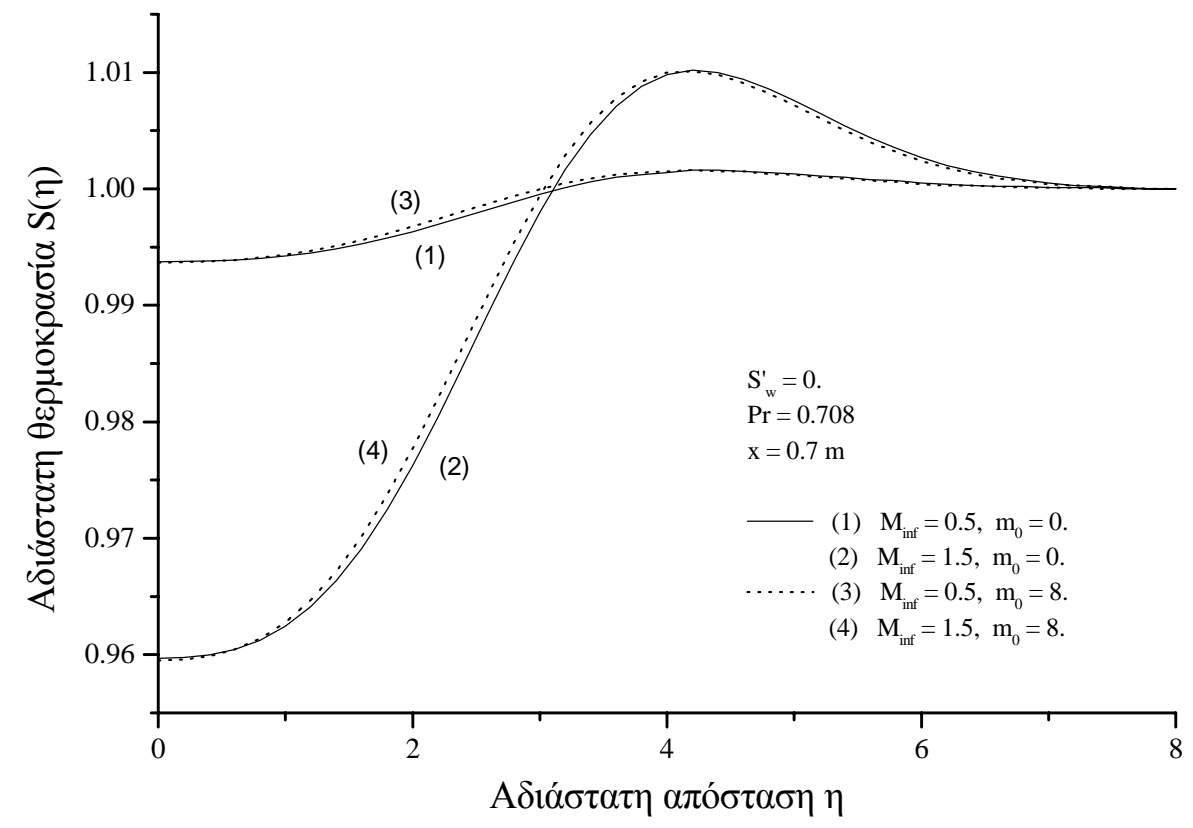

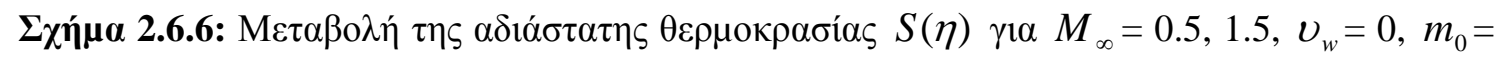
0. ка1 8. 


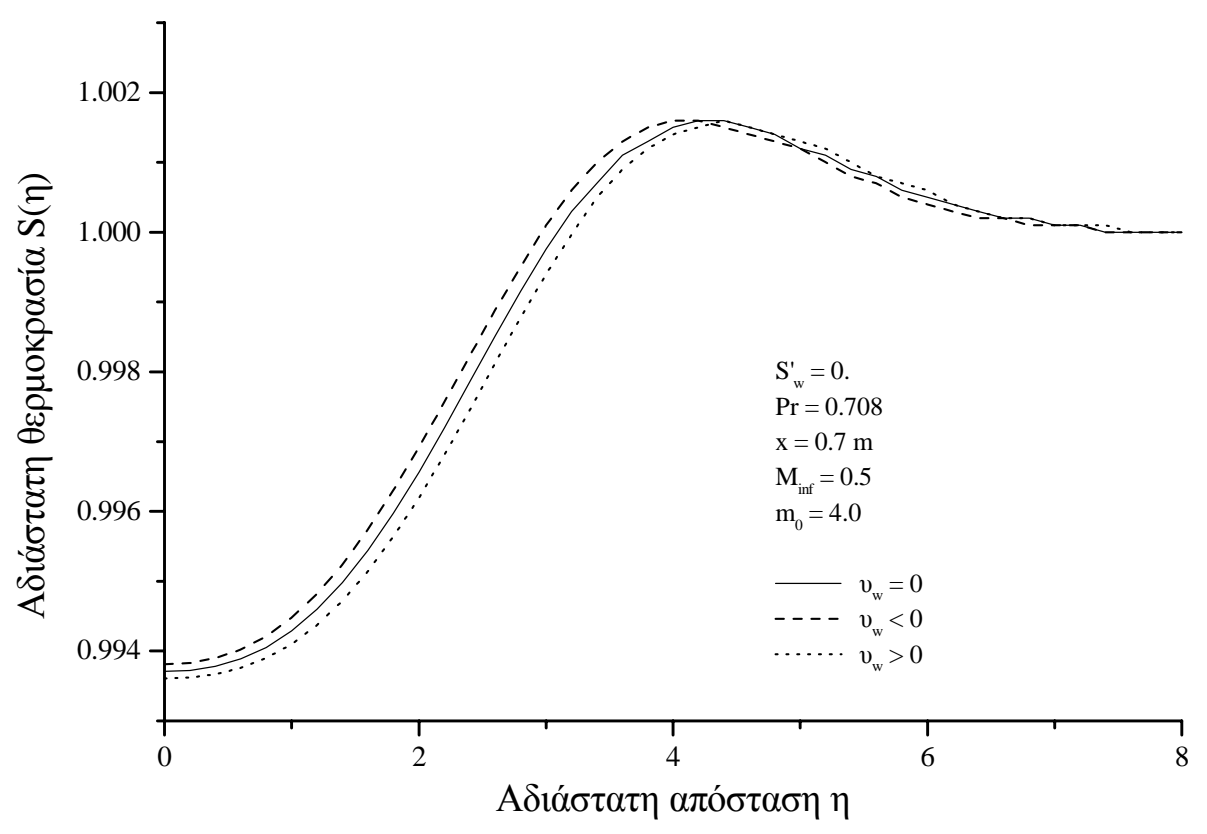

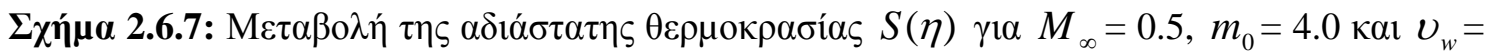
$0,<0,>0$.

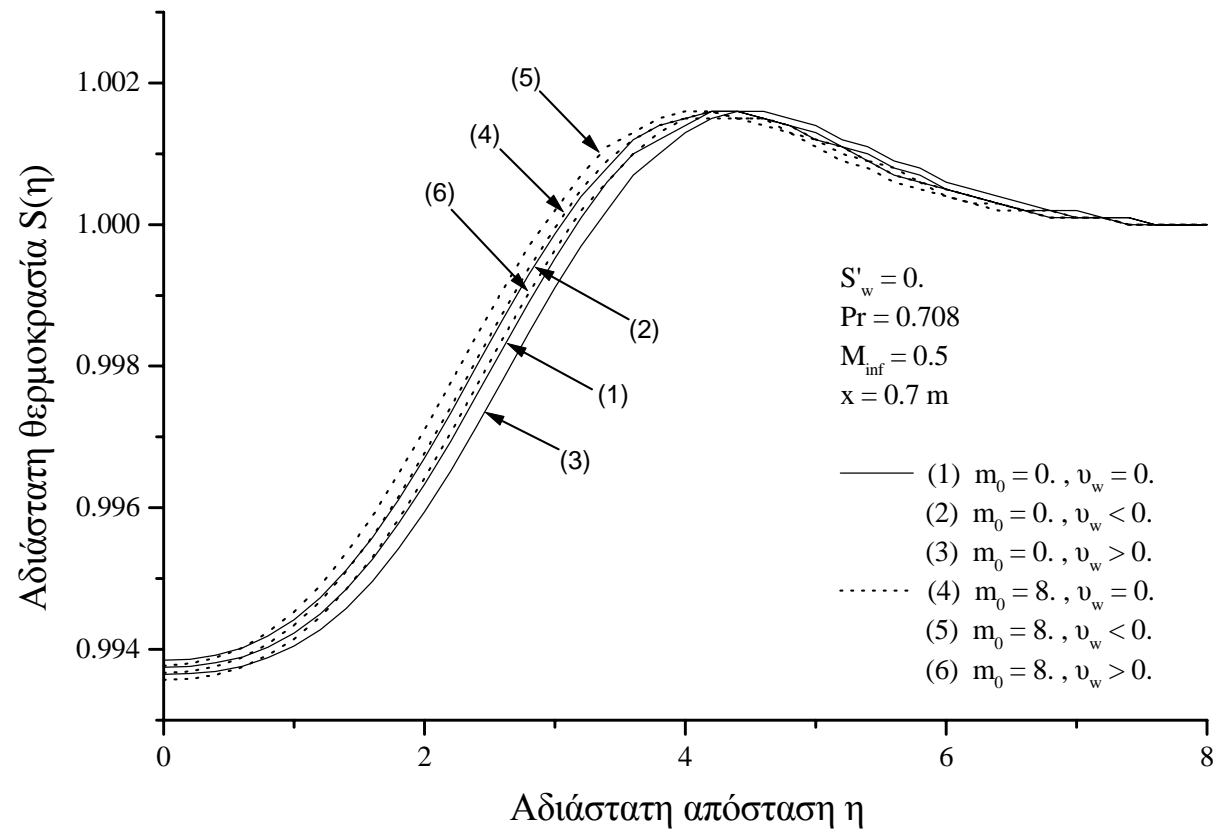

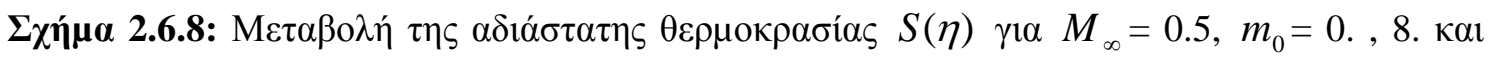
$v_{w}=0,<0,>0$. 


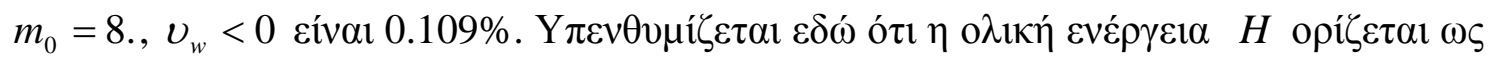

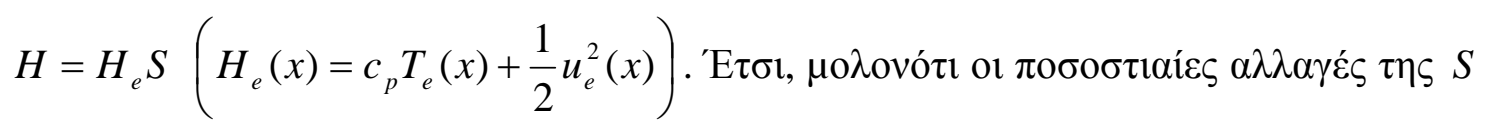

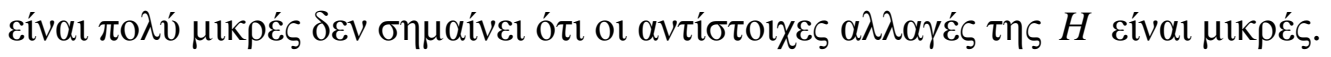

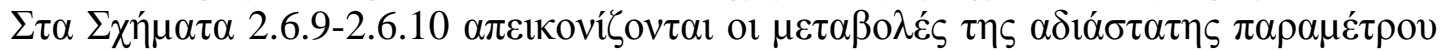

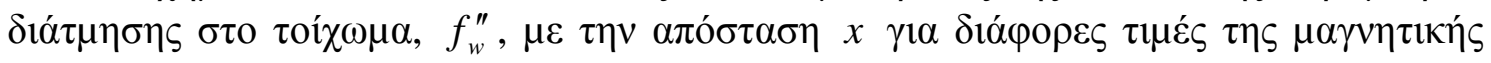

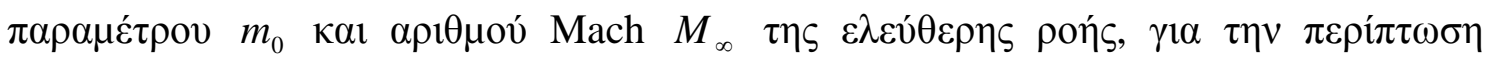

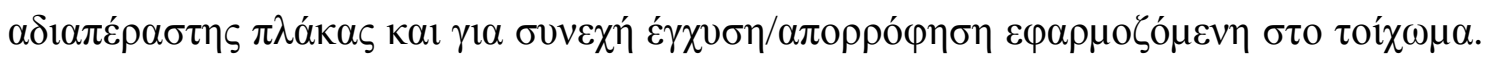

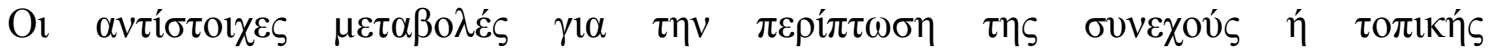

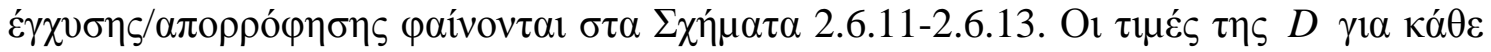

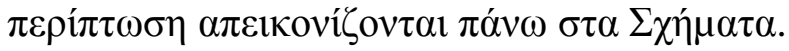

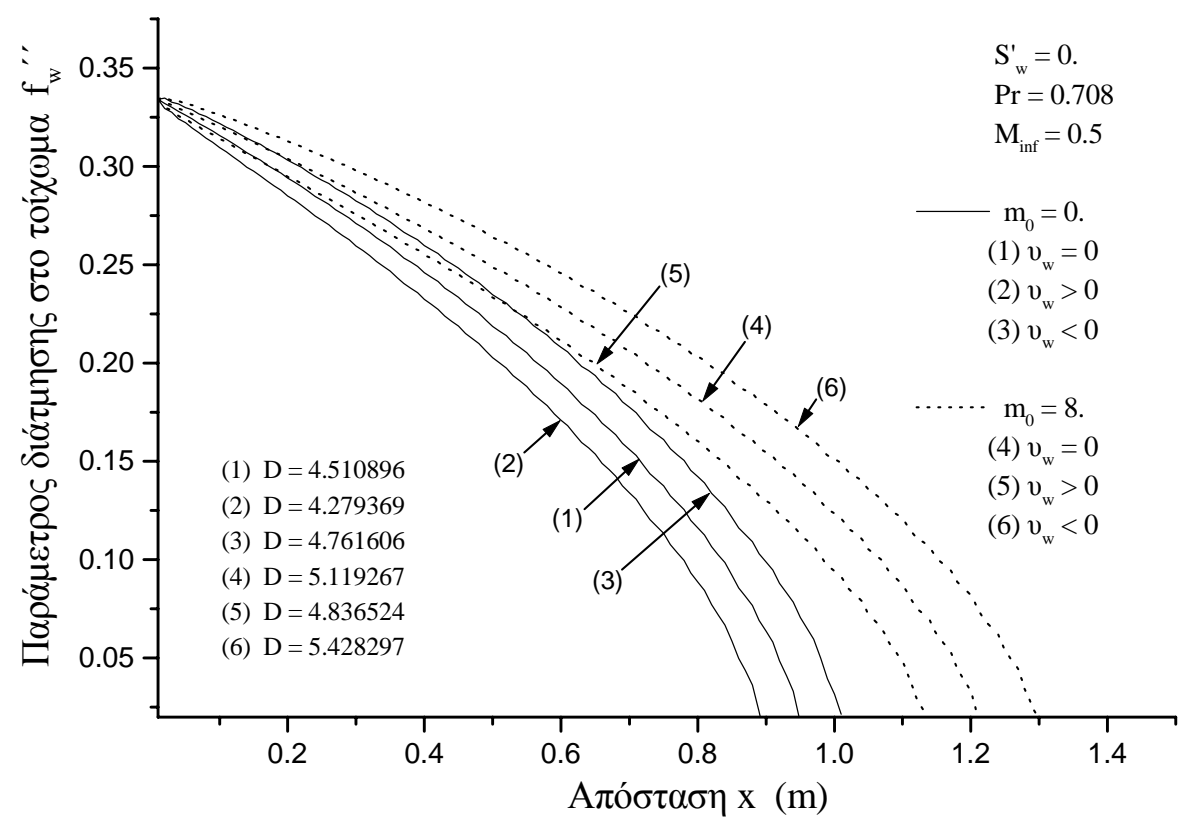

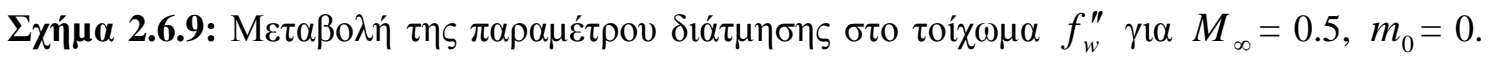
$\kappa \alpha 18 . \kappa \alpha 1 v_{w}=0,>0,<0$.

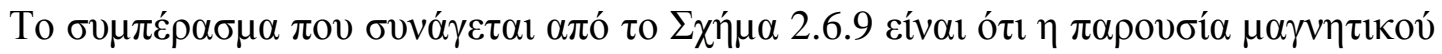

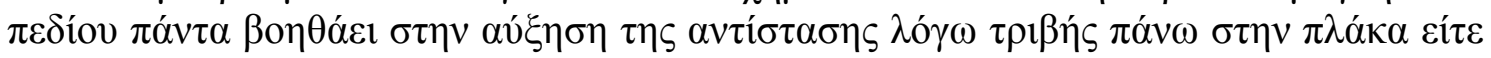

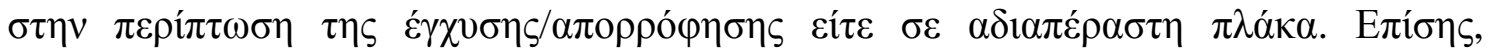

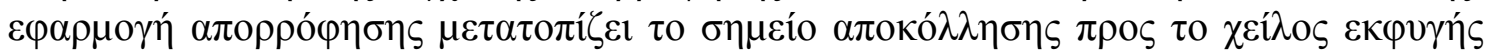

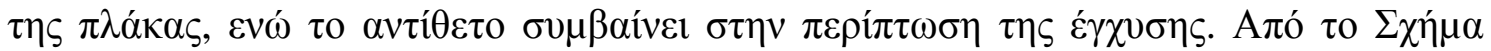

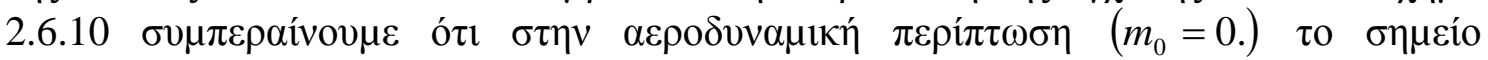

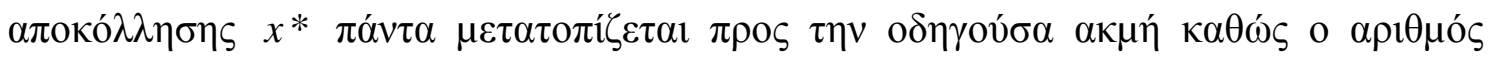

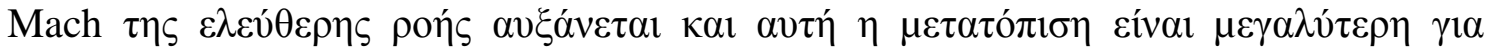

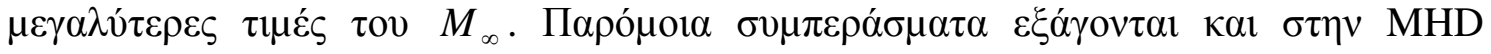

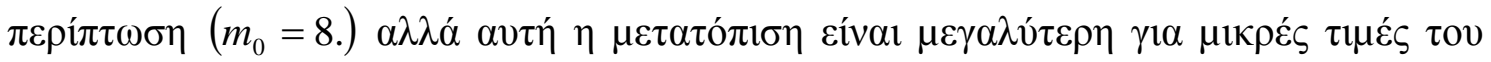

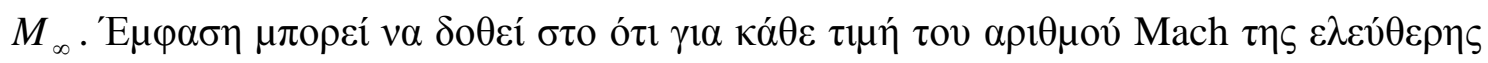

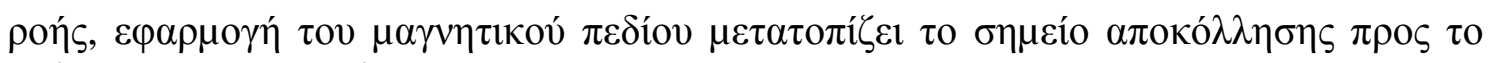

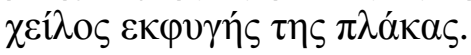




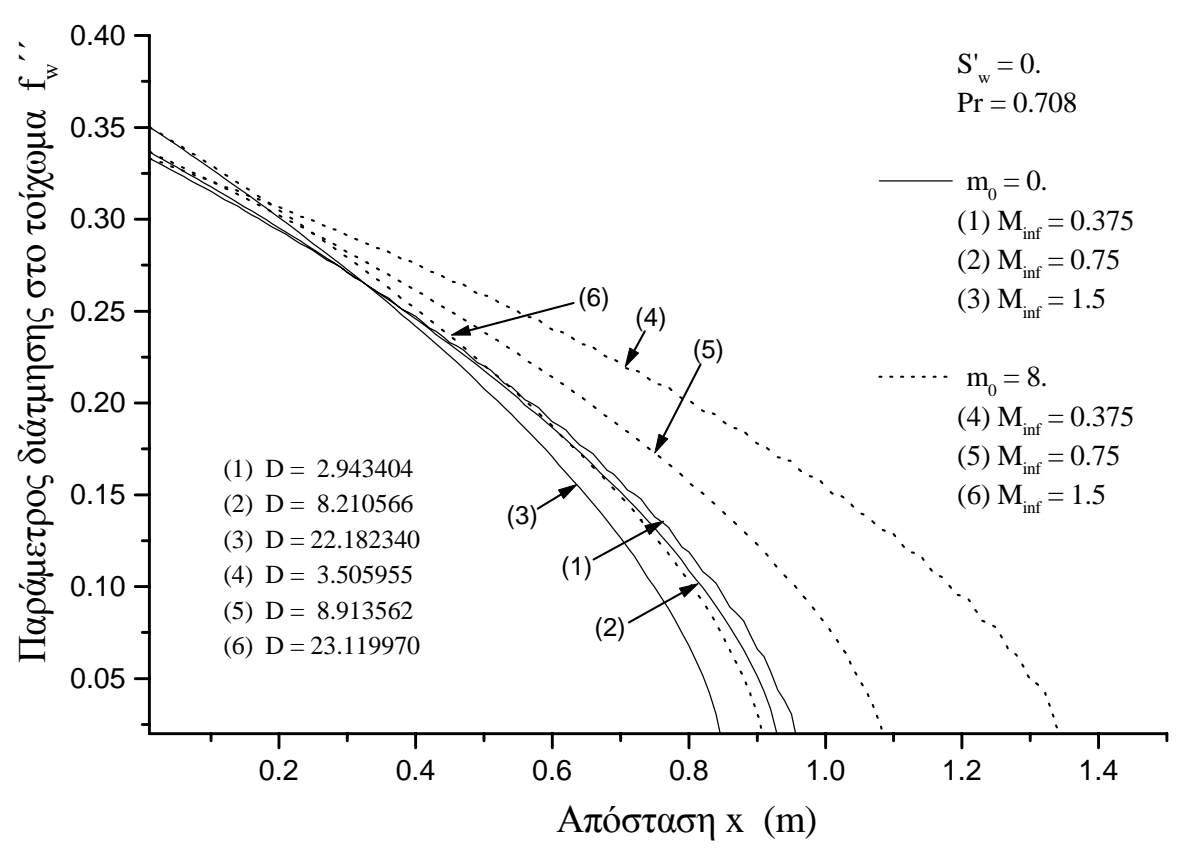

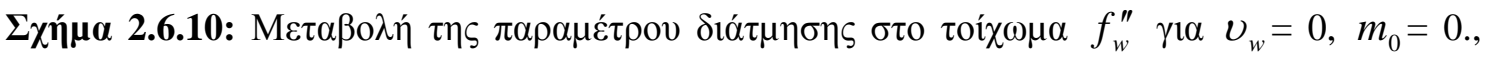
8. $\alpha \alpha 1 \gamma 1 \alpha M_{\infty}=0.375,0.75 \kappa \alpha l 1.5$.

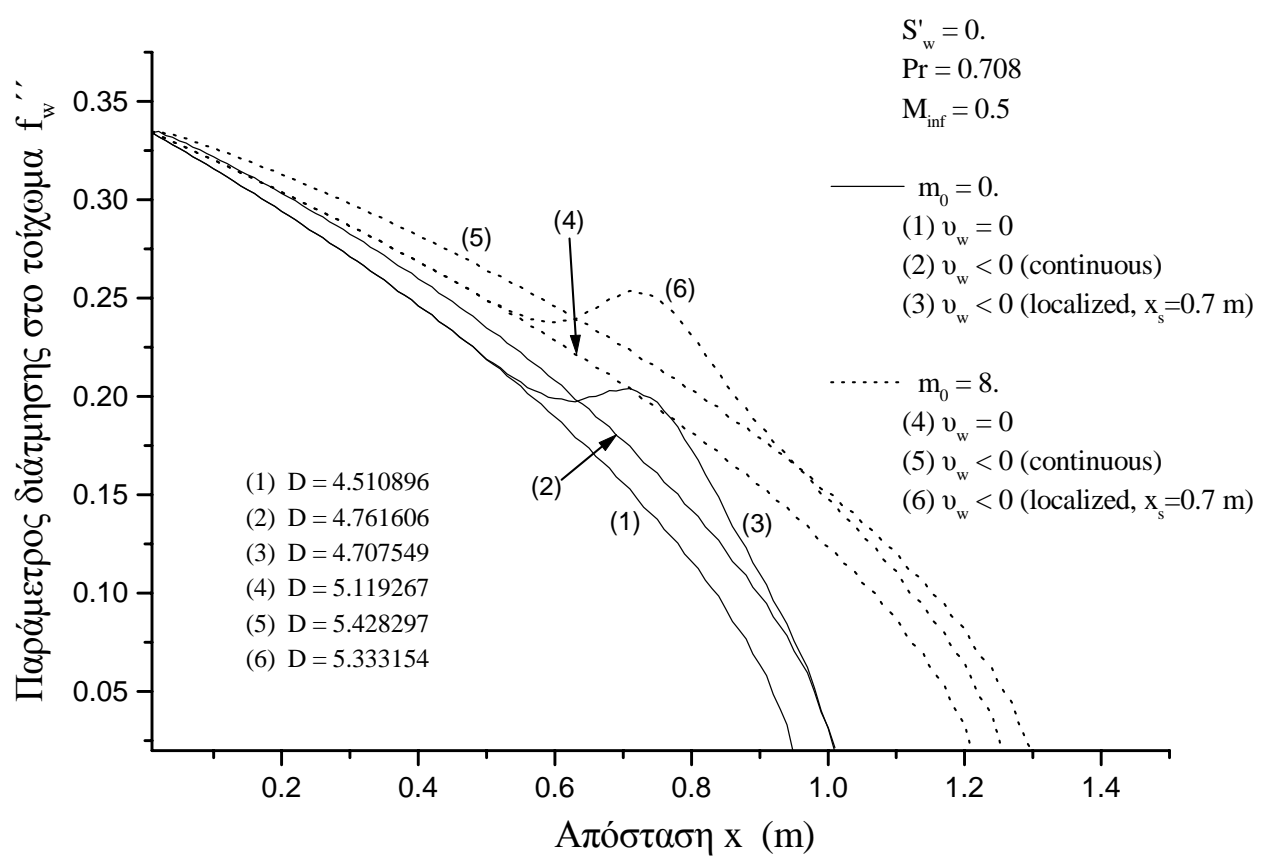

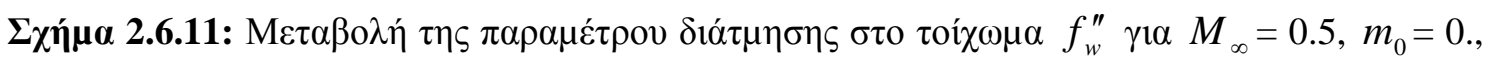

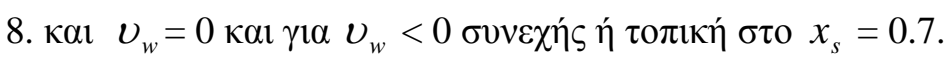




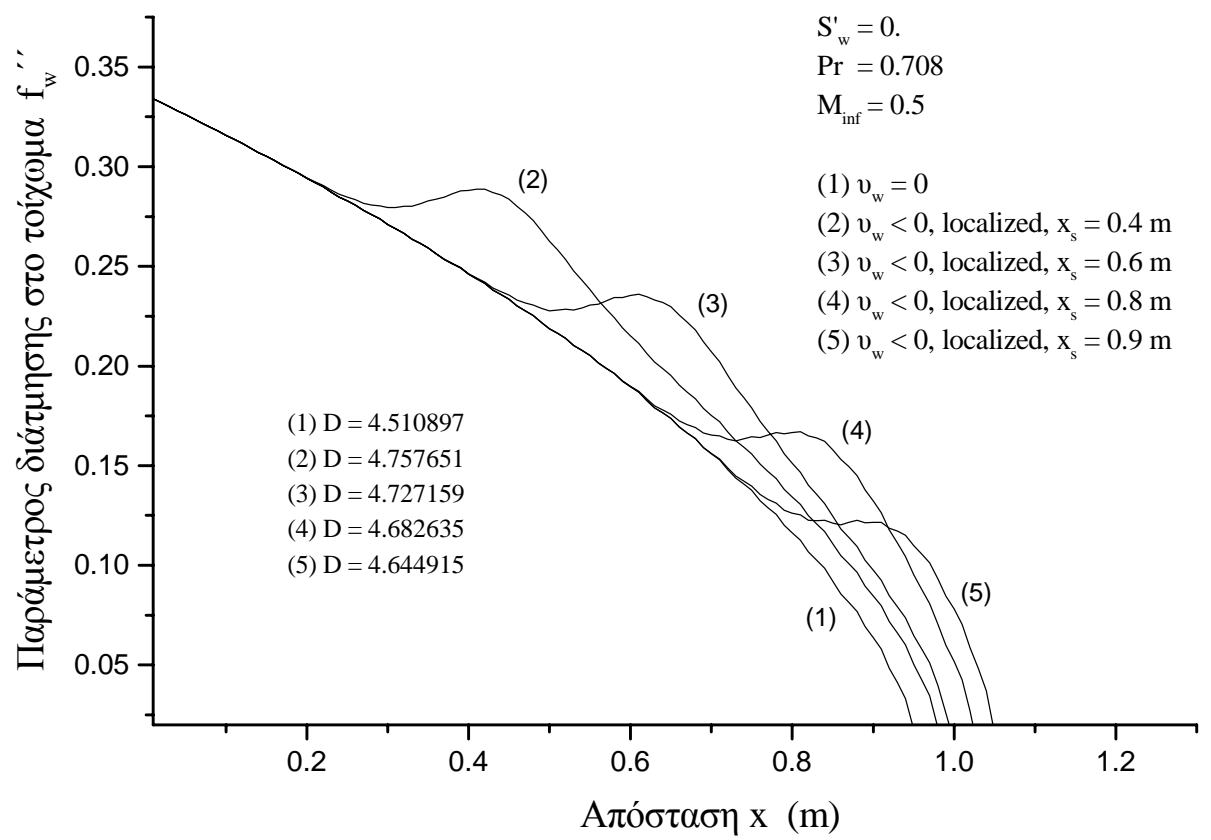

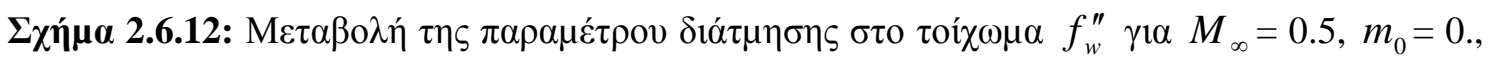

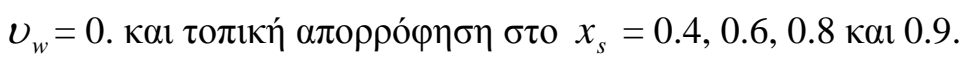

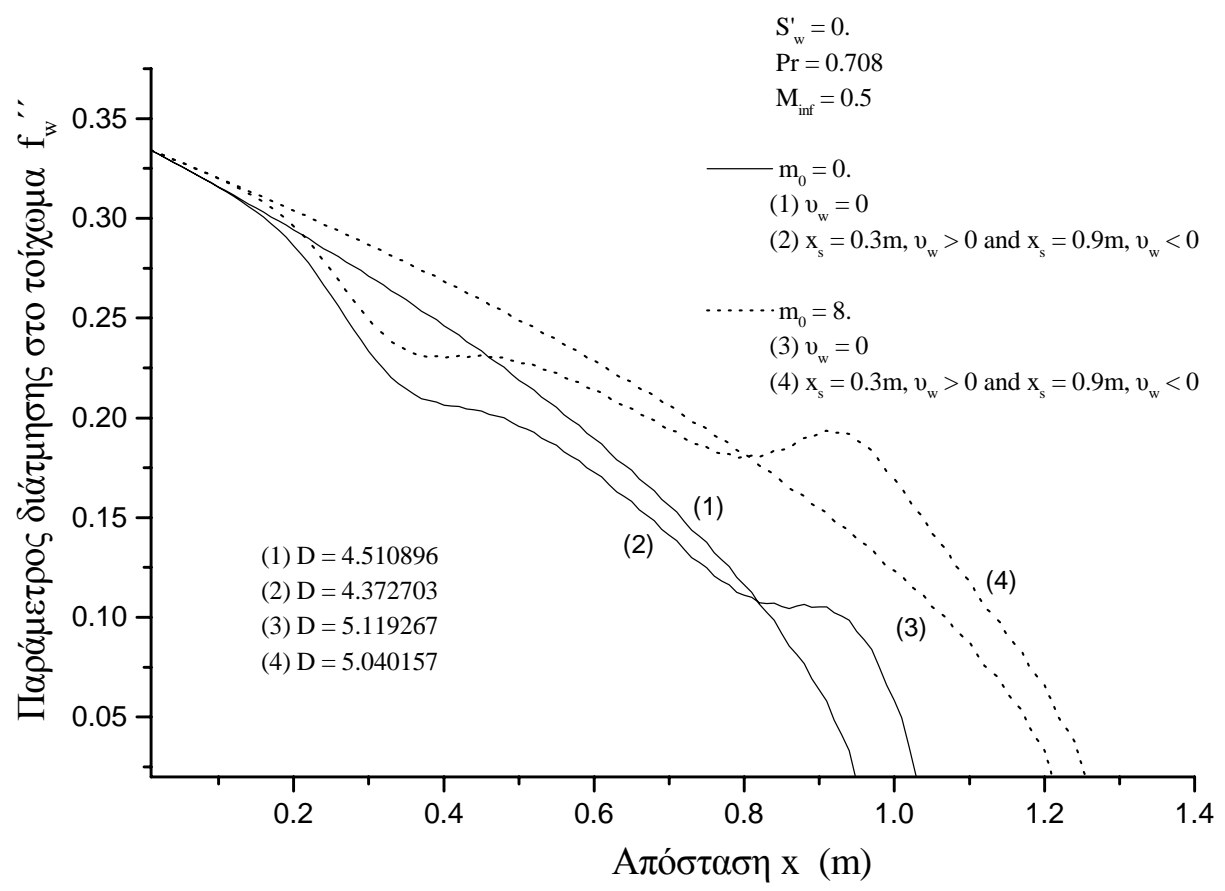

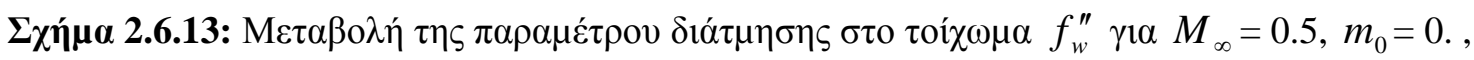

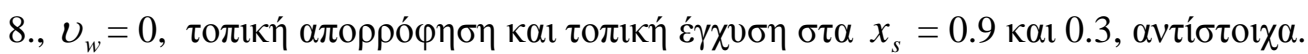




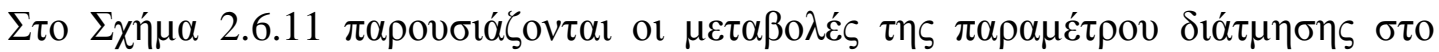

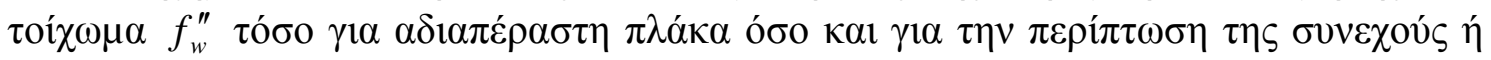

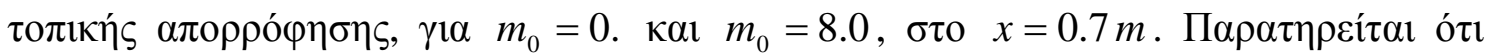

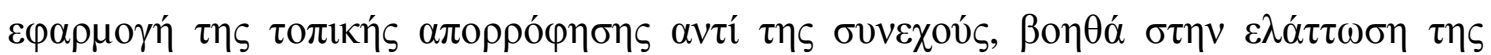

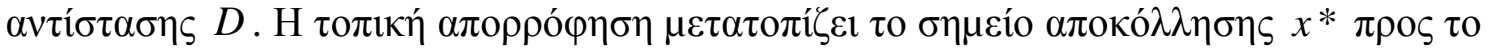

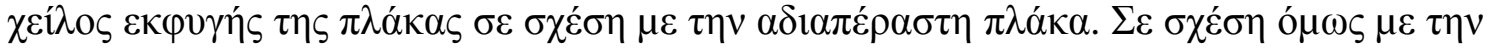

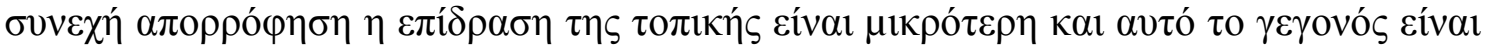

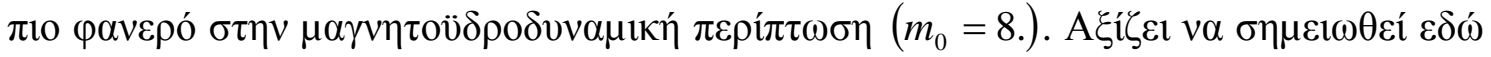

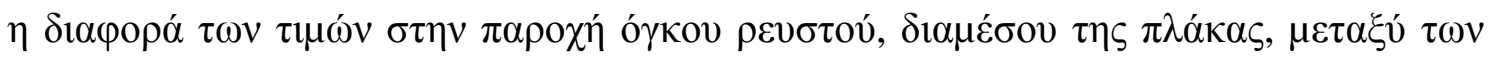

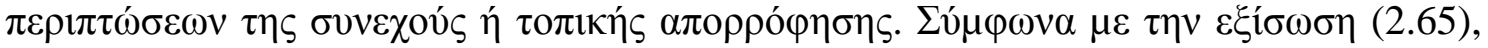

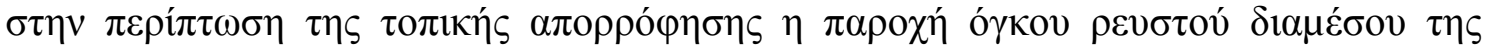

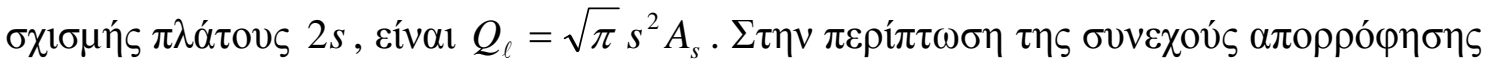

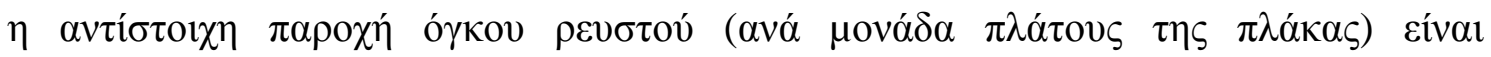

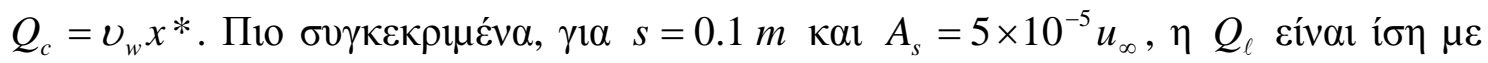

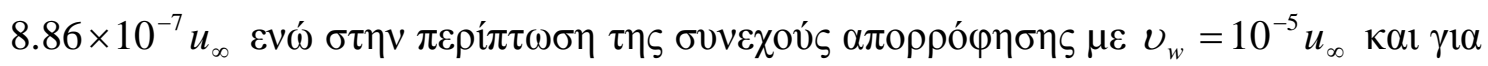

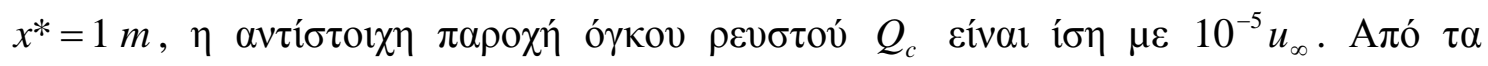

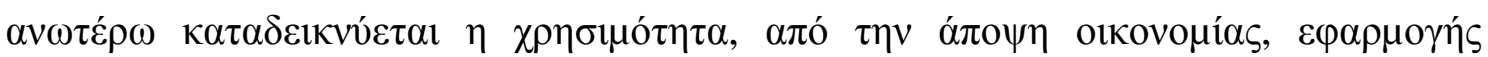

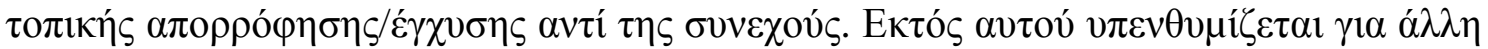

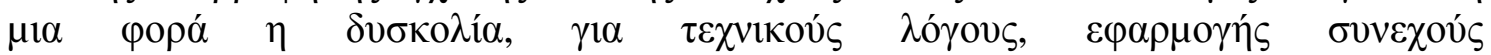

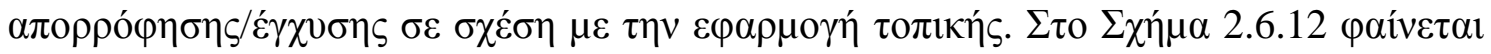

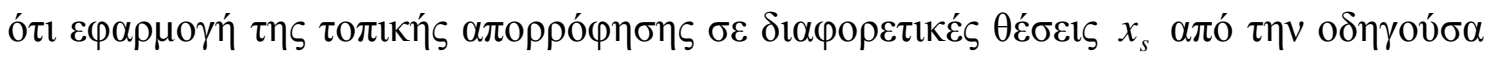

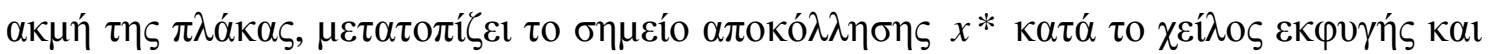

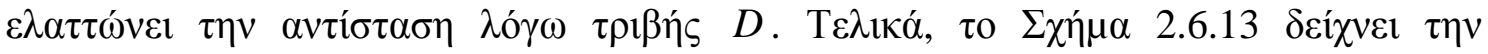

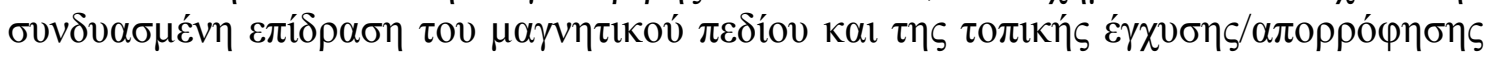

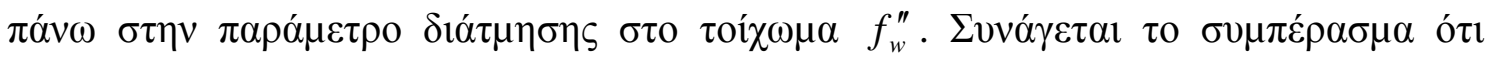

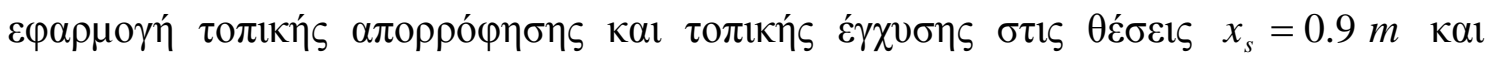

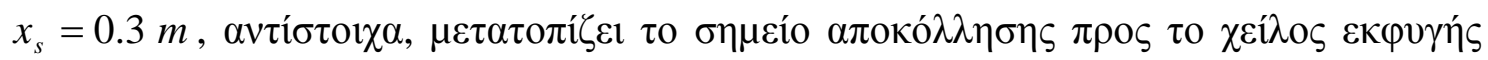

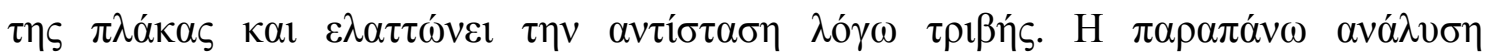

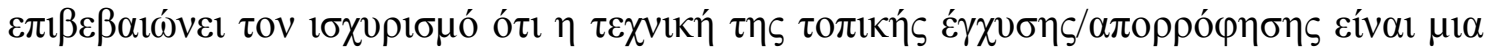

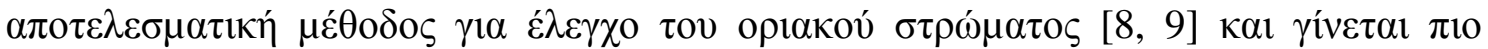

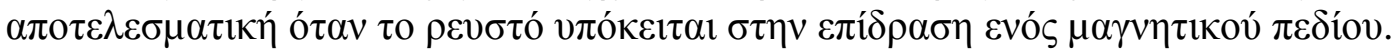

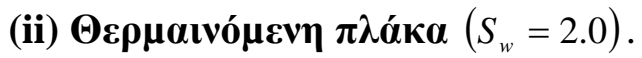

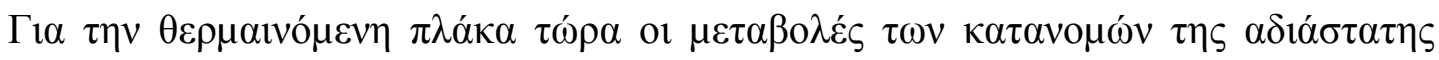

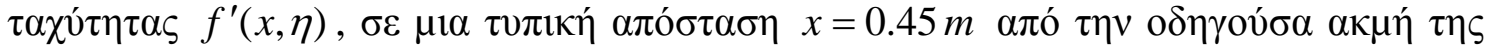

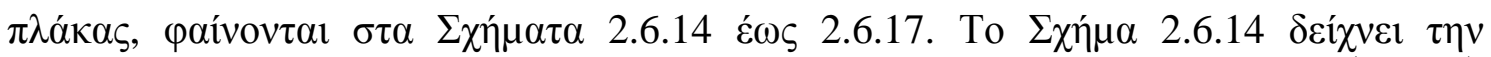

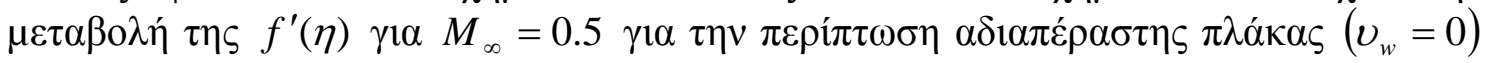

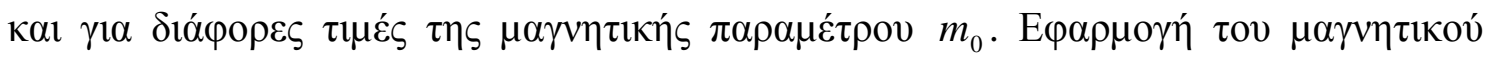

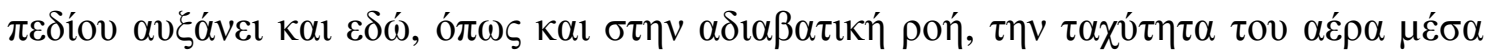

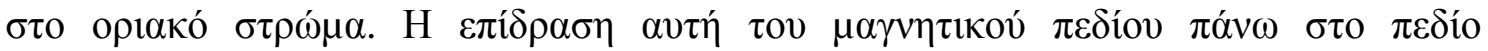

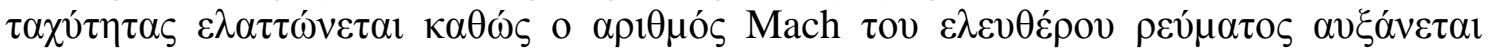

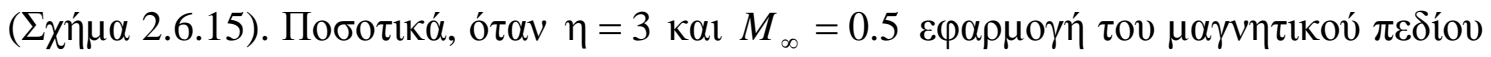

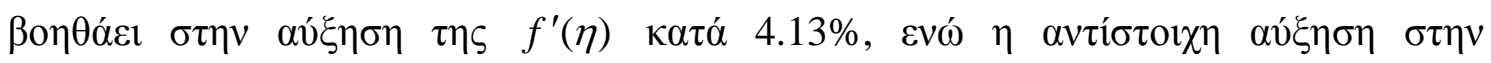

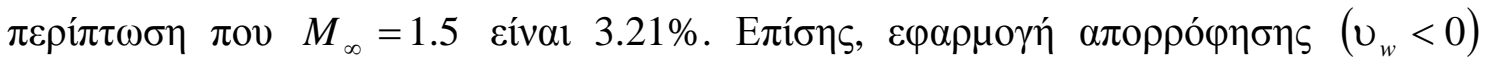




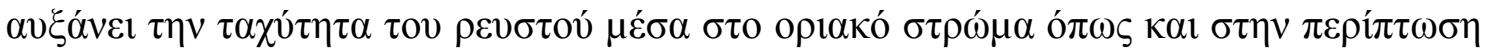

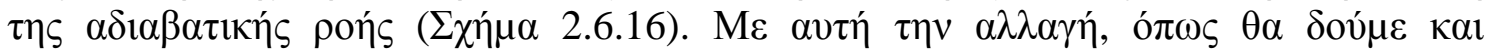

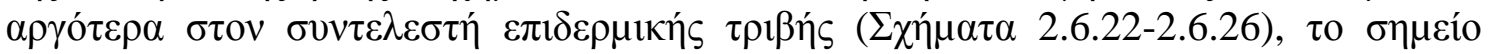

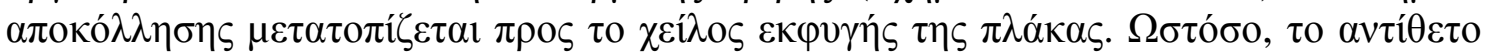

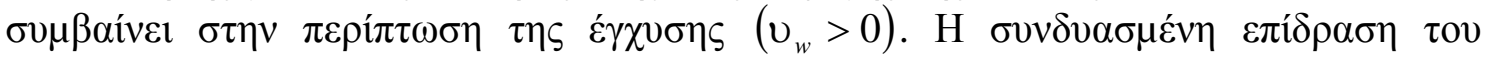

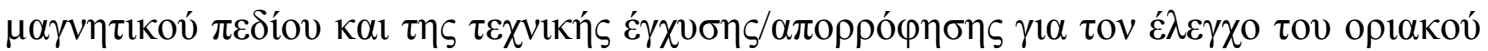

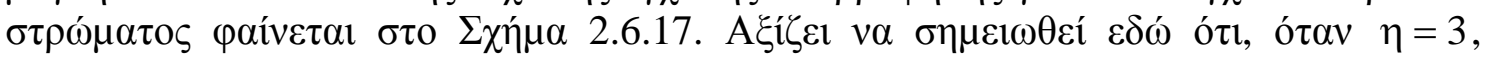

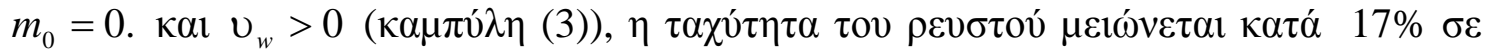

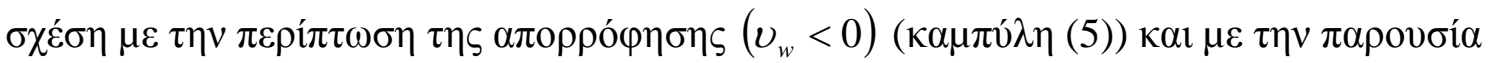

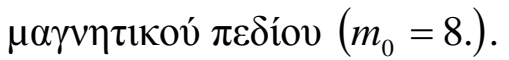

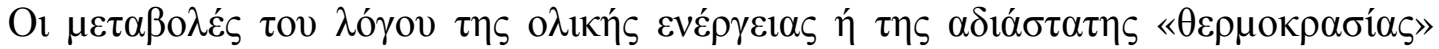

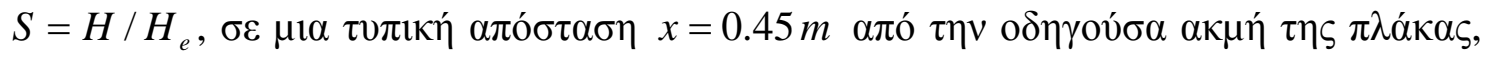

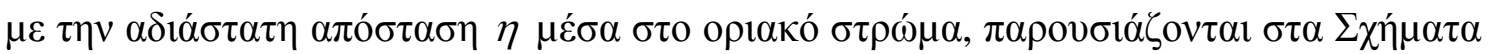

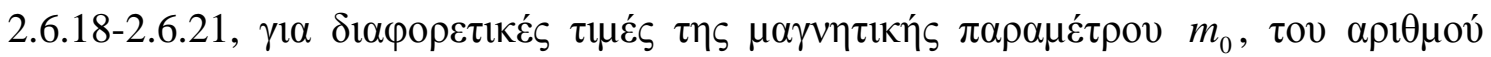

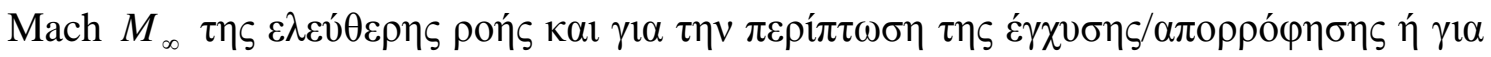

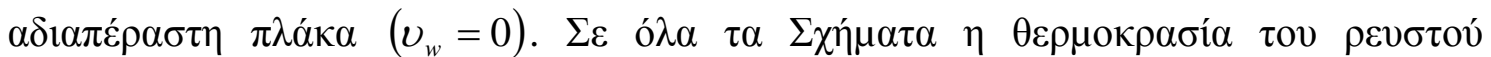

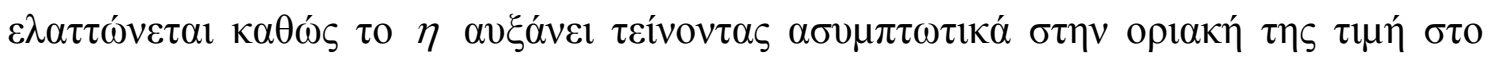

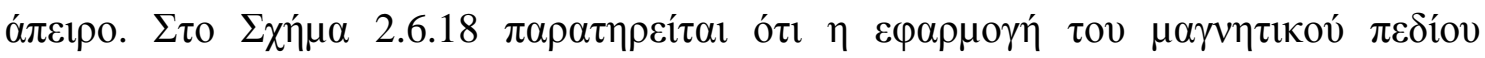

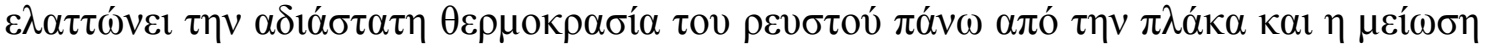

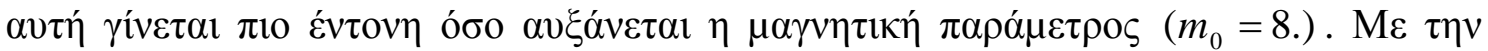

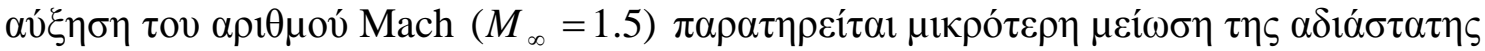

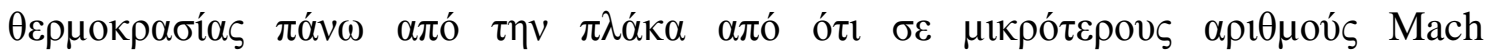

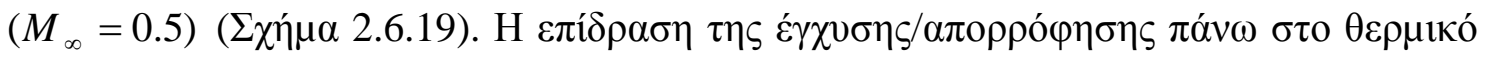

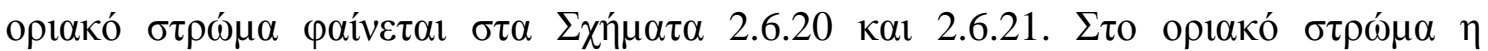

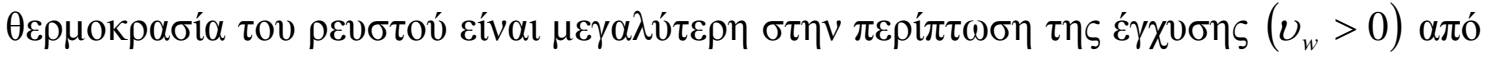

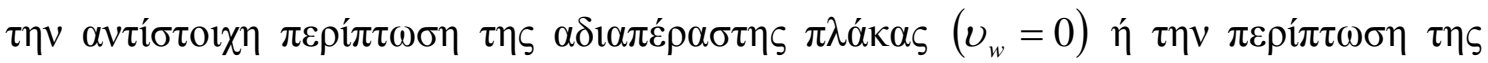

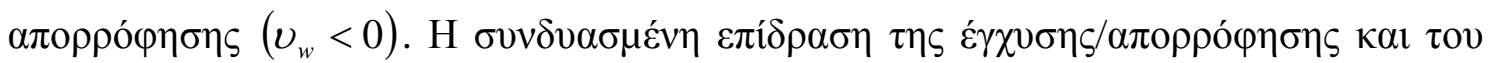

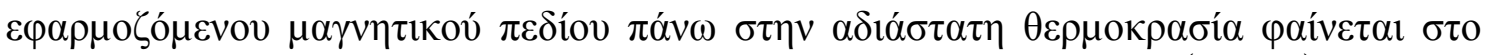

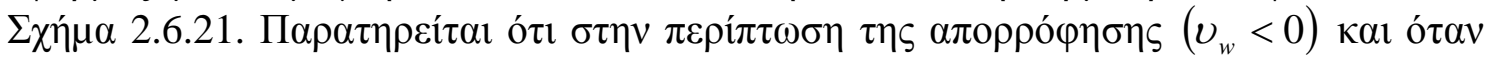

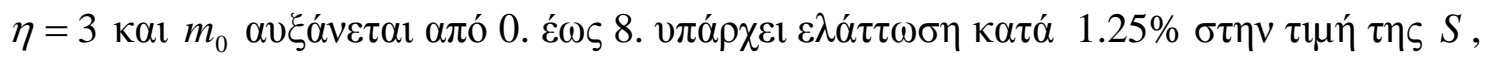

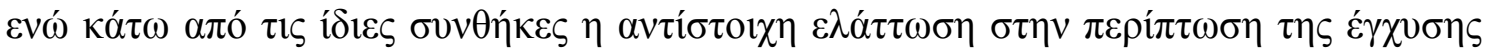

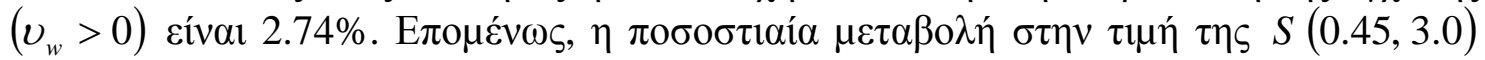

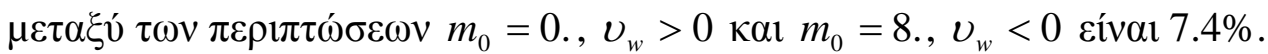

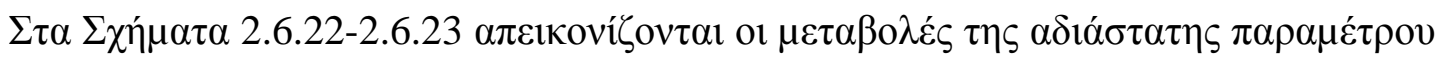

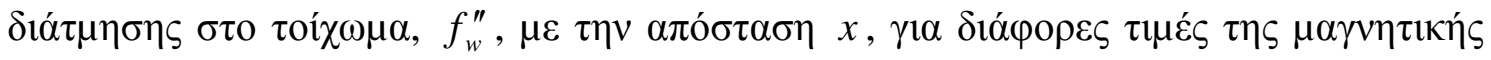

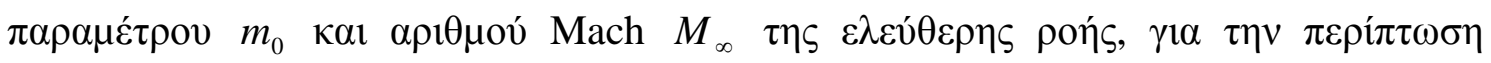

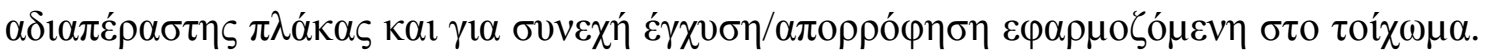

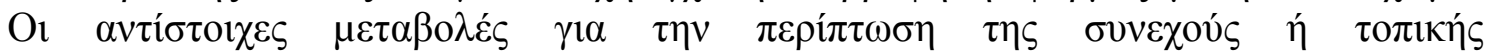

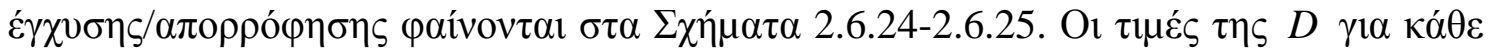

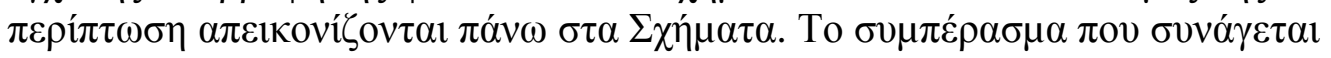




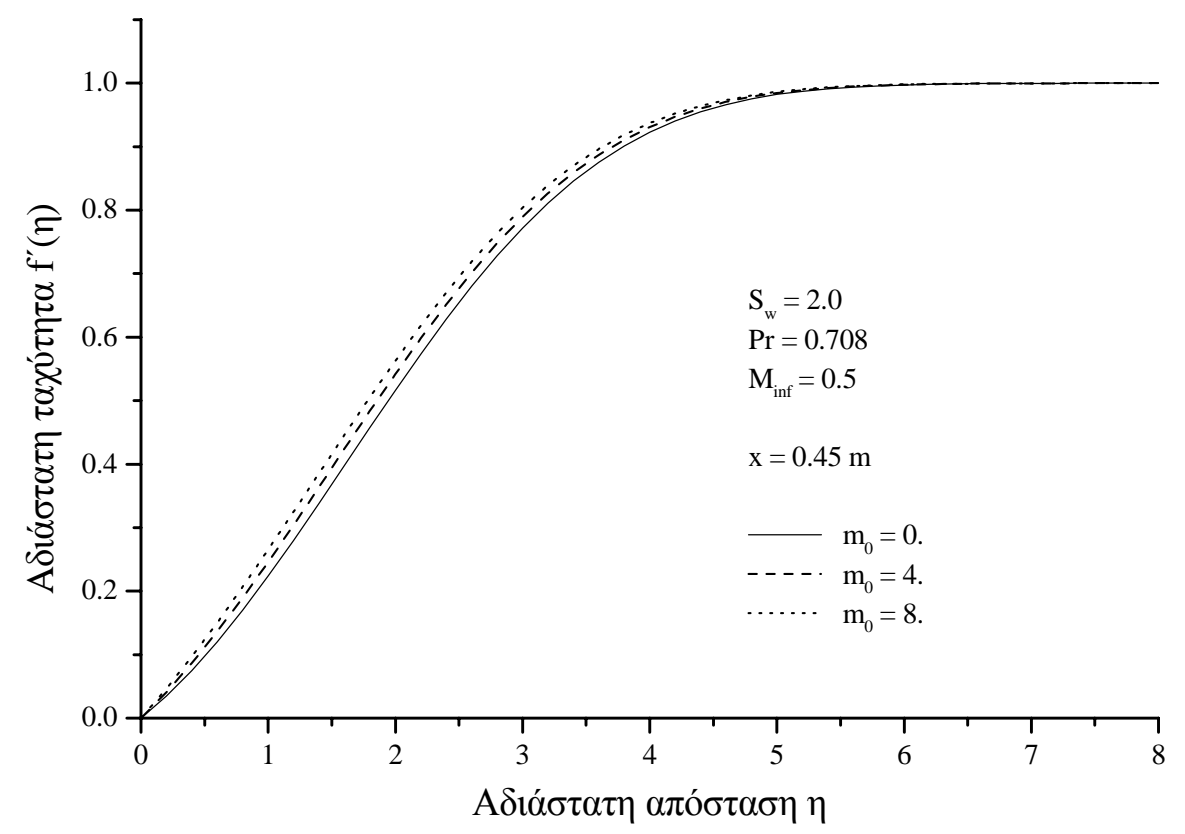

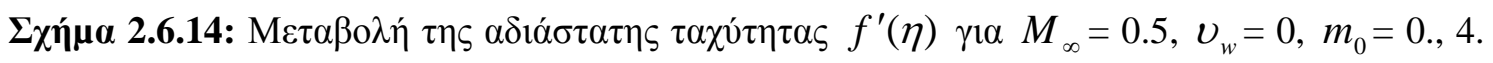
кol 8.

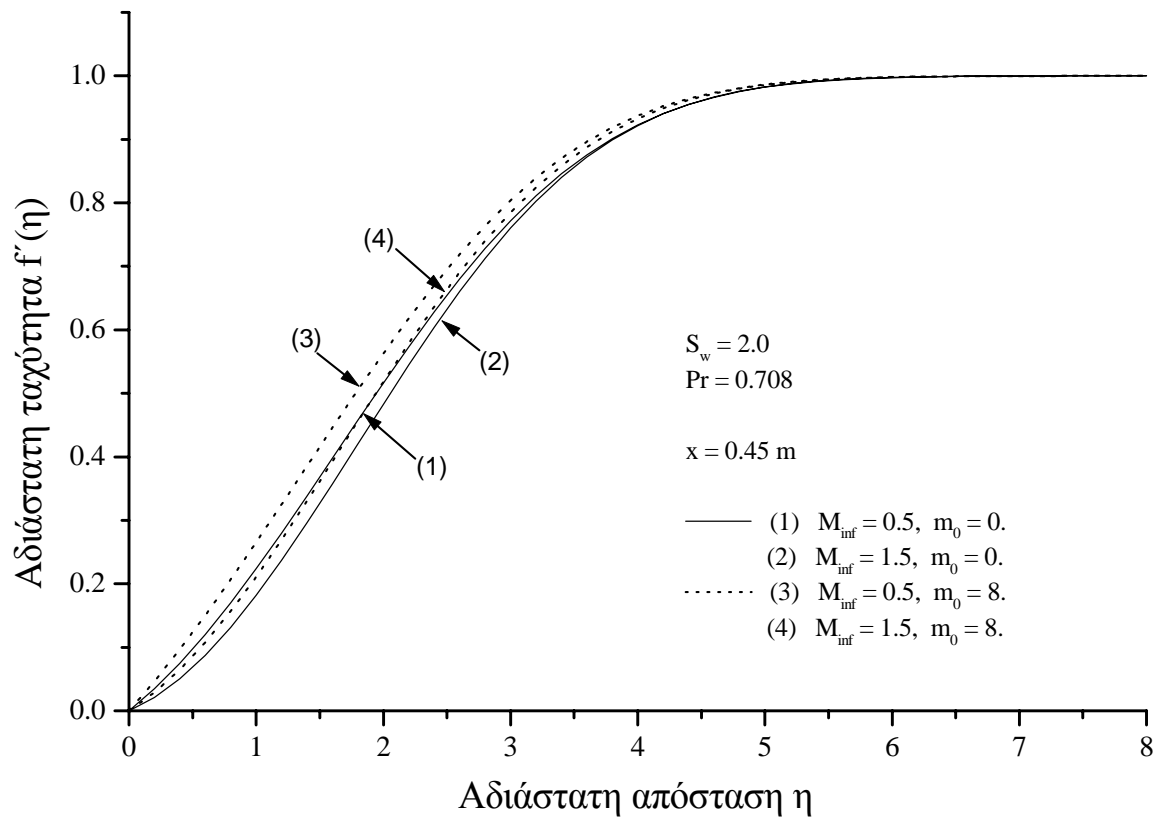

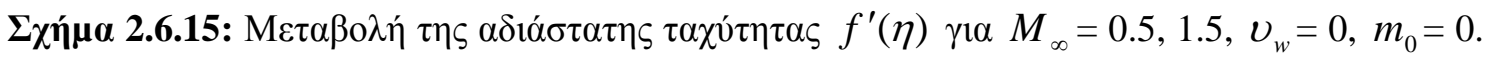
ка1 8 . 


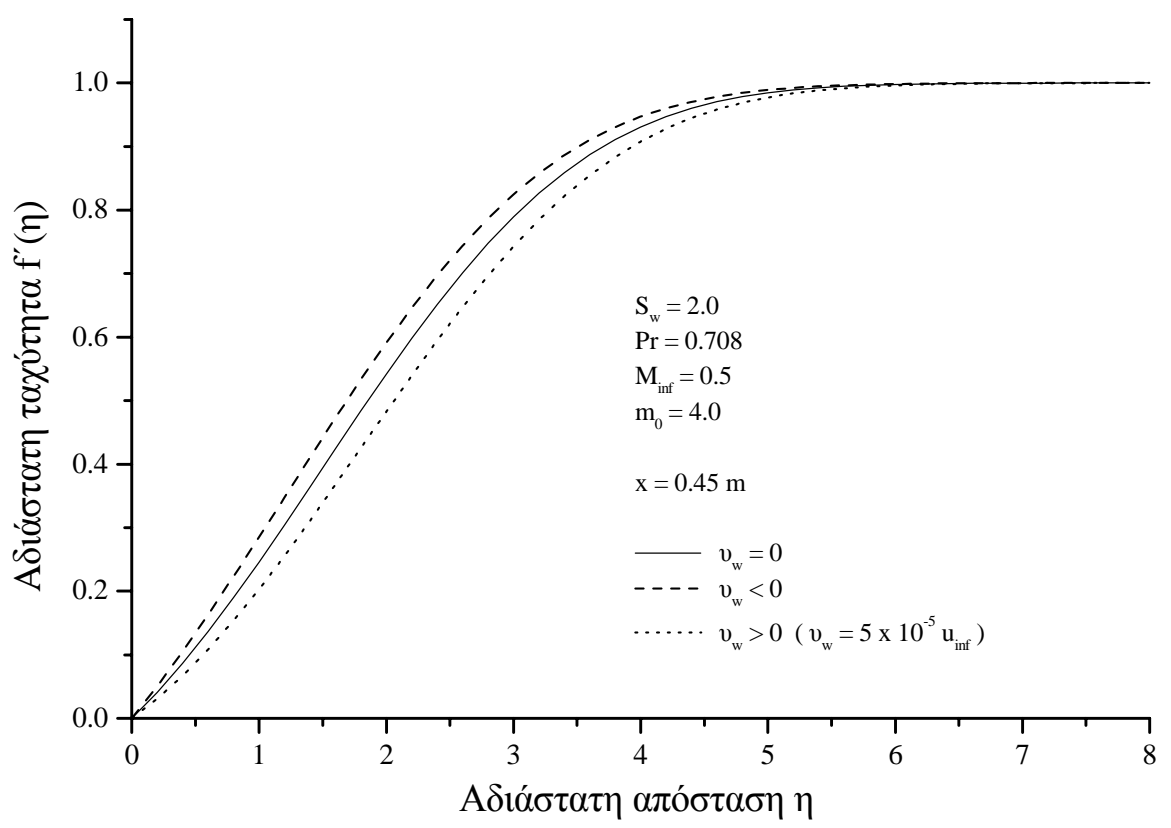

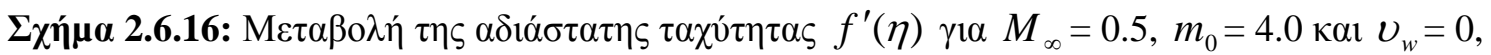
$<0,>0$.

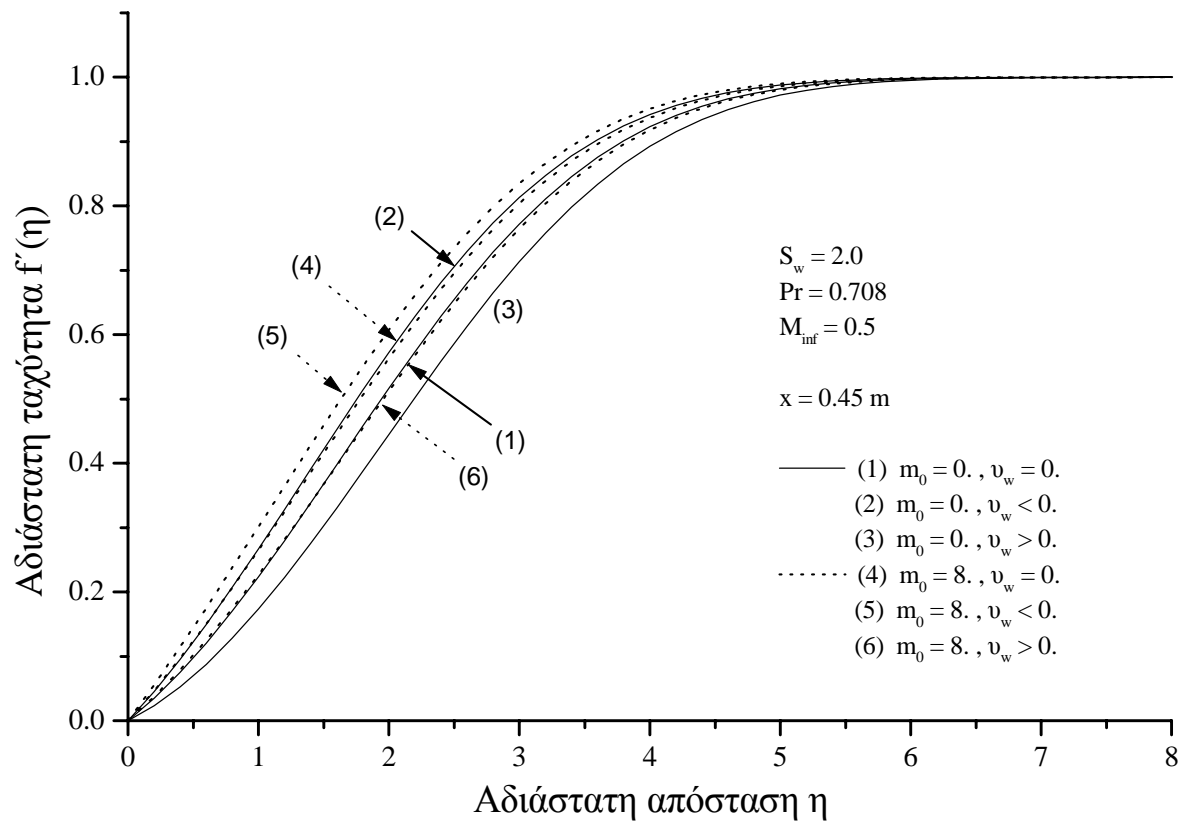

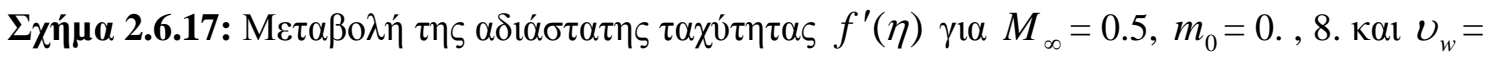
$0,<0,>0$. 


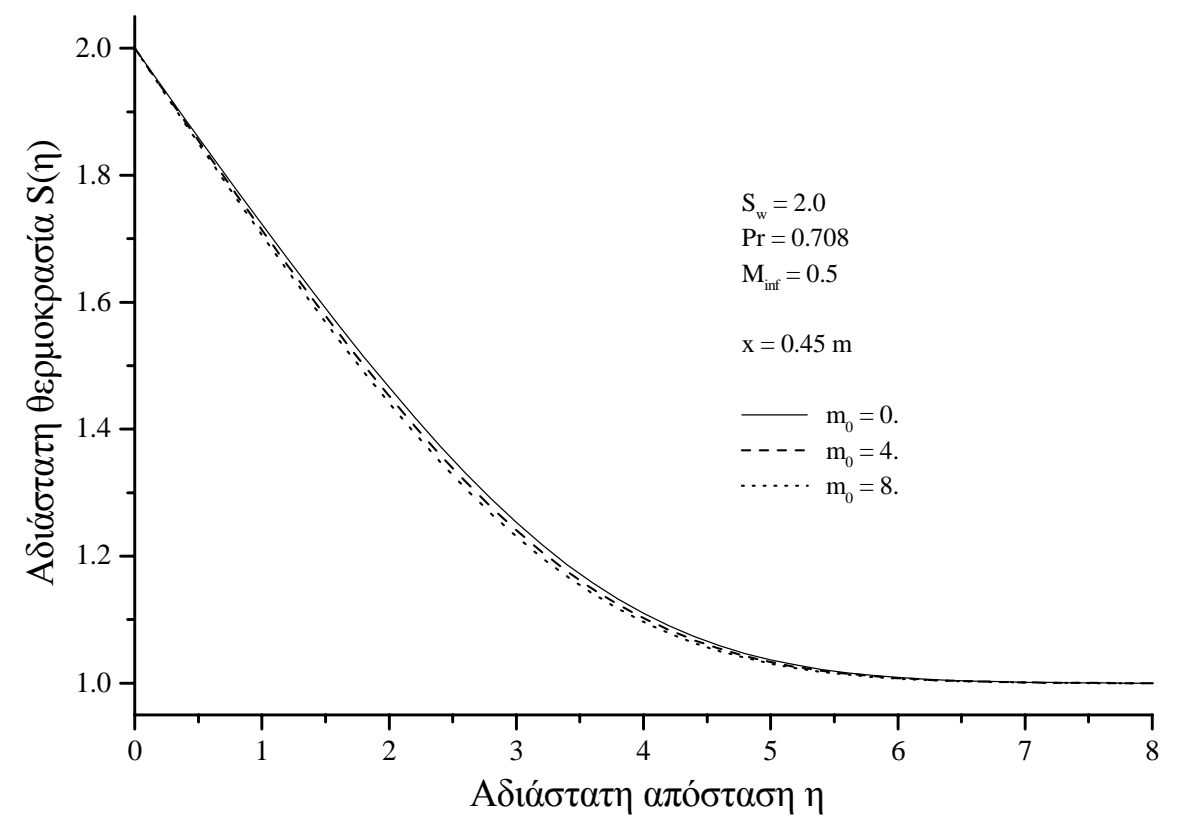

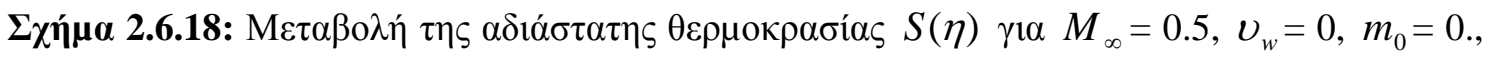
4. $\kappa \alpha 18$.

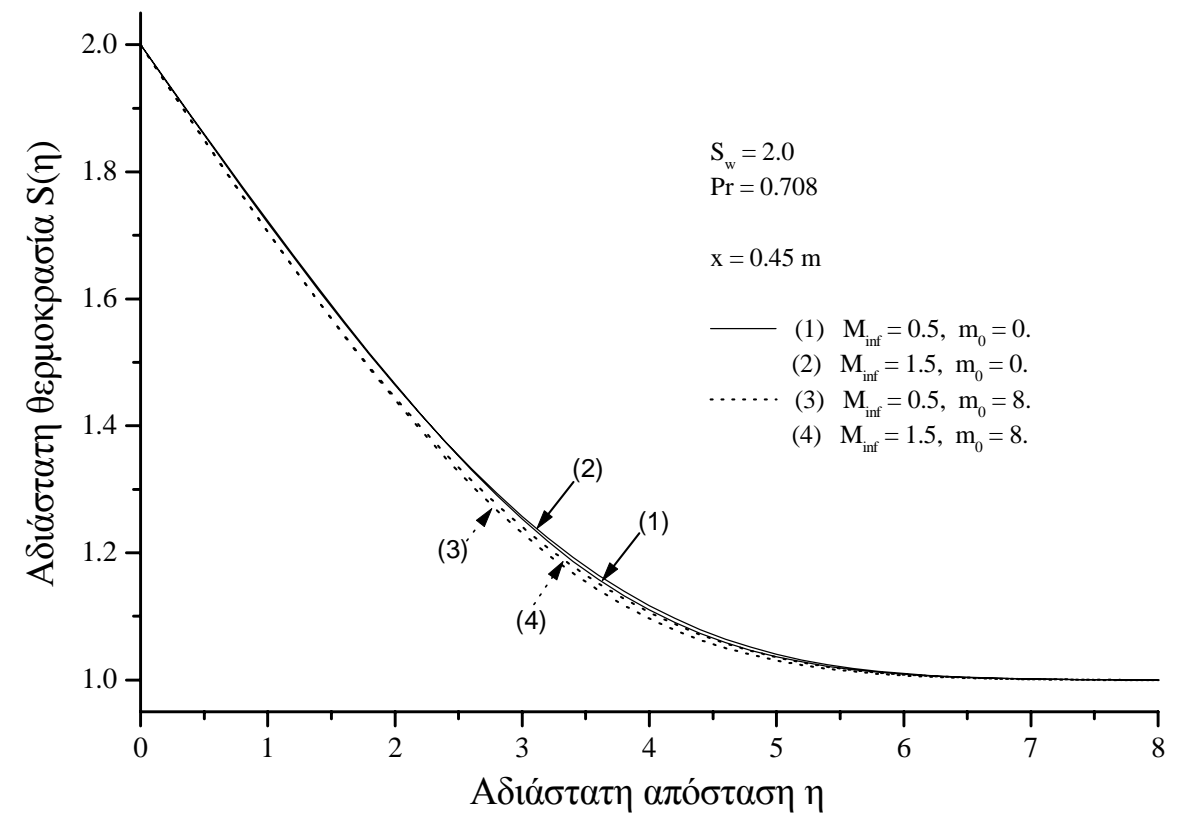

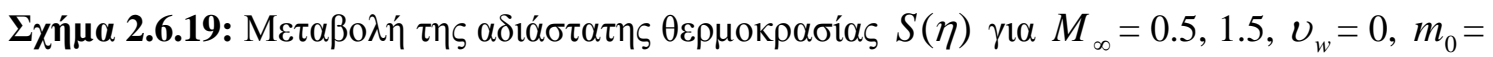
0. ка1 8. 


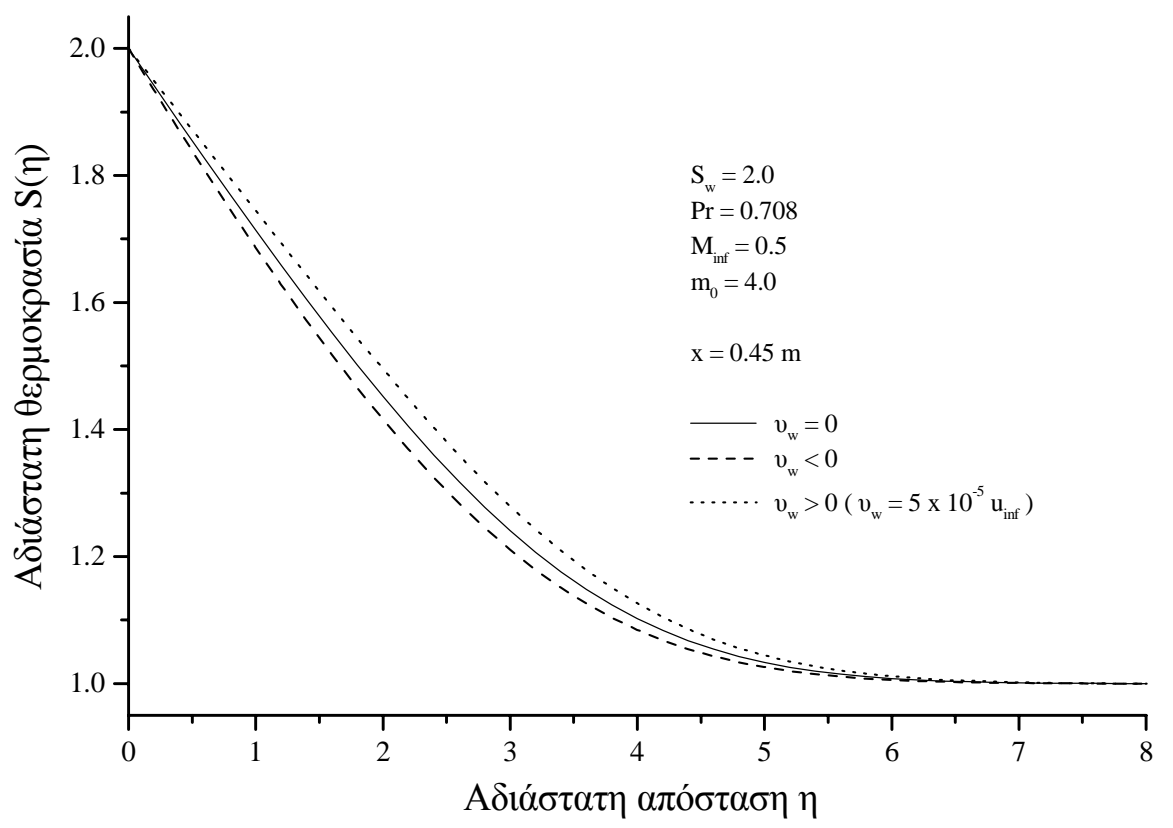

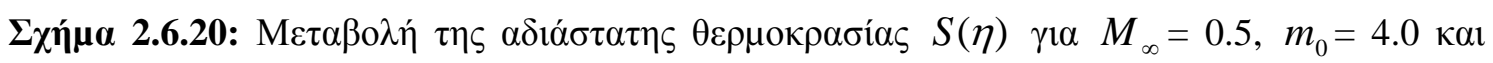
$v_{w}=0,<0,>0$.

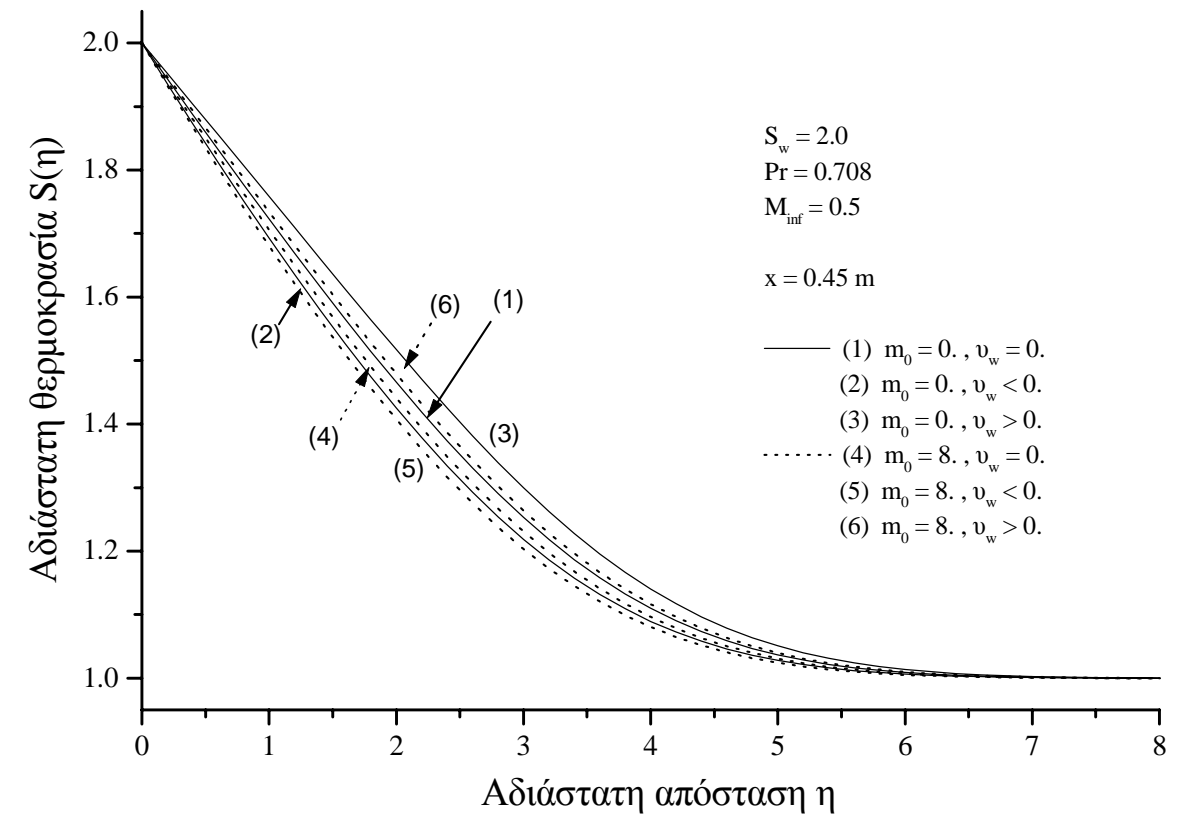

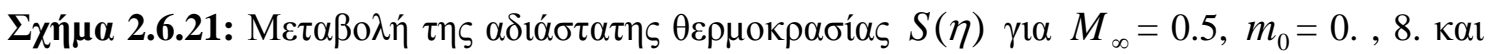
$v_{w}=0,<0,>0$. 


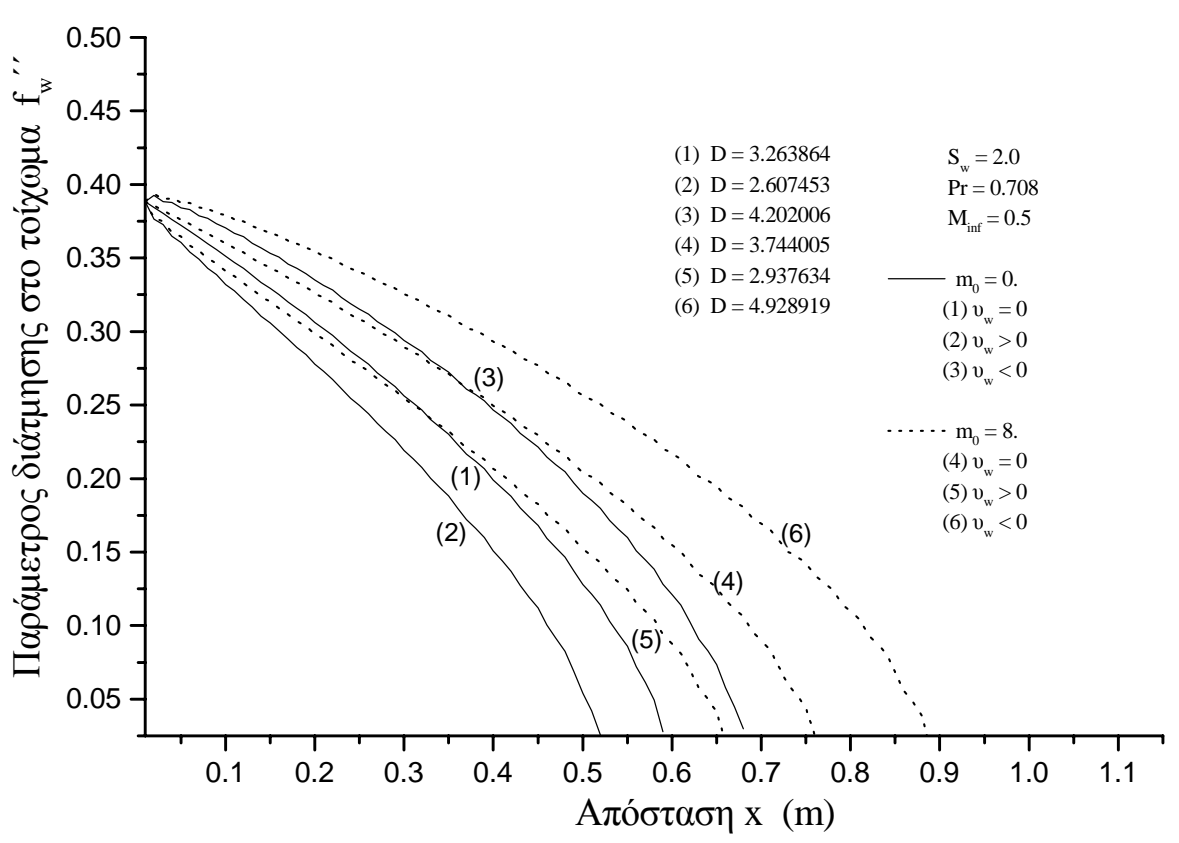

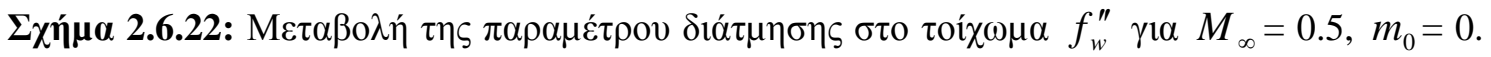
ка1 8. $\kappa \alpha 1 v_{w}=0,>0,<0$.

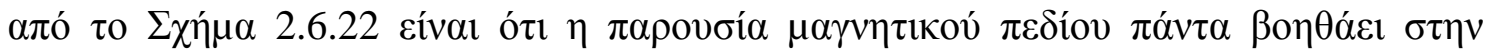

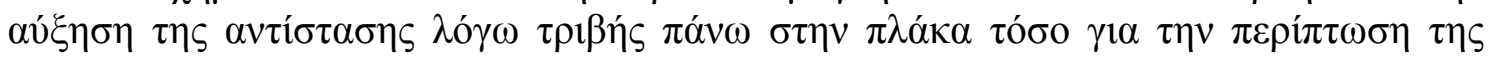

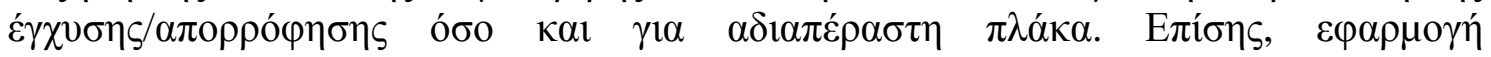

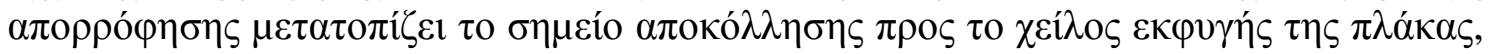

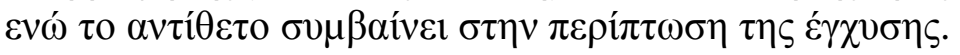

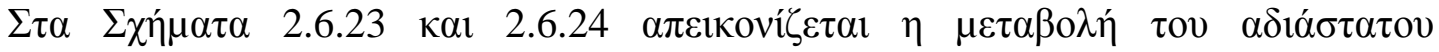

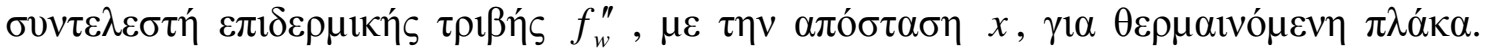

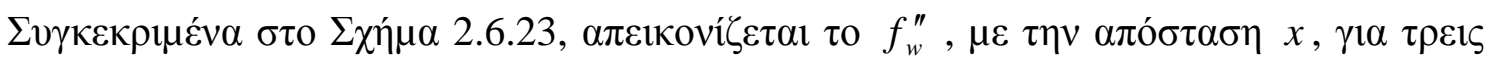

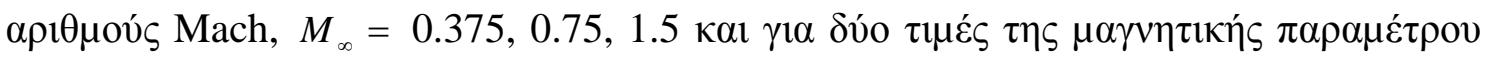

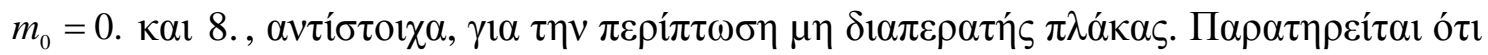

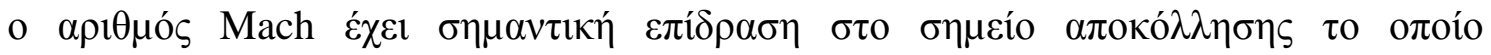

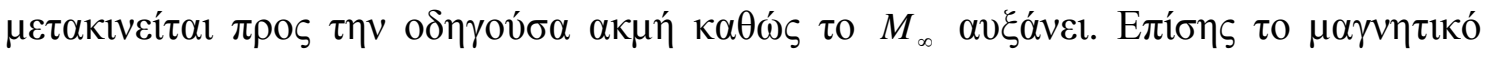

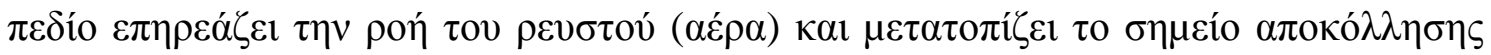

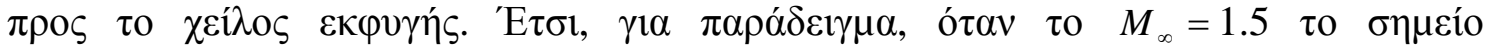

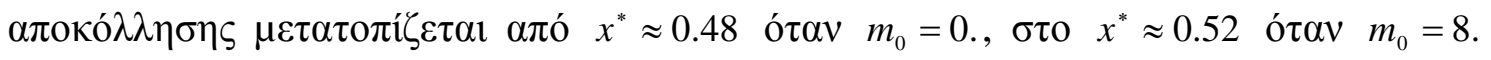

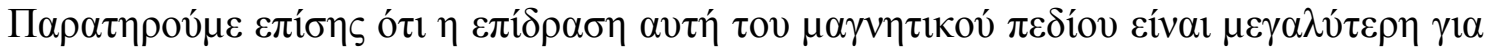

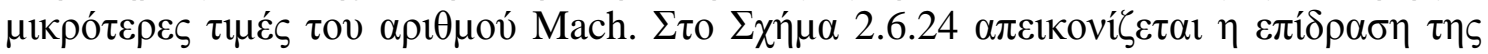

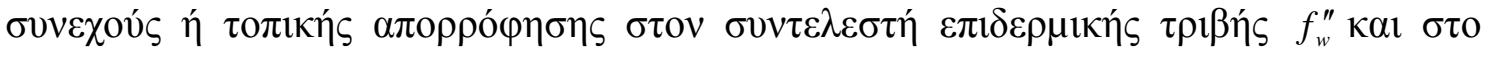

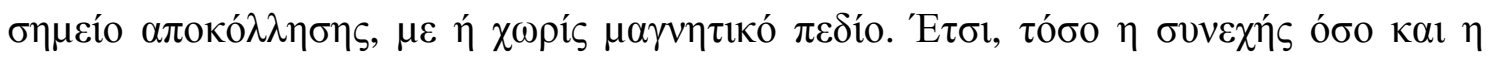

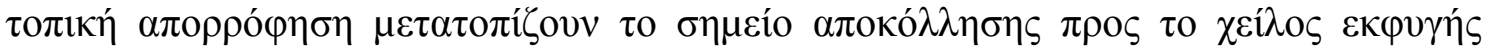

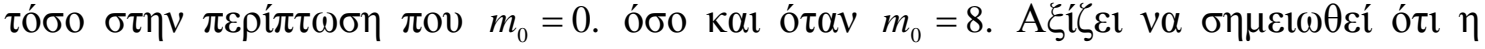

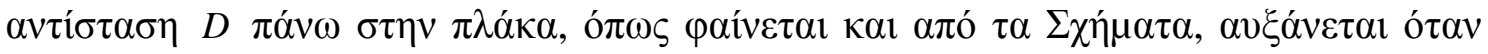

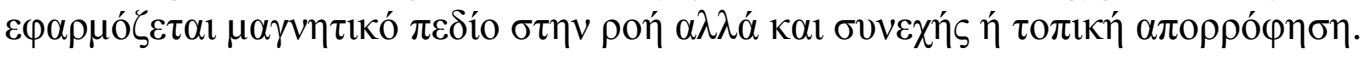




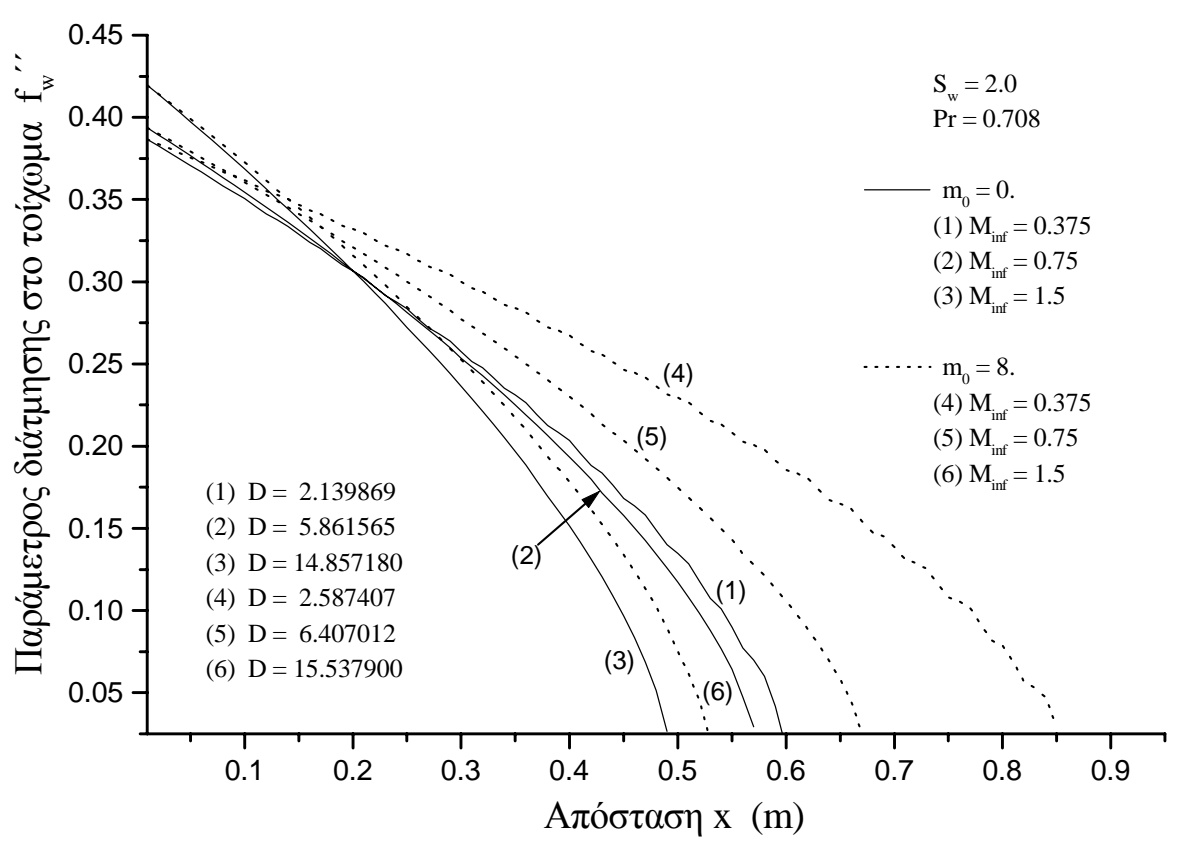

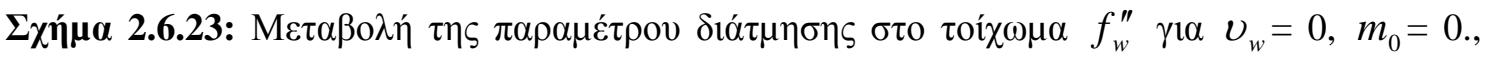

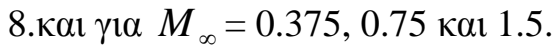

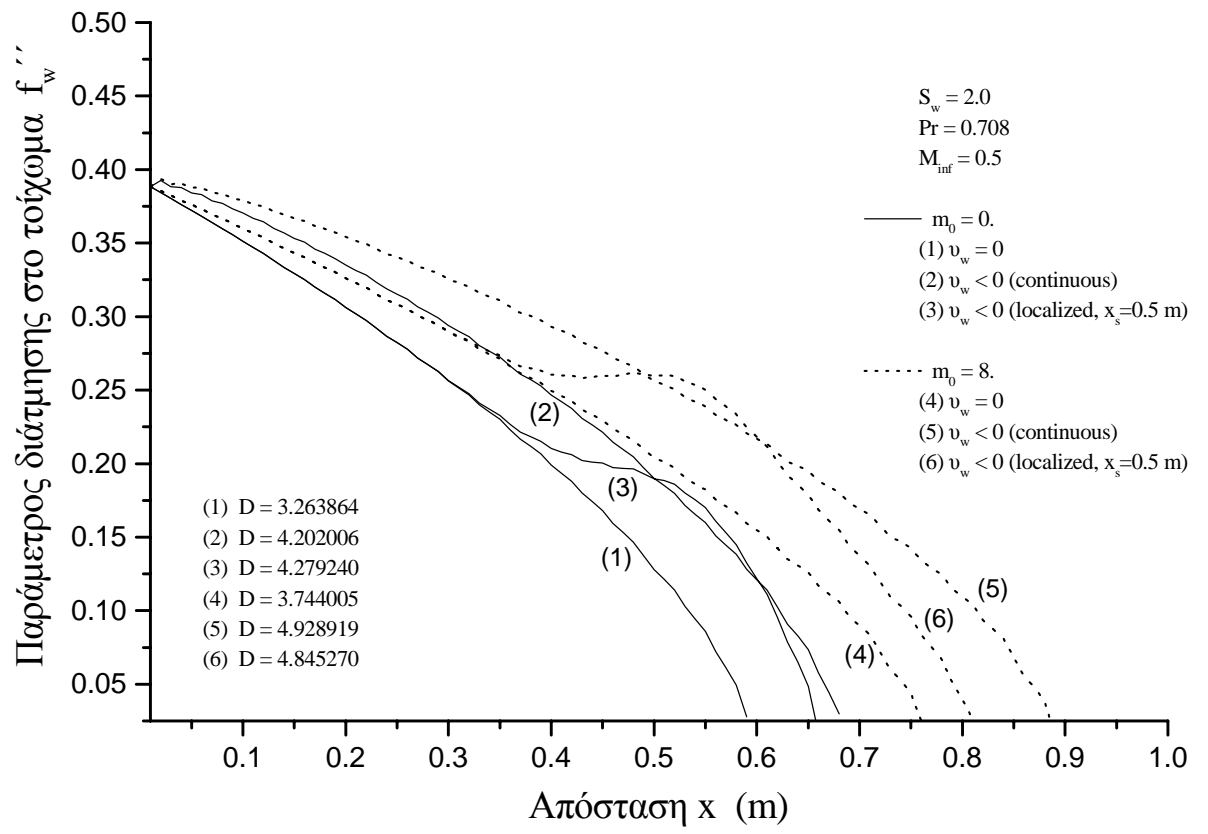

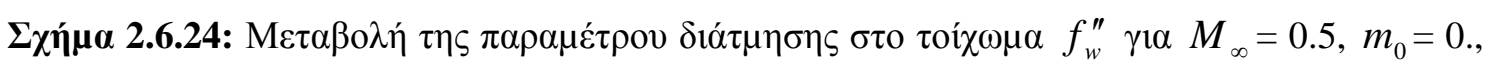

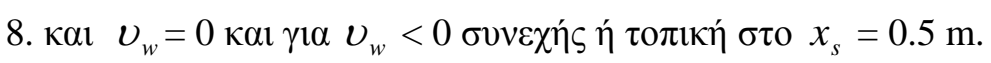




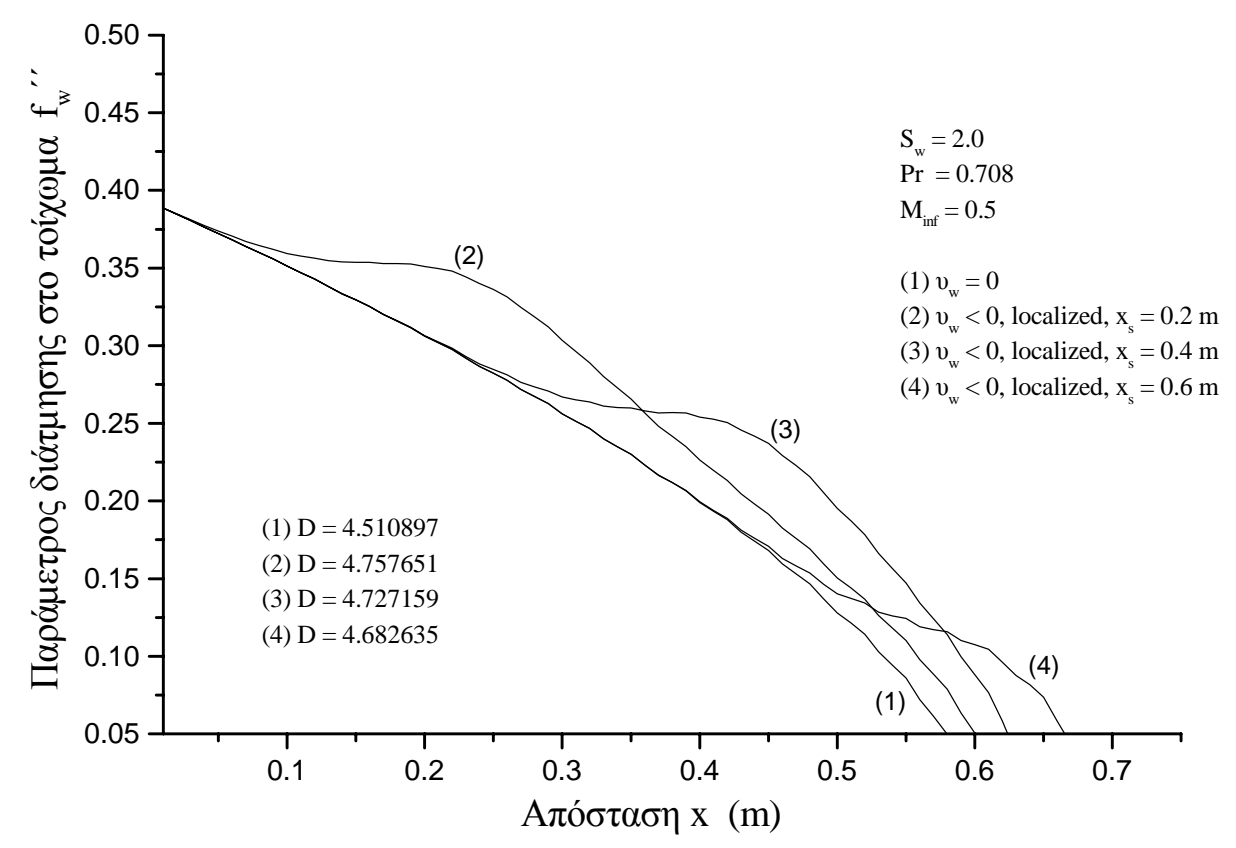

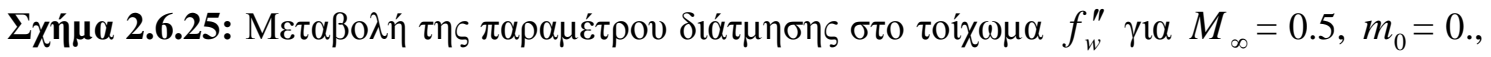

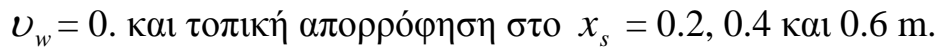

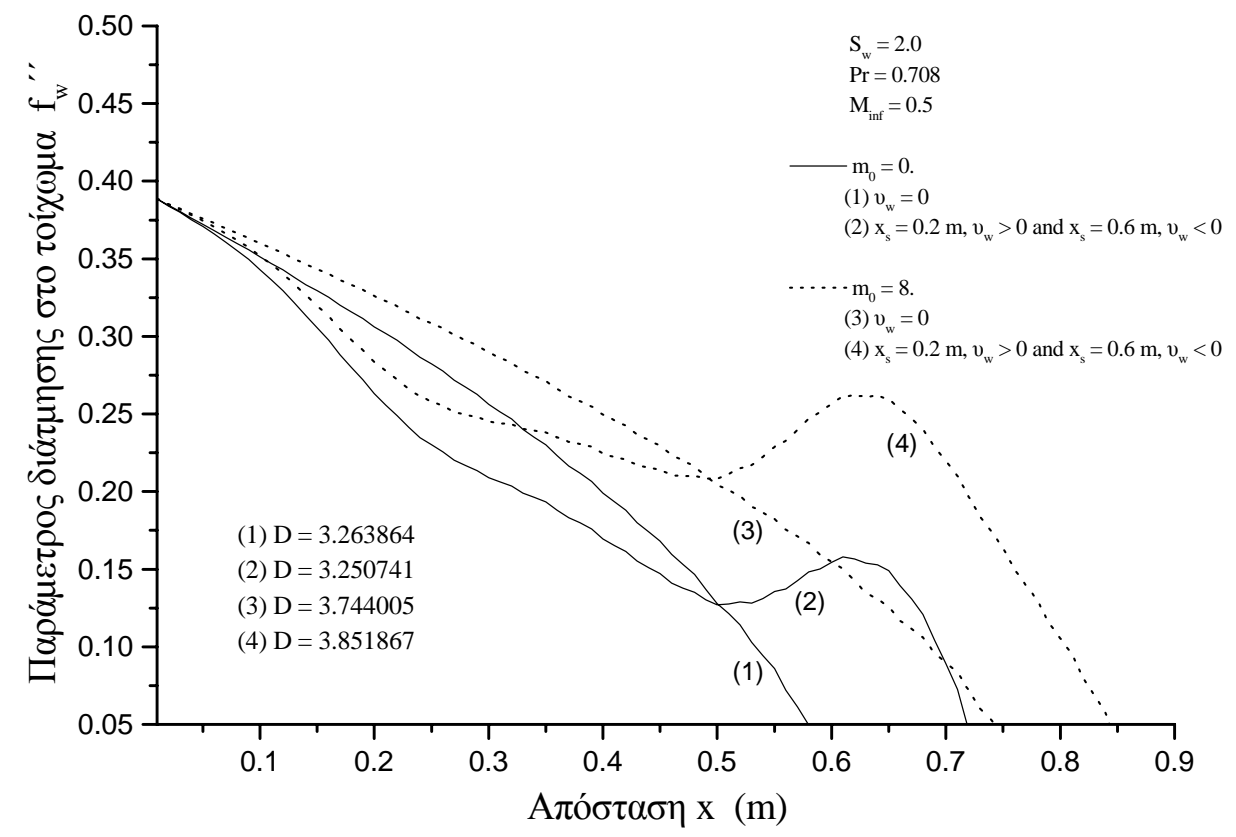

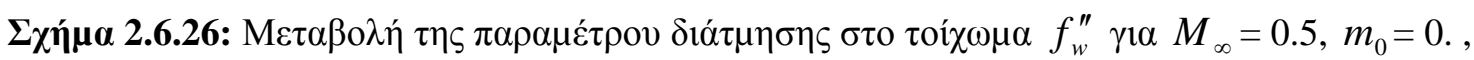

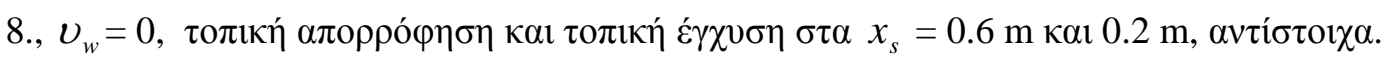




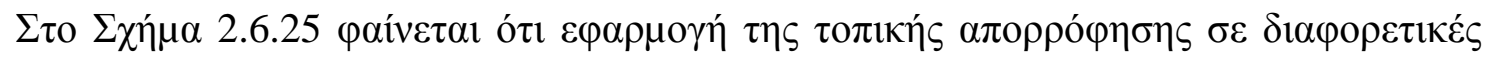

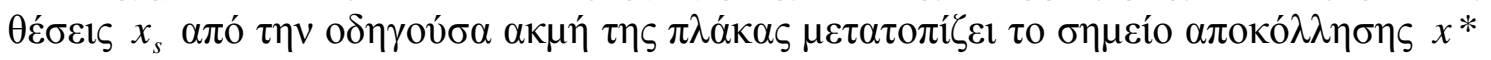

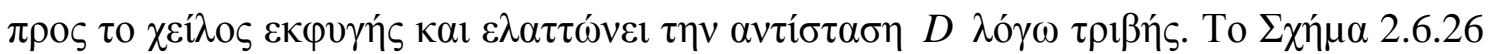

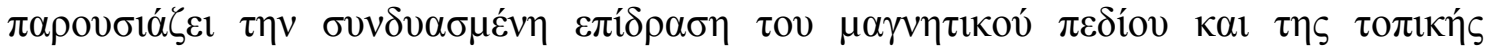

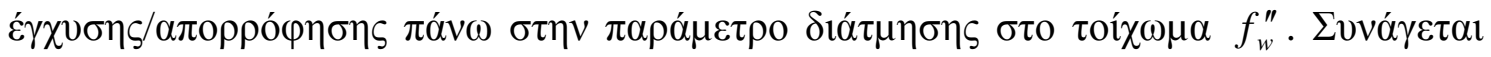

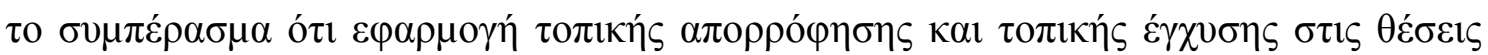

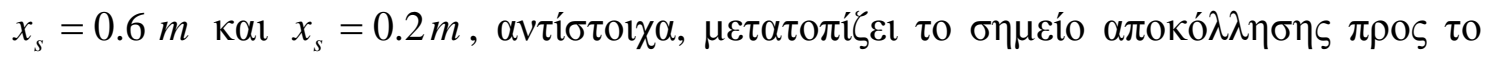

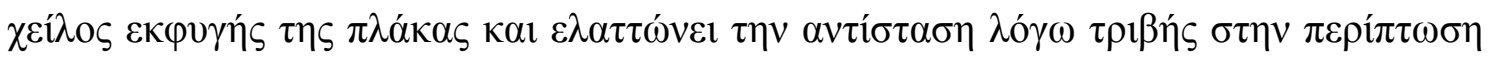

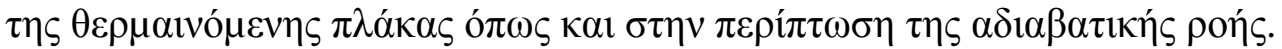

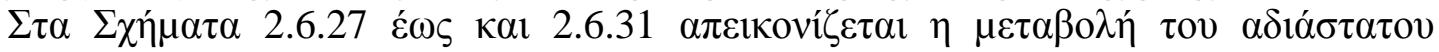

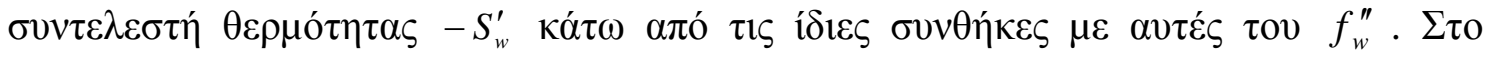

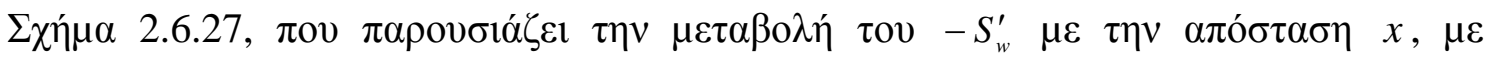

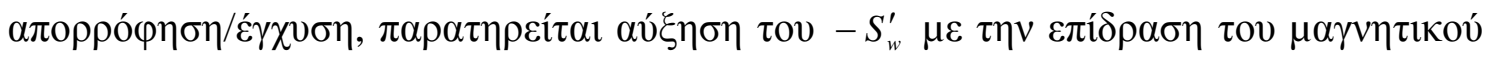

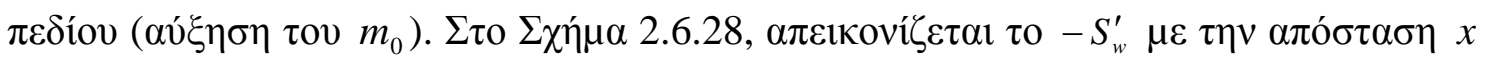

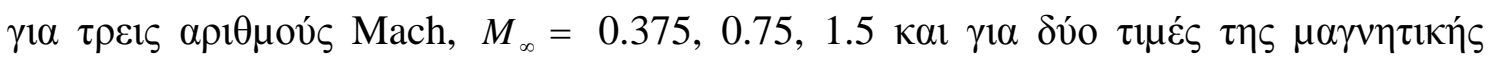

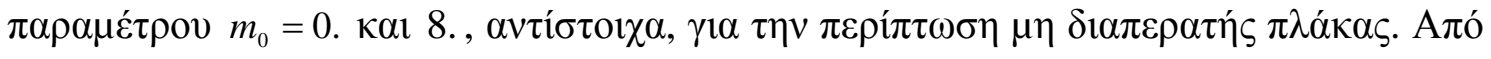

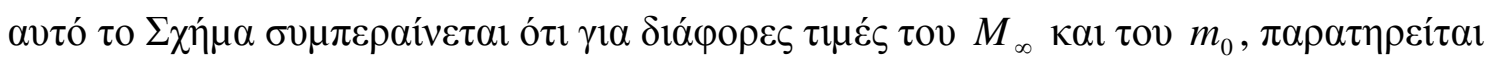

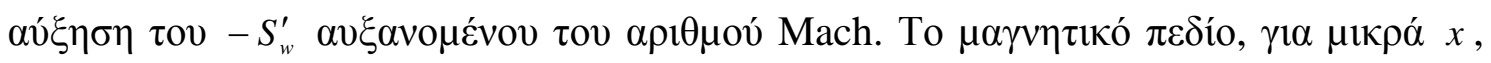

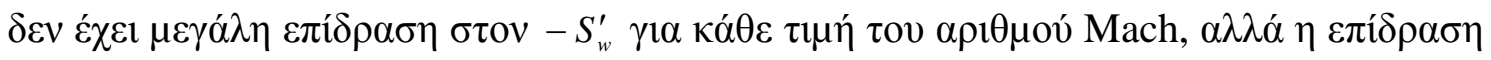

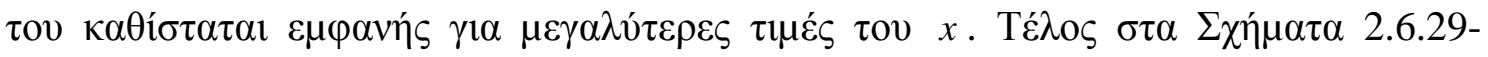

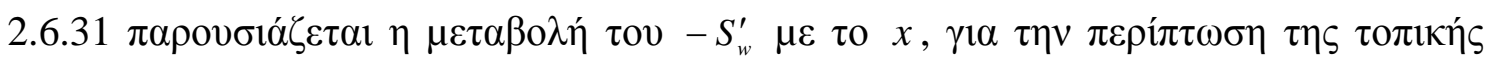

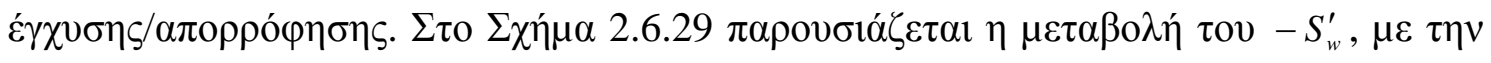

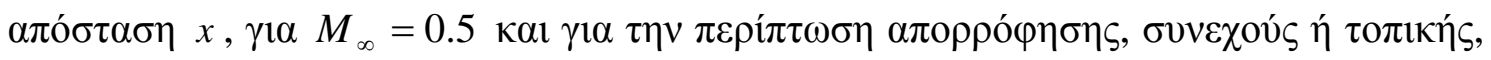

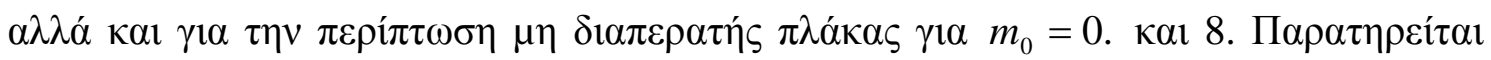

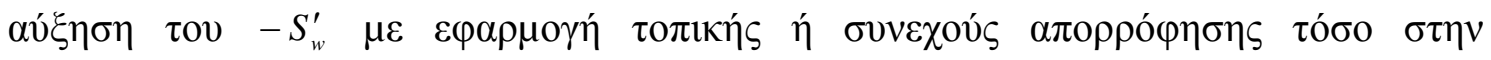

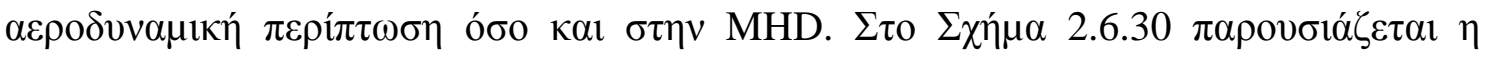

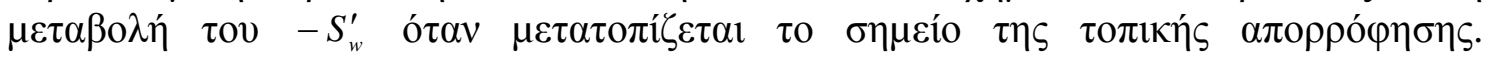

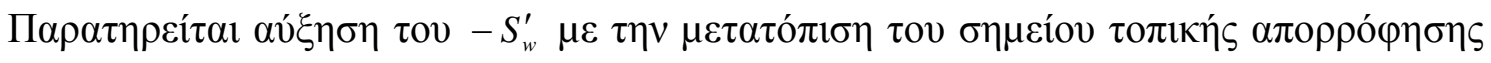

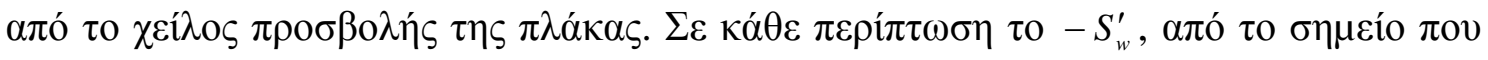

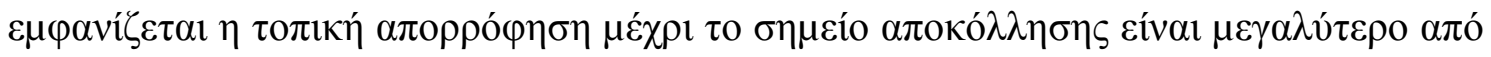

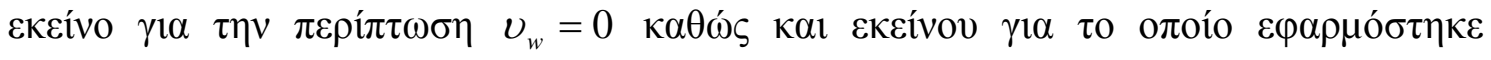

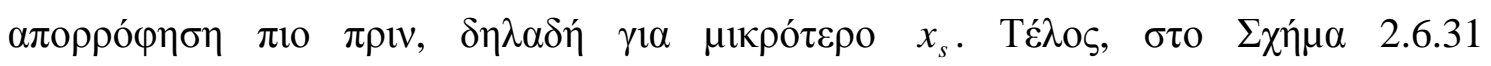

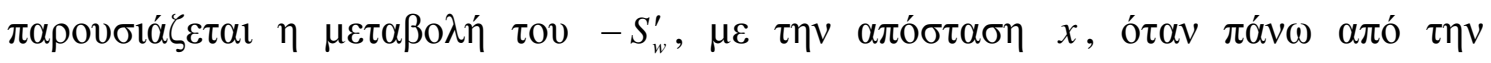

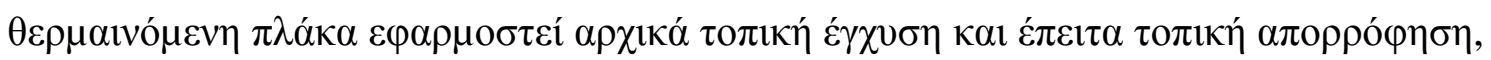

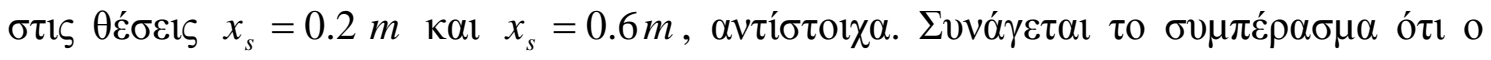

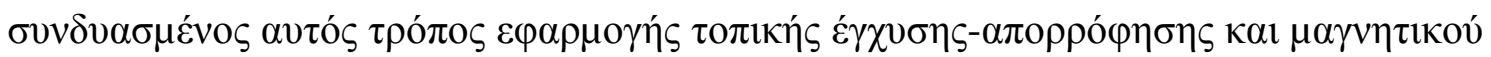

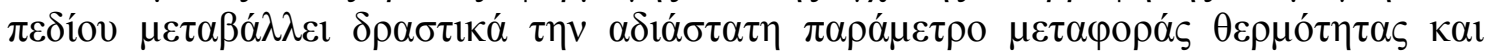

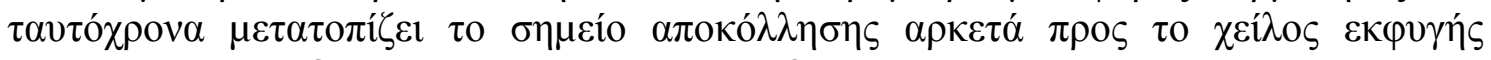

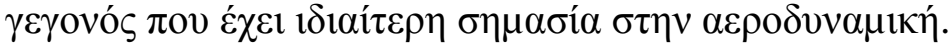




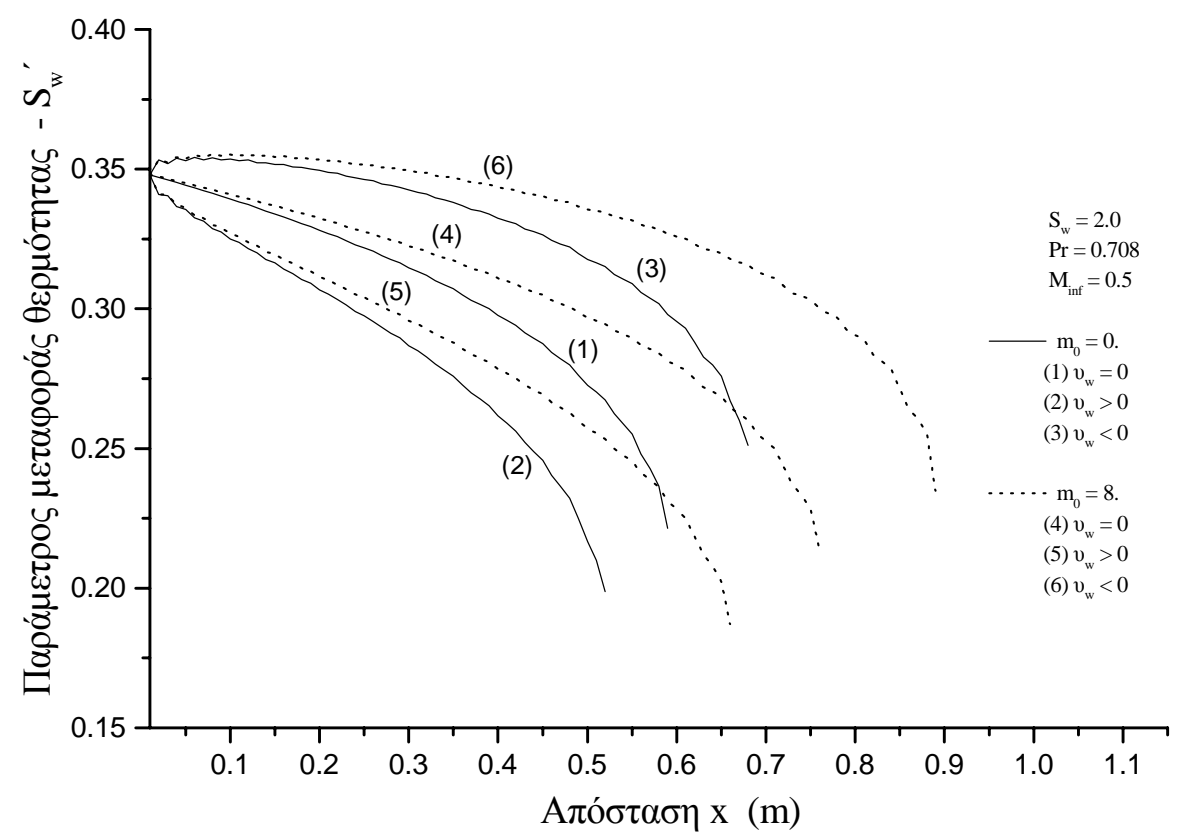

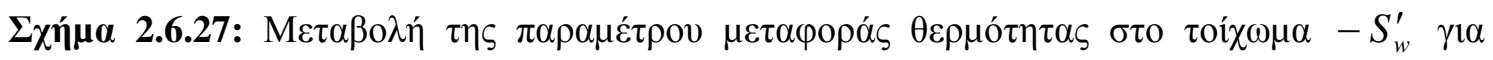
$M_{\infty}=0.5, m_{0}=0 . \kappa \alpha 18 . \kappa \alpha 1 \quad v_{w}=0,>0,<0$.

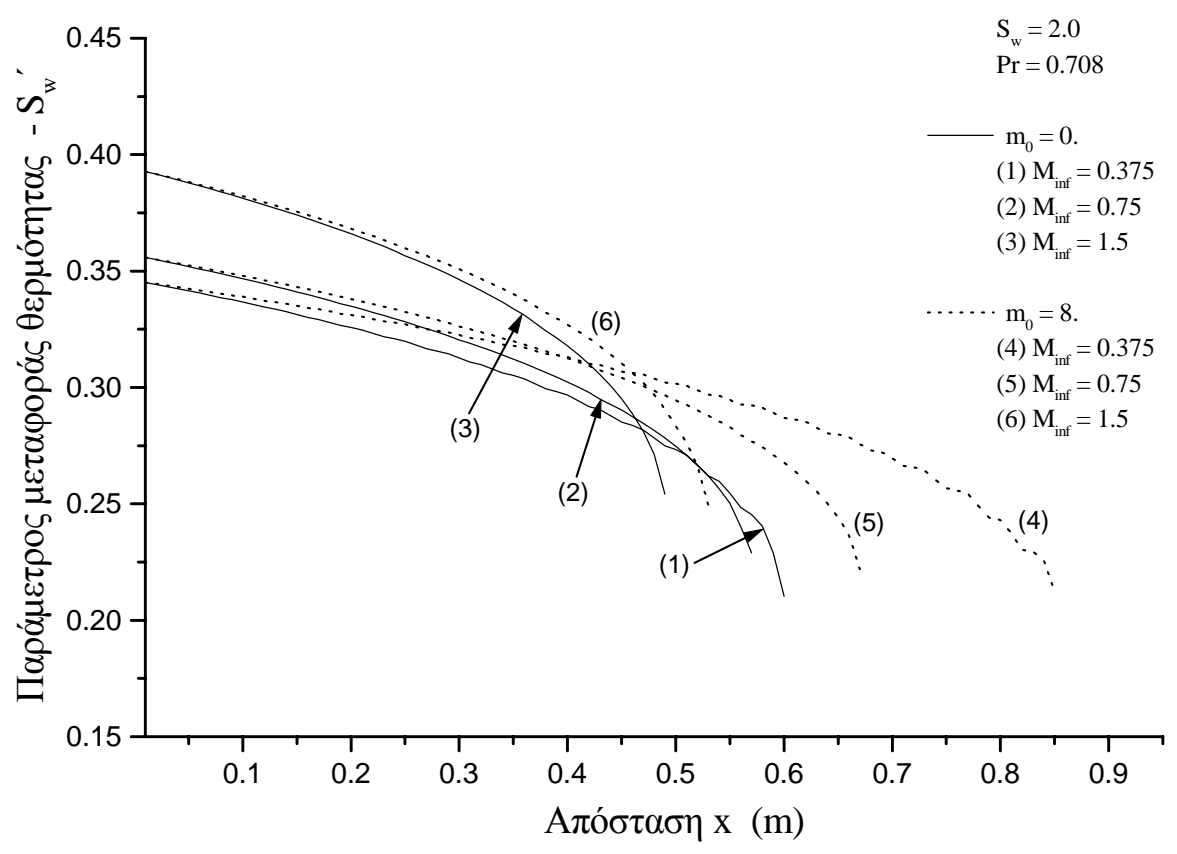

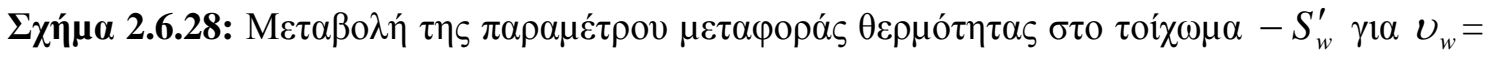
$0, m_{0}=0 ., 8 . \kappa \alpha \mathrm{l} \gamma 1 \alpha M_{\infty}=0.375,0.75 \kappa \alpha 1$ 1.5. 


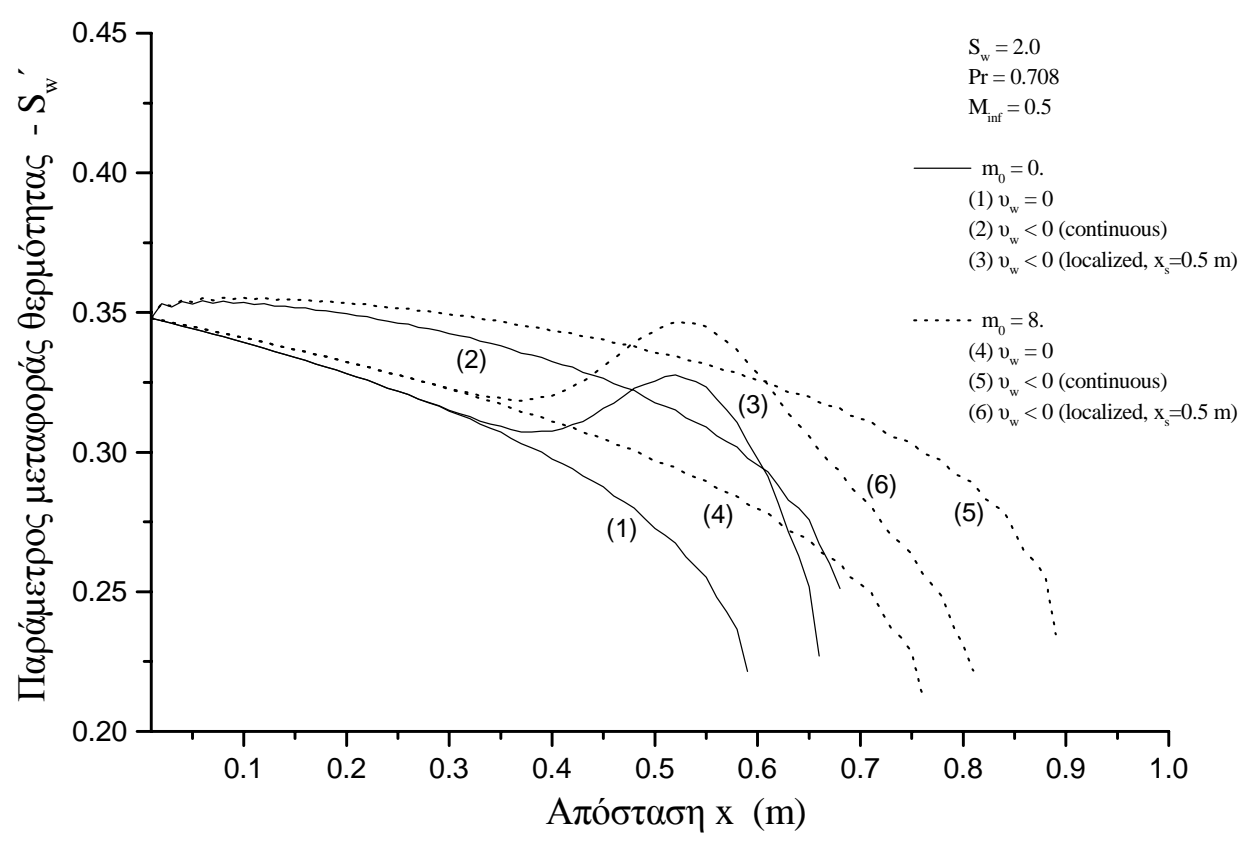

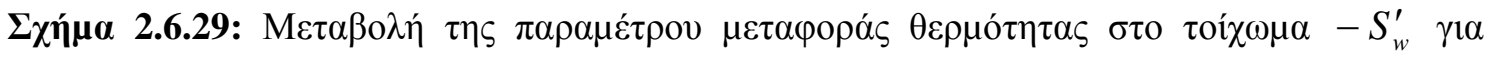

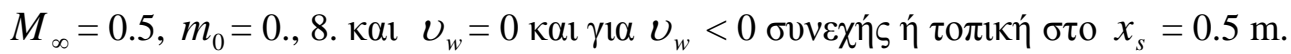

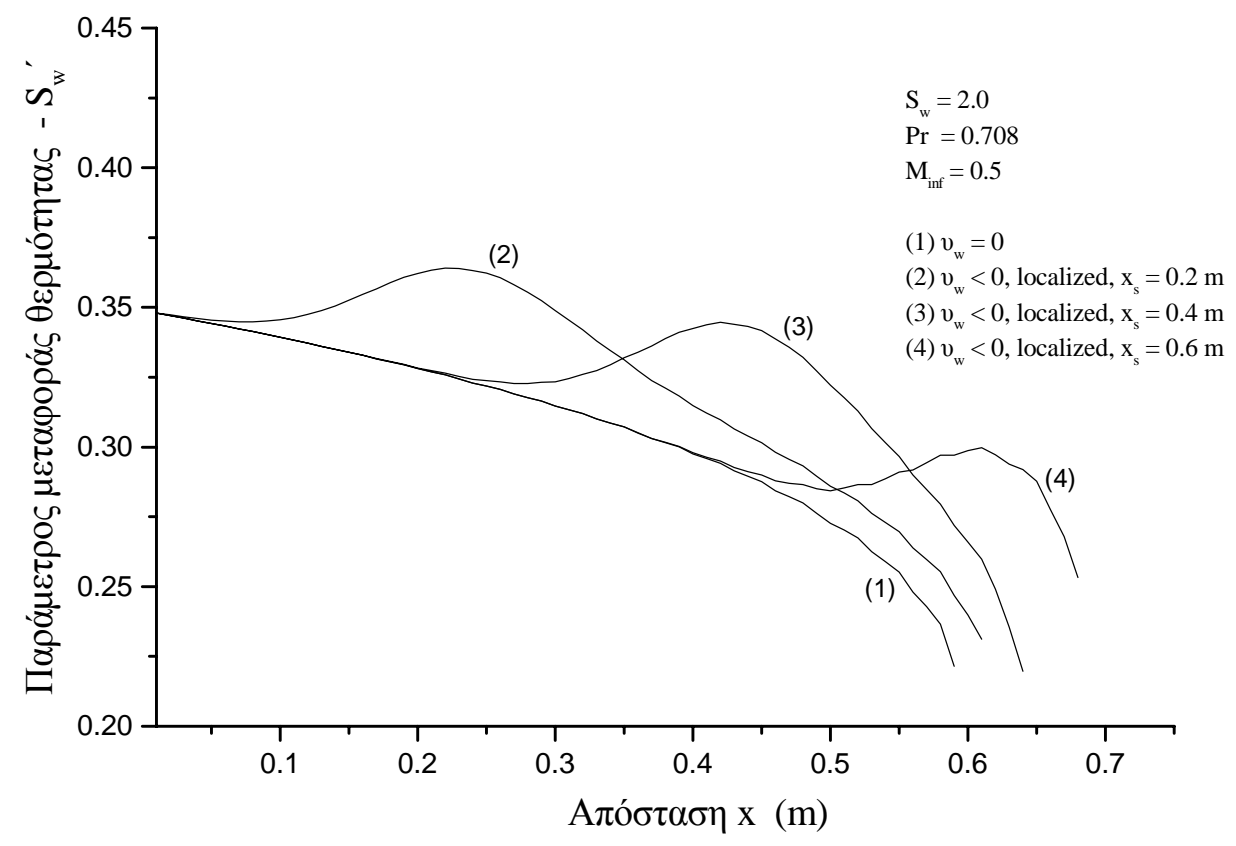

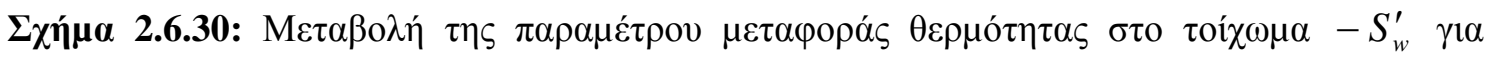

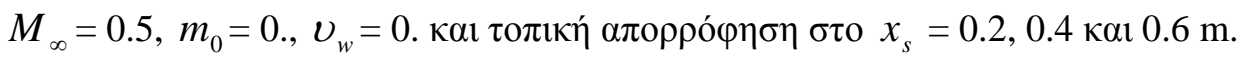




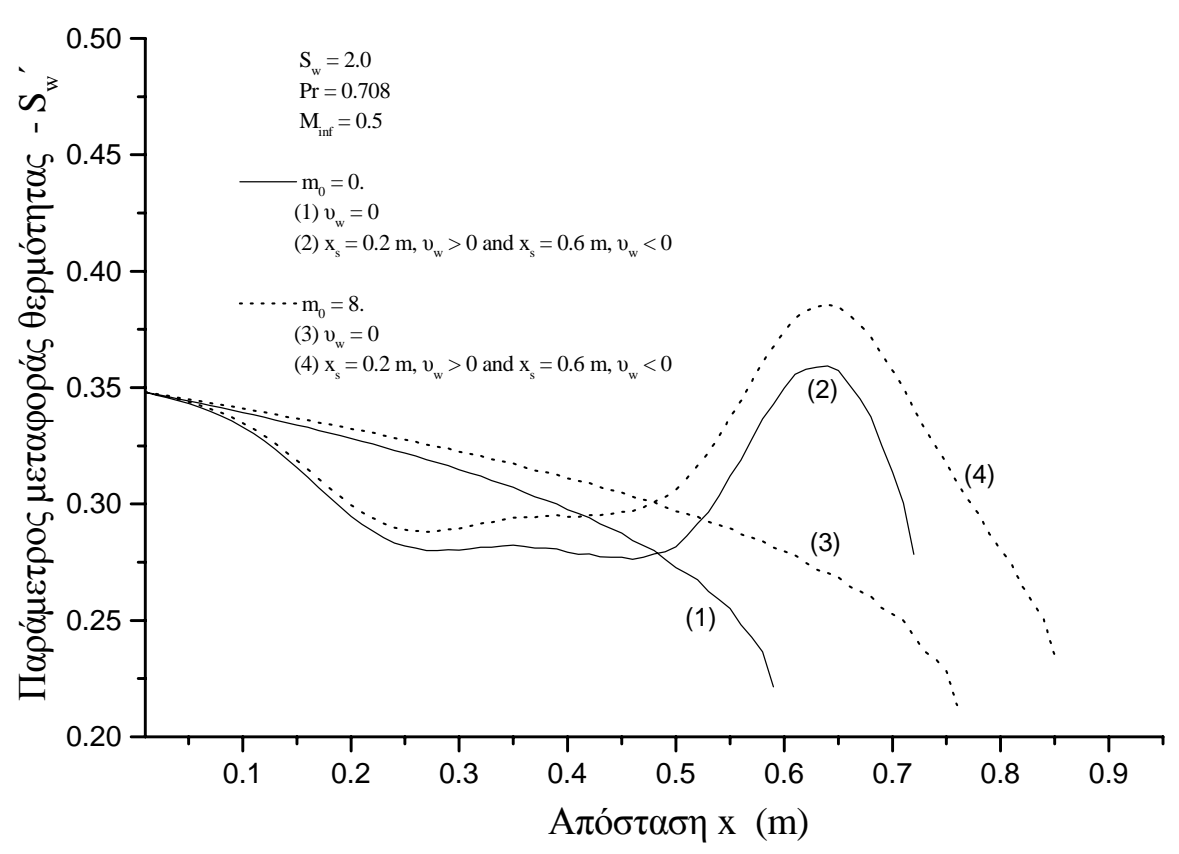

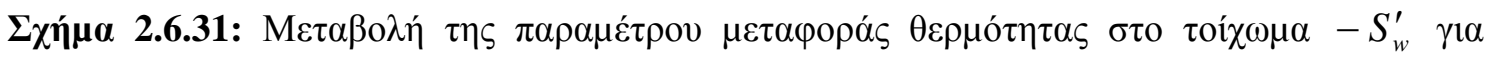

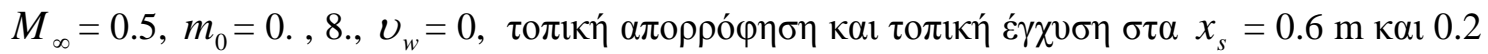
$\mathrm{m}, \alpha v \tau i ́ \sigma \tau o \imath \alpha \alpha$

(iii) $\Psi \mathbf{v} \chi \hat{o} \boldsymbol{\mu} \varepsilon v \boldsymbol{\eta} \boldsymbol{\pi} \lambda \boldsymbol{\alpha} \boldsymbol{\alpha} \boldsymbol{\alpha}\left(S_{w}=0.5\right)$.

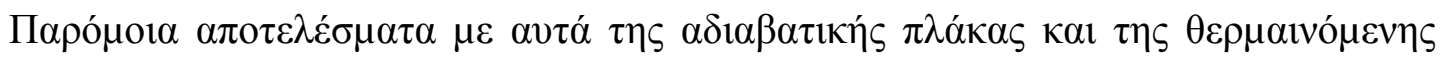

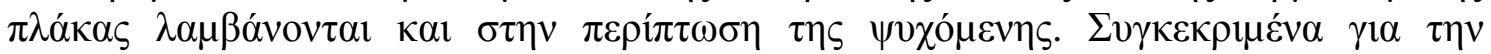

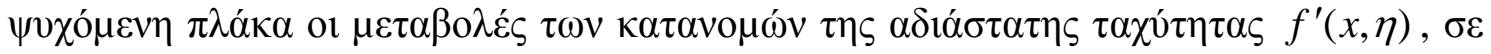

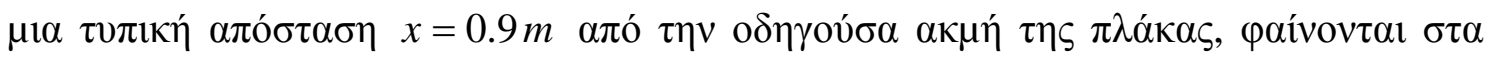

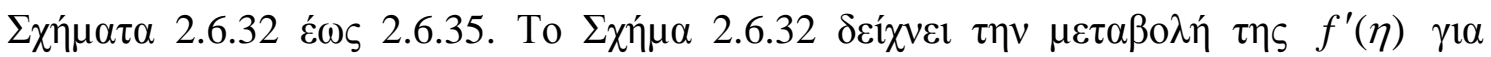

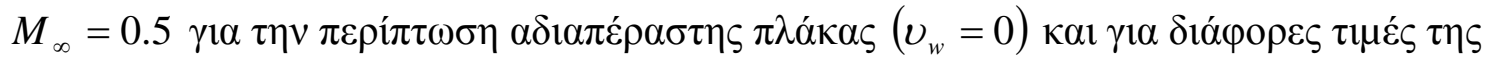

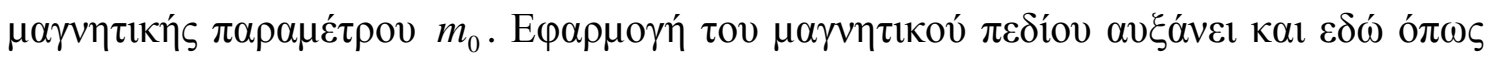

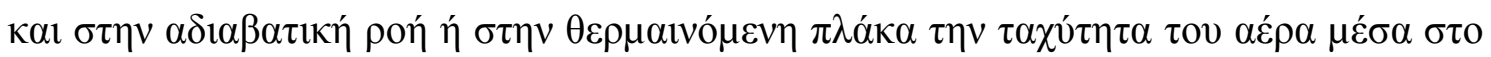

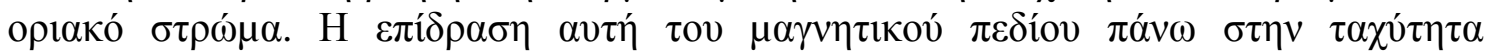

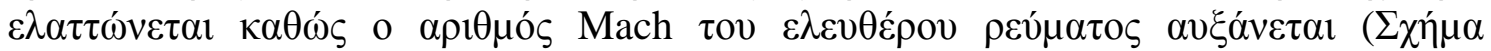

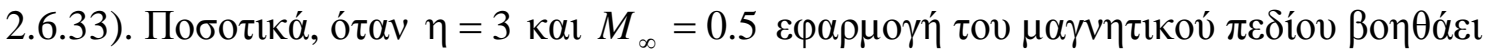

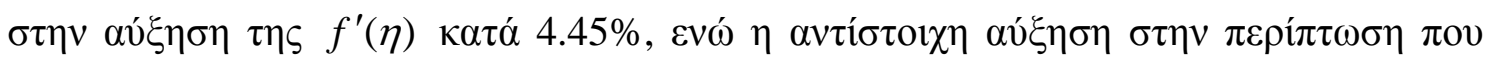

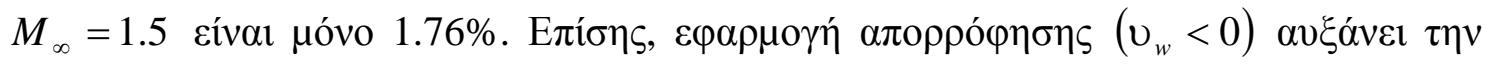

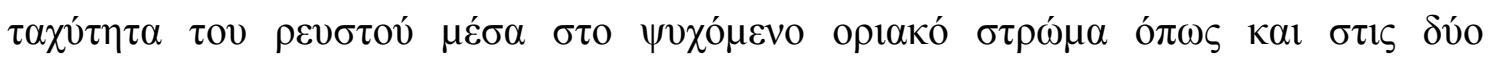

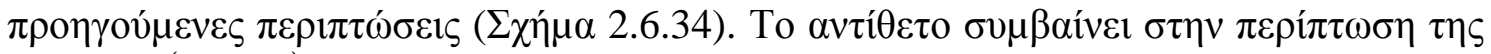

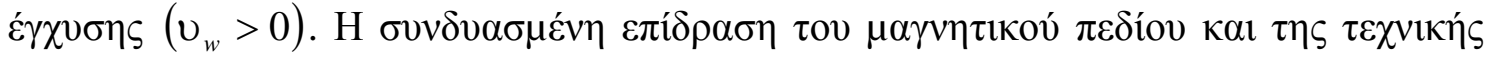

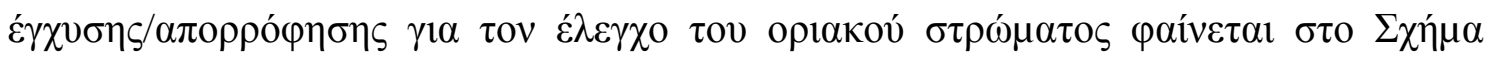

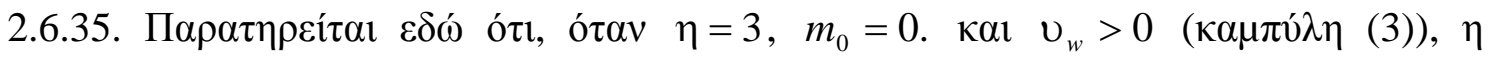

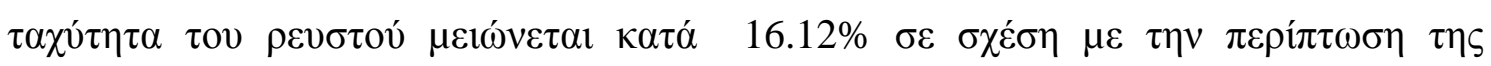




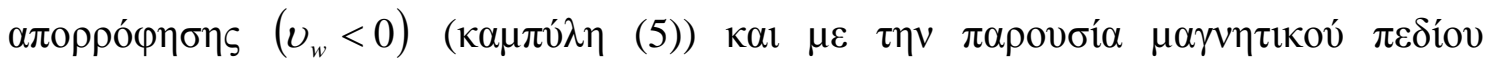
$\left(m_{0}=8\right.$.).

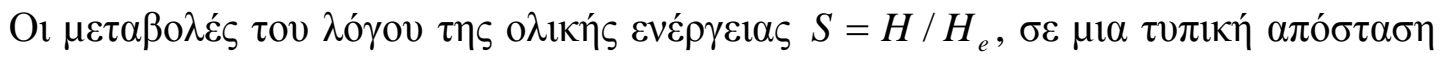

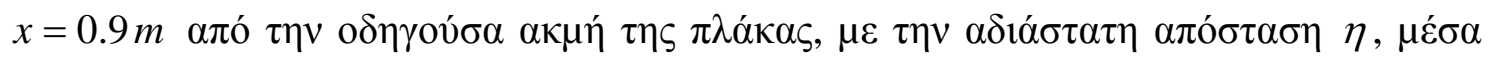

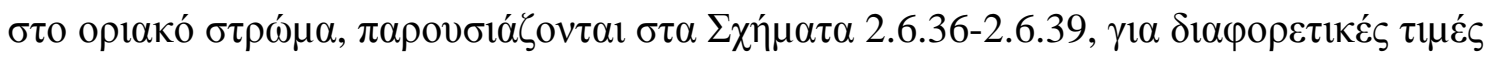

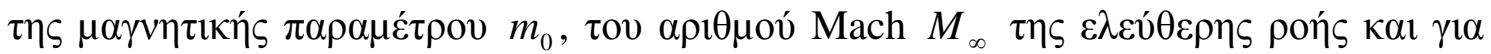

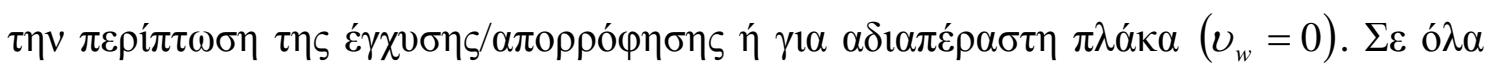

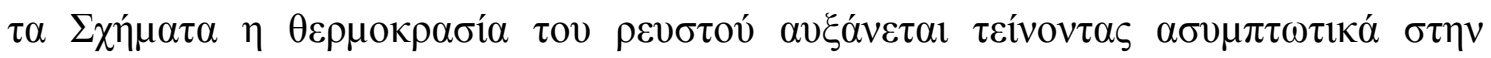

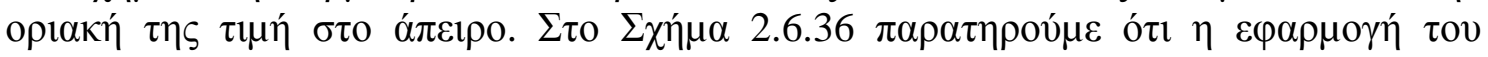

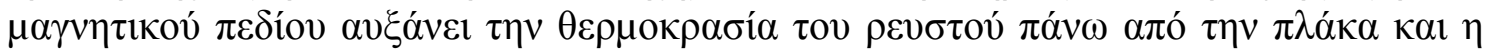

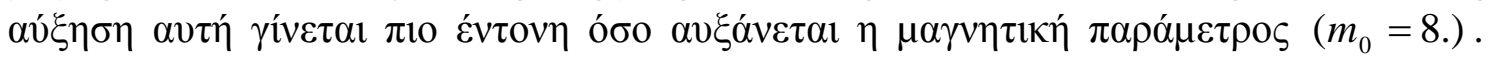

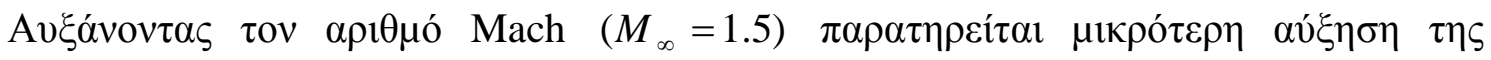

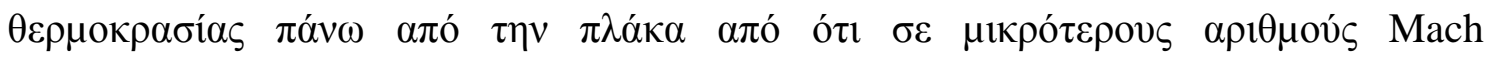

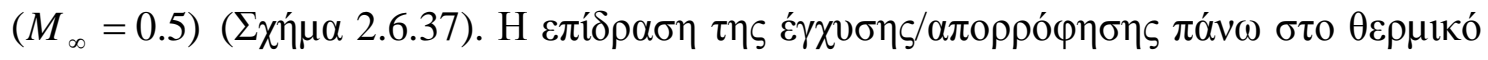

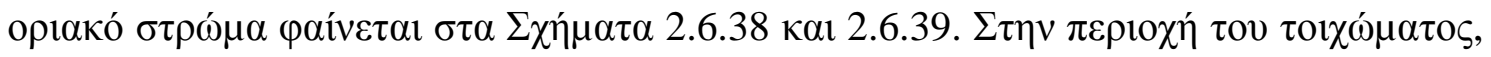

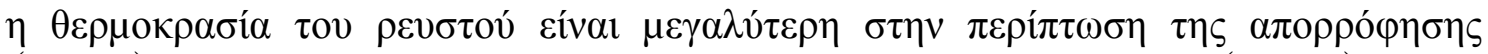

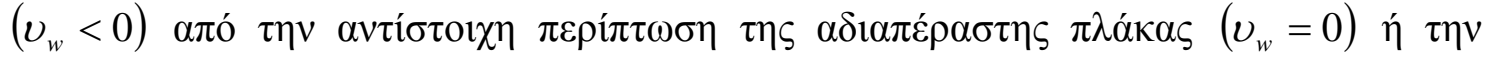

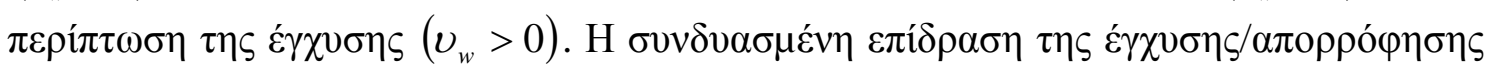

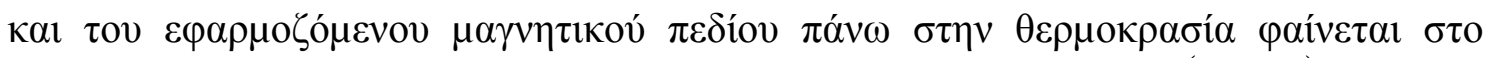

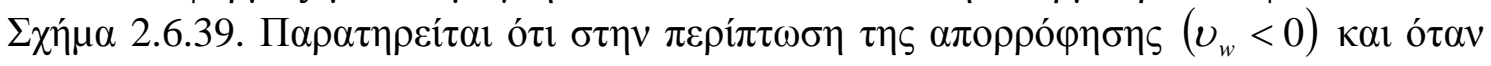

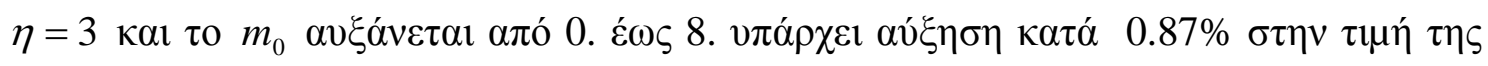

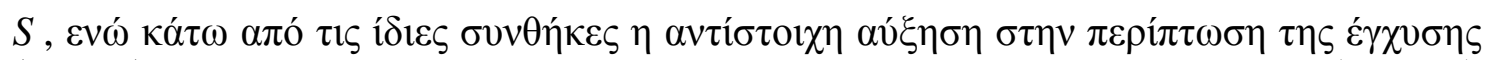

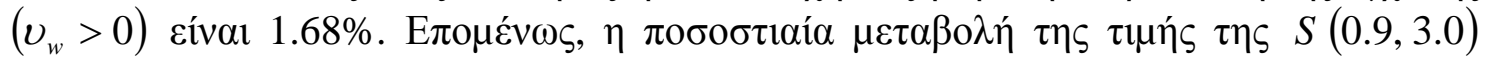

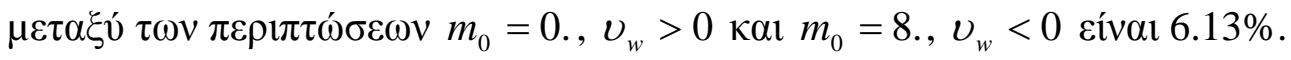

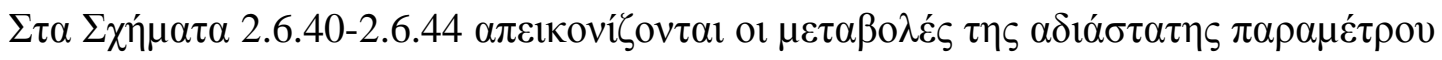

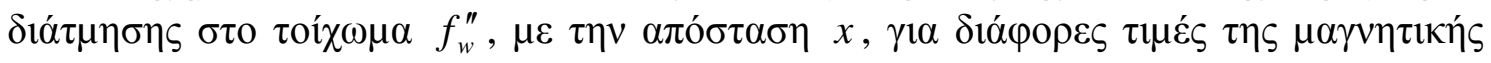

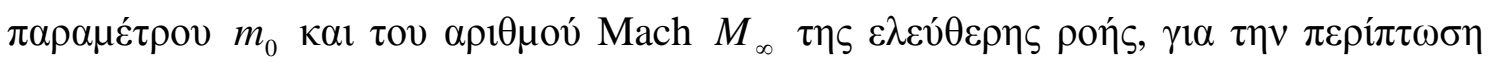

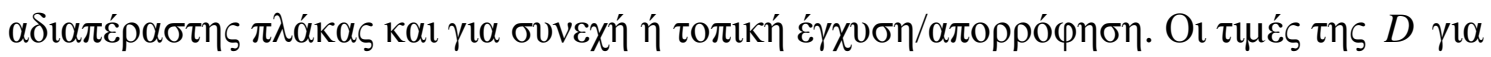
$\kappa \alpha ́ \theta \varepsilon \pi \varepsilon \rho i ́ \pi \tau \omega \sigma \eta \alpha \pi \varepsilon 1 \kappa o v i ́ \zeta o v \tau \alpha 1 ~ \pi \alpha ́ v \omega ~ \sigma \tau \alpha \Sigma \chi \eta ́ \mu \alpha \tau \alpha$

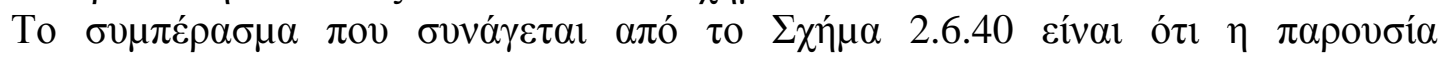

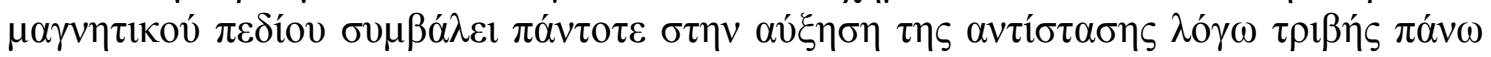

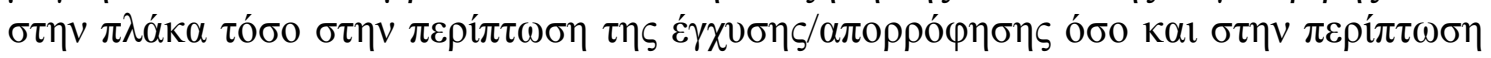

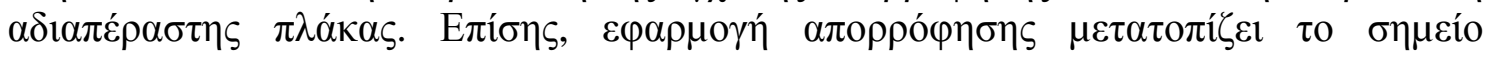

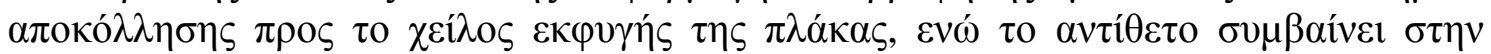

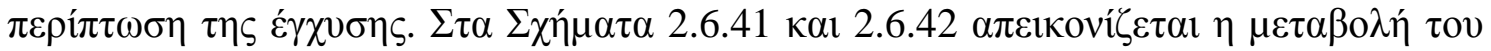

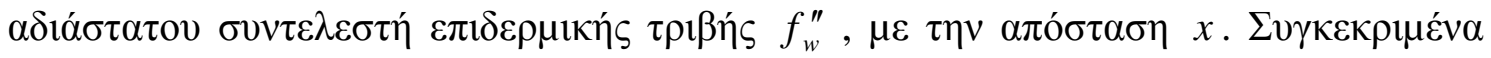

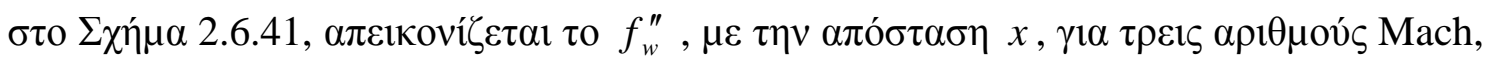

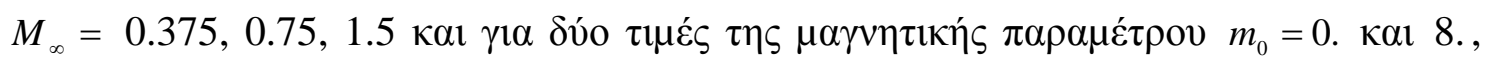

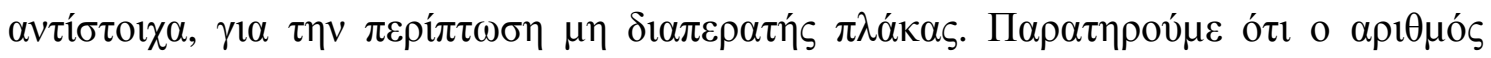

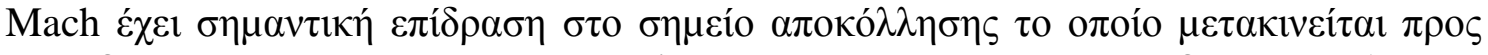

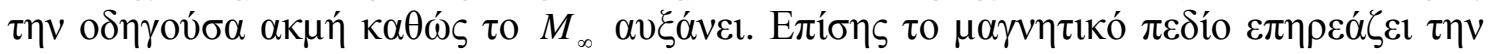


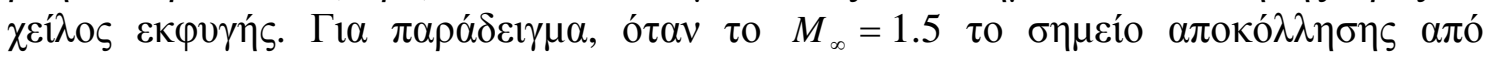

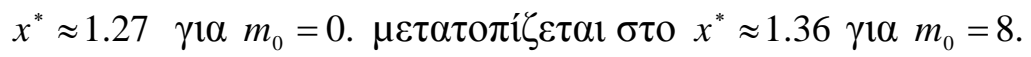




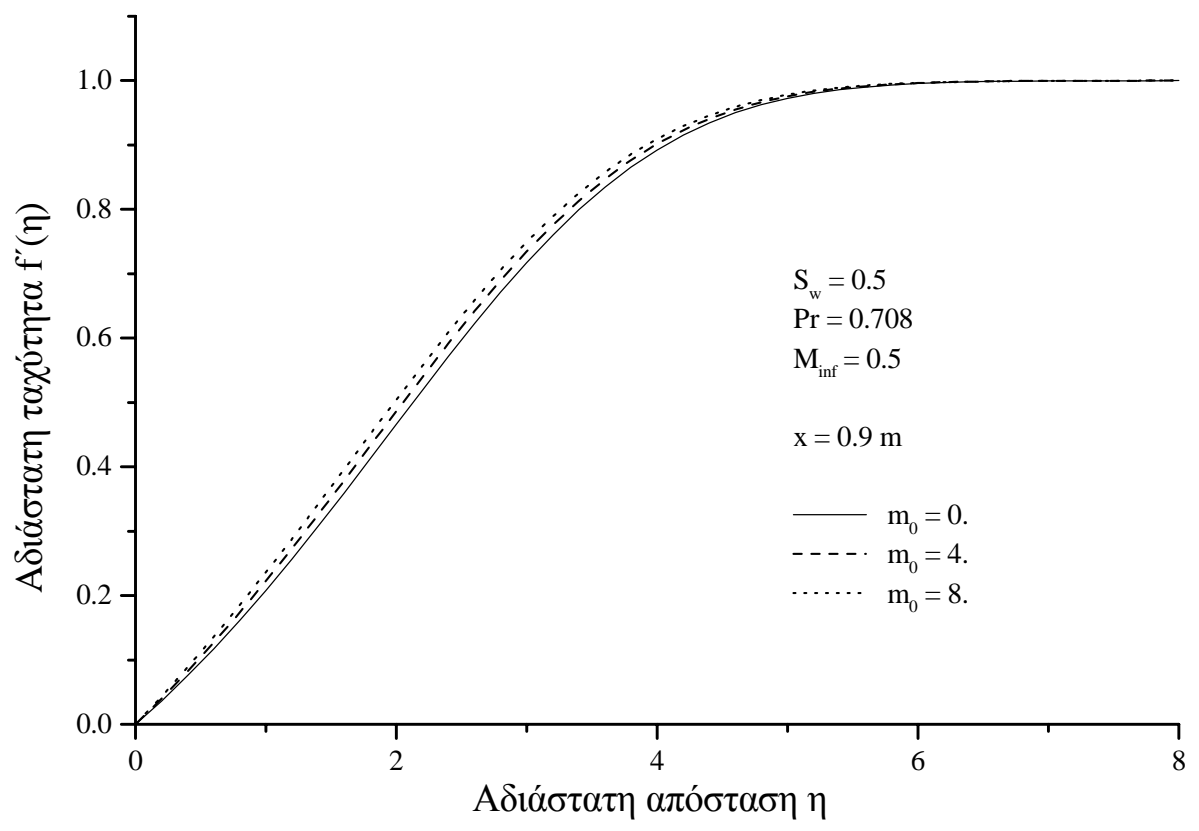

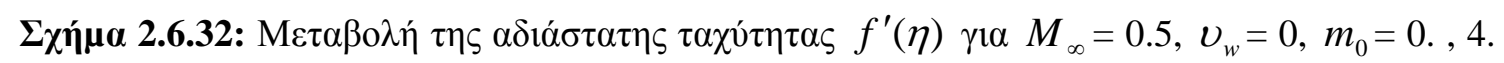
каl 8.

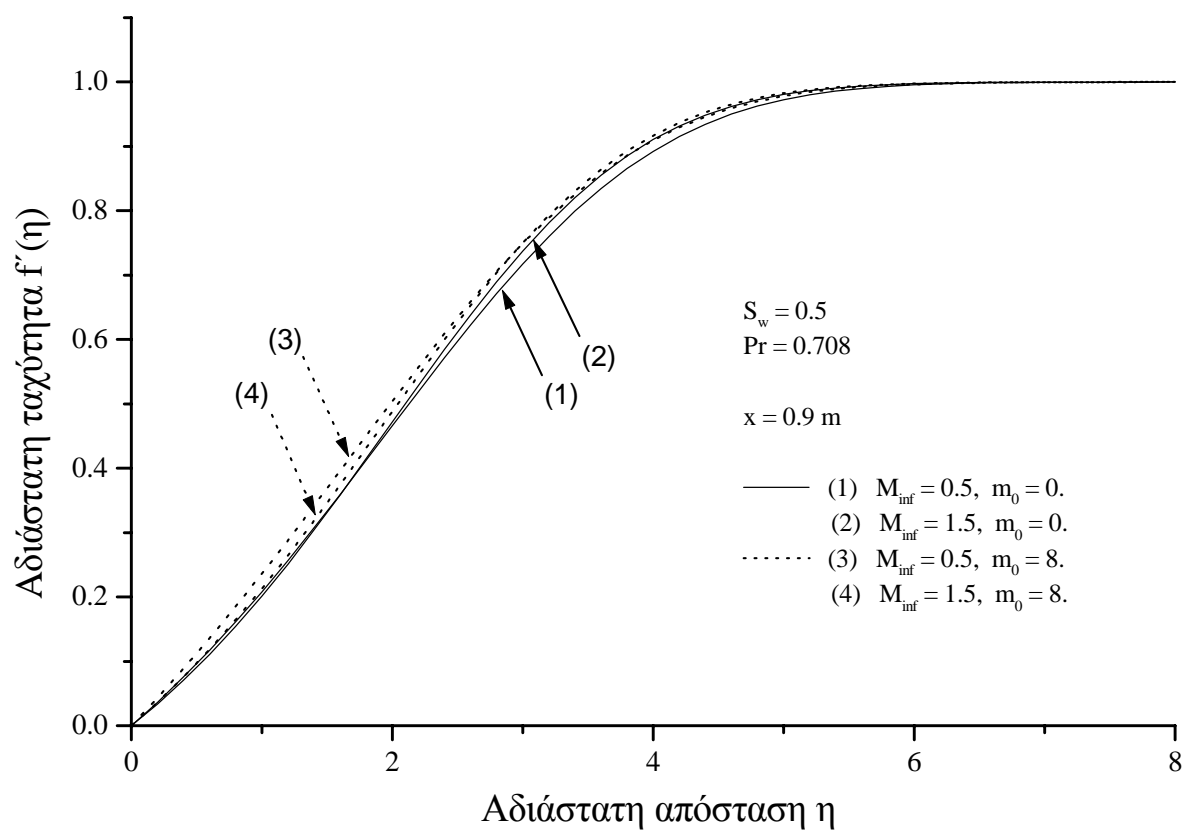

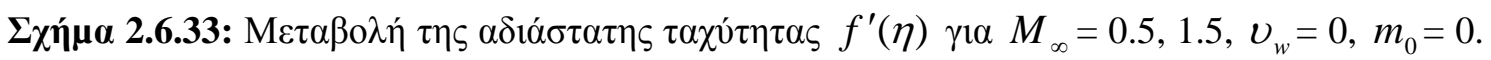
ка1 8. 


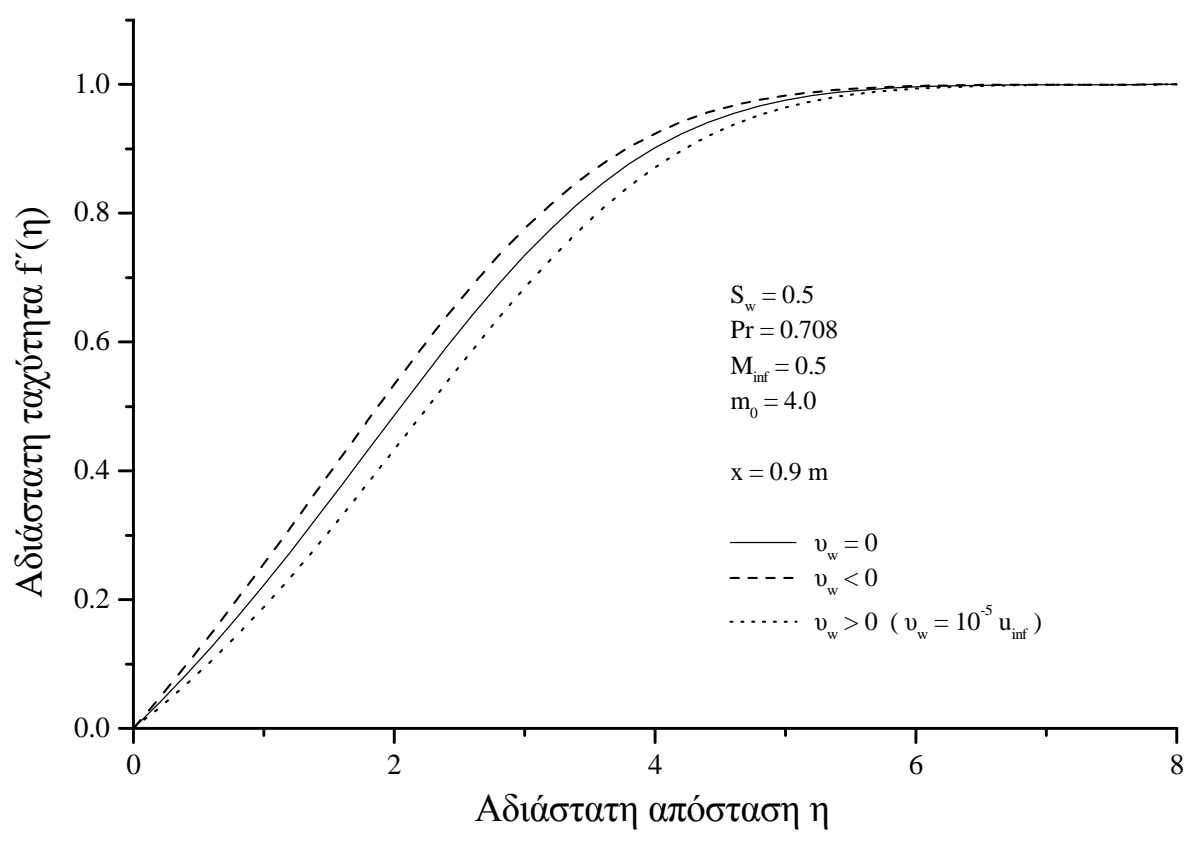

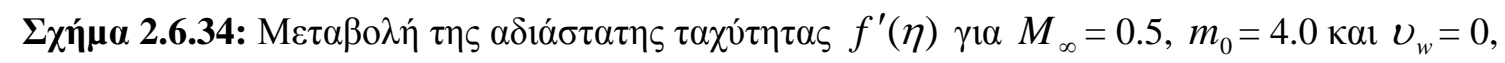
$<0,>0$.

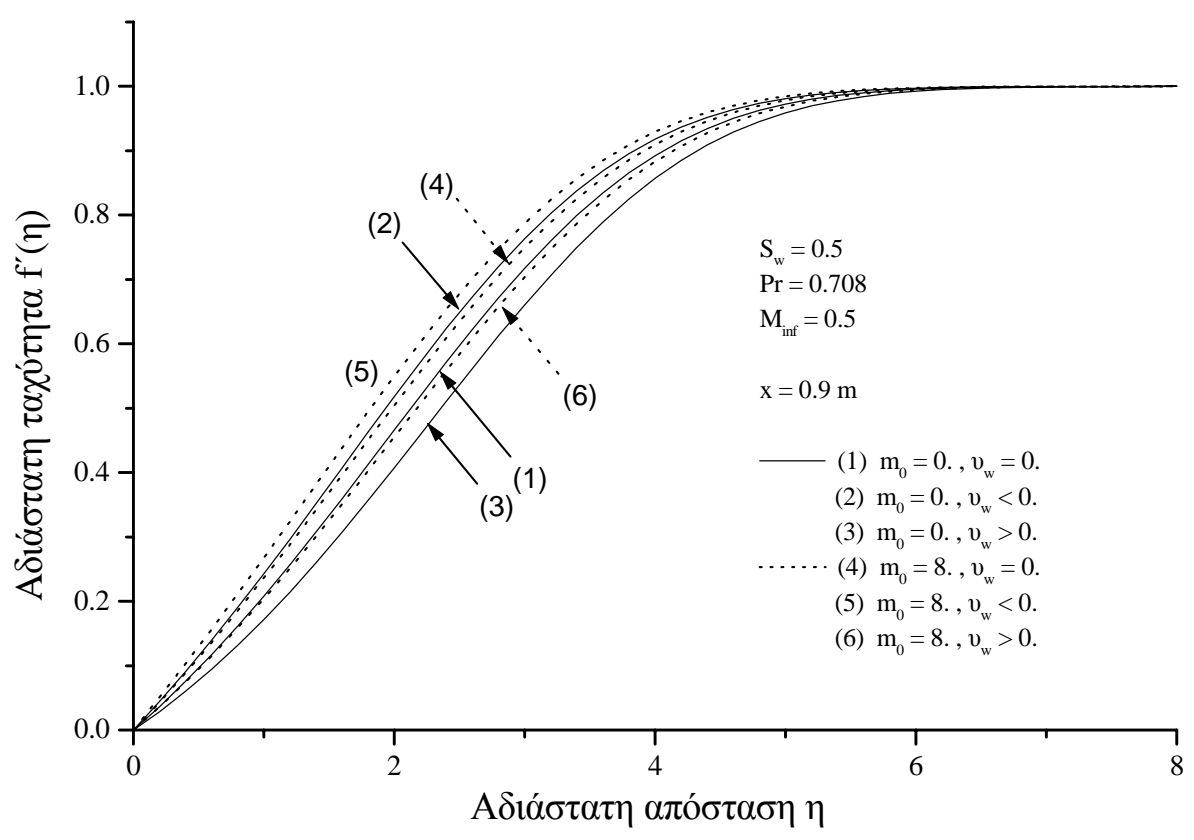

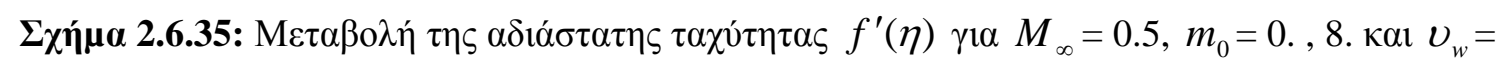
$0,<0,>0$. 


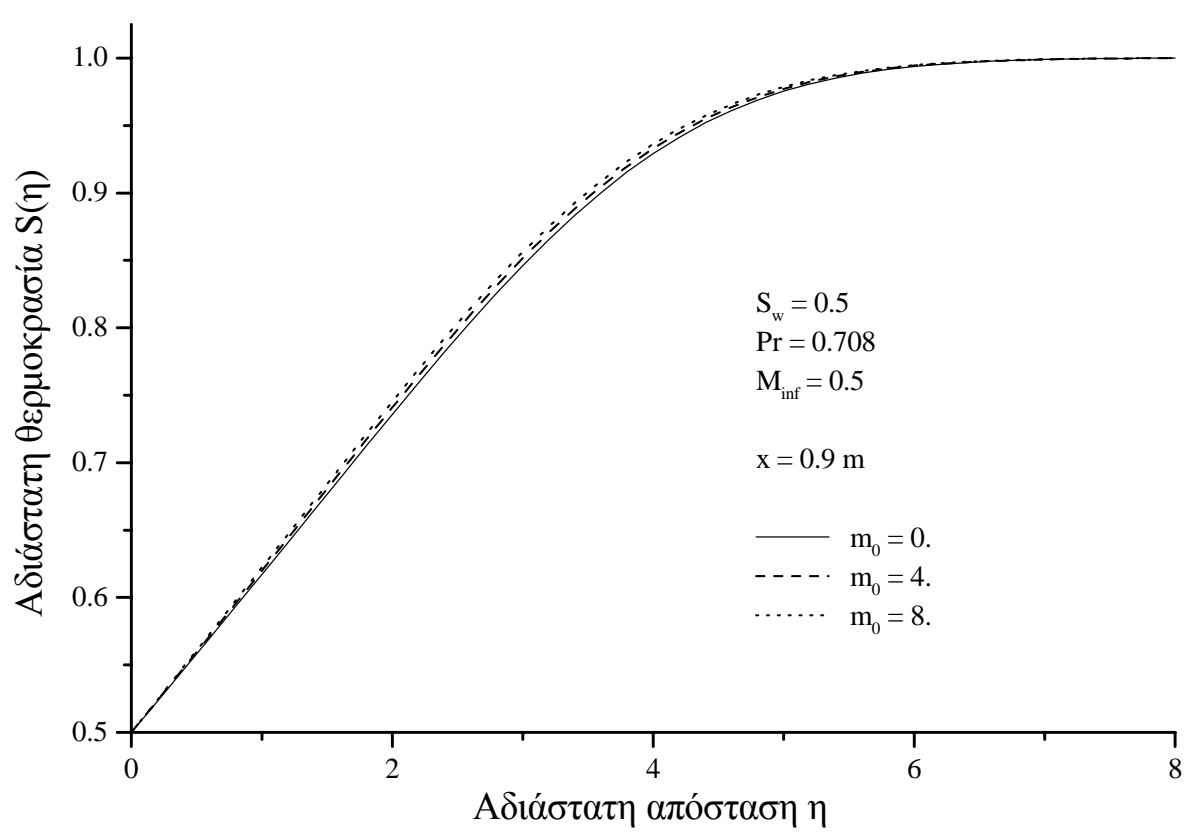

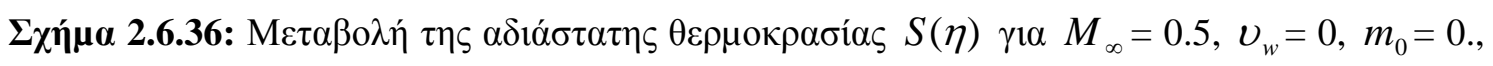
4. кal 8.

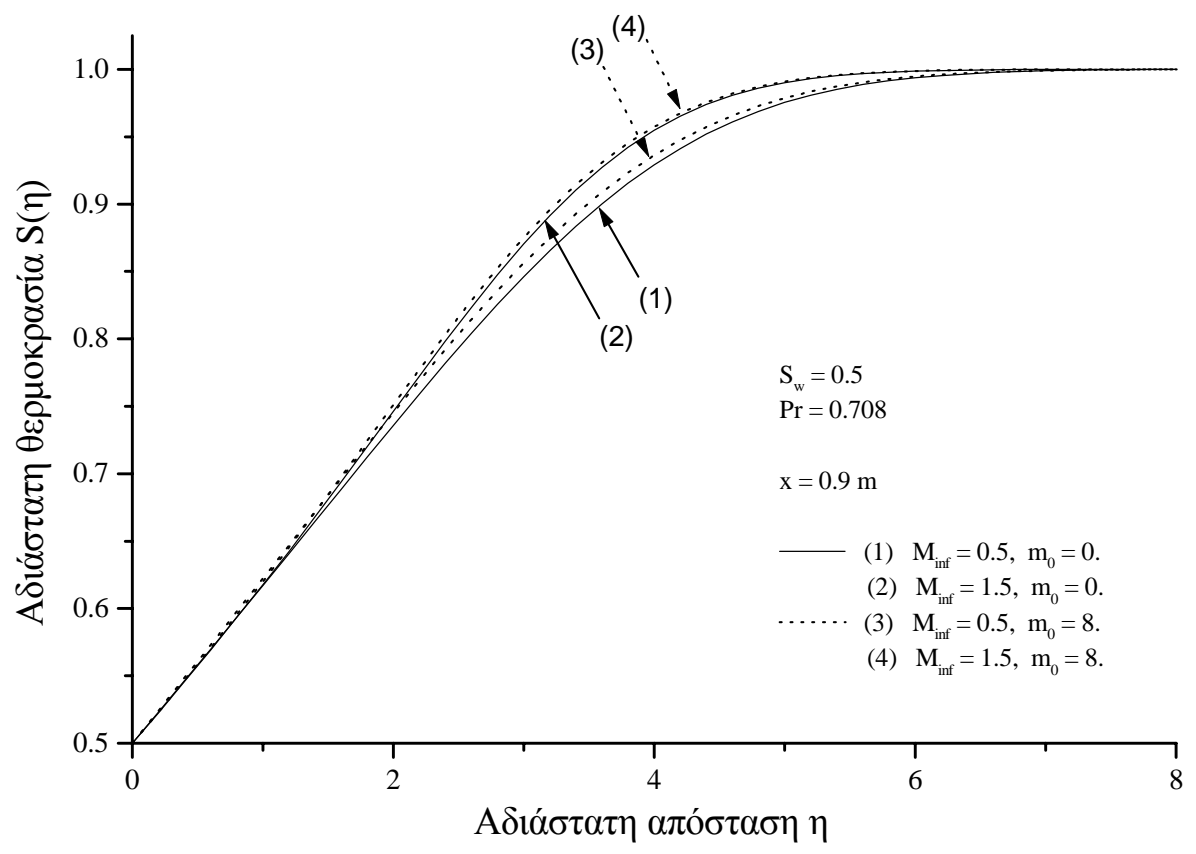

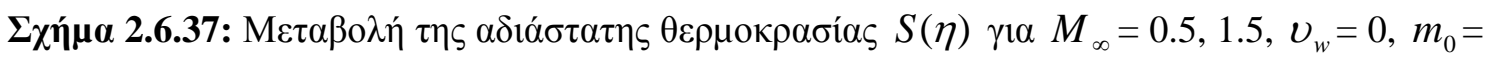
0. кal 8. 


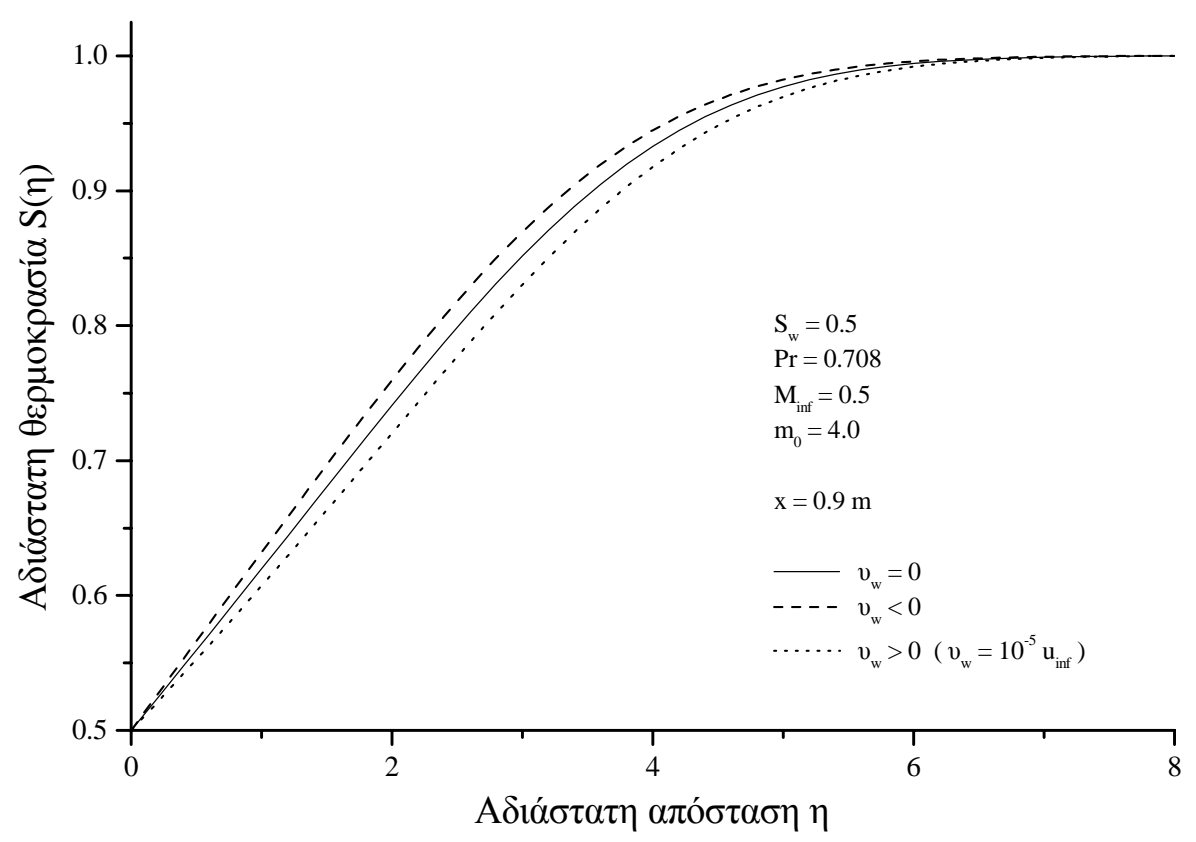

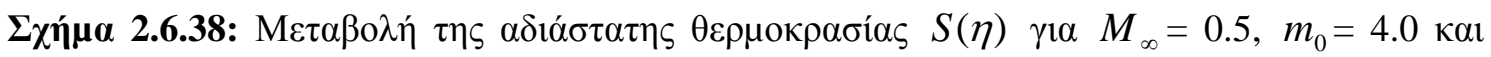
$v_{w}=0,<0,>0$.

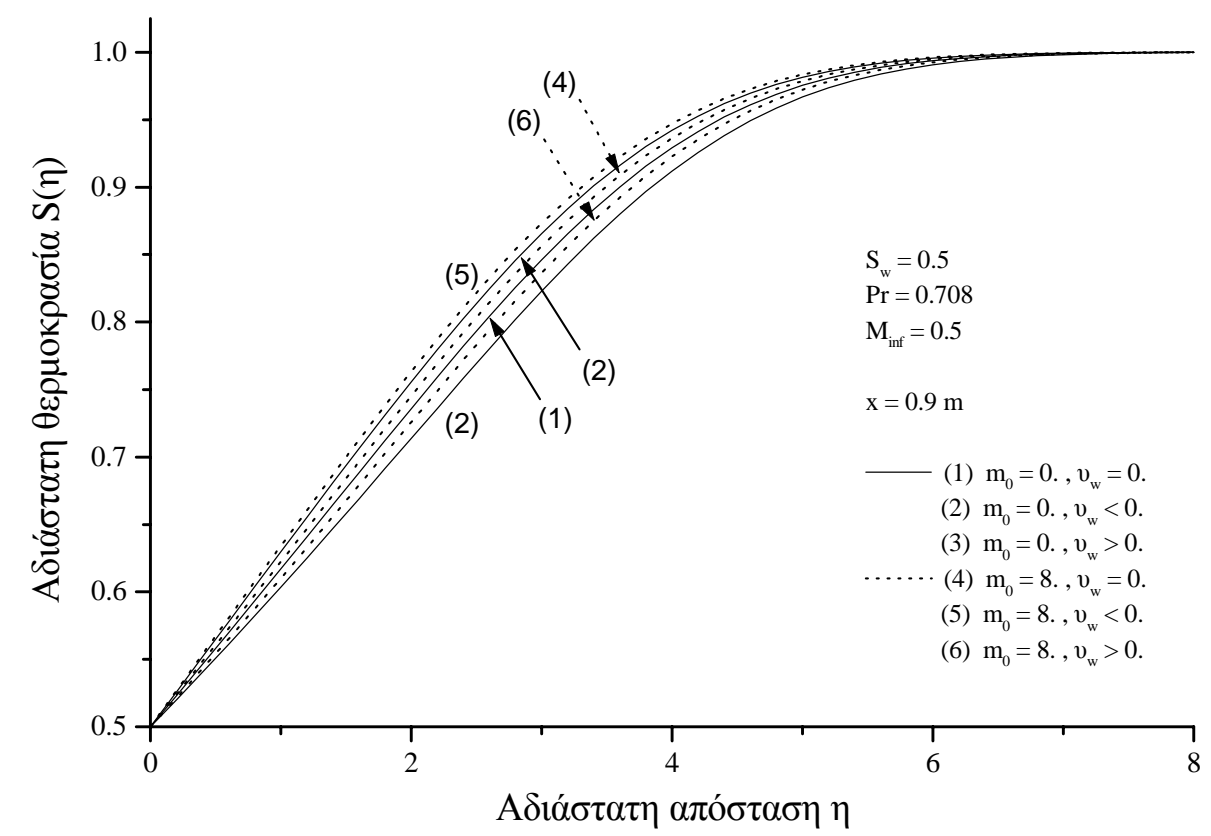

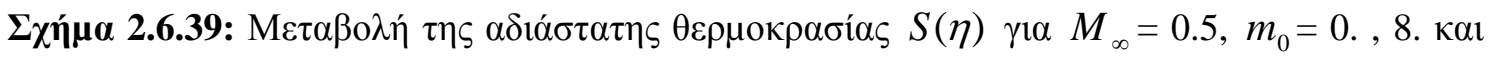
$v_{w}=0,<0,>0$. 


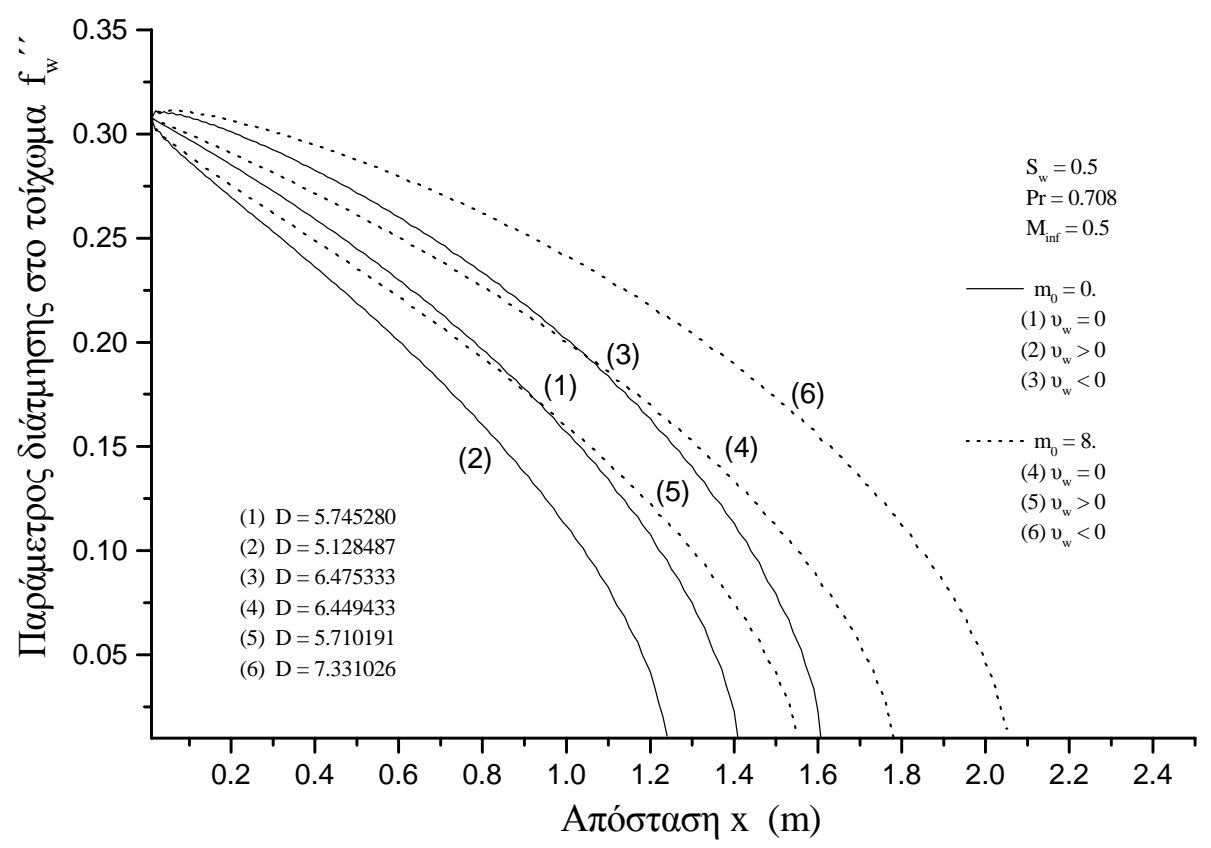

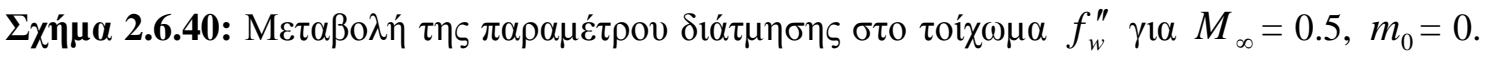
$\kappa \alpha 18 . \kappa \alpha 1 v_{w}=0,>0,<0$.

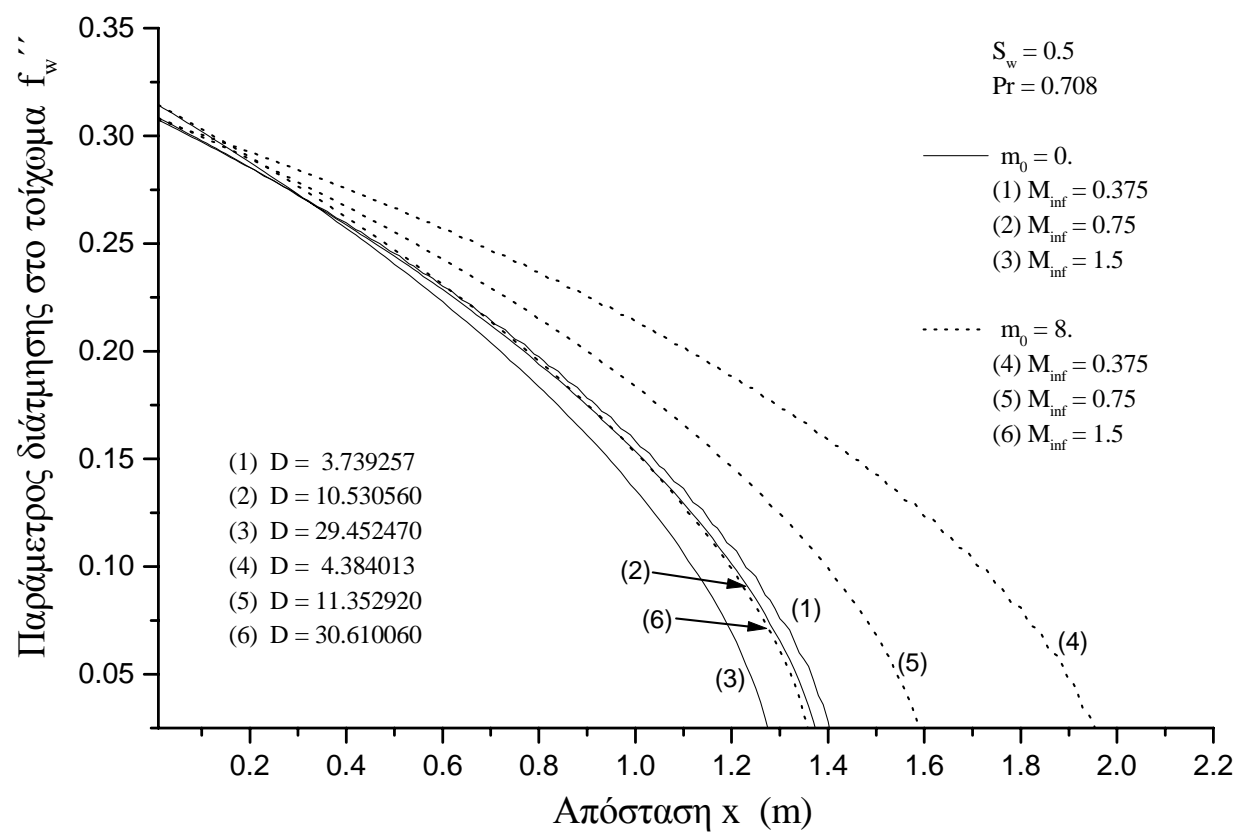

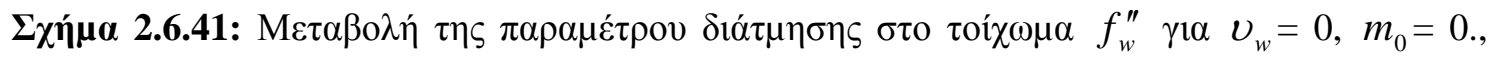

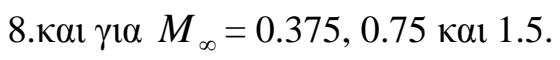




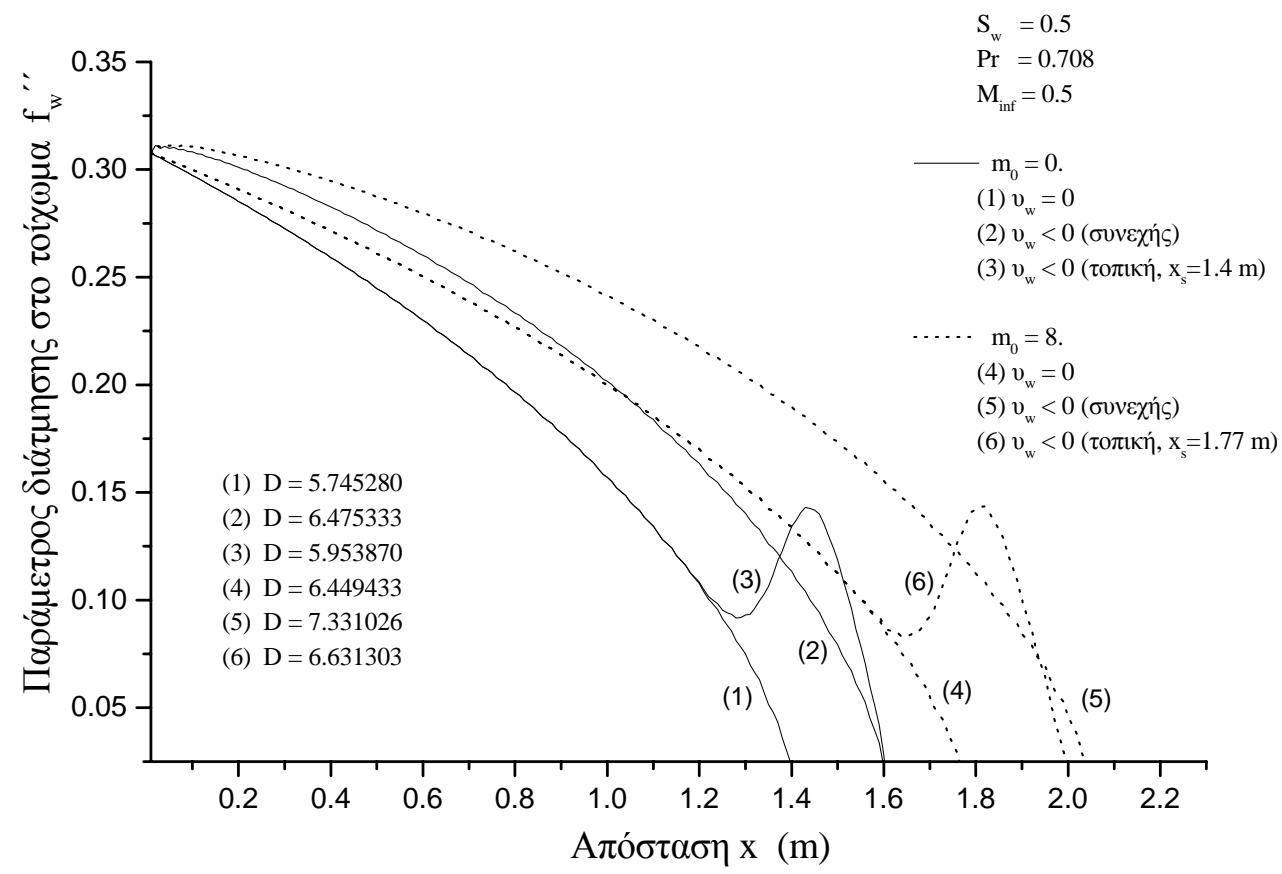

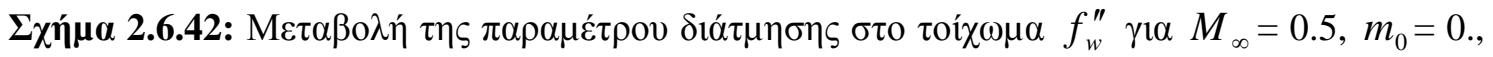

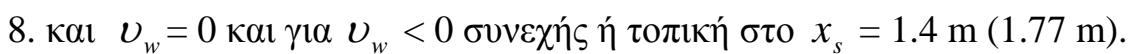

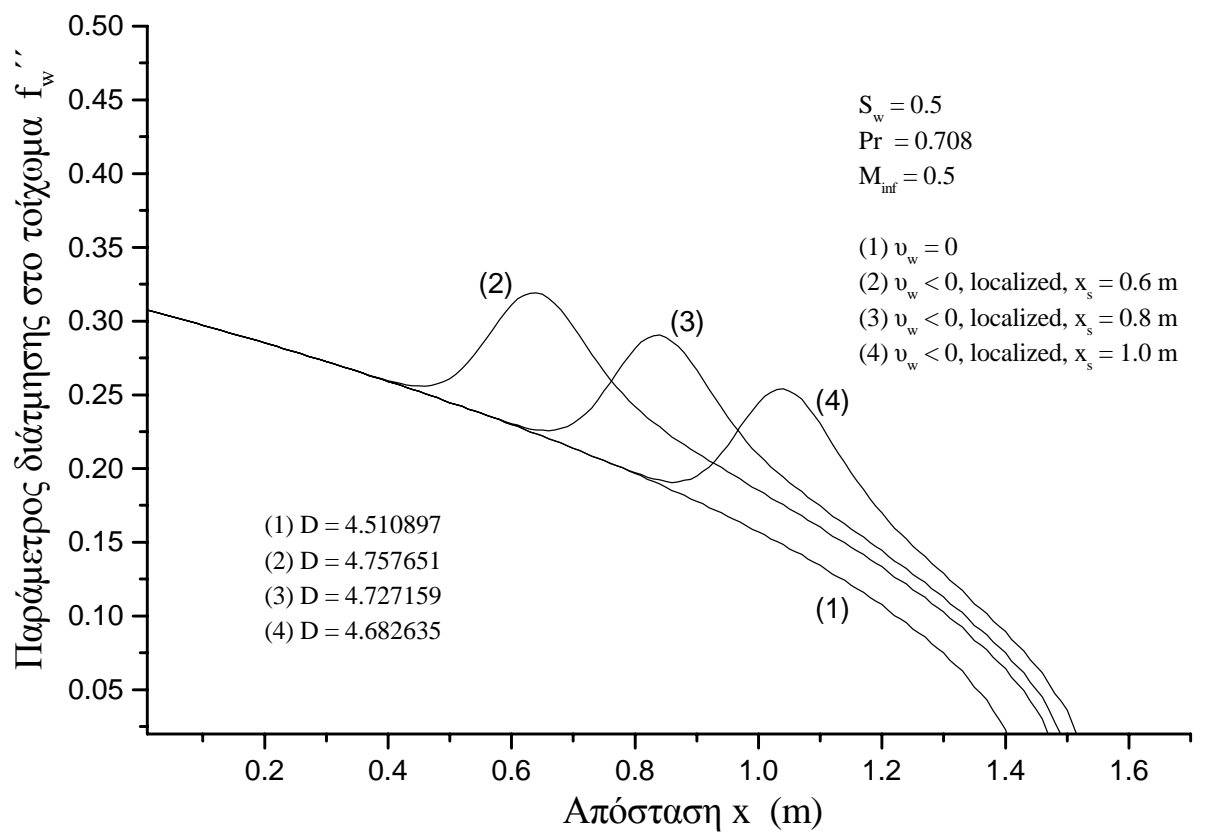

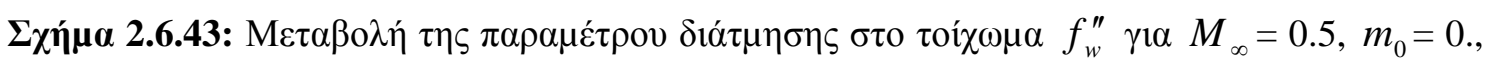

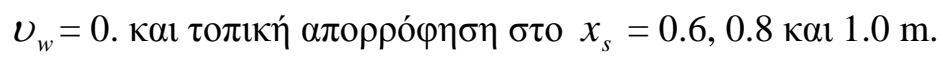




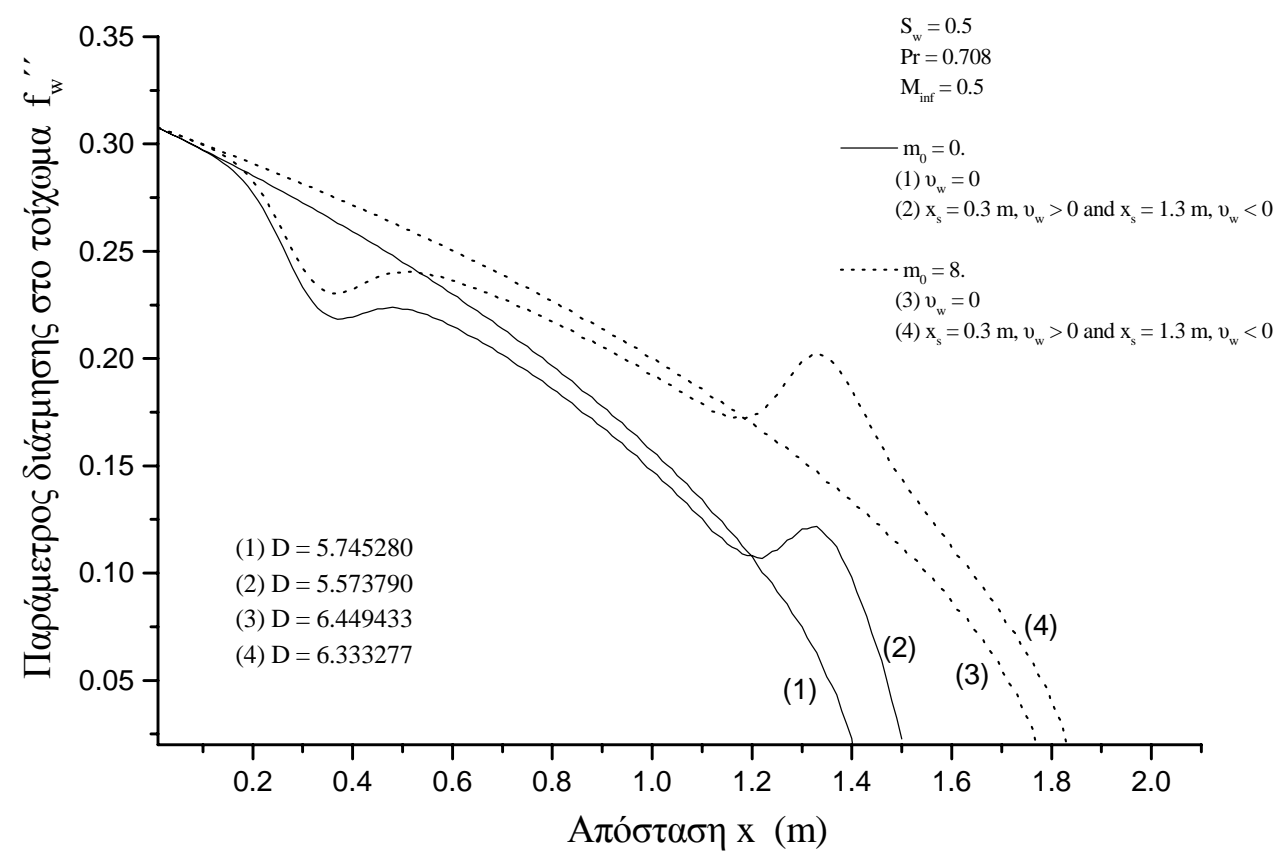

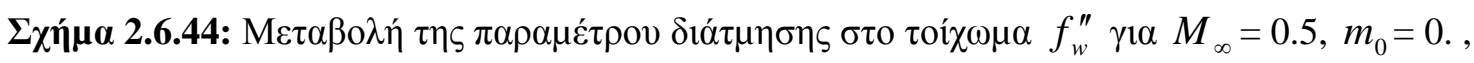

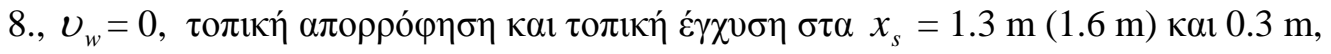

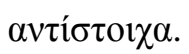

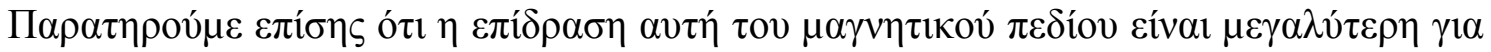

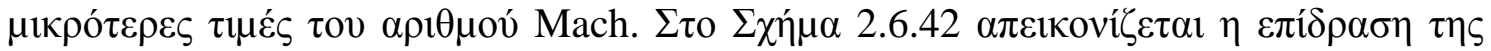

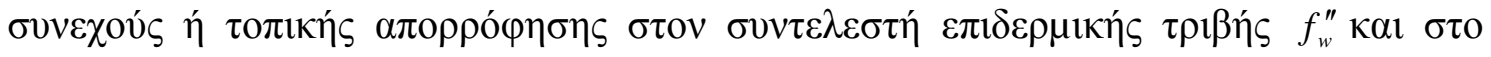

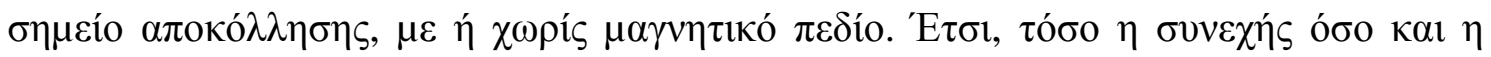

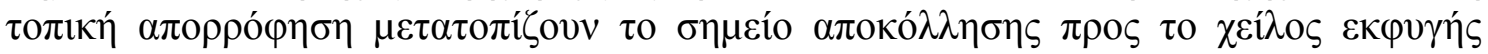

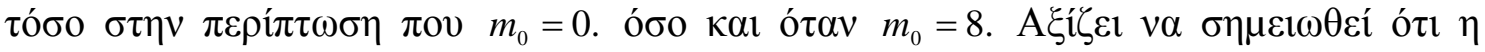

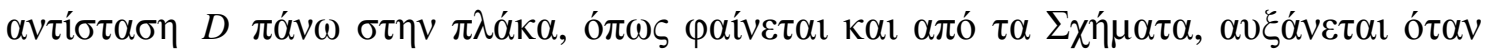

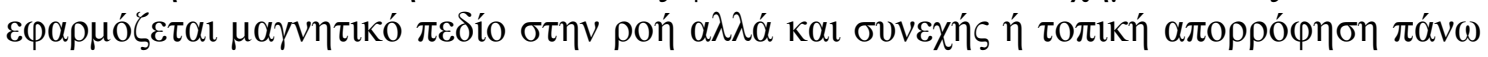

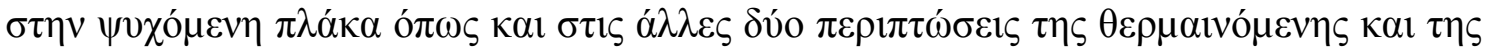

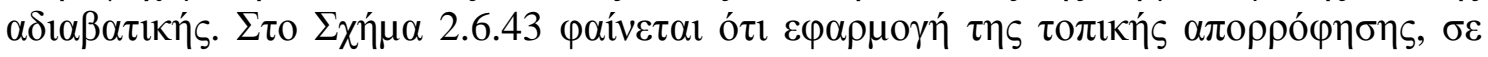

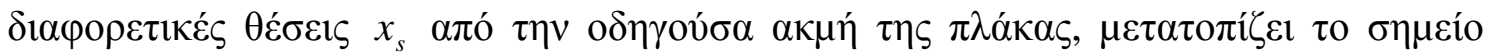

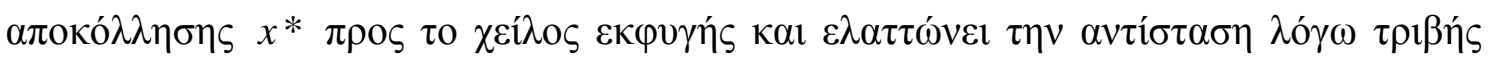

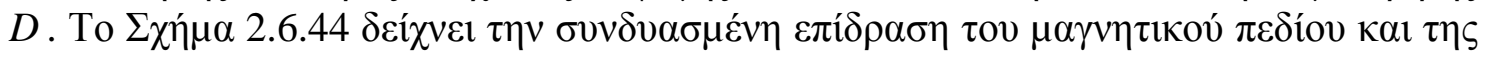

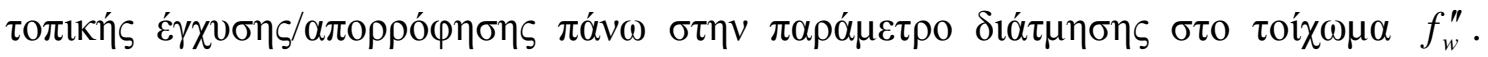

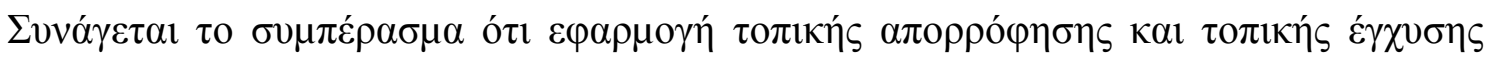

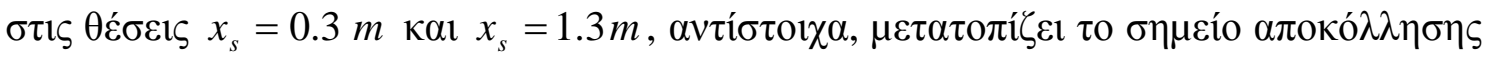

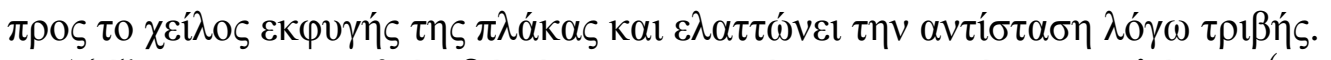

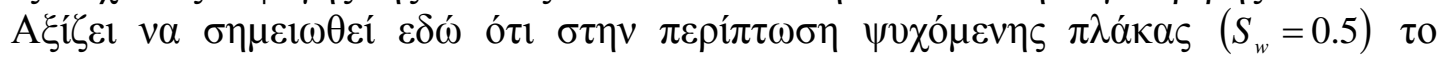

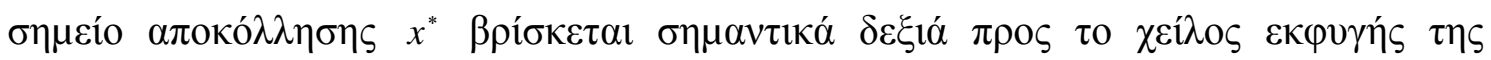

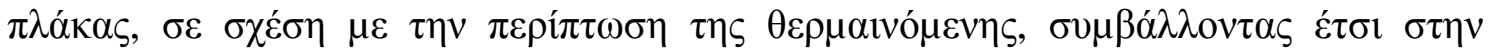

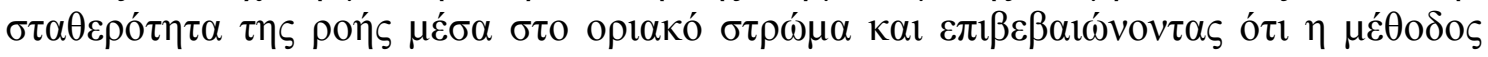

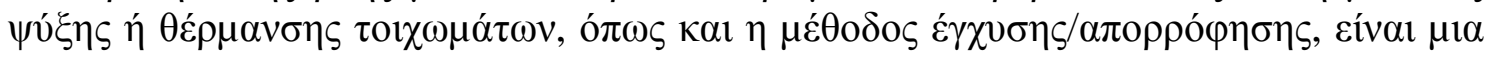




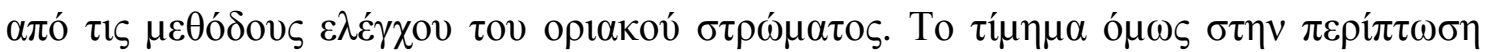

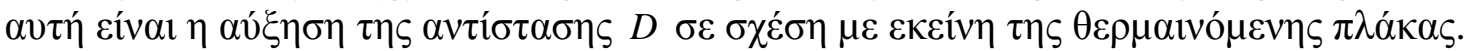

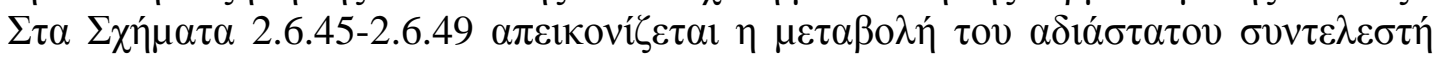

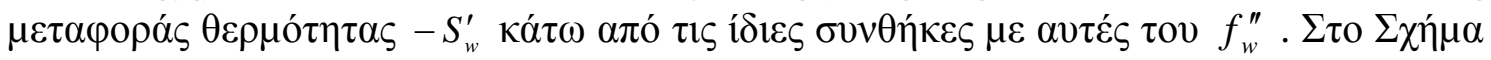

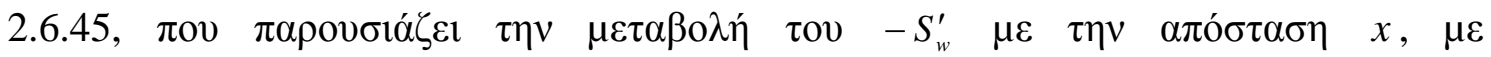

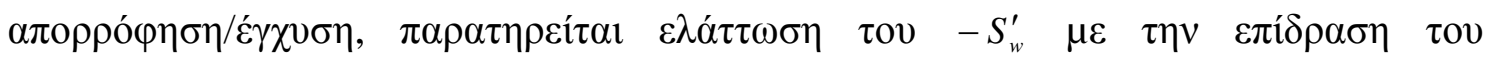

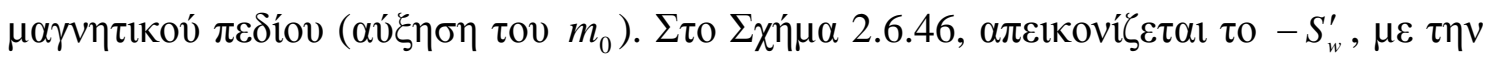

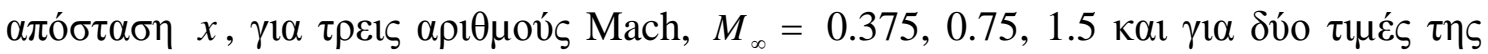

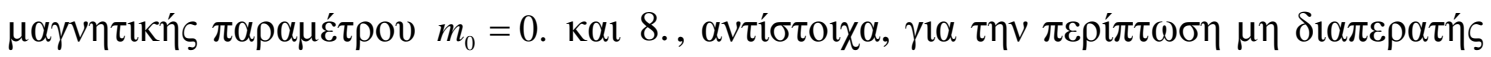

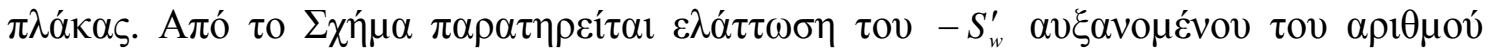

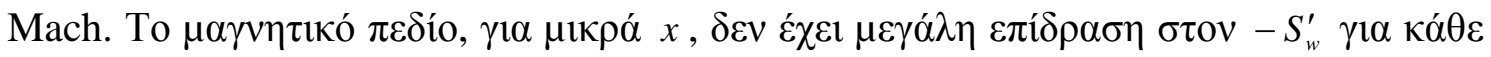

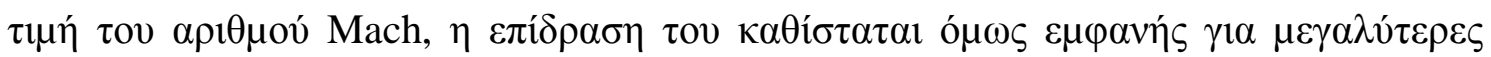

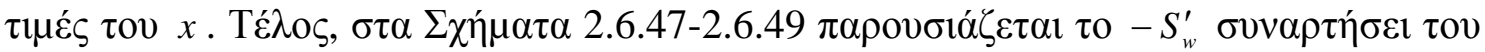

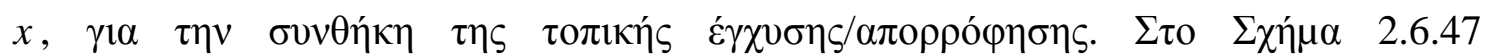

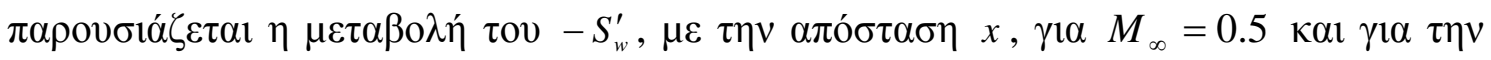

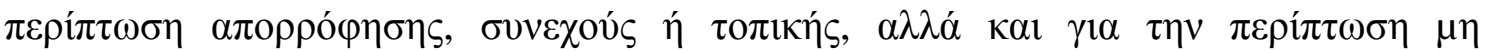

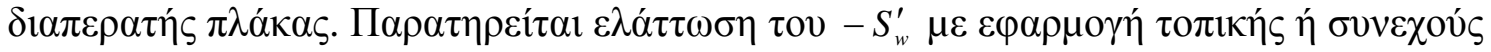

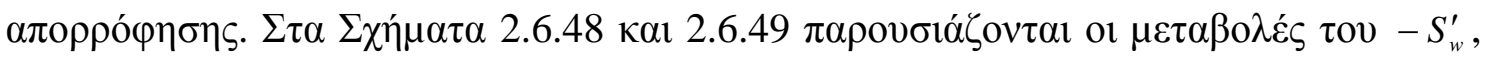

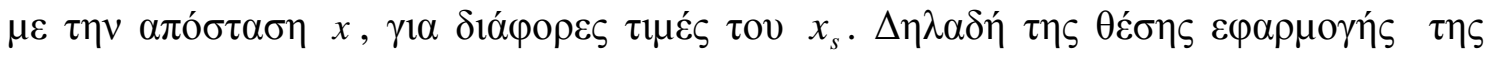

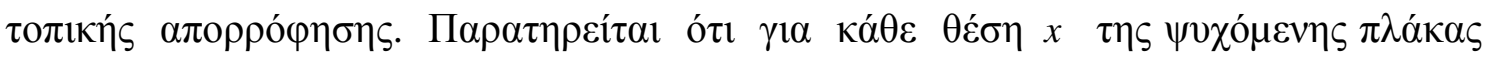

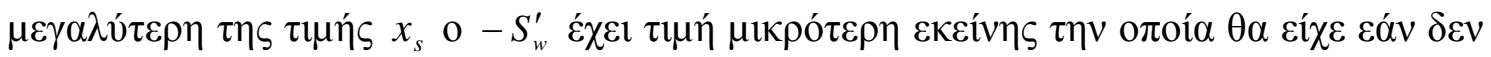

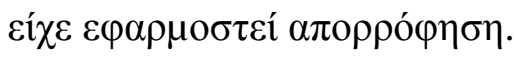

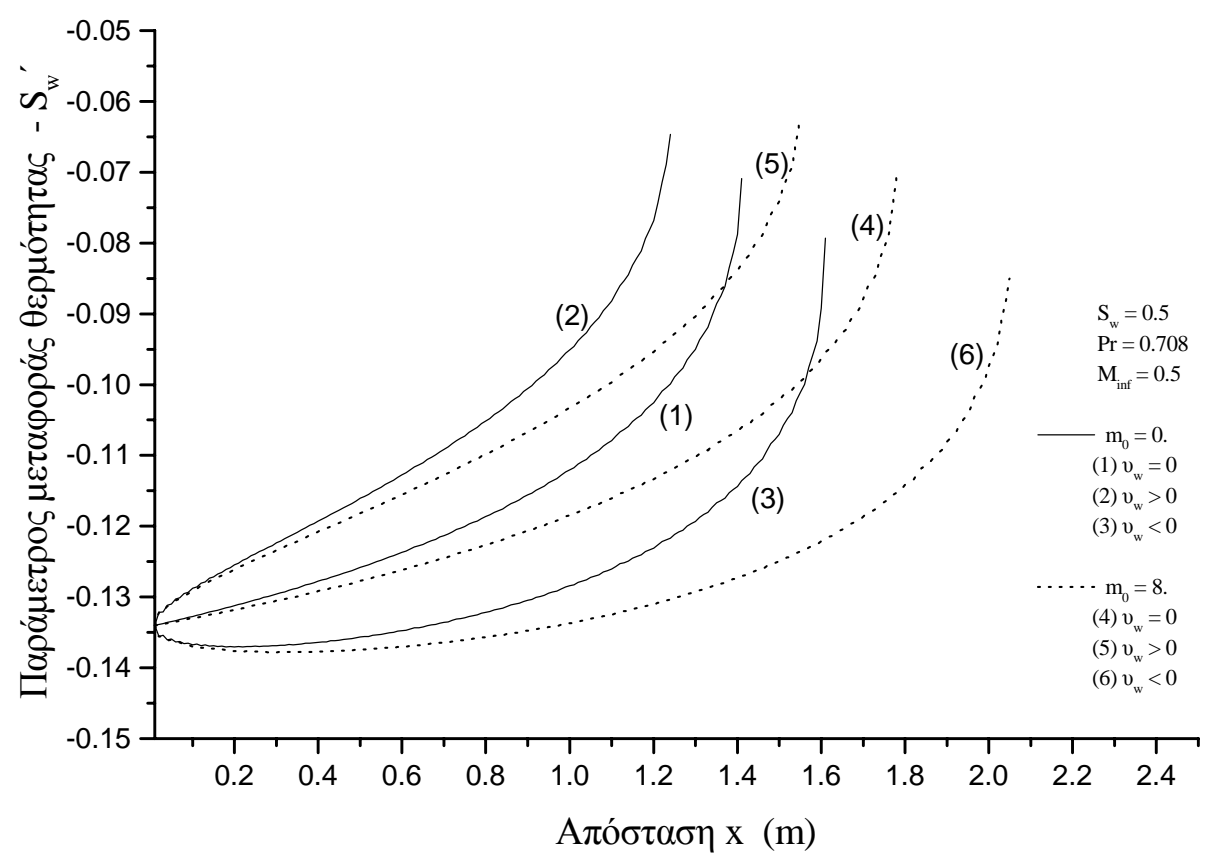

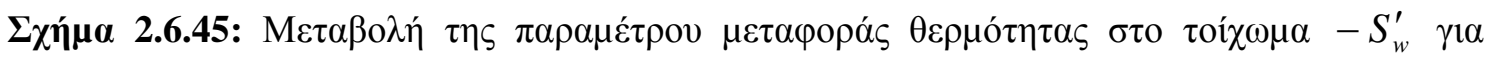
$M_{\infty}=0.5, m_{0}=0 . \kappa \alpha 18 . \kappa \alpha \mathrm{l} v_{w}=0,>0,<0$. 


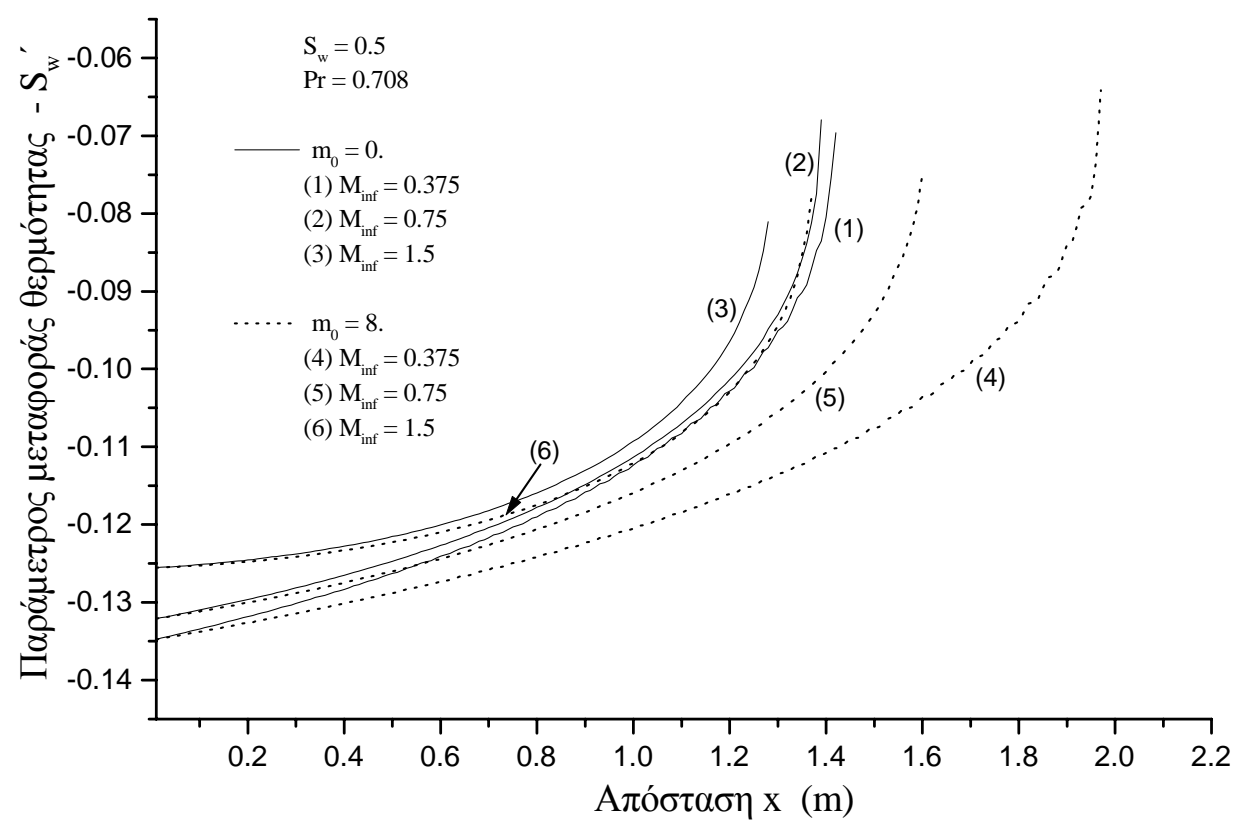

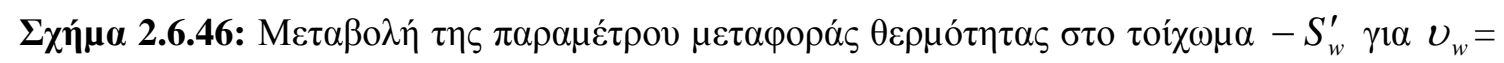
$0, m_{0}=0 ., 8 . \kappa \alpha \mathrm{l} \gamma 1 \alpha M_{\infty}=0.375,0.75 \kappa \alpha 1$ 1.5.

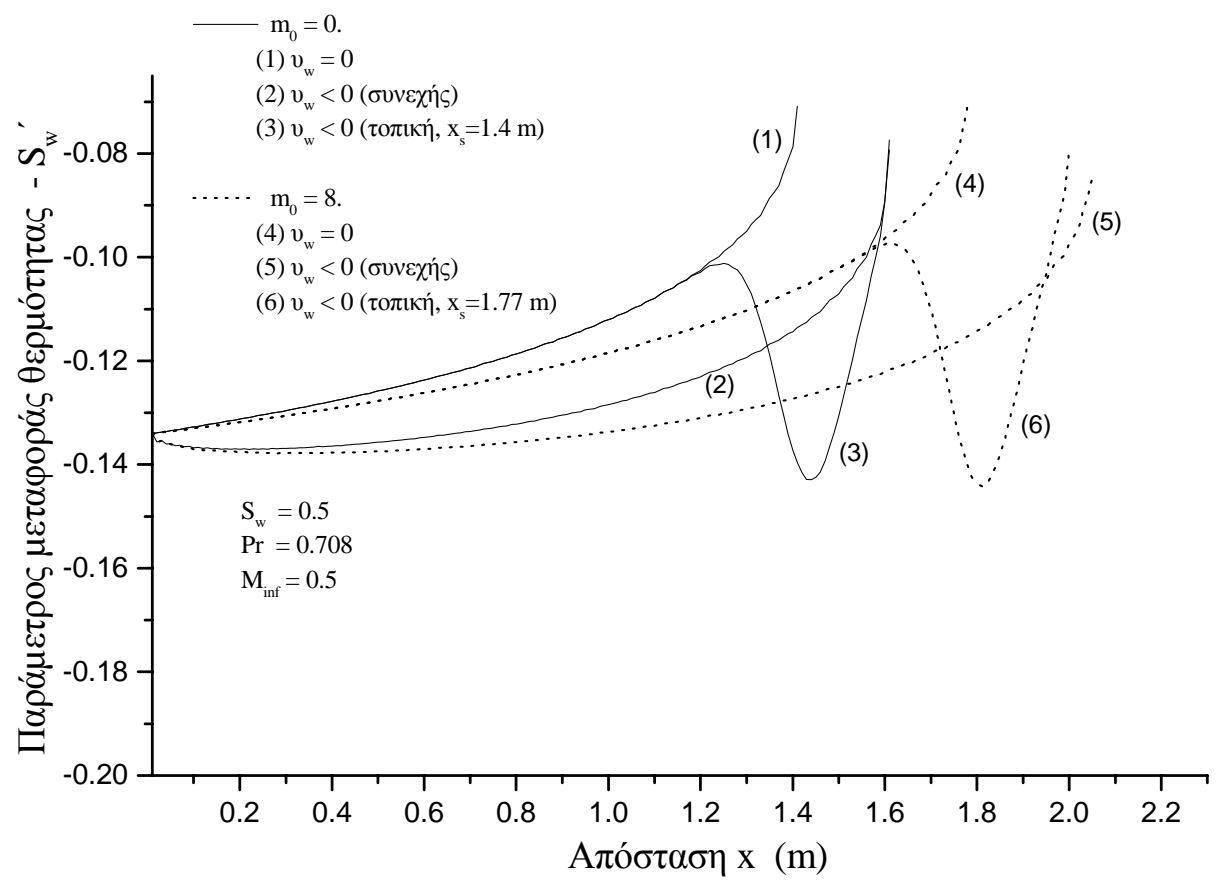

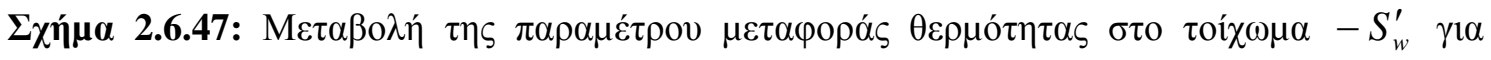

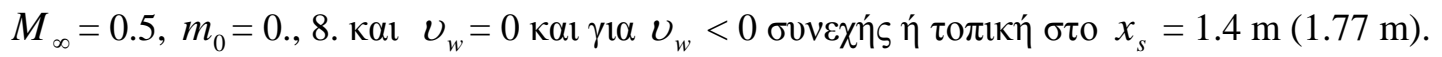




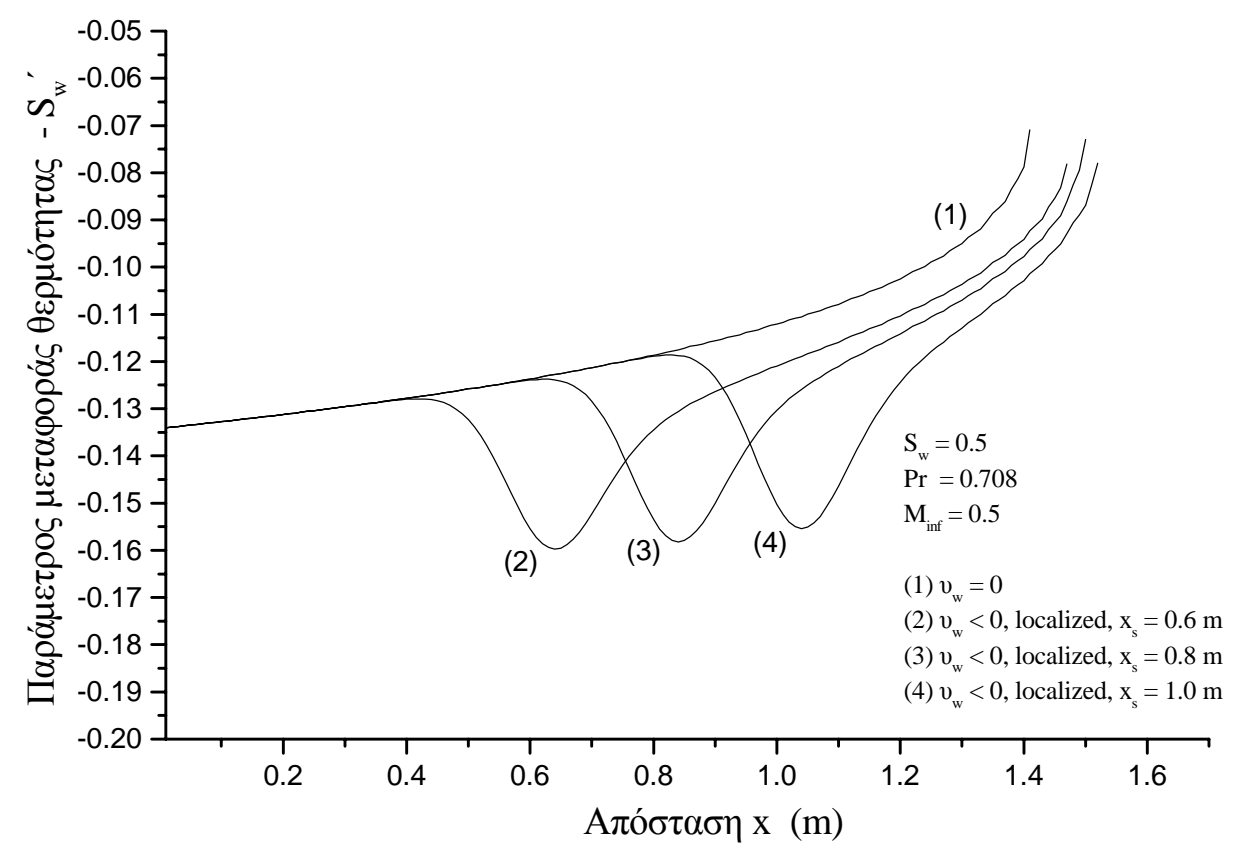

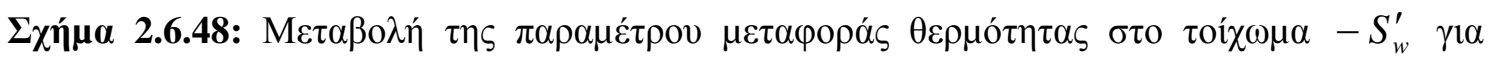

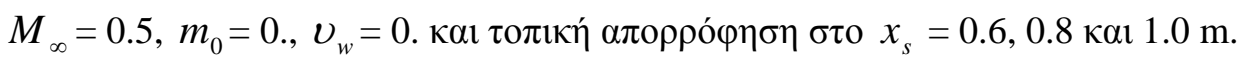

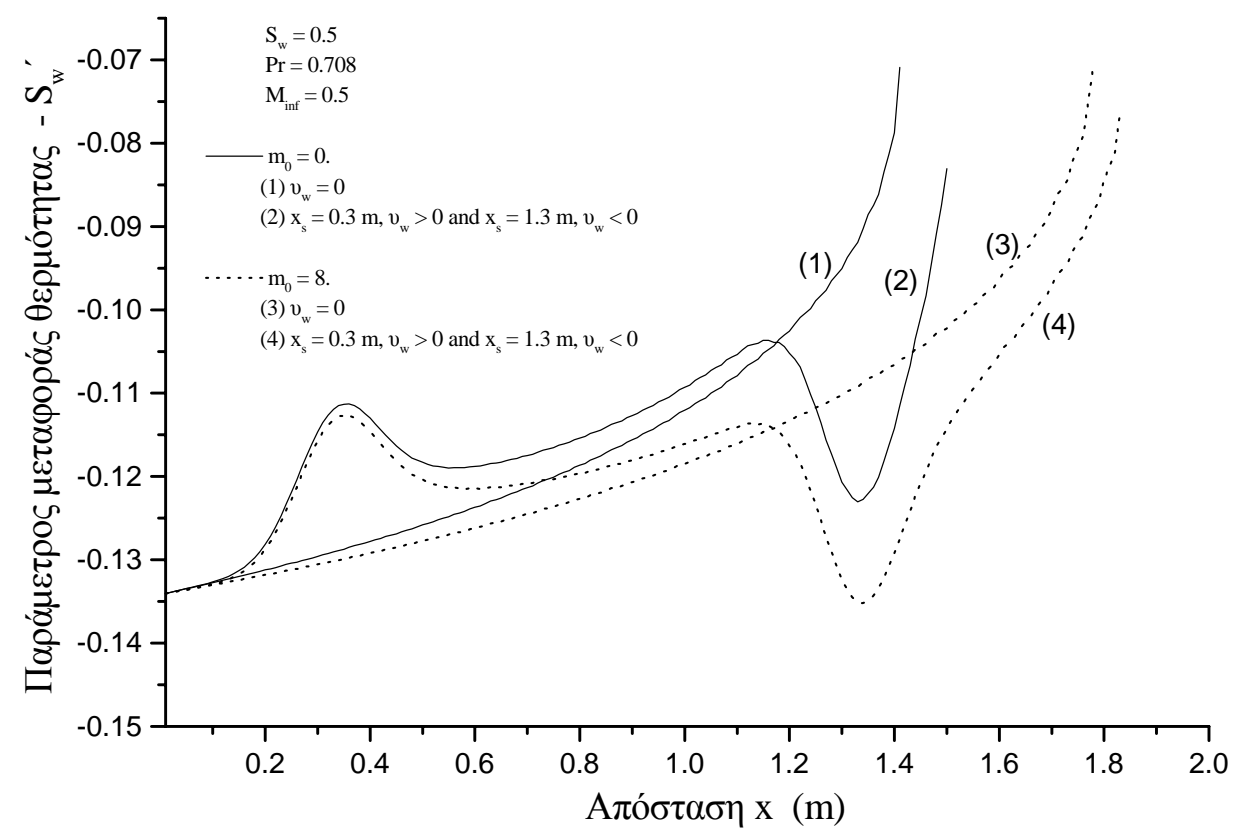

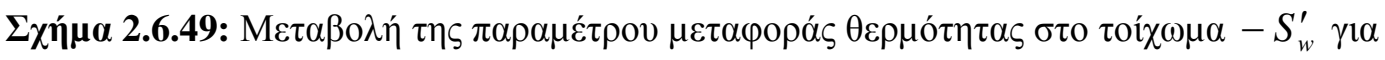

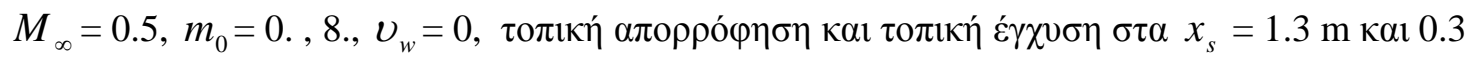
m, $\alpha \nu \tau i ́ \sigma \tau o \iota \chi \alpha$. 


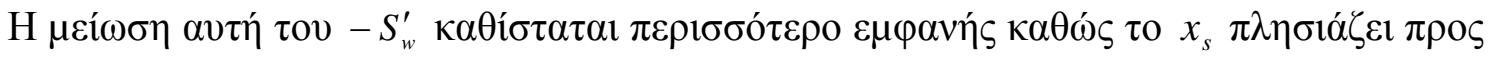

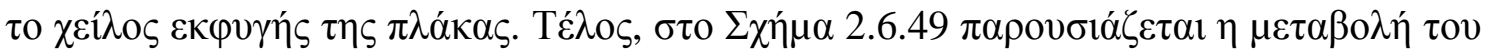

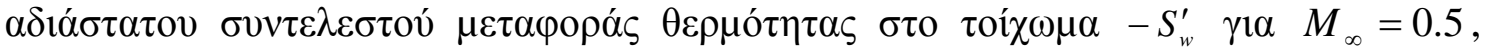

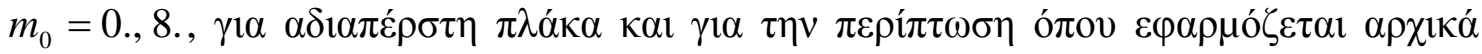

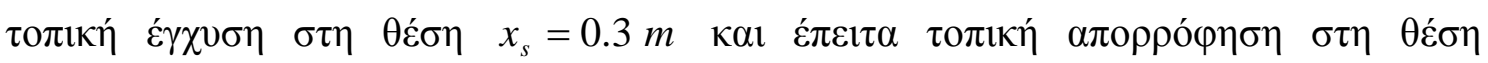

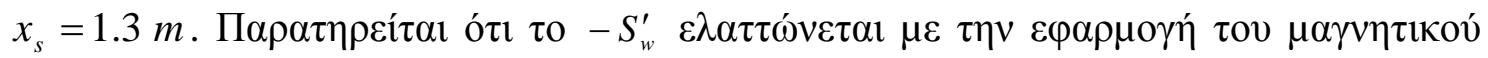

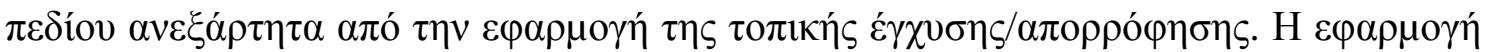

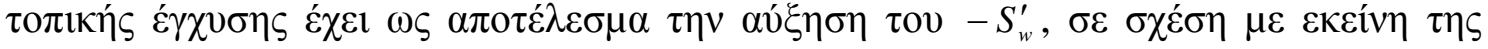

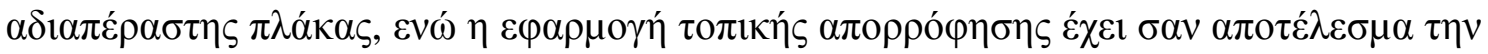

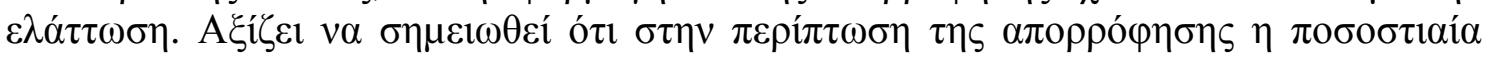

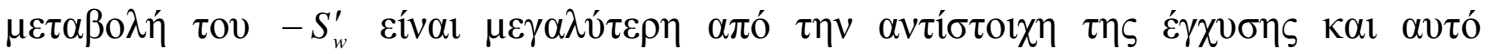

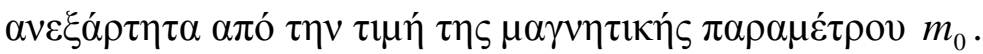

\section{$2.7 \Sigma v \mu \pi \varepsilon \rho \alpha ́ \sigma \mu \alpha \tau \alpha$}

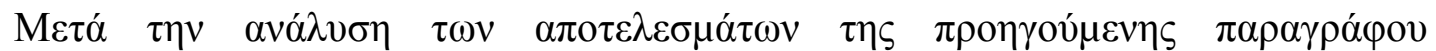

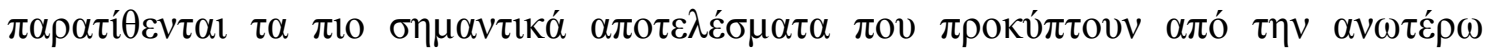

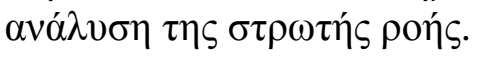

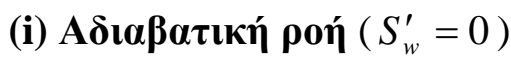

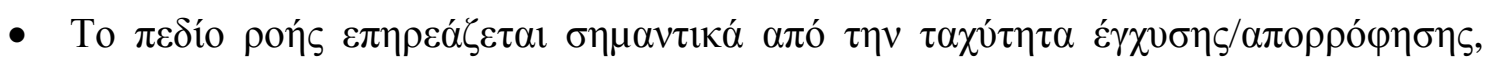

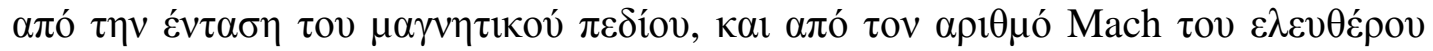
$\rho \varepsilon u ́ \mu \alpha \tau o \varsigma$.

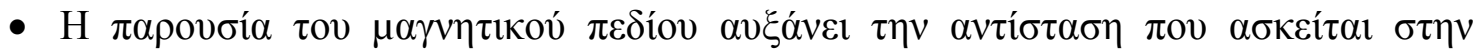

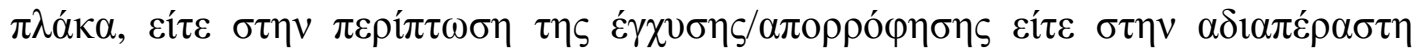

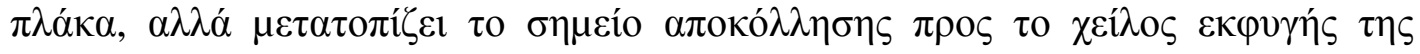
$\pi \lambda \alpha \dot{\alpha} \alpha$.

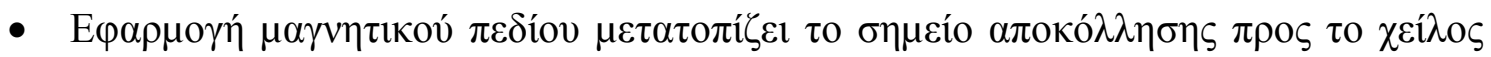

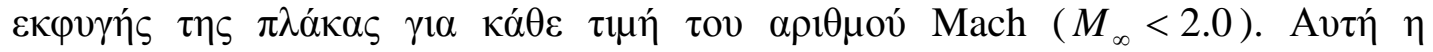

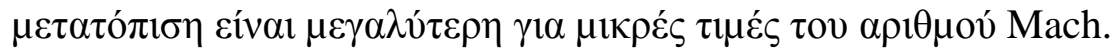

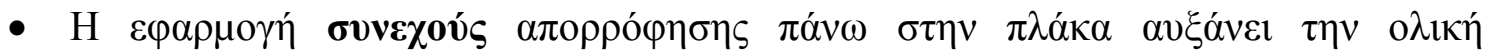

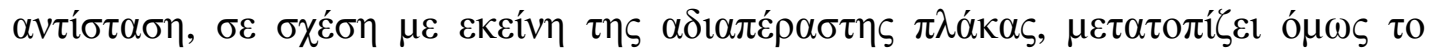

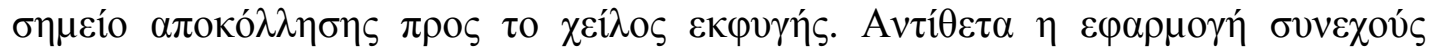

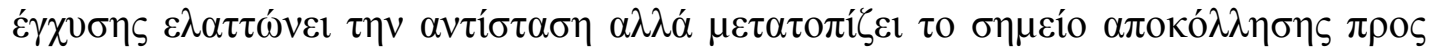

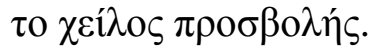

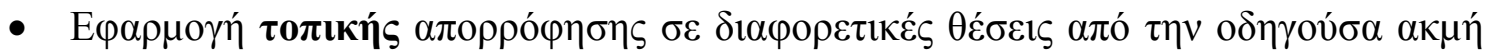

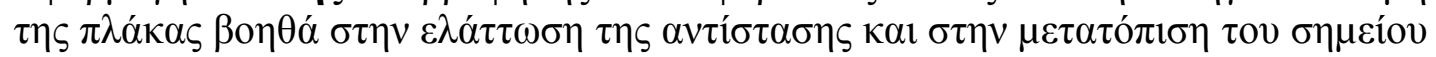

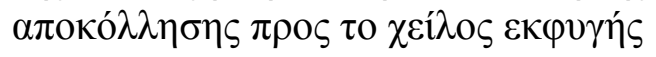

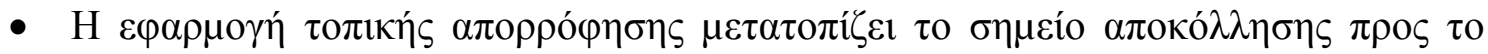

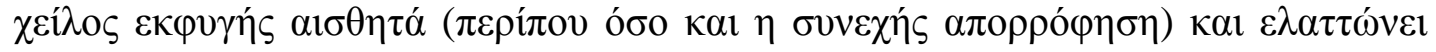




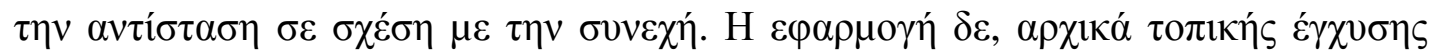

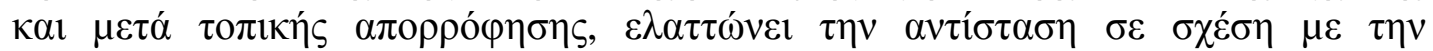

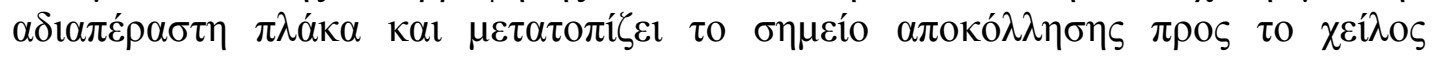

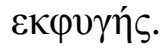

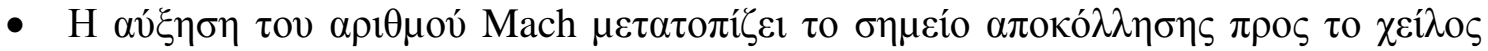

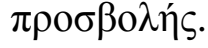

(ii) $\Theta \varepsilon \rho \mu \alpha t v o ́ \mu \varepsilon v \eta \pi \lambda \alpha ́ \kappa \alpha\left(S_{\mathrm{w}}=2.0\right)$

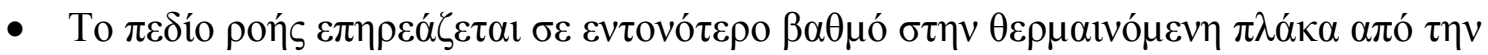

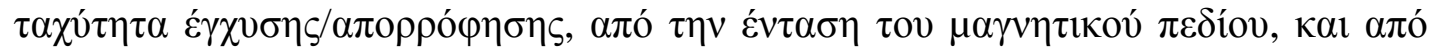

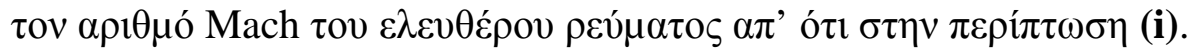

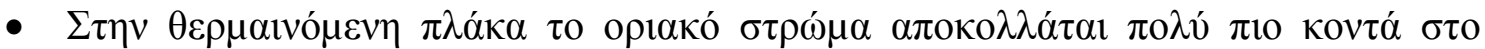

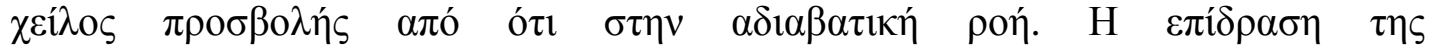

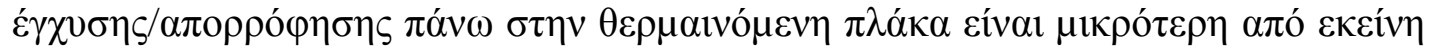

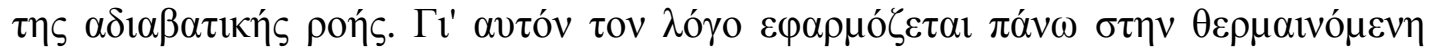

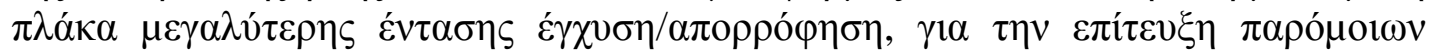
$\alpha \pi \mathrm{o} \varepsilon \lambda \varepsilon \varepsilon \sigma \mu \alpha \dot{\tau} \tau \omega v$.

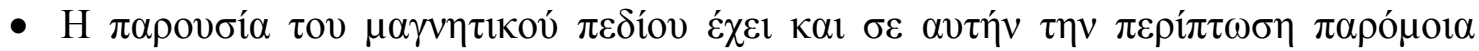

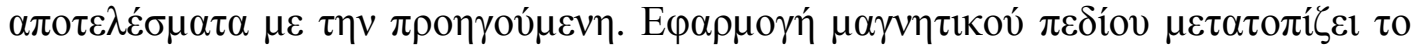

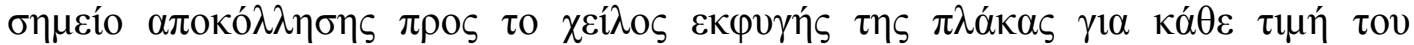

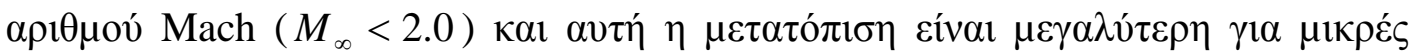

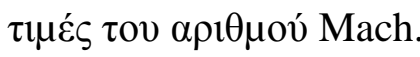

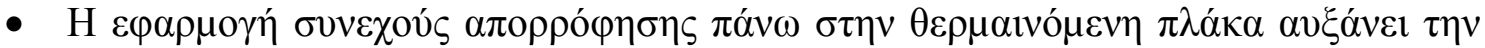

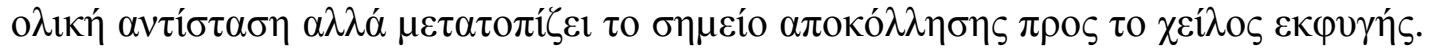

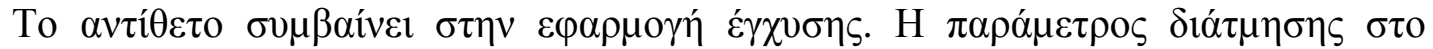

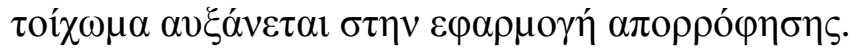

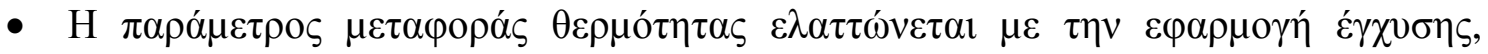

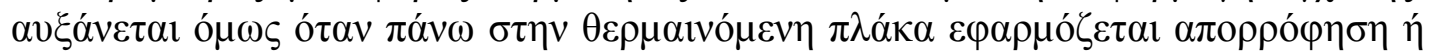

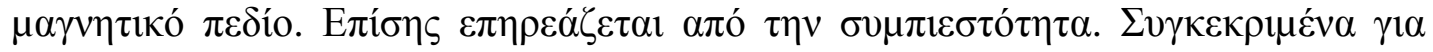

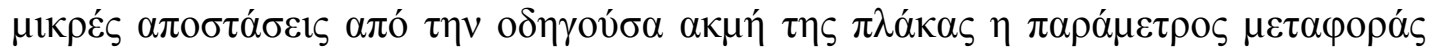

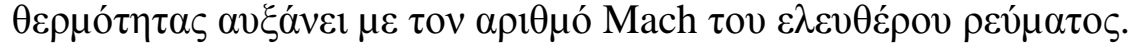

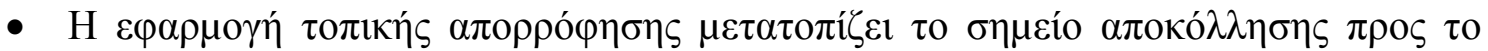

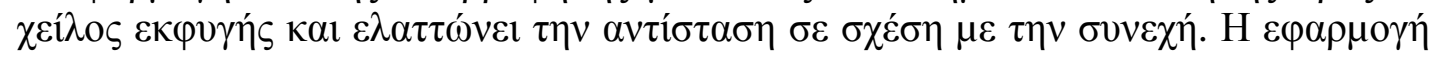

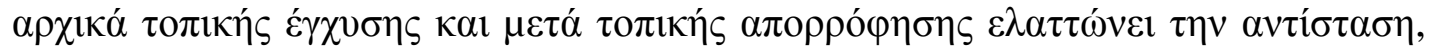

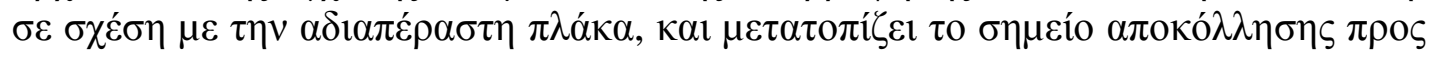

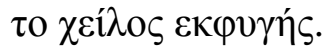

(iii) $\Psi v \chi o ́ \mu \varepsilon v \eta \pi \lambda \alpha ́ \kappa \alpha\left(S_{\mathrm{w}}=0.5\right)$

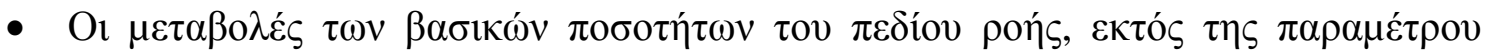

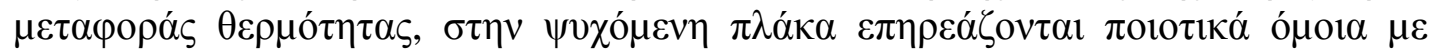

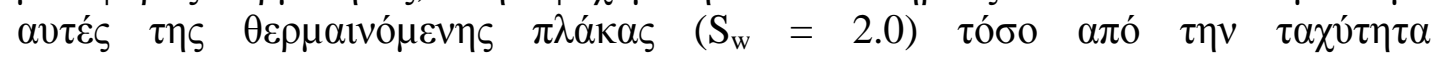




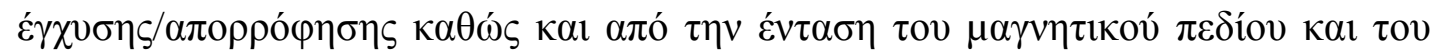

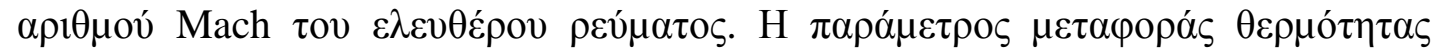

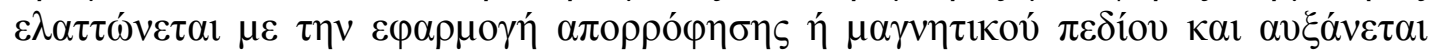

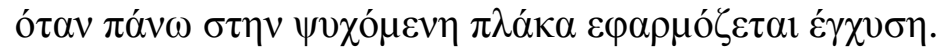

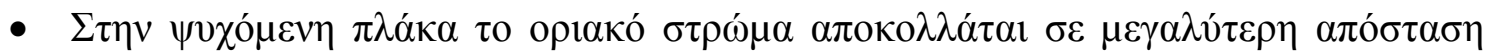

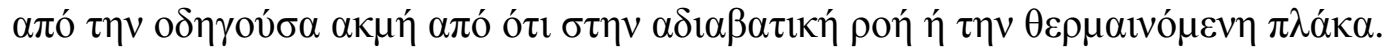

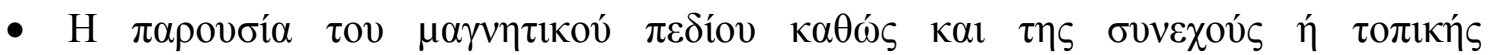

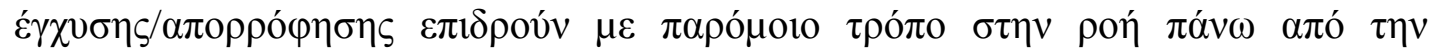

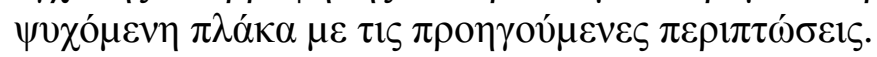

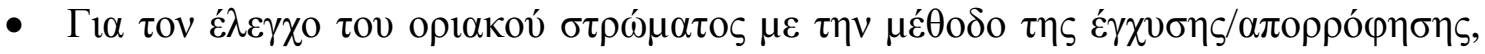

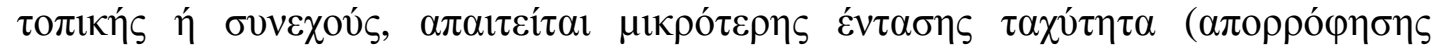

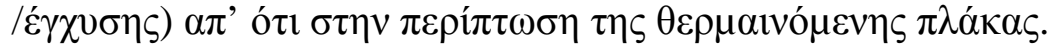

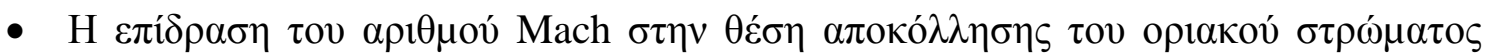

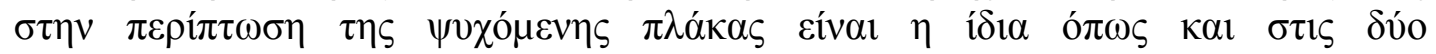
$\pi \rho \circ \eta \gamma о v ́ \mu \varepsilon v \varepsilon \varsigma \pi \varepsilon \rho i \pi \tau \omega ́ \sigma \varepsilon 1 \varsigma$. 


\section{КЕФАААIO III}

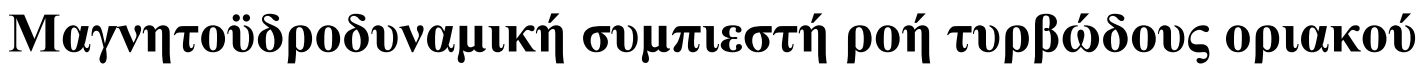

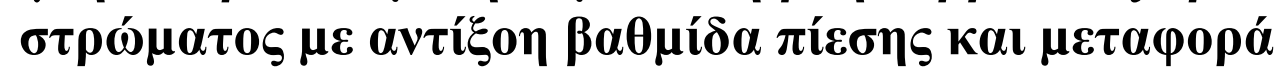

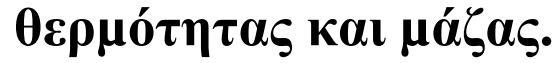

\section{Пері́ $\eta \psi \eta$}

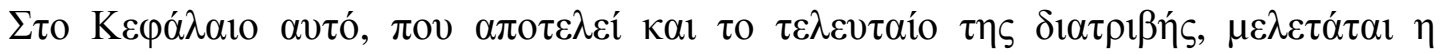
$\mu \alpha \gamma v \eta \tau о \ddot{\delta} \delta \rho \delta$

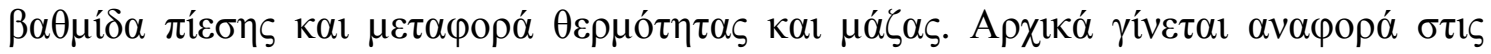

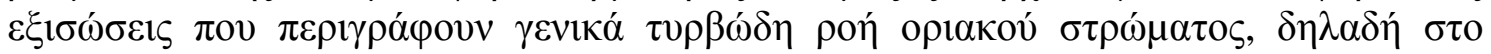

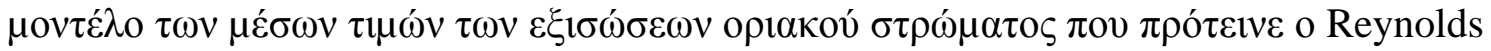

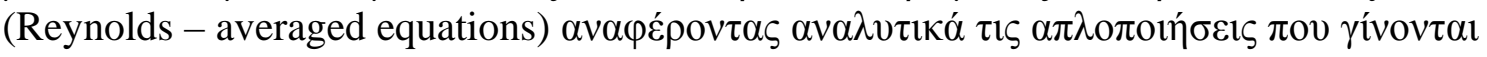

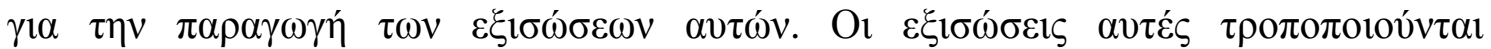

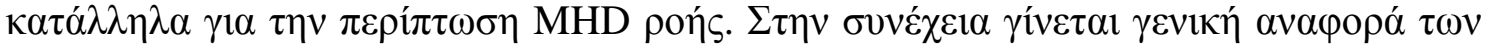

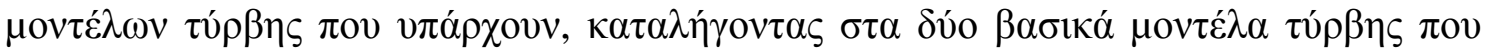

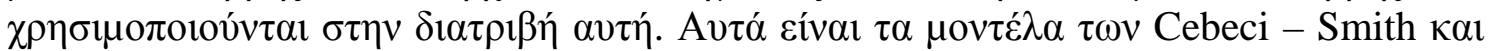

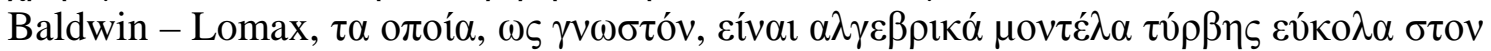

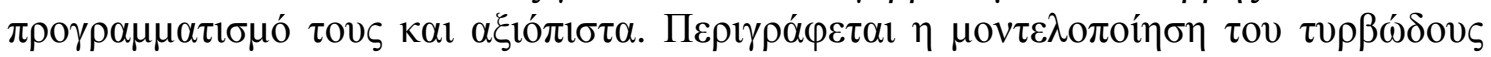

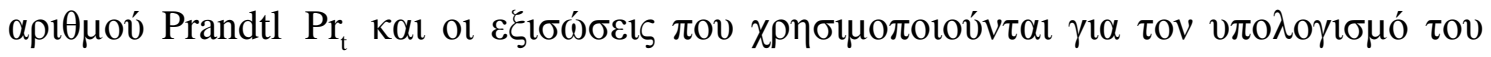

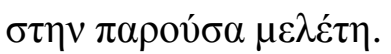

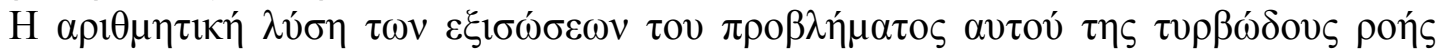

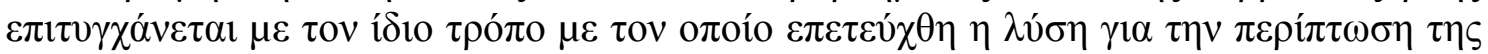

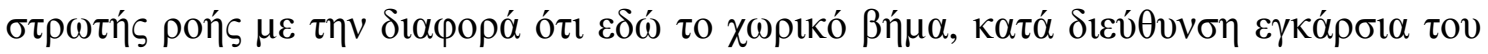

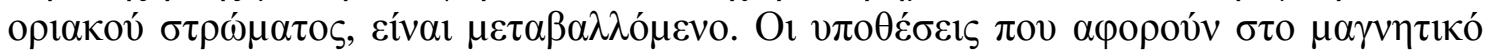

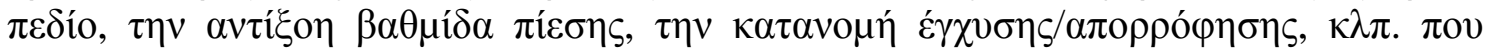

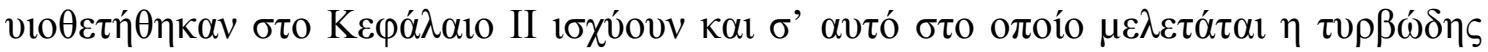

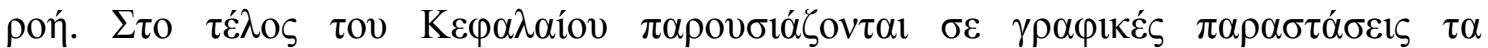

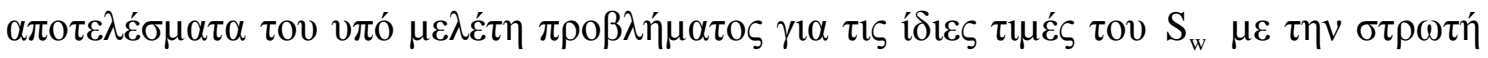

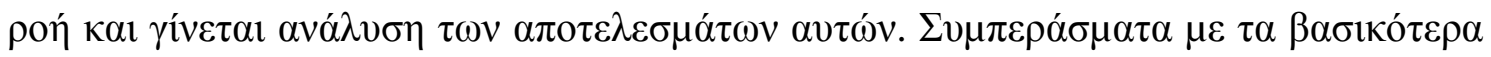

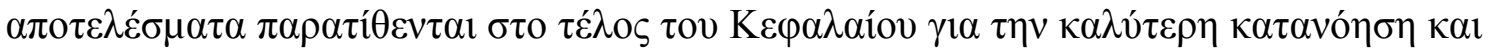
$\alpha \xi$ io

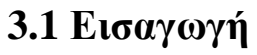

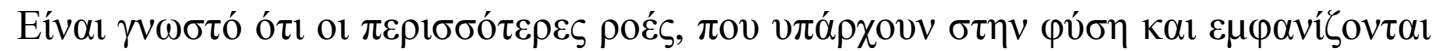

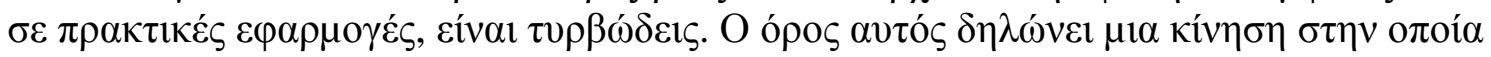

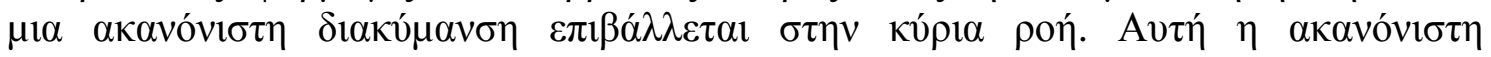

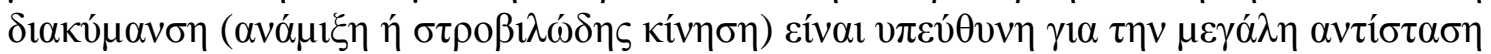

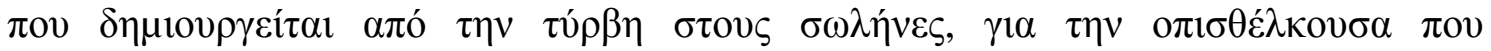

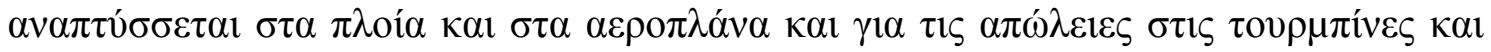

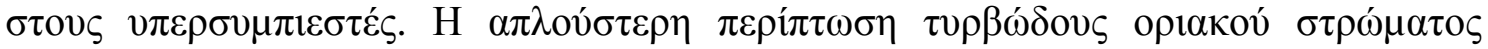

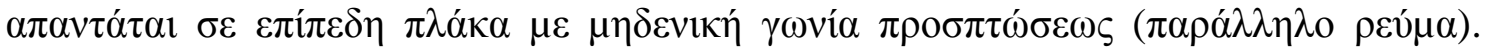




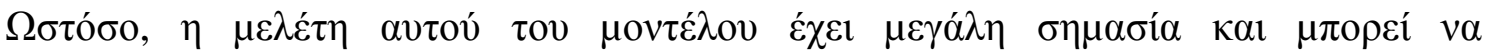

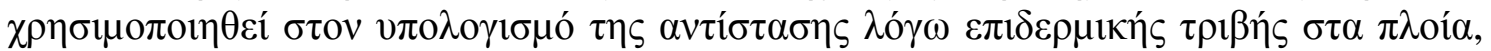

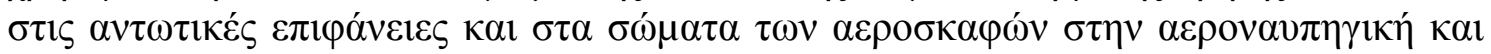

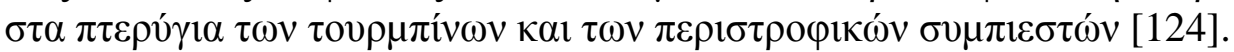

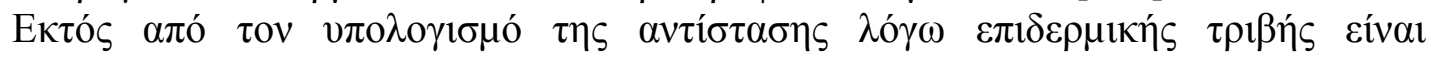

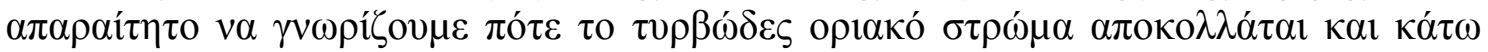

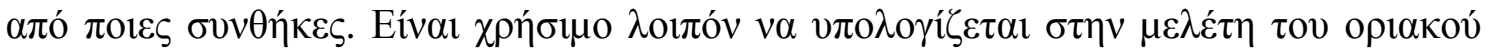

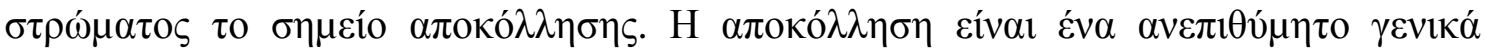

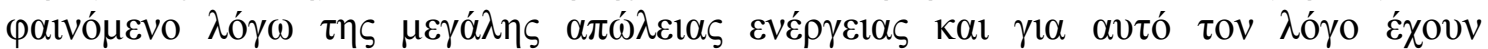

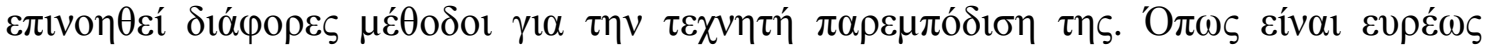

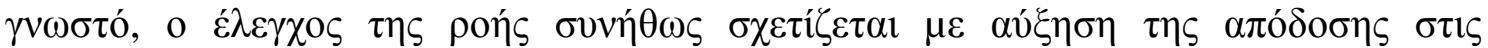

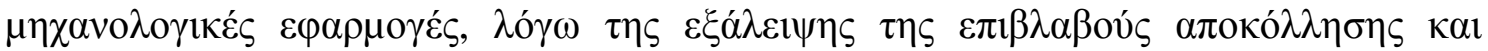

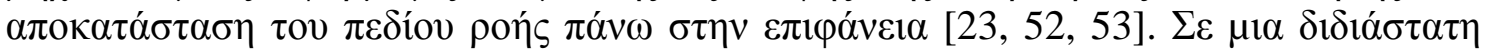

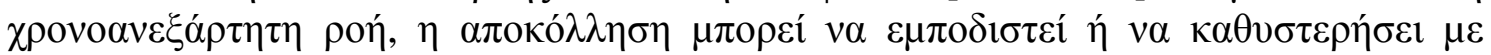

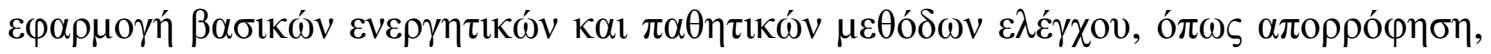

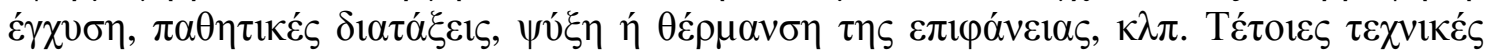

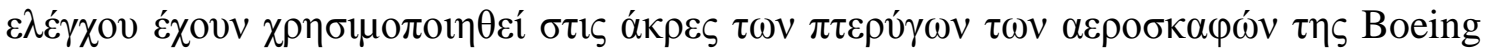

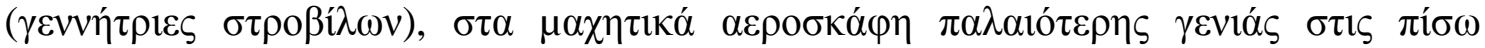

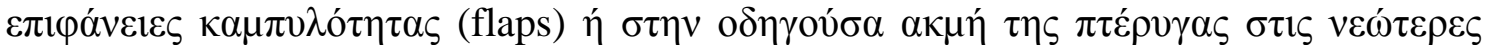

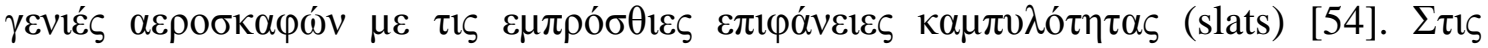

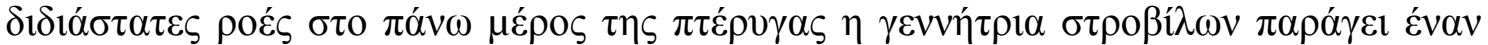

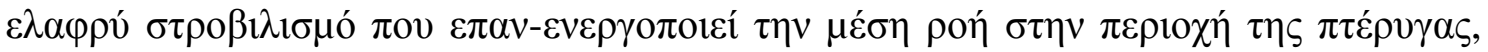

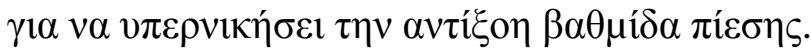

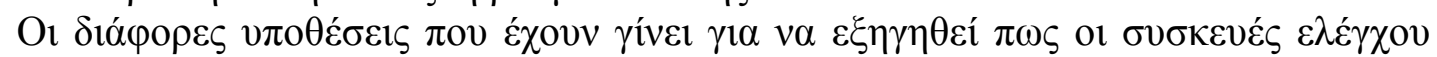

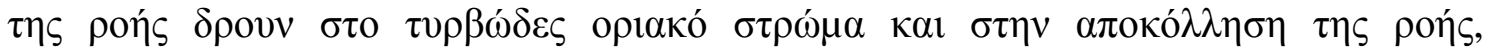

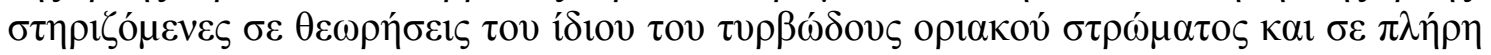

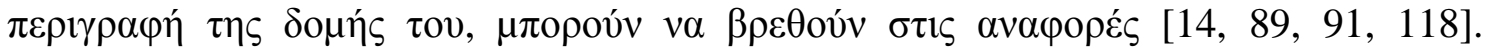

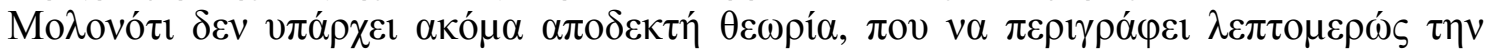

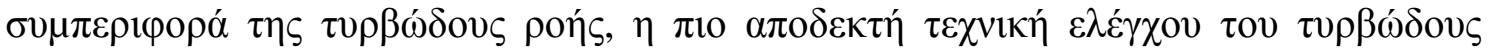

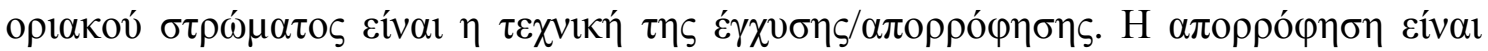

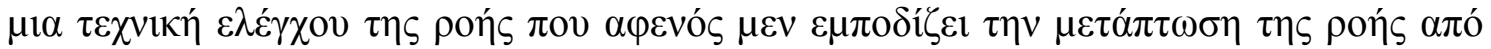

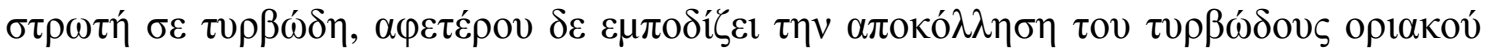

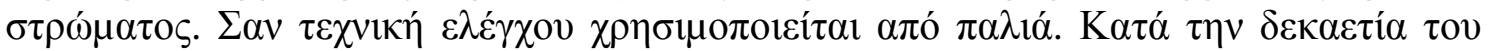

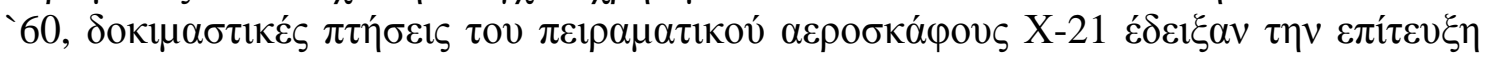

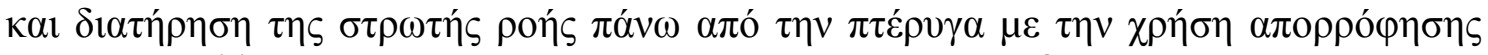

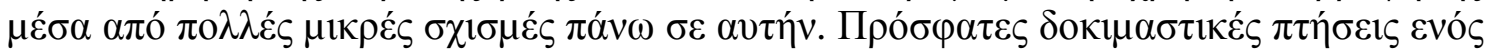

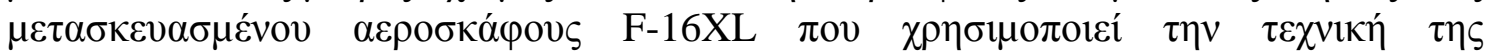

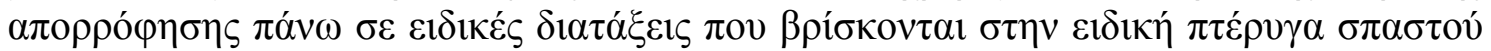

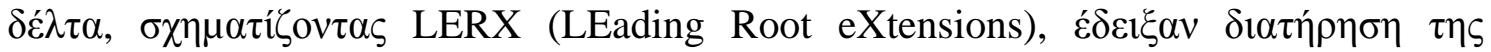

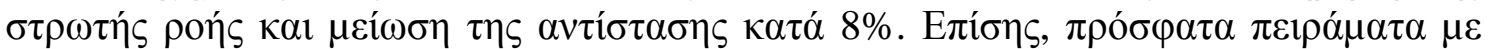

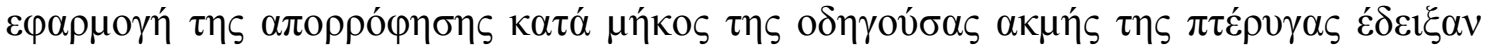

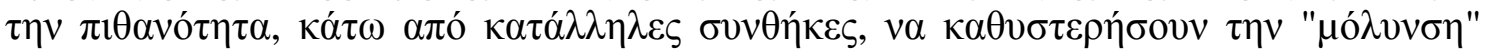

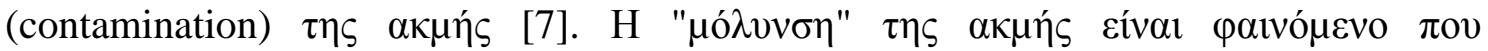

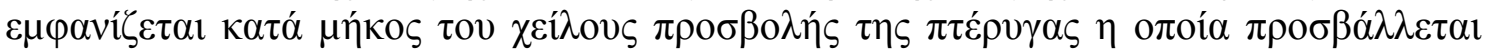

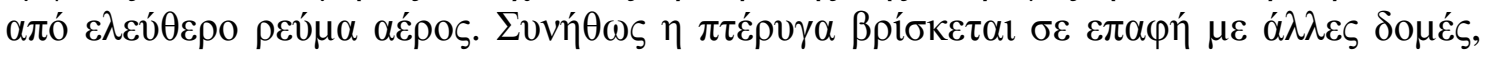

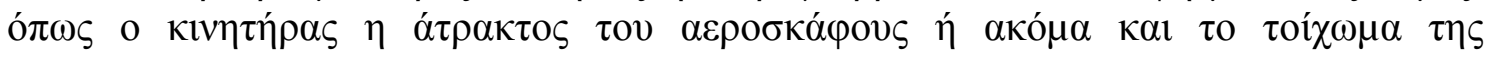

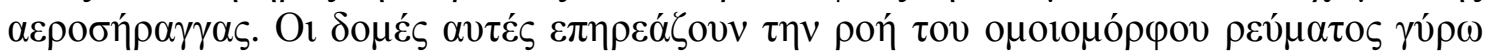

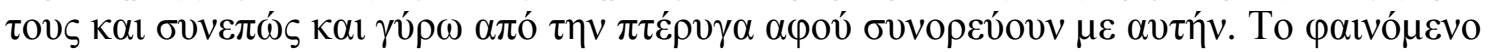

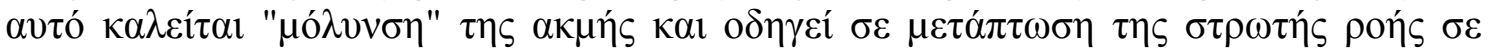

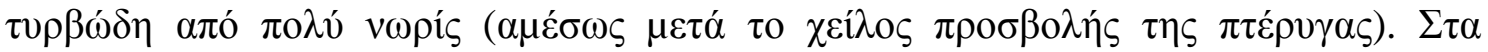




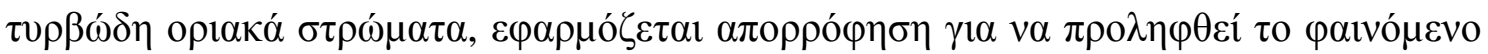

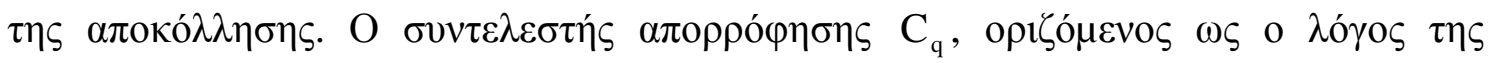

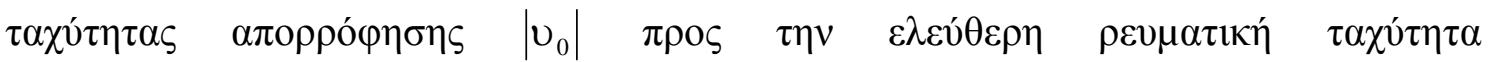

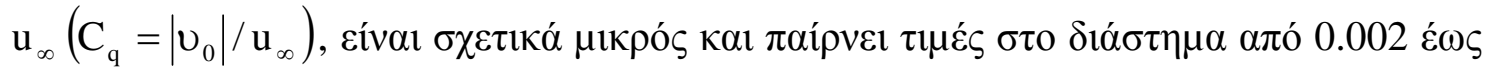

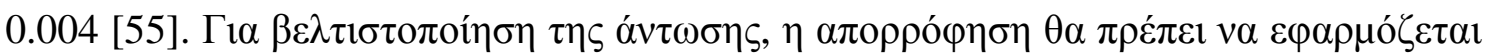

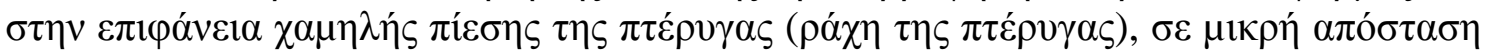

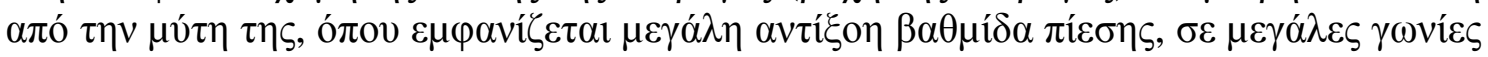

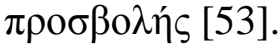

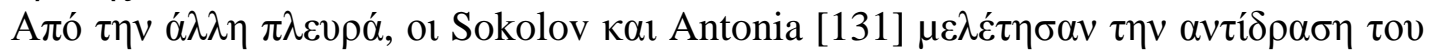

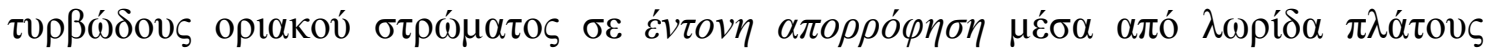

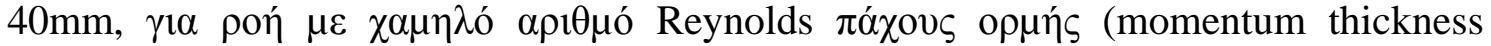

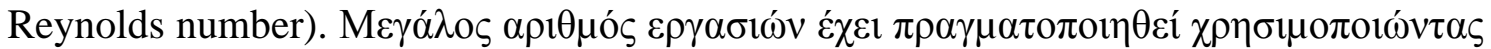

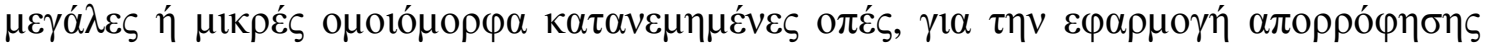

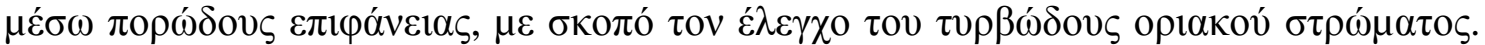

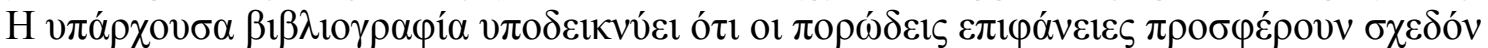
оноіо́

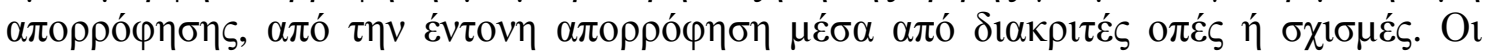

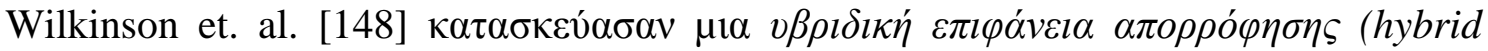

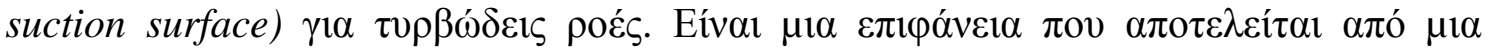

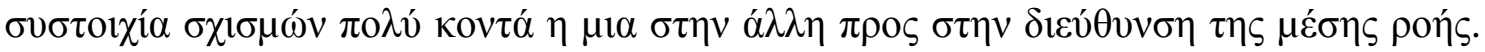

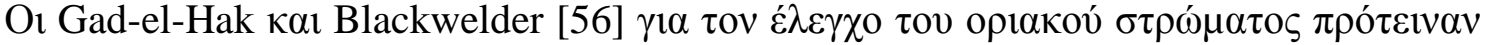

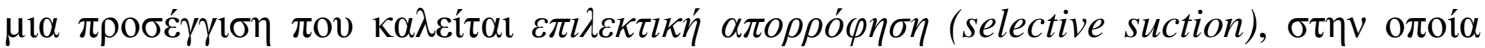

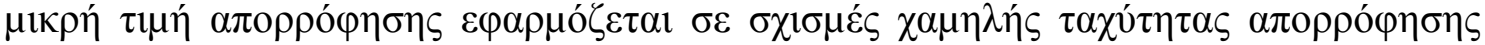

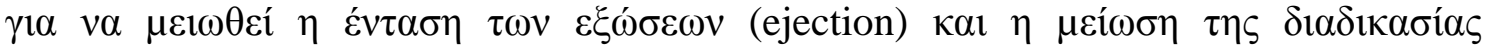

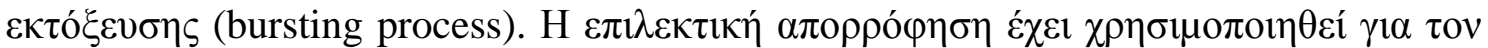

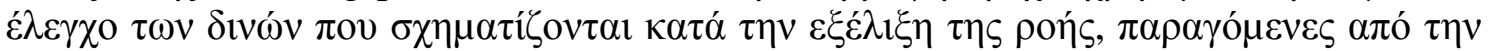

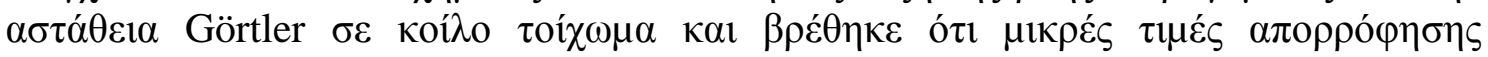

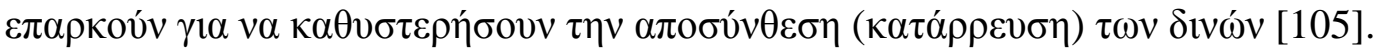

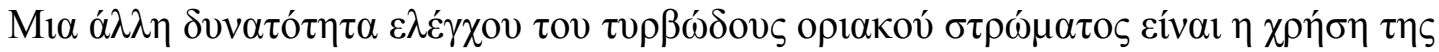

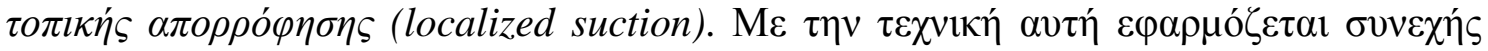

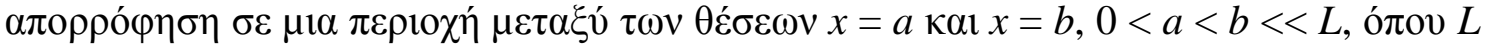

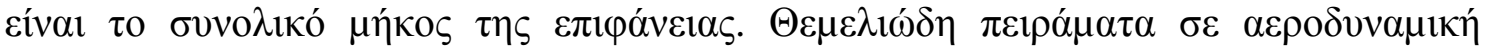

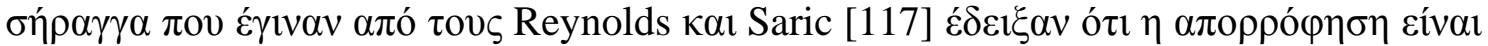

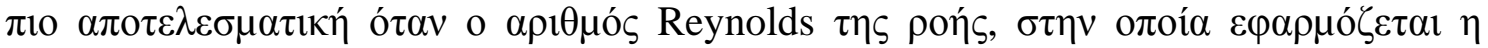

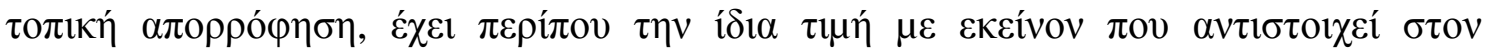

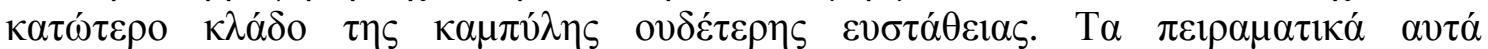

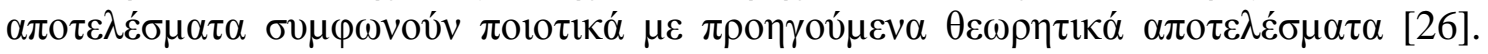

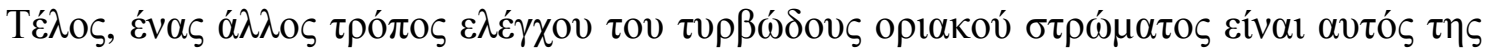

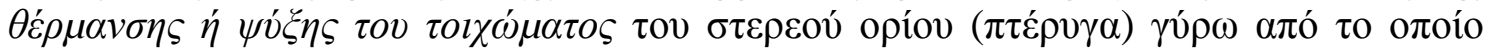

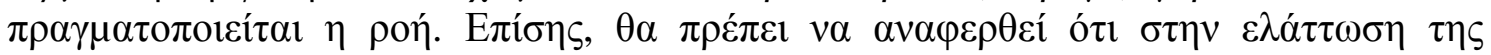

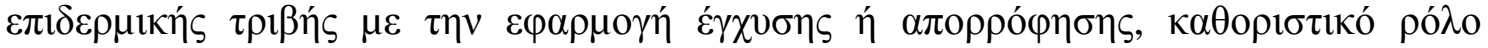

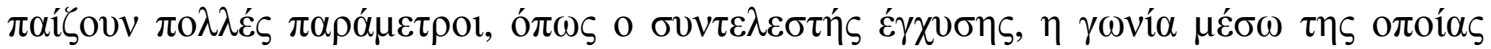

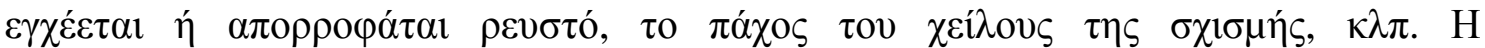

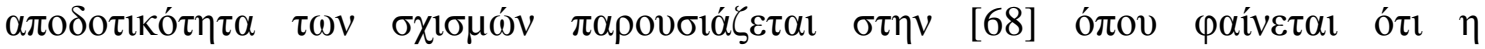

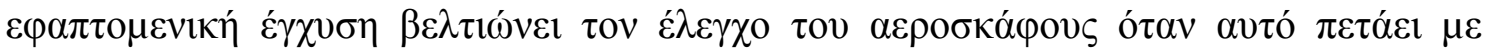

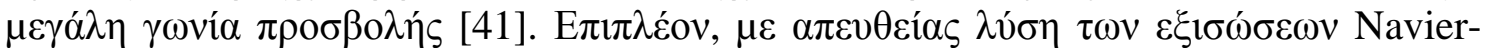
Stokes (Direct Numerical Simulation (DNS)) $\varepsilon \xi \varepsilon \tau \alpha ́ \sigma \tau \eta \kappa \varepsilon ~ \eta \pi \lambda \eta ́ \rho \omega \varsigma ~ \alpha v \alpha \pi \tau v \gamma \mu \varepsilon ́ v \eta$

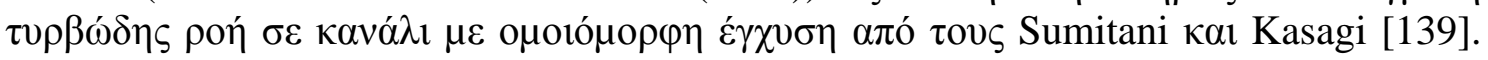

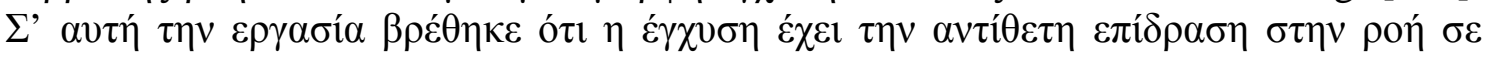




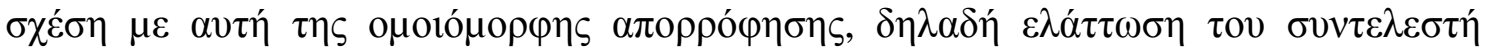

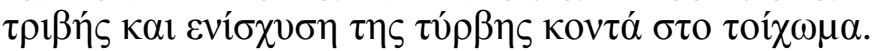

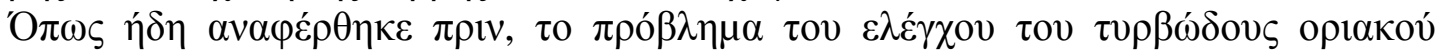

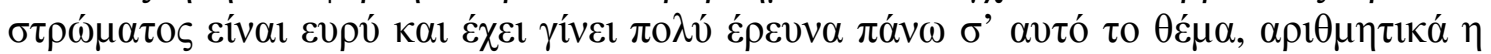

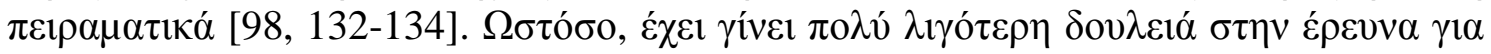

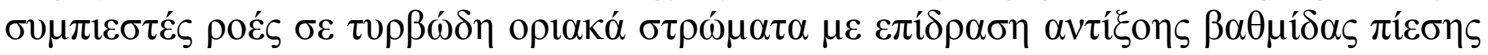

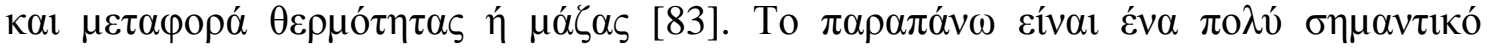

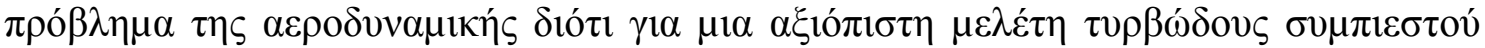

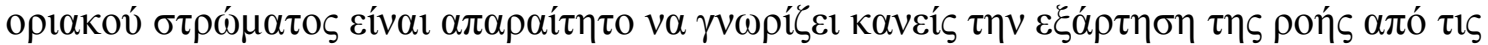
$\delta$ iá

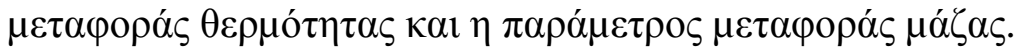

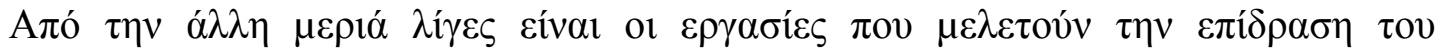
$\mu \alpha \gamma v \eta \tau ı \kappa o v ́ ~ \pi \varepsilon \delta i ́ o v ~ \sigma \tau \eta v ~ \tau v \rho \beta \omega ́ \delta \eta ~ \rho о \eta ́ . ~ П \alpha \lambda \alpha ı ́ \tau \varepsilon \rho \varepsilon \varsigma ~ \varepsilon \rho \gamma \alpha \sigma i ́ \varepsilon \varsigma ~ \mu \varepsilon \lambda \varepsilon \tau o v ́ \sigma \alpha v ~ \alpha \pi \lambda \omega ́ \varsigma \tau \eta v$

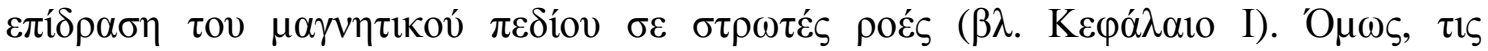

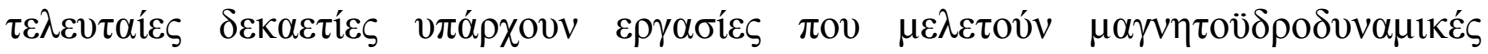

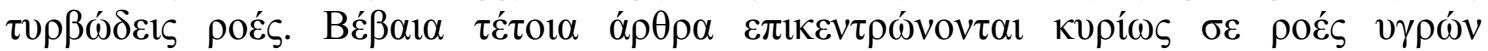

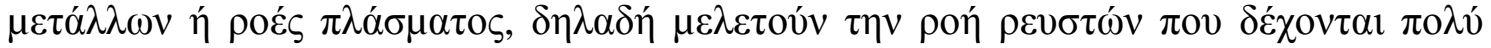

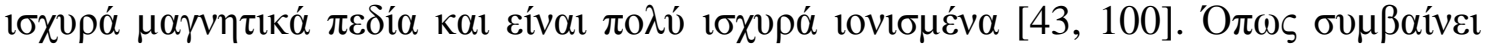

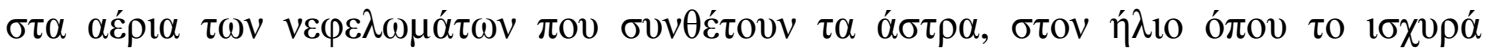

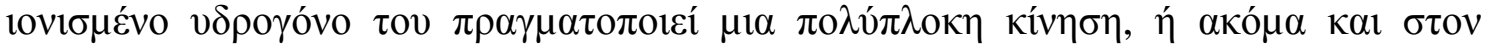

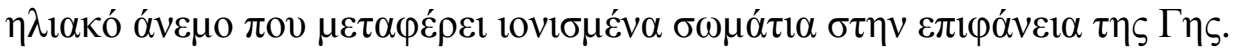

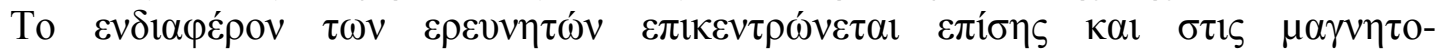

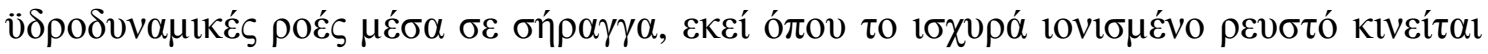

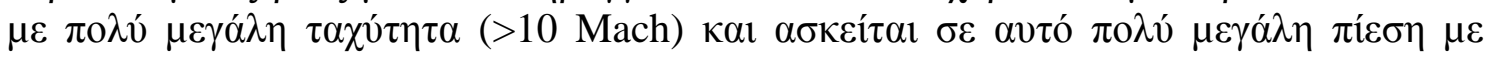

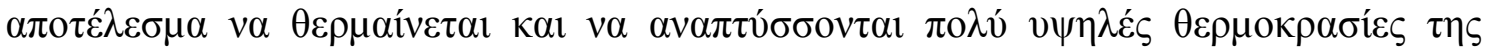

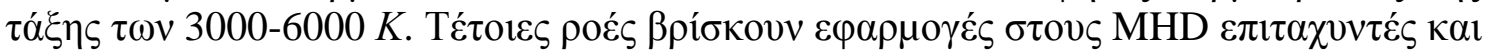

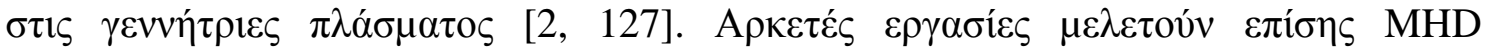

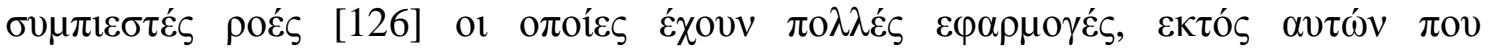

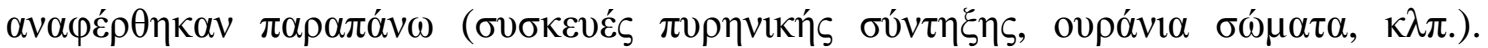

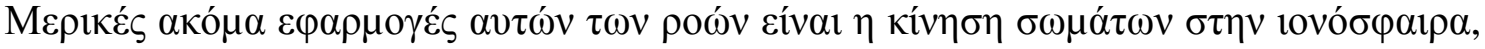

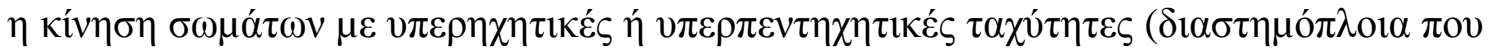

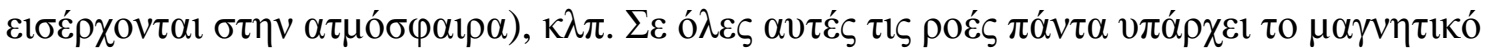

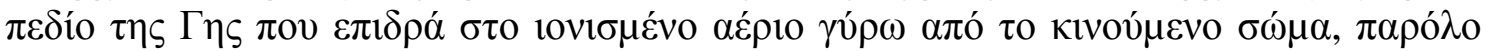

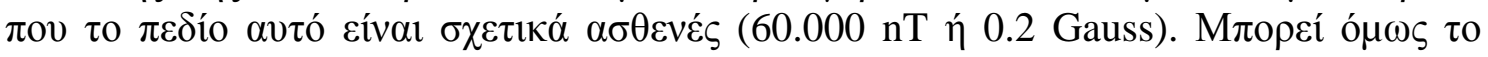

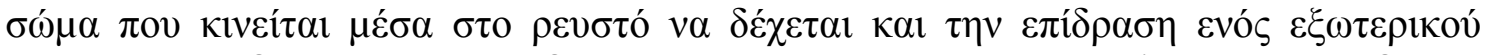

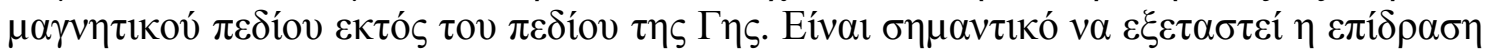

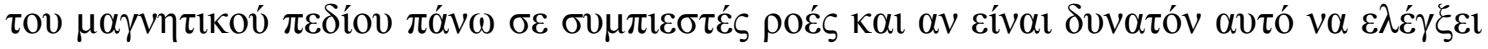

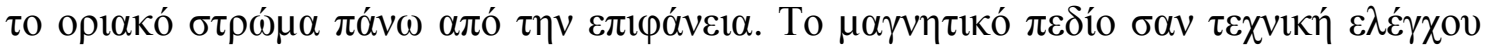

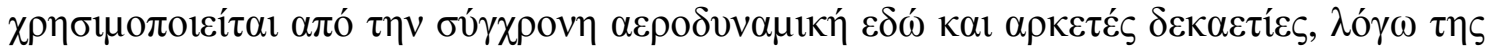

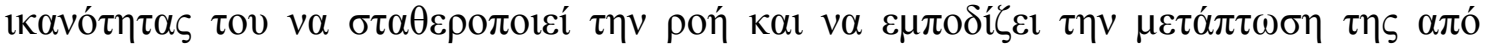

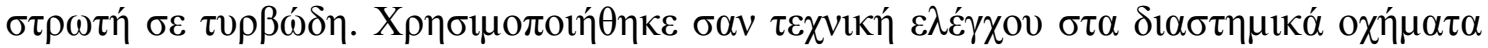

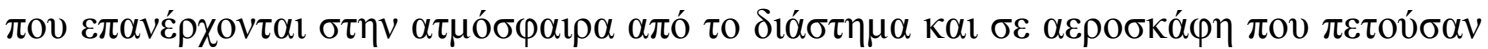

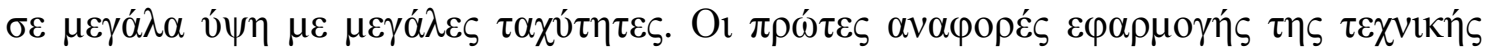

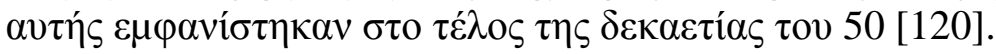

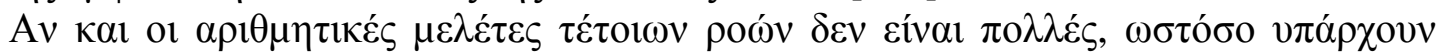

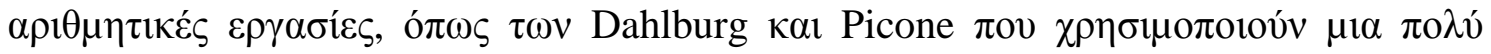

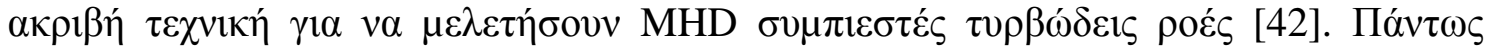

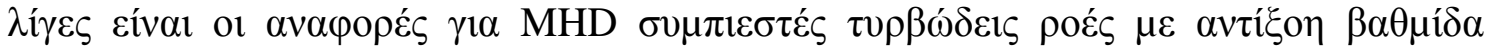

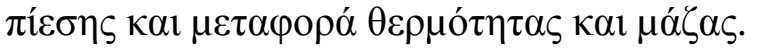




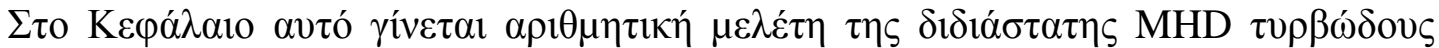

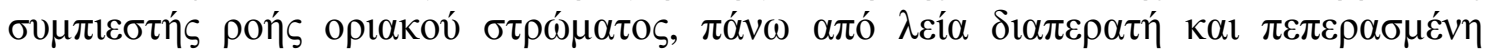

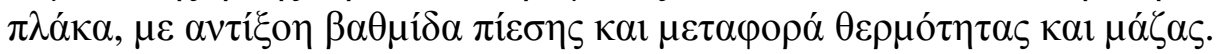

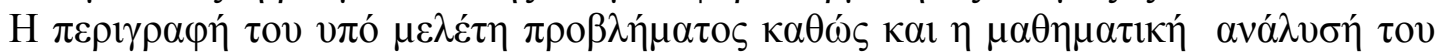

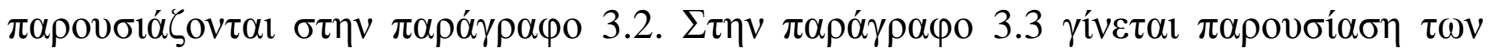

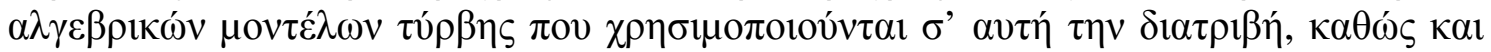

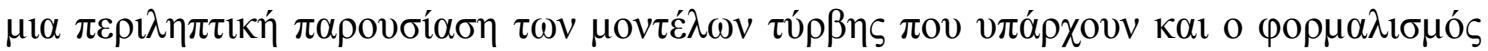

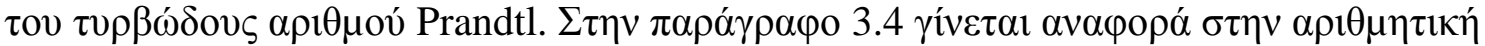

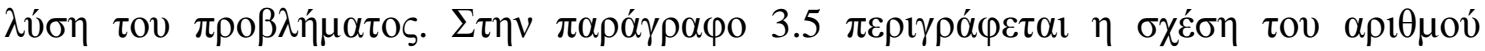

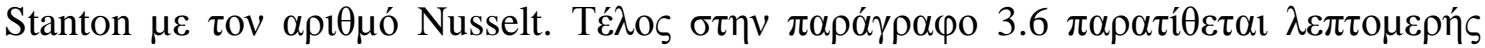

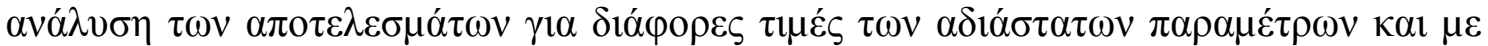

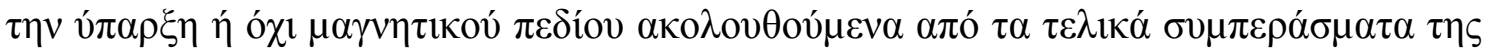

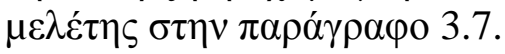

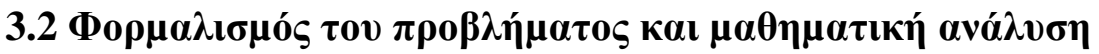

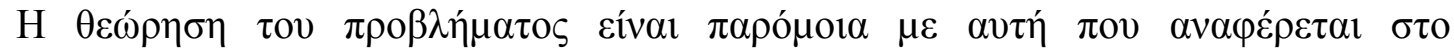

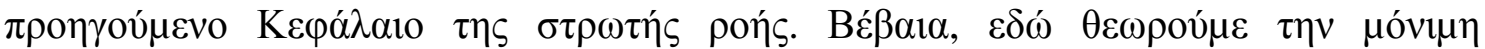

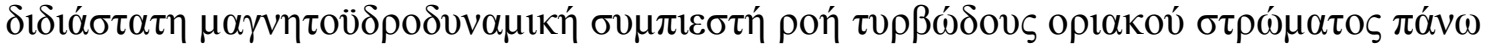

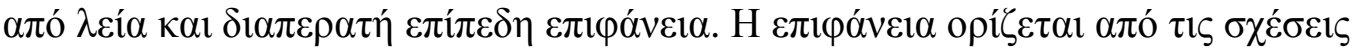

$$
y=0, \quad 0 \leq x \leq L, \quad-\infty<z<+\infty
$$

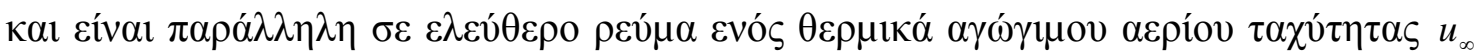

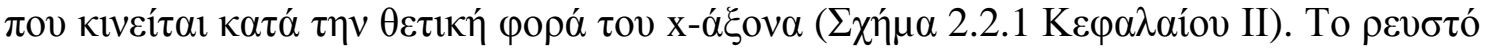

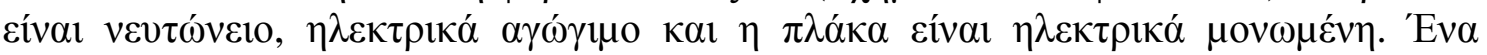

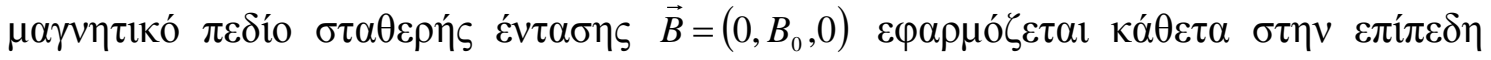

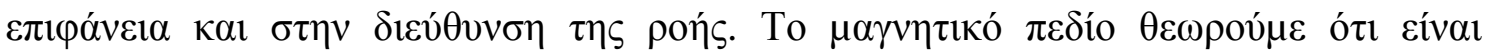

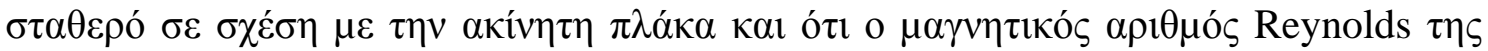

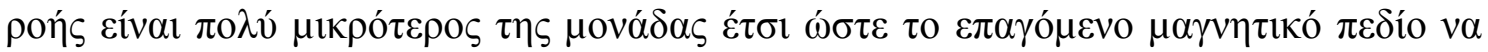

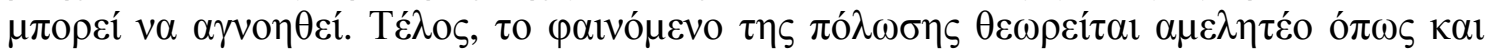

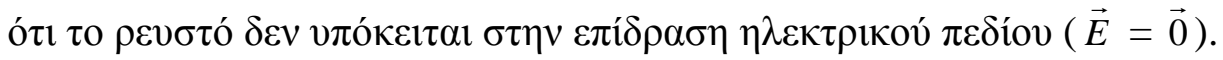

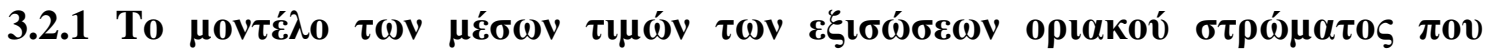

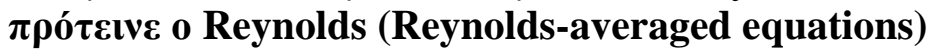

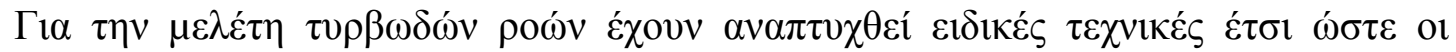

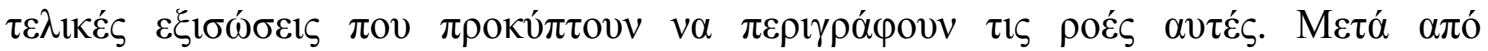

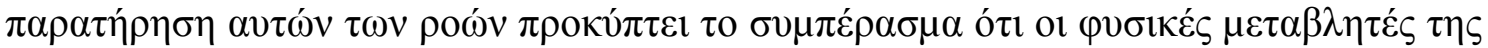

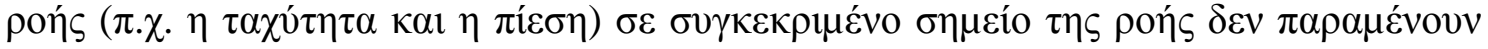

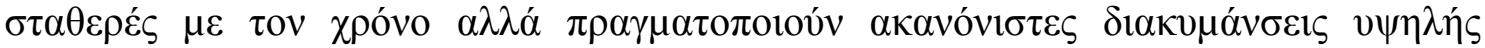

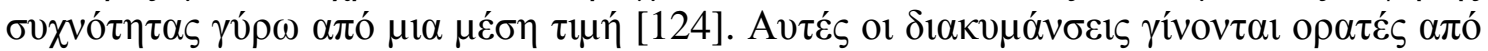

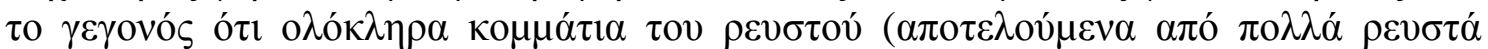

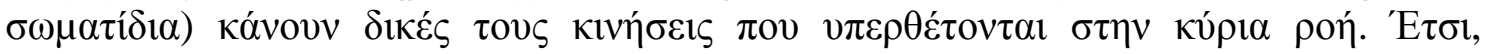

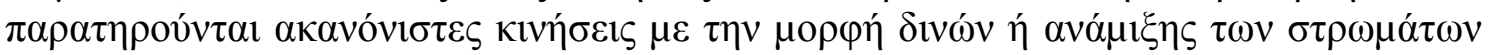

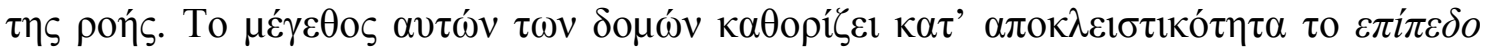

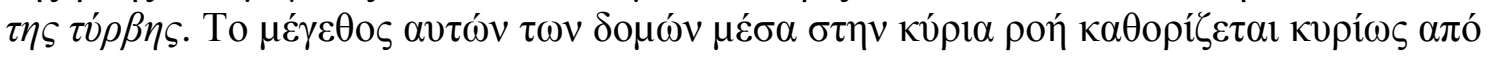




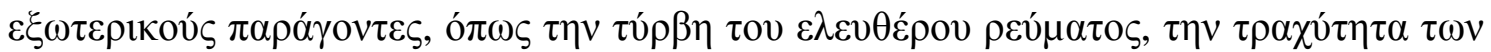

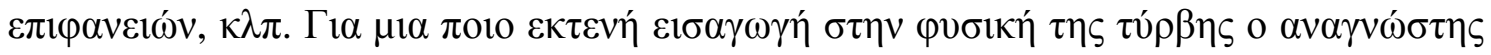

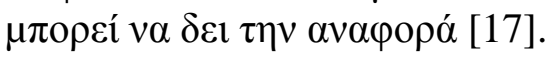

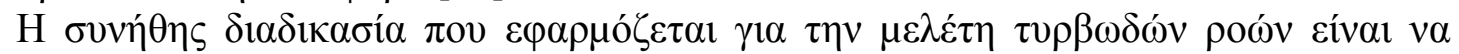

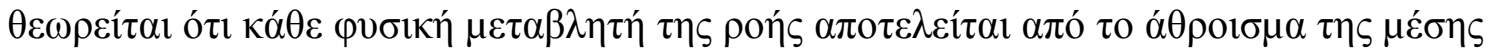

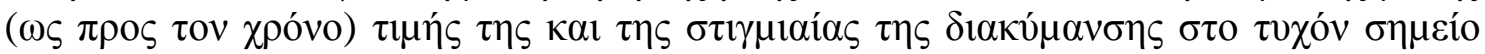

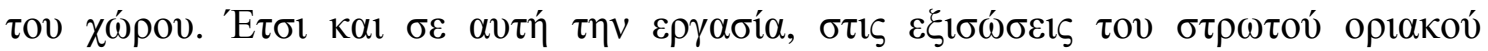

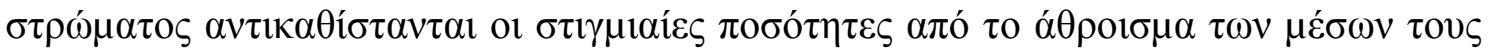

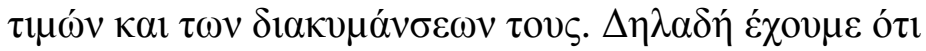

$$
\mathrm{u}=\overline{\mathrm{u}}+\mathrm{u}^{\prime}, \mathrm{v}=\overline{\mathrm{v}}+\mathrm{v}^{\prime}, \mathrm{p}=\overline{\mathrm{p}}+\mathrm{p}^{\prime}
$$

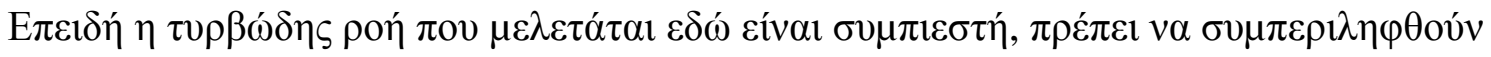

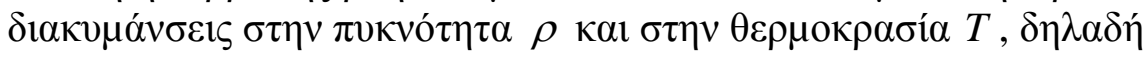

$$
\rho=\bar{\rho}+\rho^{\prime}, \quad T=\bar{T}+T^{\prime} .
$$

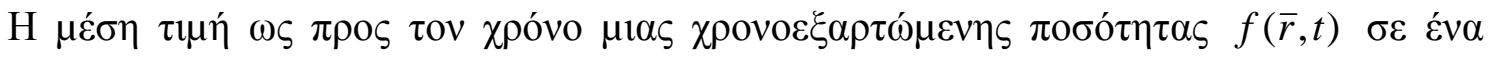

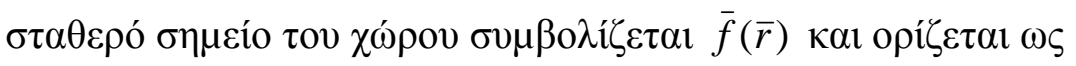

$$
\bar{f}(\bar{r})=\lim _{T \rightarrow \infty} \frac{1}{T} \int_{0}^{T} f(\bar{r}, t) d t .
$$

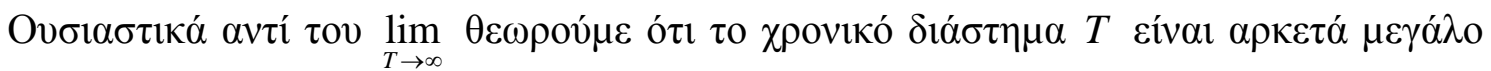

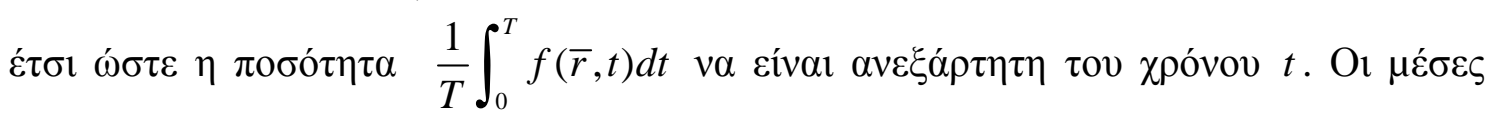

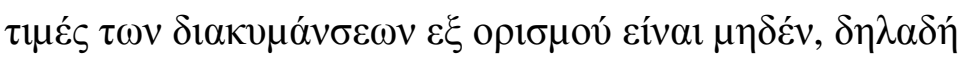

$$
\overline{u^{\prime}}=0, \quad \overline{v^{\prime}}=0, \quad \overline{p^{\prime}}=0, \quad \overline{\rho^{\prime}}=0, \quad \overline{T^{\prime}}=0
$$

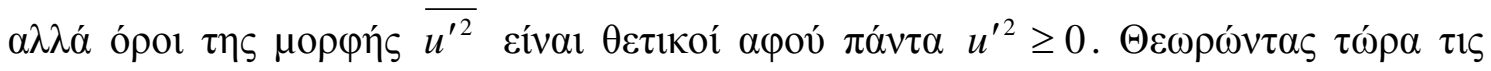

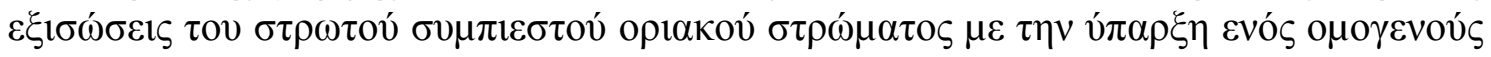

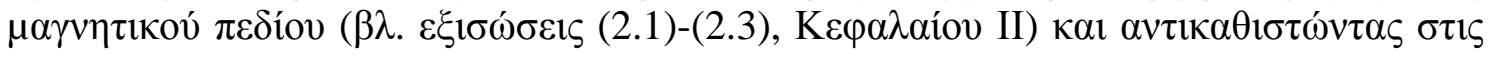

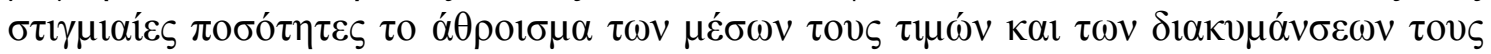

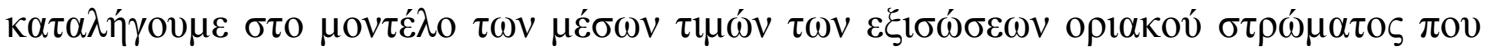

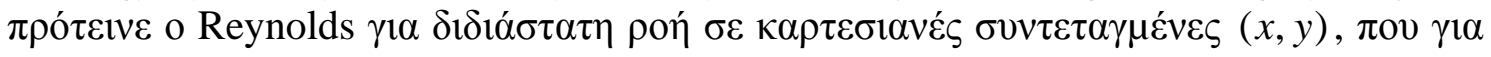

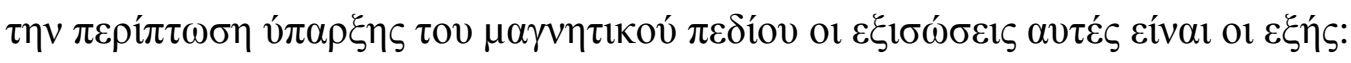

$\varepsilon \xi \dot{i} \sigma \omega \sigma \eta \sigma v v \varepsilon ́ \chi \varepsilon 1 \alpha \varsigma$

$$
\frac{\partial}{\partial x}\left(\bar{\rho} \bar{u}+\overline{\rho^{\prime} u^{\prime}}\right)+\frac{\partial}{\partial y}\left(\bar{\rho} \bar{v}+\overline{\rho^{\prime} v^{\prime}}\right)=0
$$

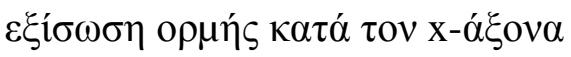

$$
\left(\bar{\rho} \bar{u}+\overline{\rho^{\prime} u^{\prime}}\right) \frac{\partial \bar{u}}{\partial x}+\left(\bar{\rho} \bar{v}+\overline{\rho^{\prime} v^{\prime}}\right) \frac{\partial \bar{u}}{\partial y}=-\frac{\partial \bar{p}}{\partial x}+\frac{\partial}{\partial y}\left[\mu \frac{\partial \bar{u}}{\partial y}-\left(\bar{\rho} \overline{u^{\prime} v^{\prime}}+\overline{\rho^{\prime} u^{\prime} v^{\prime}}\right)\right]-\sigma B_{0}^{2} \bar{u}
$$

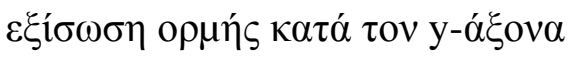




$$
\frac{\partial \bar{p}}{\partial y}=0
$$

$\varepsilon \xi \dot{i} \sigma \omega \sigma \eta \varepsilon v \varepsilon \dot{\rho} \gamma \varepsilon 1 \alpha \varsigma$

$$
\begin{aligned}
& c_{p}\left[\left(\bar{\rho} \bar{u}+\overline{\rho^{\prime} u^{\prime}}\right)\right.\left.\frac{\partial \bar{T}}{\partial x}+\left(\bar{\rho} \bar{v}+\overline{\rho^{\prime} v^{\prime}}\right) \frac{\partial \bar{T}}{\partial y}\right]= \\
&\left.\frac{\partial}{\partial y}\left[k \frac{\partial \bar{T}}{\partial y}-c_{p} \bar{\rho} \overline{T^{\prime} v^{\prime}}-c_{p} \overline{\rho^{\prime} T^{\prime} v^{\prime}}\right]+\bar{u} \frac{\partial \bar{p}}{\partial x}+\mu\left(\frac{\partial \bar{u}}{\partial y}\right)^{2}+\sigma B_{0}^{2} \overline{\left(\bar{u}+u^{\prime}\right)}\right)^{2} .
\end{aligned}
$$

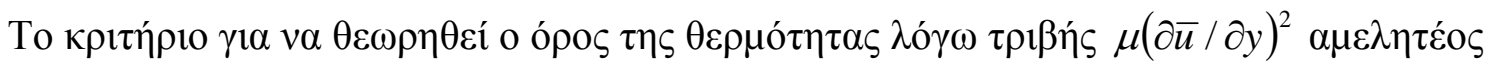

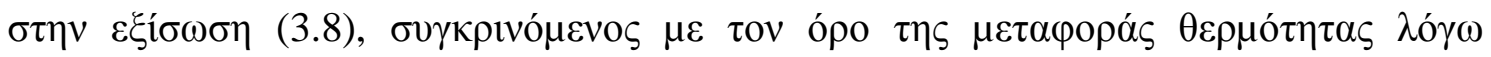

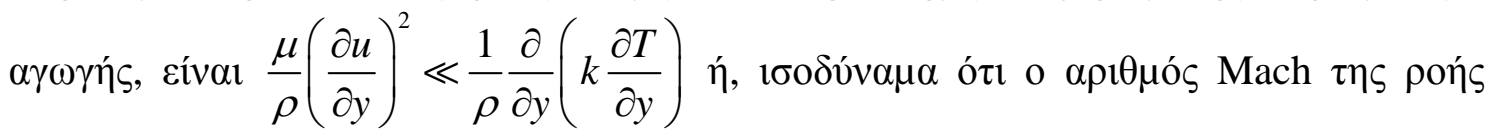

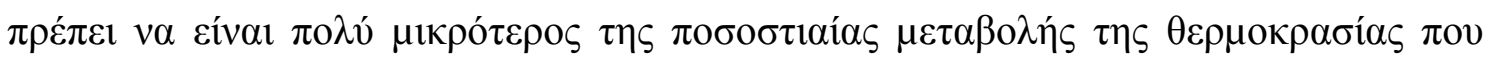

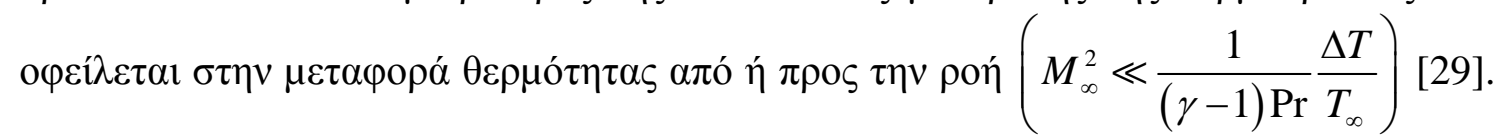

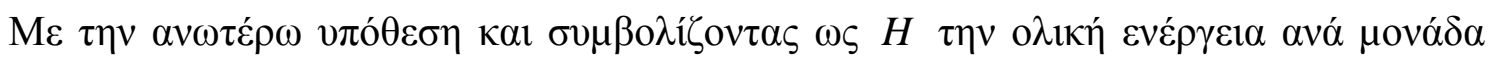

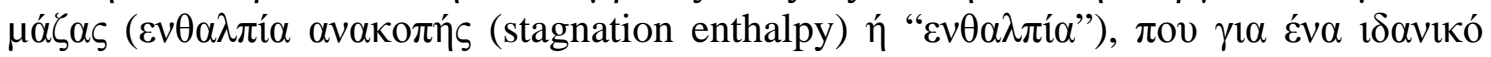

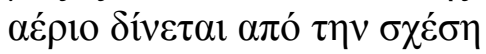

$$
H=c_{p} T+\frac{1}{2} u^{2}
$$

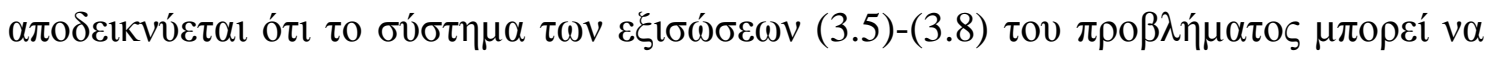

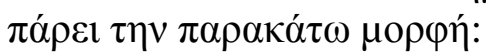

$\varepsilon \xi i ́ \sigma \omega \sigma \eta \sigma v v \varepsilon ́ \chi \varepsilon 1 \alpha \varsigma$

$$
\frac{\partial}{\partial x}\left(\bar{\rho} \bar{u}+\overline{\rho^{\prime} u^{\prime}}\right)+\frac{\partial}{\partial y}\left(\bar{\rho} \bar{v}+\overline{\rho^{\prime} v^{\prime}}\right)=0
$$

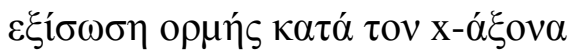

$$
\left(\bar{\rho} \bar{u}+\overline{\rho^{\prime} u^{\prime}}\right) \frac{\partial \bar{u}}{\partial x}+\left(\bar{\rho} \bar{v}+\overline{\rho^{\prime} v^{\prime}}\right) \frac{\partial \bar{u}}{\partial y}=-\frac{\partial \bar{p}}{\partial x}+\frac{\partial}{\partial y}\left[\mu \frac{\partial \bar{u}}{\partial y}-\left(\bar{\rho} \overline{u^{\prime} v^{\prime}}+\overline{\rho^{\prime} u^{\prime} v^{\prime}}\right)\right]-\sigma B_{0}^{2} \bar{u},
$$

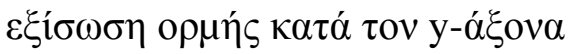

$$
\frac{\partial \bar{p}}{\partial y}=0
$$

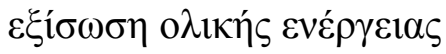

$$
\begin{aligned}
\left(\bar{\rho} \bar{u}+\overline{\rho^{\prime} u^{\prime}}\right) \frac{\partial H}{\partial x}+\left(\bar{\rho} \bar{v}+\overline{\rho^{\prime} v^{\prime}}\right) \frac{\partial H}{\partial y}= & \\
& \frac{\partial}{\partial y}\left[k \frac{\partial \bar{T}}{\partial y}-c_{p} \bar{\rho} \overline{T^{\prime} v^{\prime}}-c_{p} \overline{\rho^{\prime} T^{\prime} v^{\prime}}+\bar{u}\left(\mu \frac{\partial \bar{u}}{\partial y}-\bar{\rho} \overline{u^{\prime} v^{\prime}}-\overline{\rho^{\prime} u^{\prime} v^{\prime}}\right)\right] .
\end{aligned}
$$




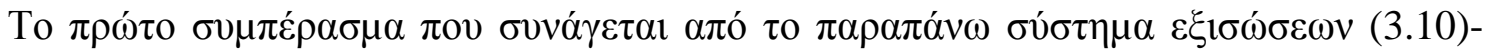

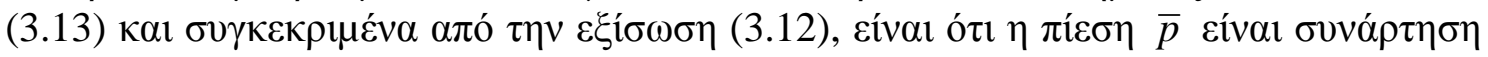

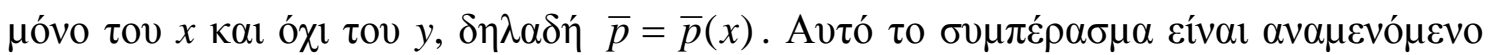

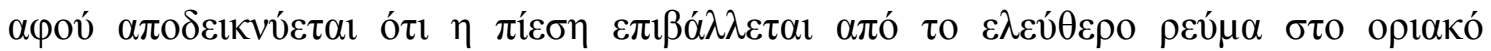
$\sigma \tau \rho \omega ́ \mu \alpha$.

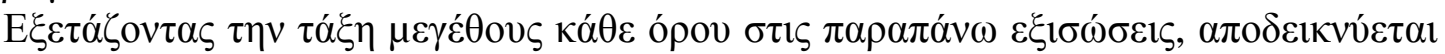

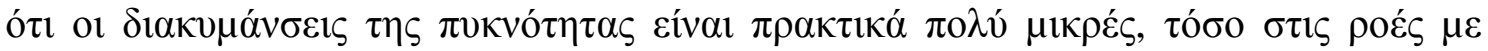

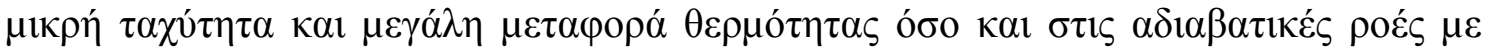

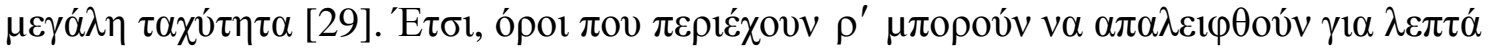

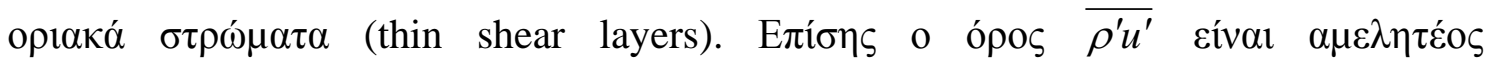

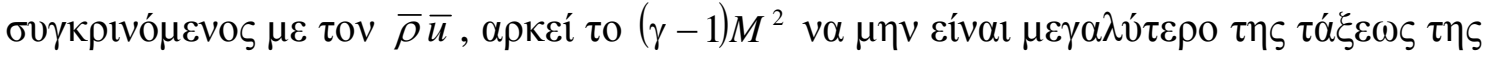

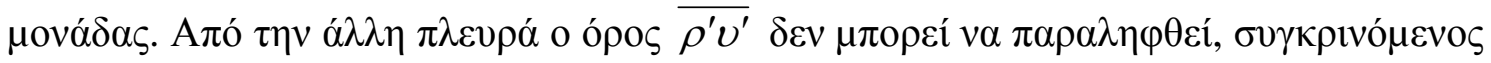

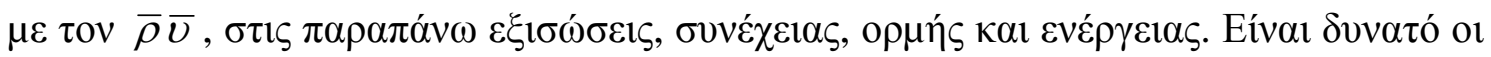

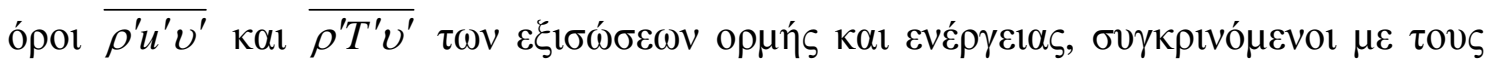

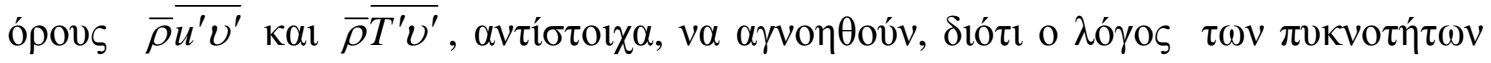

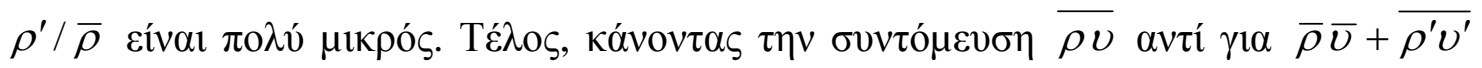

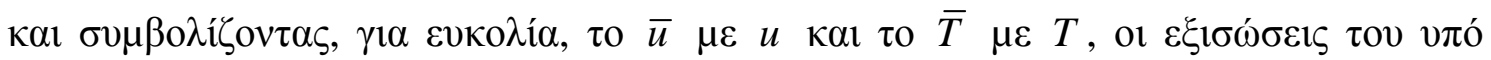

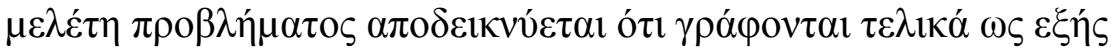

$$
\begin{gathered}
\frac{\partial}{\partial x}(\rho u)+\frac{\partial}{\partial y}(\overline{\rho v})=0 \\
\rho u \frac{\partial u}{\partial x}+\overline{\rho v} \frac{\partial u}{\partial y}=-\frac{d \bar{p}}{d x}+\frac{\partial}{\partial y}\left[\mu \frac{\partial u}{\partial y}-\rho \overline{u^{\prime} v^{\prime}}\right]-\sigma B_{0}^{2} u \\
\rho u \frac{\partial H}{\partial x}+\overline{\rho v} \frac{\partial H}{\partial y}=\frac{\partial}{\partial y}\left[k \frac{\partial T}{\partial y}-c_{p} \rho \overline{T^{\prime} v^{\prime}}+u\left(\mu \frac{\partial u}{\partial y}-\rho \overline{u^{\prime} v^{\prime}}\right)\right] .
\end{gathered}
$$

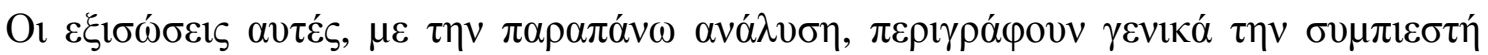

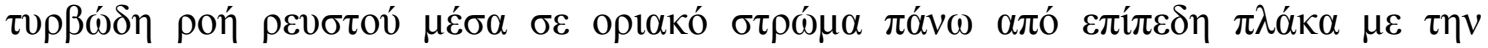

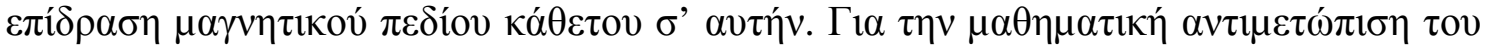

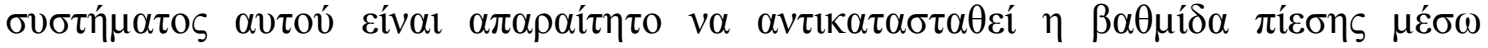

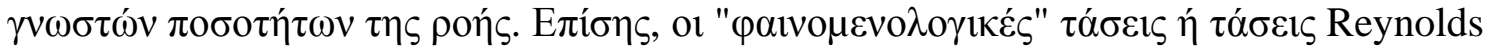

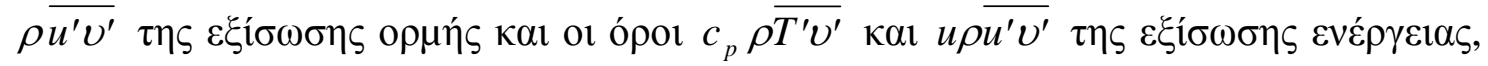

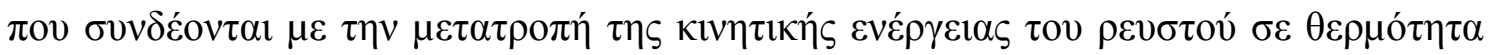

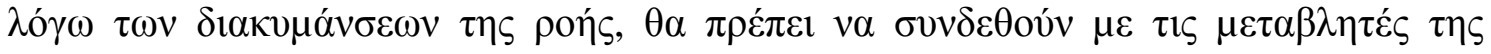

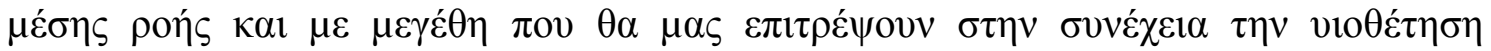

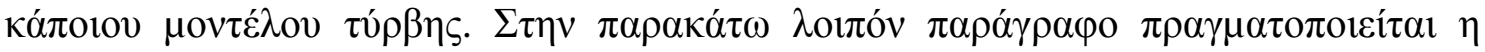

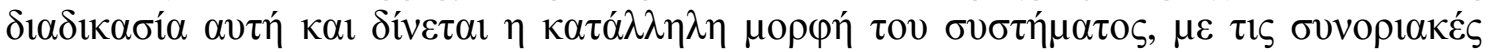

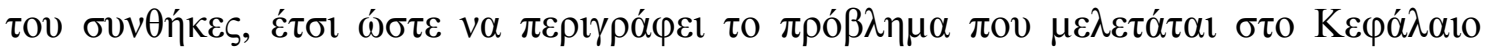

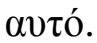




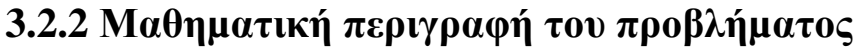

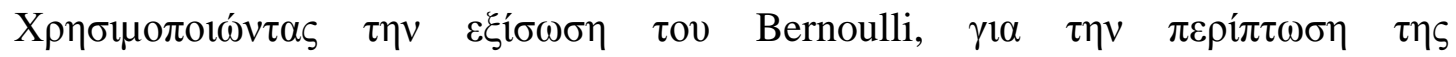

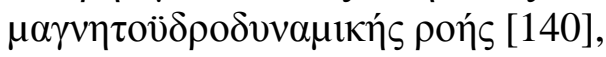

$$
-\frac{d p}{d x}=\rho_{e} u_{e} \frac{d u_{e}}{d x}+\sigma B_{0}^{2} u_{e}
$$

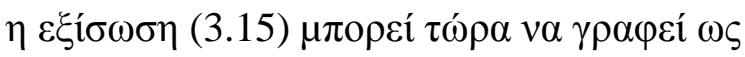

$$
\rho u \frac{\partial u}{\partial x}+\overline{\rho v} \frac{\partial u}{\partial y}=\rho_{e} u_{e} \frac{d u_{e}}{d x}+\sigma B_{0}^{2} u_{e}+\frac{\partial}{\partial y}\left[\mu \frac{\partial u}{\partial y}-\rho \overline{u^{\prime} v^{\prime}}\right]-\sigma B_{0}^{2} u \text {. }
$$

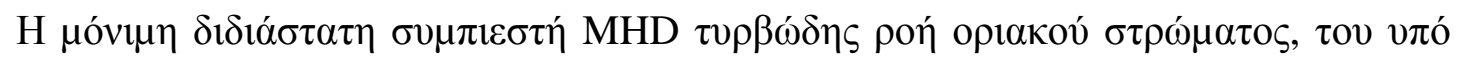

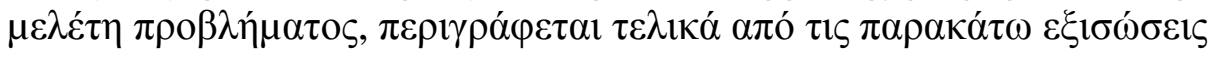

$$
\begin{gathered}
\frac{\partial}{\partial x}(\rho u)+\frac{\partial}{\partial y}(\overline{\rho v})=0 \\
\rho u \frac{\partial u}{\partial x}+\overline{\rho v} \frac{\partial u}{\partial y}=\rho_{e} u_{e} \frac{d u_{e}}{d x}+\frac{\partial}{\partial y}\left[\mu \frac{\partial u}{\partial y}-\rho \overline{u^{\prime} v^{\prime}}\right]-\sigma B_{0}^{2}\left(u-u_{e}\right) \\
\rho u \frac{\partial H}{\partial x}+\overline{\rho v} \frac{\partial H}{\partial y}=\frac{\partial}{\partial y}\left[k \frac{\partial T}{\partial y}-c_{p} \rho \overline{T v^{\prime}}+u\left(\mu \frac{\partial u}{\partial y}-\rho \overline{u^{\prime} v^{\prime}}\right)\right] .
\end{gathered}
$$

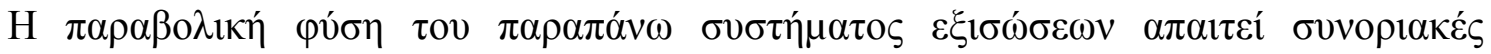

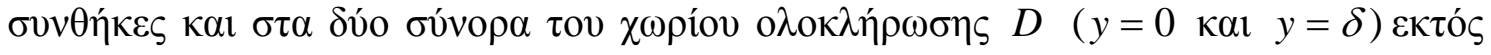

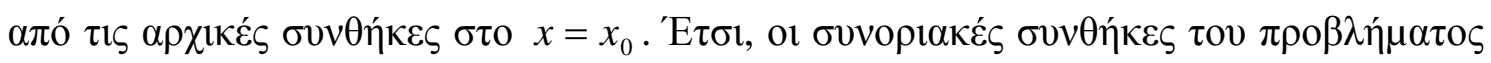

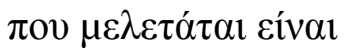

$$
\begin{gathered}
y=0: u=0, v=v_{w}(x), H=H_{w}(x), \\
y=\delta: u=u_{e}(x), H=H_{e}(x),
\end{gathered}
$$

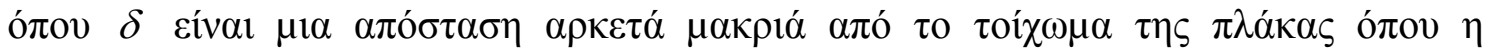

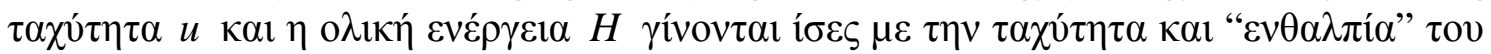

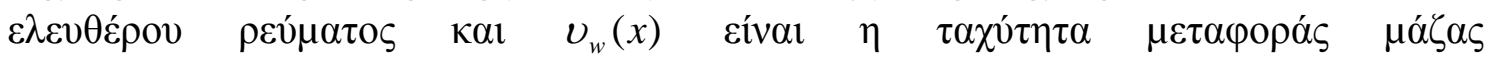

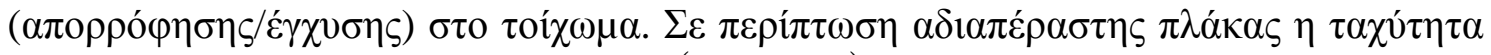

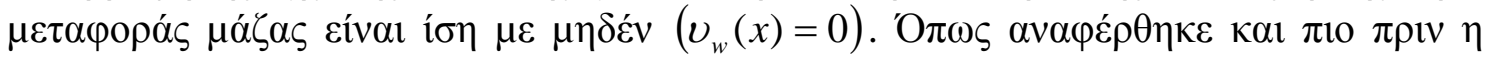

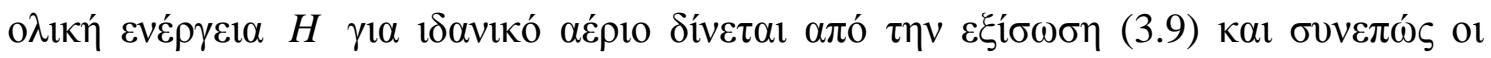

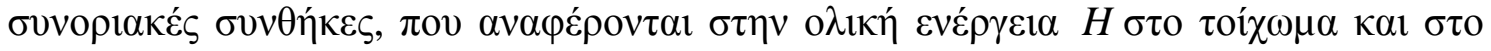

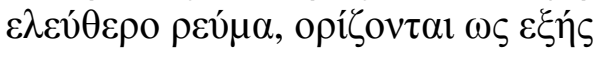

$$
y=0: H=H_{w}(x)=c_{p} T_{w}(x), \quad y=\delta: H=H_{e}(x)=c_{p} T_{e}(x)+\frac{1}{2} u_{e}^{2}(x) .
$$




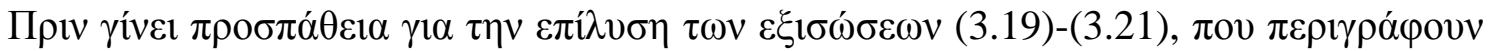

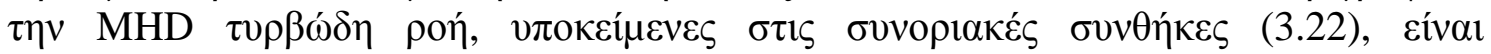

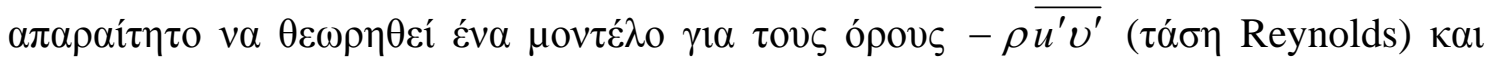

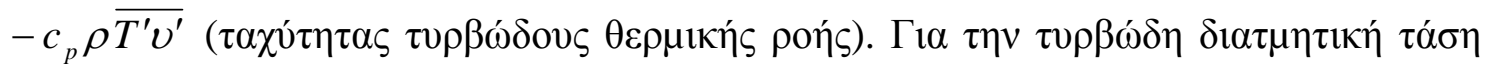

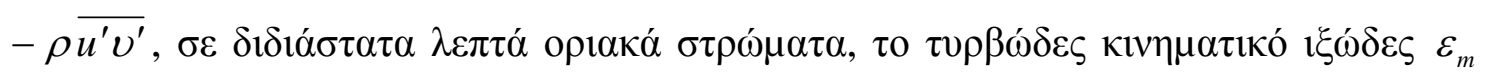

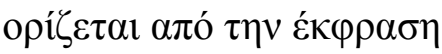

$$
\varepsilon_{m}=\frac{-\overline{u^{\prime} v^{\prime}}}{\frac{\partial u}{\partial y}}
$$

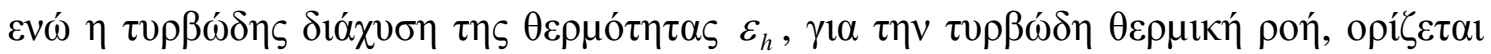
$\alpha \pi$ ó $\tau \eta \nu \sigma \chi \varepsilon ́ \sigma \eta$

$$
\varepsilon_{h}=\frac{-\overline{T^{\prime} v^{\prime}}}{\frac{\partial T}{\partial y}}
$$

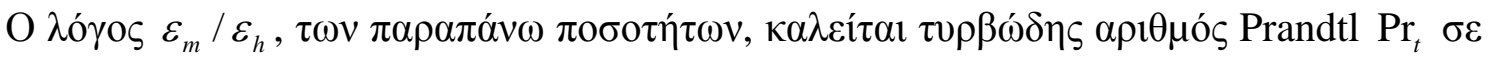

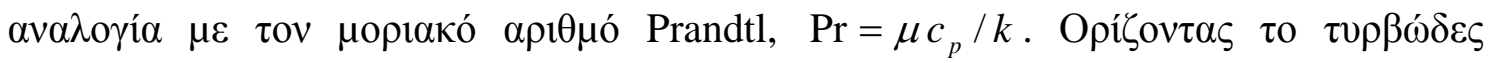

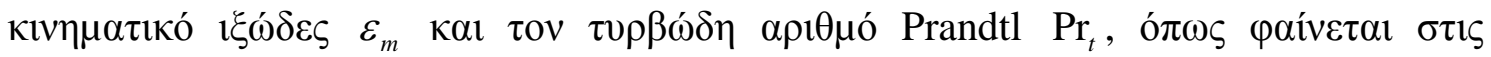
$\pi \alpha \rho \alpha \kappa \alpha ́ \tau \omega ~ \varepsilon \kappa \varphi \rho \alpha ́ \sigma \varepsilon 1 \varsigma$

$$
-\overline{u^{\prime} v^{\prime}}=\varepsilon_{m} \frac{\partial u}{\partial y}, \quad-\overline{T^{\prime} v^{\prime}}=\frac{\varepsilon_{m}}{\operatorname{Pr}_{t}} \frac{\partial T}{\partial y}
$$

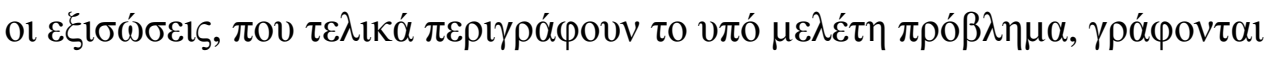

$$
\begin{gathered}
\frac{\partial}{\partial x}(\rho u)+\frac{\partial}{\partial y}(\overline{\rho v})=0 \\
\rho u \frac{\partial u}{\partial x}+\overline{\rho v} \frac{\partial u}{\partial y}=\rho_{e} u_{e} \frac{d u_{e}}{d x}+\frac{\partial}{\partial y}\left[\left(\mu+\rho \varepsilon_{m}\right) \frac{\partial u}{\partial y}\right]-\sigma B_{0}^{2}\left(u-u_{e}\right), \\
\rho u \frac{\partial H}{\partial x}+\overline{\rho v} \frac{\partial H}{\partial y}=\frac{\partial}{\partial y}\left\{\left(\frac{\mu}{\operatorname{Pr}}+\rho \frac{\varepsilon_{m}}{\operatorname{Pr}_{t}}\right) \frac{\partial H}{\partial y}+\left[\mu\left(1-\frac{1}{\operatorname{Pr}}\right)+\rho \varepsilon_{m}\left(1-\frac{1}{\operatorname{Pr}_{t}}\right)\right] u \frac{\partial u}{\partial y}\right\},
\end{gathered}
$$

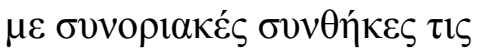

$$
\begin{gathered}
y=0: u=0, v=v_{w}(x), H=H_{w}(x), \\
y=\delta: u=u_{e}(x), H=H_{e}(x) .
\end{gathered}
$$




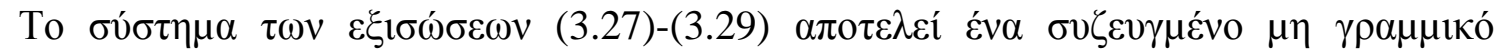

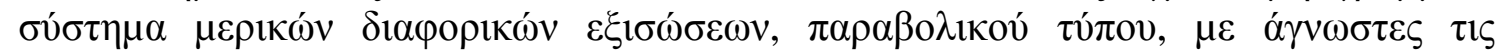

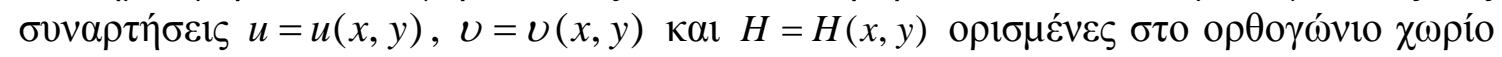

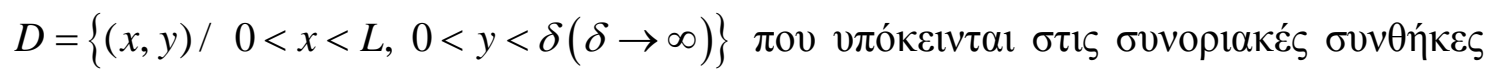

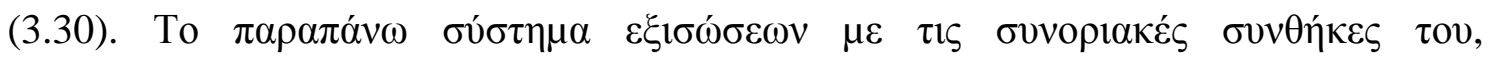

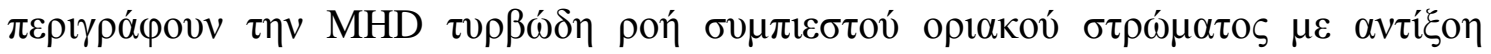

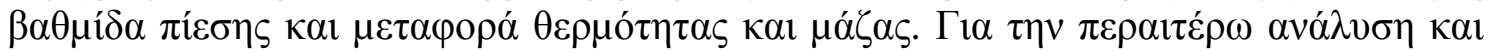

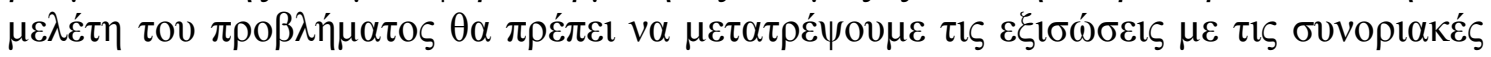

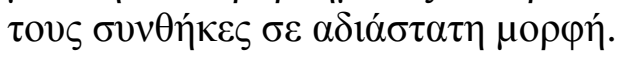

\subsection{3 А}

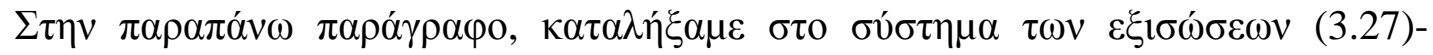

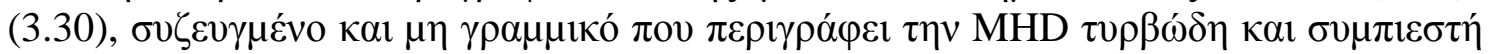

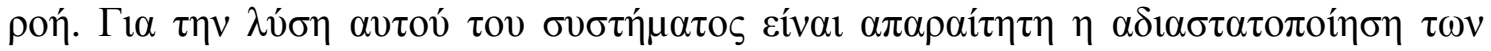

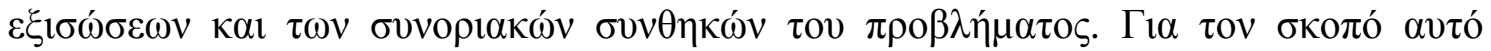

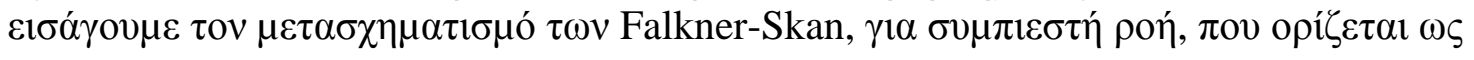

$$
\begin{gathered}
\eta(x, y)=\int_{0}^{y}\left(\frac{u_{e}(x)}{v_{e}(x) x}\right)^{1 / 2} \frac{\rho(x, y)}{\rho_{e}(x)} d y \\
\psi(x, y)=\left(\rho_{e} \mu_{e} u_{e} x\right)^{1 / 2} f(x, \eta)
\end{gathered}
$$

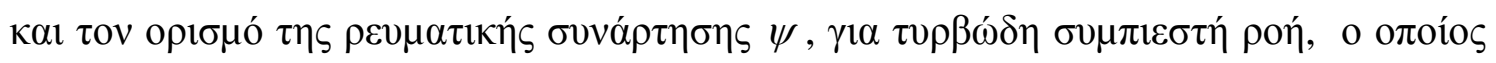

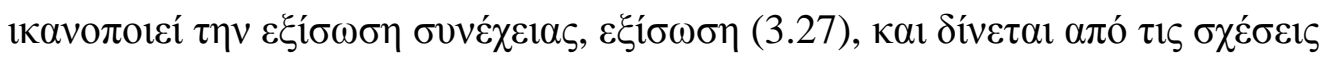

$$
\rho u=\frac{\partial \psi}{\partial y}, \overline{\rho v}=-\frac{\partial \psi}{\partial x}
$$

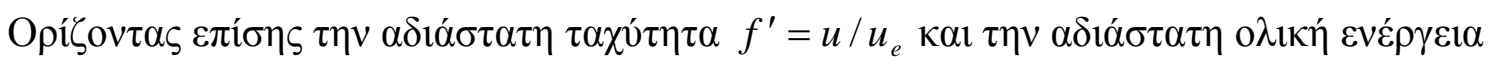

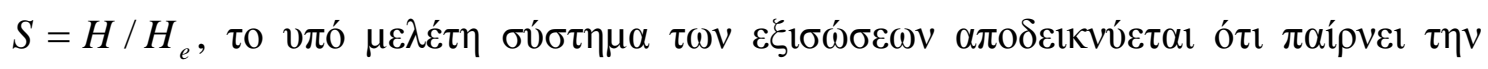
норфи́

$$
\begin{gathered}
\left(b f^{\prime \prime}\right)^{\prime}+m_{1} f f^{\prime \prime}+m_{2}\left[c-\left(f^{\prime}\right)^{2}\right]=x\left\{m_{3}\left(f^{\prime}-1\right)+f^{\prime} \frac{\partial f^{\prime}}{\partial x}-f^{\prime \prime} \frac{\partial f}{\partial x}\right\}, \\
\left(e S^{\prime}+d f^{\prime} f^{\prime \prime}\right)^{\prime}+m_{1} f S^{\prime}=x\left\{f^{\prime} \frac{\partial S}{\partial x}-S^{\prime} \frac{\partial f}{\partial x}\right\}, \\
\eta=0: f^{\prime}=0, f_{w}(x)=f(x, 0)=-\frac{1}{\left(u_{e} \mu_{e} \rho_{e} x\right)^{1 / 2}} \int_{0}^{x} \rho_{w}(x, 0) v_{w}(x) d x, S=S_{w}(x, 0), \\
\eta=\eta_{e}: \quad f^{\prime}=1, \quad S=1,
\end{gathered}
$$




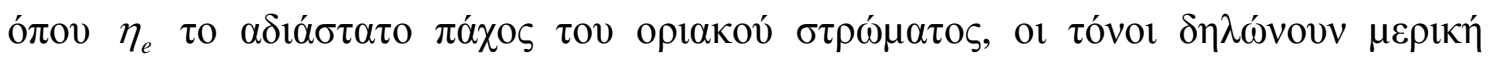

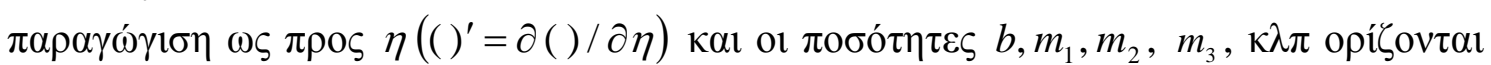
$\omega \varsigma$

$$
\begin{gathered}
b=C\left(1+\varepsilon_{m}^{+}\right), \quad C=\frac{\rho(x, \eta) \mu(x, \eta)}{\rho_{e}(x) \mu_{e}(x)}, \quad c=\frac{\rho_{e}(x)}{\rho(x, \eta)}, \\
d=\frac{C u_{e}^{2}(x)}{H_{e}(x)}\left[1-\frac{1}{\operatorname{Pr}}+\varepsilon_{m}^{+}\left(1-\frac{1}{\operatorname{Pr}_{t}}\right)\right], \quad \varepsilon_{m}^{+}=\frac{\varepsilon_{m}}{v(x, \eta)}, \\
e=\frac{C}{\operatorname{Pr}}\left(1+\varepsilon_{m}^{+} \frac{\operatorname{Pr}}{\operatorname{Pr}_{t}}\right), m_{2}=\frac{x}{u_{e}(x)} \frac{d u_{e}(x)}{d x}, \quad R_{x}=\frac{u_{e}(x) x}{\nu_{e}(x)} \\
m_{1}=\frac{1}{2}\left[1+m_{2}+\frac{x}{\rho_{e}(x) \mu_{e}(x)} \frac{d}{d x}\left(\rho_{e} \mu_{e}\right)\right], \\
m_{3}=\frac{m_{0} c}{\rho_{e} u_{e}}, \quad m_{0}=\sigma B_{0}^{2} .
\end{gathered}
$$

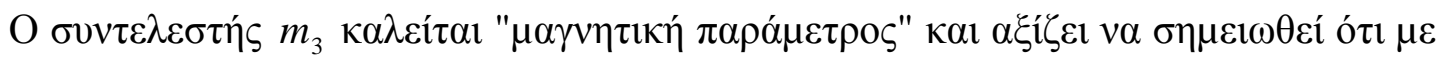

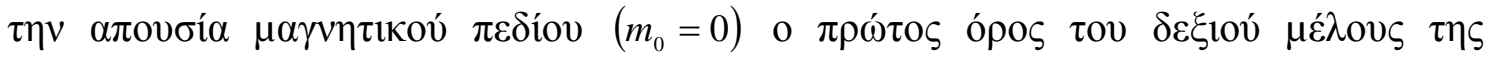

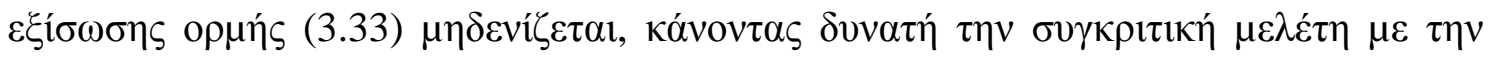

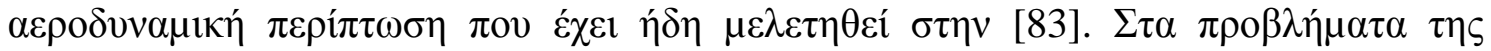

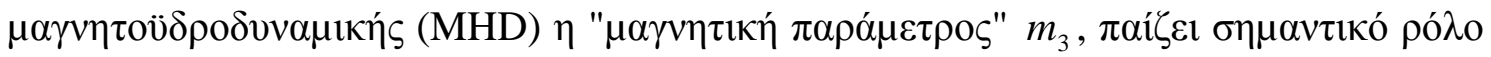

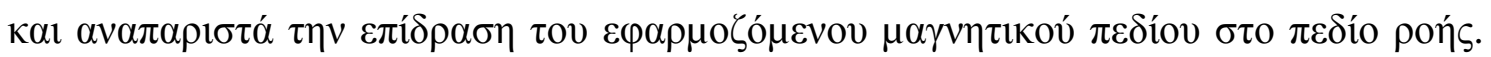

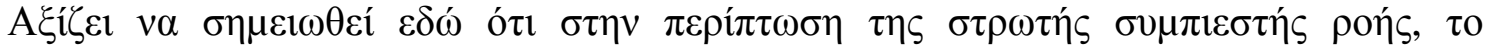

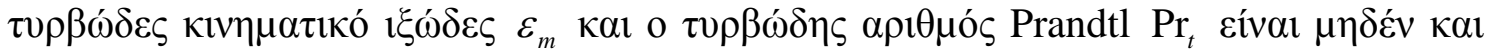

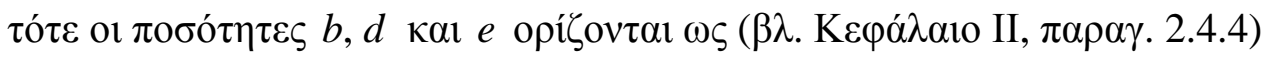

$$
b=C, \quad d=\frac{C u_{e}^{2}(x)}{H_{e}(x)}\left(1-\frac{1}{\operatorname{Pr}}\right) \quad \kappa \alpha \iota \quad e=\frac{b}{\operatorname{Pr}} .
$$

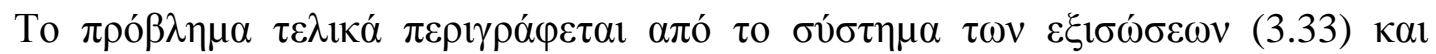

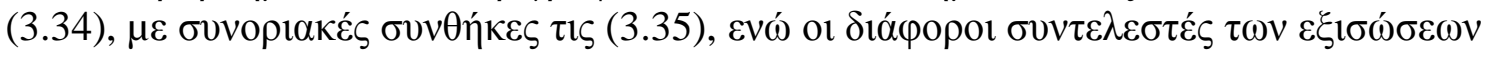

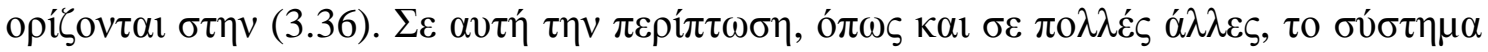

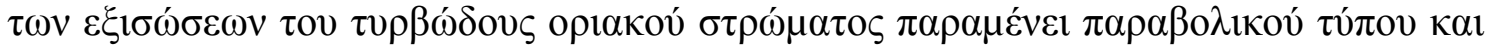

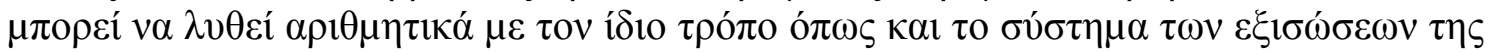

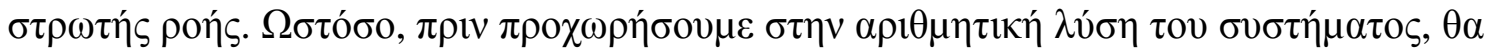

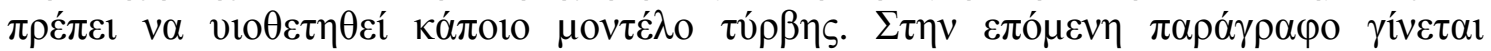

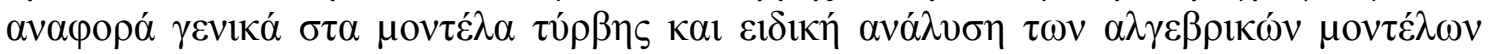

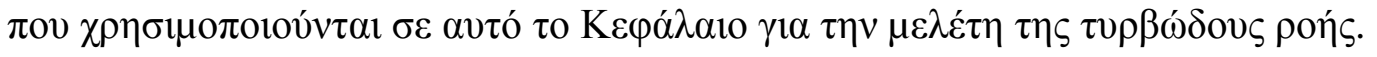




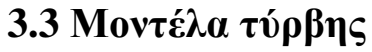

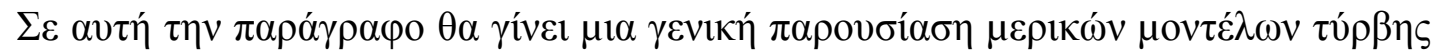

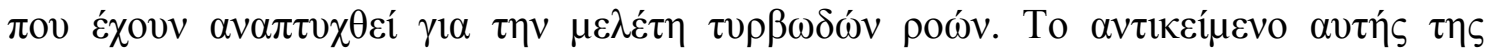

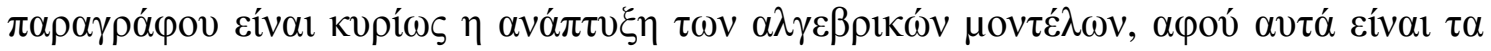

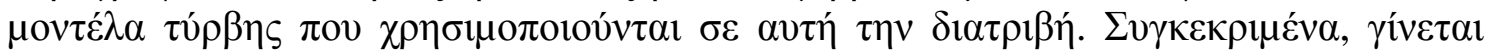

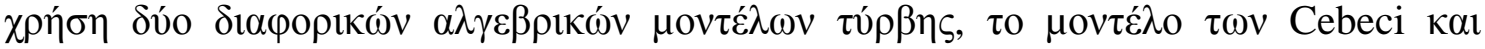

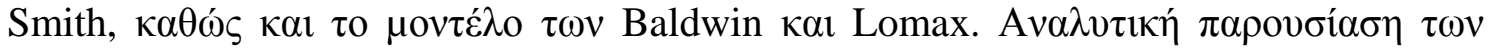

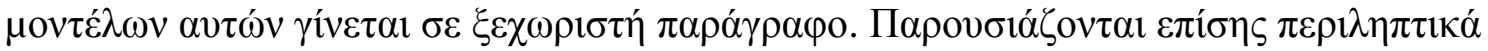

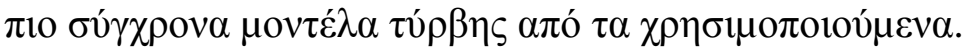

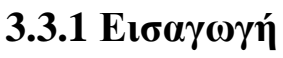

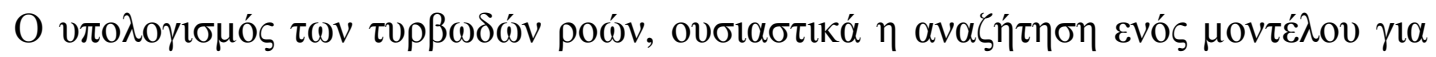

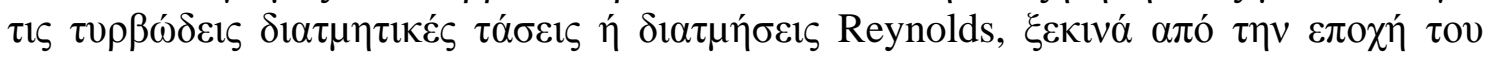

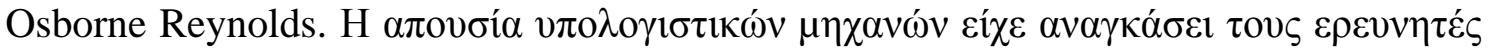

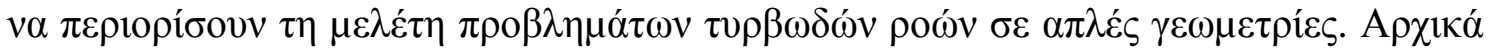

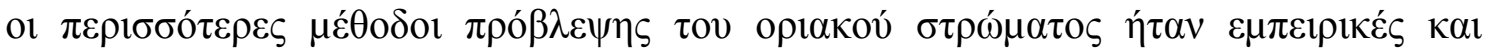

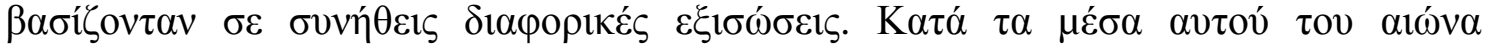

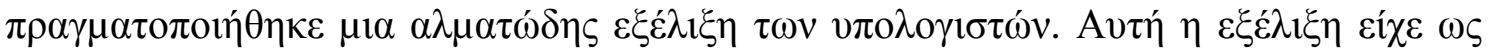

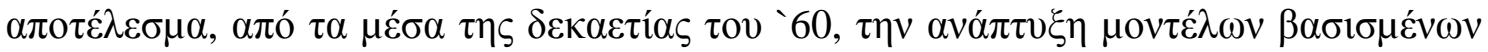

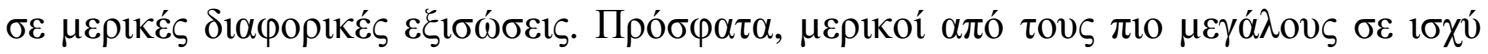

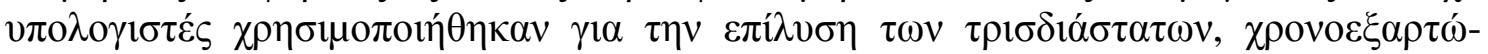

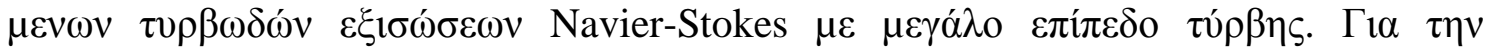

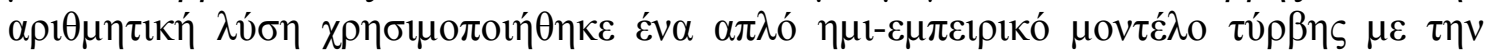

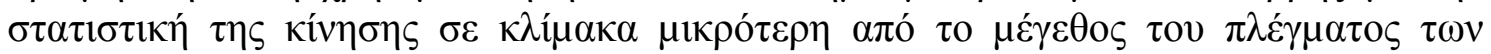

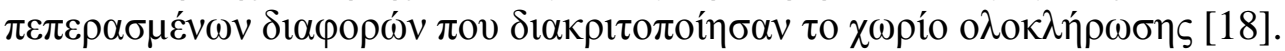

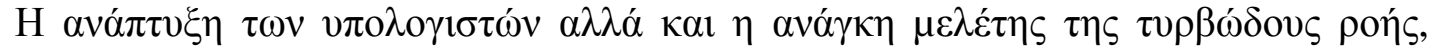

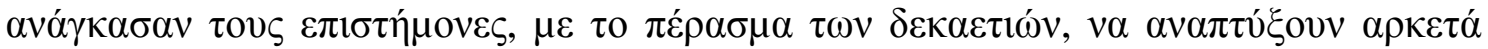

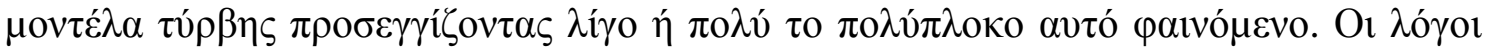

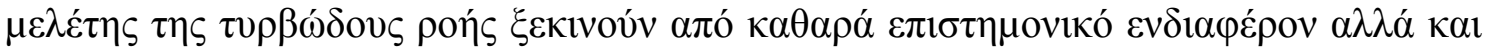

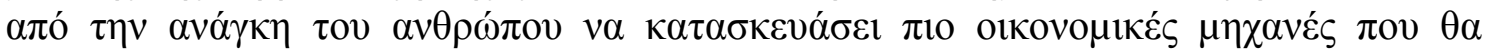

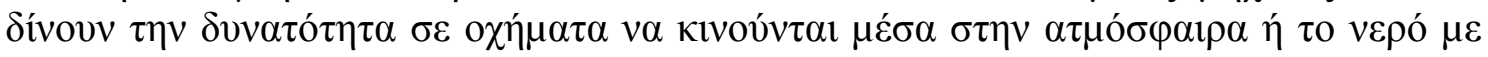

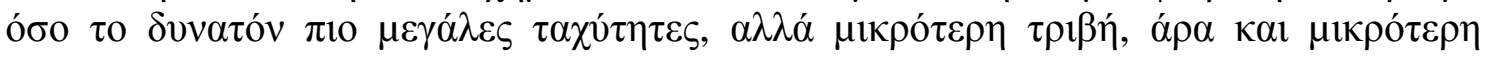

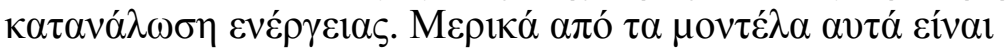

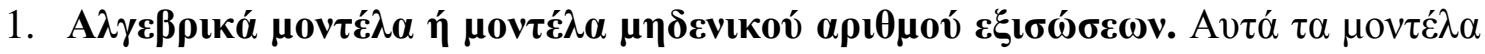

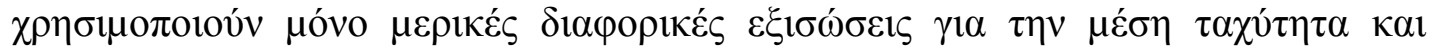

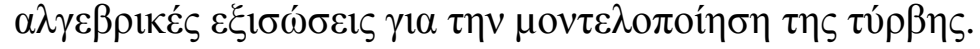

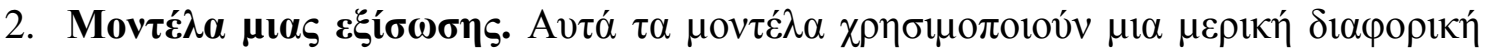

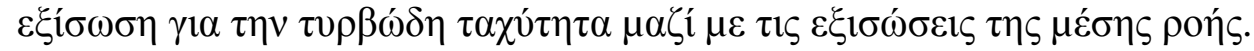

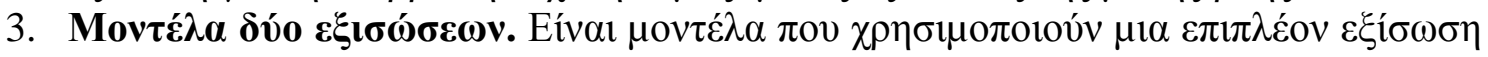

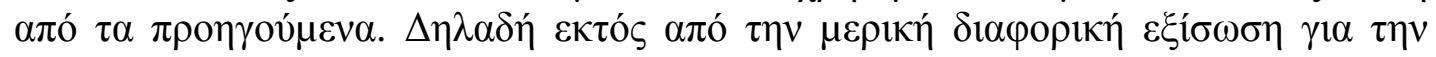

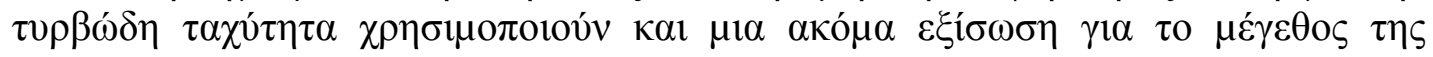
$\tau u ́ \rho \beta \eta$ (turbulence length scale).

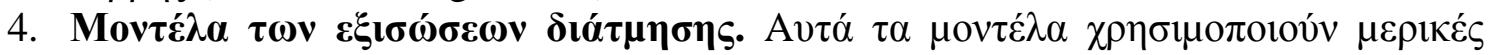

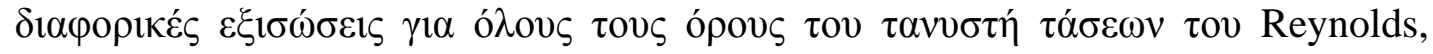

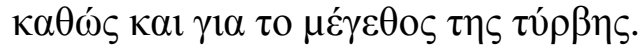




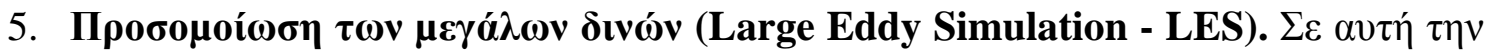

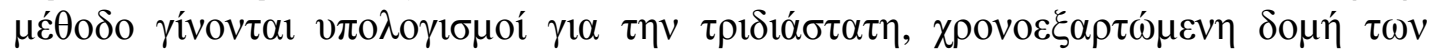

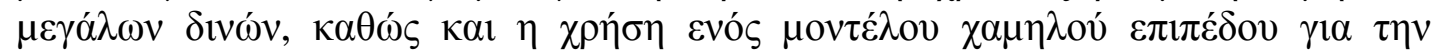

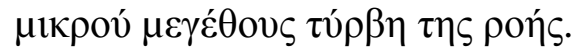

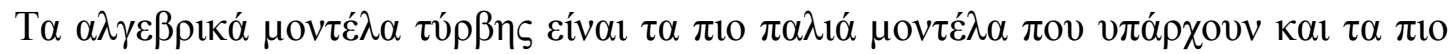

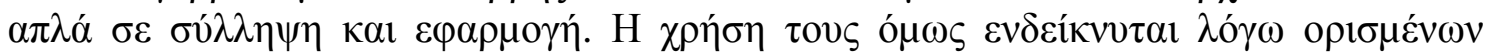

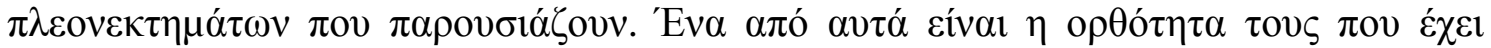

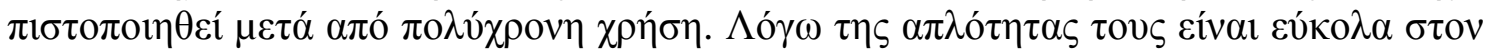

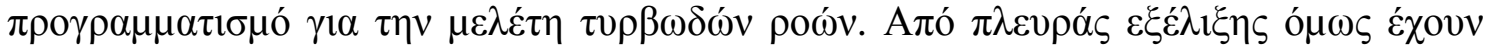

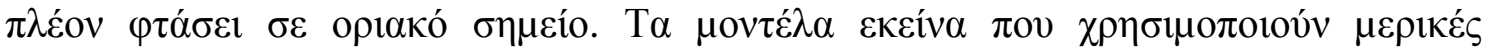

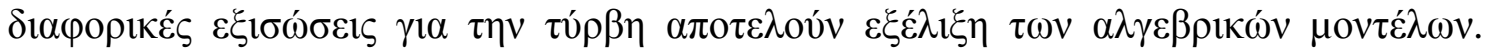

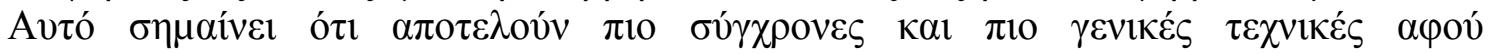

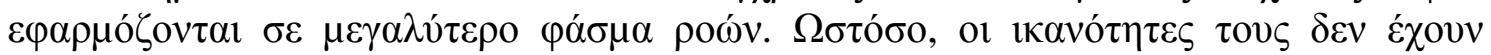

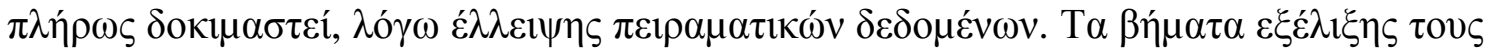

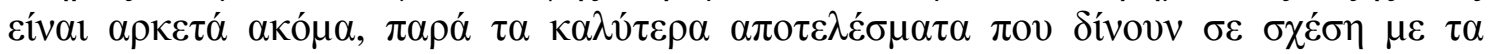

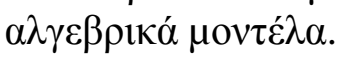

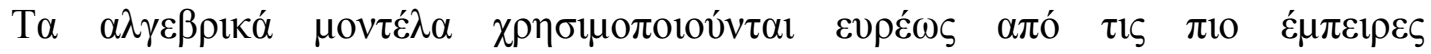

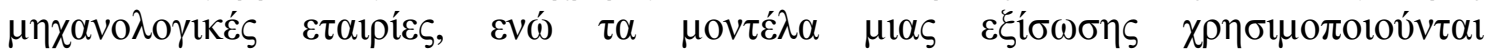

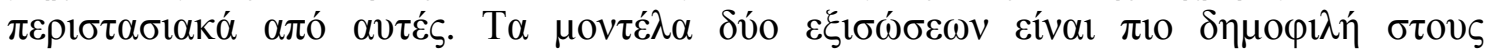

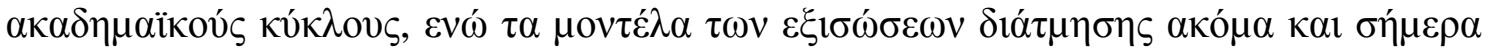

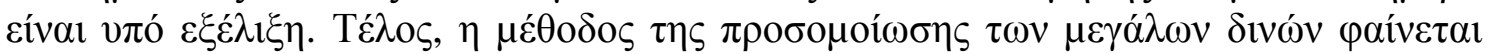

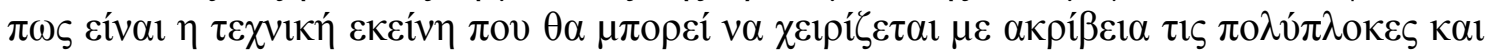

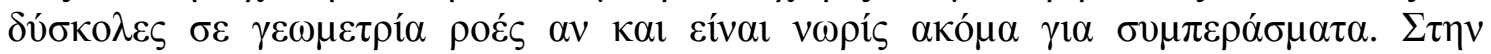

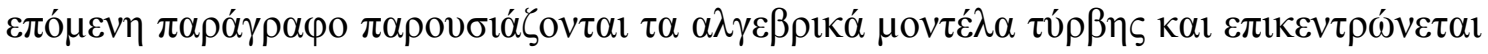

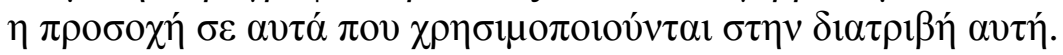

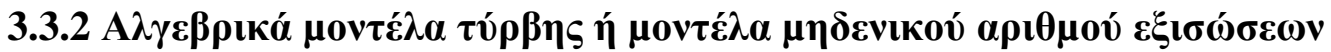

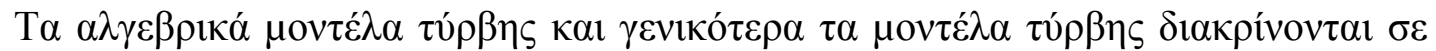

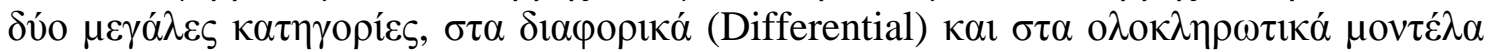

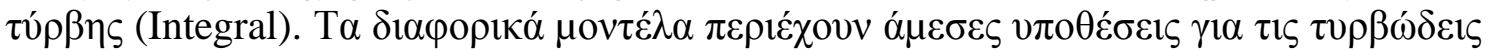

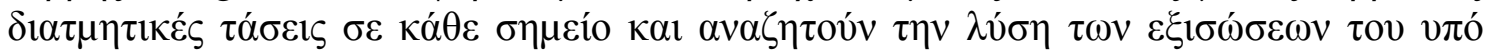

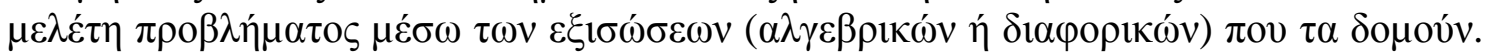

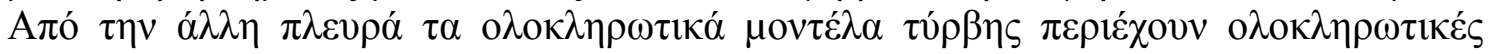

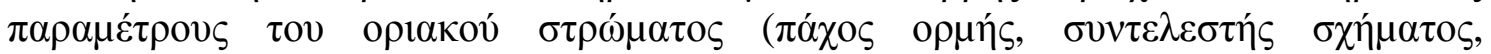

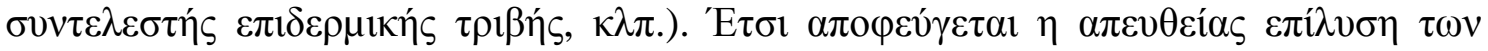

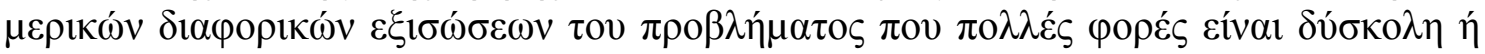

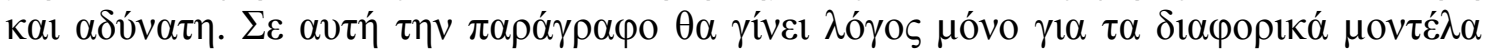

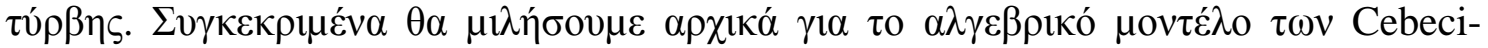

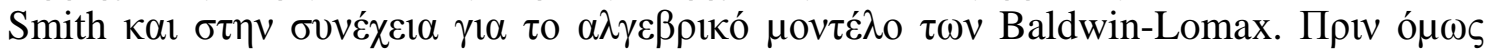

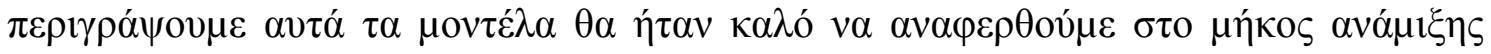
(mixing length).

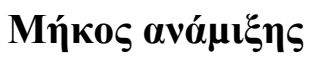

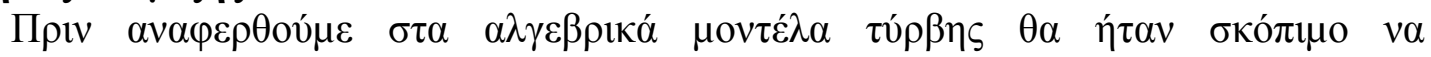

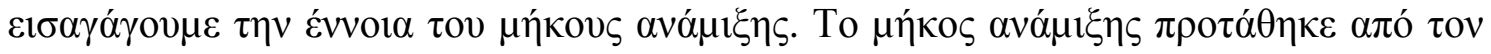

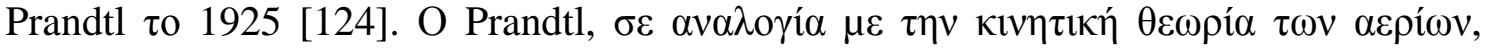




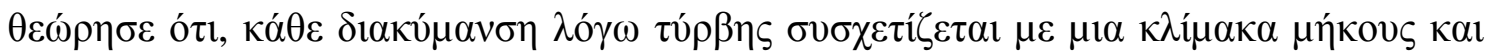

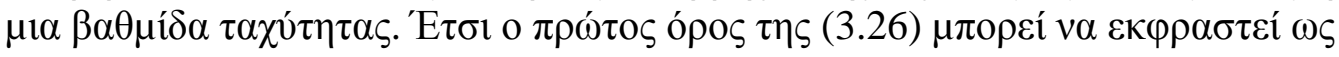

$$
-\overline{u^{\prime} v^{\prime}} \sim C\left(l_{1} \frac{\partial \bar{u}}{\partial y}\right)\left(l_{2} \frac{\partial \bar{u}}{\partial y}\right),
$$

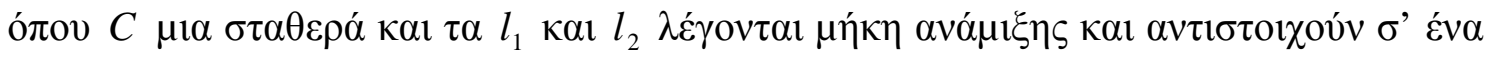

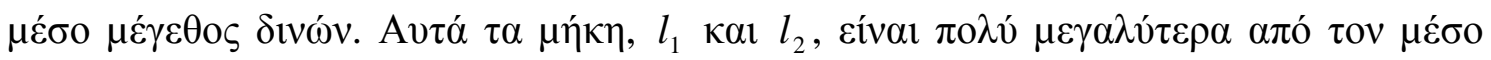

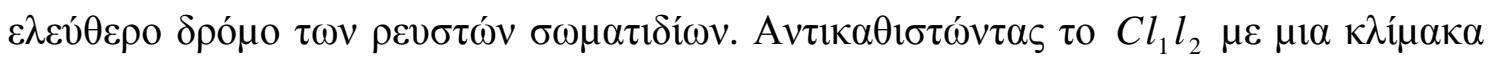

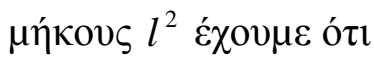

$$
\varepsilon_{m} \sim l^{2}\left|\frac{\partial \bar{u}}{\partial y}\right| .
$$

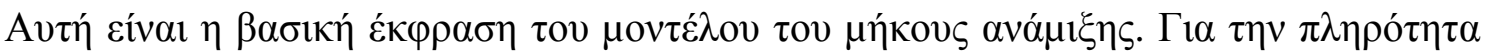

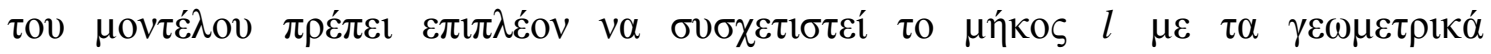

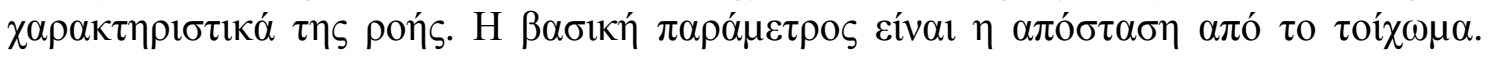

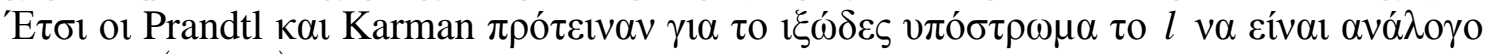

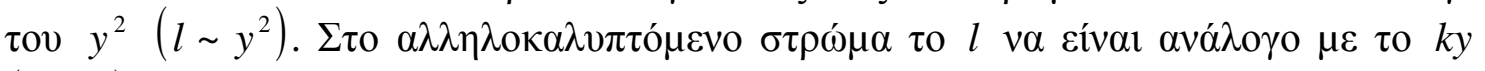

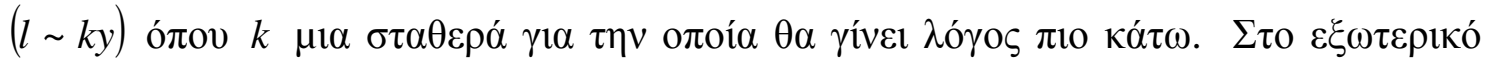

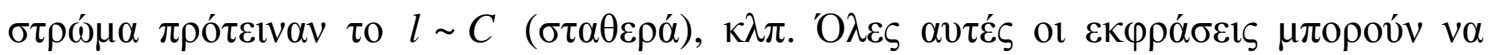

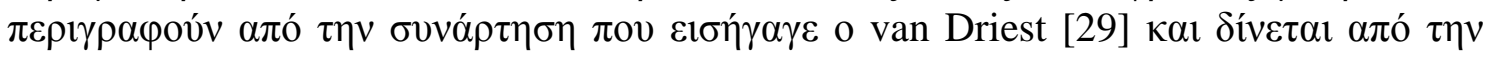
$\sigma \chi \varepsilon \dot{\sigma} \eta$

$$
l \sim k y\left[1-\exp \left(-\frac{y}{A}\right)\right],
$$

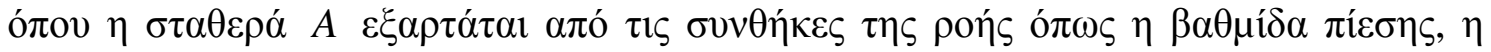

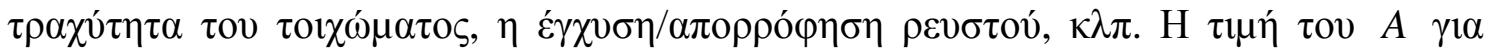

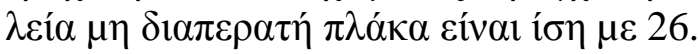

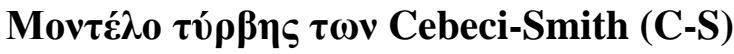

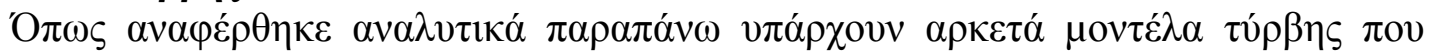

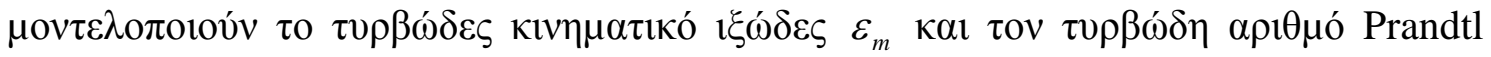

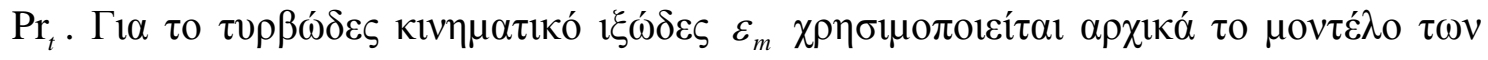

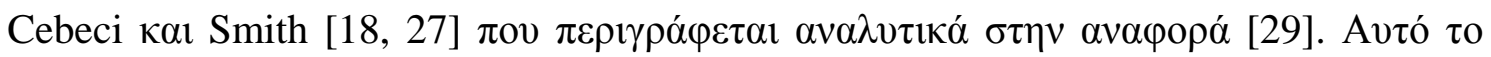

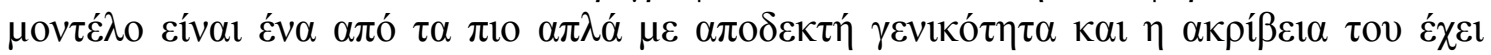

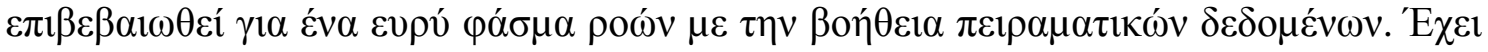

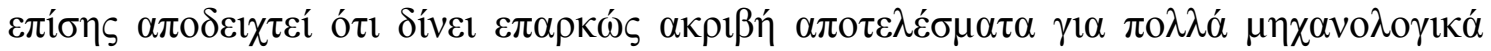
$\pi \rho \circ \beta \lambda \eta \dot{\mu} \alpha \tau \alpha[29,101]$.

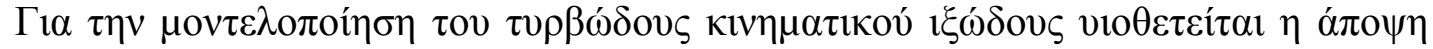

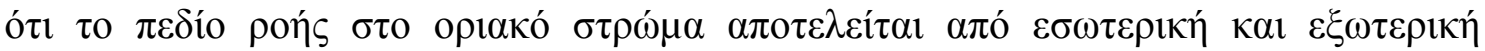

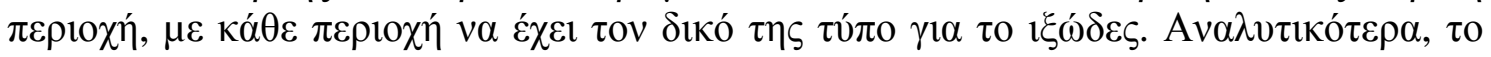

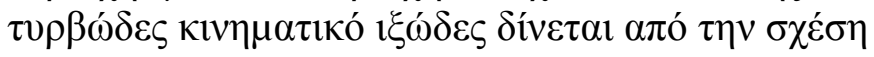




$$
\varepsilon_{m}= \begin{cases}\left(\varepsilon_{m}\right)_{i}, & \left(\varepsilon_{m}\right)_{i} \leq\left(\varepsilon_{m}\right)_{o} \\ \left(\varepsilon_{m}\right)_{o}, & \left(\varepsilon_{m}\right)_{i} \geq\left(\varepsilon_{m}\right)_{o}\end{cases}
$$

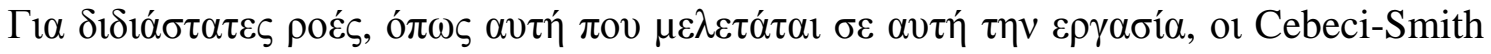

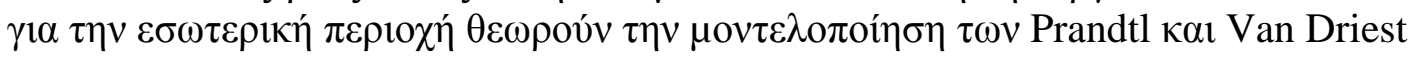

$$
\left(\varepsilon_{m}\right)_{i}=l^{2}\left|\frac{\partial u}{\partial y}\right| \gamma_{t r} \gamma
$$

ó $\pi$ ov

$$
\begin{gathered}
l=k y\left[1-\exp \left(-\frac{y}{A}\right)\right], \\
\mu \varepsilon A=26\left(\frac{\rho}{\rho_{w}}\right)^{1 / 2} \frac{v_{e}}{N} u_{\tau}^{-1}, \quad u_{\tau}=\left(\frac{\tau_{w}}{\rho_{w}}\right)^{1 / 2} .
\end{gathered}
$$

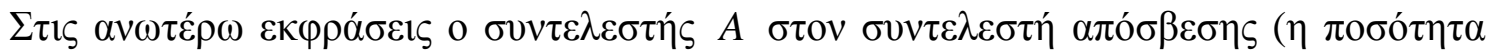

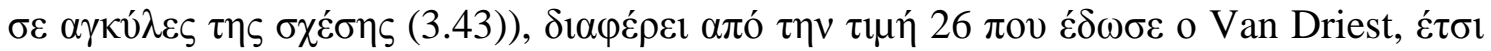

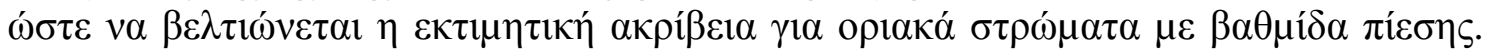

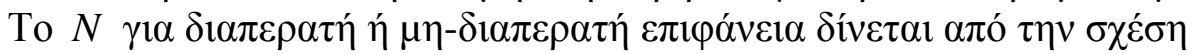

$$
\begin{gathered}
N=\left\{\frac{\mu}{\mu_{e}}\left(\frac{\rho_{e}}{\rho_{w}}\right)^{2}\left(\frac{p^{+}}{v_{w}^{+}}\right)\left[1-\exp \left(11.8 \frac{\mu_{w}}{\mu} v_{w}^{+}\right)\right]+\exp \left(11.8 \frac{\mu_{w}}{\mu} v_{w}^{+}\right)\right\}^{1 / 2}, \\
\text { о́ } \pi \text { ov } p^{+}=\frac{v_{e} u_{e}}{u_{\tau}^{3}} \frac{d u_{e}}{d x} \kappa \alpha v_{w}^{+}=\frac{v_{w}}{u_{\tau}},
\end{gathered}
$$

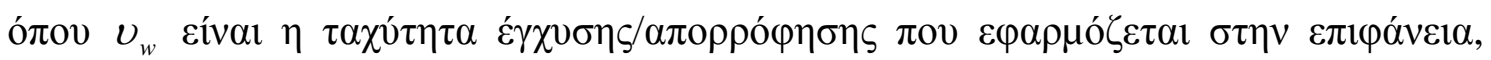

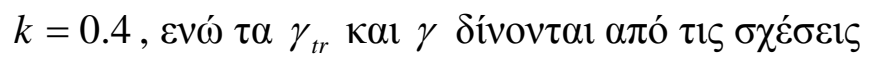

$$
\begin{aligned}
& \gamma_{t r}=1-\exp \left[-G_{t r}\left(x-x_{t r}\right) \int_{x_{t r}}^{x} \frac{d x}{u_{e}}\right], \gamma=\left[1+5.5\left(\frac{y}{\delta}\right)^{6}\right]^{-1}, \alpha v \tau i ́ \sigma \tau o \iota \chi \alpha \\
& \mu \varepsilon \quad G_{t r}=8.33 \times 10^{-4} \frac{u_{e}^{3}}{v_{e}^{2}}\left(R_{x}\right)^{-1.34}, \quad R_{x}=\frac{u_{e} x}{v_{e}} .
\end{aligned}
$$

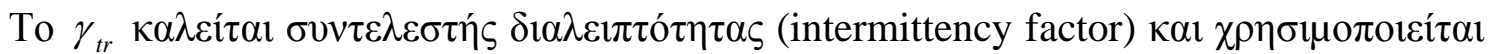

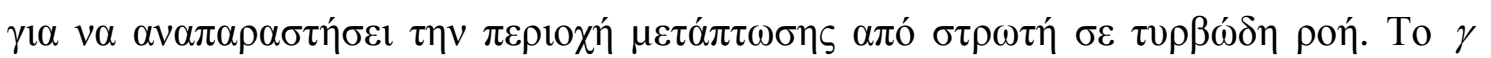

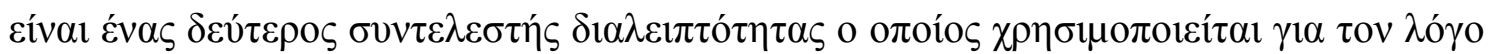

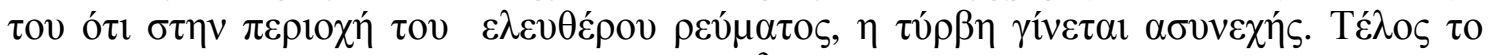

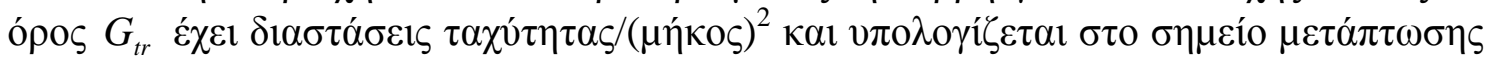

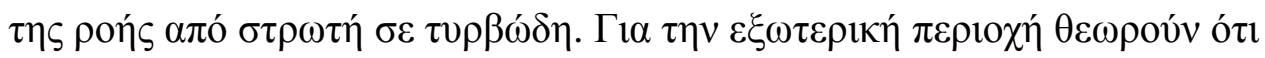




$$
\left(\varepsilon_{m}\right)_{o}=K\left|\int_{0}^{\infty}\left(u_{e}-u\right) d y\right| \gamma_{t r} \gamma
$$

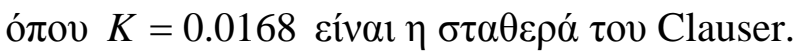

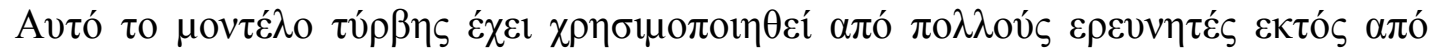

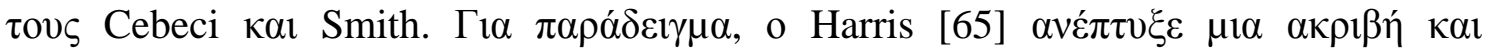

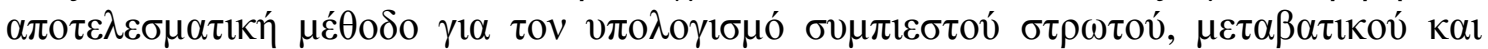

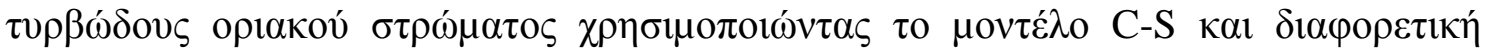

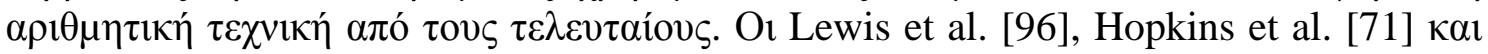

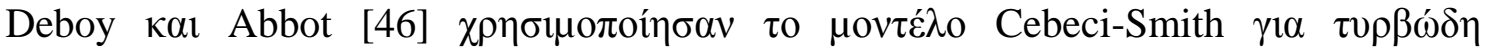

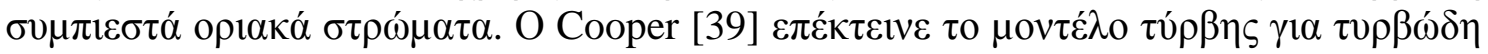

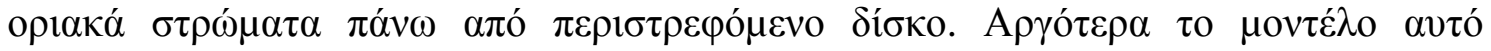

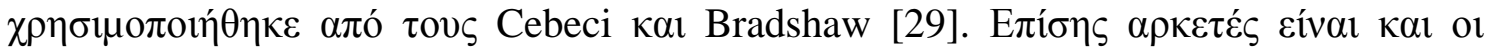

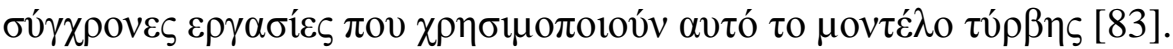

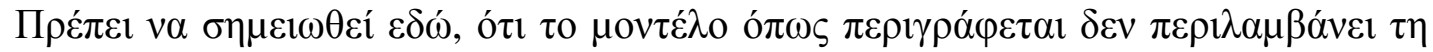

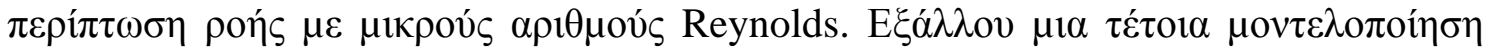

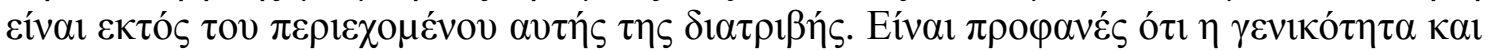

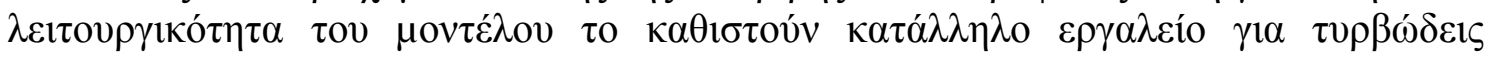

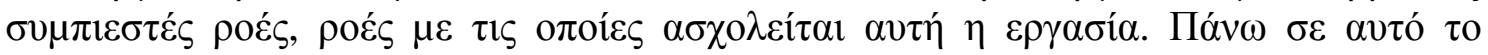

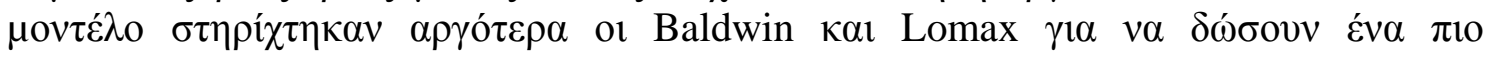

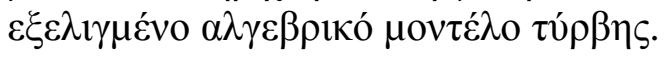

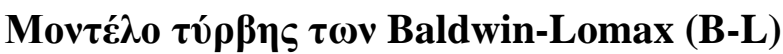

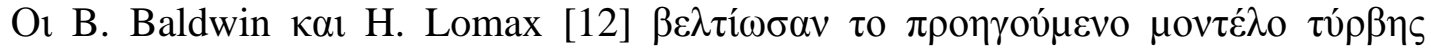

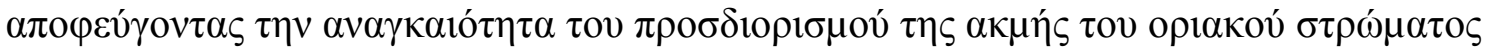

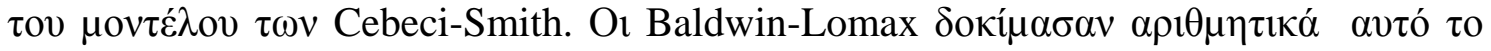

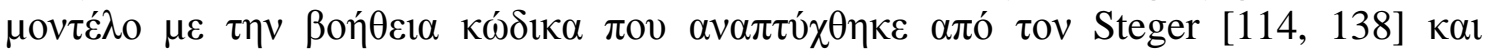

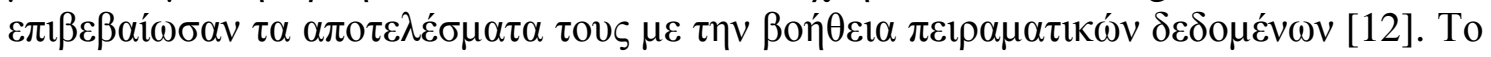

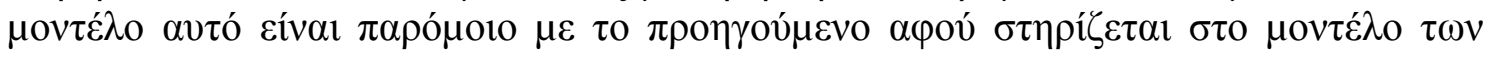
Cebeci-Smith. A

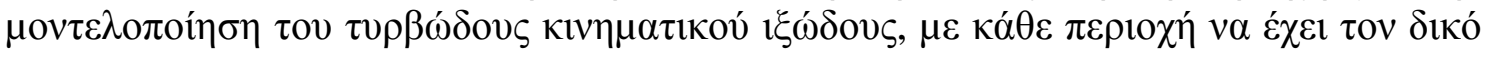

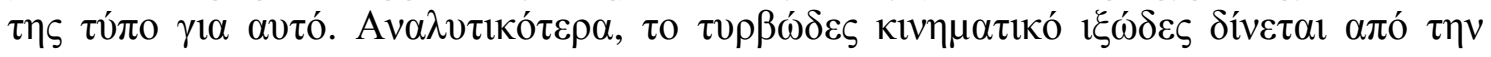
$\sigma \chi \varepsilon \dot{\sigma} \sigma \eta$

$$
\varepsilon_{m}= \begin{cases}\left(\varepsilon_{m}\right)_{i}, & \left(\varepsilon_{m}\right)_{i} \leq\left(\varepsilon_{m}\right)_{o} \\ \left(\varepsilon_{m}\right)_{o}, & \left(\varepsilon_{m}\right)_{i} \geq\left(\varepsilon_{m}\right)_{o}\end{cases}
$$

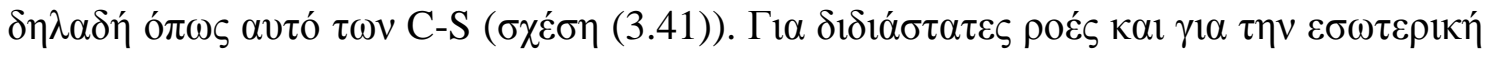

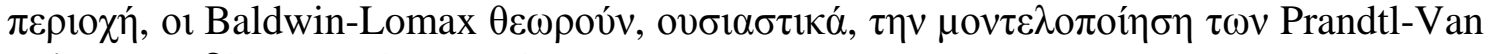

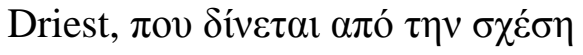

$$
\left(\varepsilon_{m}\right)_{i}=l^{2}|\omega|
$$

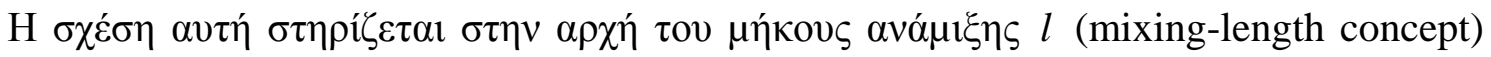

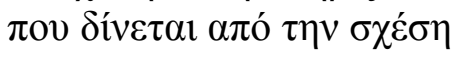




$$
l=k y\left[1-\exp \left(-\frac{y^{+}}{A^{+}}\right)\right]
$$

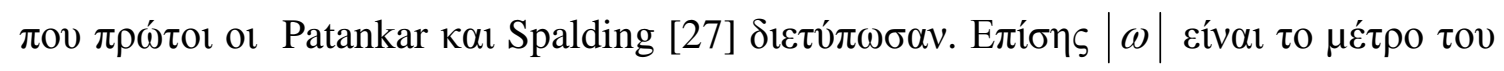

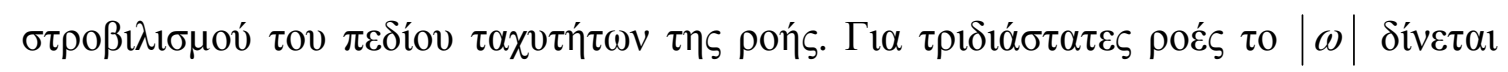
$\alpha \pi$ $\tau \eta v \sigma \chi \varepsilon \dot{\sigma}\rceil$

$$
|\omega|=\sqrt{\left(\frac{\partial u}{\partial y}-\frac{\partial v}{\partial x}\right)^{2}+\left(\frac{\partial v}{\partial z}-\frac{\partial w}{\partial y}\right)^{2}+\left(\frac{\partial w}{\partial x}-\frac{\partial u}{\partial z}\right)^{2}}
$$

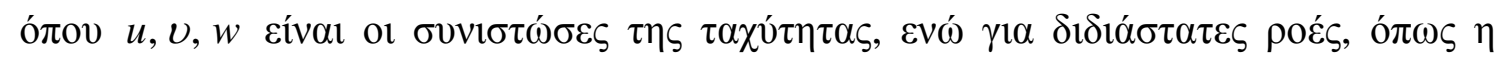

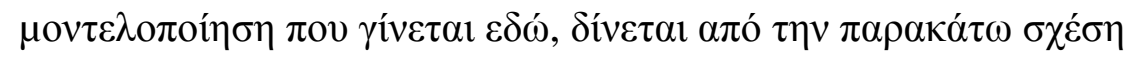

$$
|\omega|=\left|\frac{\partial u}{\partial y}-\frac{\partial v}{\partial x}\right|
$$

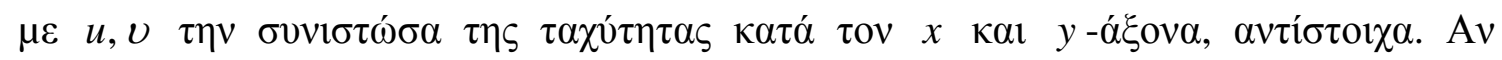

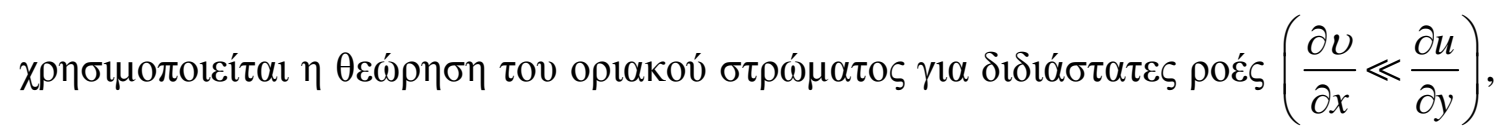

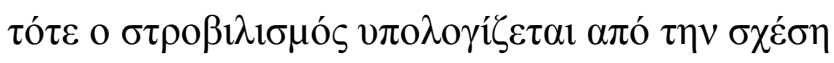

$$
|\omega|=\left|\frac{\partial u}{\partial y}\right|
$$

To $y^{+} \iota \sigma o v ́ \tau \alpha \iota \mu \varepsilon$

$$
y^{+}=\frac{\rho_{w} u_{\tau} y}{\mu_{w}}=\frac{\sqrt{\rho_{w} \tau_{w}} y}{\mu_{w}},
$$

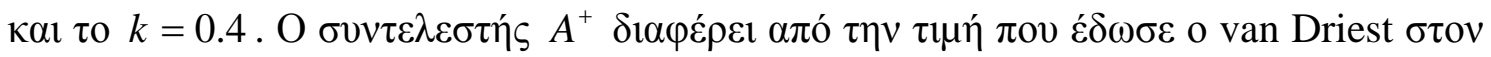

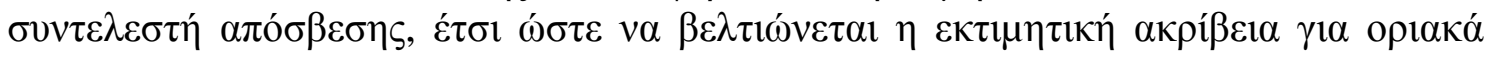

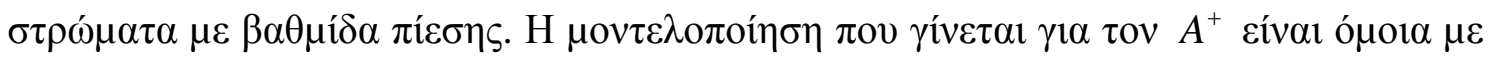

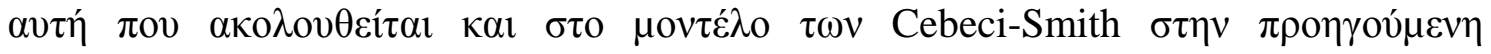

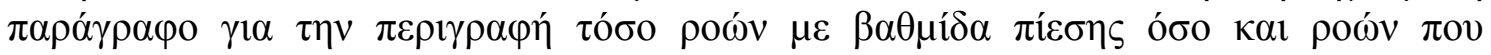

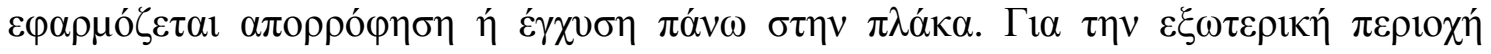

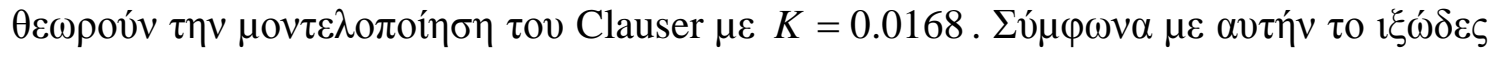

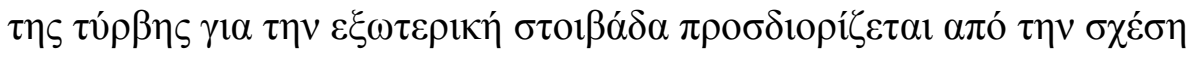

$$
\left(\varepsilon_{m}\right)_{o}=K C_{C P} F_{W A K E} F_{K L E B}(y),
$$

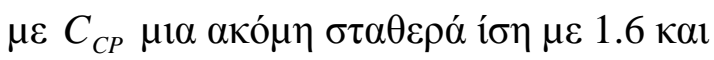




$$
F_{W A K E}=\left\{\begin{array}{c}
y_{M A X} F_{M A X} \\
\dot{\eta} \\
C_{W K} y_{M A X} U_{D I F}^{2} / F_{M A X}
\end{array}\right.
$$

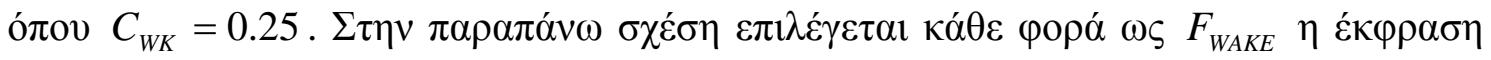

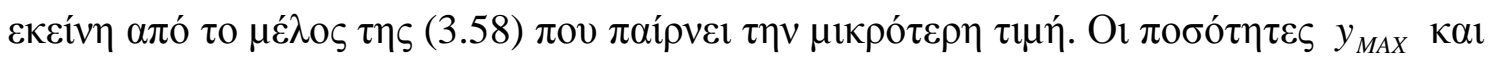

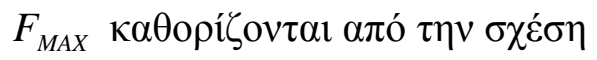

$$
F(y)=y|\omega|\left[1-\exp \left(-\frac{y^{+}}{A^{+}}\right)\right] .
$$

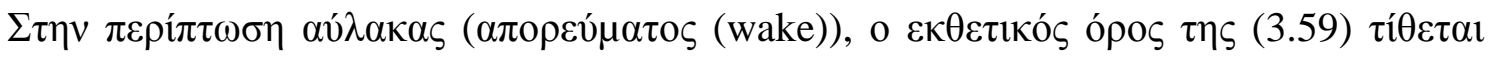

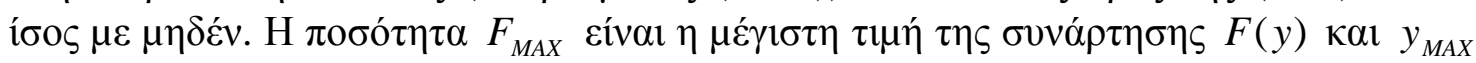

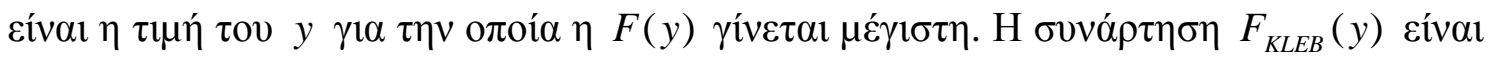

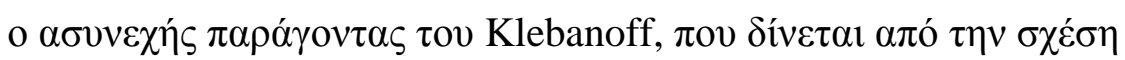

$$
F_{K L E B}(y)=\left[1+5.5\left(\frac{C_{K L E B} y}{y_{M A X}}\right)^{6}\right]^{-1},
$$

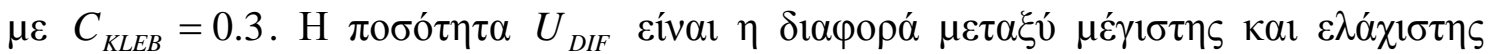

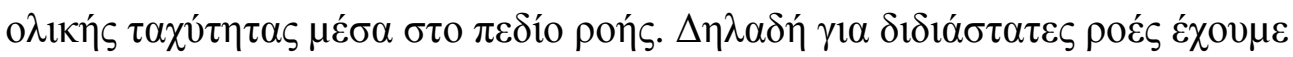

$$
U_{D I F}=\left(\sqrt{u^{2}+v^{2}}\right)_{M A X}-\left(\sqrt{u^{2}+v^{2}}\right)_{M I N} \text {. }
$$

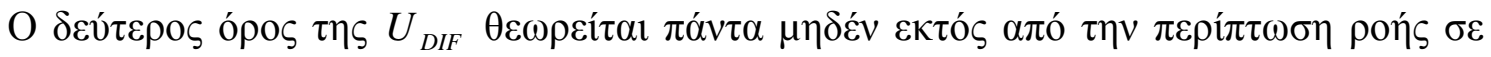
$\alpha v ́ \lambda \alpha \kappa \alpha$.

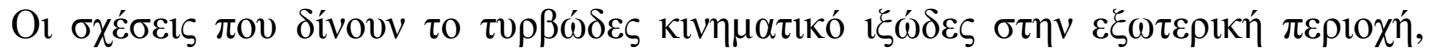

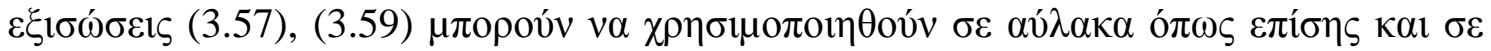

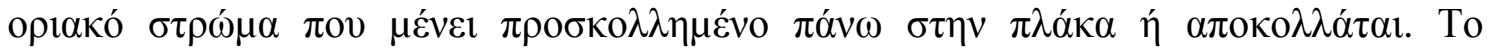

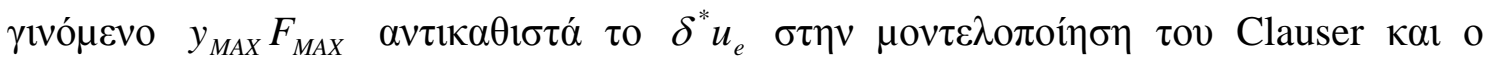

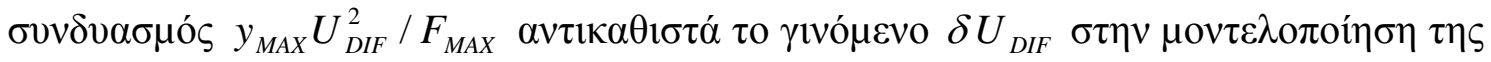

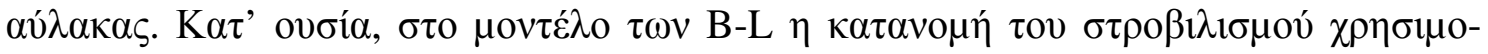

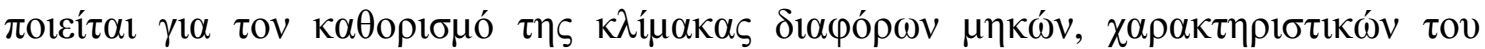

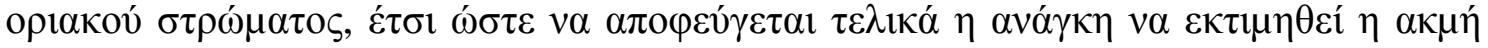

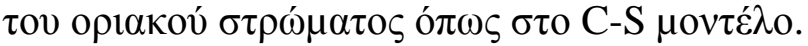

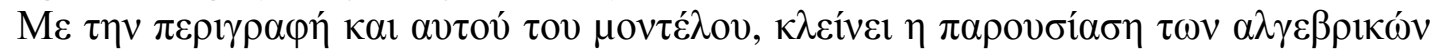

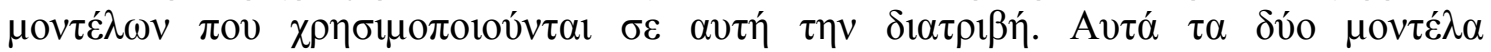

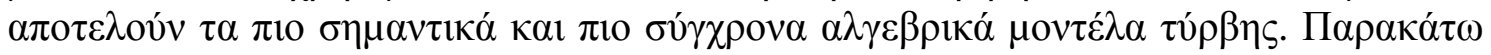

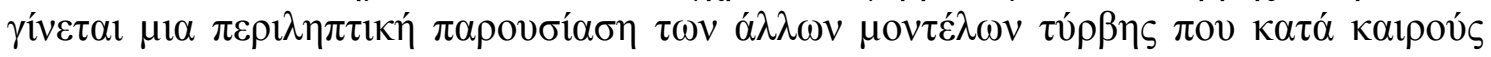

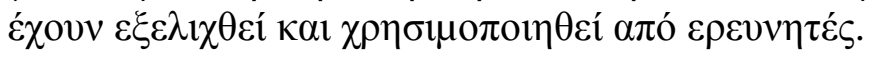




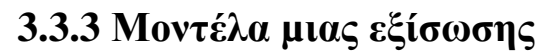

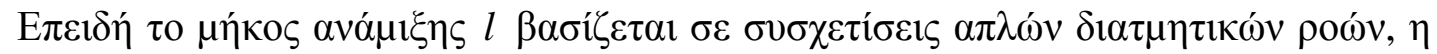

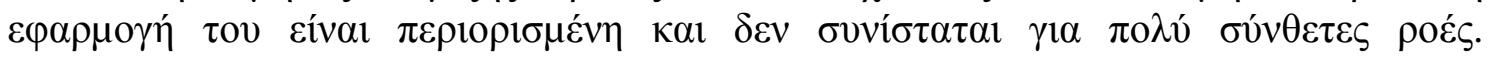

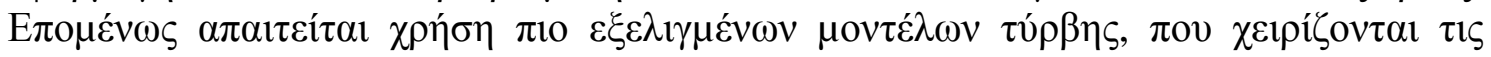

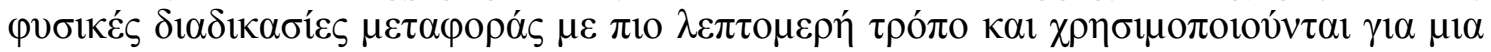

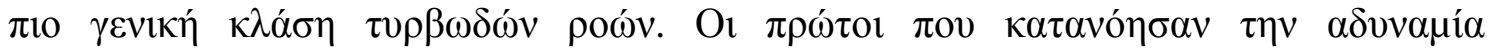

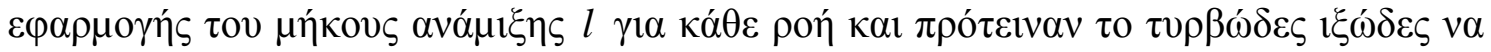

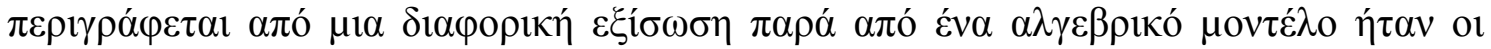

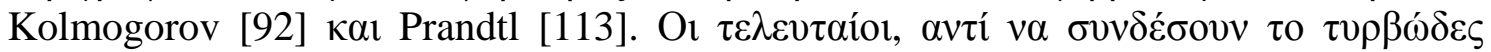

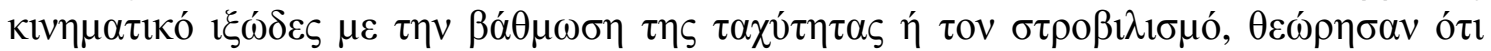

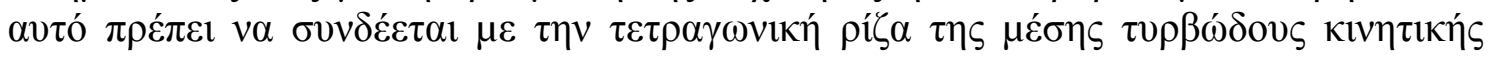

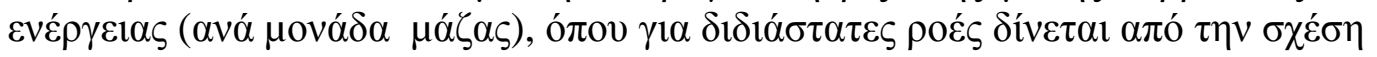

$$
\sqrt{k}=\sqrt{\frac{1}{2}\left({\overline{u^{\prime}}}^{2}+{\overline{v^{\prime}}}^{2}\right)} .
$$

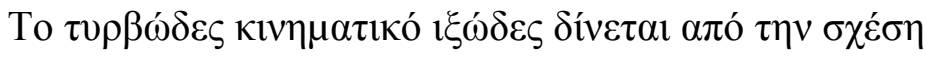

$$
\varepsilon_{m}=C_{\mu} \sqrt{k} l
$$

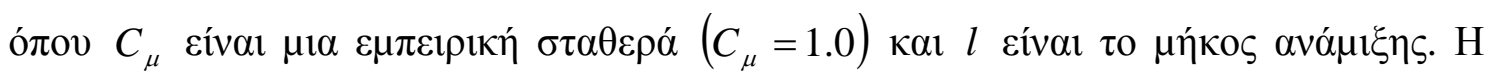


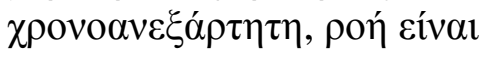

$$
u \frac{\partial k}{\partial x}+v \frac{\partial k}{\partial y}=\frac{\partial}{\partial y}\left[\left(\frac{\varepsilon_{m}}{\sigma_{k}}\right) \frac{\partial k}{\partial y}\right]+\varepsilon_{m}\left(\frac{\partial u}{\partial y}\right)^{2}-\varepsilon
$$

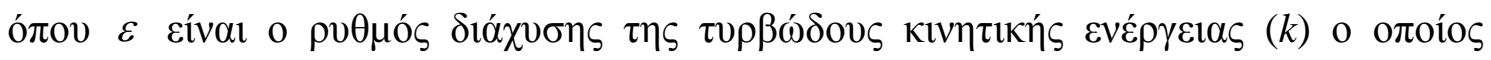
$\varepsilon \kappa \varphi \rho \alpha ́ \zeta \varepsilon \tau \alpha \iota \omega$

$$
\varepsilon=C_{D} k^{3 / 2} / l
$$

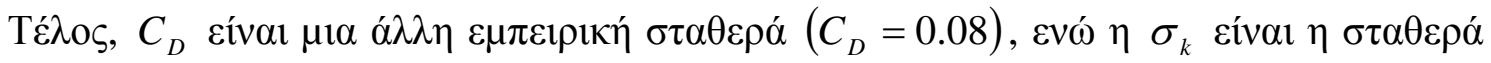

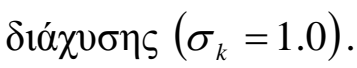

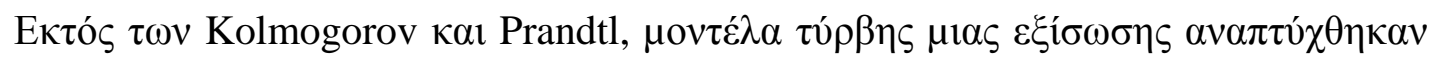

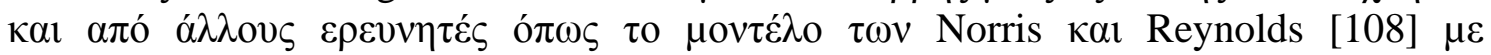

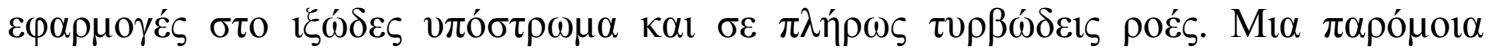

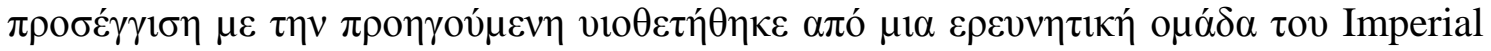

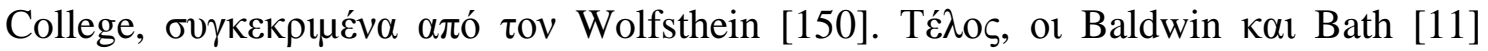

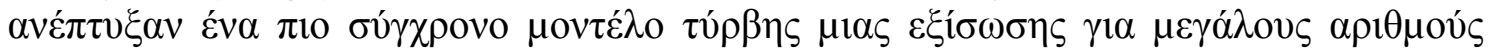

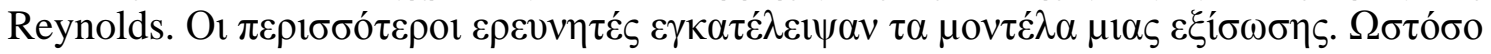

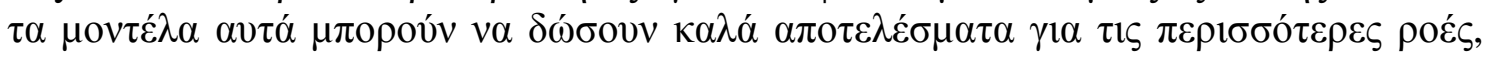

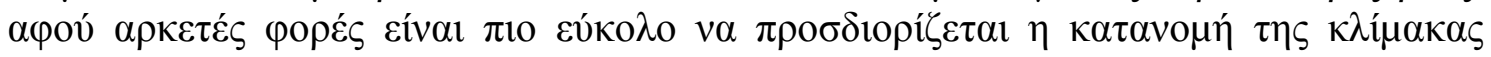

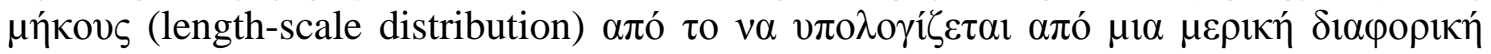

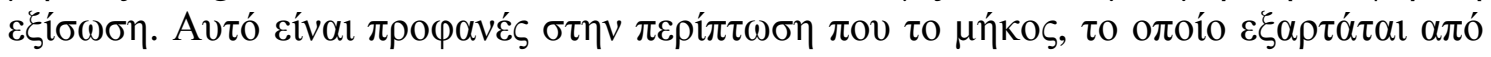

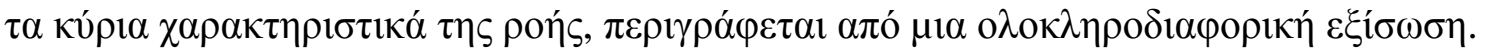




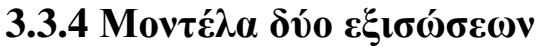

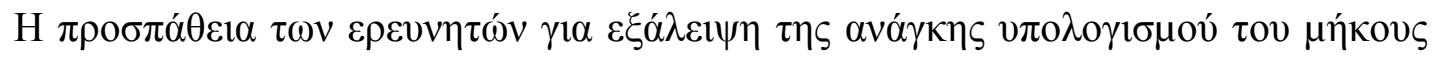

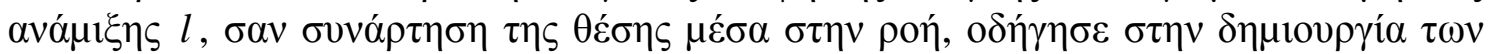

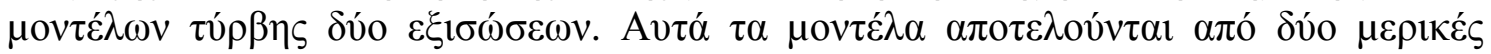

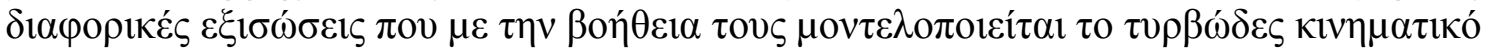

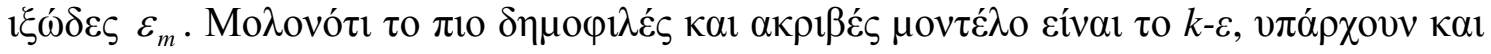

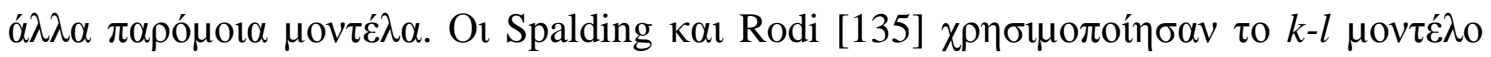

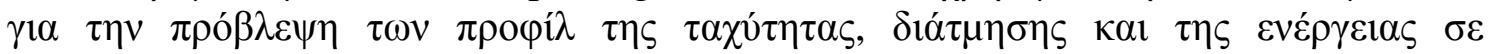

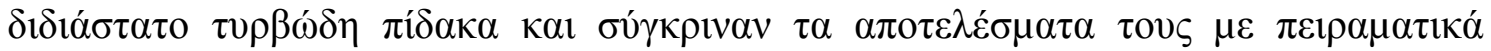

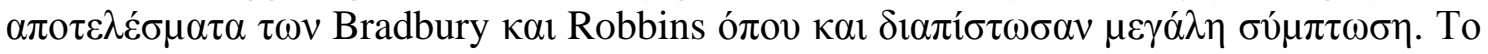

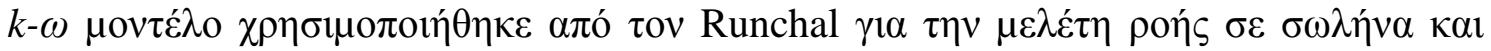

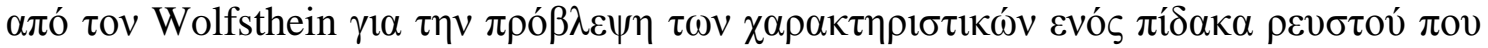

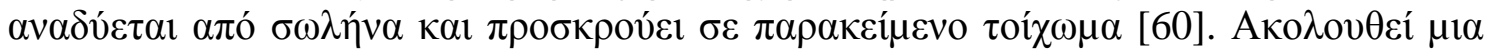

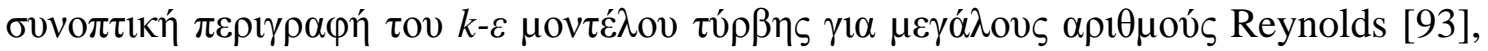

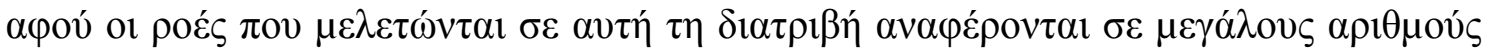
Reynolds (

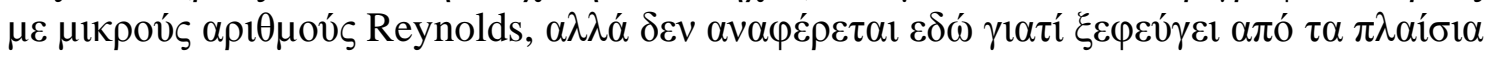

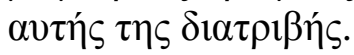

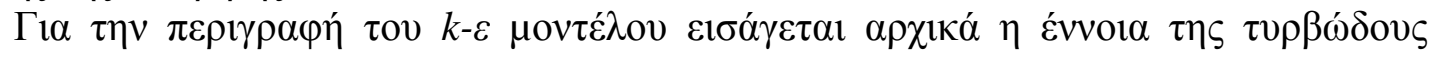

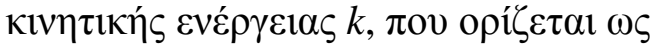

$$
k=\frac{1}{2}\left({\overline{u^{\prime}}}^{2}+{\overline{v^{\prime}}}^{2}\right),
$$

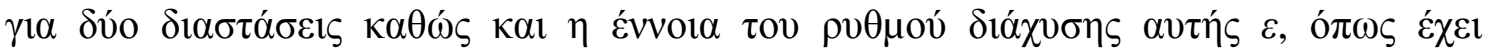

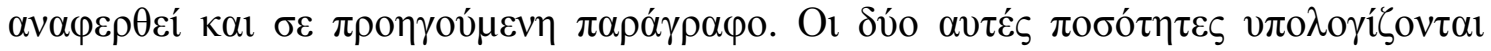

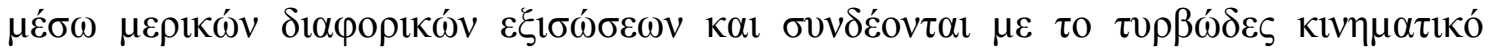

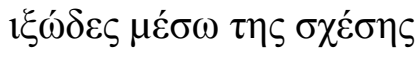

$$
\varepsilon_{m}=C_{\mu} \frac{k^{2}}{\varepsilon}
$$

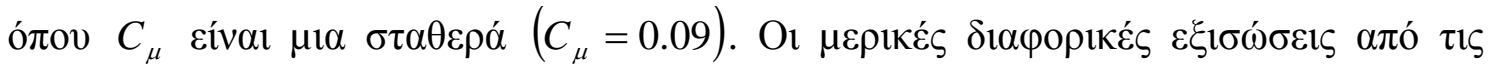

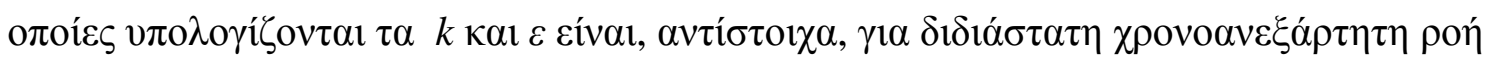

$$
\begin{gathered}
u \frac{\partial k}{\partial x}+v \frac{\partial k}{\partial y}=\frac{\partial}{\partial x}\left[\left(v+\frac{\varepsilon_{m}}{\sigma_{k}}\right) \frac{\partial k}{\partial x}\right]+\frac{\partial}{\partial y}\left[\left(v+\frac{\varepsilon_{m}}{\sigma_{k}}\right) \frac{\partial k}{\partial y}\right]+\varepsilon_{m}\left(\frac{\partial u}{\partial y}\right)^{2}-\varepsilon \\
u \frac{\partial \varepsilon}{\partial x}+v \frac{\partial \varepsilon}{\partial y}=\frac{\partial}{\partial x}\left[\left(v+\frac{\varepsilon_{m}}{\sigma_{\varepsilon}}\right) \frac{\partial \varepsilon}{\partial x}\right]+\frac{\partial}{\partial y}\left[\left(v+\frac{\varepsilon_{m}}{\sigma_{\varepsilon}}\right) \frac{\partial \varepsilon}{\partial y}\right]+C_{1} \varepsilon_{m} \frac{\varepsilon}{k}\left(\frac{\partial u}{\partial y}\right)^{2}-C_{2} \frac{\varepsilon^{2}}{k}
\end{gathered}
$$

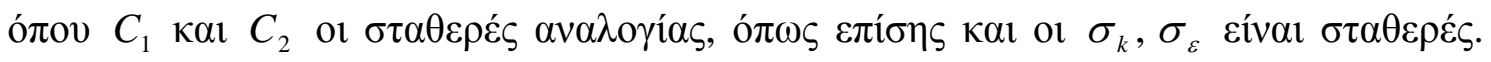

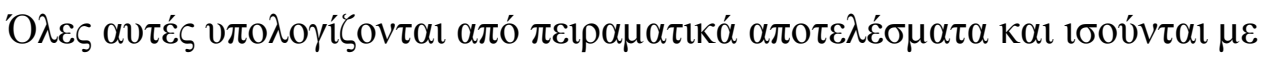

$$
C_{1}=1.55, \quad C_{2}=2.0, \quad \sigma_{k}=1.0, \quad \sigma_{\varepsilon}=1.3 .
$$




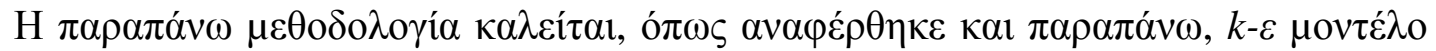

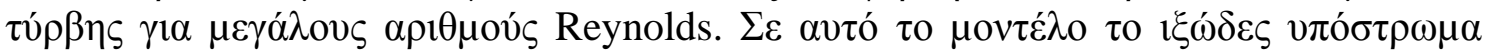

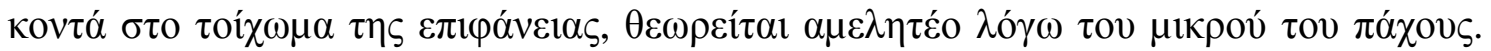

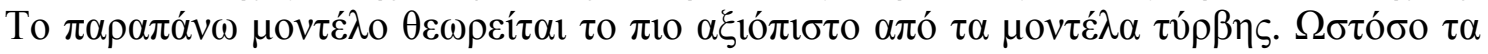

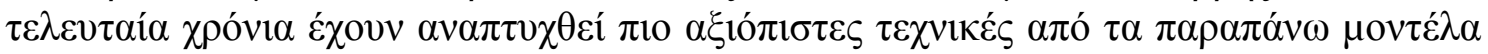

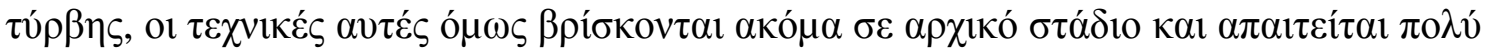

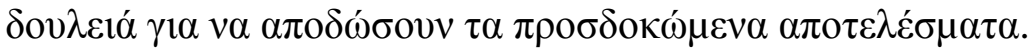

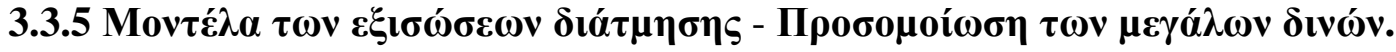

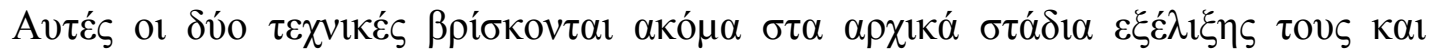

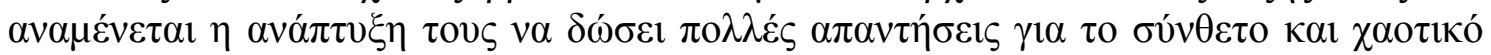

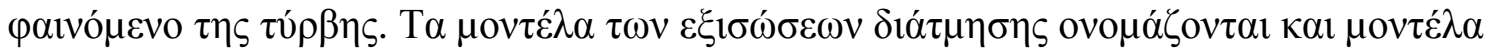

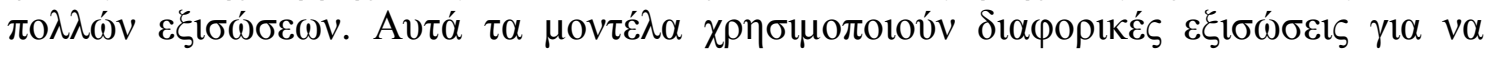

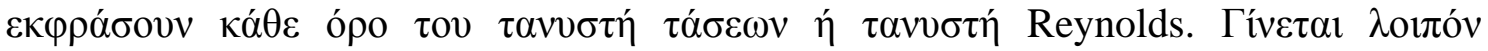

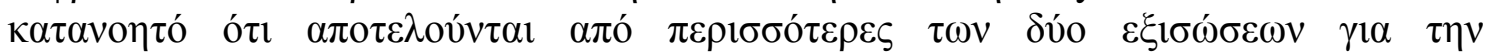

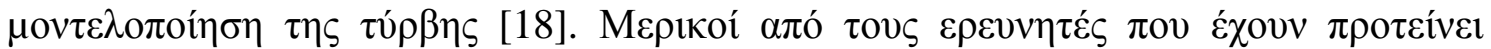

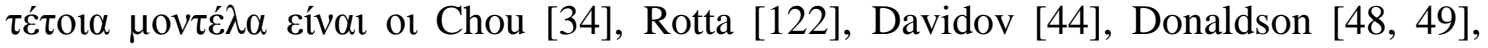

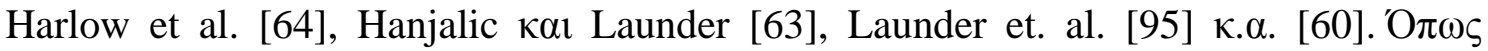

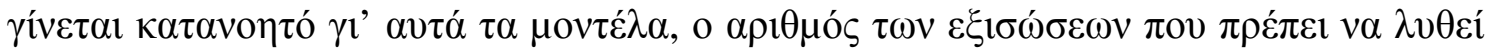

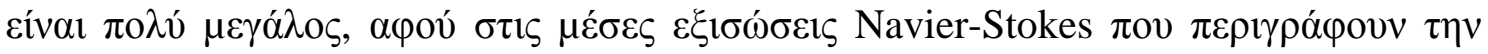

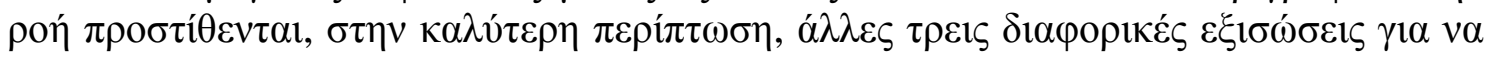

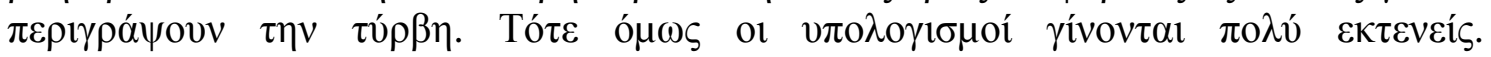

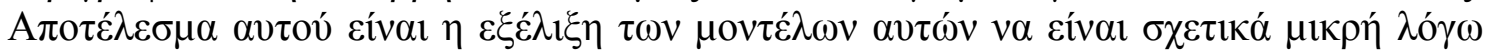

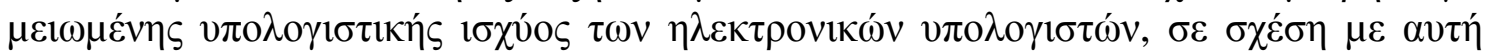

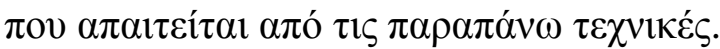

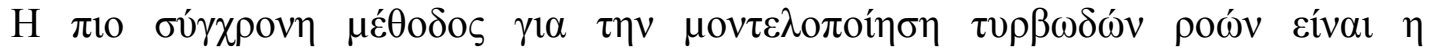

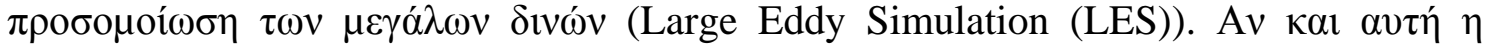

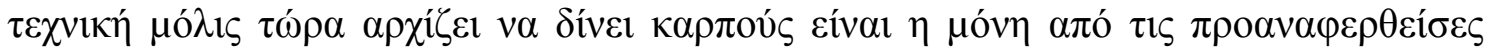

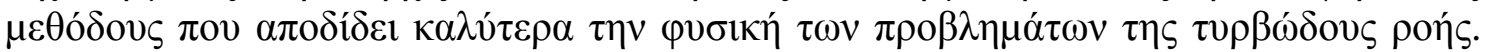

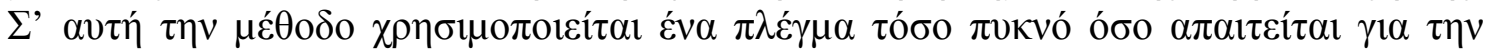

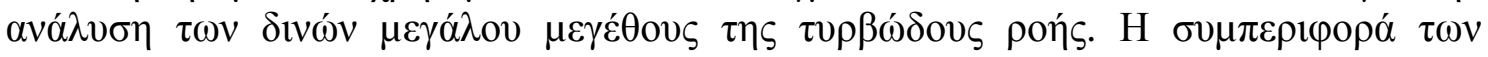

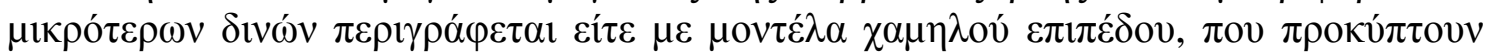

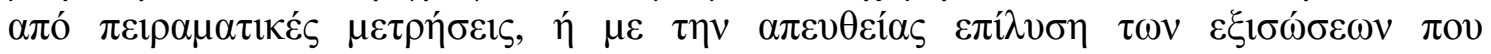

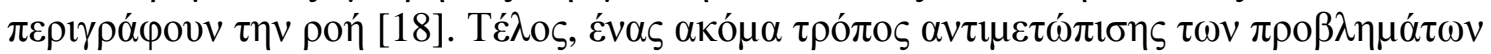

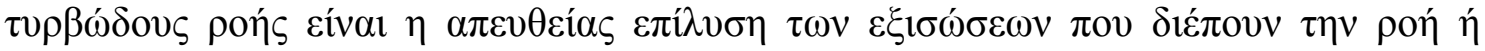

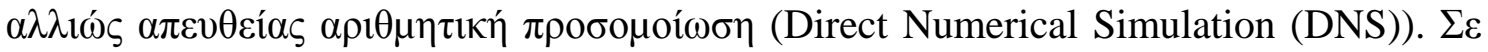

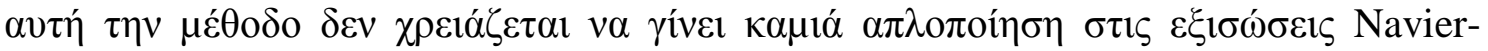

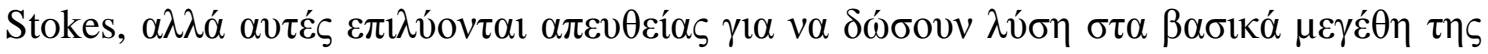

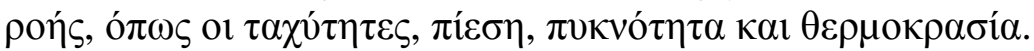

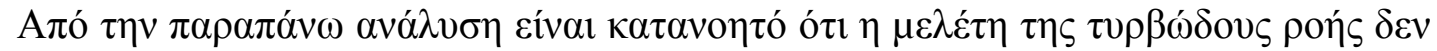

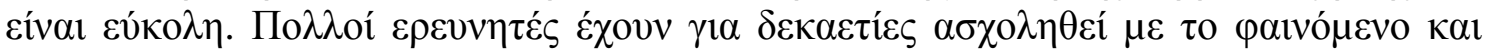

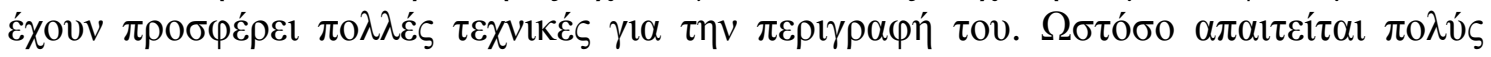

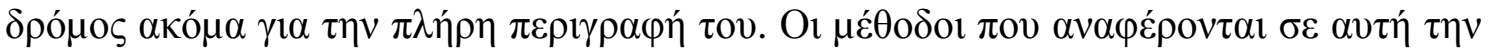

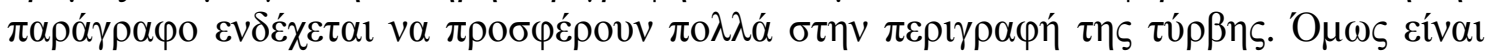

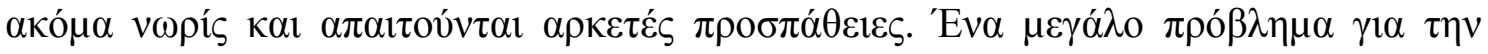

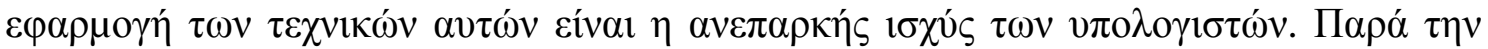

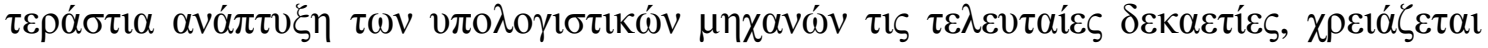




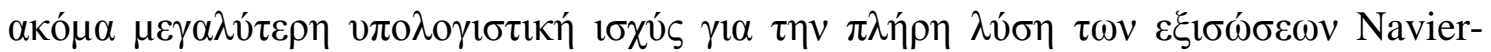

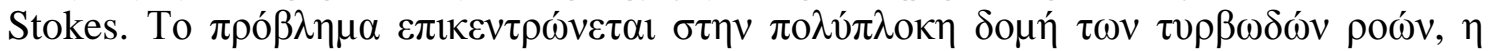

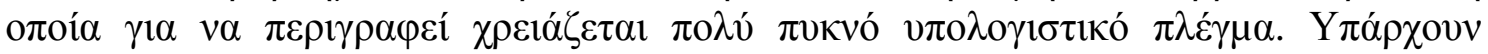

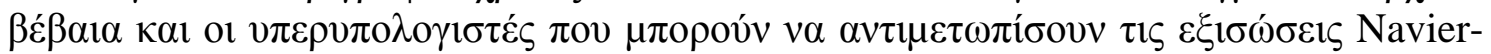

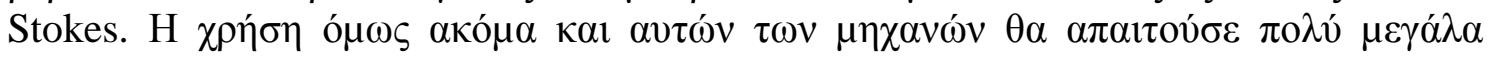

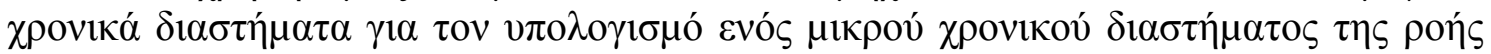

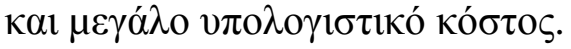

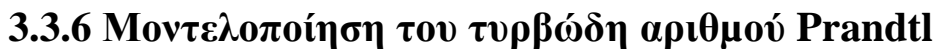

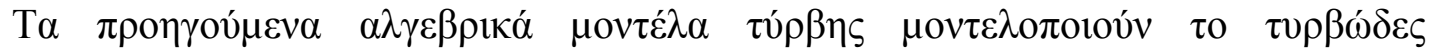

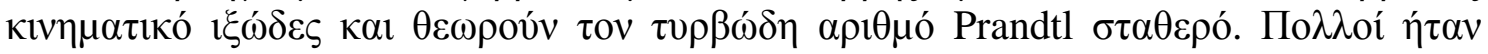

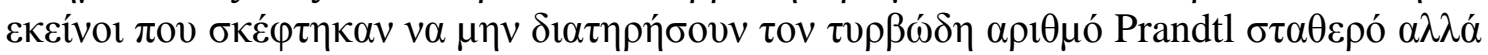

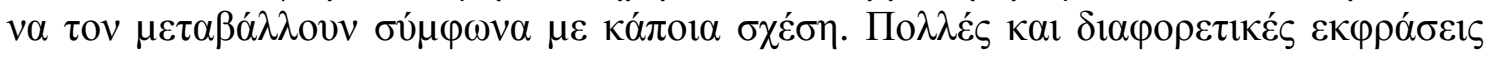

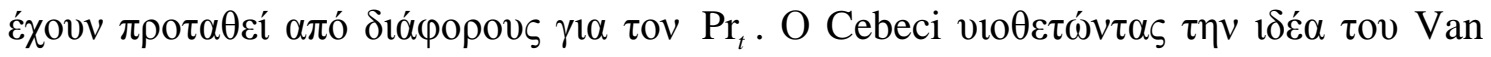

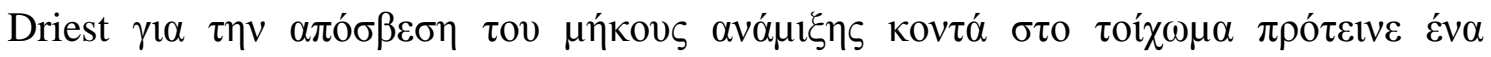

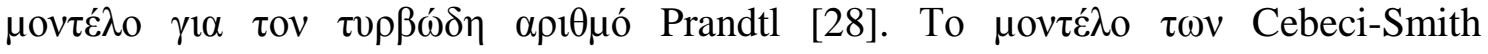

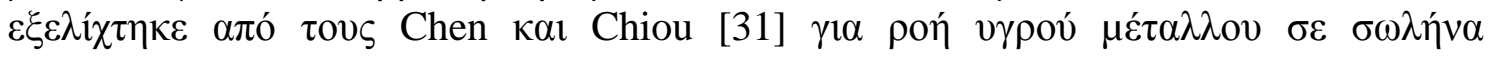

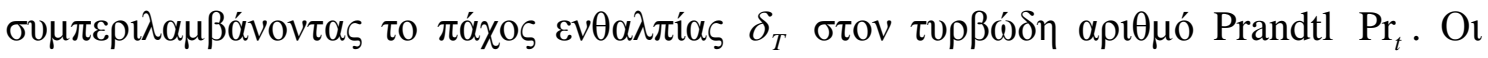

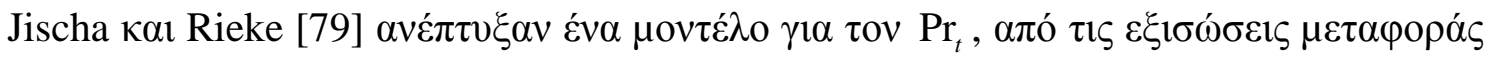

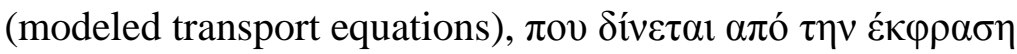

$$
\operatorname{Pr}_{t}=0.9+\frac{182.4}{\operatorname{Pr} R_{x}^{0.888}}
$$

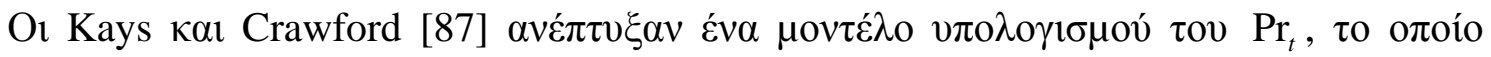

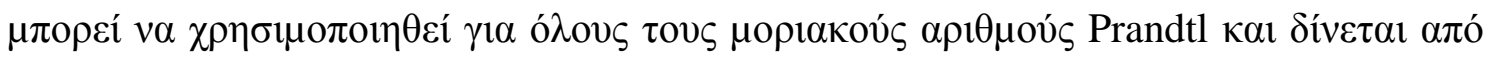
$\tau \eta \nu \sigma \chi \varepsilon \dot{\varepsilon} \sigma \eta$

$$
\operatorname{Pr}_{t}=1 /\left\{\frac{1}{2 \operatorname{Pr}_{t \infty}}+C P e_{t} \sqrt{\frac{1}{\operatorname{Pr}_{t \infty}}}-\left(C P e_{t}\right)^{2}\left[1-\exp \left(-\frac{1}{C P e_{t} \sqrt{\operatorname{Pr}_{t \infty}}}\right)\right]\right\},
$$

о́

$$
P e_{t}=\operatorname{Pr} \varepsilon_{m}^{+}
$$

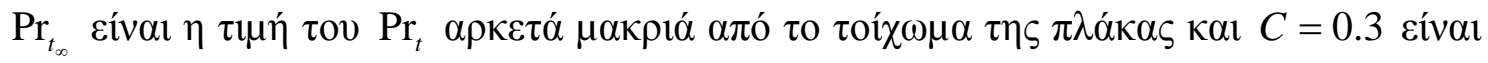

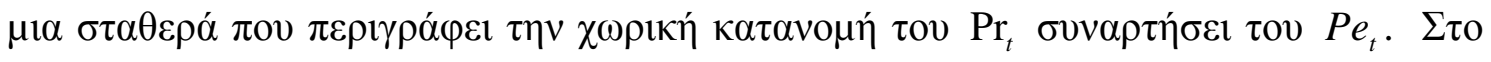

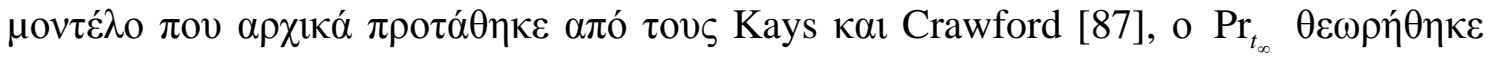

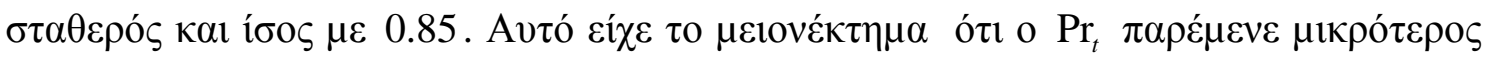

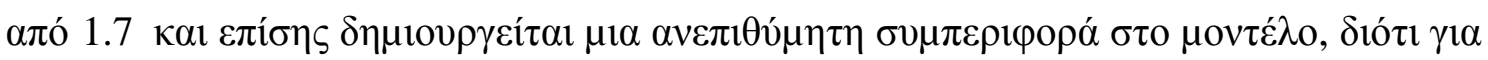

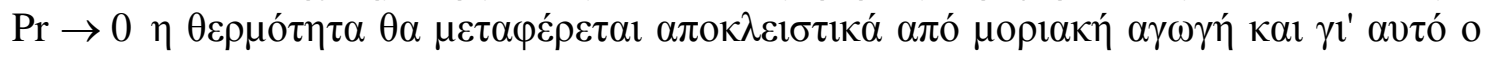

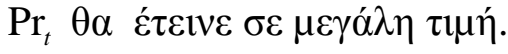

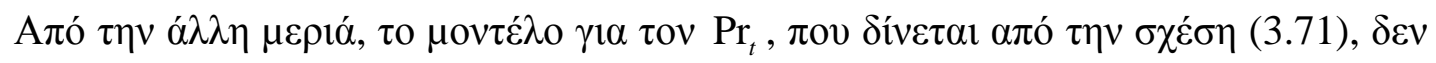

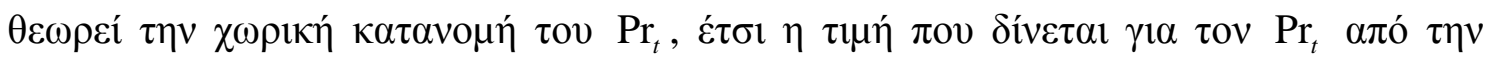

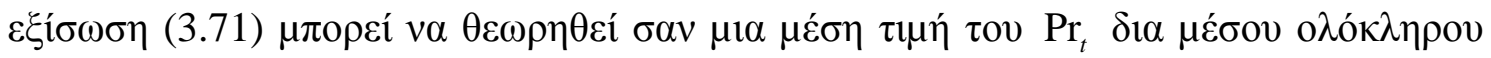




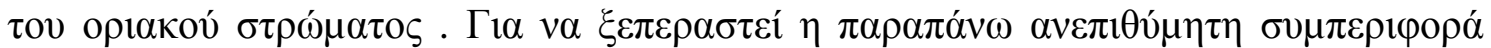

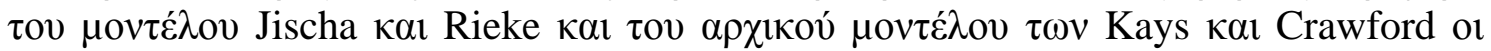

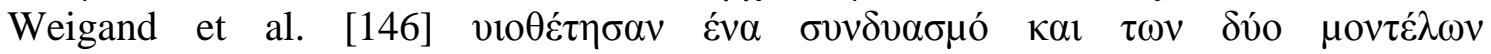

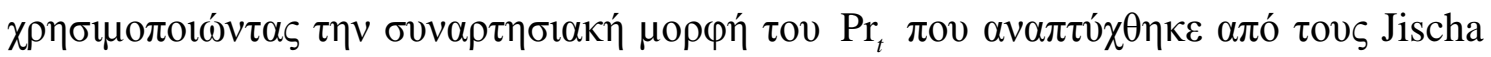

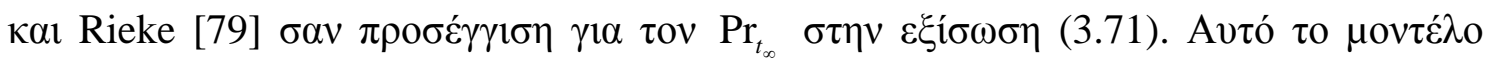

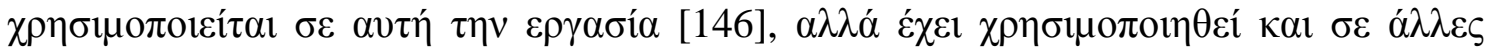

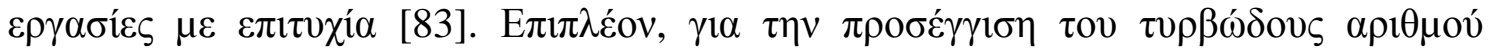

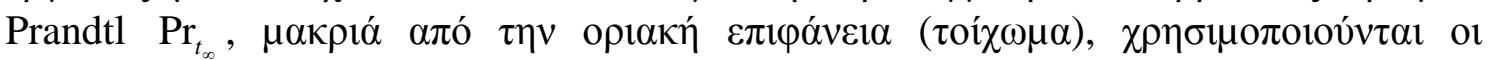
$\varepsilon \kappa \varphi \rho \alpha ́ \sigma \varepsilon ı \varsigma$

$$
\begin{gathered}
\operatorname{Pr}_{t_{\infty}}=0.9+\frac{182.4}{\operatorname{Pr} R_{\infty, x}^{0.888}}, \\
\text { о́лоv } \quad R_{\infty, x}=\frac{u_{\infty} x}{v_{\infty}} .
\end{gathered}
$$

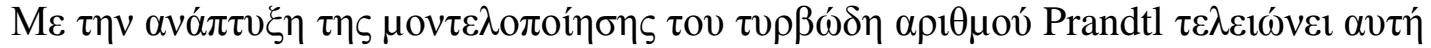

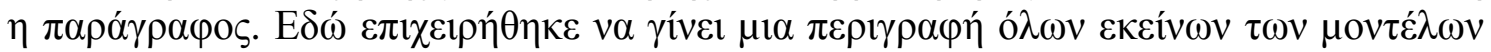

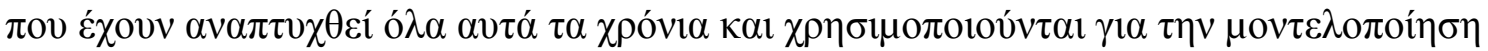

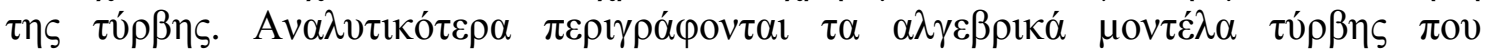

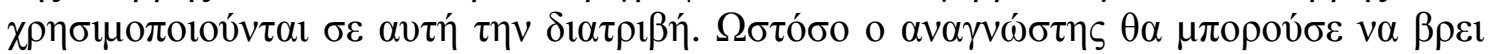

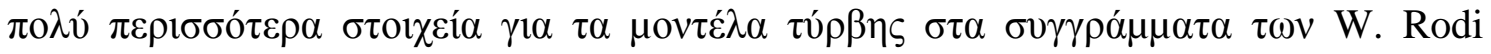
[119], Cebeci каı Bradshaw [29], Cebeci кaı Smith [27].

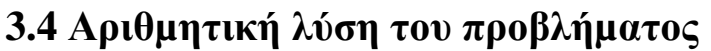

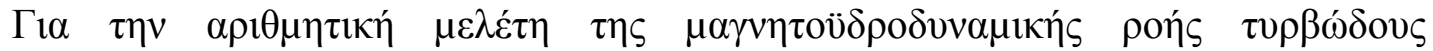

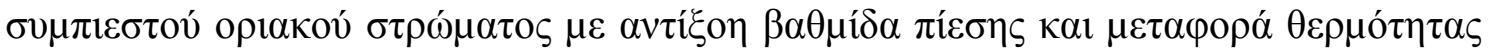

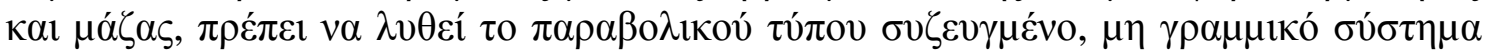

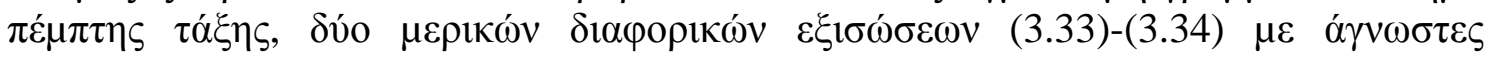

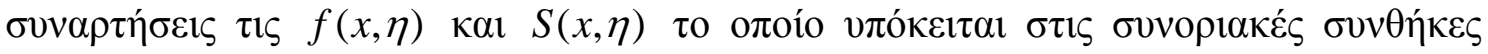

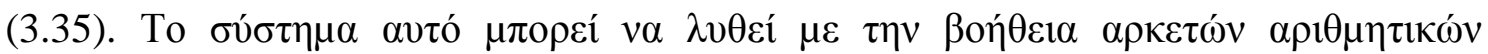

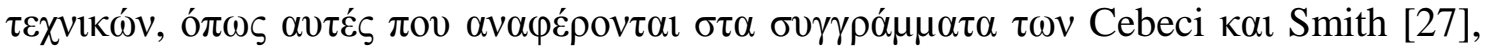
Cebeci кal Bradshaw [29], Schreier [125], Fletcher [50], Minkowycz et al. [101] $\eta$

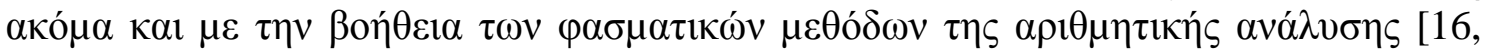

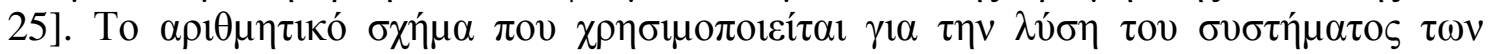

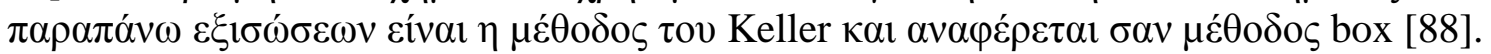

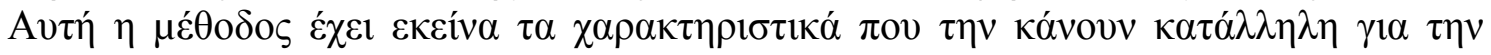

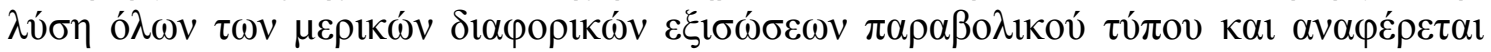

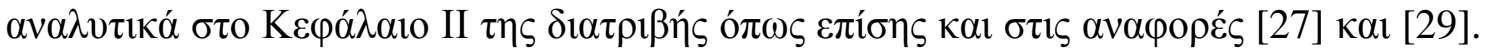

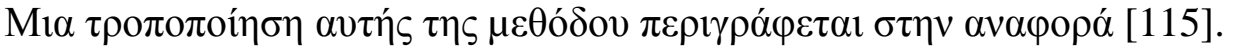

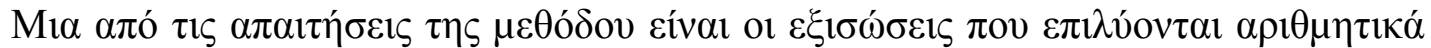

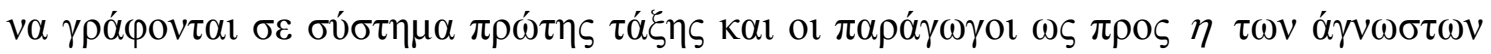

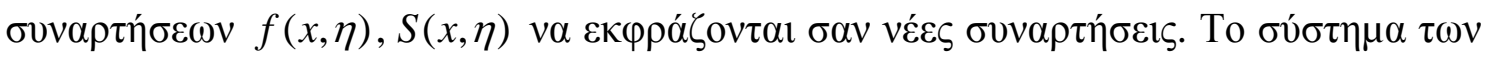

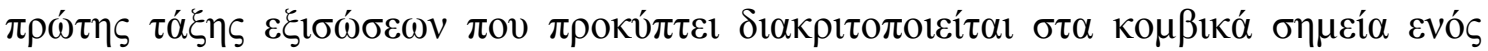

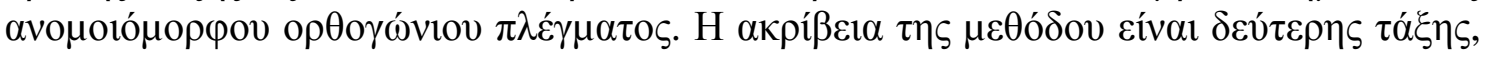




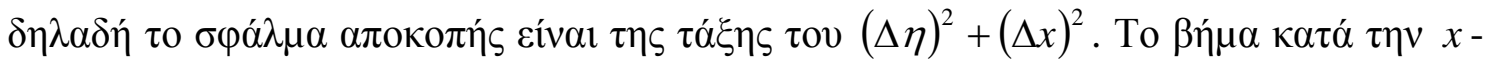

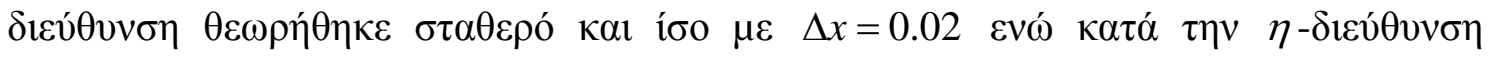

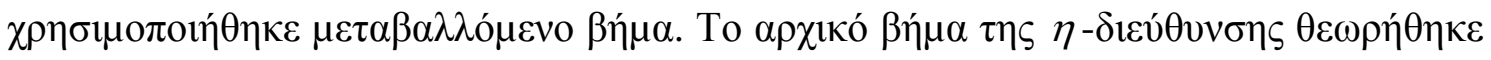

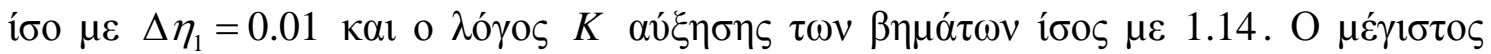

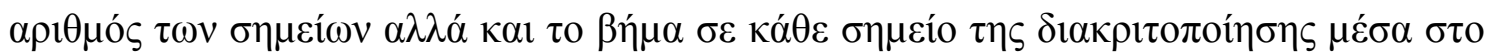

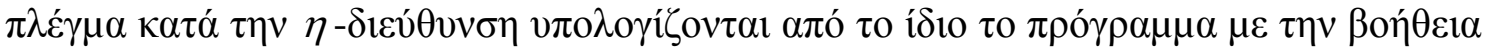
$\tau \omega \nu \pi \alpha \rho \alpha \kappa \alpha ́ \tau \omega \sigma \chi \varepsilon ́ \sigma \varepsilon \omega \nu$

$$
\begin{gathered}
J=\frac{\ln \left[1+(K-1)\left(\eta_{e} / \Delta \eta_{1}\right)\right]}{\ln K}, \\
\Delta \eta_{j}=\eta_{j}-\eta_{j-1}, \text { ó } \pi \text { ov } \eta_{j}=\Delta \eta_{1} \frac{K^{j}-1}{K-1}, j=1,2,3, \ldots ., J, K>1,
\end{gathered}
$$

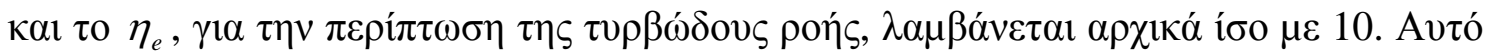

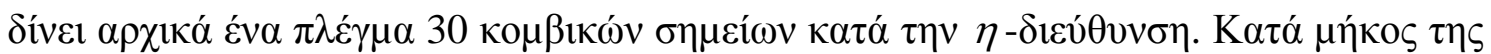

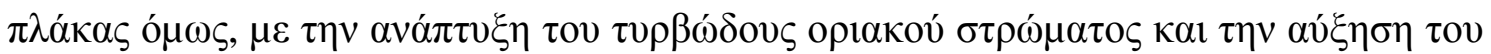

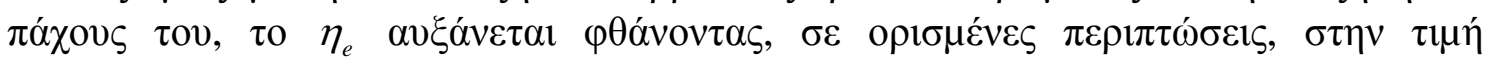

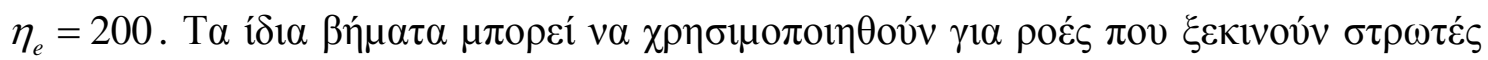

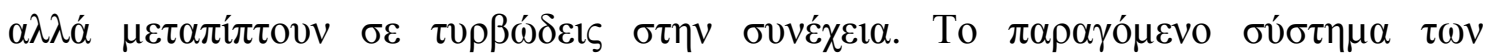

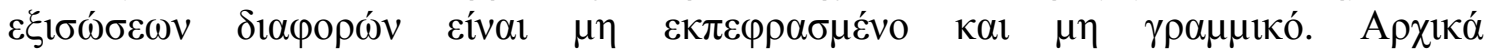

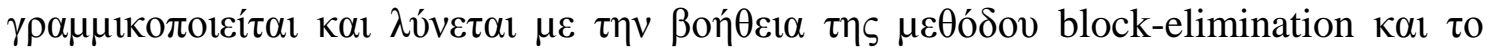

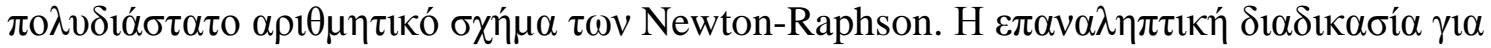

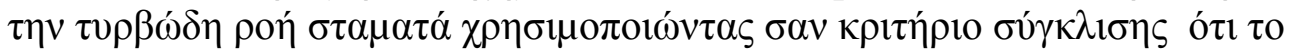

$$
\left|\frac{\delta f_{w}^{\prime \prime}}{f_{w}^{\prime \prime}+\delta f_{w}^{\prime \prime} / 2}\right|<0.02,
$$

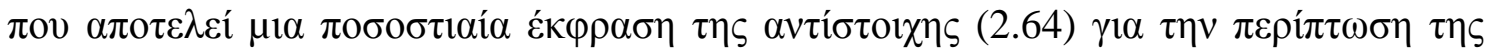

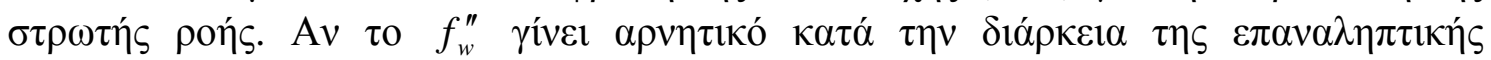

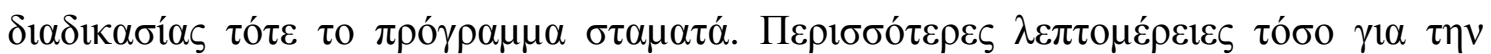

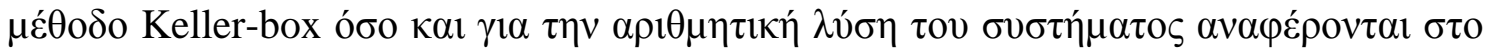

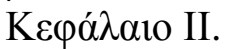

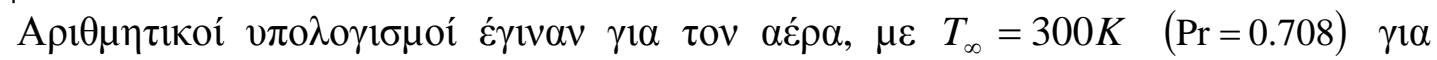

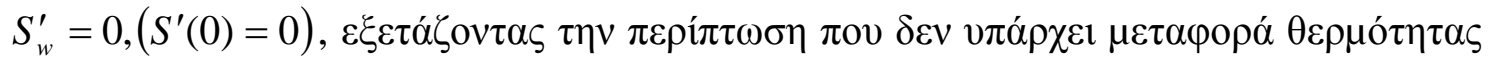

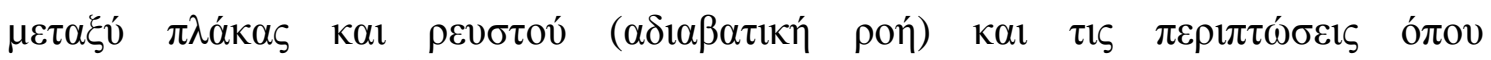

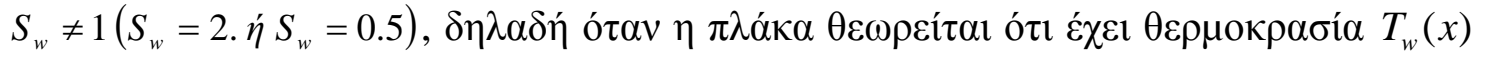

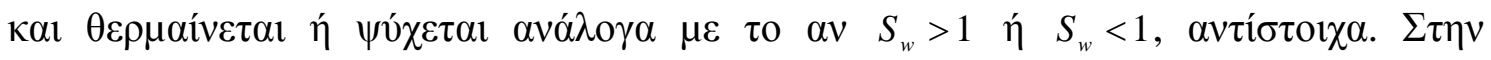

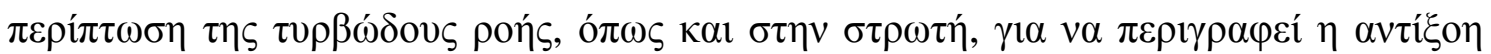

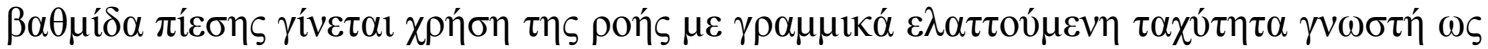

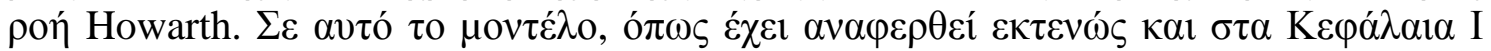

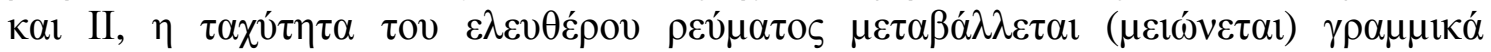

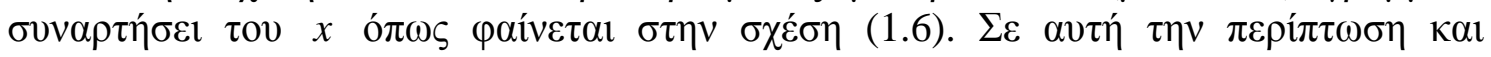

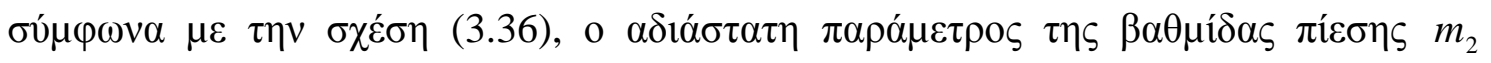

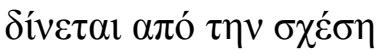




$$
m_{2}=m_{2}(x)=-\frac{x}{(8-x)}, \quad 0<x<8
$$

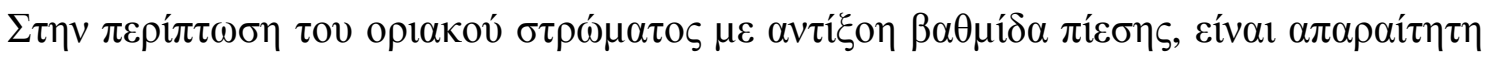

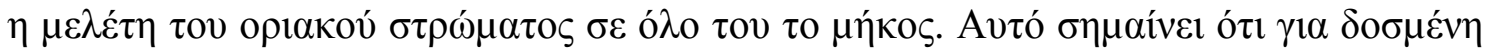

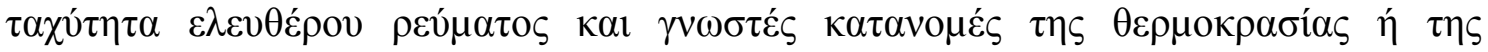

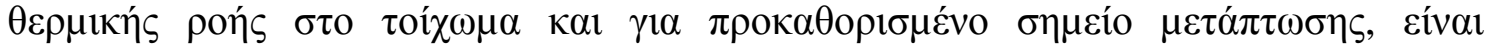

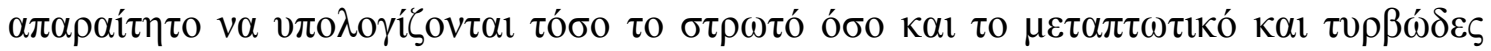

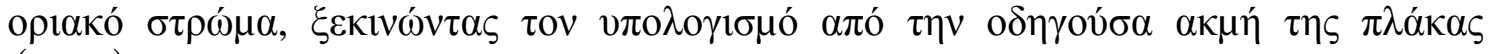
$(x=0)$.

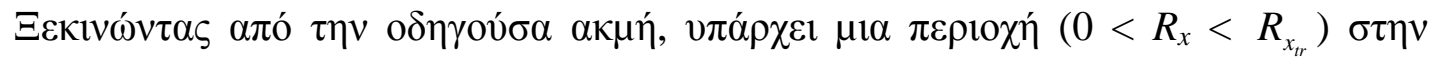

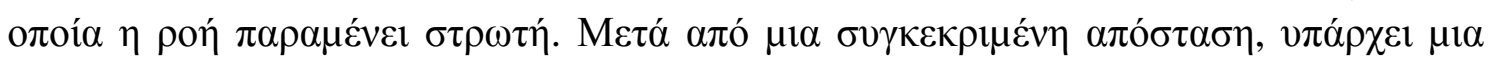

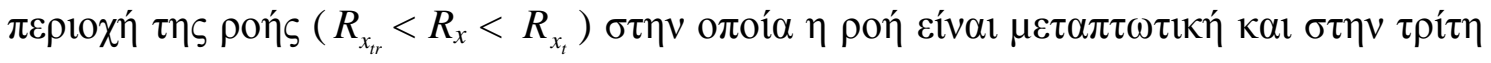

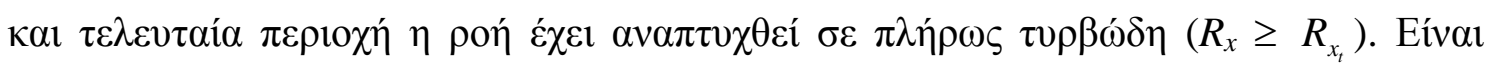

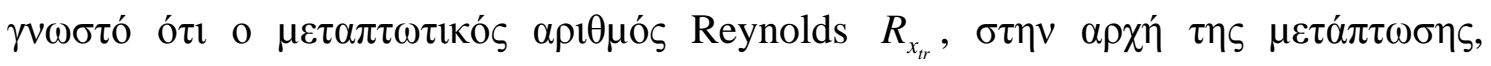

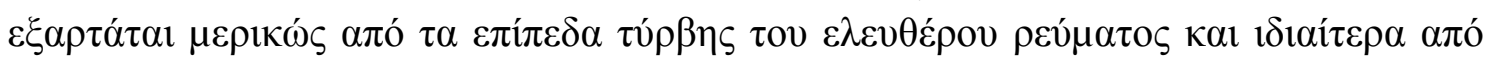

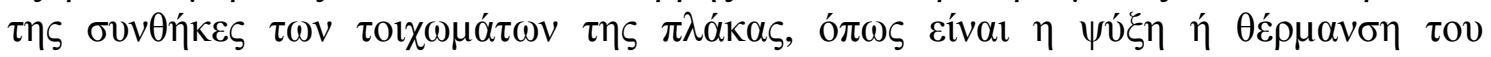

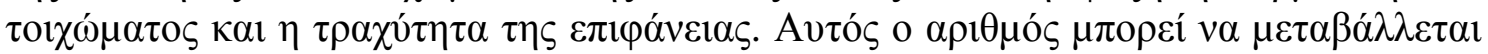
$\alpha \pi$ ó $4 \times 10^{5}$ źç $4 \times 10^{6}$ [29].

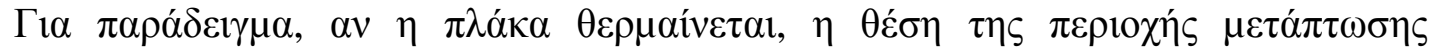

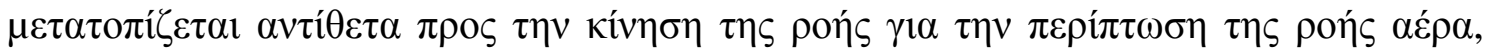

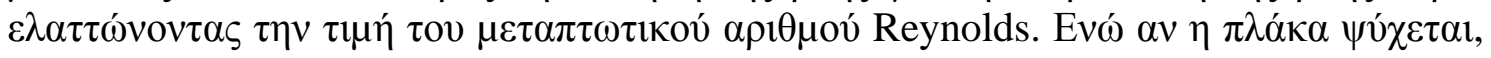

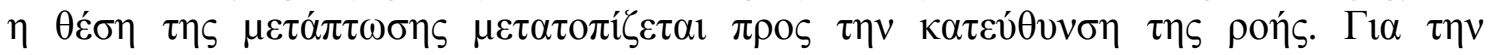

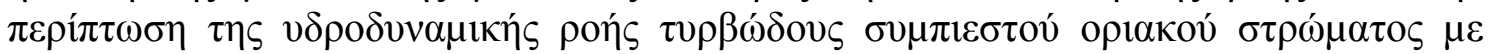

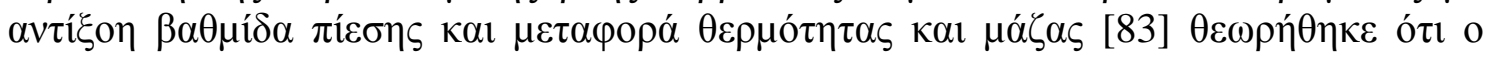

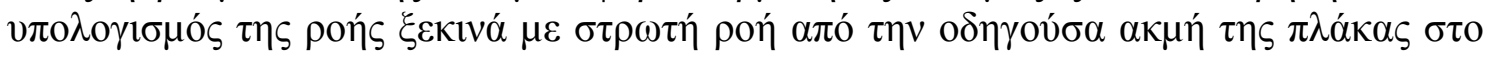

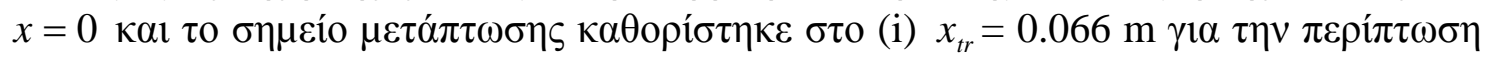
$\pi \mathrm{ov} S_{w}^{\prime}=0, \quad\left(\mathrm{R}_{\mathrm{x}_{\mathrm{tr}}}=22 \times 10^{5}\right)$, (ii) $x_{t r}=0.012 \gamma 1 \alpha \tau \eta v \pi \varepsilon \rho i ́ \tau \tau \omega \sigma \eta S_{w}=2 .\left(\mathrm{R}_{\mathrm{x}_{\mathrm{tr}}}=4 \times\right.$

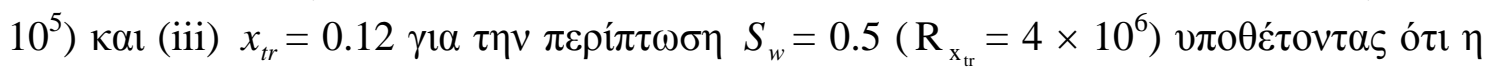

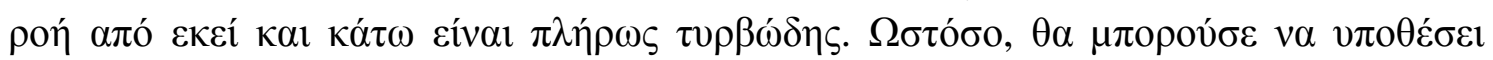

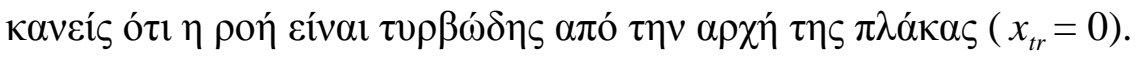

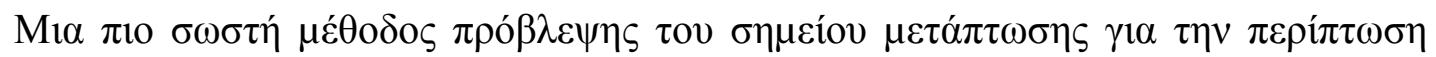

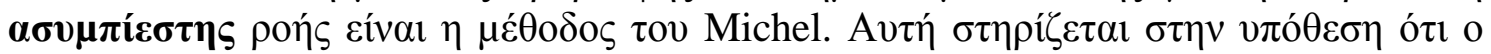

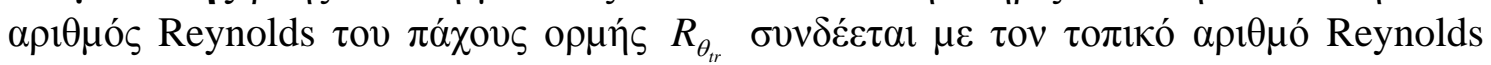

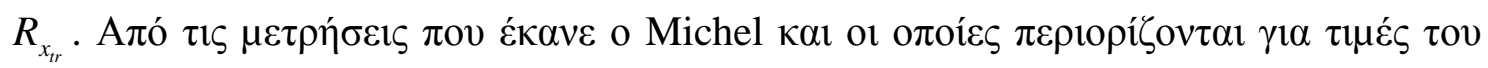

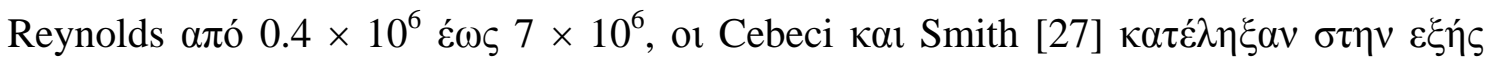
$\sigma \chi \varepsilon \dot{\sigma} \sigma \eta$

$$
R_{\theta_{t r}}=1.174\left[1+\left(22.400 / R_{x}\right)\right] R_{x}^{0.46}, \quad 0.1 \times 10^{6} \leq R_{x} \leq 40 \times 10^{6}
$$

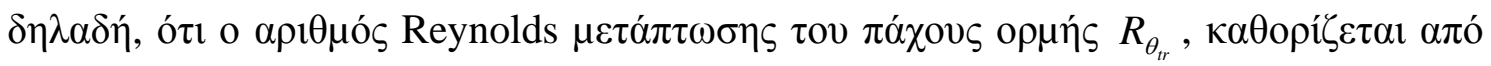

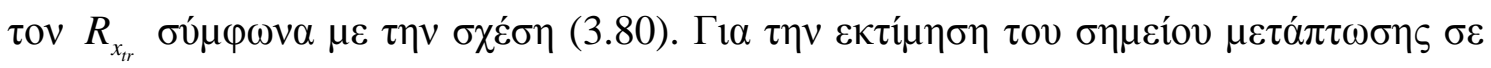

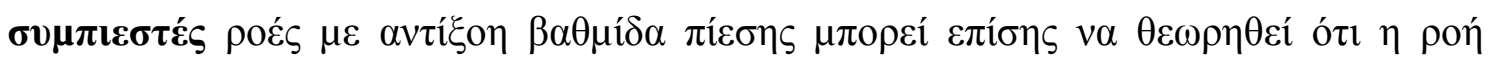




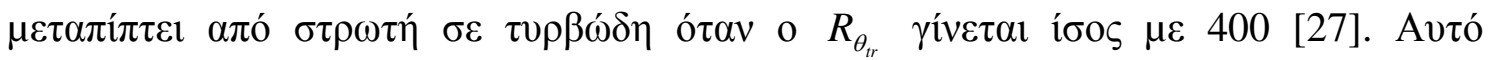

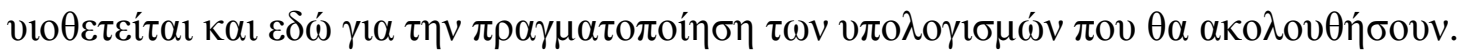

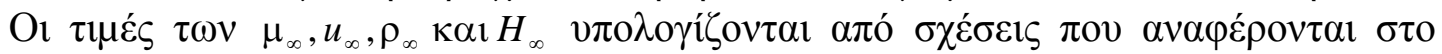

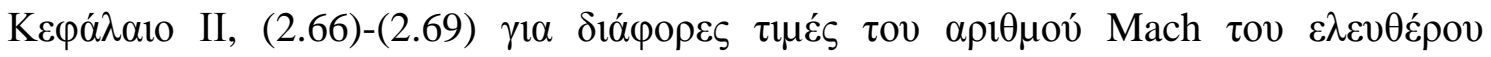

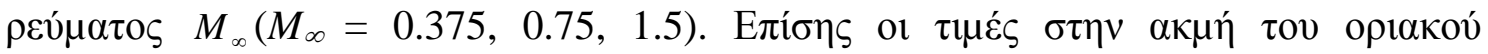

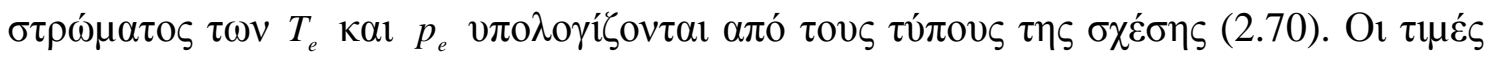

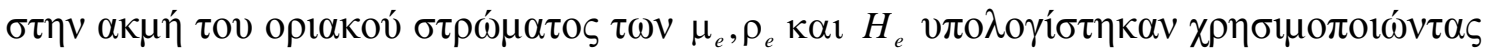

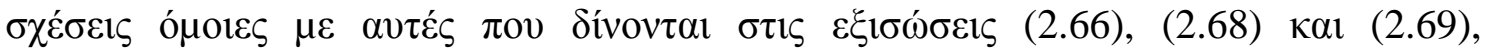

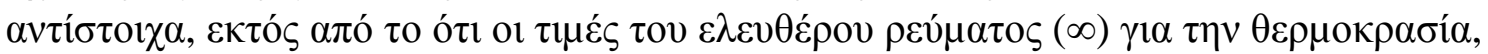

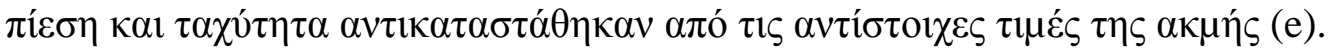

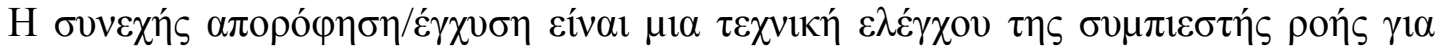

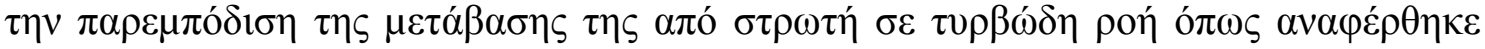

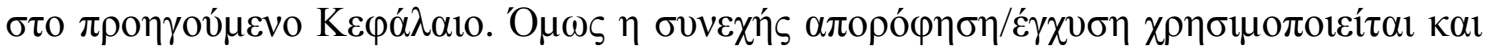

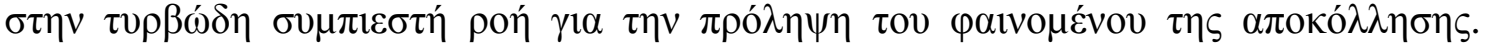

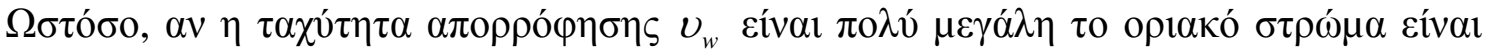

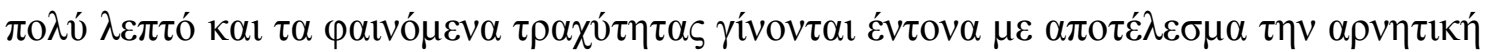

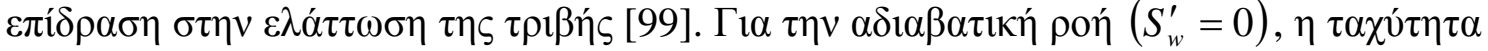

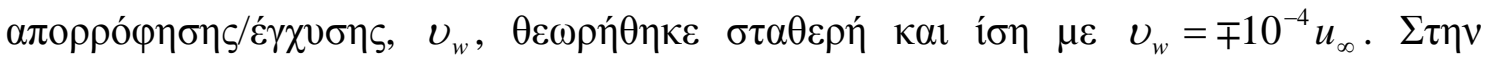

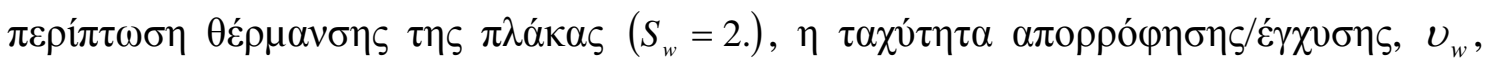

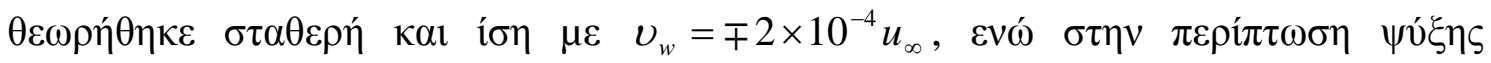

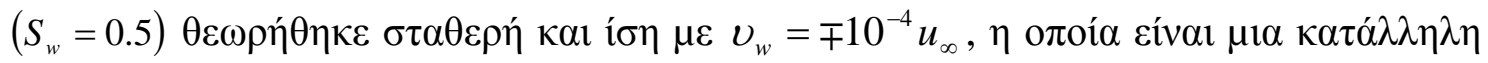

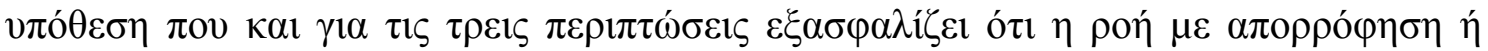

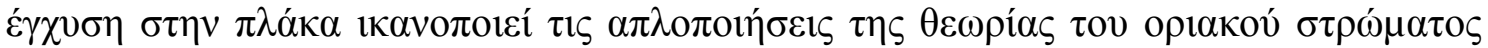

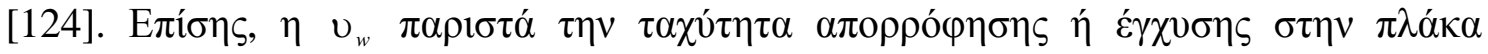

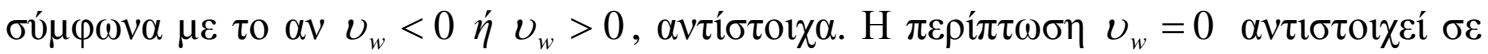
$\alpha \delta 1 \alpha \pi \varepsilon ́ \rho \alpha \sigma \tau \eta \pi \lambda \alpha \dot{\kappa} \alpha \alpha(\chi \omega \rho i ́ \varsigma \alpha \pi$ $\alpha \rho o ́ \varphi \eta \sigma \eta / \varepsilon ́ \gamma \chi v \sigma \eta)$.

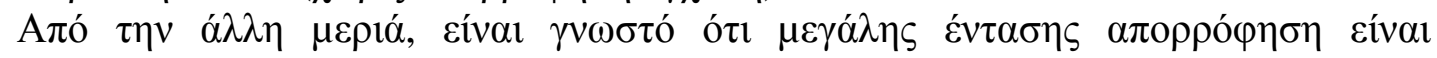

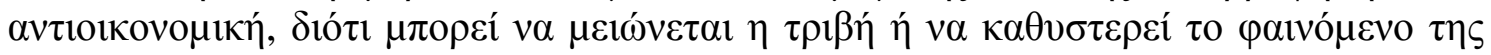

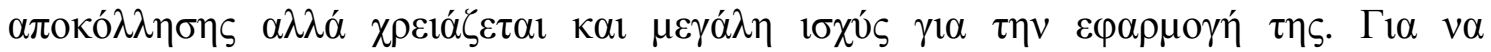

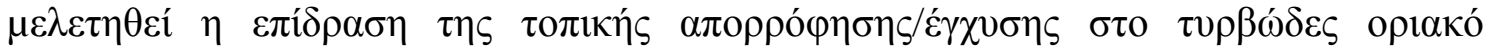

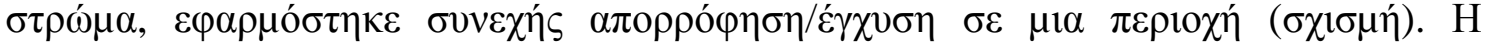

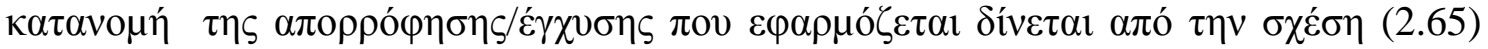

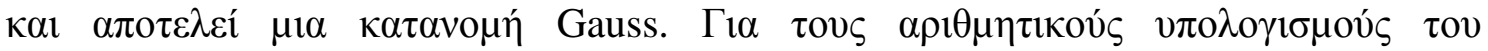

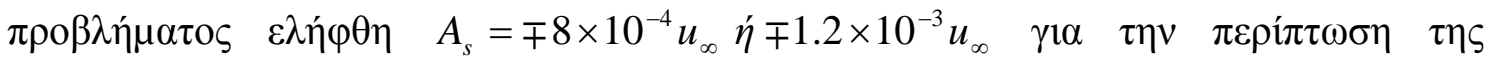

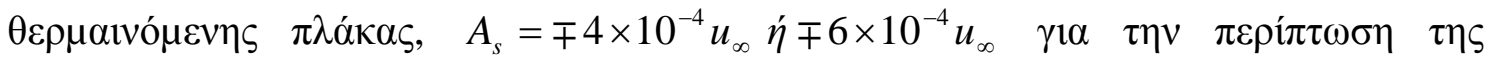

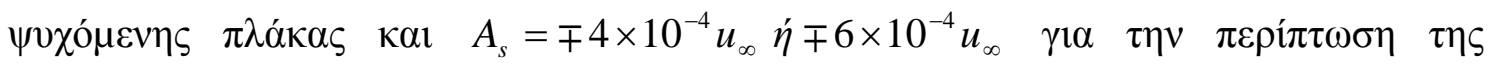

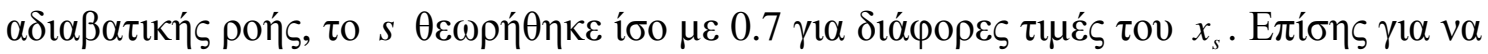

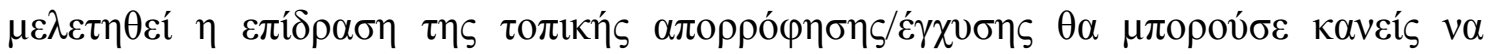

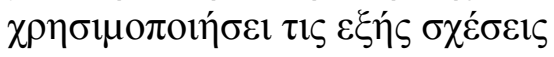

$$
v_{w}(x)=\frac{1}{2} v_{0}[1+\tanh \beta(x-a)] \quad 0<x \leq \frac{a+b}{2},
$$

$\kappa \alpha 1$

$$
v_{w}(x)=\frac{1}{2} v_{0}[1-\tanh \beta(x-b)] \quad x>\frac{a+b}{2},
$$




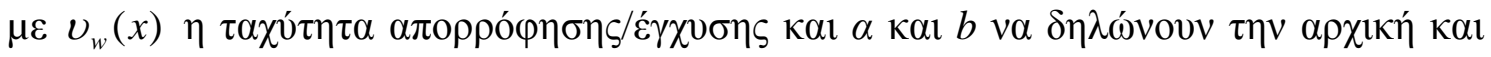

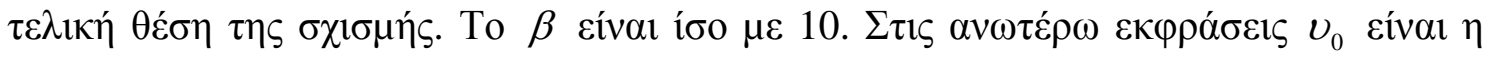

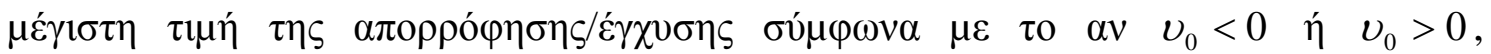

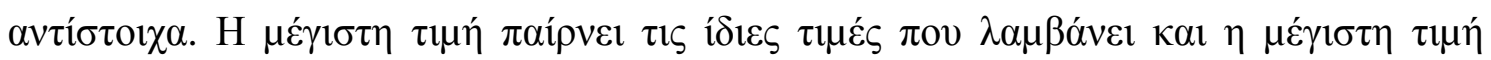

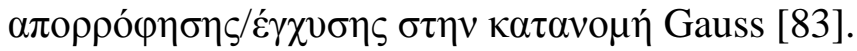

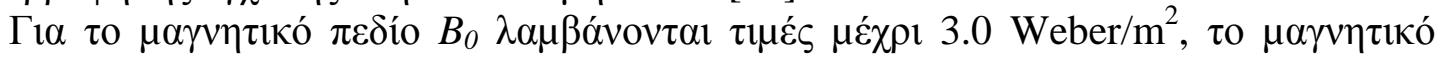

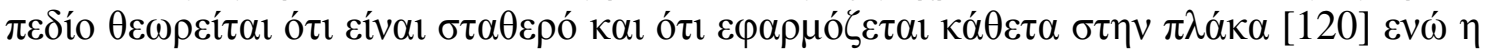

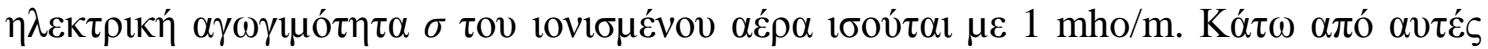

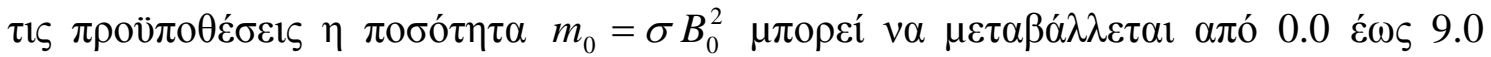

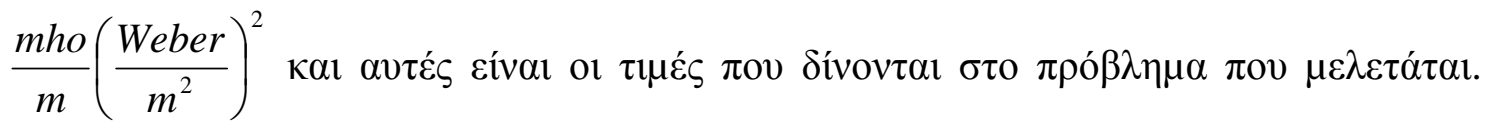

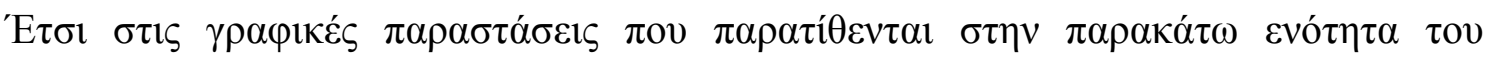

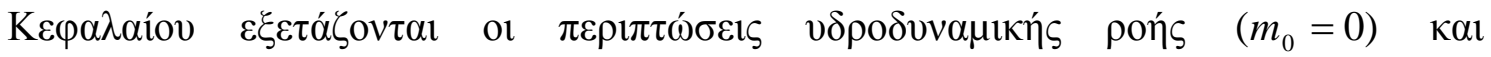

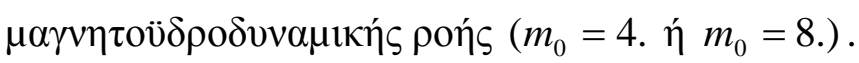

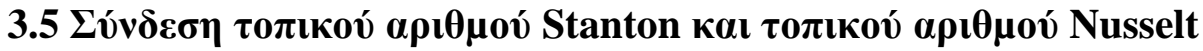

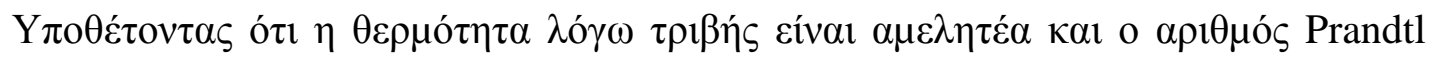

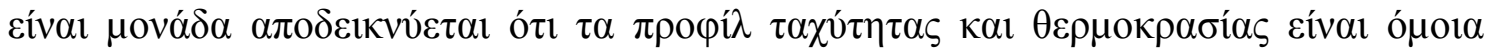

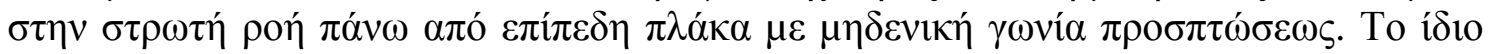

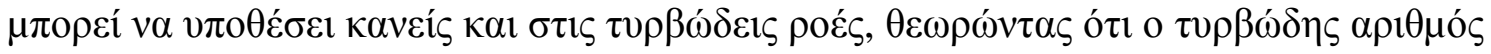

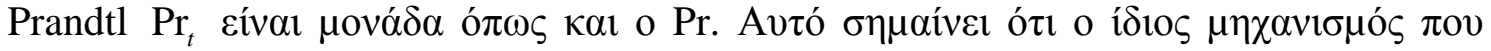

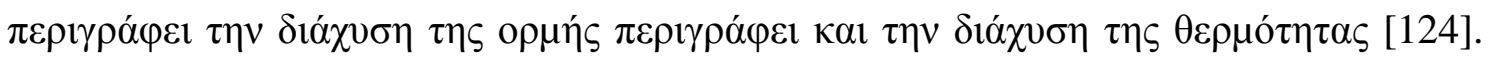

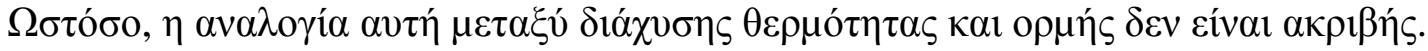

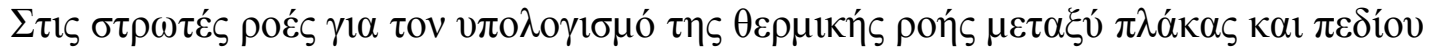

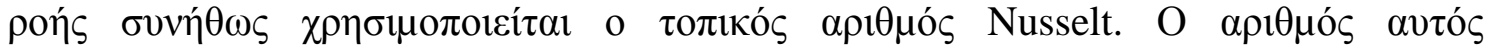

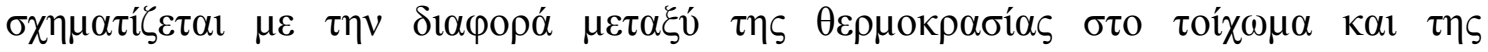

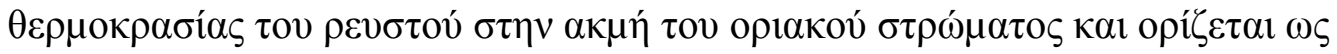

$$
N u_{x}=\frac{\dot{q}_{w} x}{k\left(T_{w}-T_{e}\right)}
$$

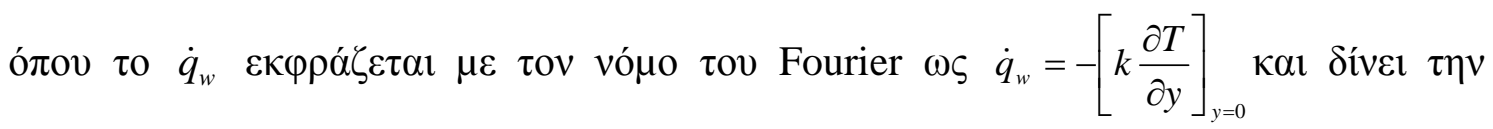

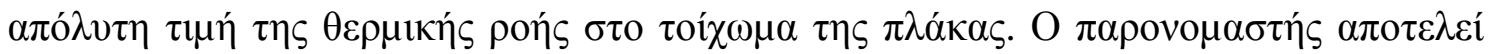

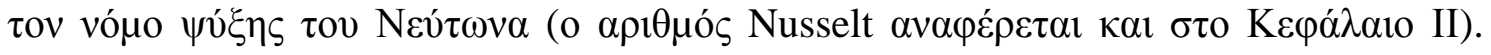

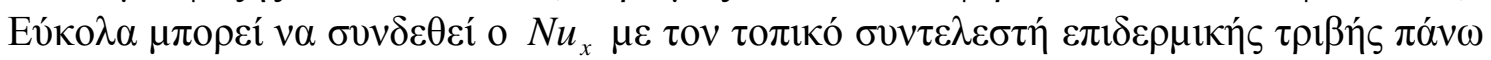
$\sigma \tau \eta \nu \pi \lambda \alpha ́ \kappa \alpha C_{f_{x}} \mu \varepsilon \dot{\varepsilon} \sigma \omega \tau \eta \varsigma \sigma \chi \varepsilon ́ \sigma \eta \varsigma$

$$
N u_{x}=\frac{1}{2} R_{x} C_{f_{x}}, \quad\left(\operatorname{Pr}=\operatorname{Pr}_{t}=1\right)
$$




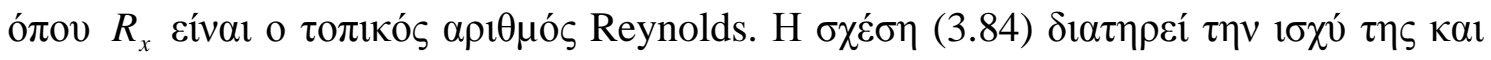

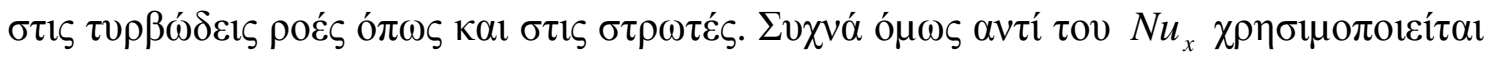

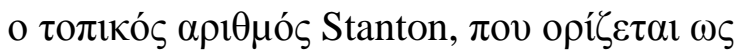

$$
S t_{x}=\frac{\dot{q}_{w}}{\rho_{e} c_{p}\left(T_{w}-T_{e}\right) u_{e}} .
$$

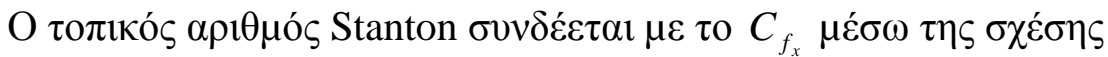

$$
S t_{x}=\frac{1}{2} C_{f_{x}}, \quad\left(\operatorname{Pr}=\operatorname{Pr}_{t}=1\right)
$$

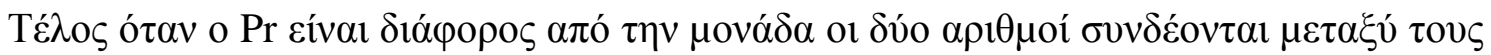
$\mu \varepsilon \dot{\sigma} \sigma \omega \tau \eta \varsigma \sigma \chi \varepsilon ́ \sigma \eta \varsigma$

$$
S t_{x}=\frac{N u_{x}}{R_{x} \operatorname{Pr}}
$$

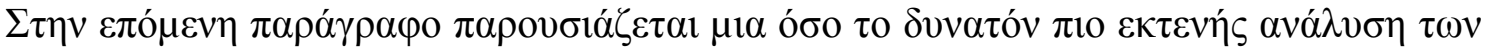

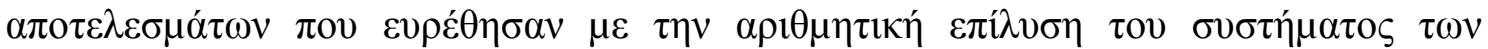

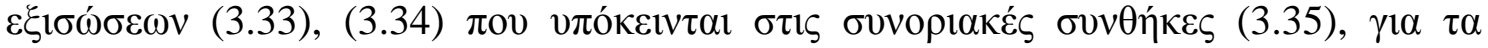

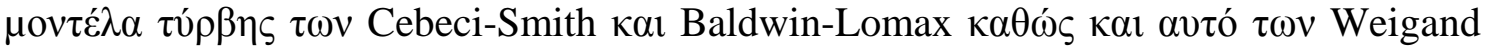

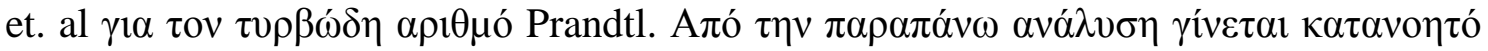

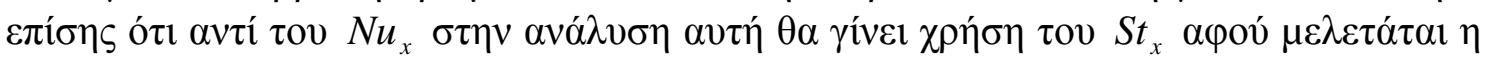

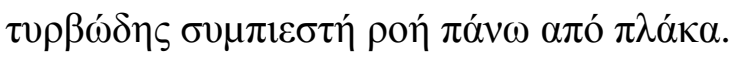

\subsection{Avód $v \sigma \eta \tau \omega v \alpha \pi 0 \tau \varepsilon \lambda \varepsilon \sigma \mu \alpha ́ \tau \omega v$}

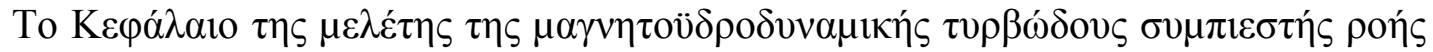

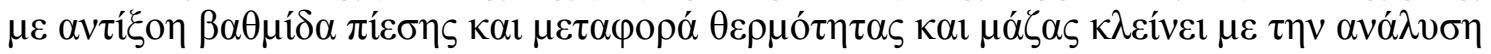

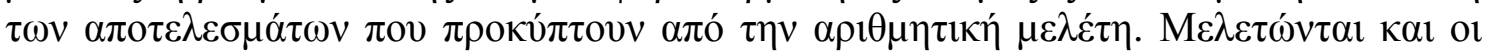

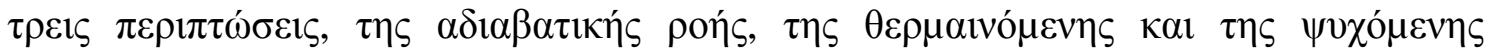

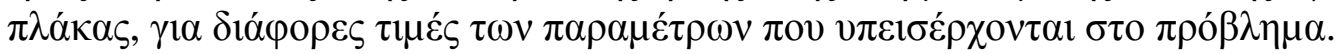

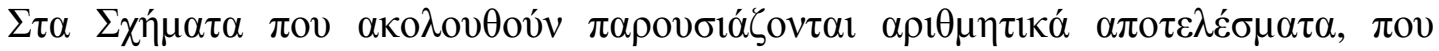

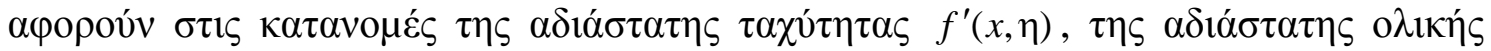

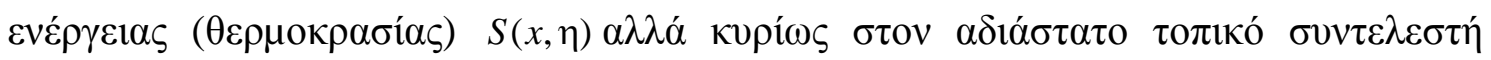

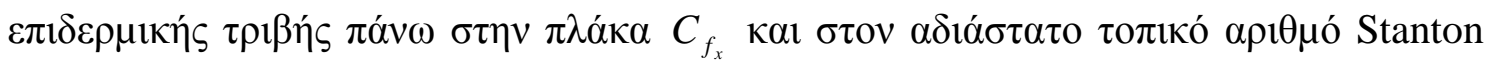

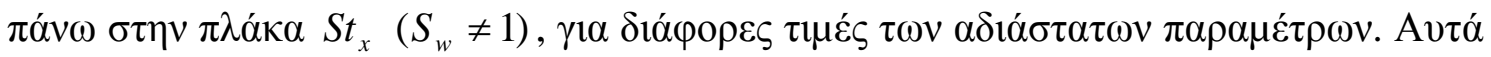

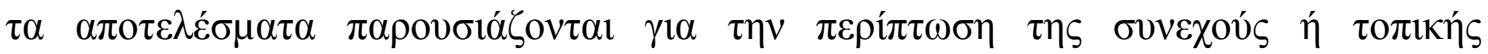

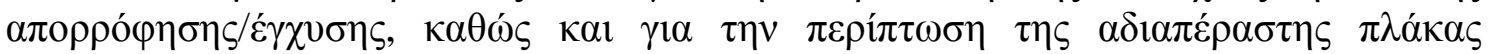

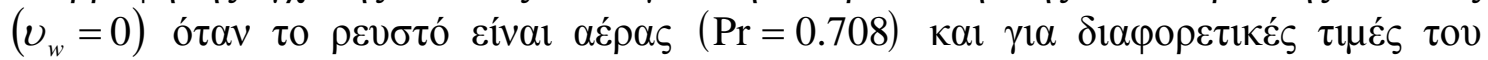

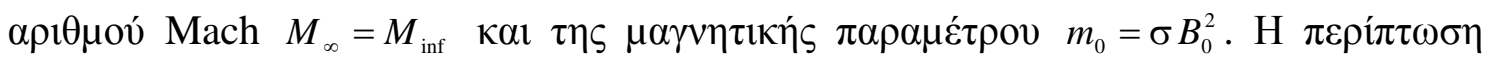

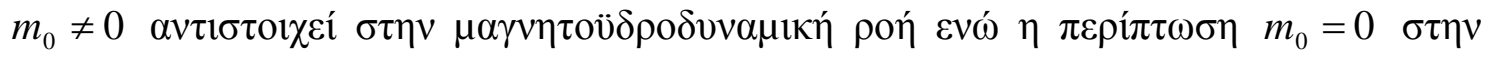

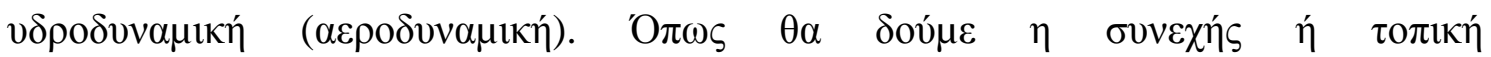

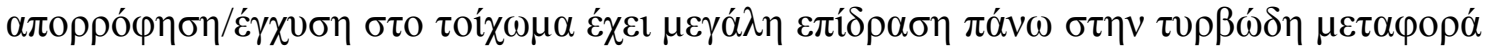




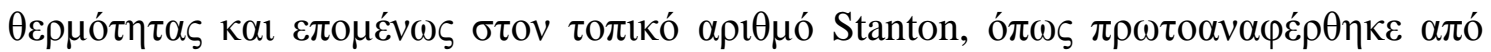

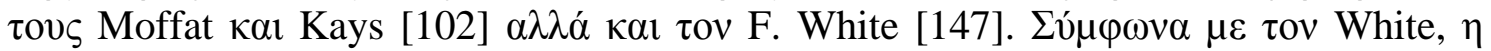

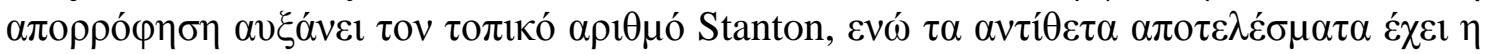

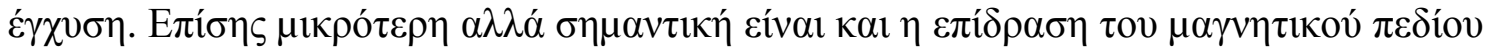

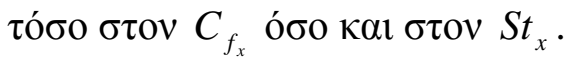

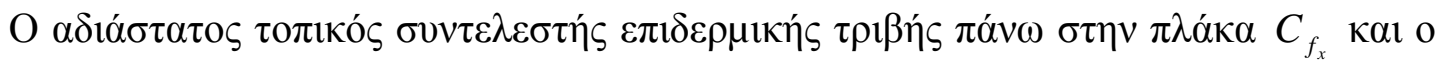

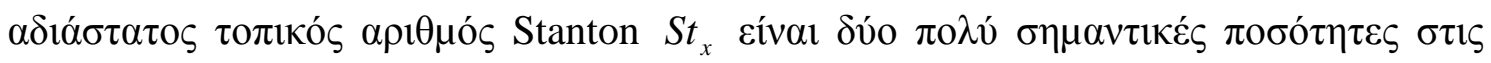

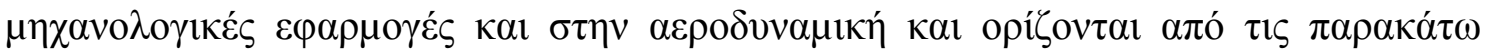
$\sigma \chi \varepsilon \dot{\sigma \varepsilon \varepsilon 1 \varsigma}$

$$
\begin{aligned}
& C_{f_{x}}=\frac{\tau_{w}}{\frac{1}{2} \rho_{e} u_{e}^{2}} \kappa \alpha \mathrm{l} S t_{x}=\frac{\dot{q}_{w}}{\rho_{e}\left(H_{w}-H_{e}\right) u_{e}}, \\
& \text { о́лоv } \tau_{w}=\left[\mu \frac{\partial u}{\partial y}\right]_{y=0} \kappa \alpha \mathrm{l} \dot{q}_{w}=-\left[k \frac{\partial T}{\partial y}\right]_{y=0} .
\end{aligned}
$$

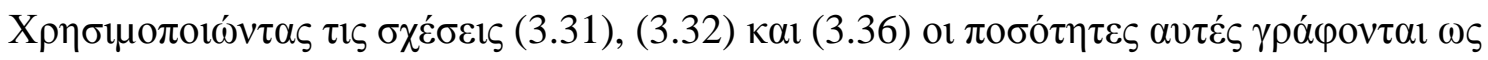

$$
C_{f_{x}}=\frac{2 C_{w}}{\sqrt{R_{x}}} f_{w}^{\prime \prime} \kappa \alpha \mathrm{l} S t_{x}=\frac{C_{w} S_{w}^{\prime}}{\operatorname{Pr} \sqrt{R_{x}}\left(1-S_{w}\right)} \quad\left(S_{w} \neq 1\right),
$$

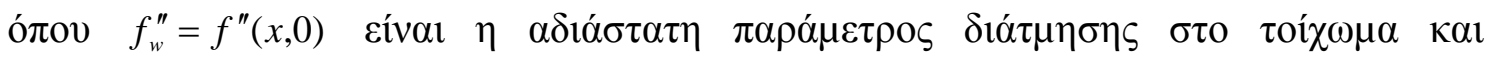

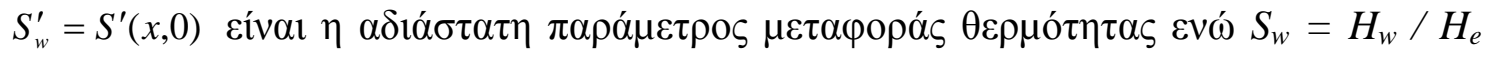

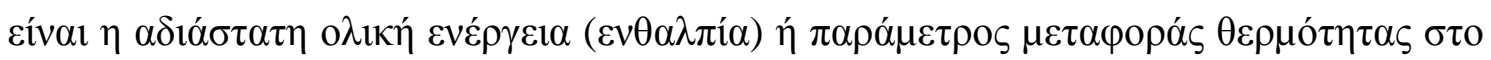
$\tau o i ́ \chi \omega \mu \alpha$.

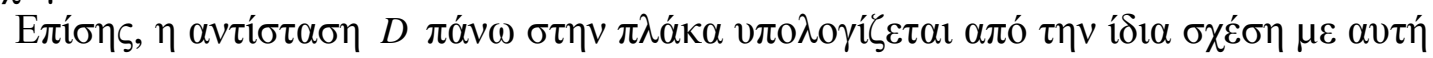

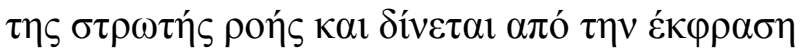

$$
D=\int_{0}^{x^{*}} \frac{C_{w} f_{w}^{\prime \prime}(x, 0)}{\sqrt{\operatorname{Re}_{x}}} \rho_{e}(x) u_{e}^{2}(x) d x,
$$

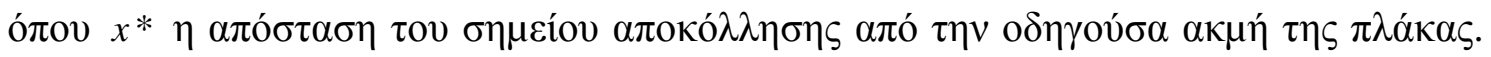

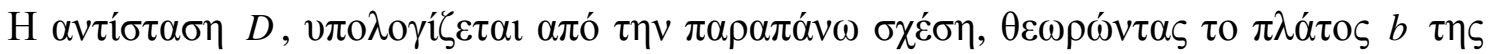

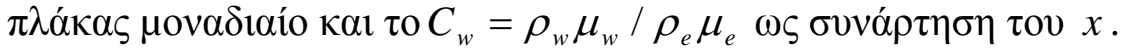

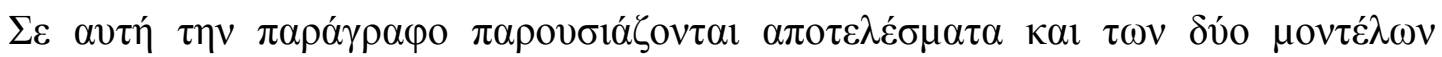

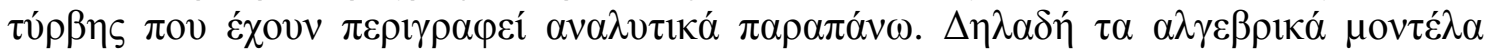

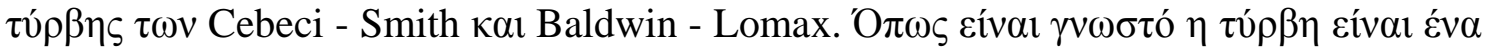

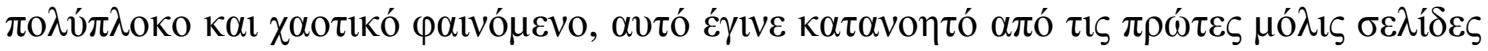

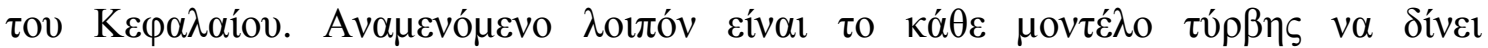

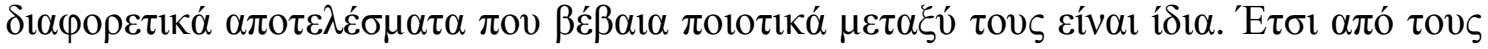

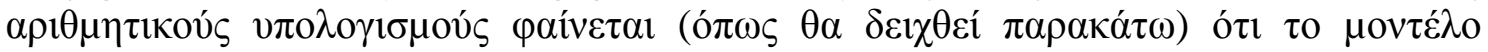

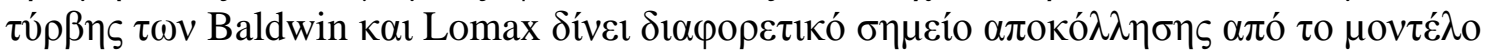

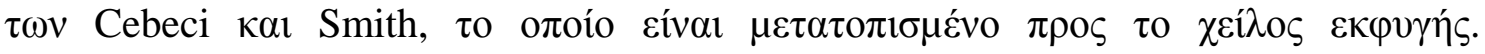

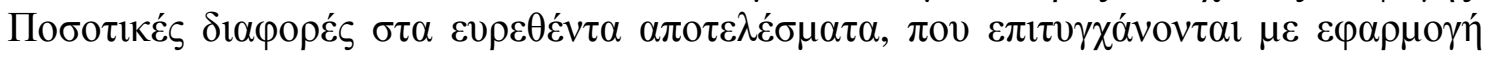




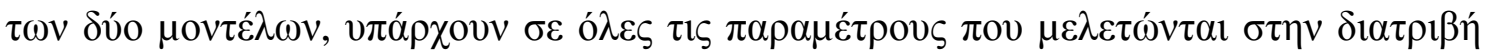

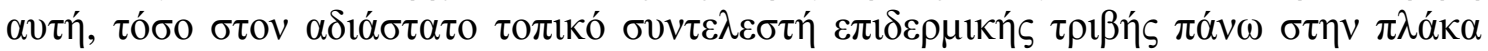

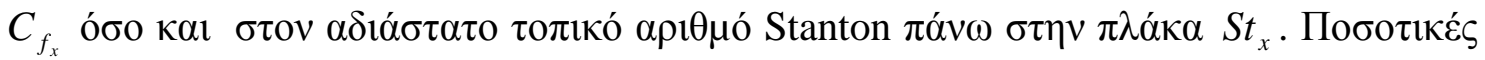

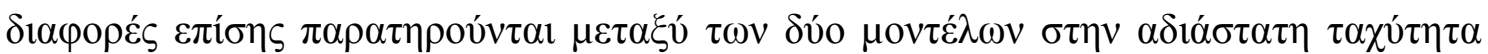

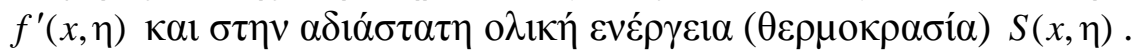

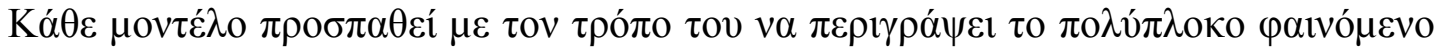

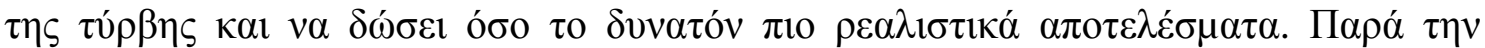

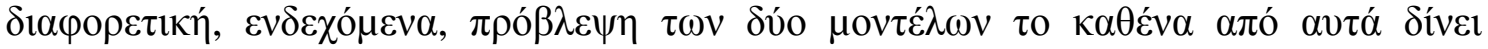

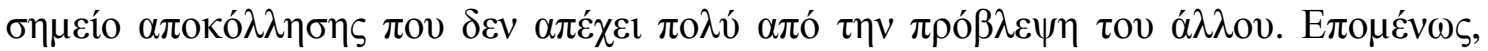

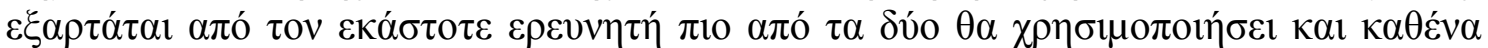

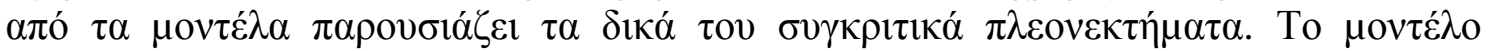

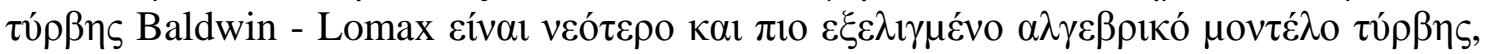

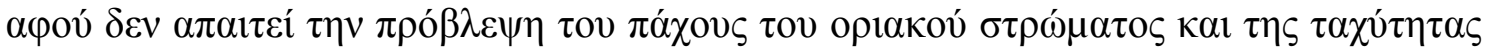

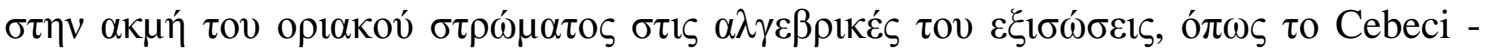

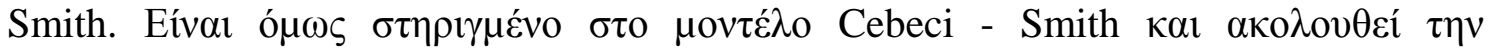

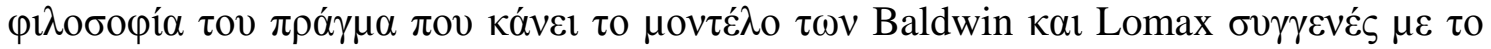

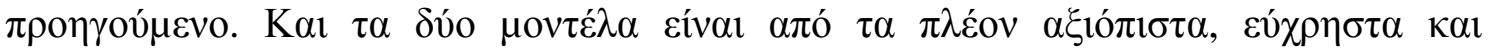

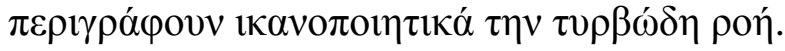

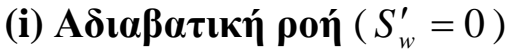

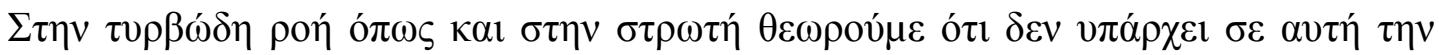

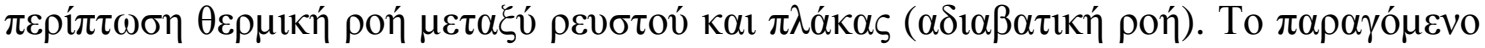

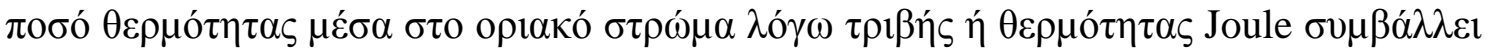

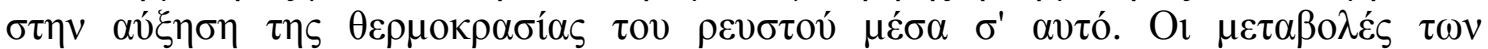

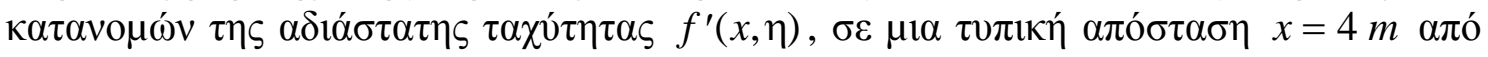

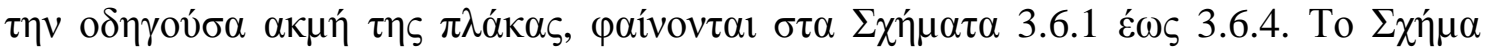

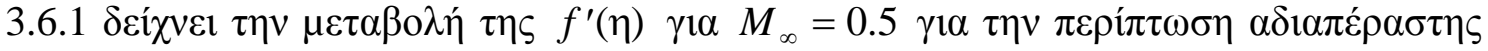

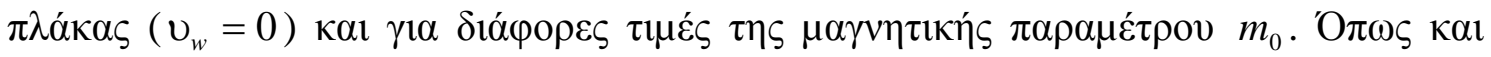

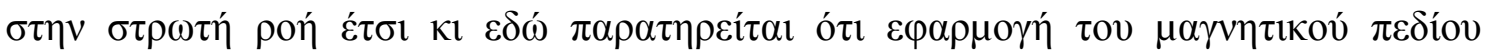

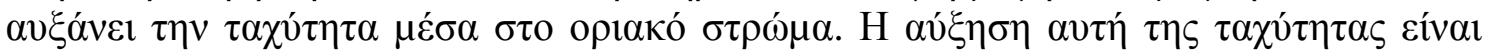

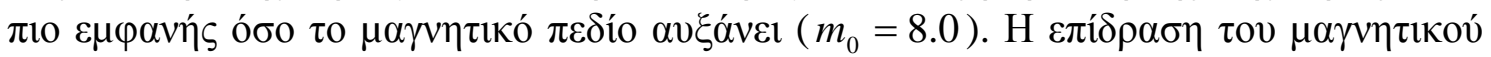

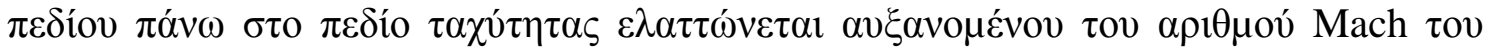

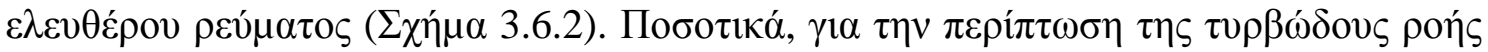

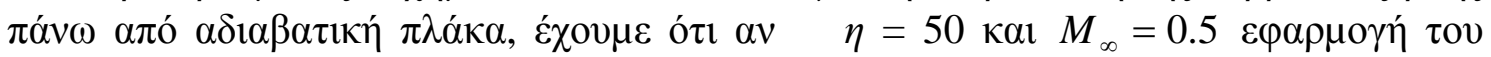

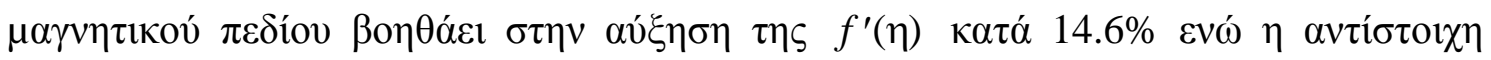

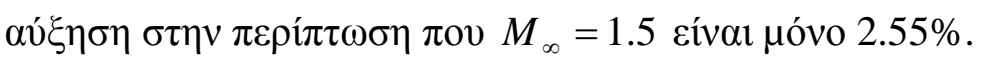

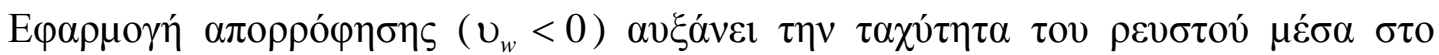

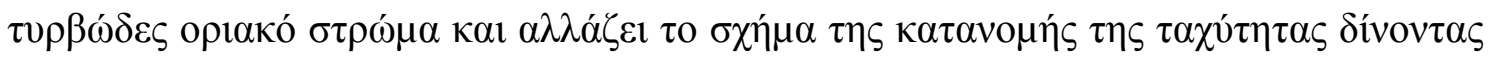

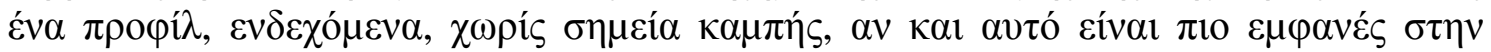

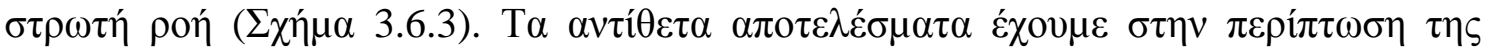

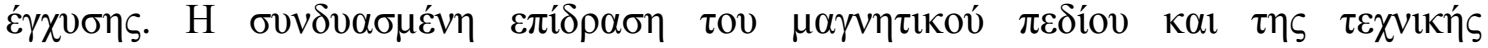

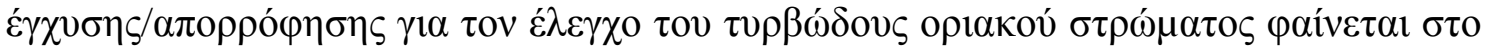

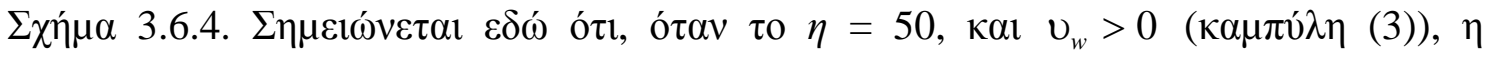

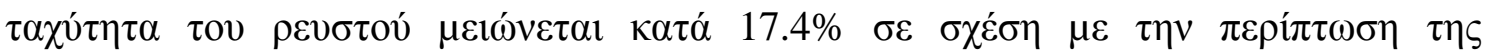

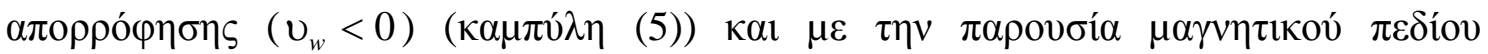
$\left(m_{0}=8.0\right)$. 


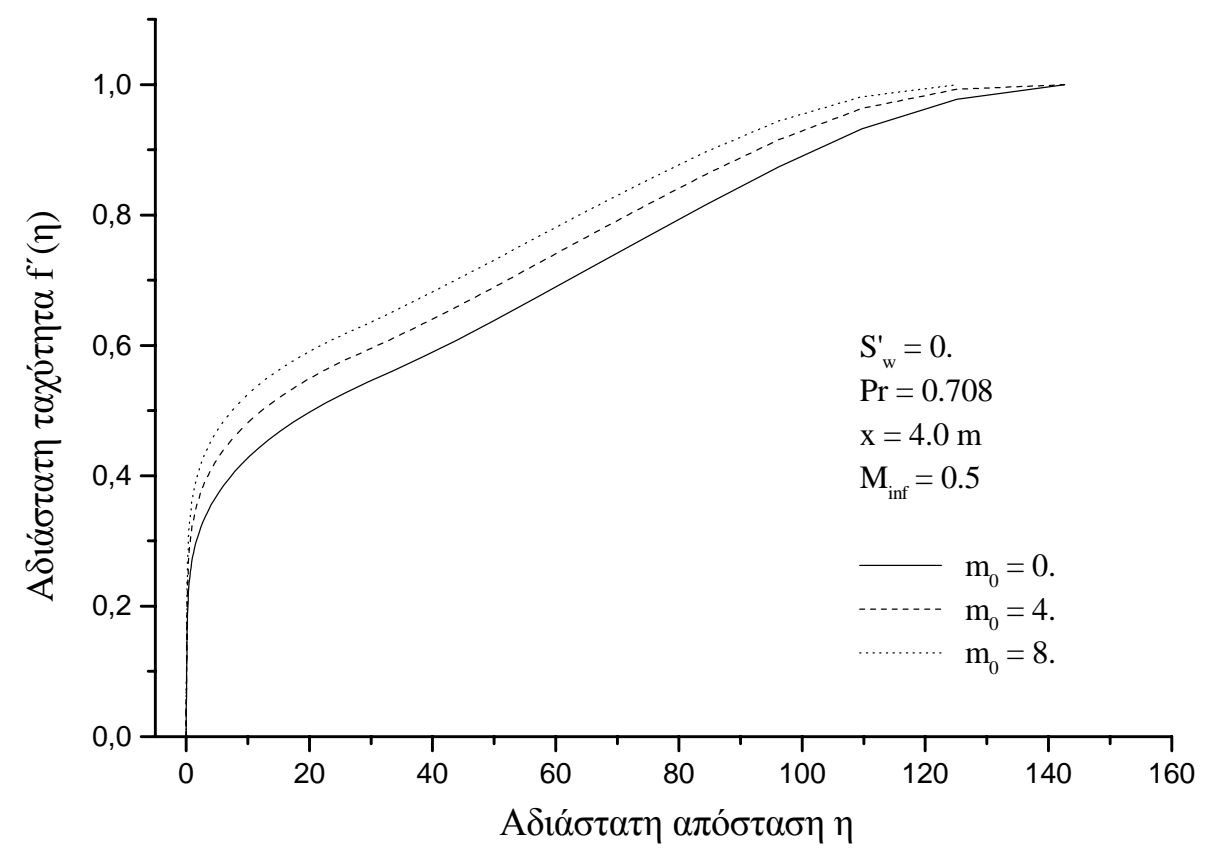

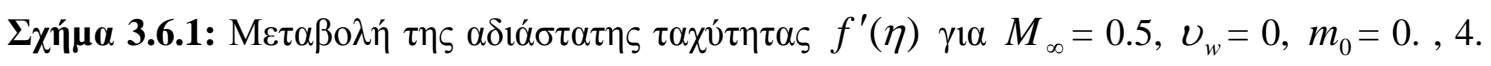

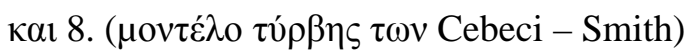

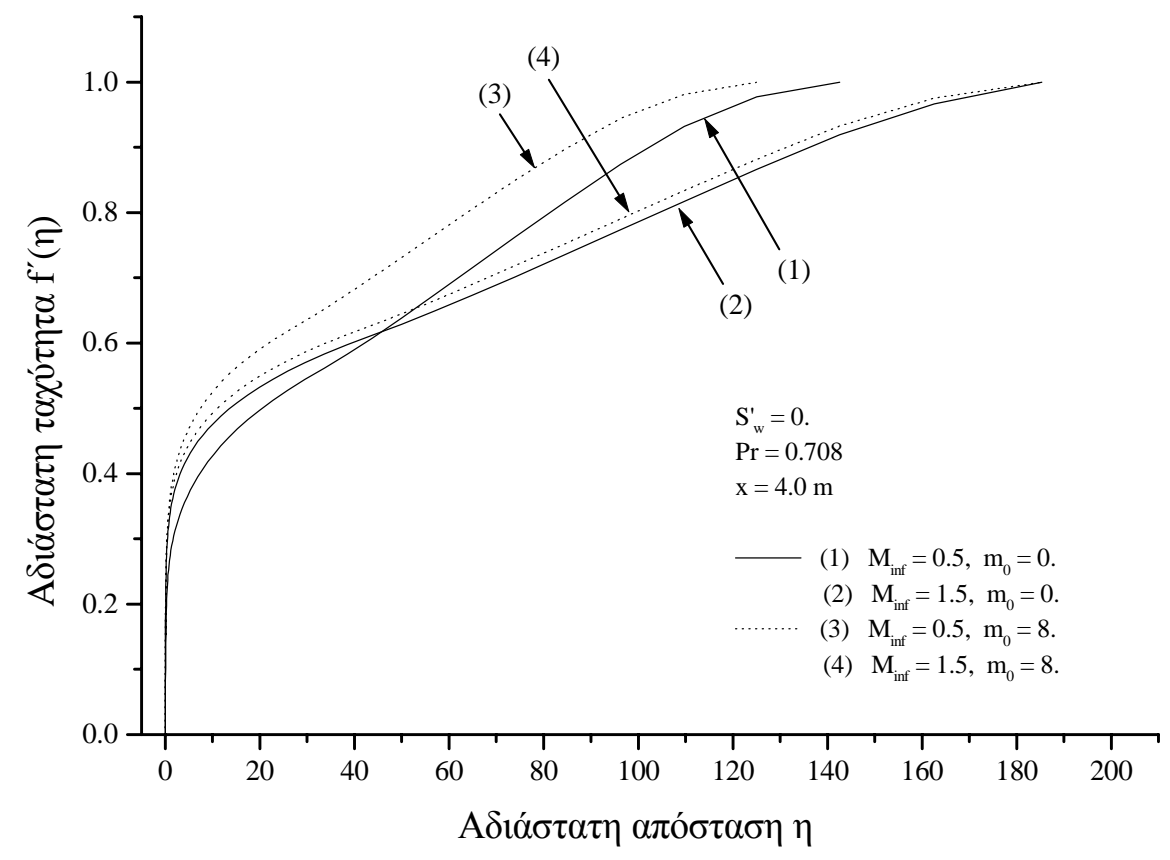

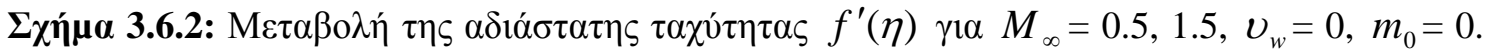

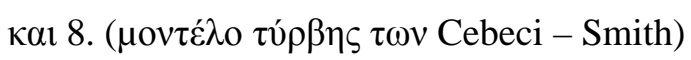




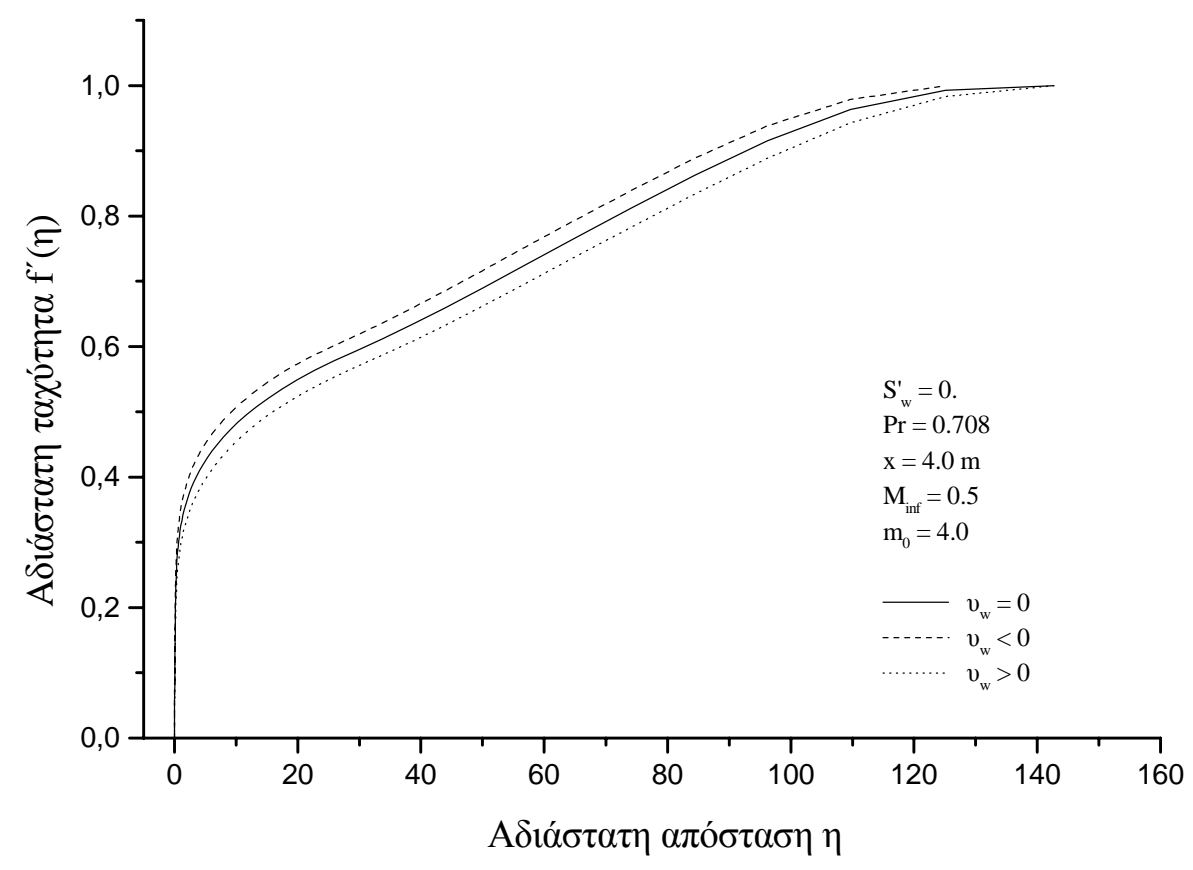

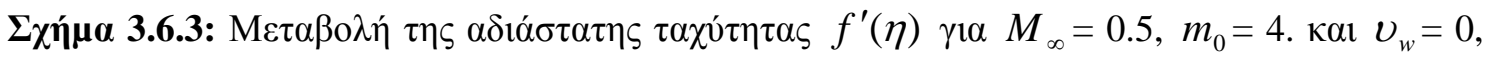

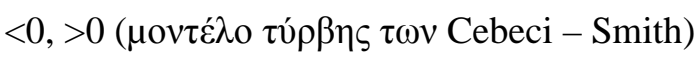

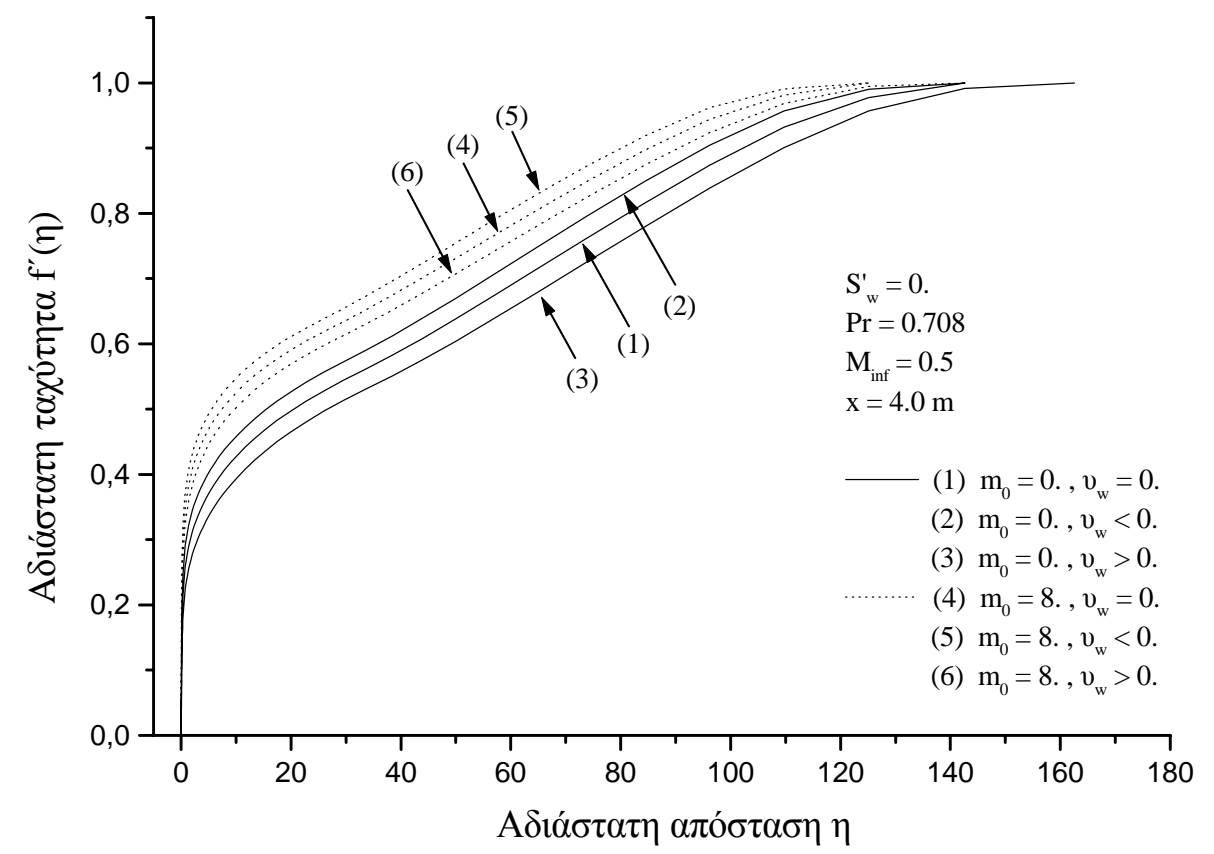

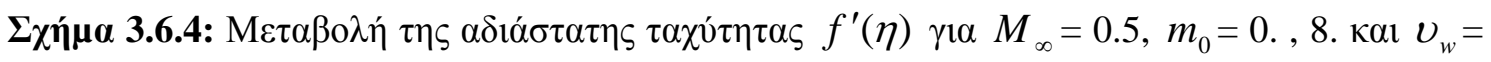

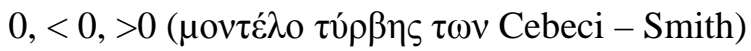




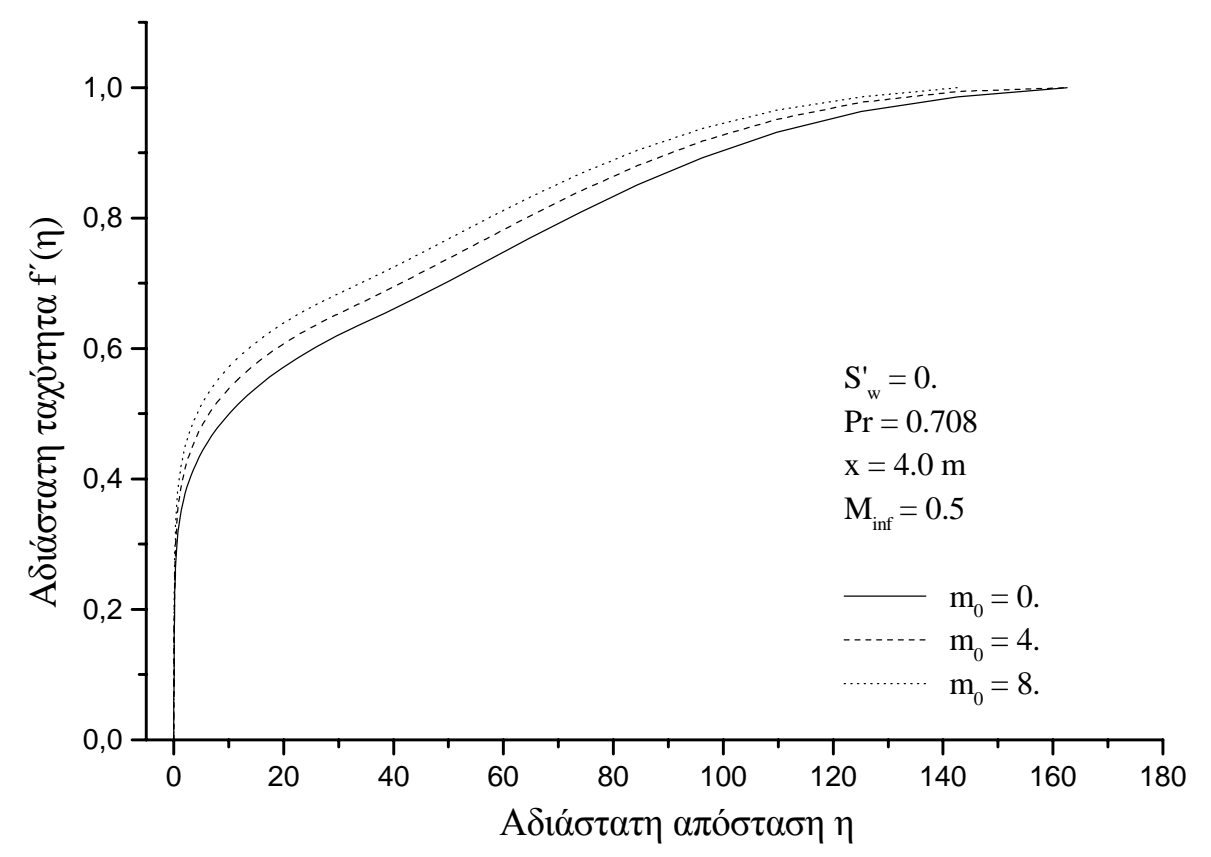

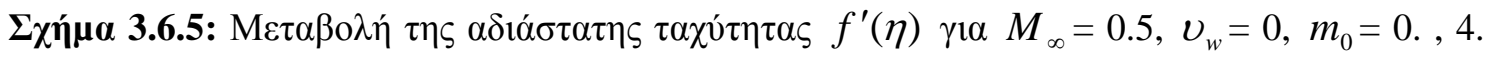

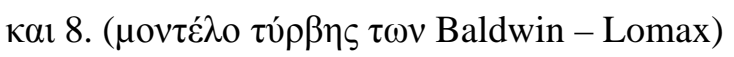

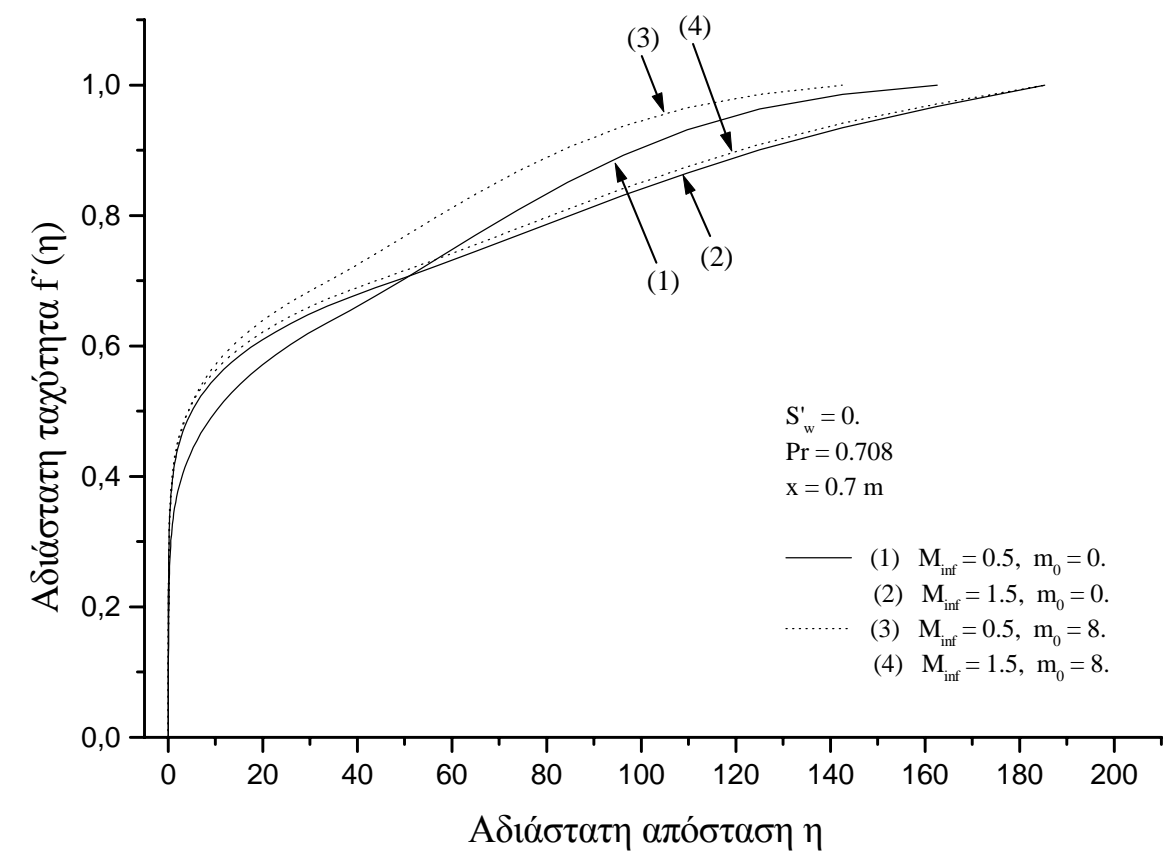

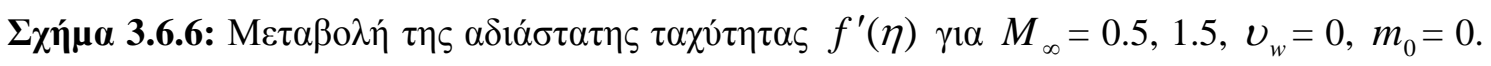

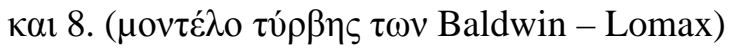




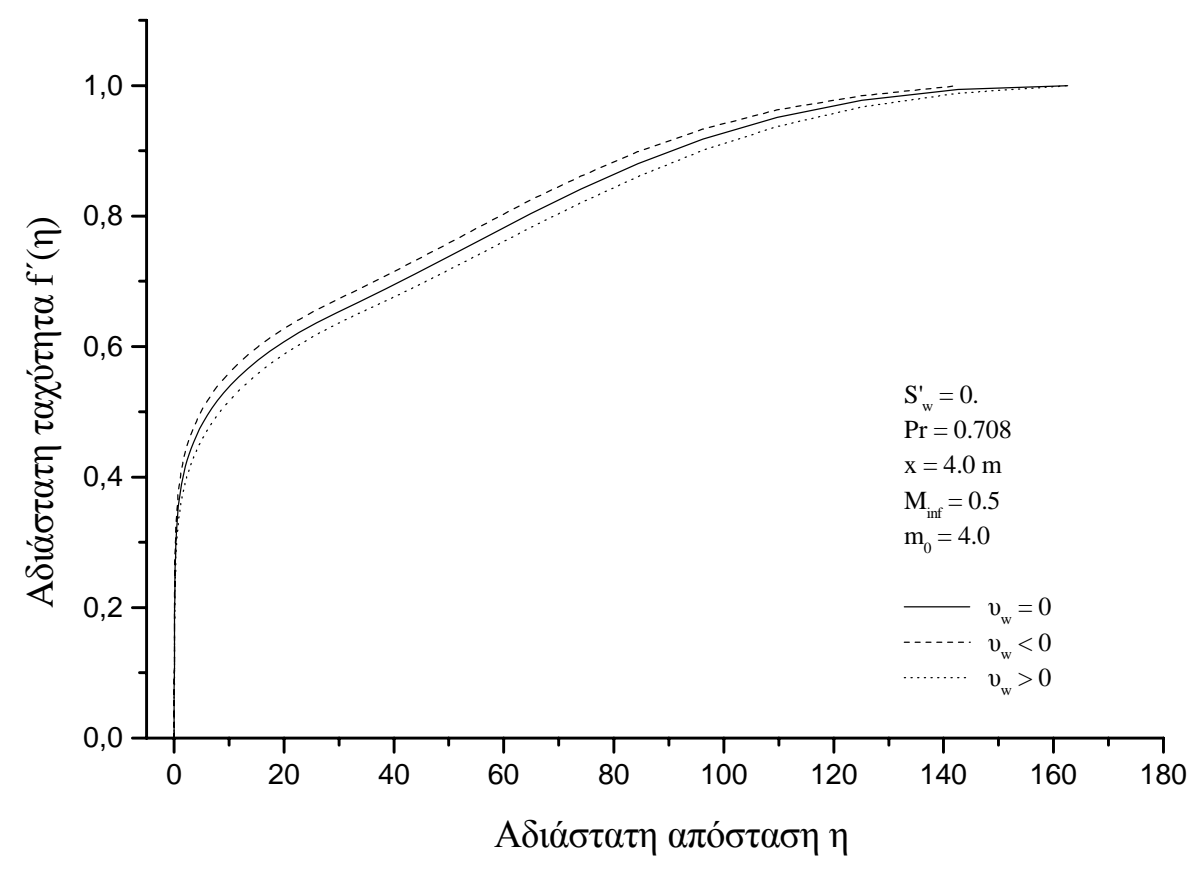

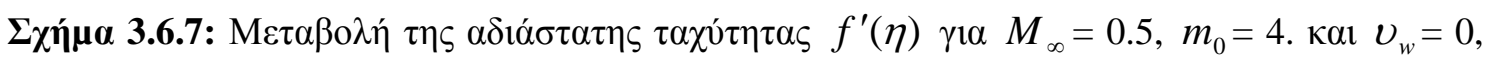

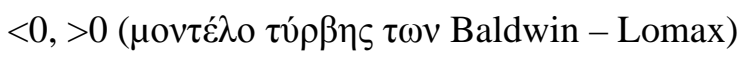

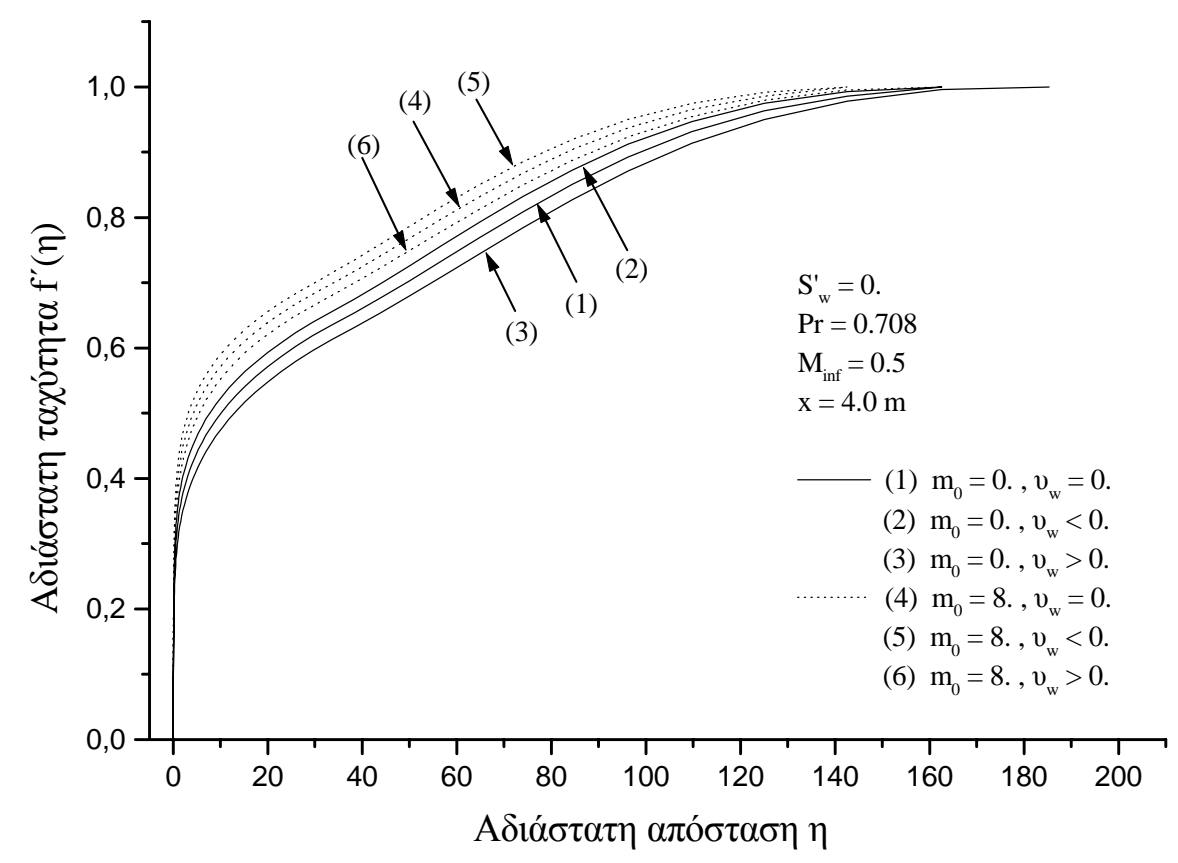

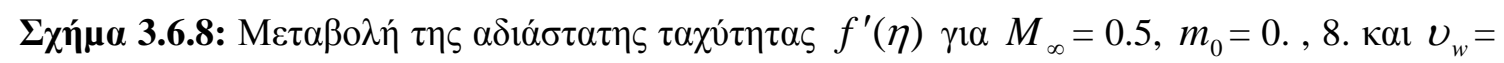

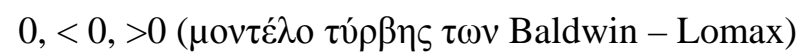




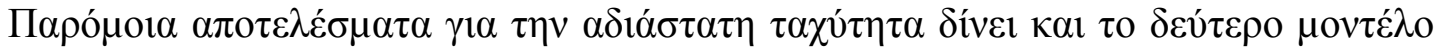

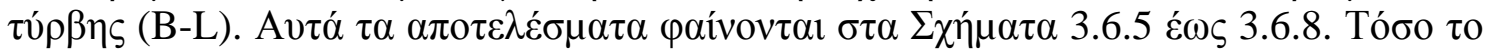

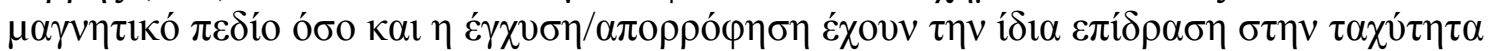

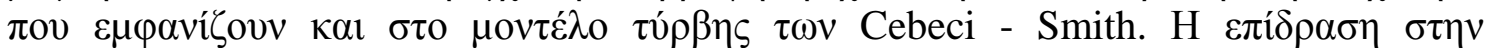

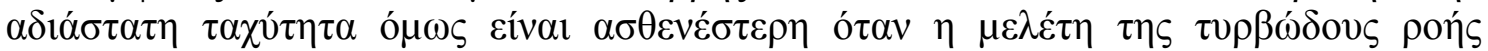

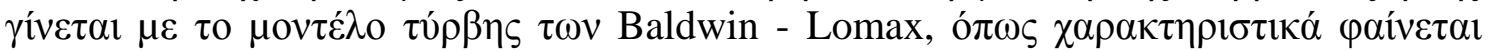

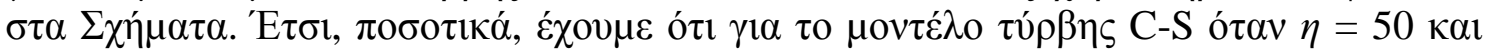

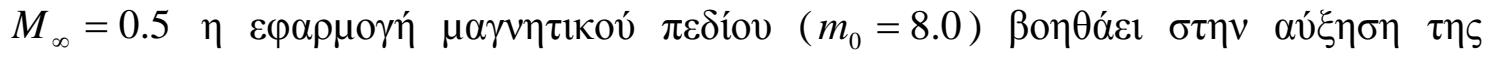

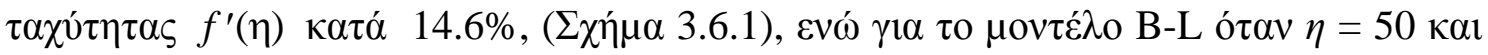

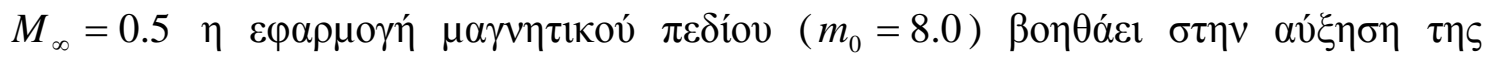

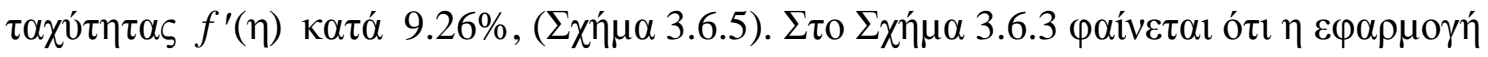

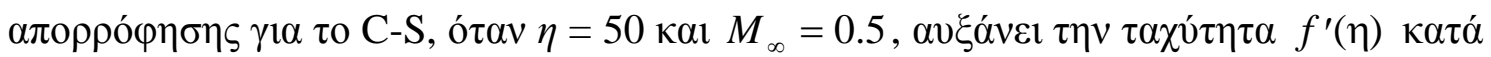

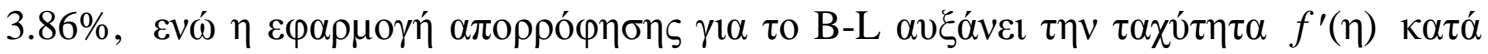

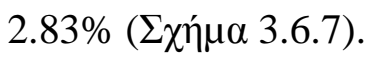

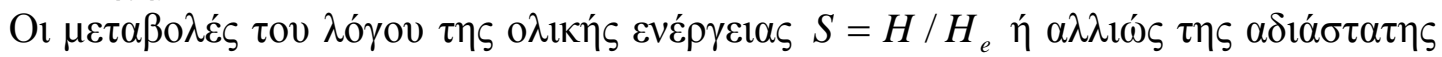

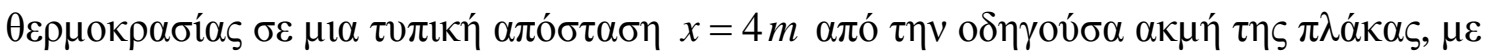

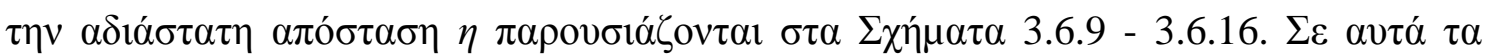

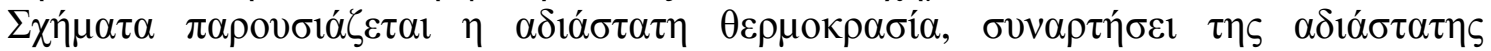

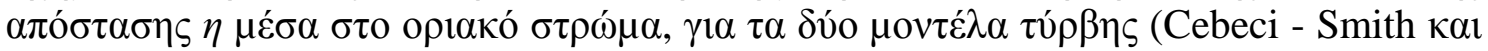

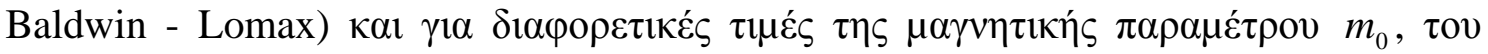

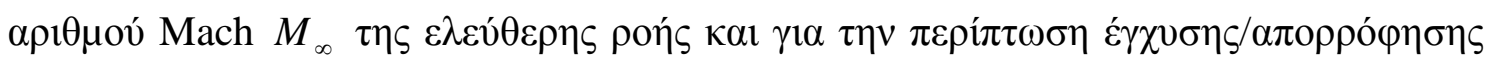

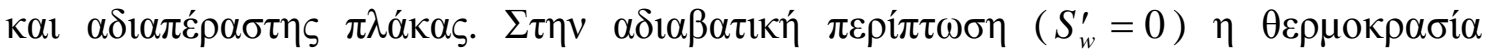

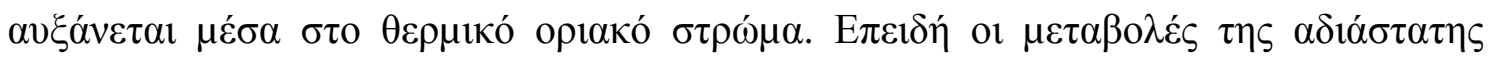

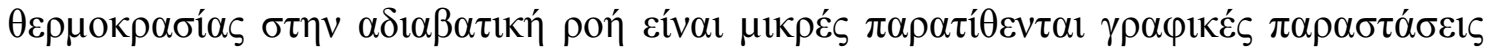

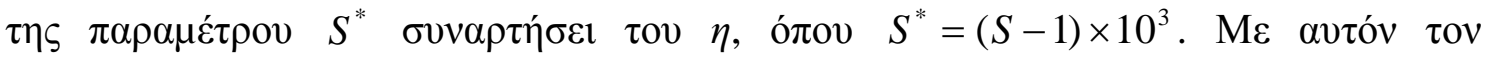

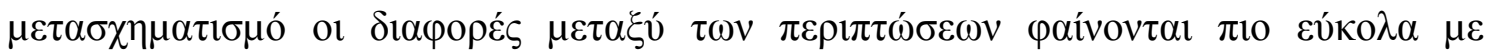

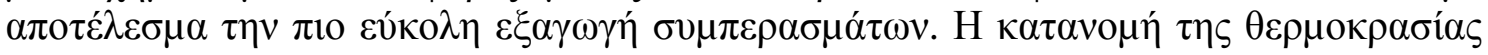

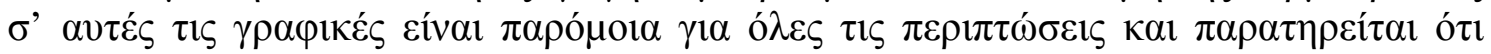
$\mu$

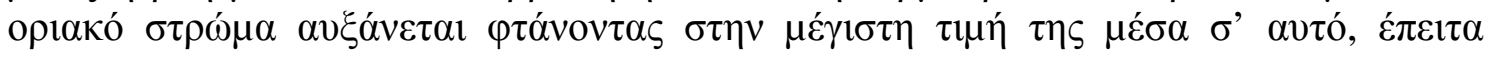

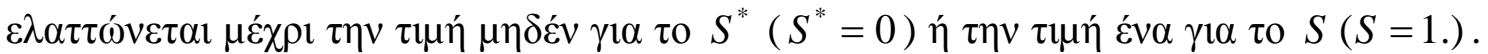

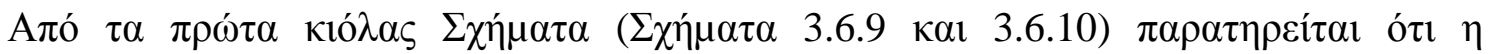

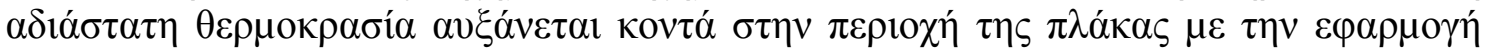

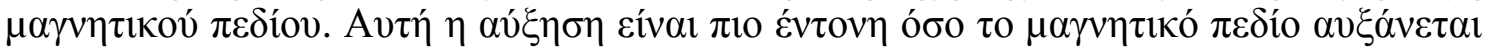

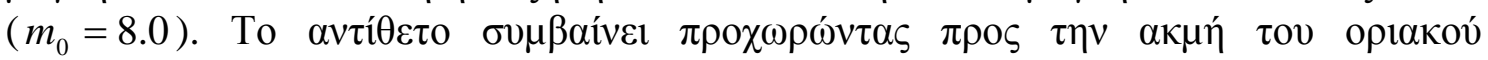

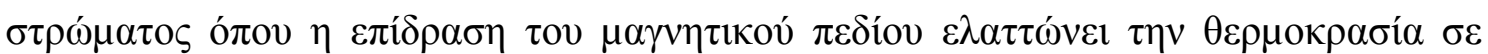

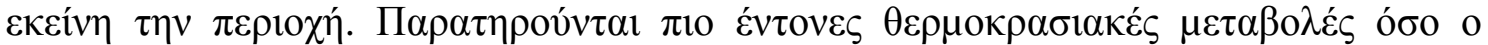

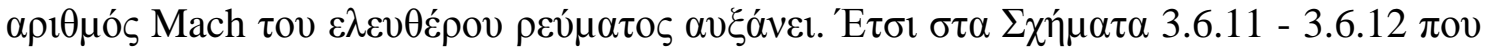

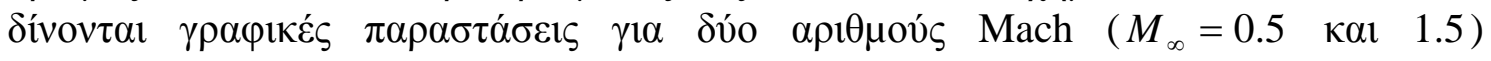

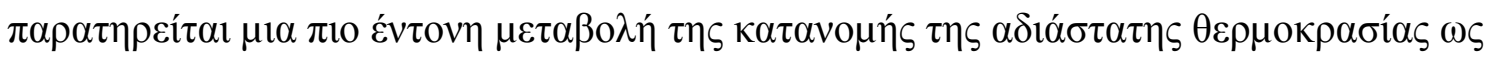

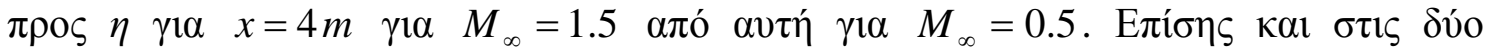

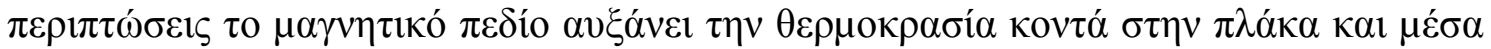

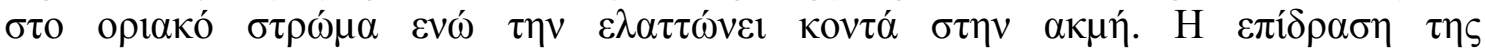

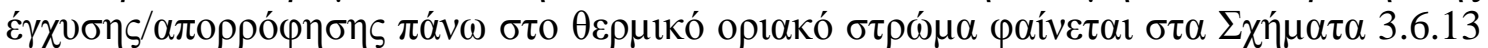

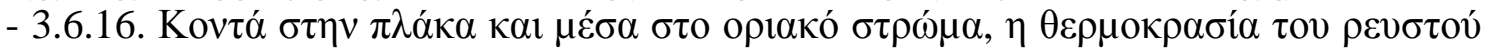

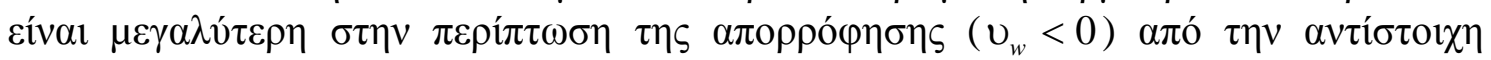

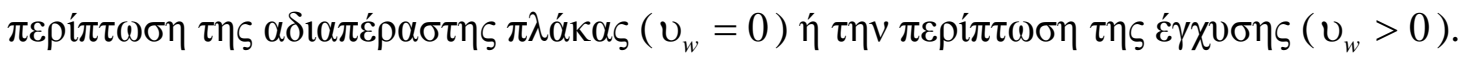




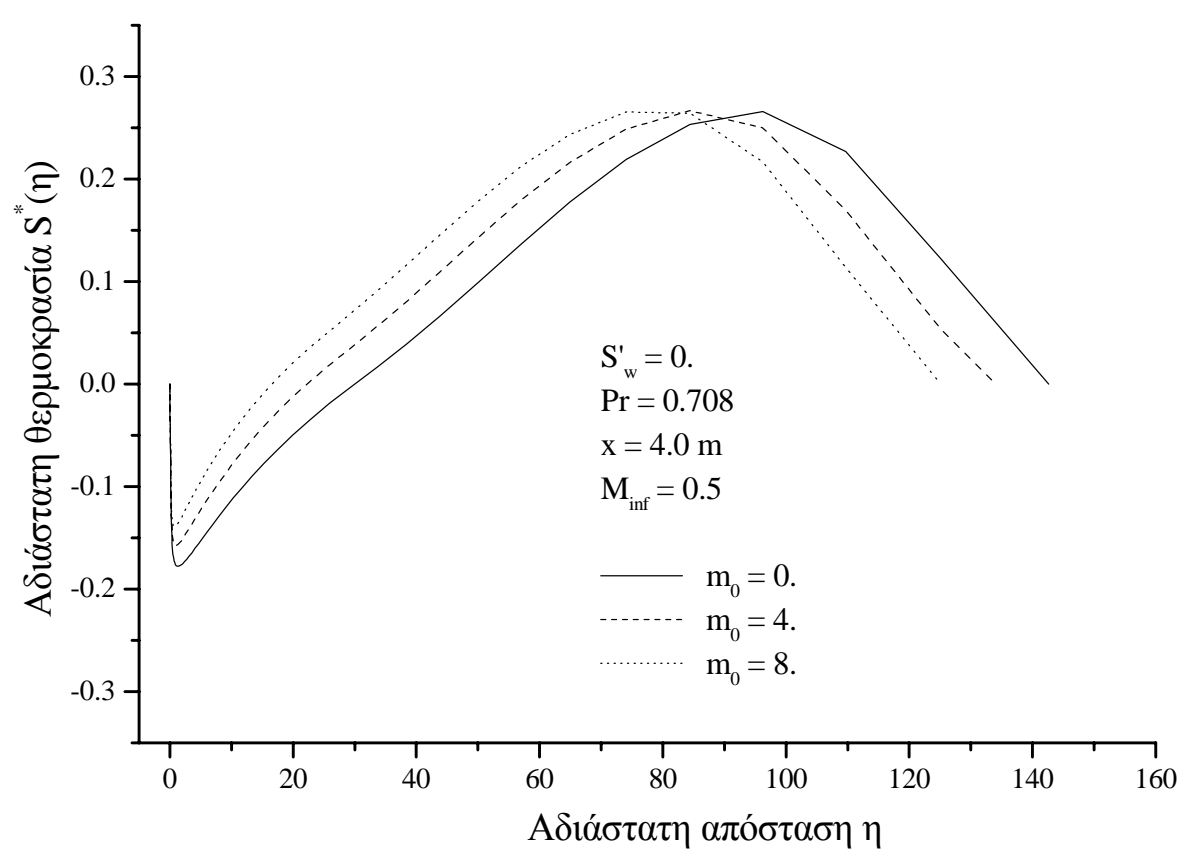

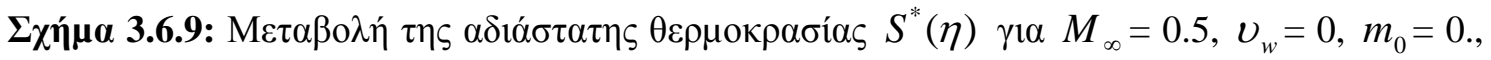

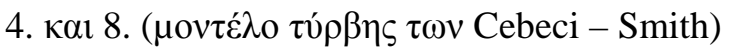

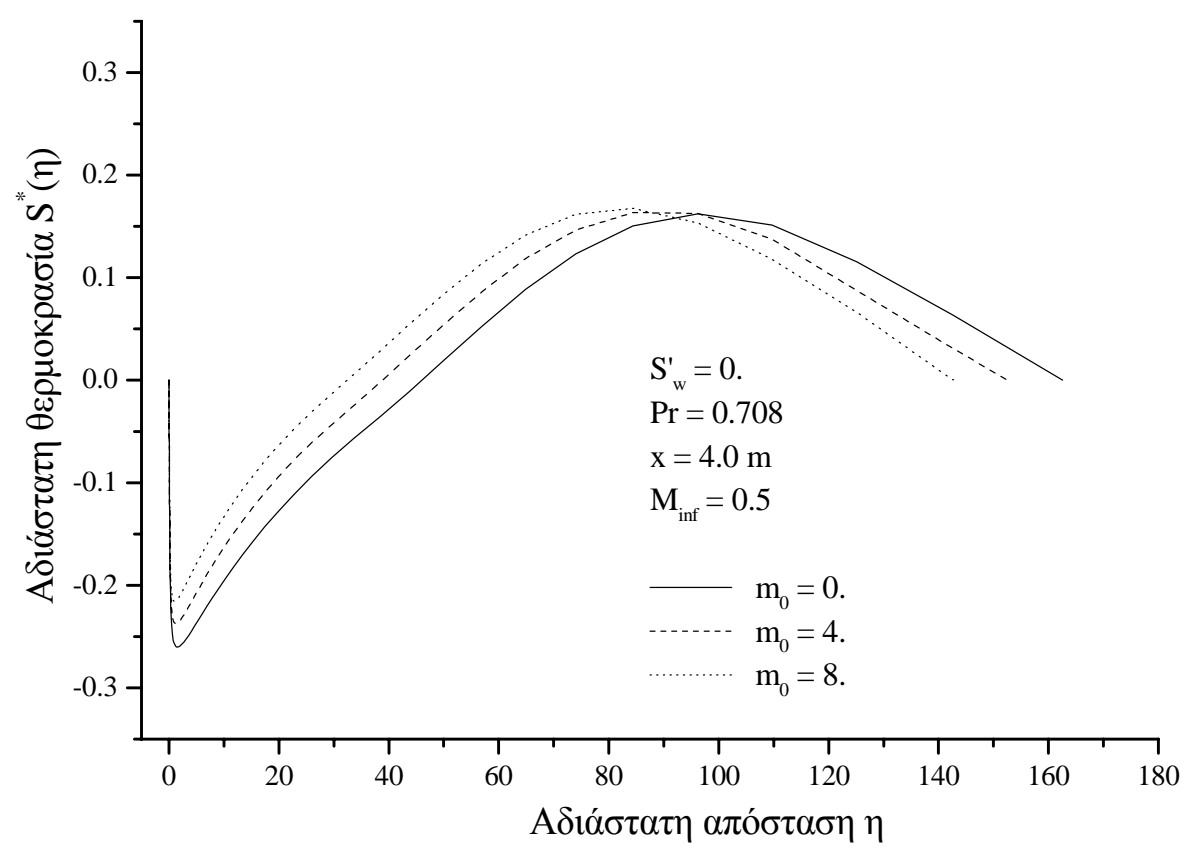

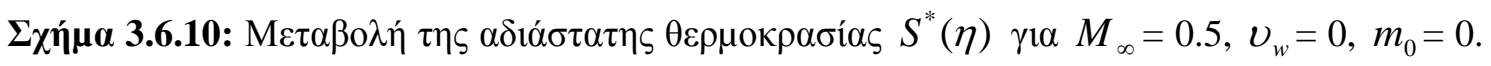

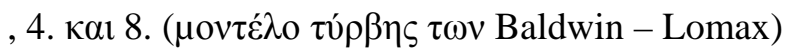




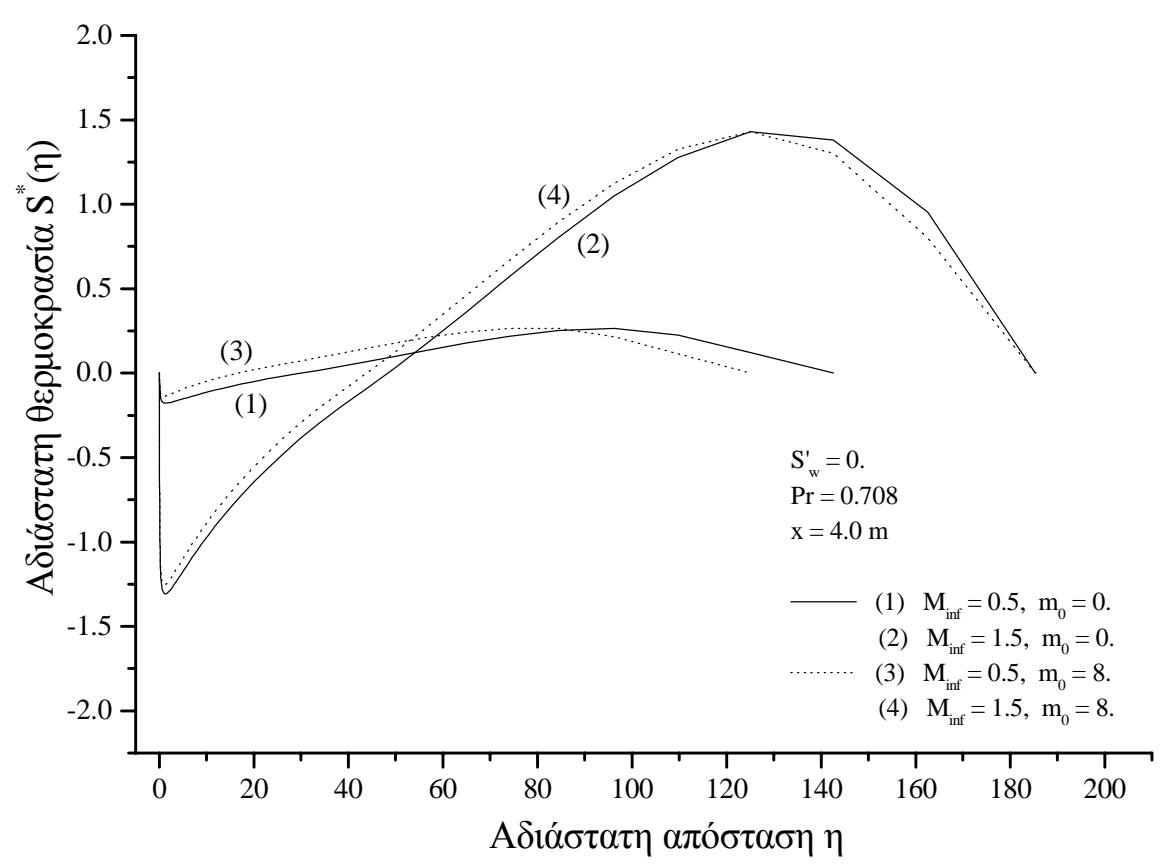

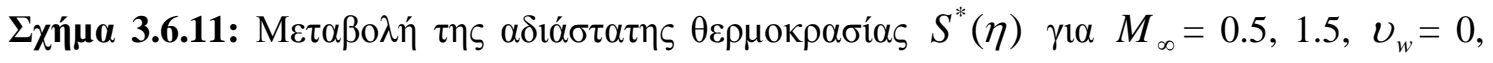

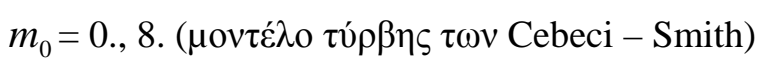

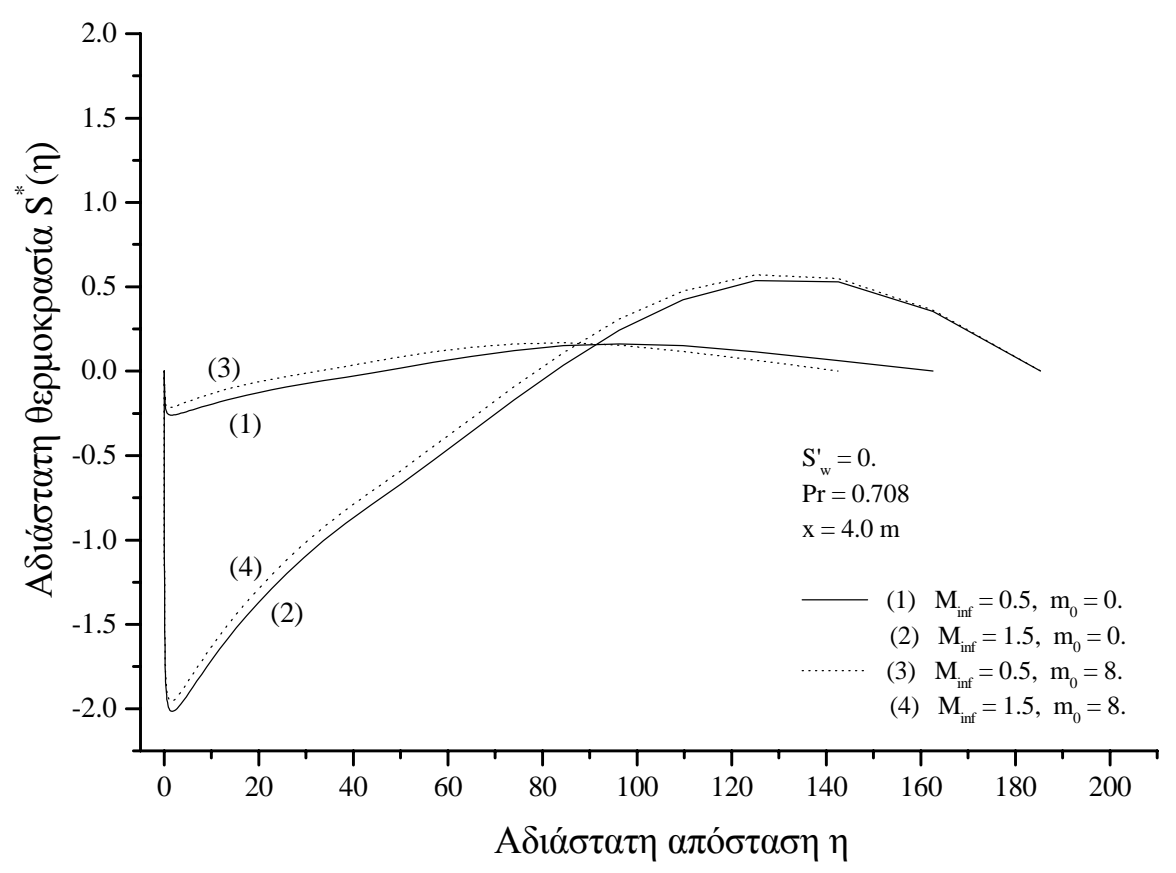

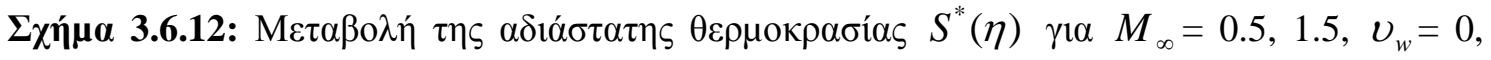

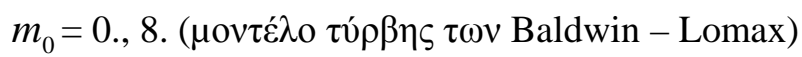




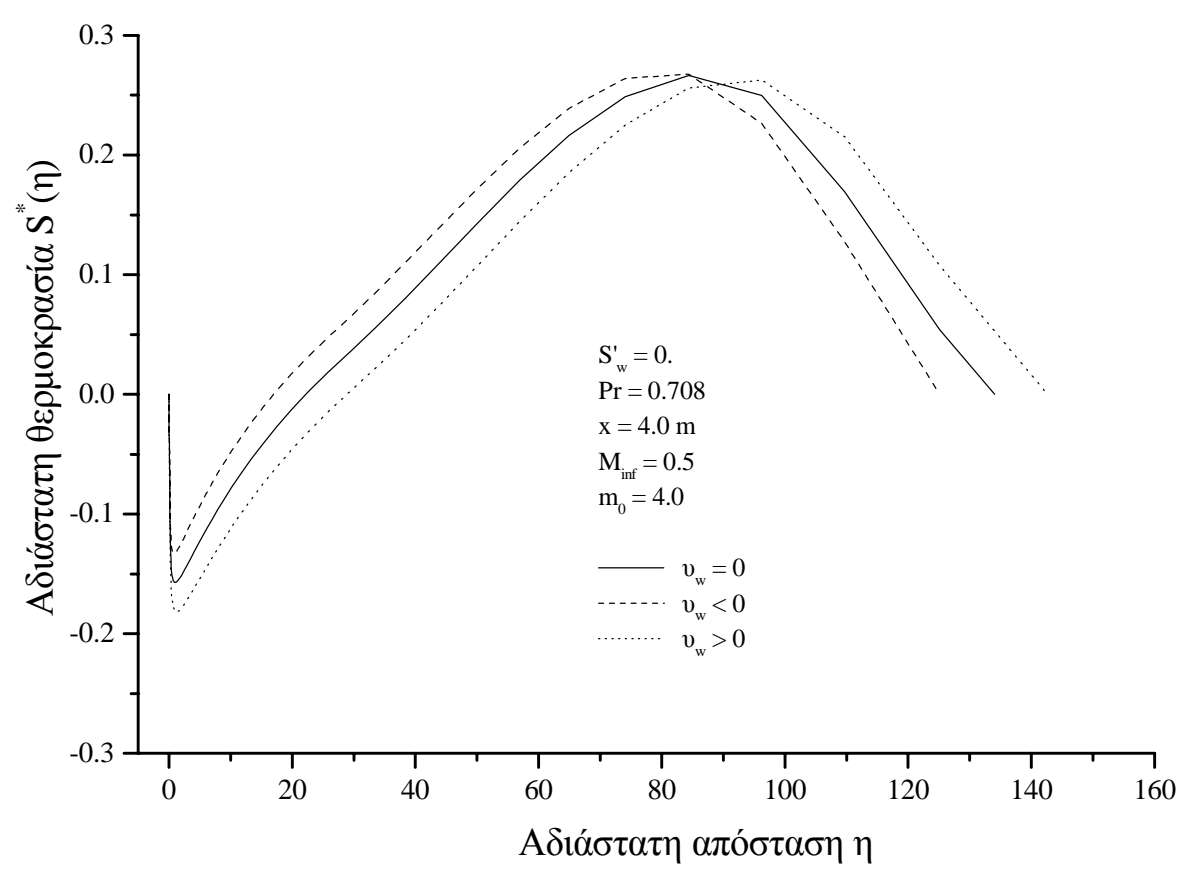

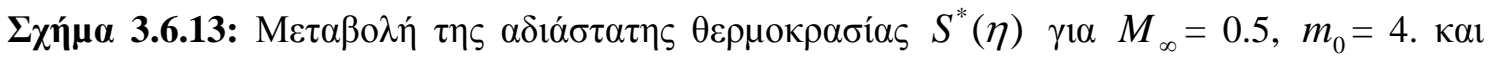

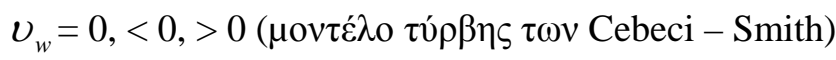

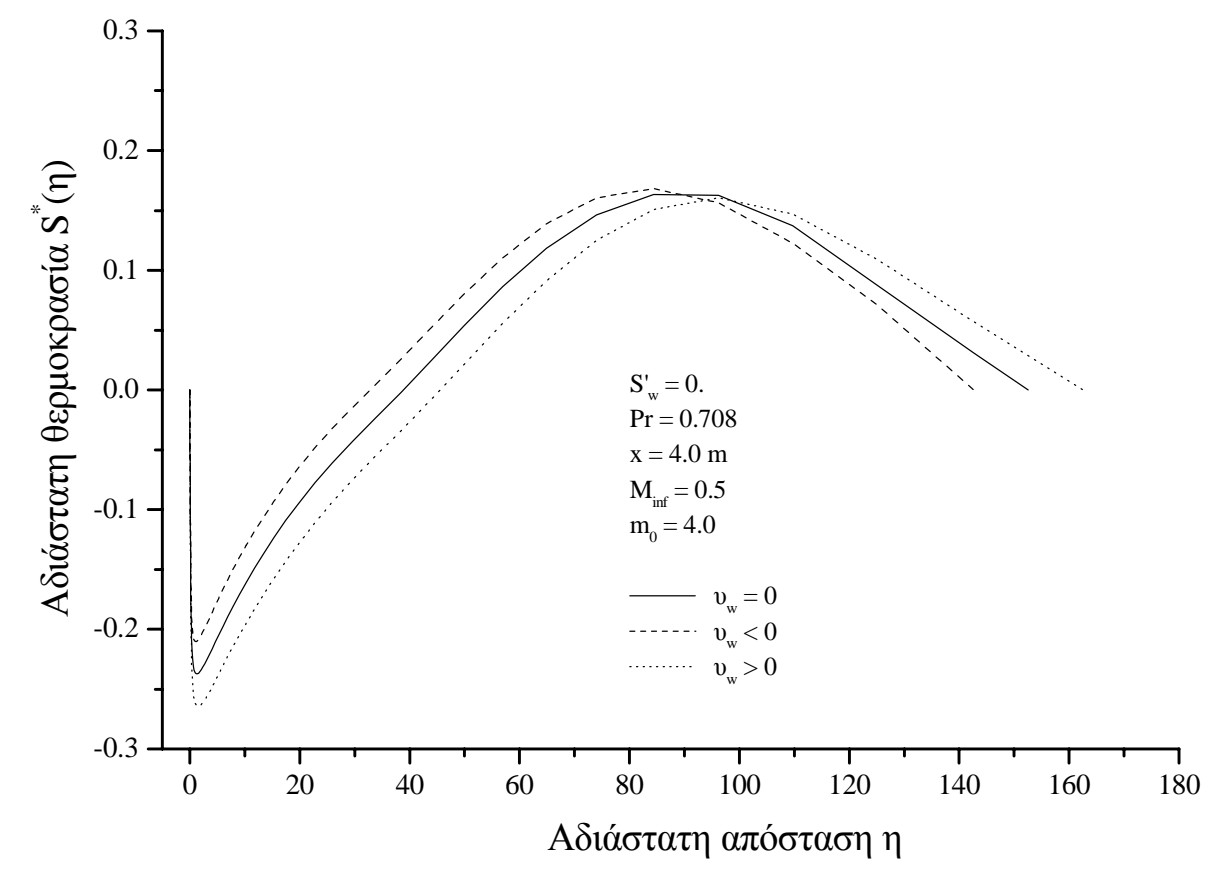

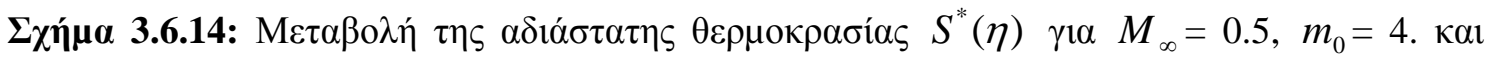

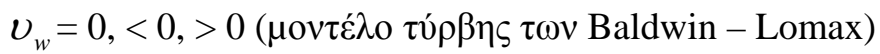




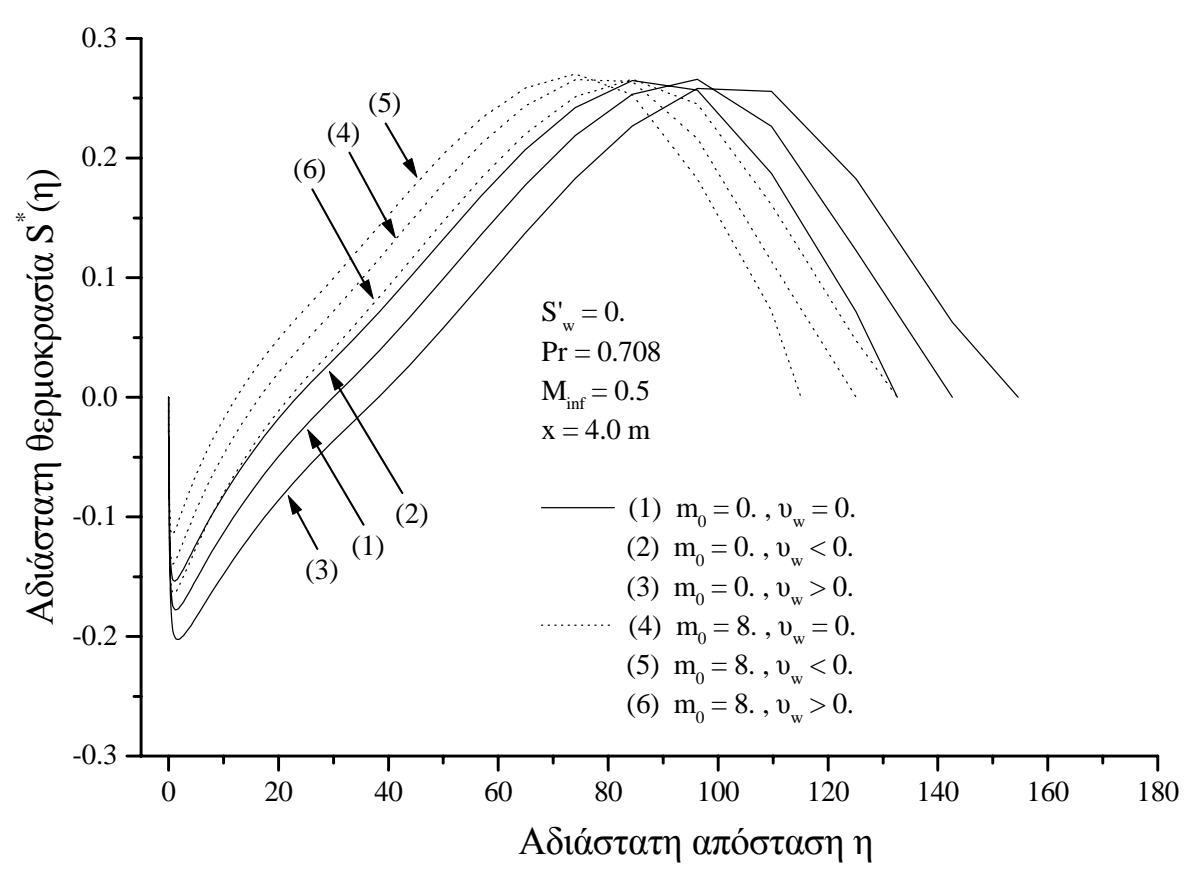

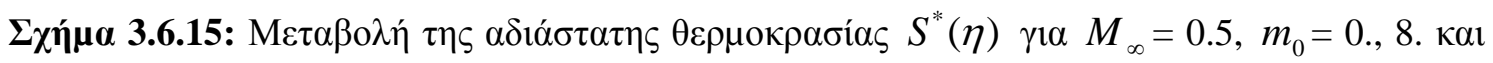

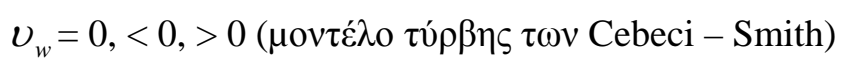

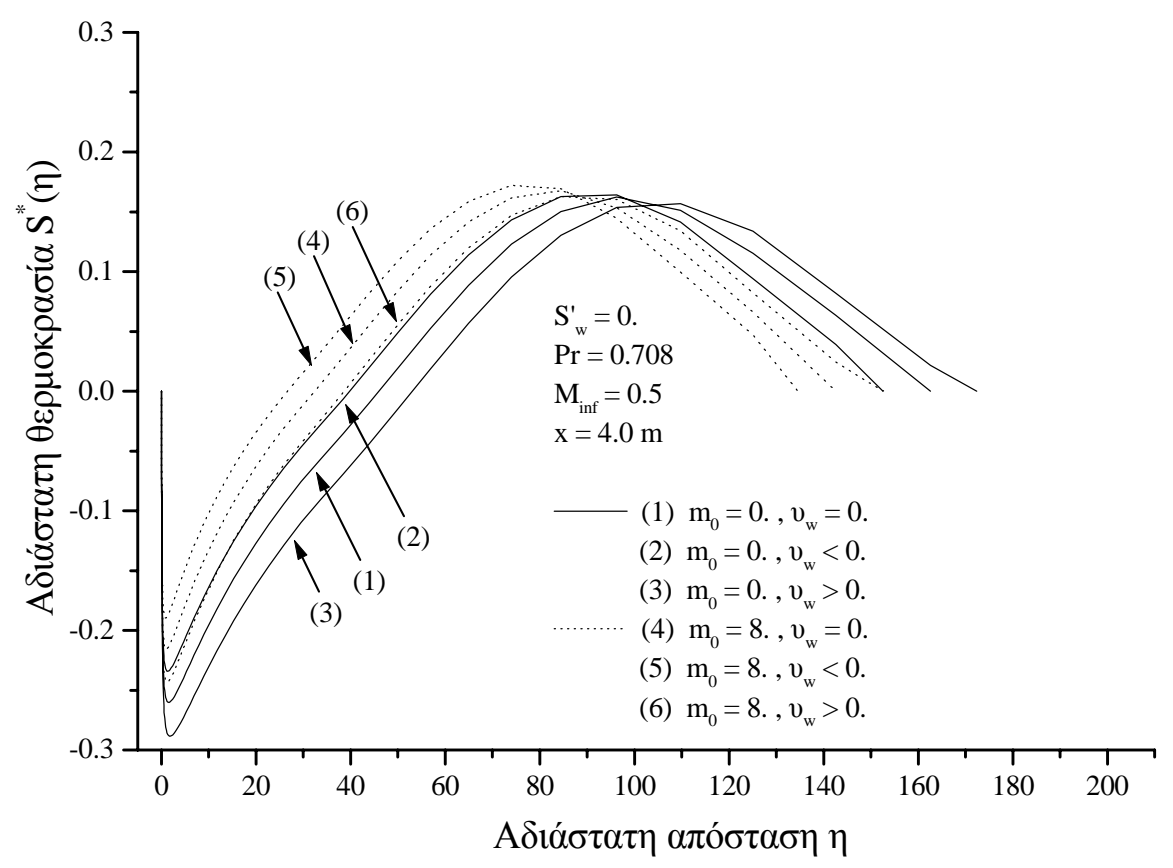

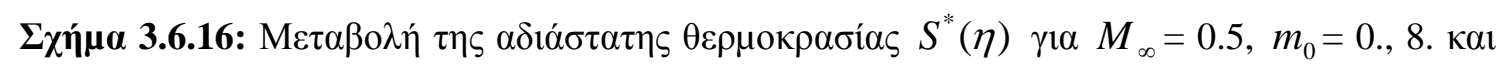

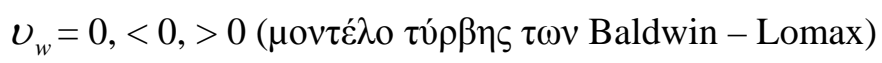




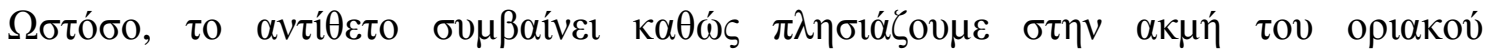

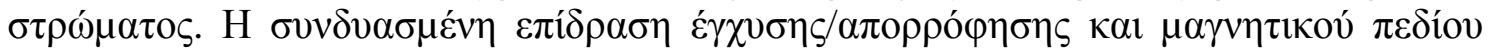

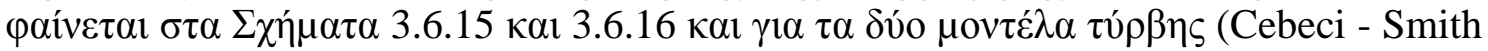

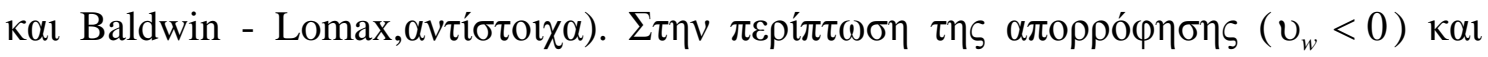

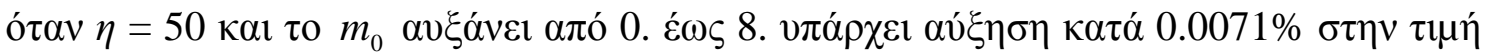

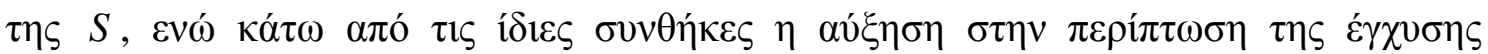

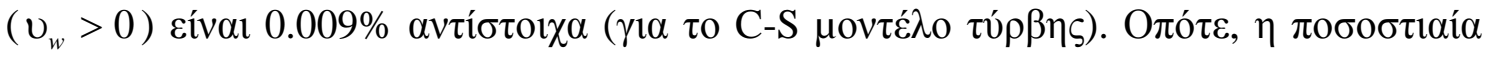

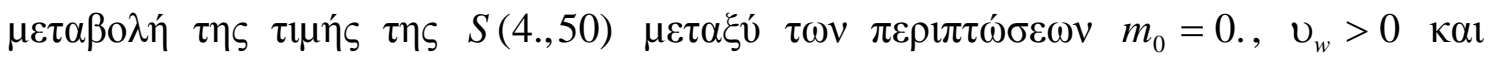

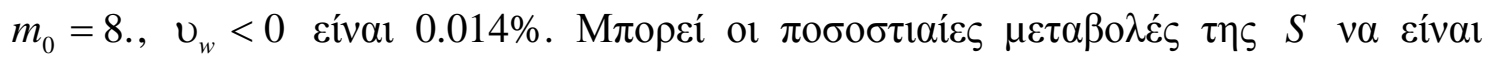

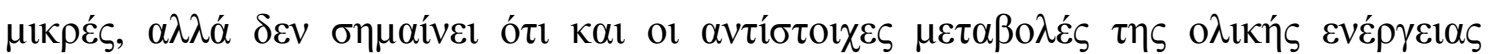

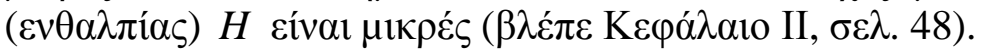

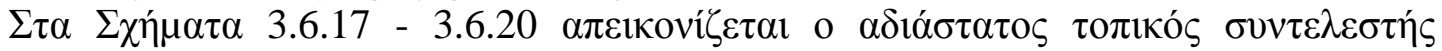

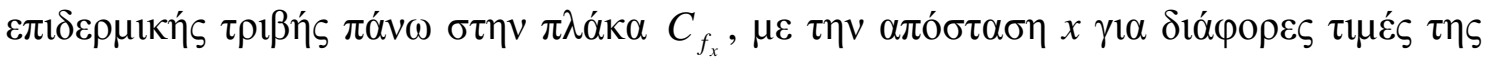

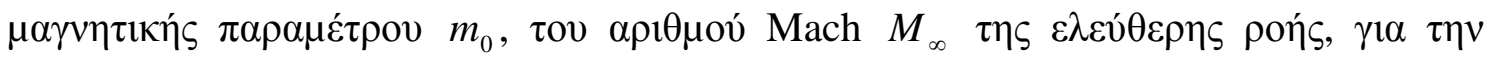

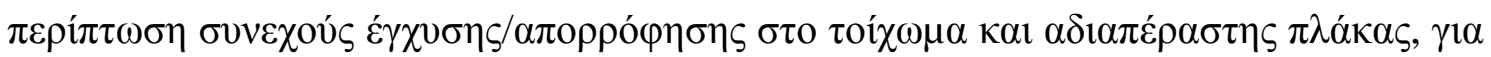

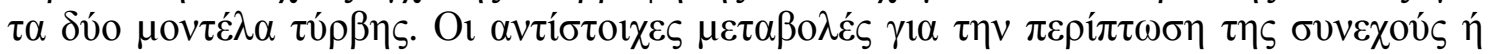

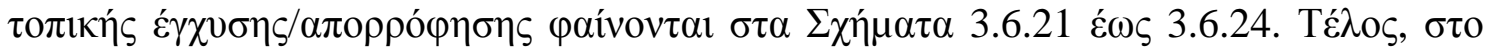

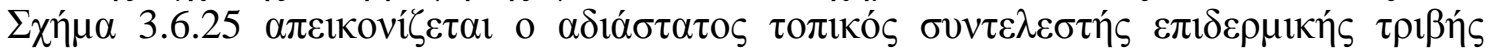

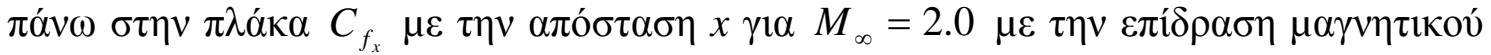

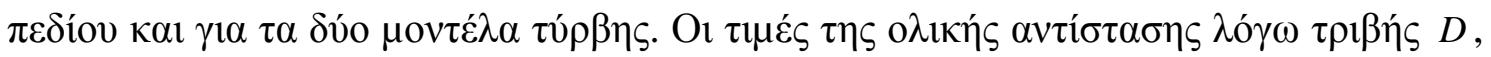

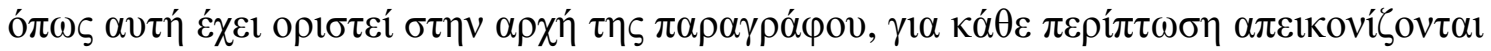

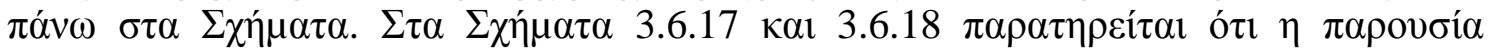

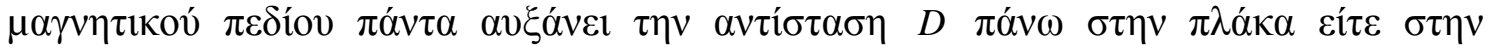

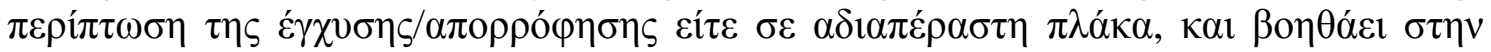

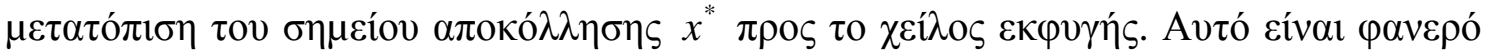

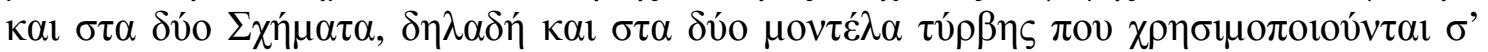

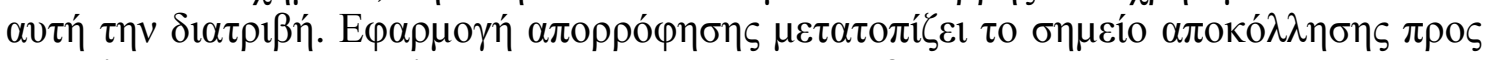

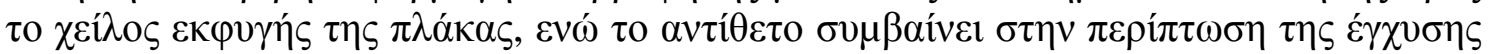

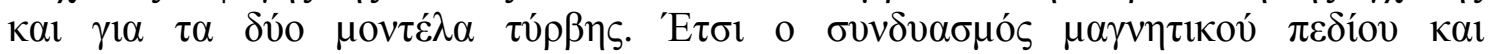

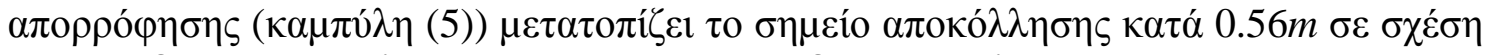

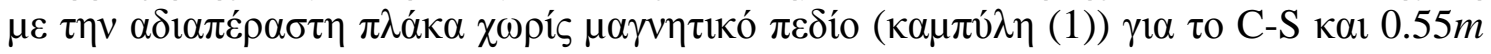

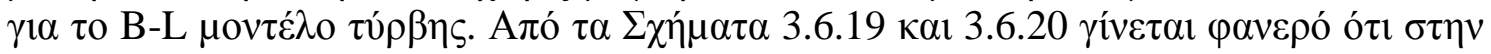

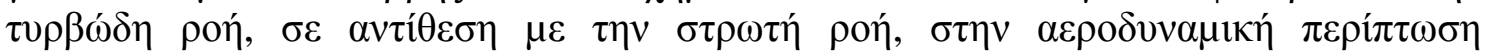

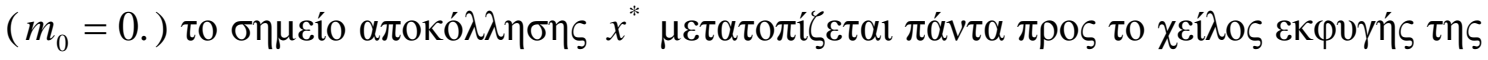

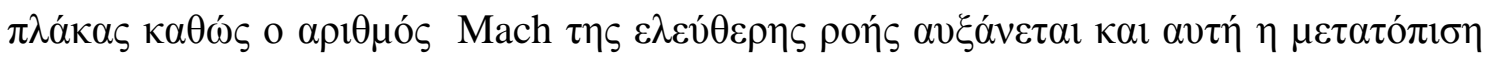

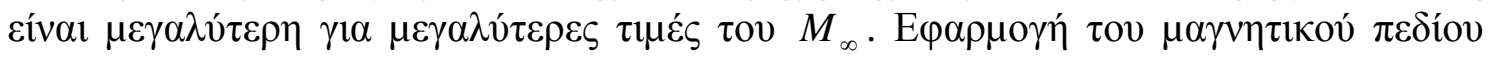

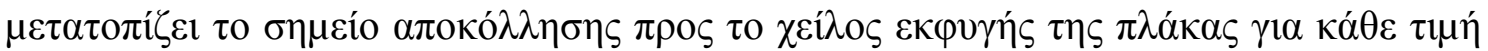

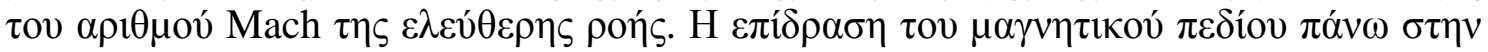

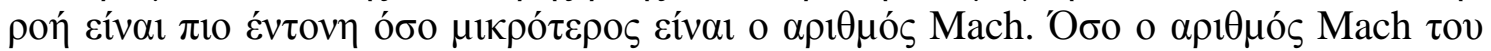

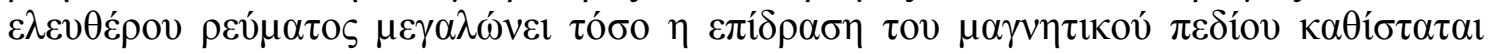
$\alpha \sigma \theta \varepsilon v \varepsilon ́ \sigma \tau \varepsilon \rho \eta$.

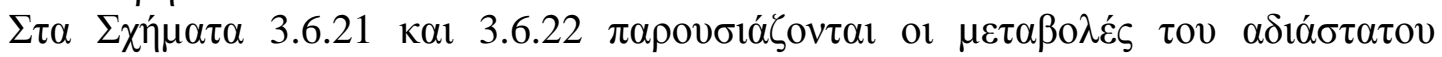

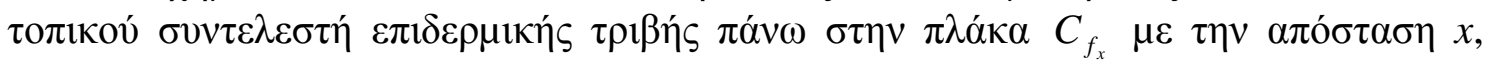

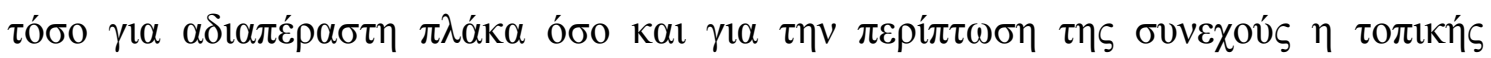

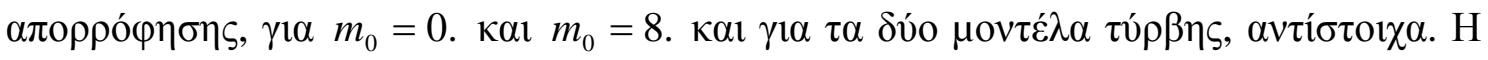

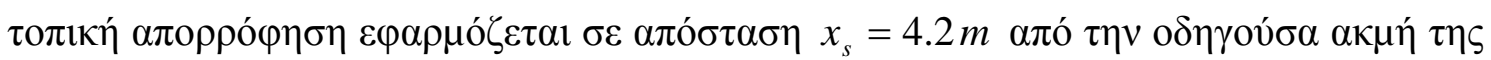
$\pi \lambda \alpha \dot{\kappa} \alpha \varsigma$. 


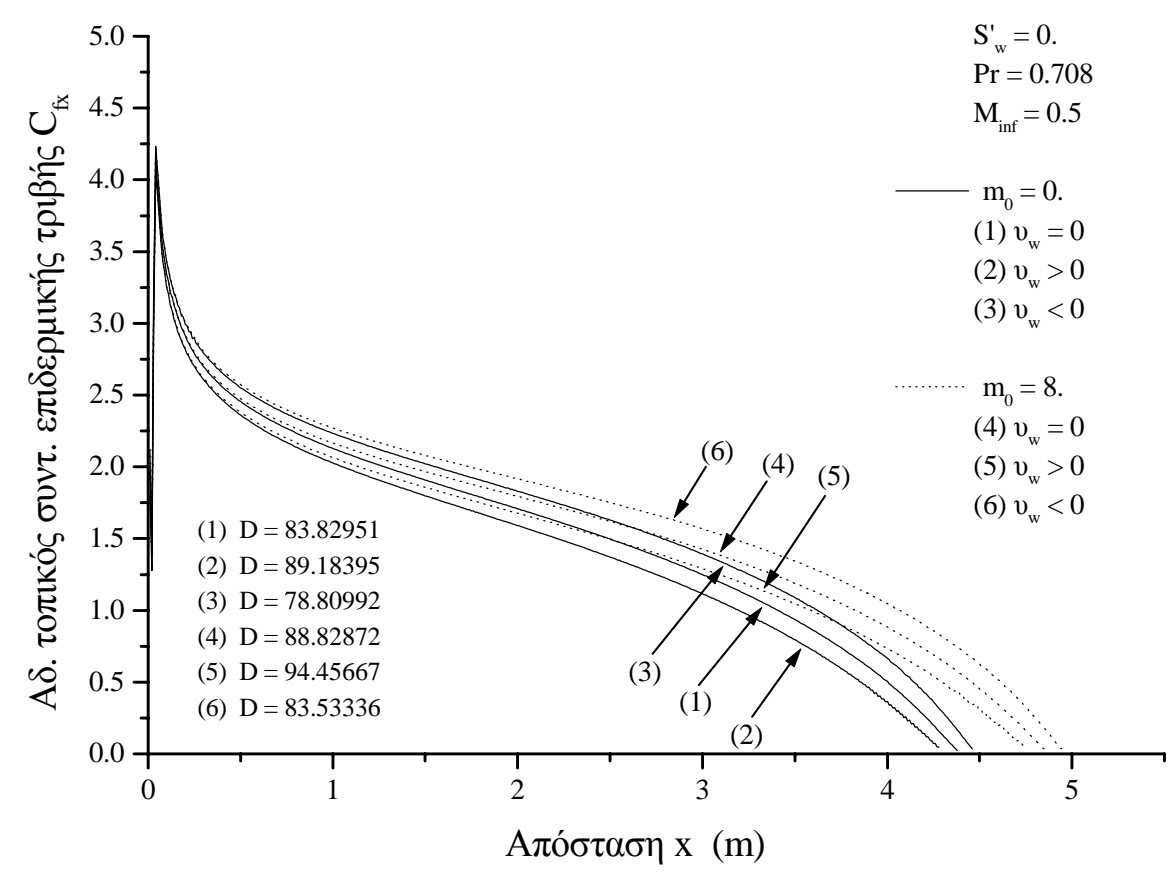

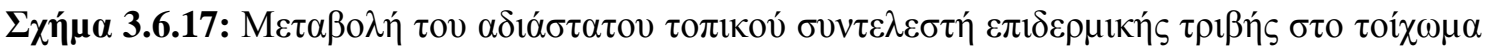

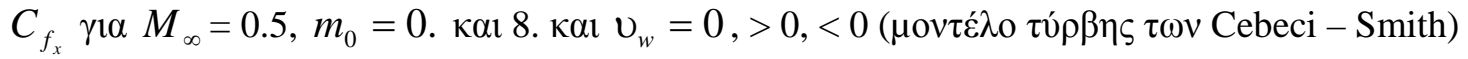

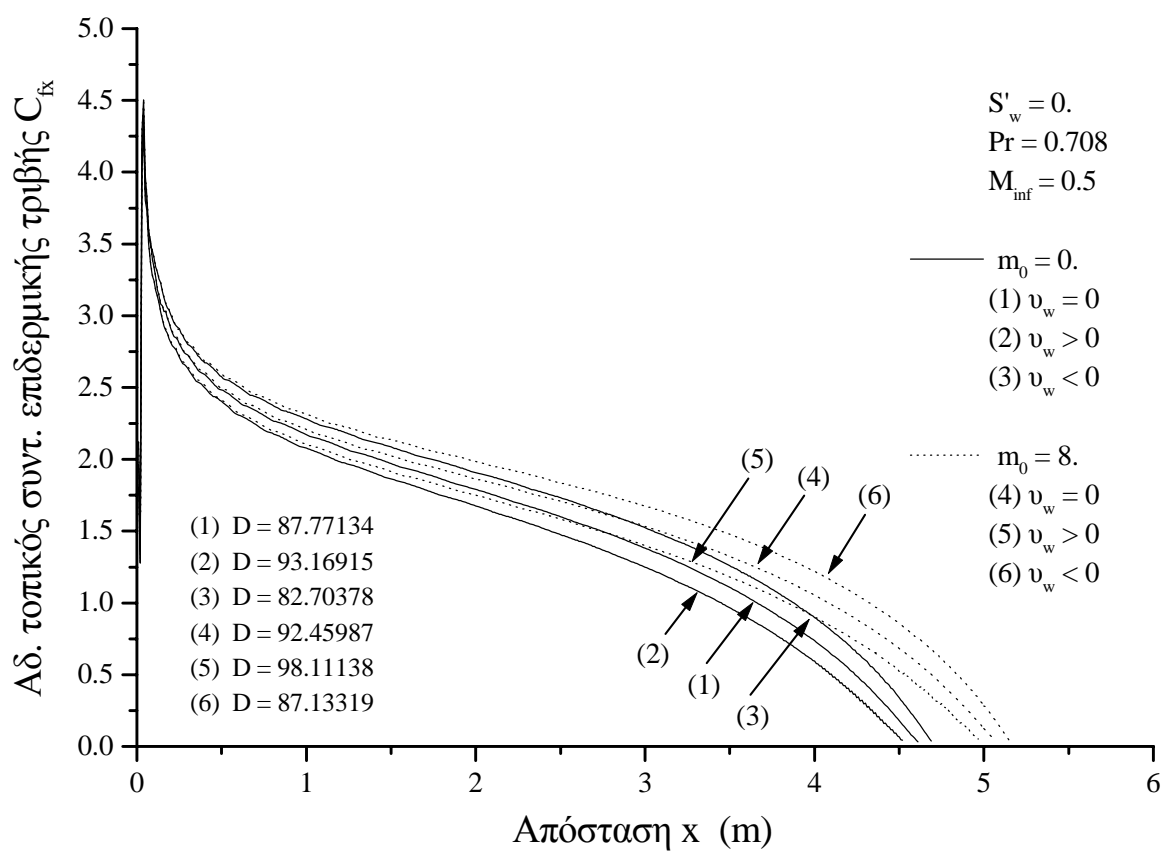

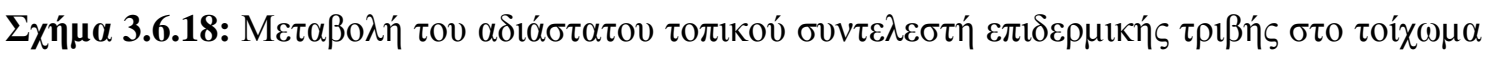

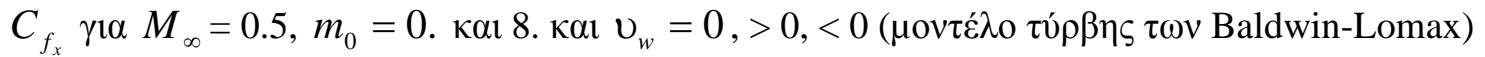




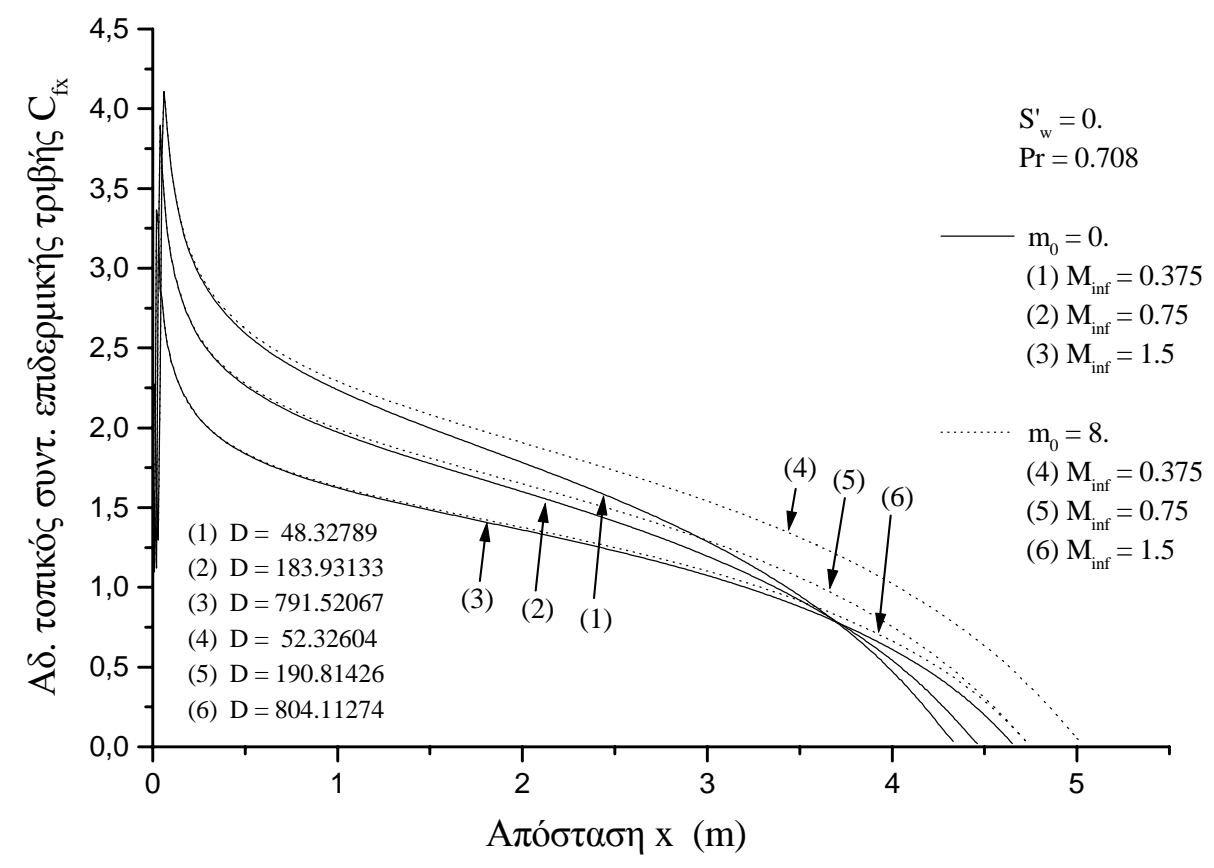

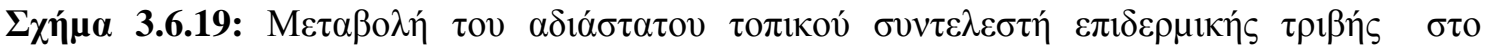

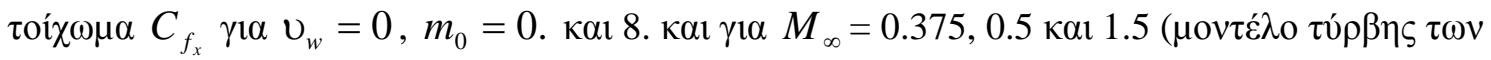
Cebeci - Smith)

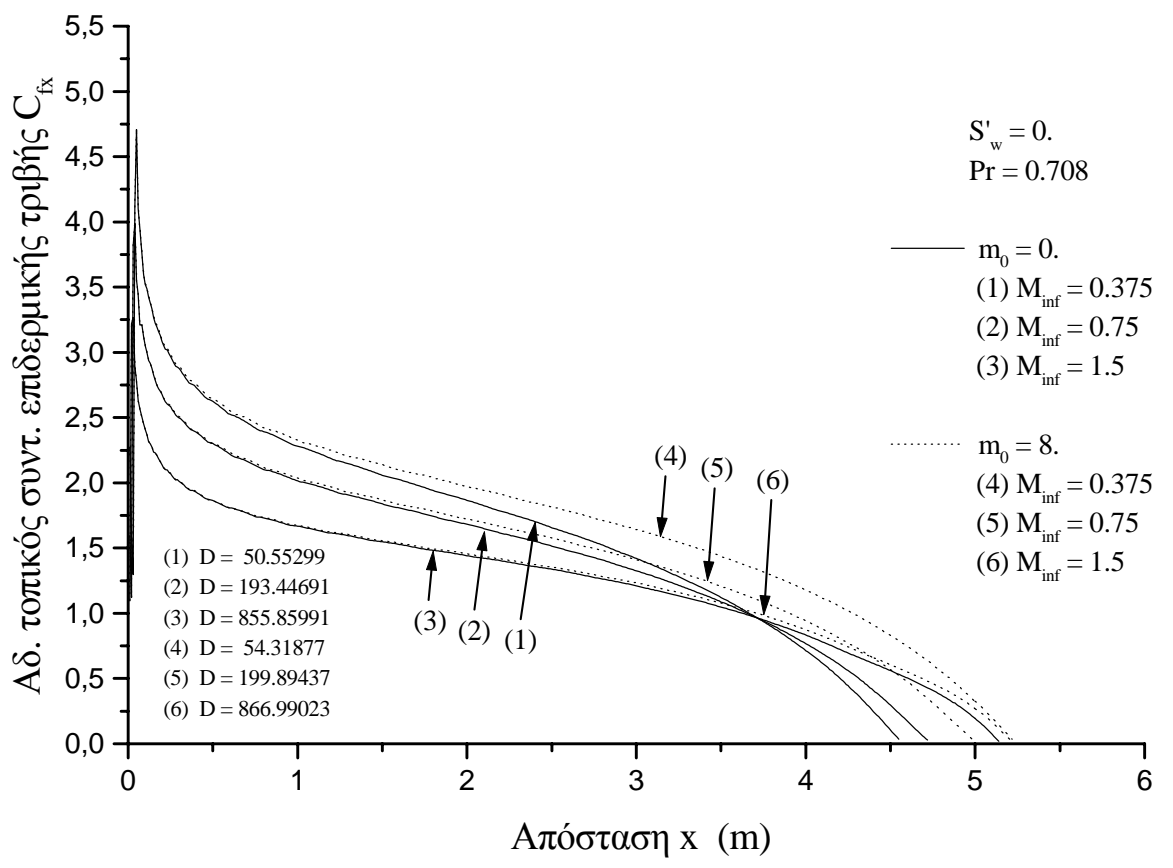

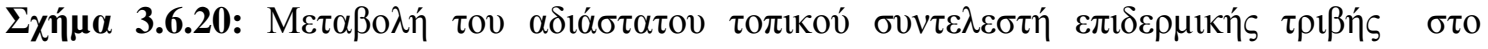

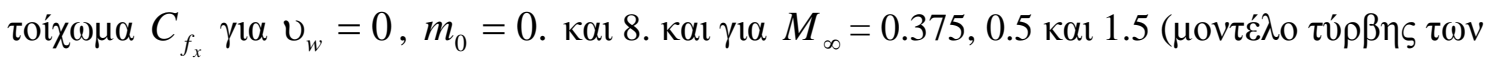
Baldwin - Lomax) 


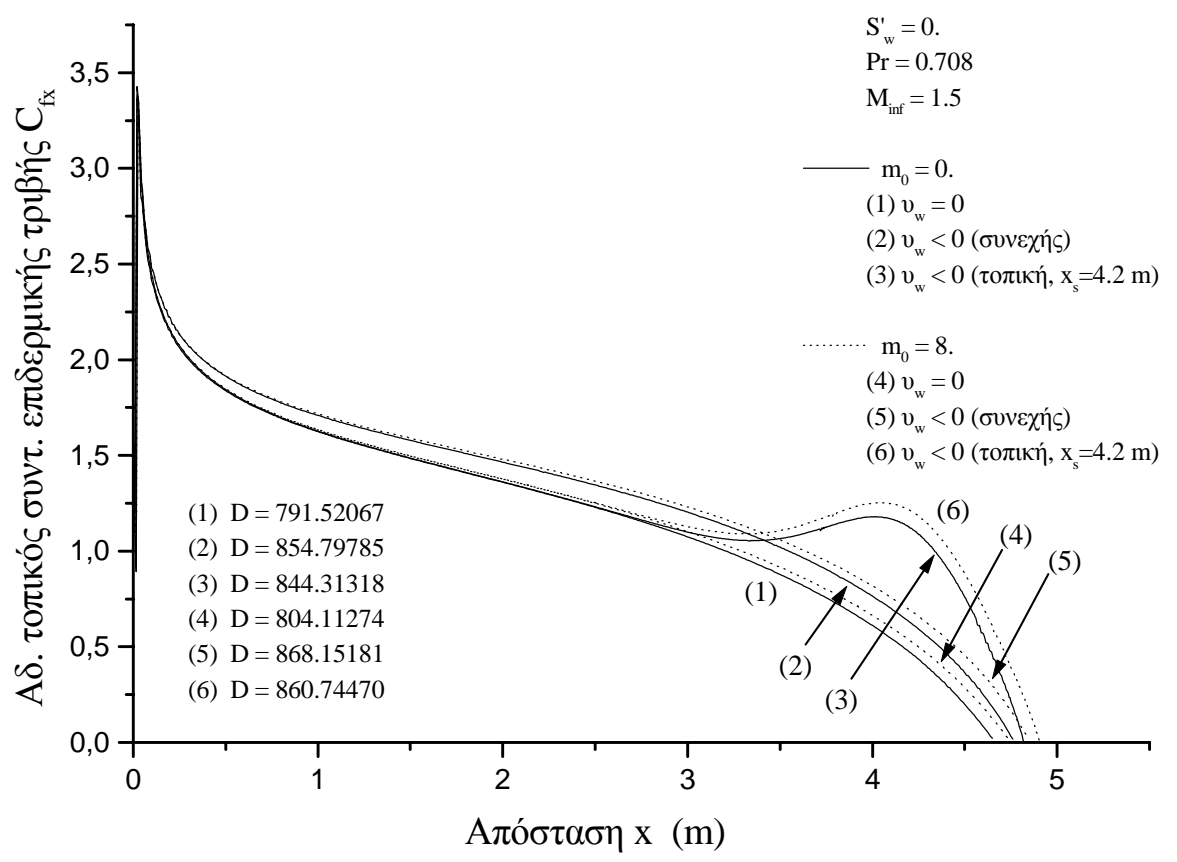

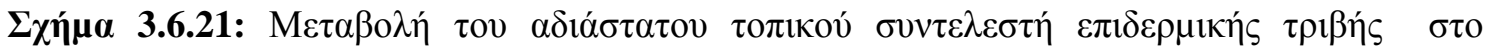

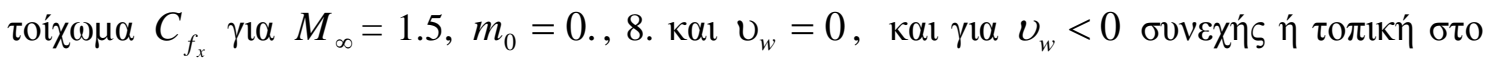

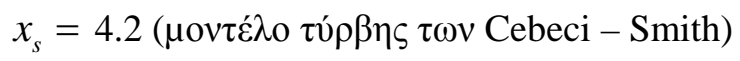

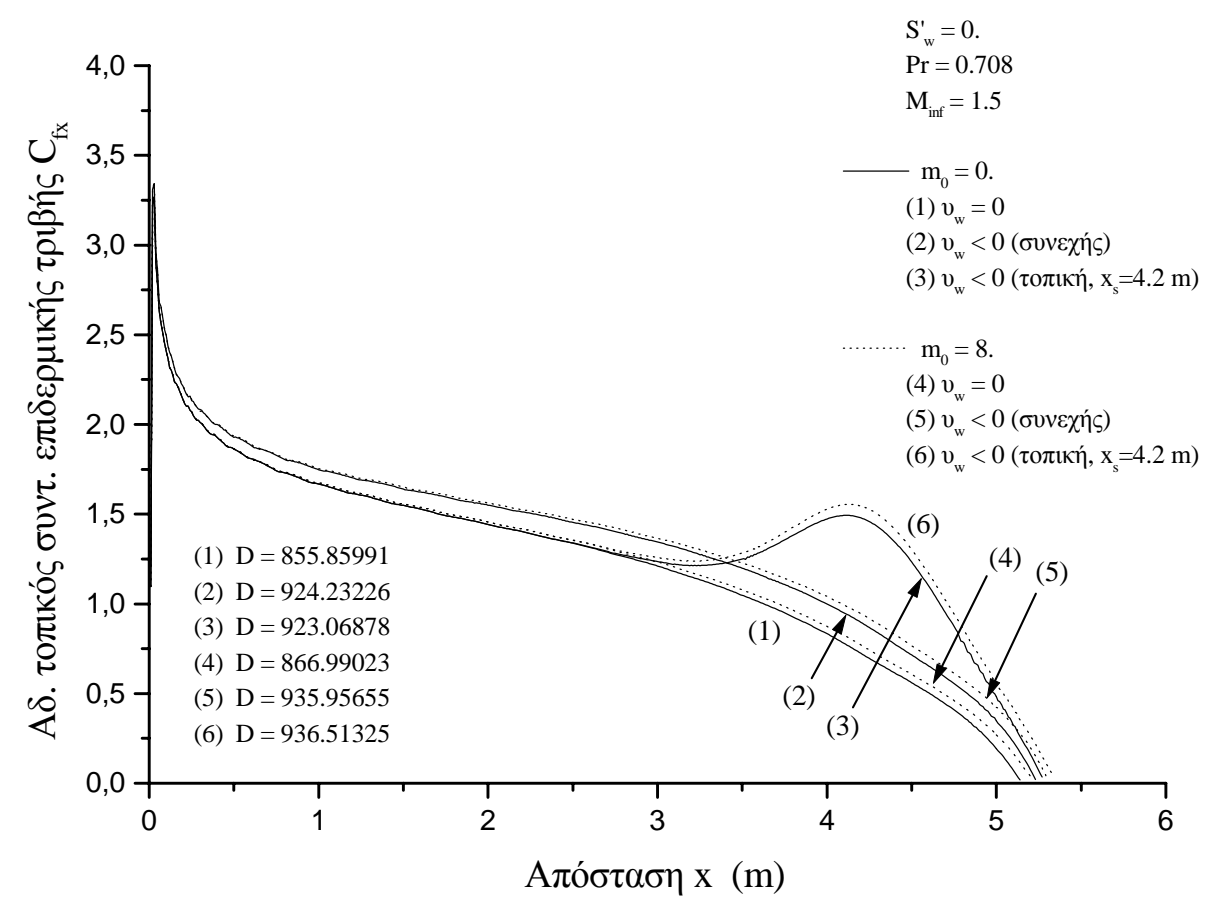

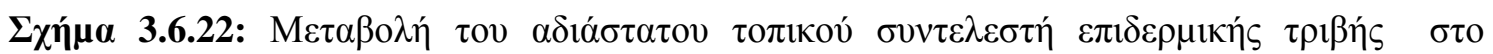

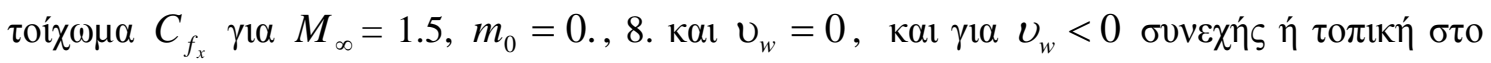

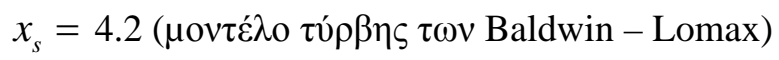




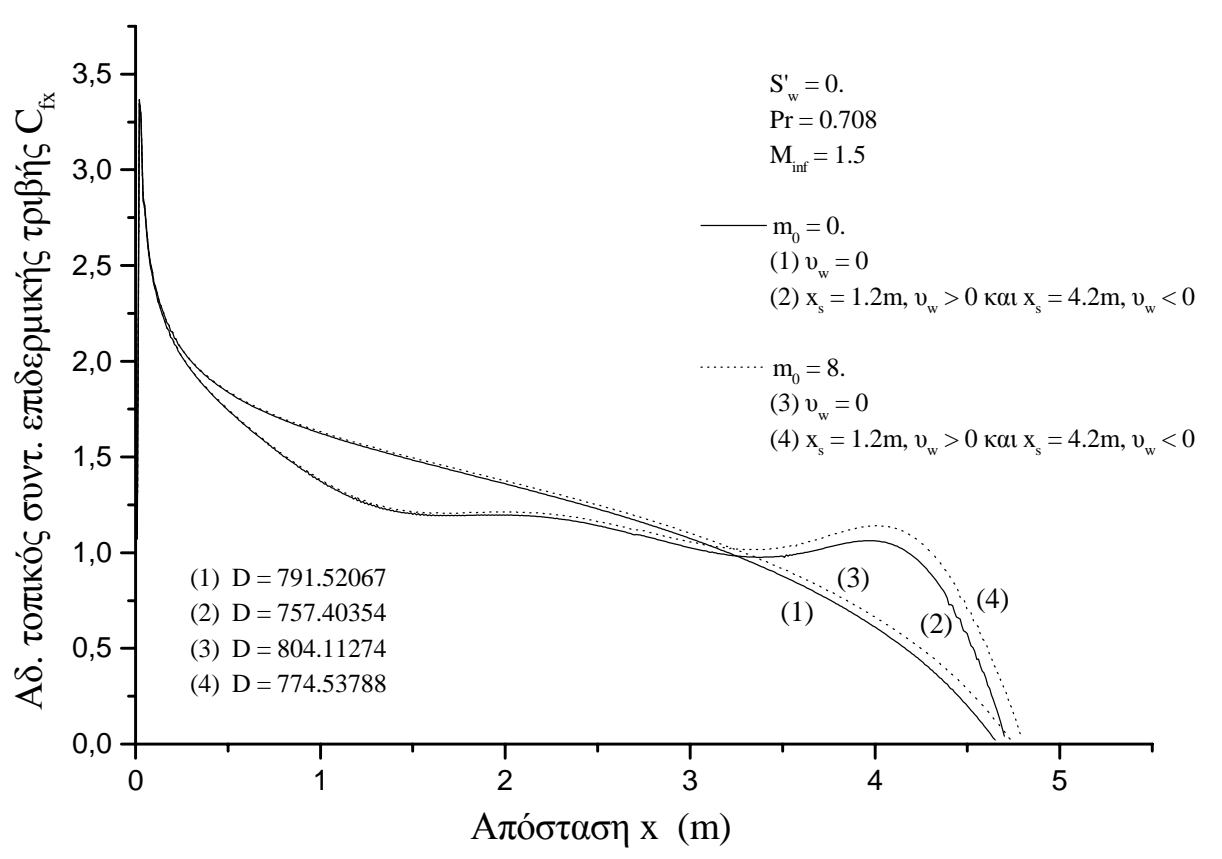

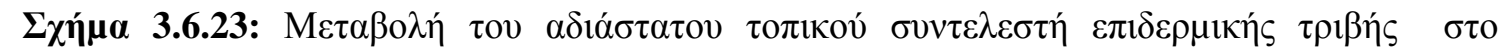

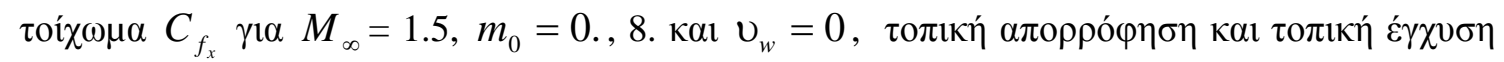

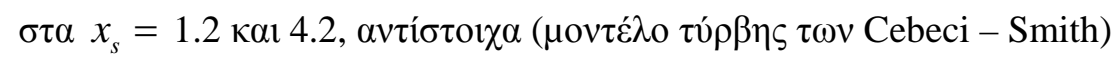

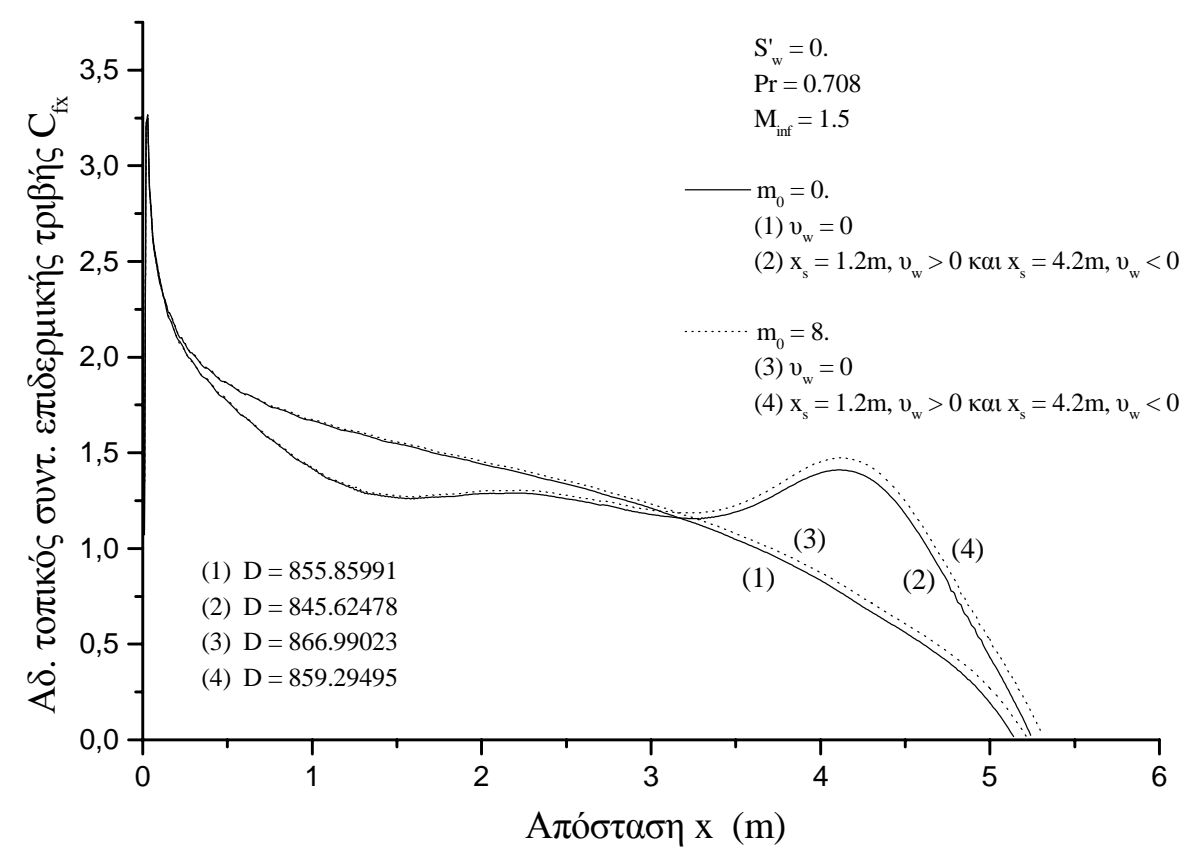

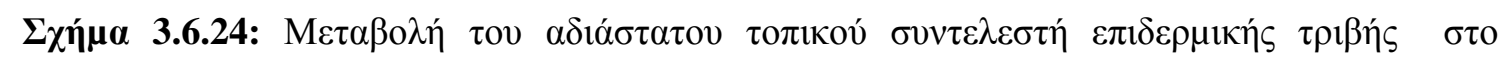

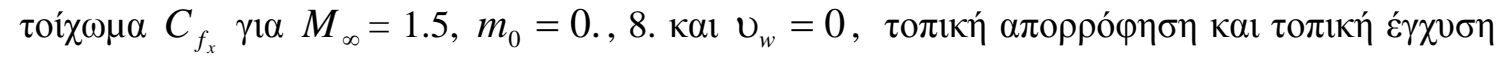

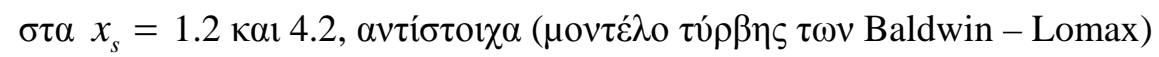




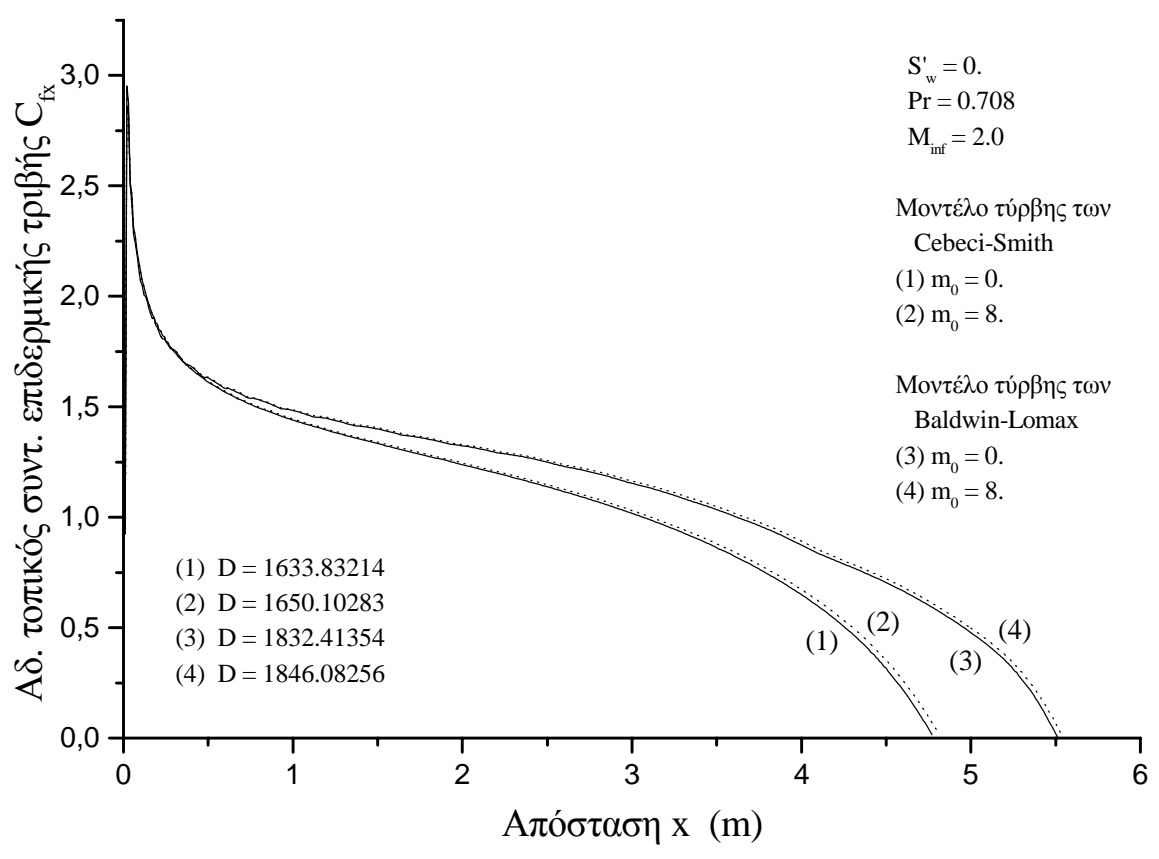

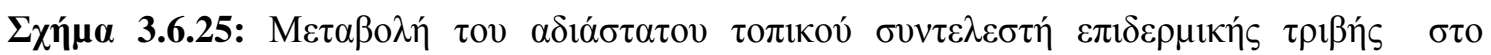

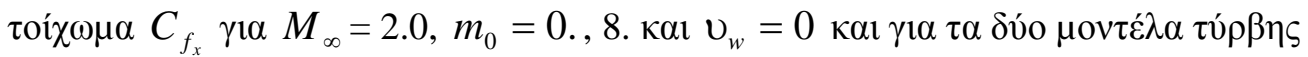

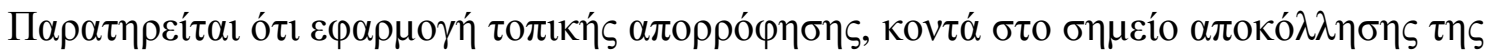

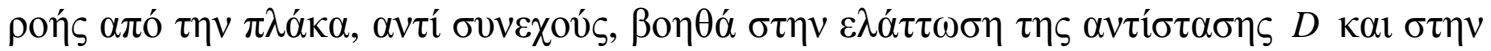

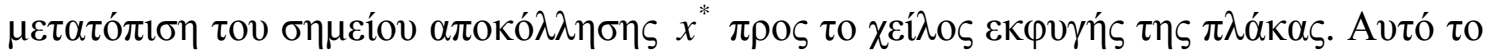

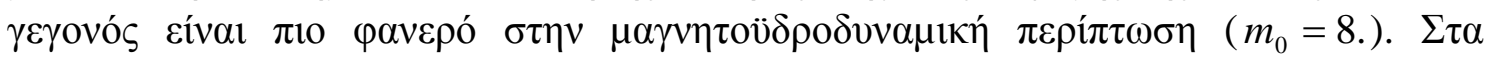

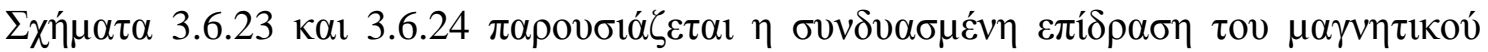

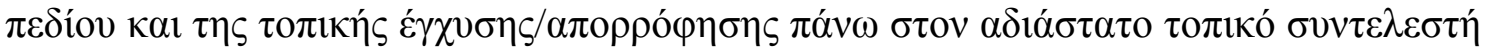

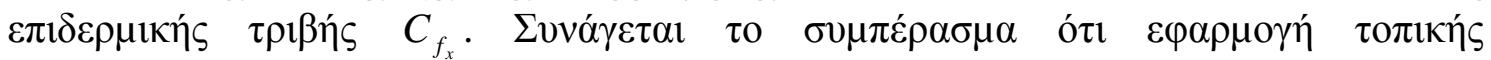

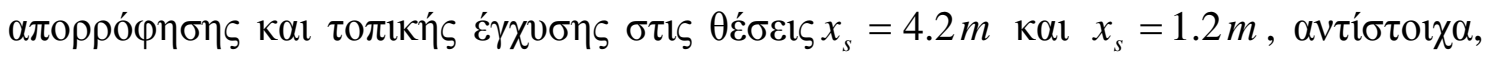

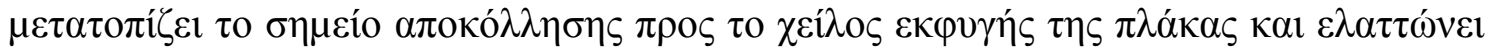

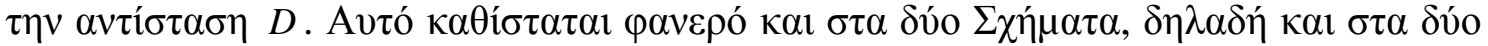

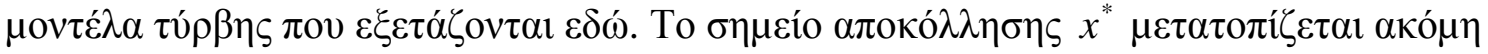

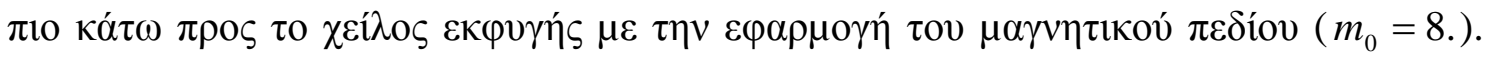

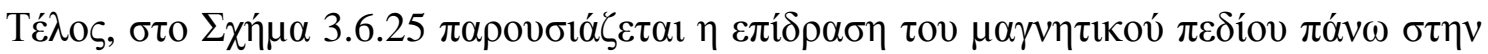

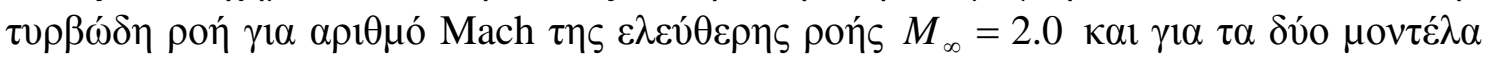

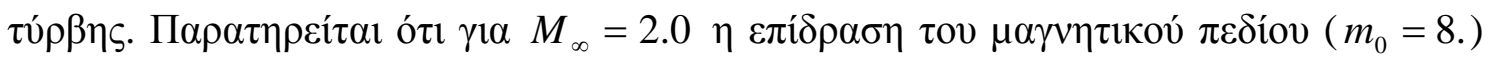

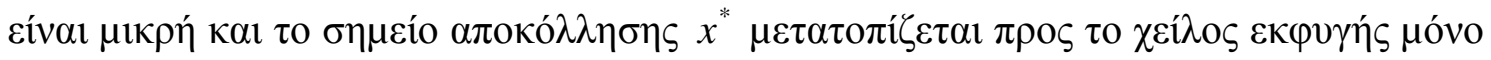

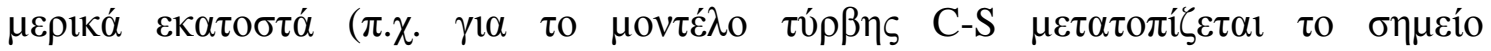

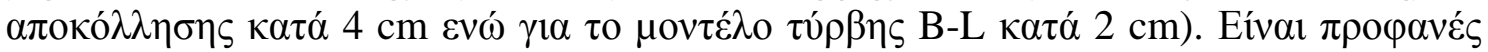

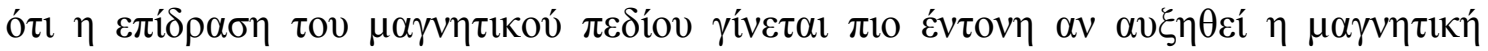

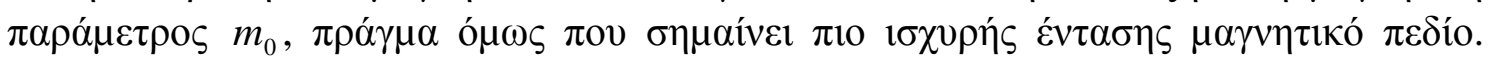

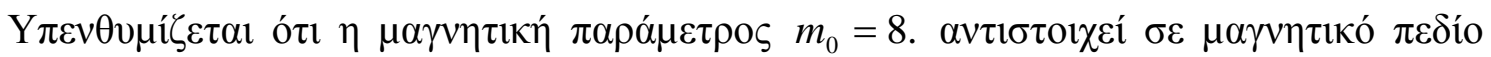

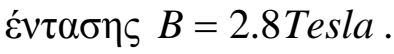




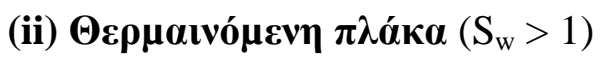

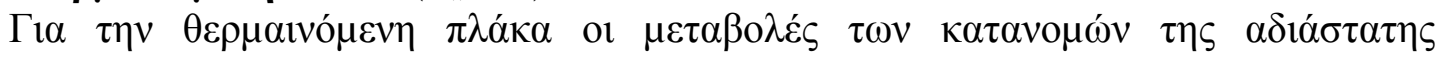

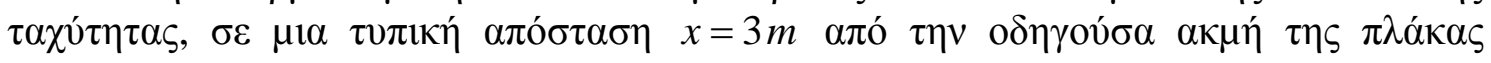

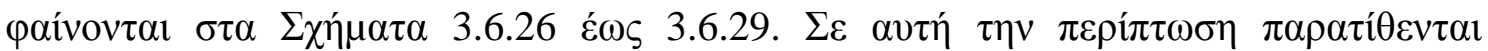

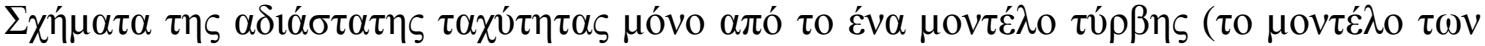

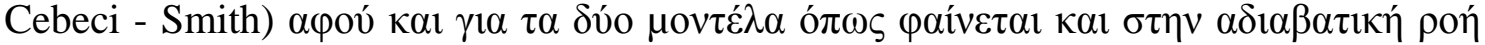

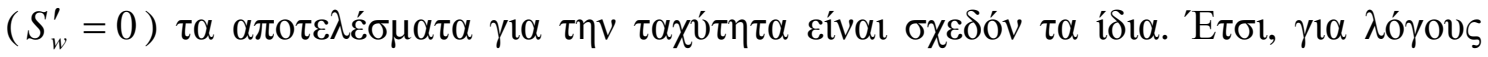

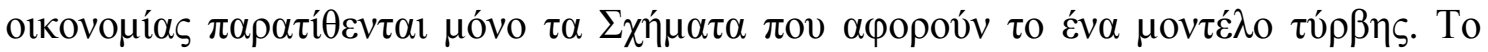

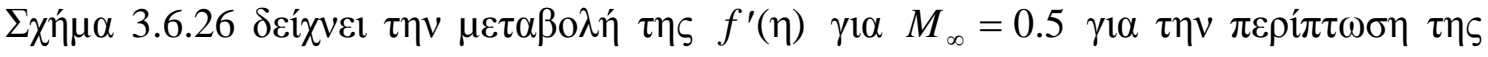

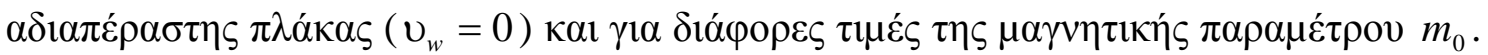

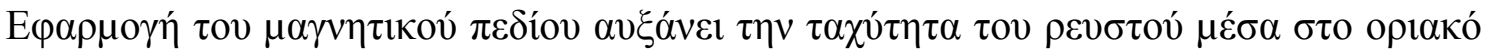

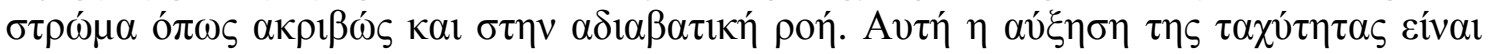

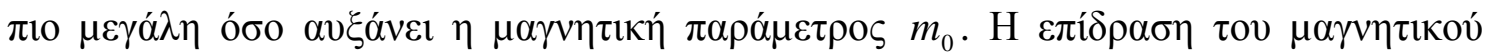

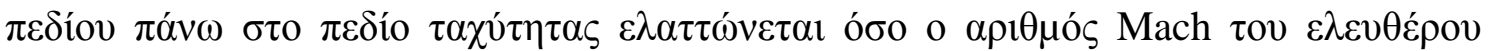

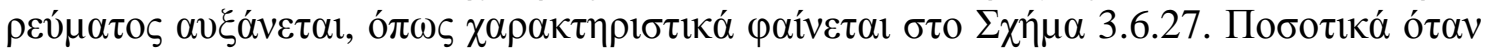

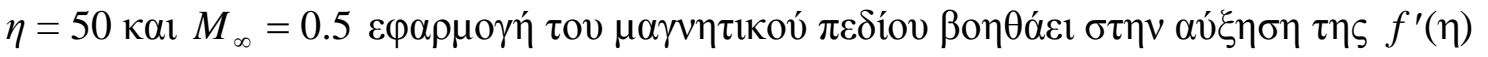

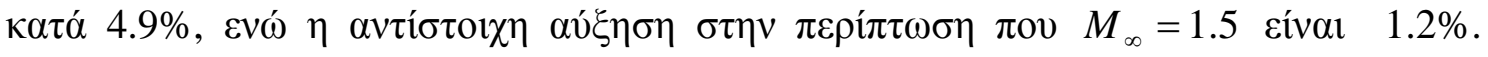

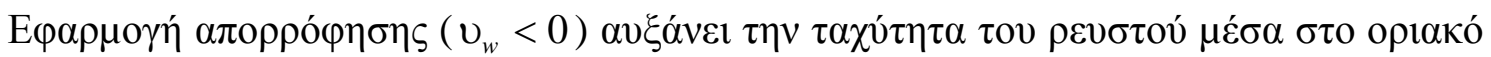

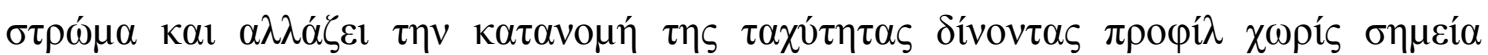

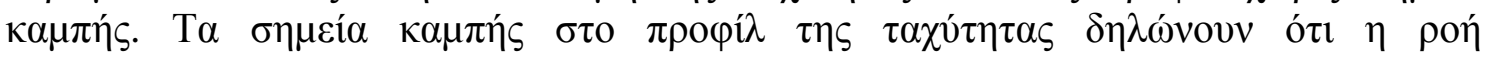

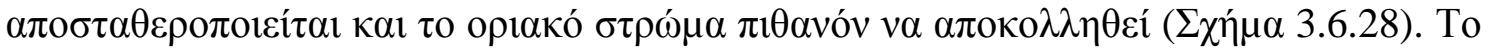

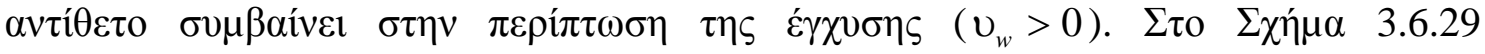

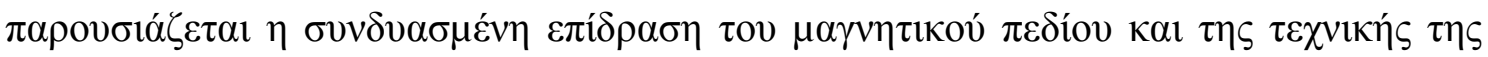

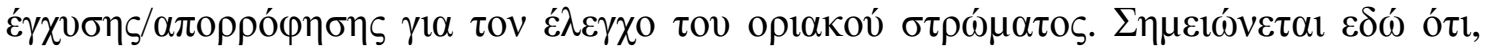

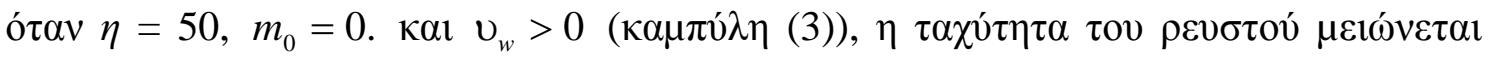

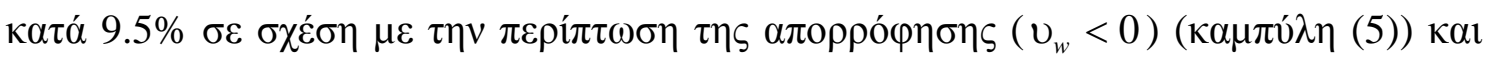

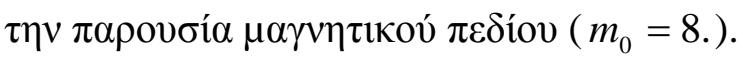

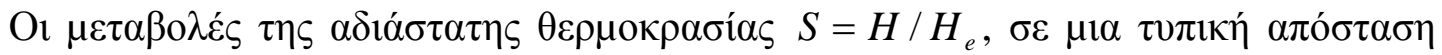

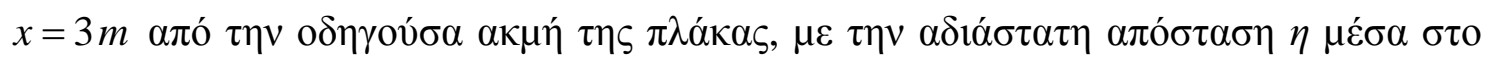

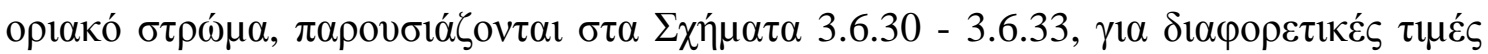

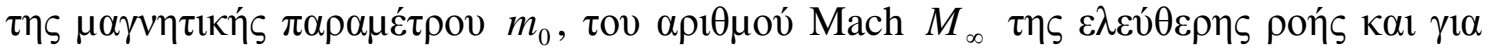

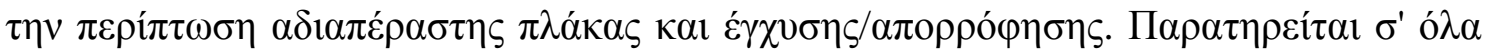

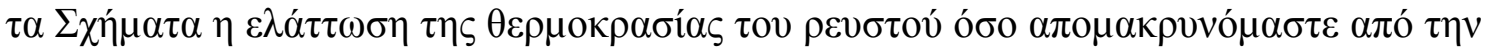

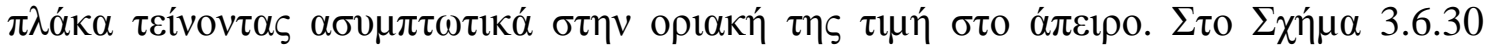

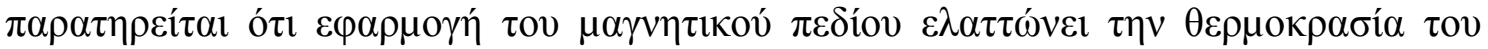

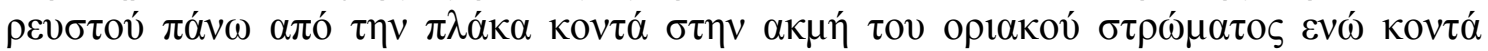

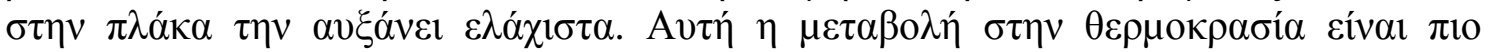

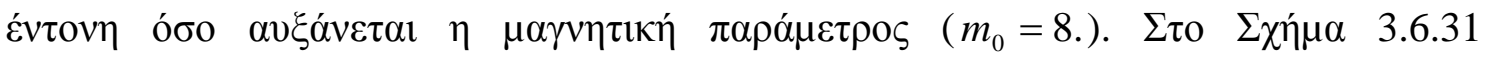

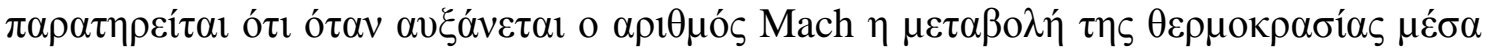

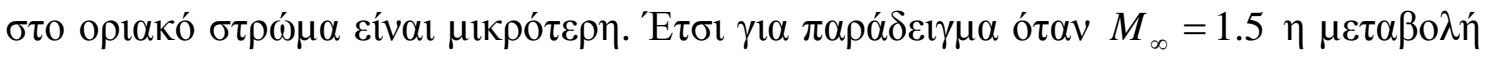

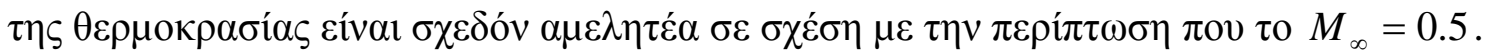

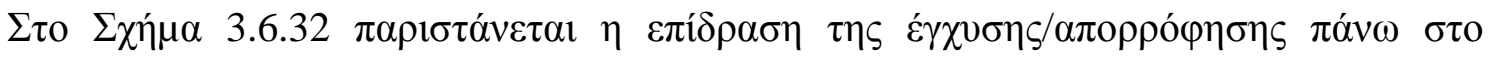

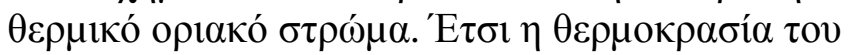




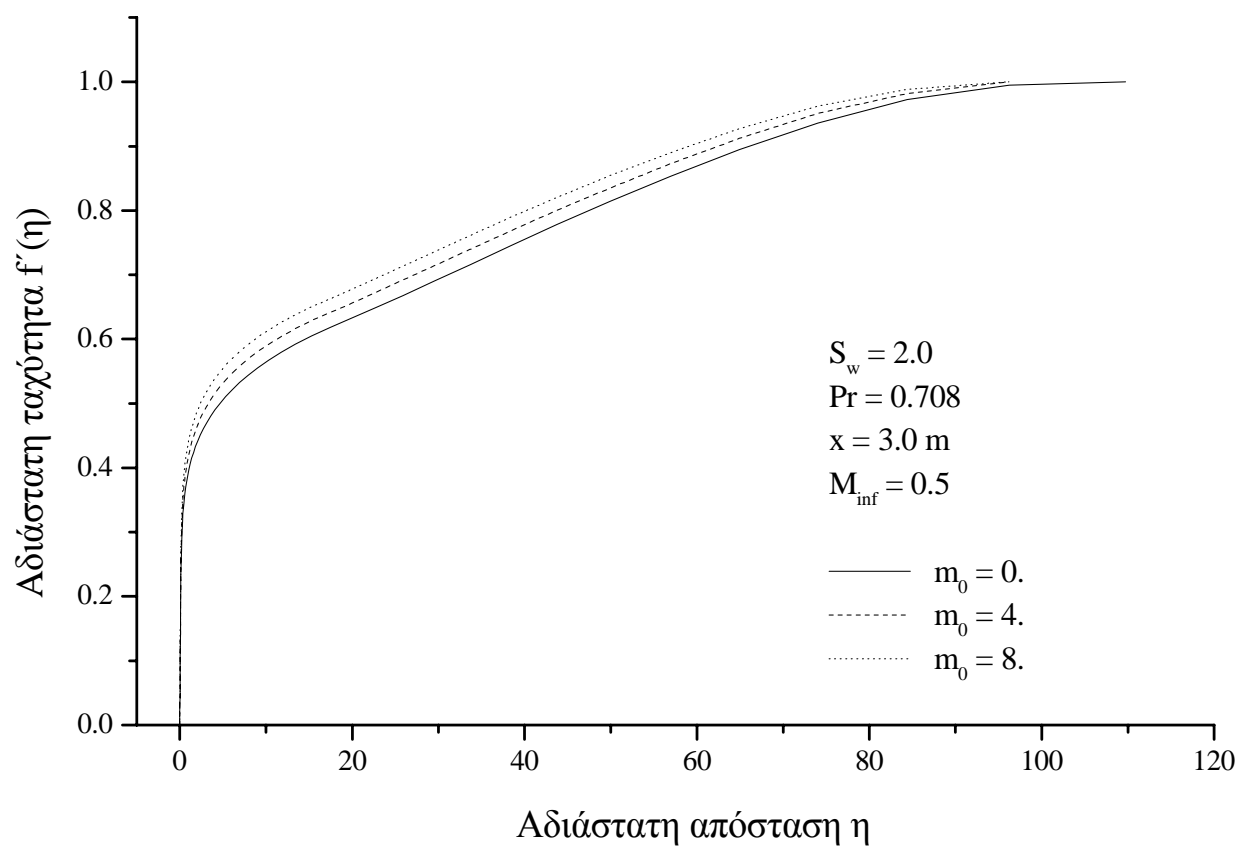

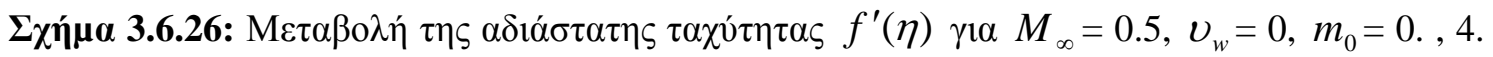

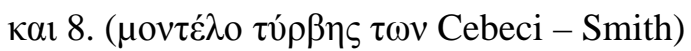

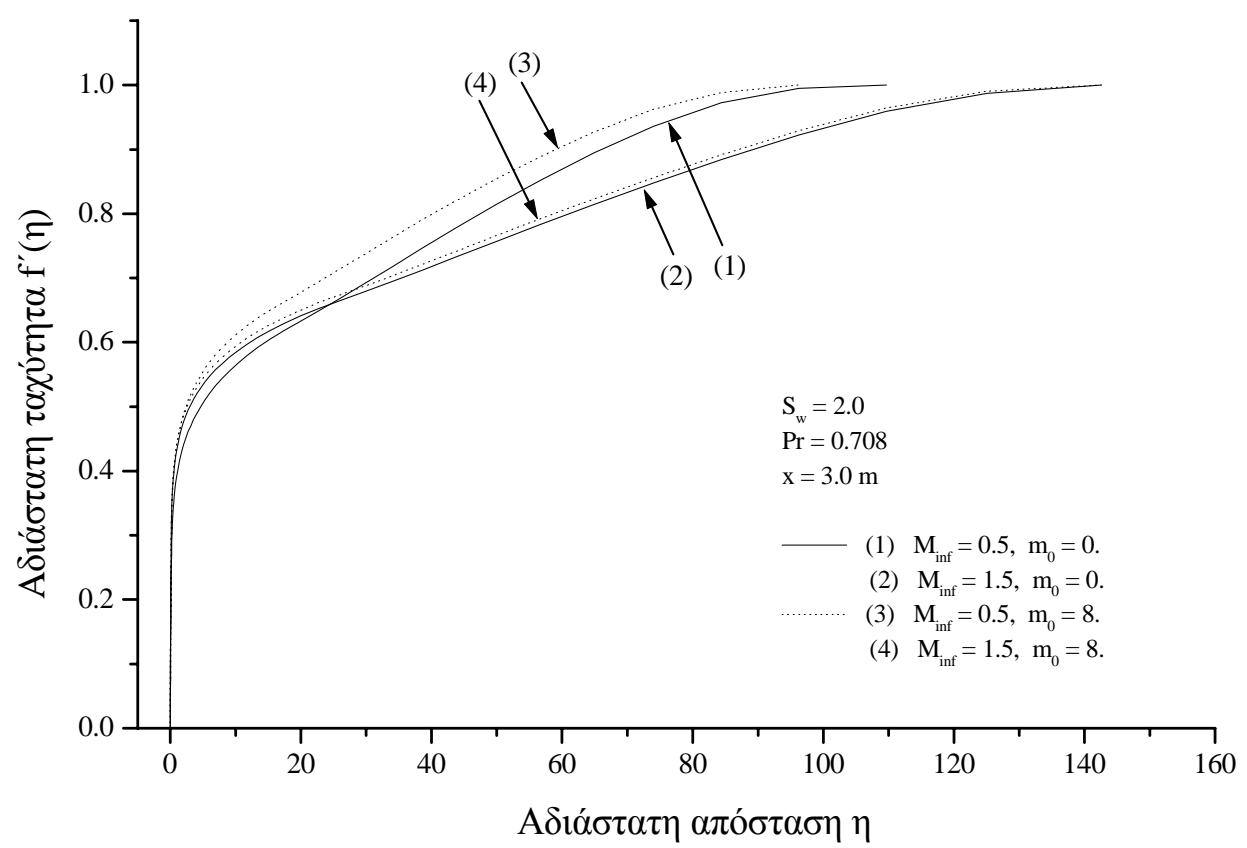

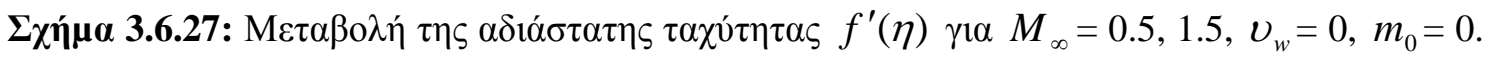

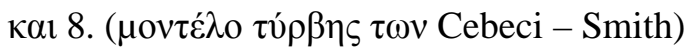




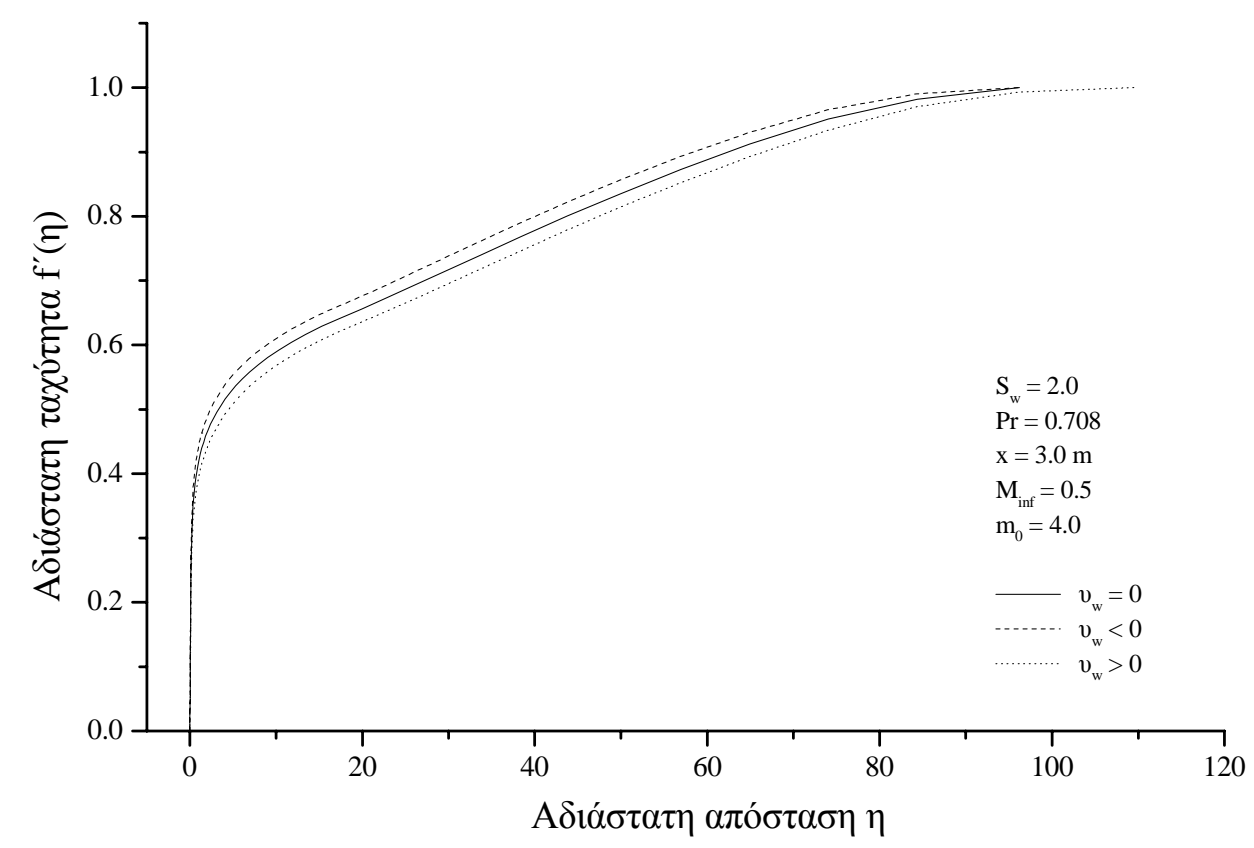

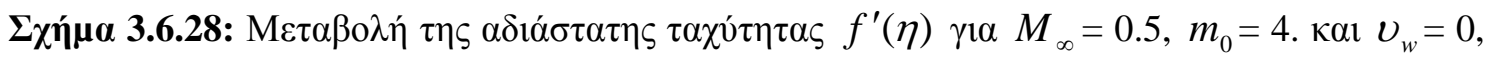

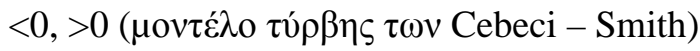

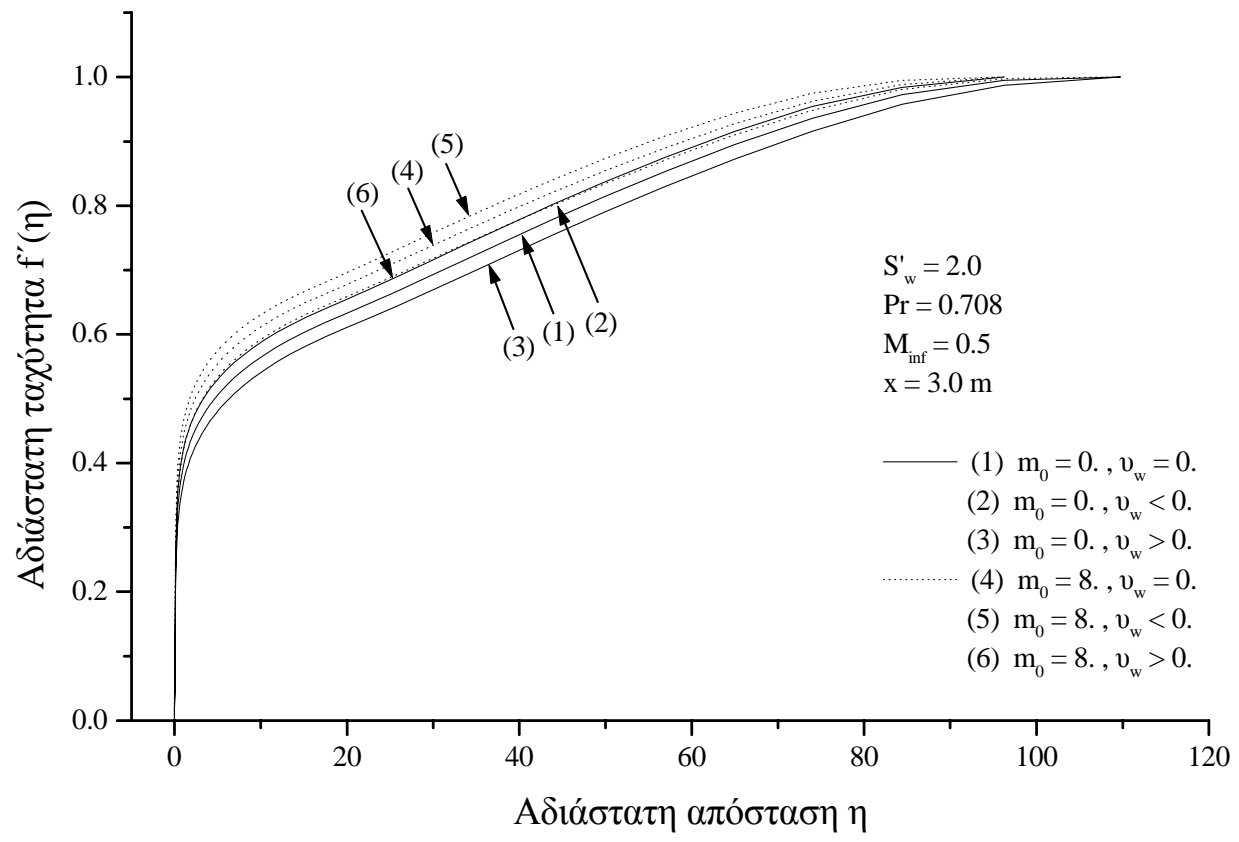

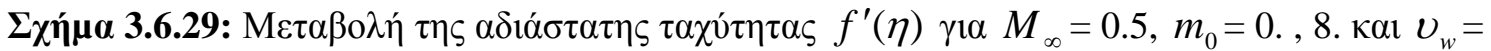

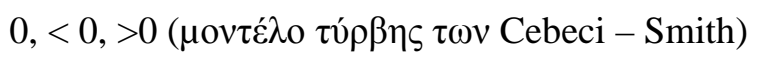




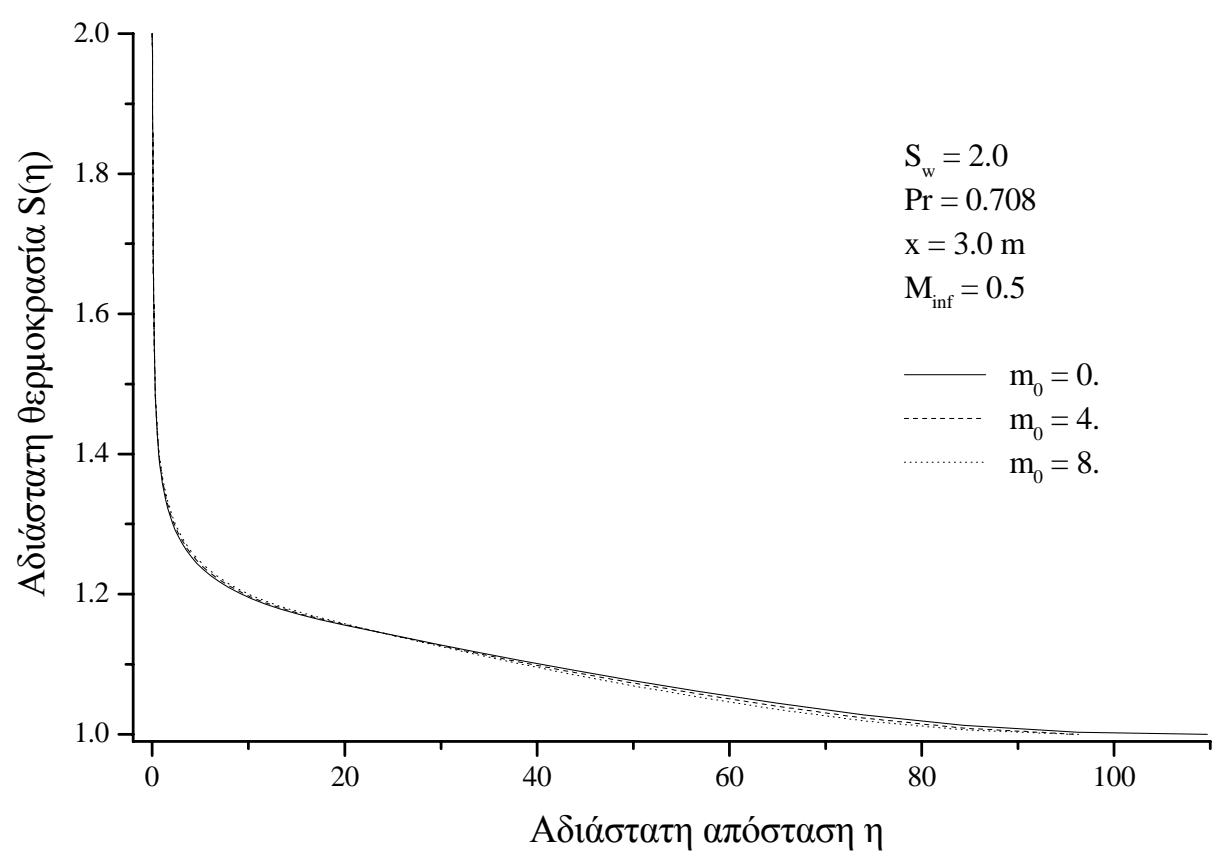

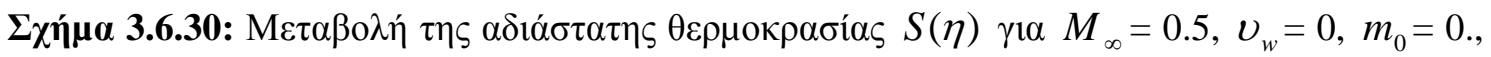

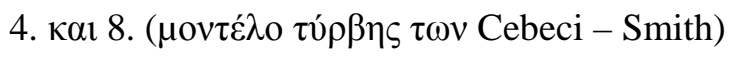

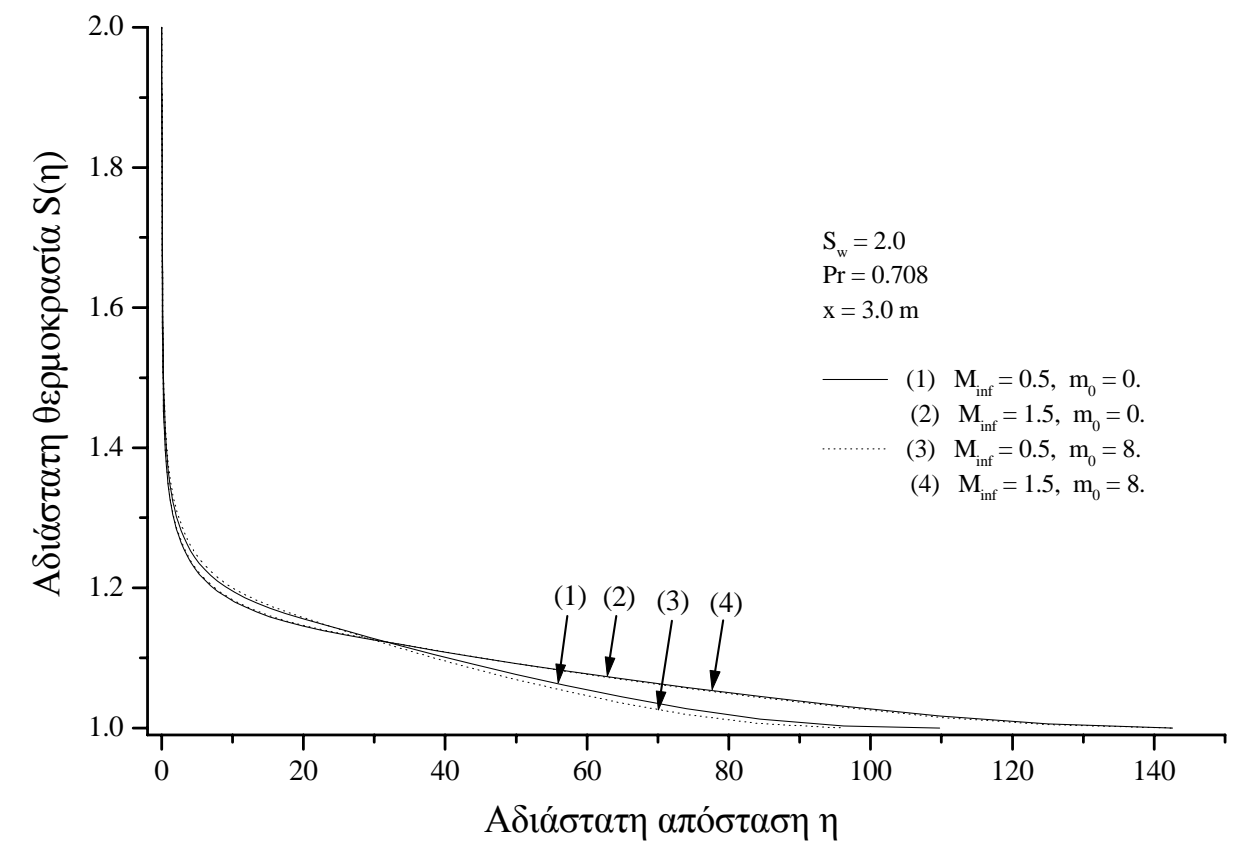

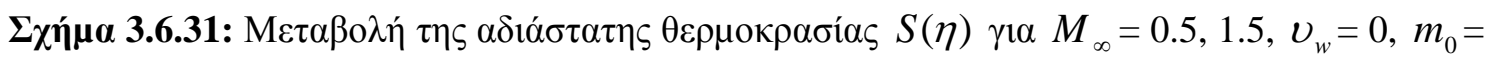

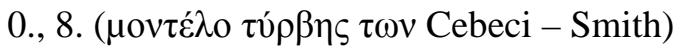




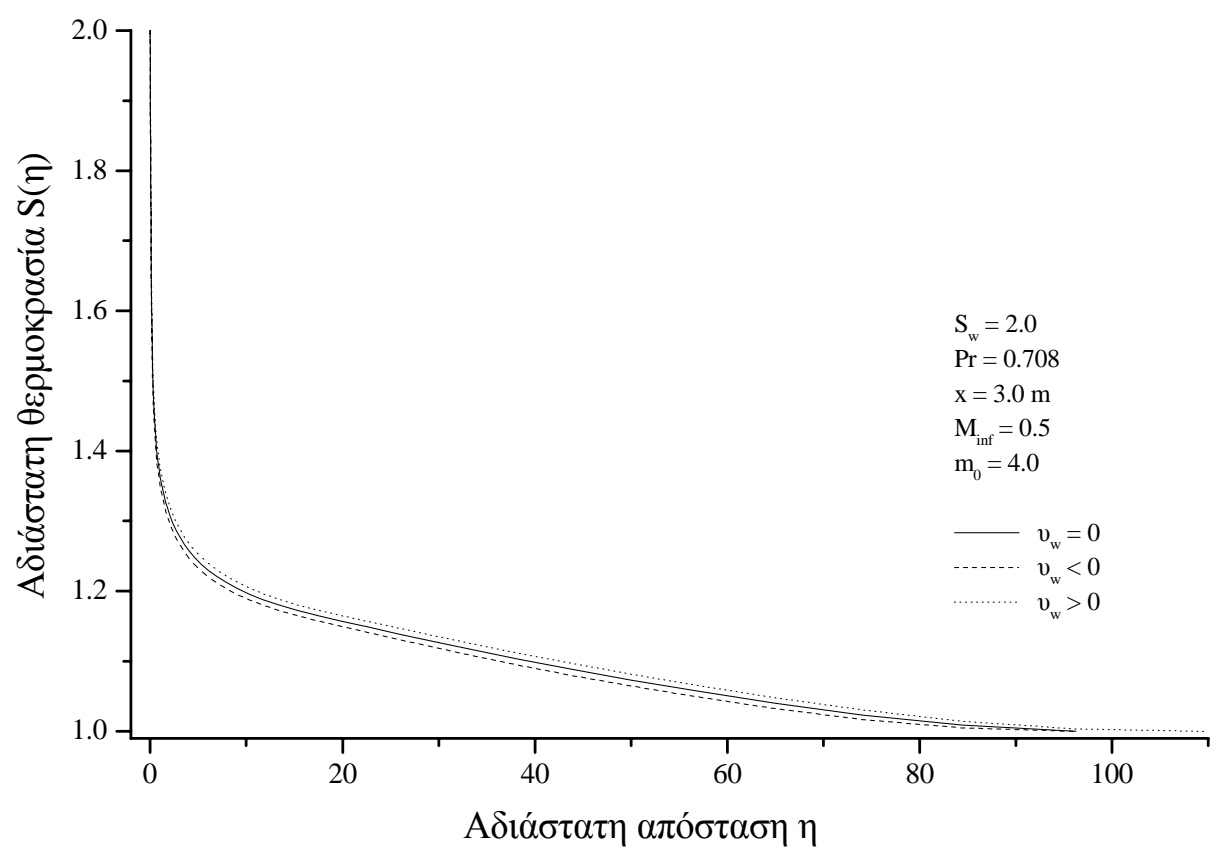

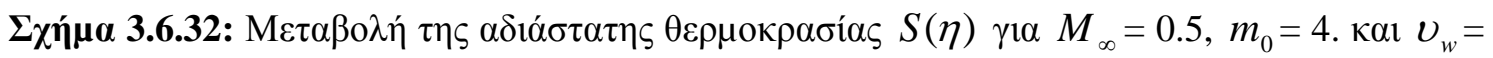

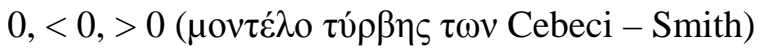

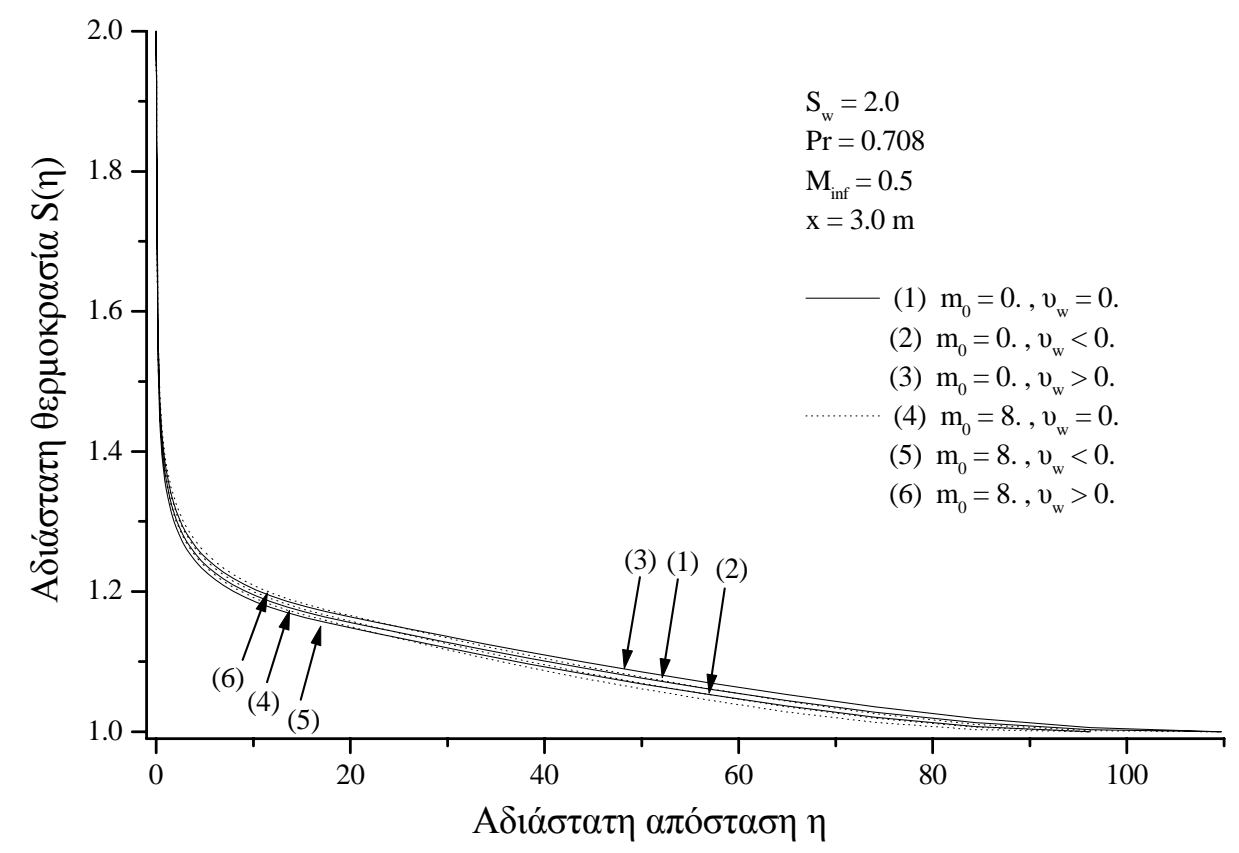

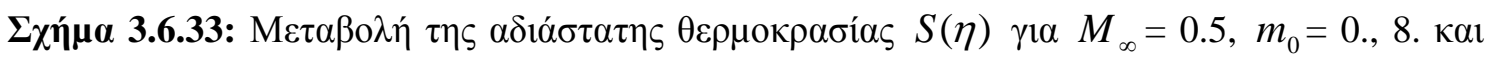

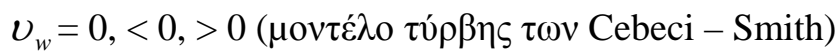




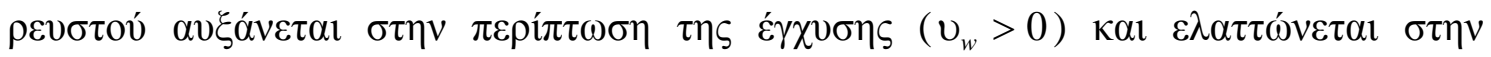

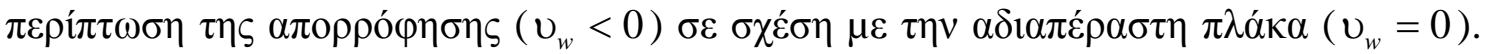

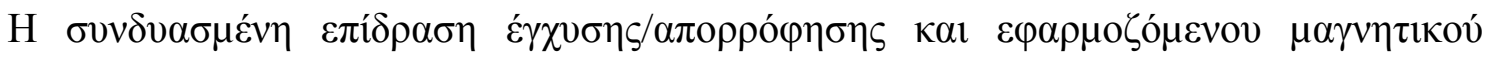

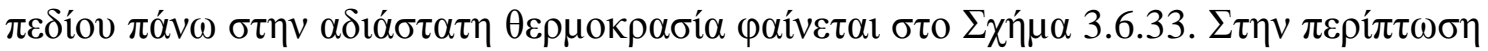

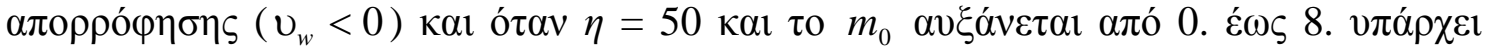

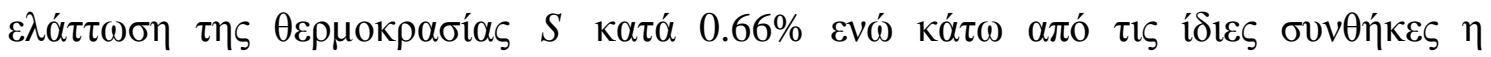

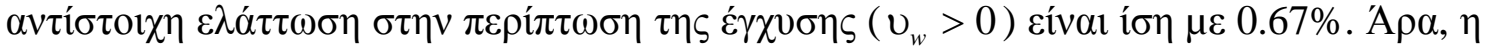

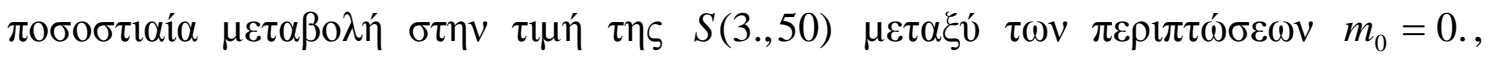
$v_{w}>0$ каı $m_{0}=8 ., v_{w}<0$ eíval $2.22 \%$.

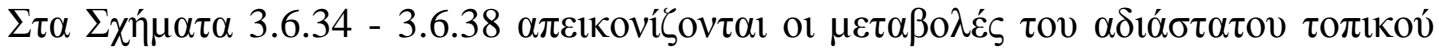

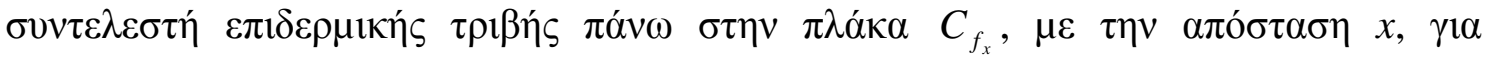

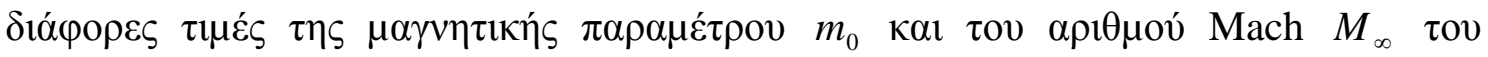

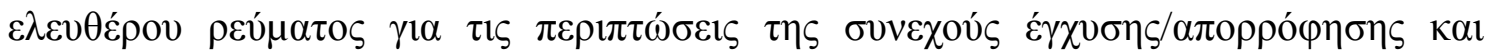

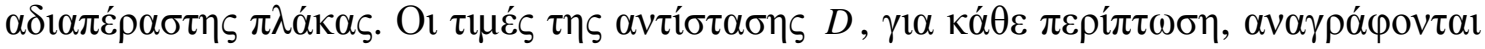

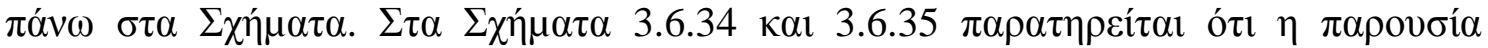

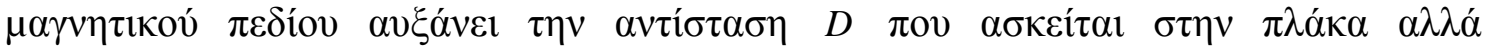

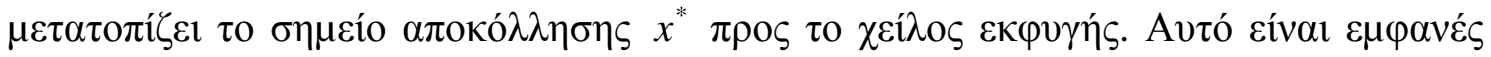

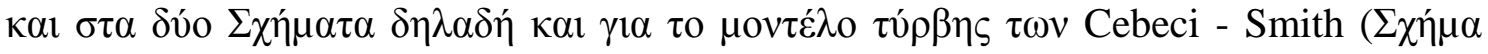

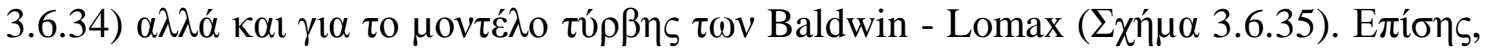

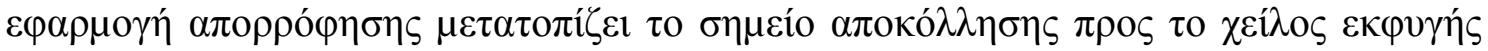

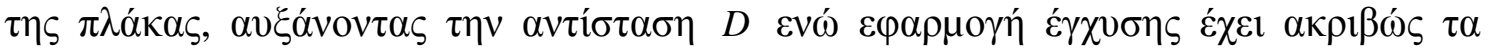

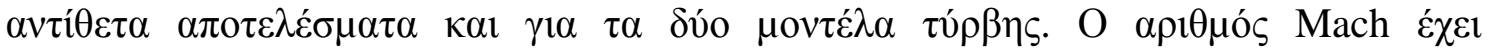

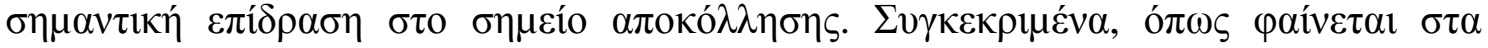

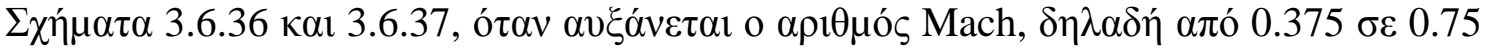

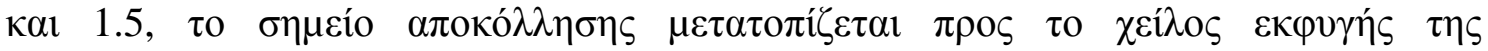

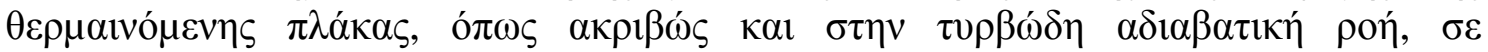

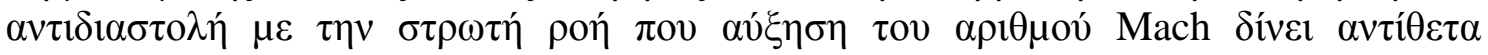

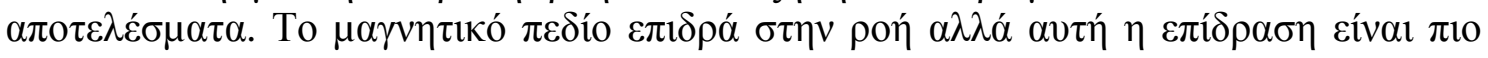

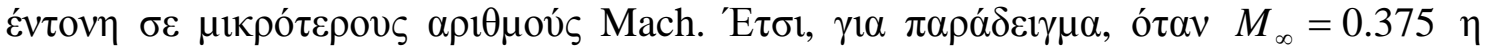

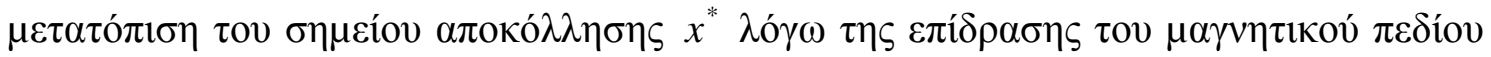

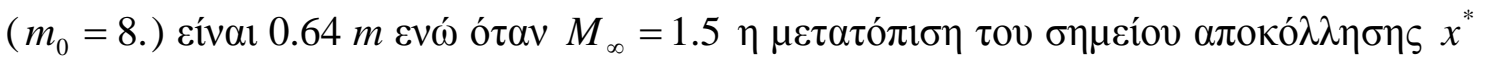

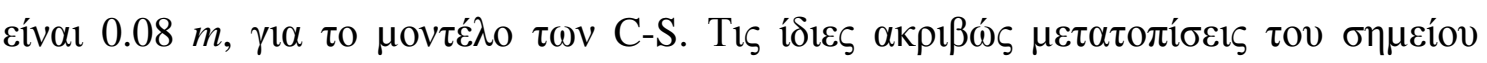

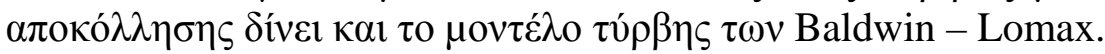

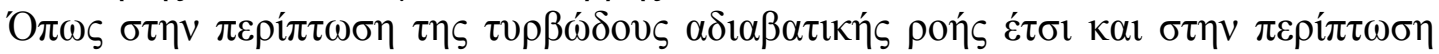

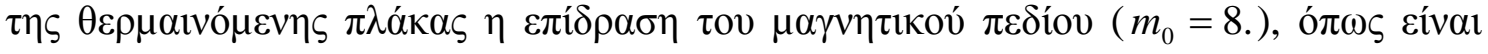

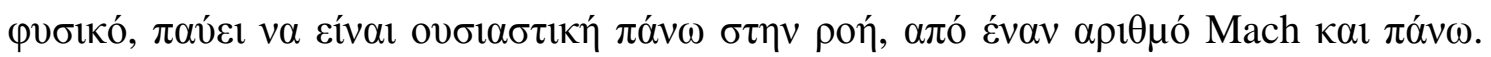

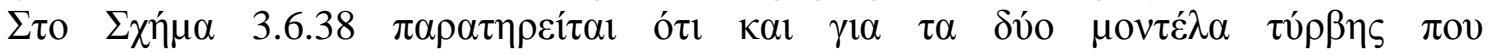

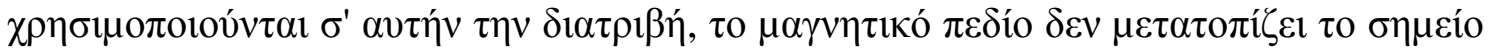

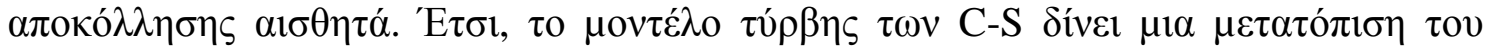

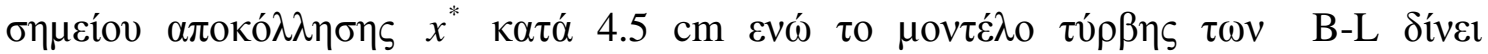

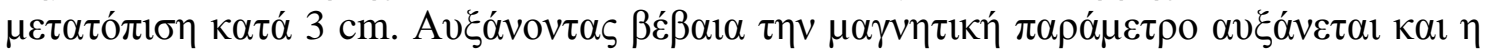

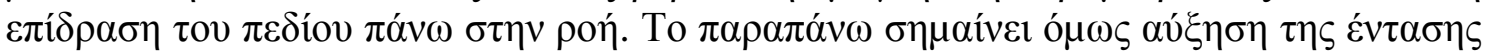

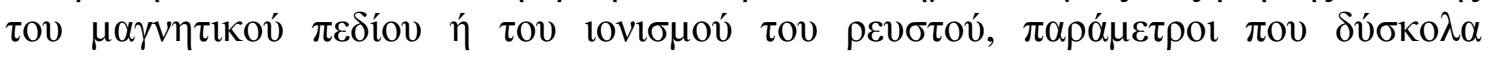

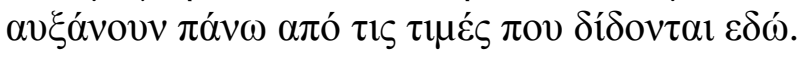




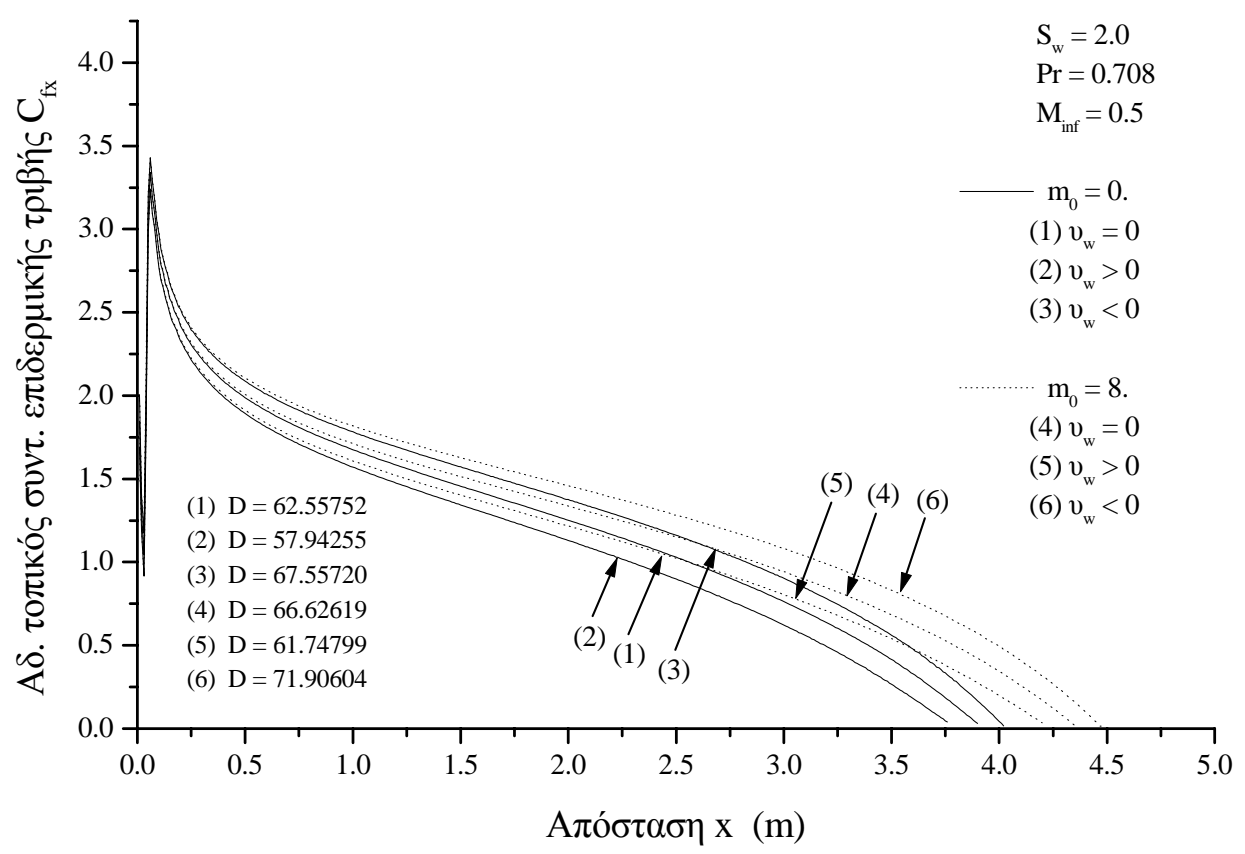

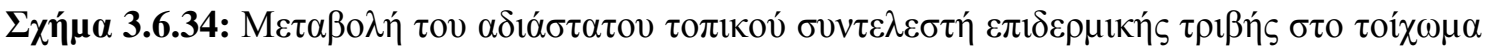

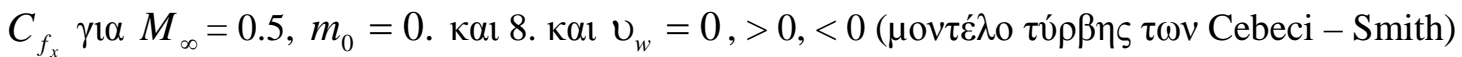

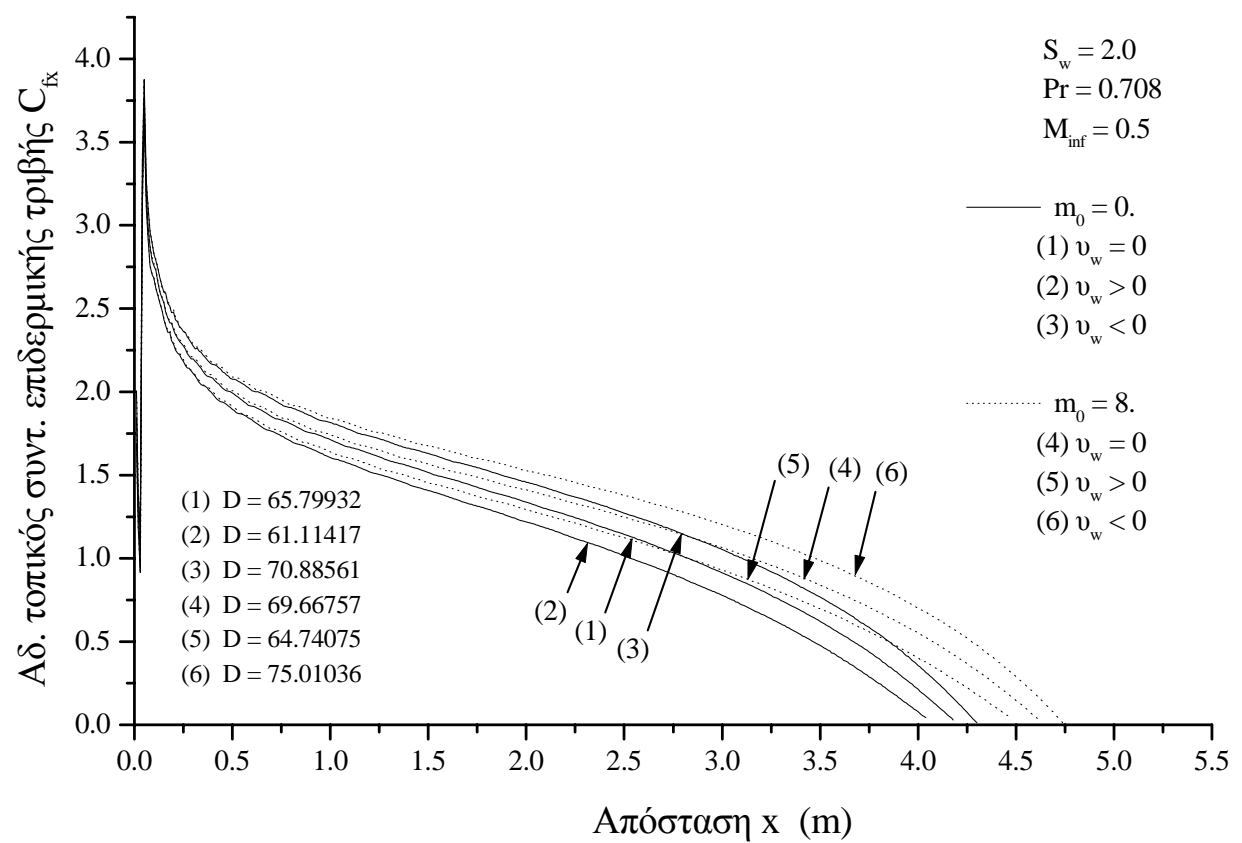

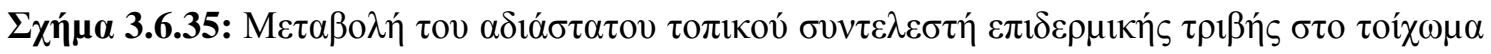

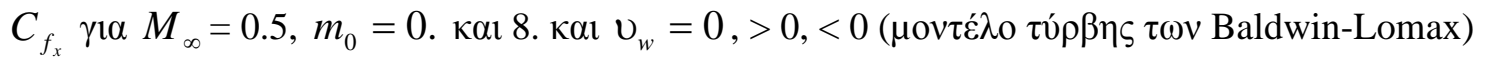




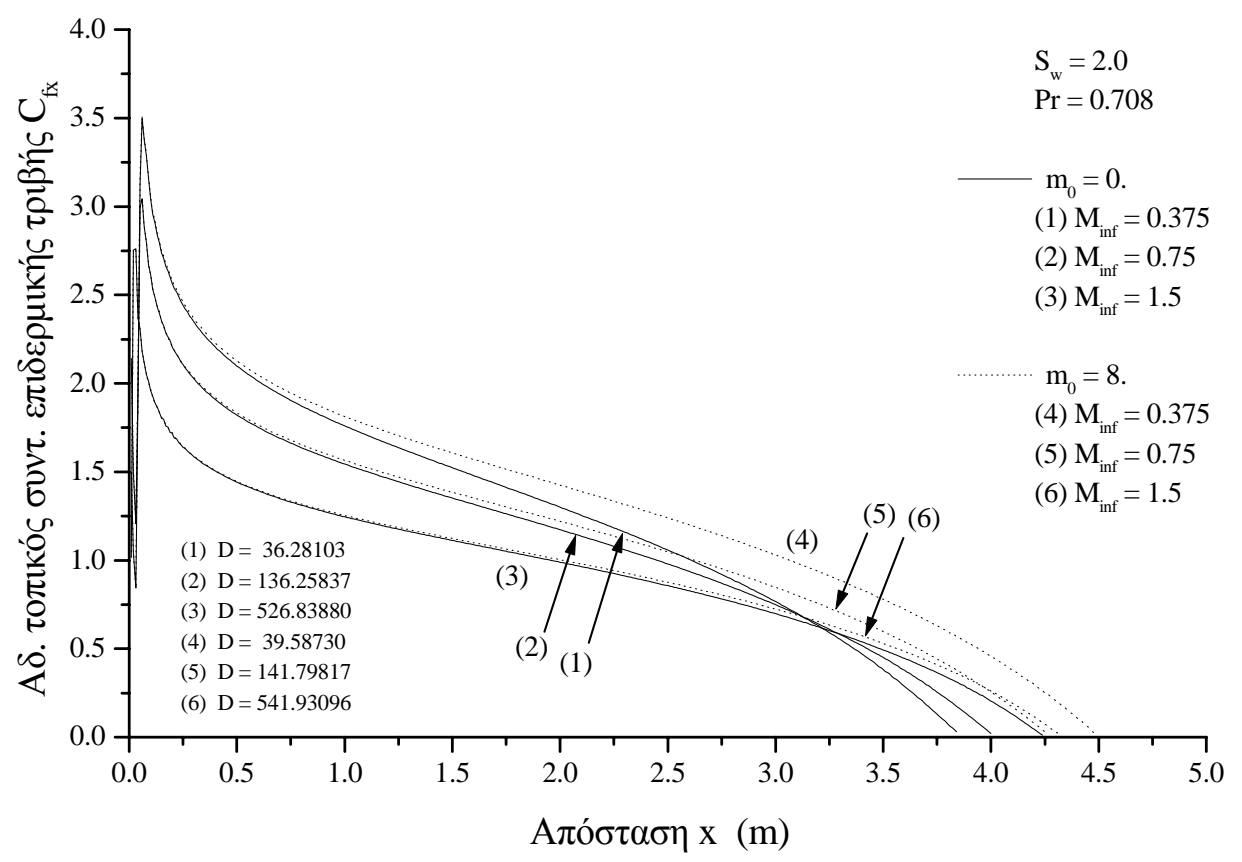

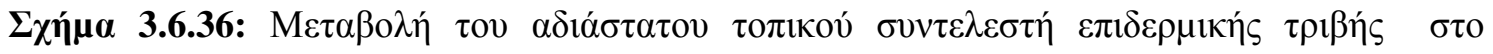

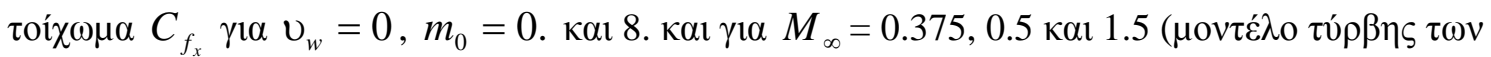
Cebeci - Smith)

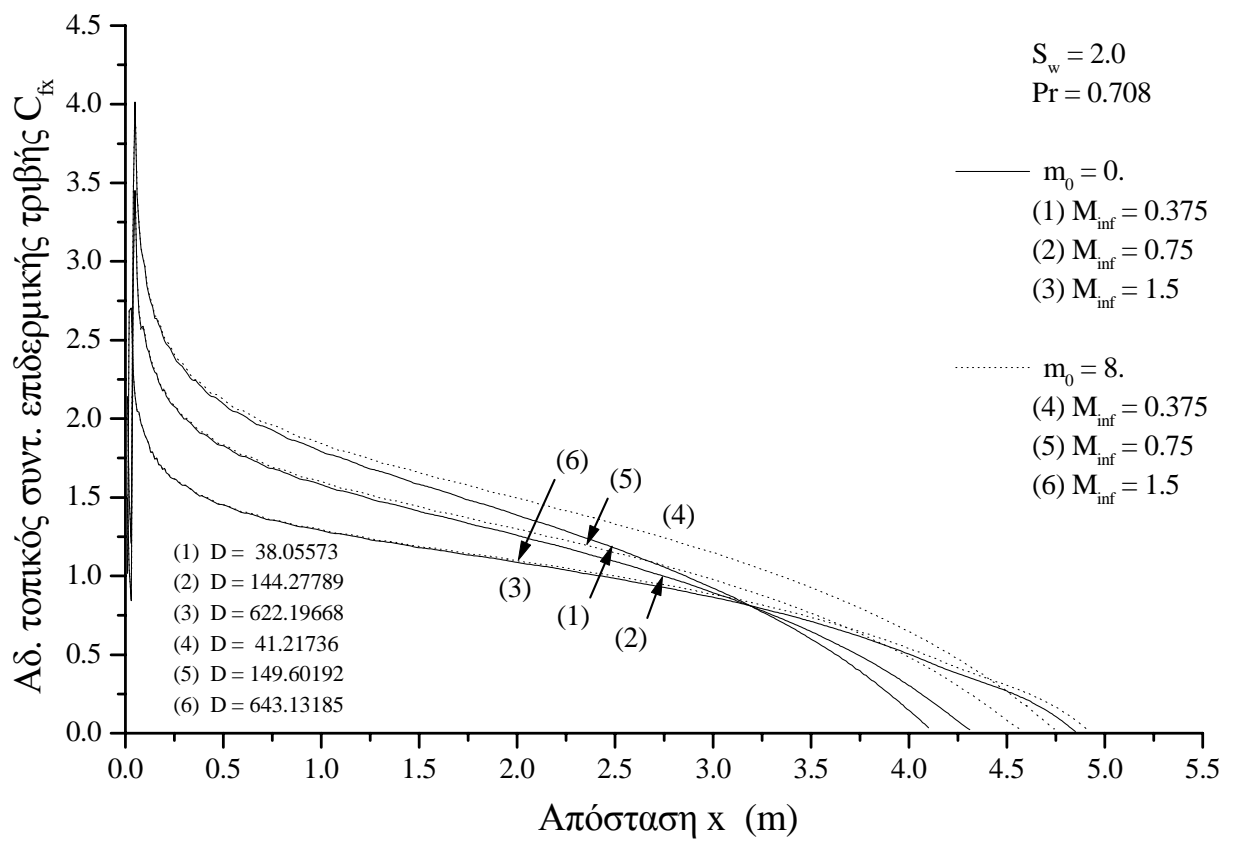

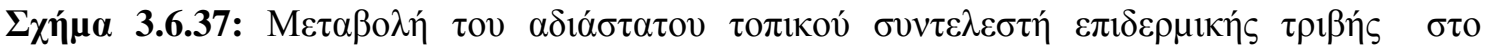

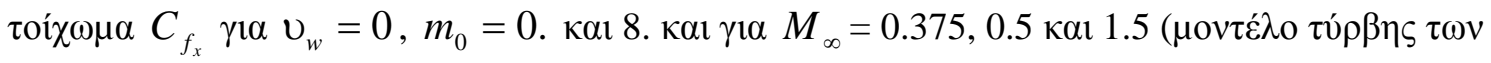
Baldwin - Lomax) 


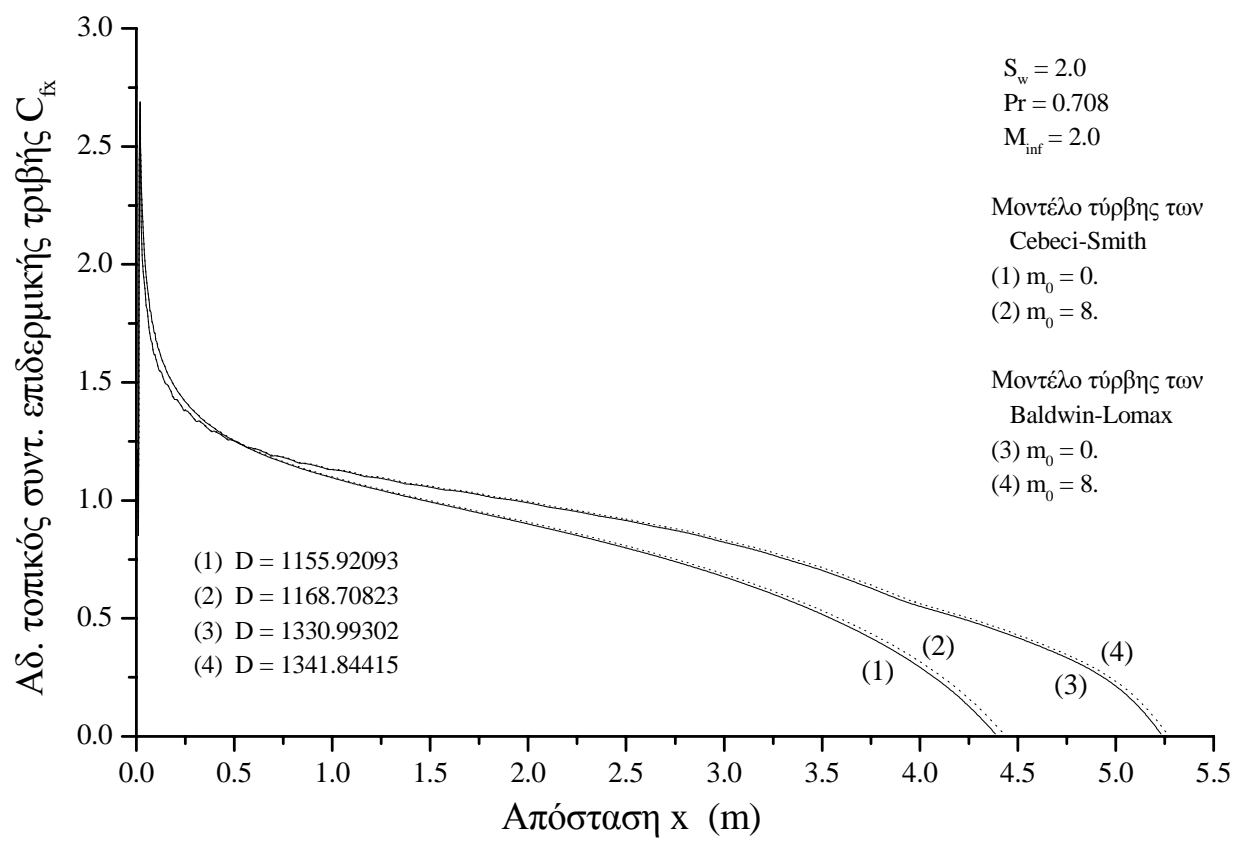

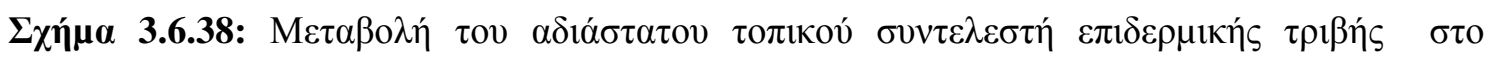

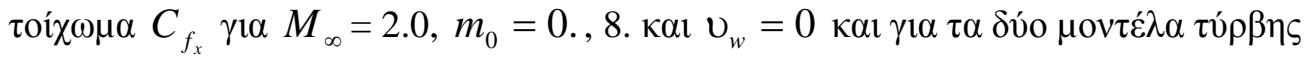

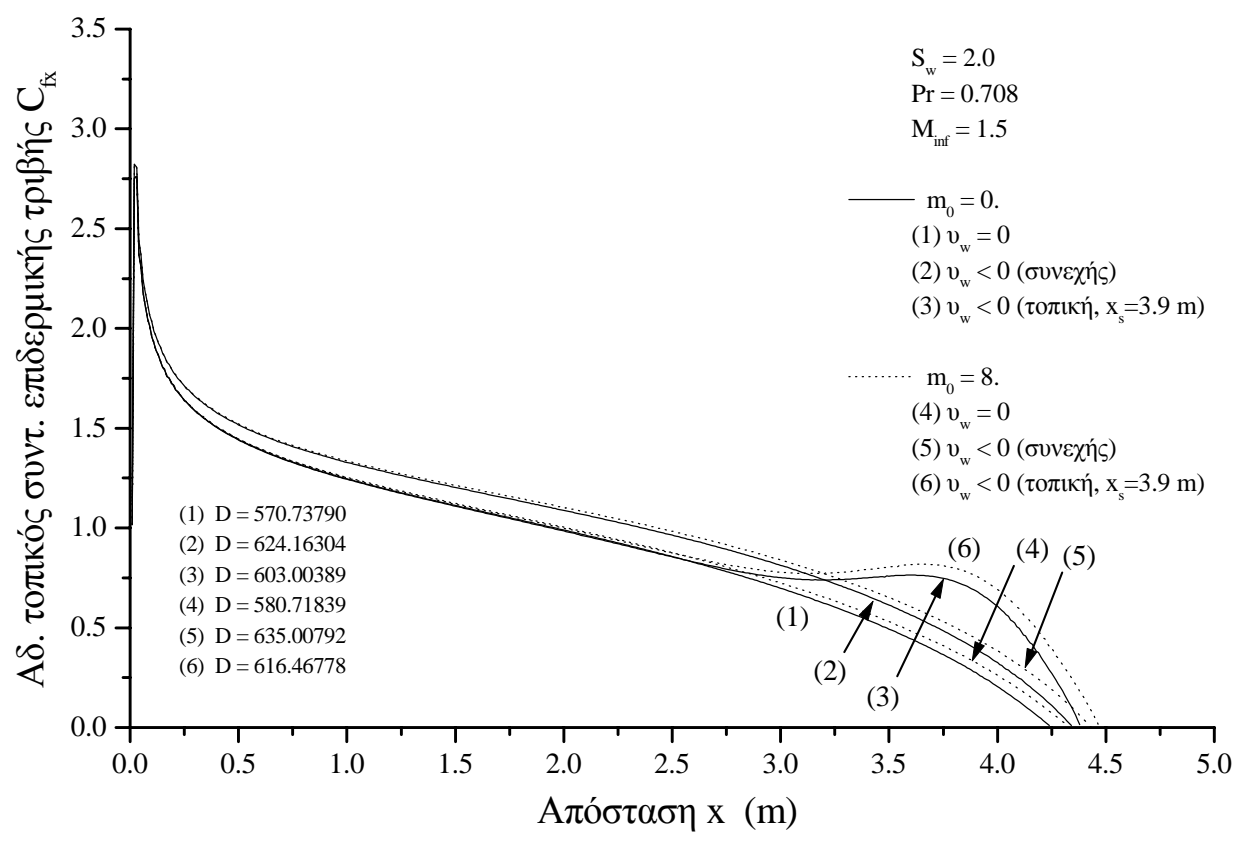

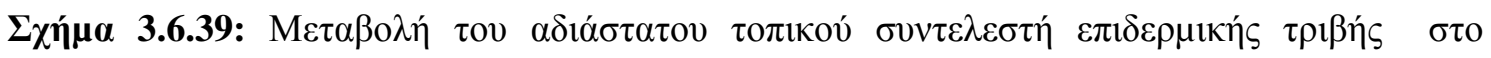

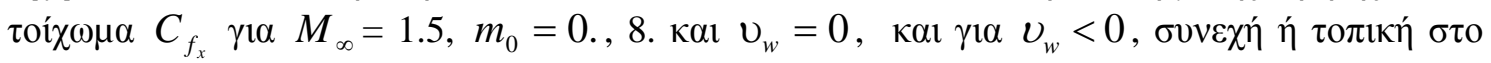

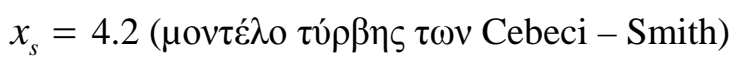




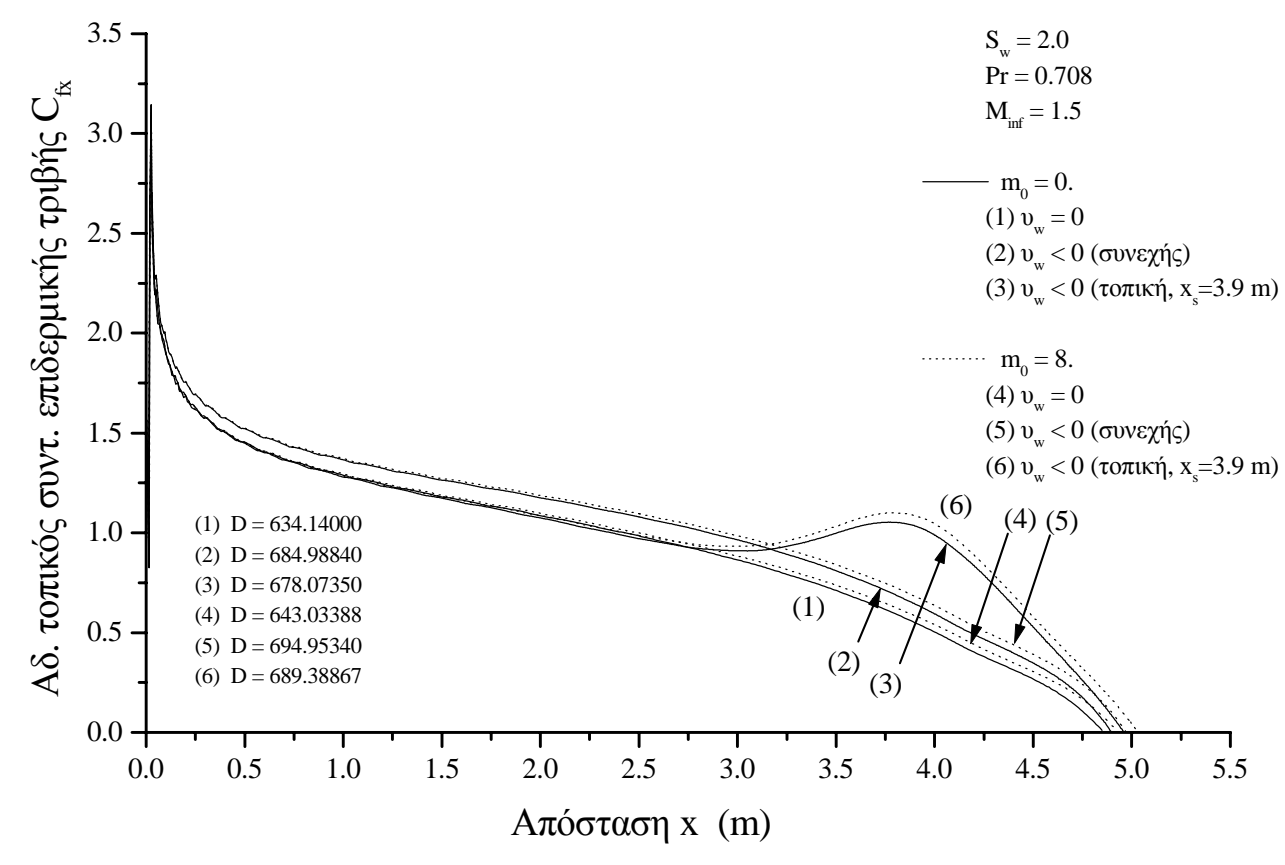

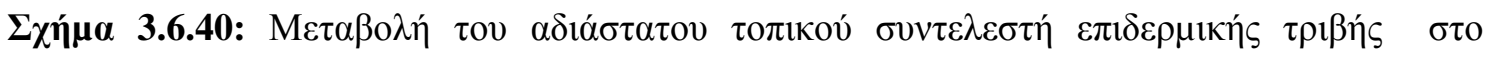

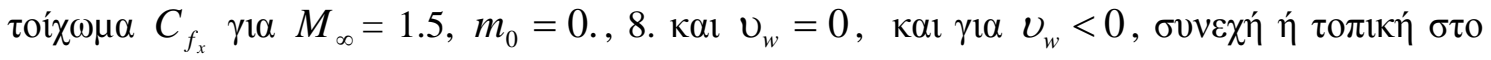

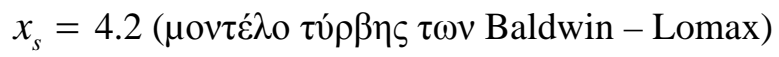

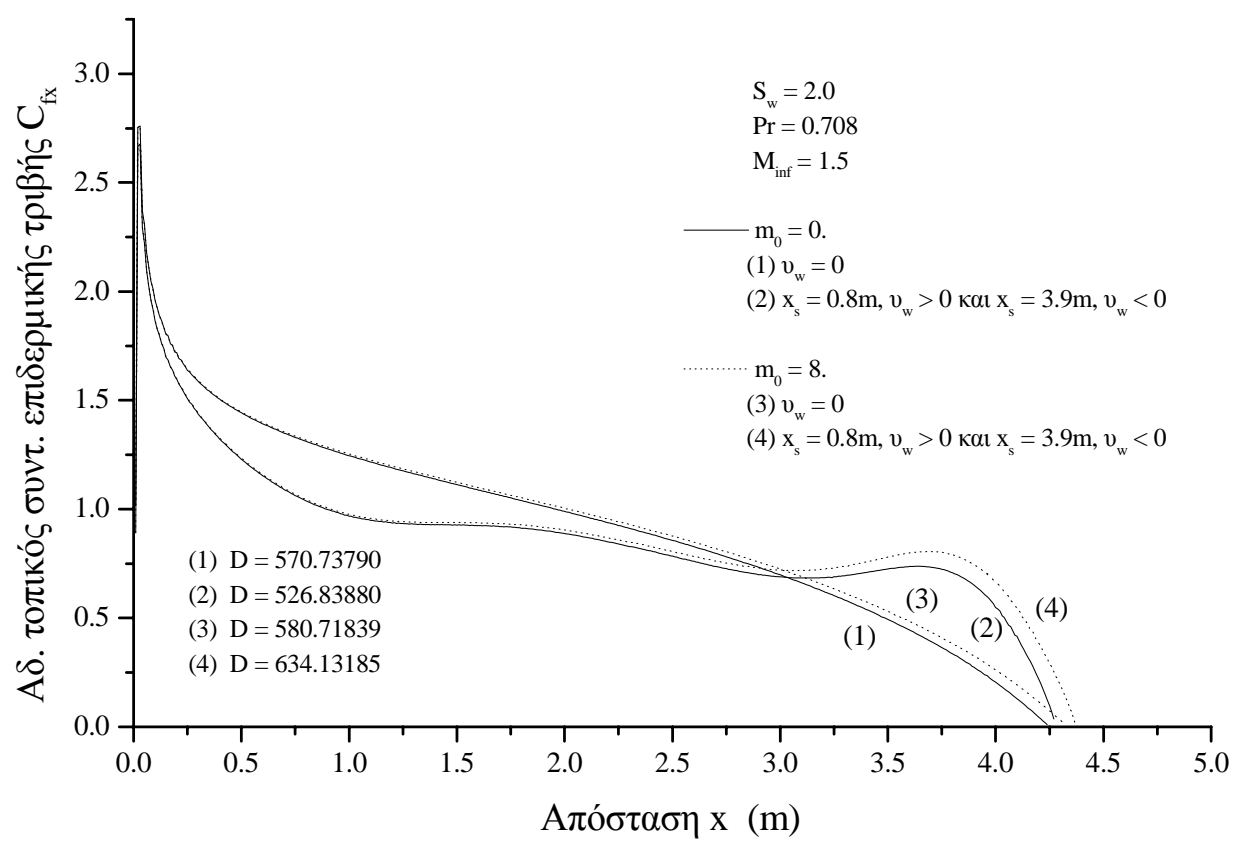

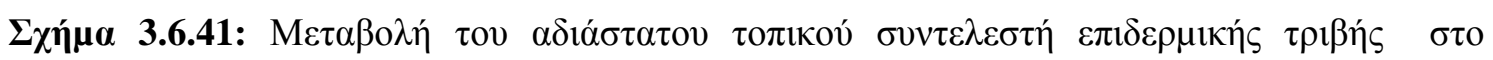

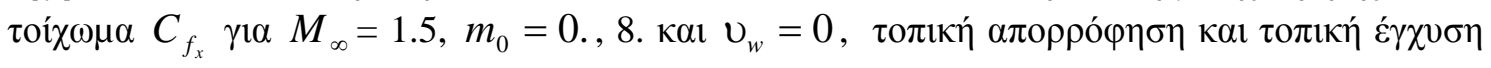

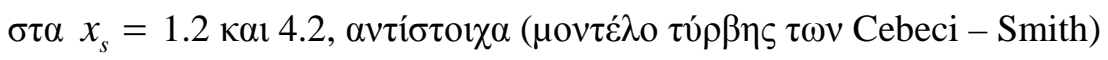




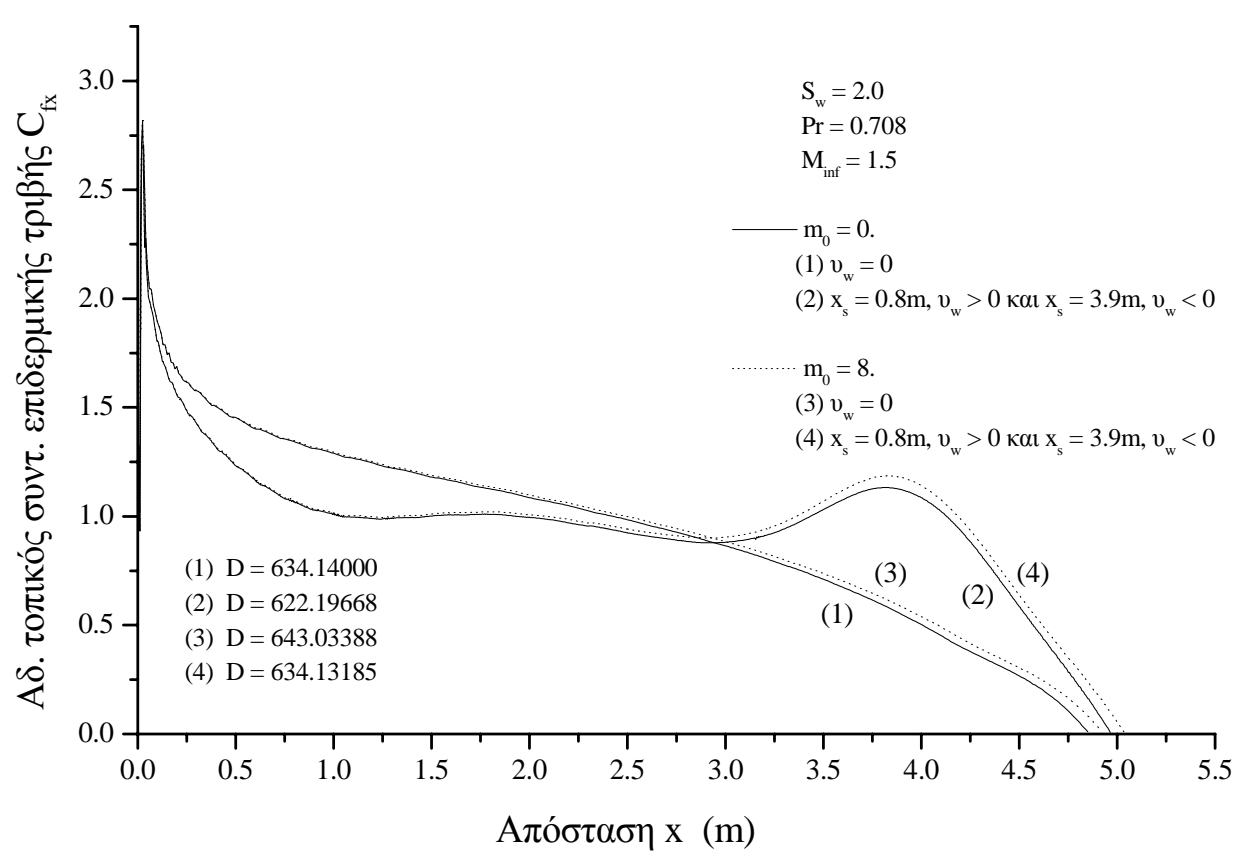

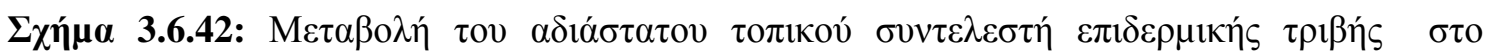

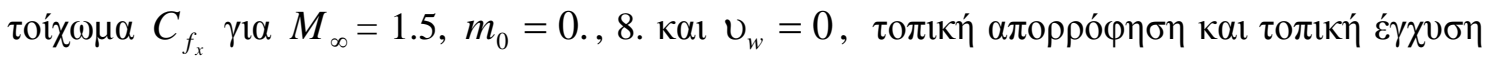

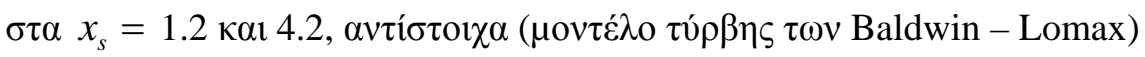

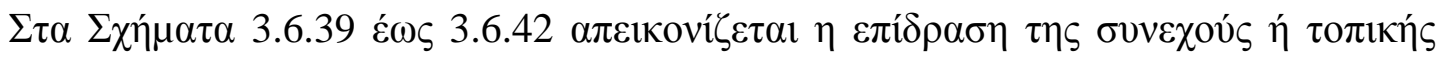

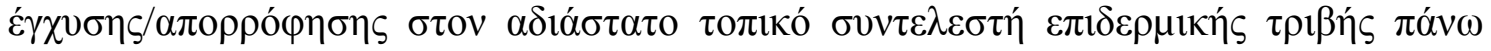

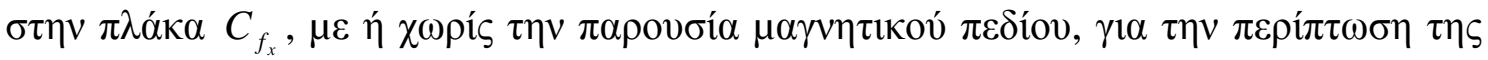

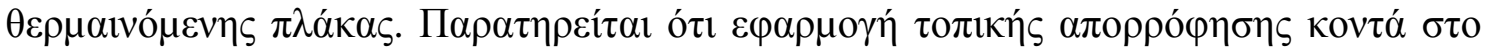

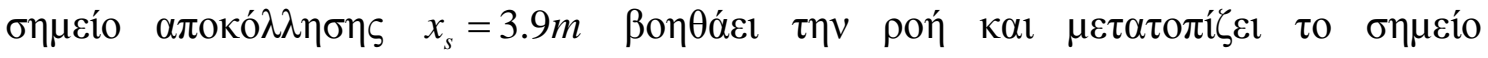

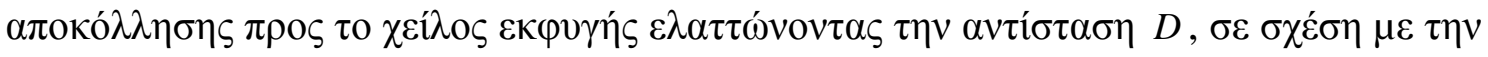

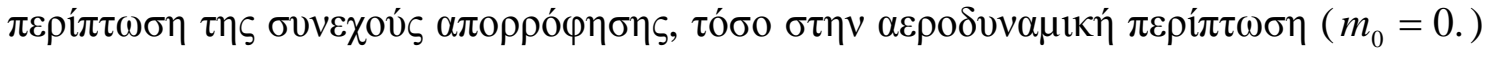

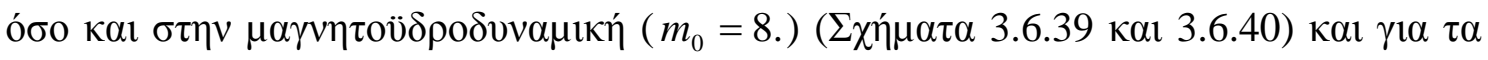

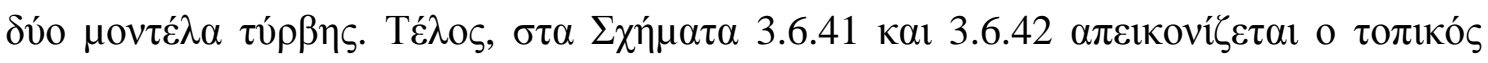

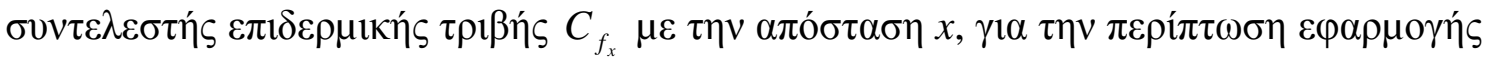

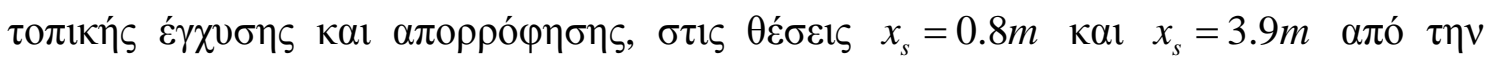

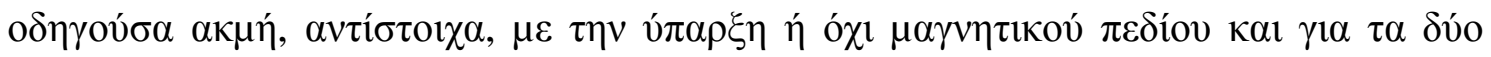

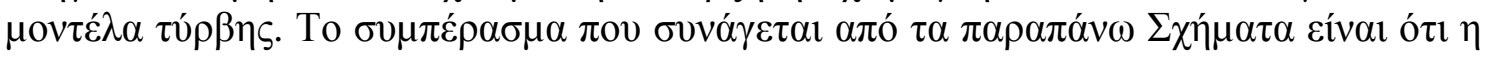

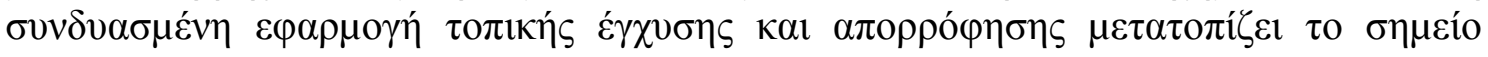

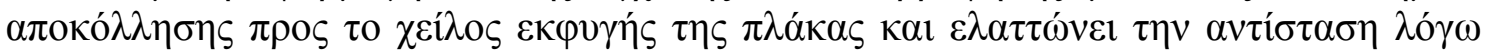

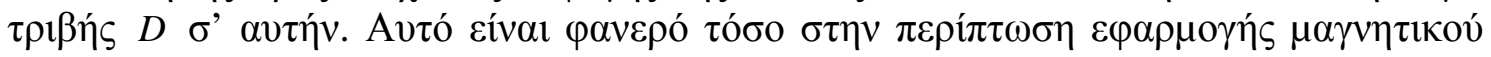

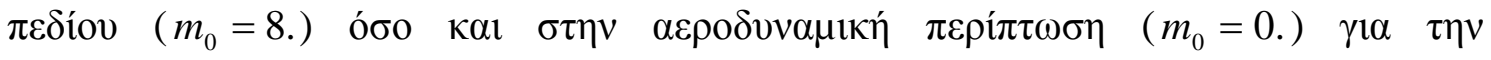

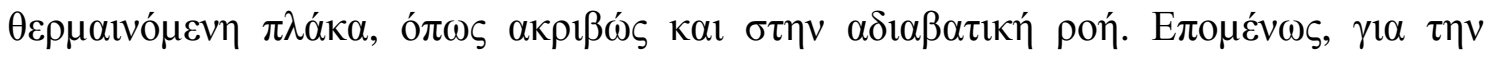

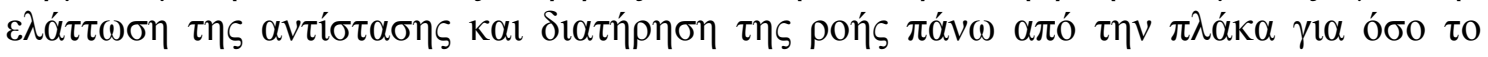

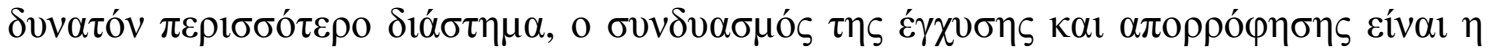

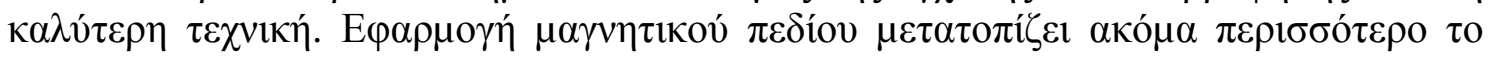




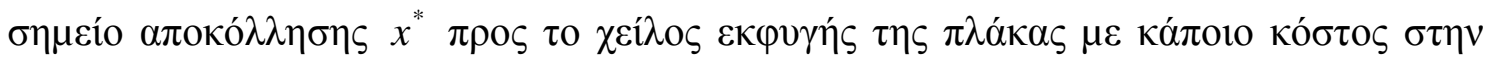

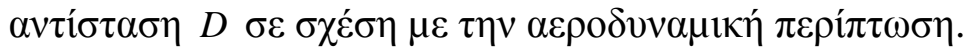

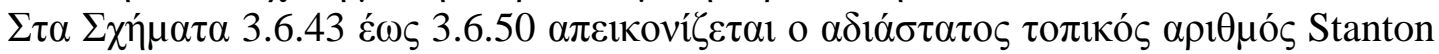

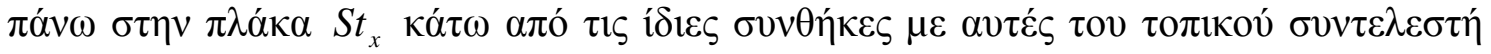

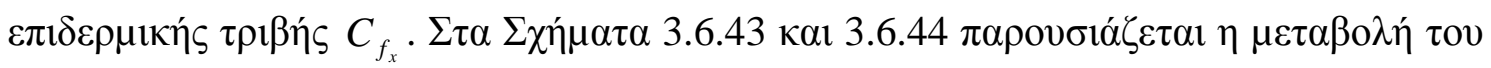

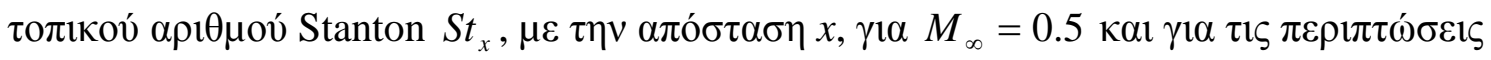

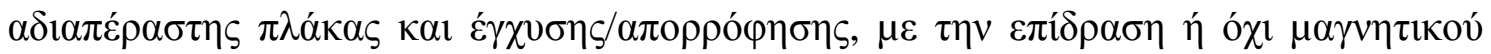

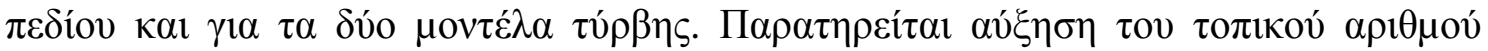

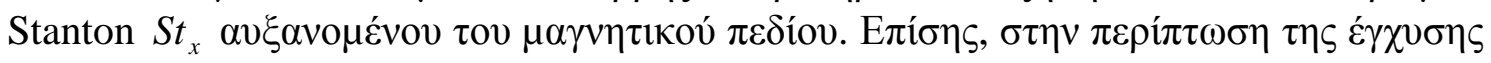

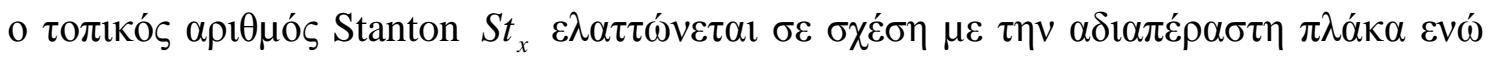

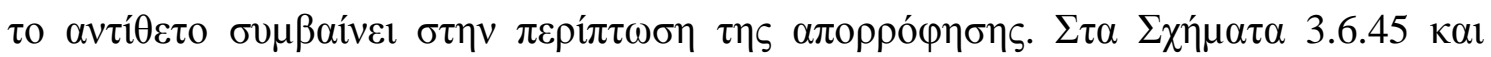

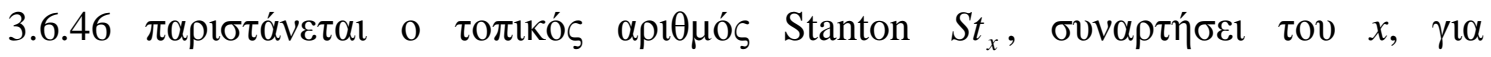

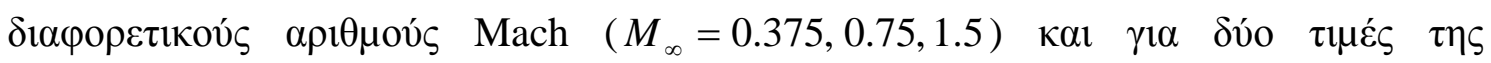

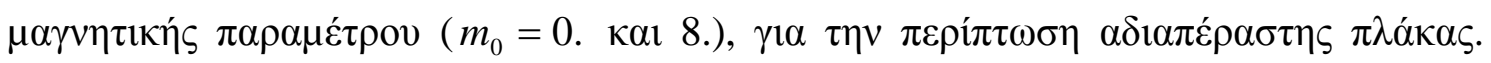

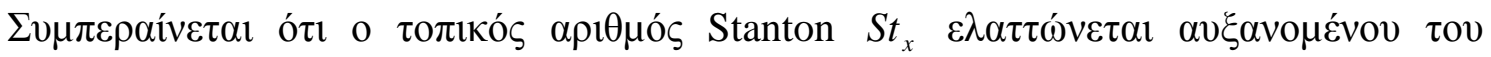

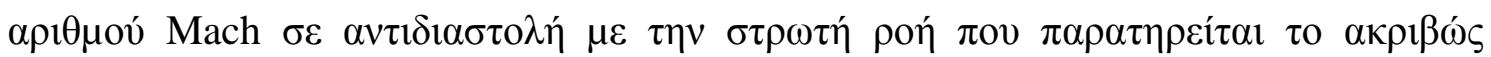

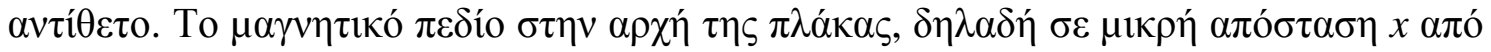

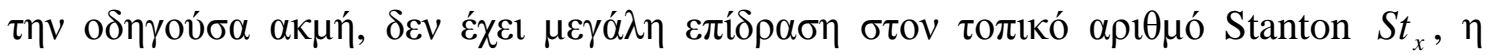

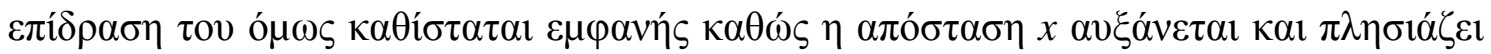

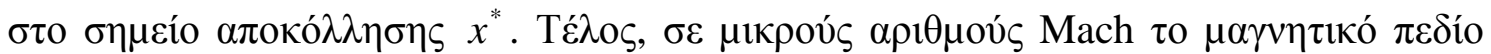

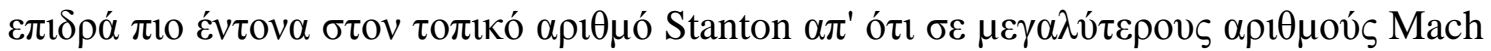

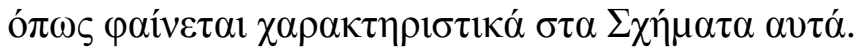

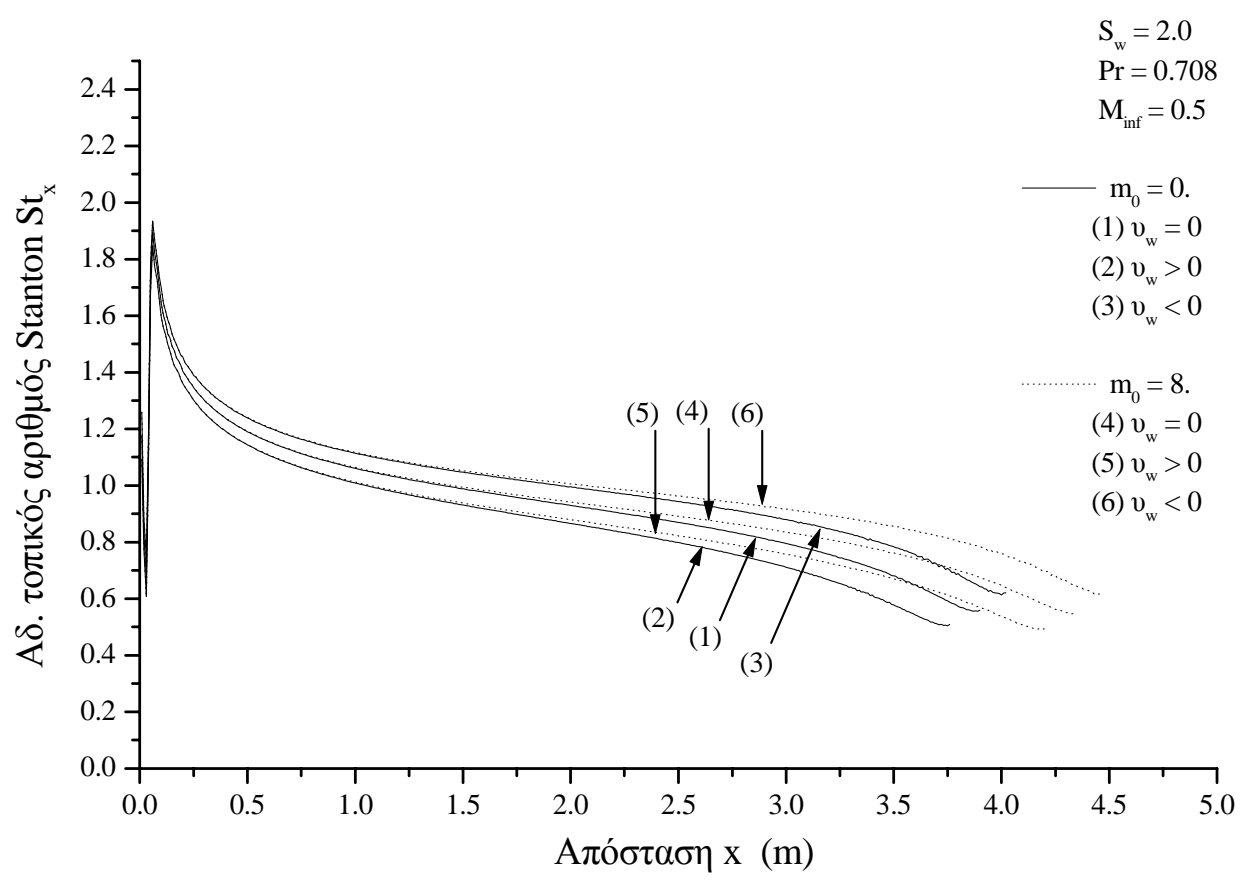

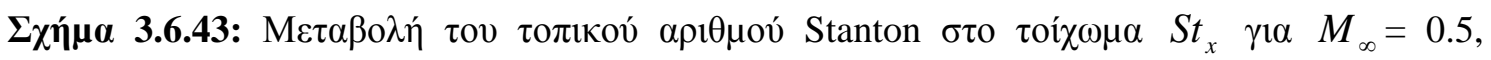

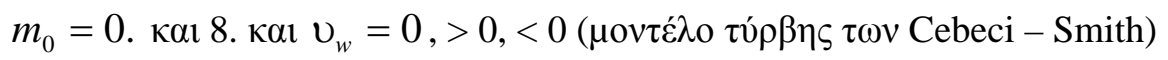




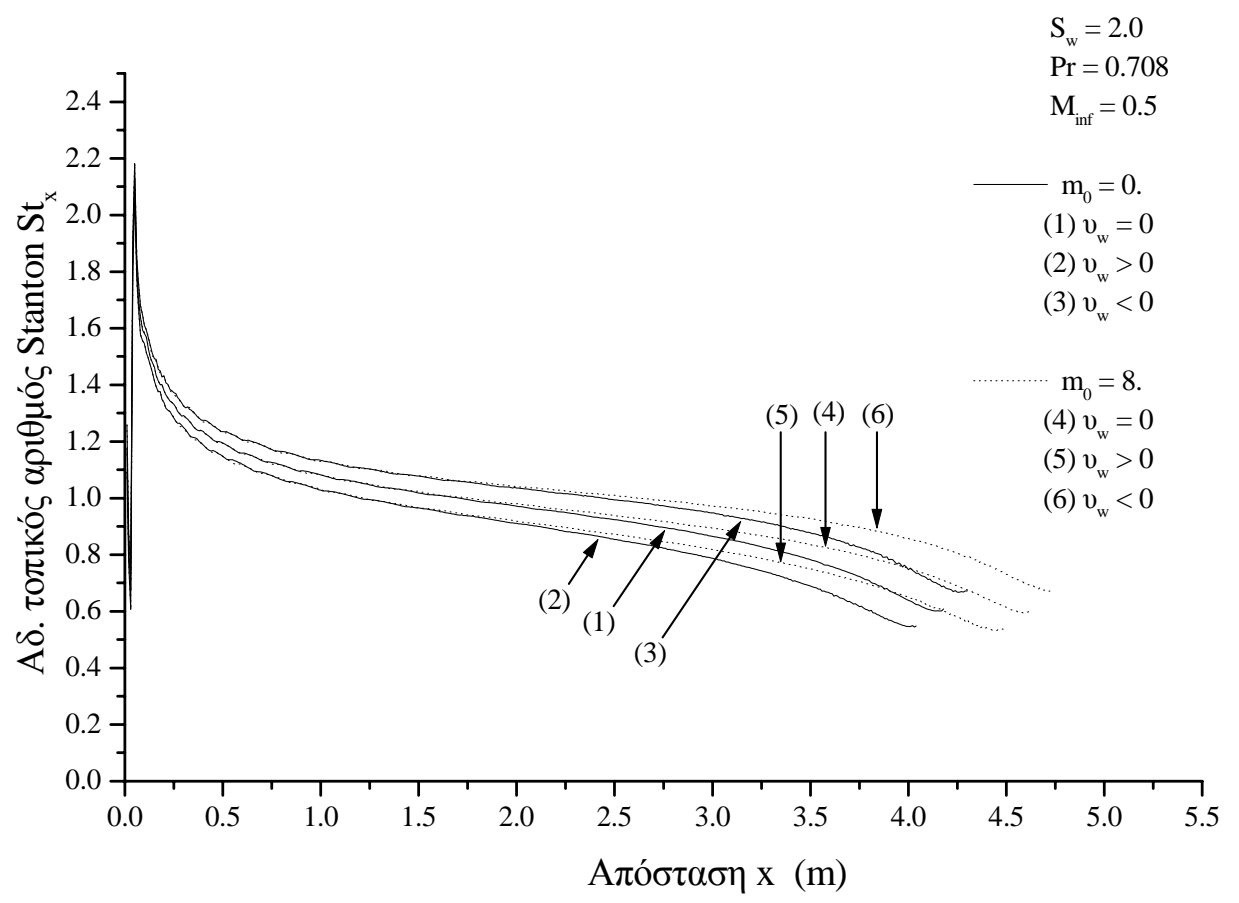

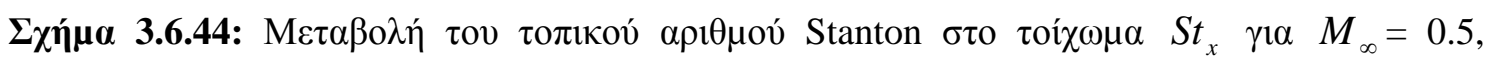

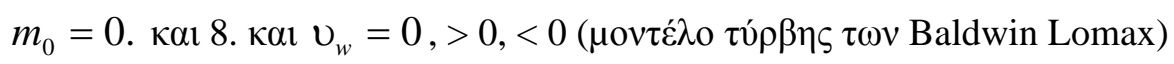

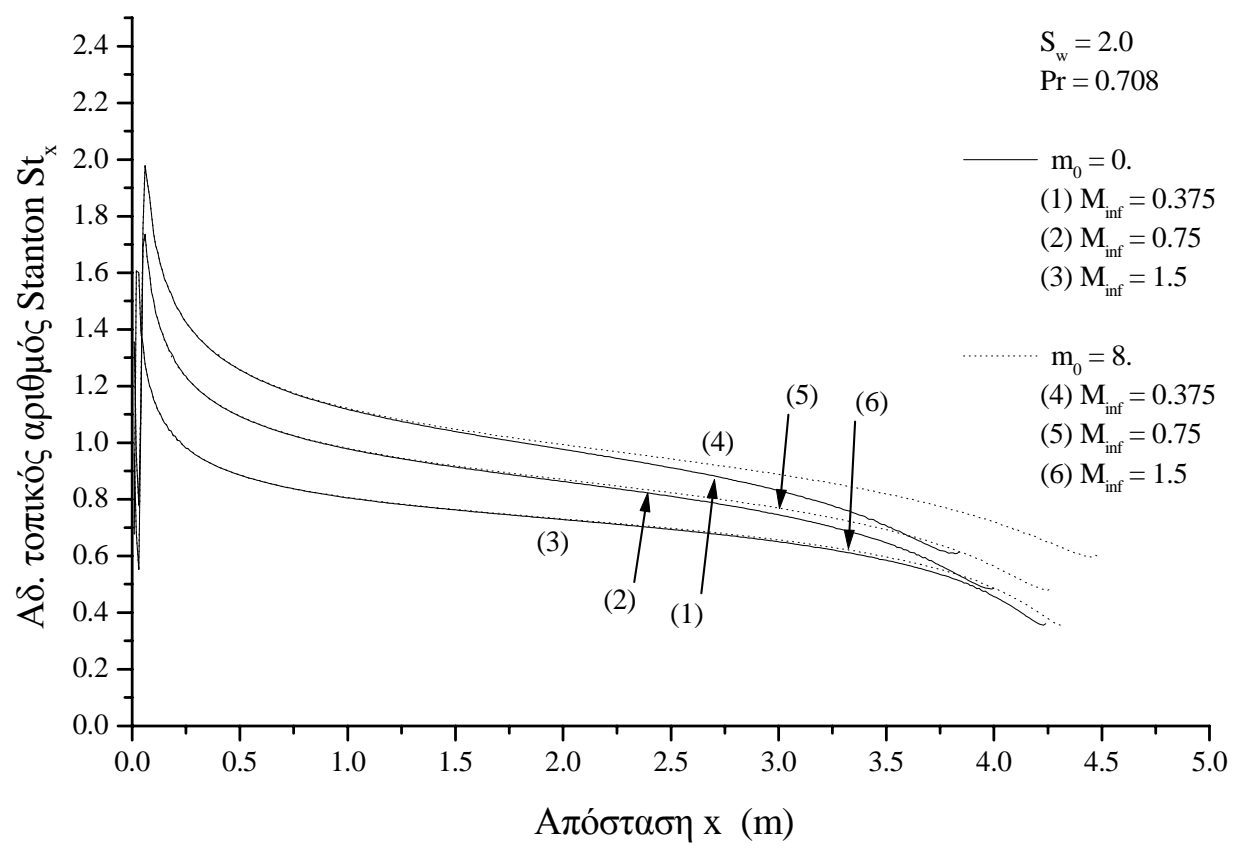

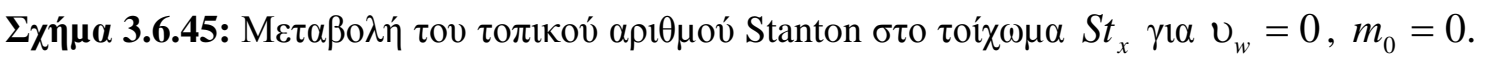

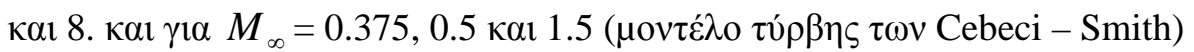




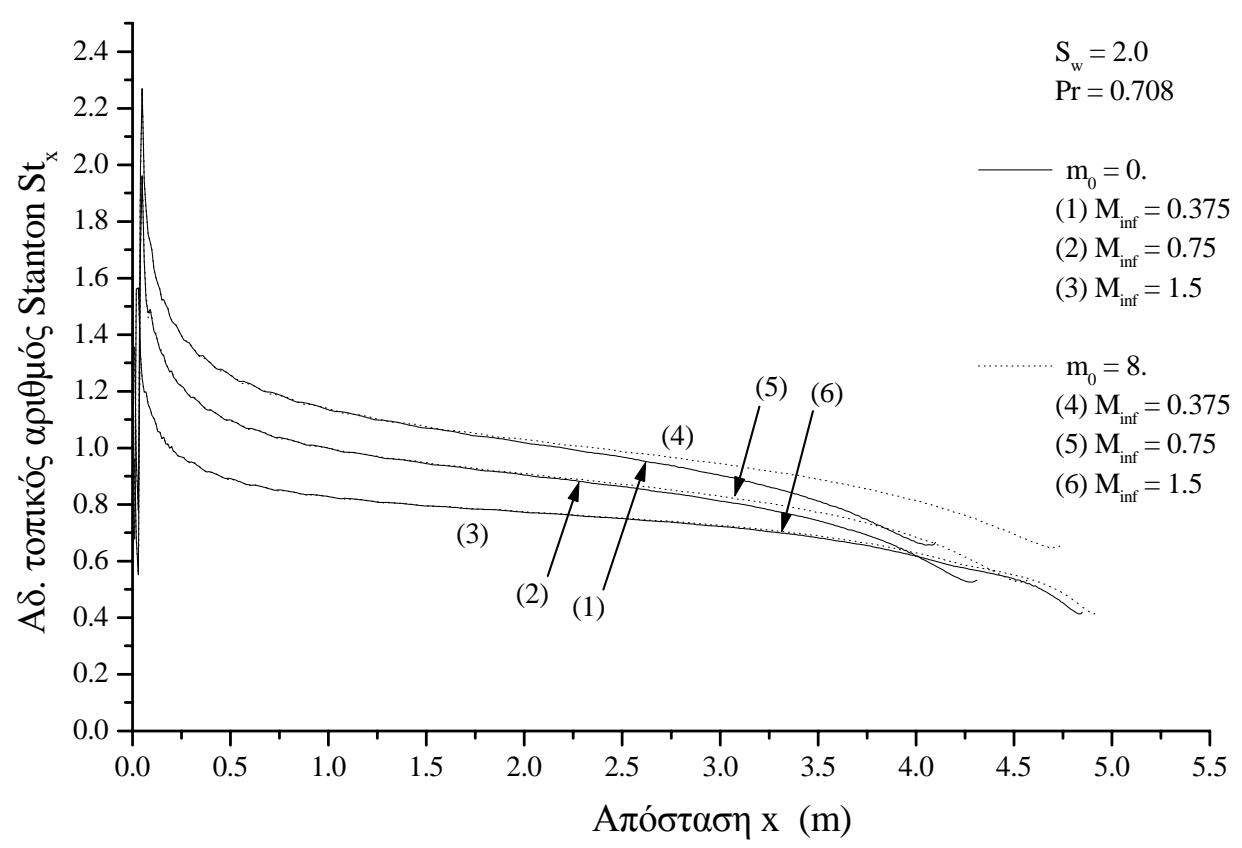

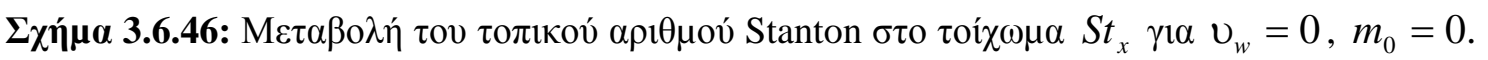

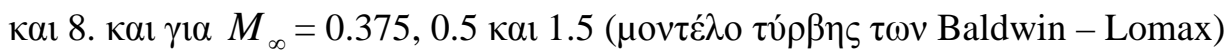

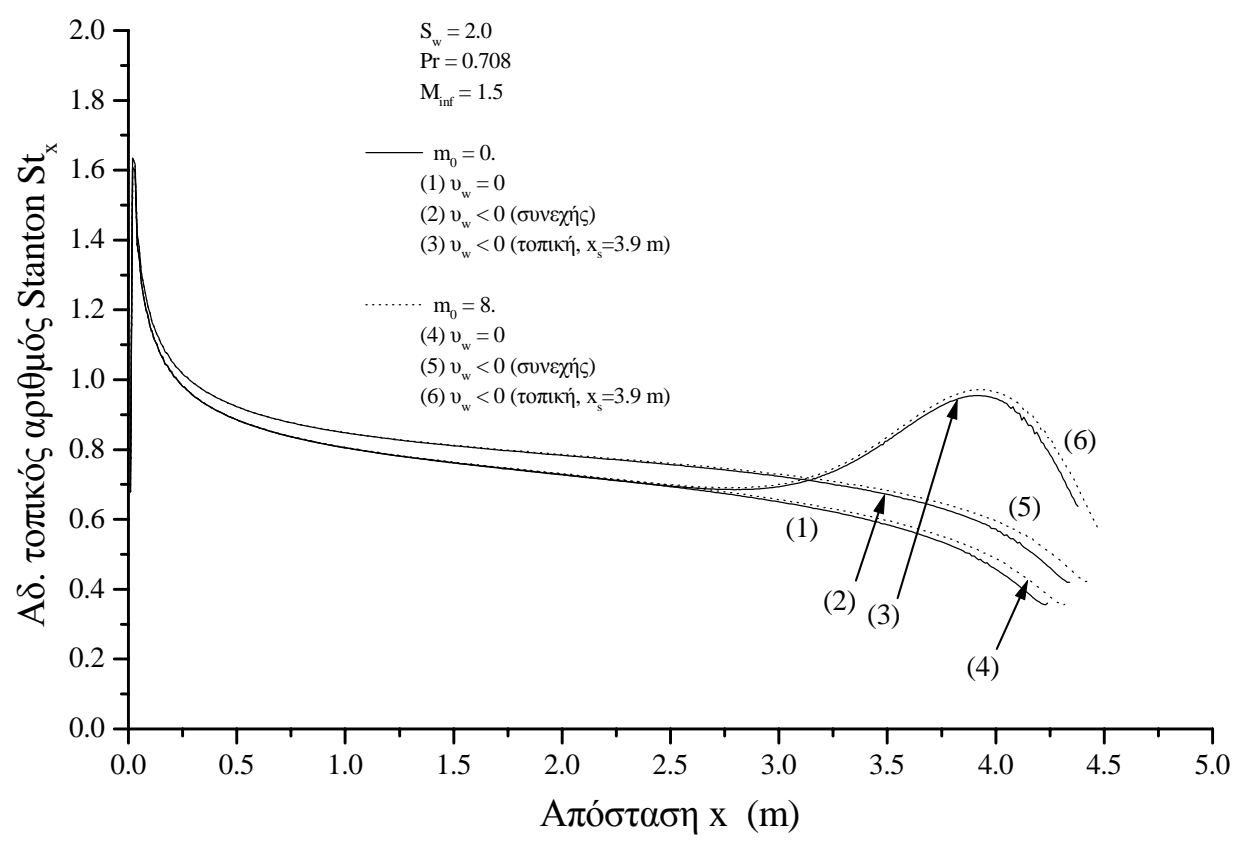

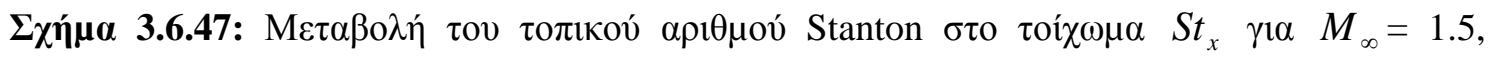

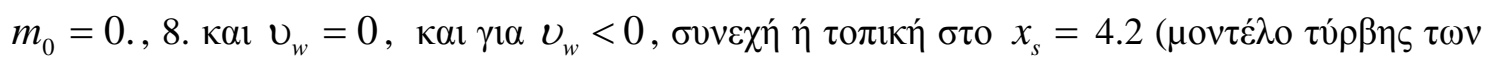
Cebeci - Smith) 


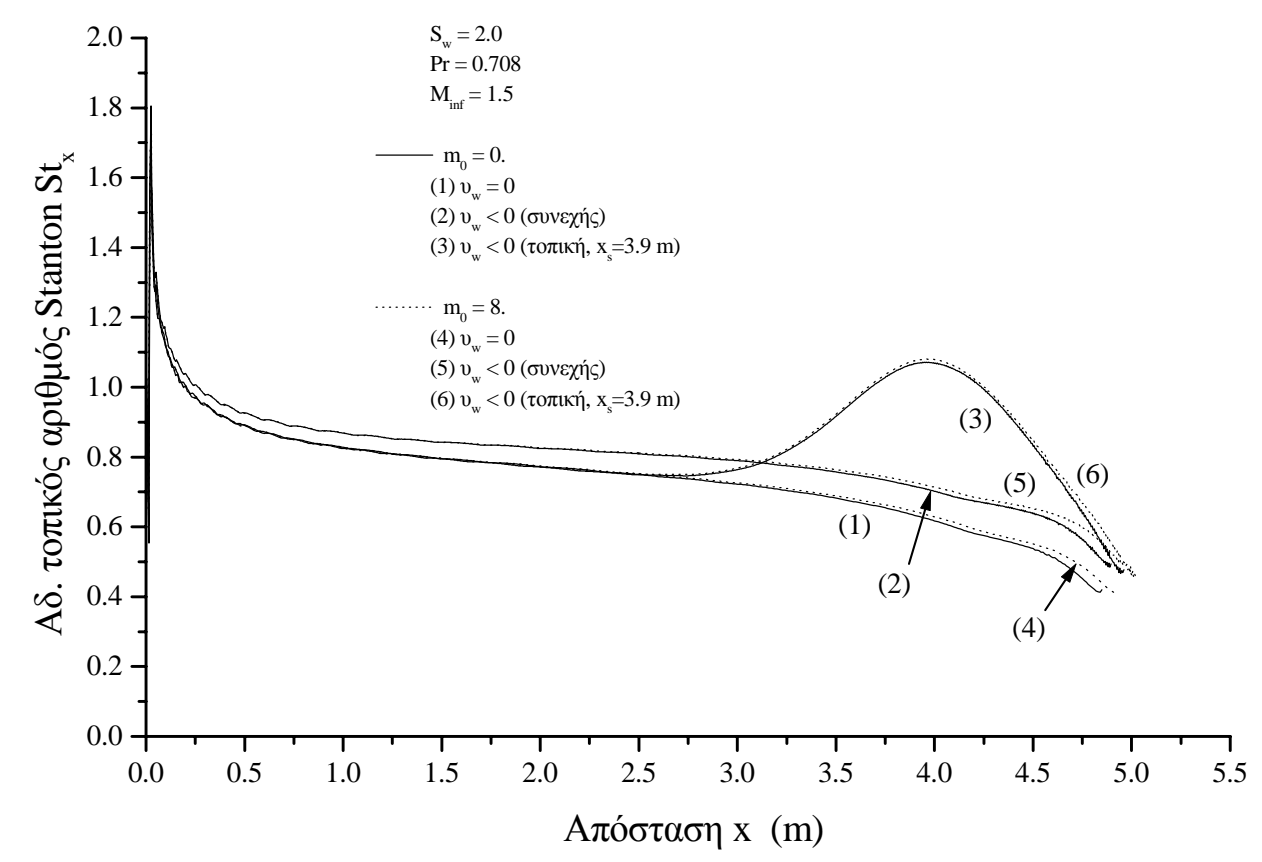

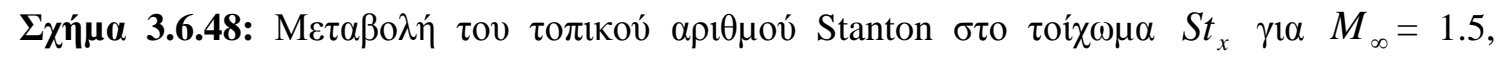

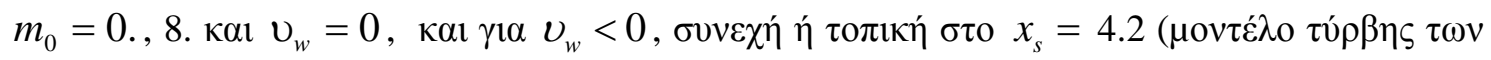
Baldwin - Lomax)

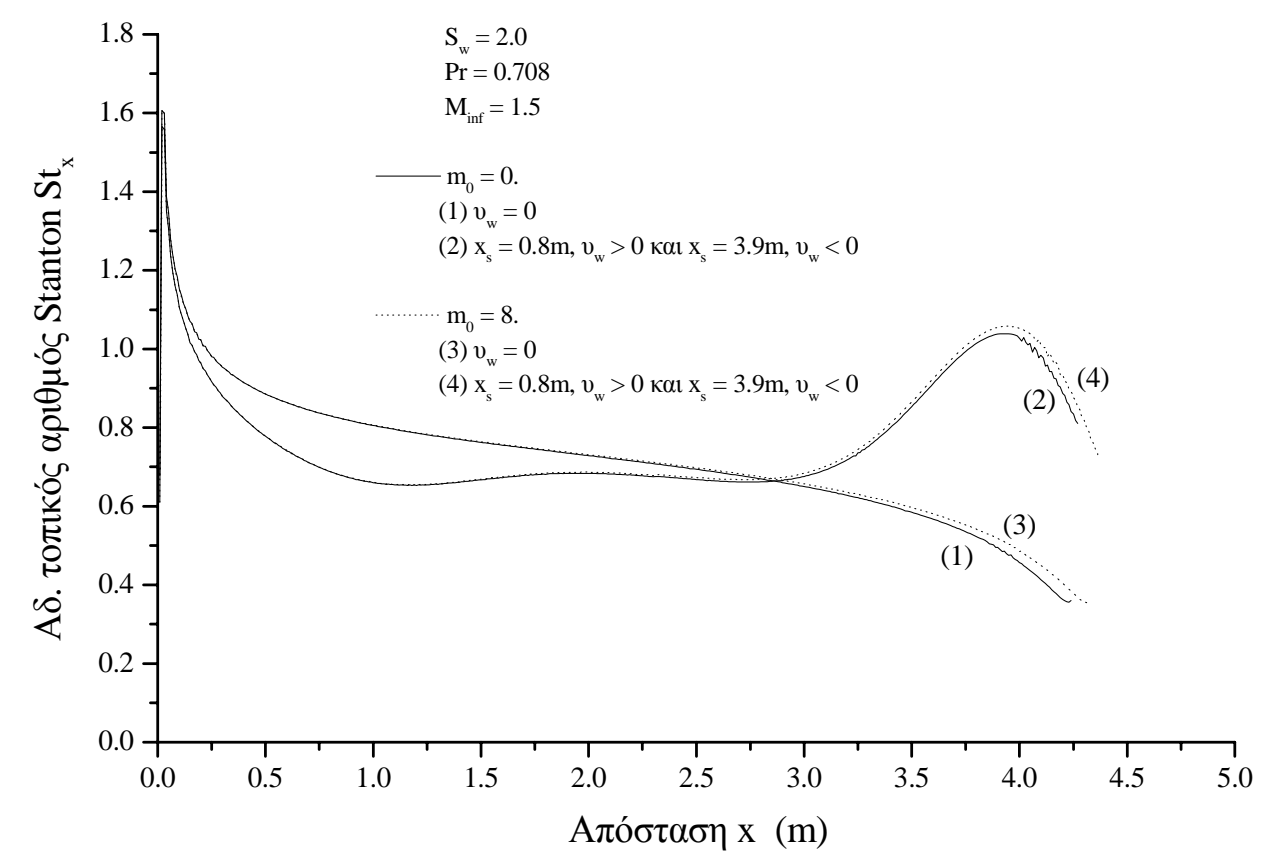

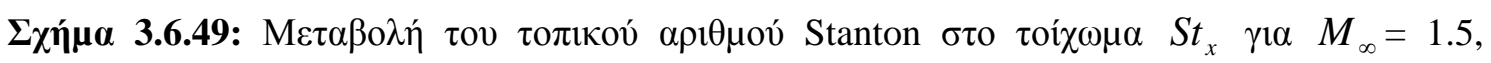

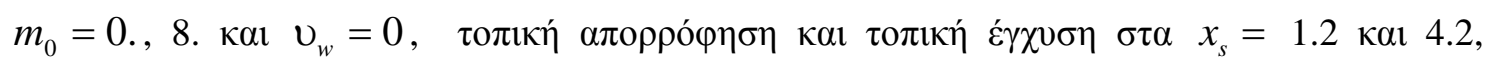

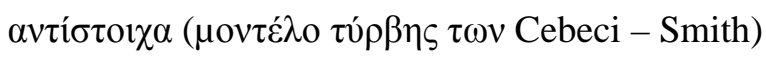




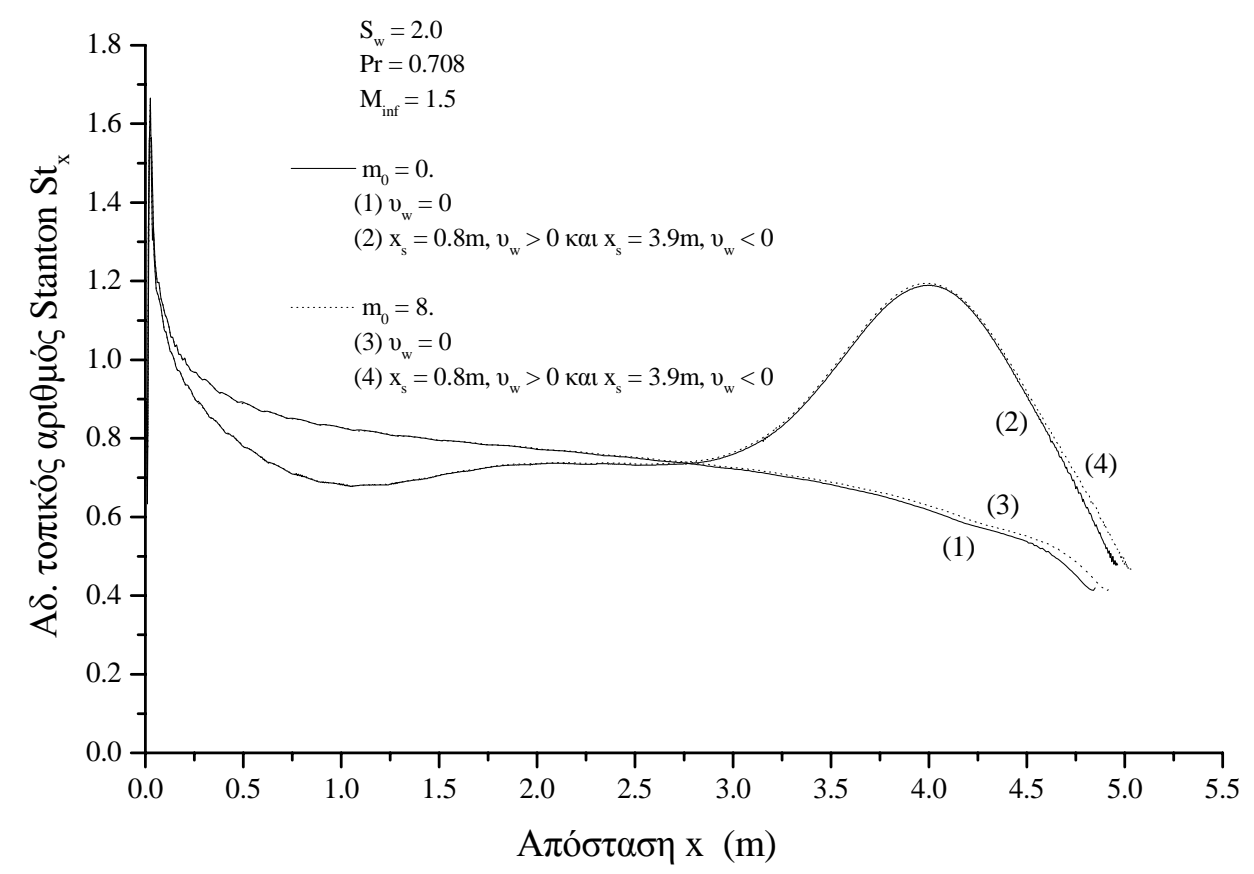

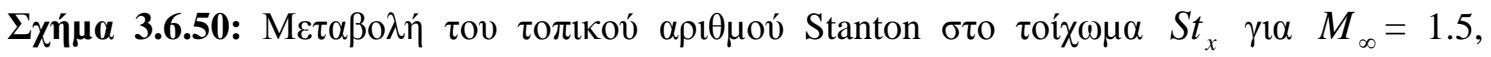

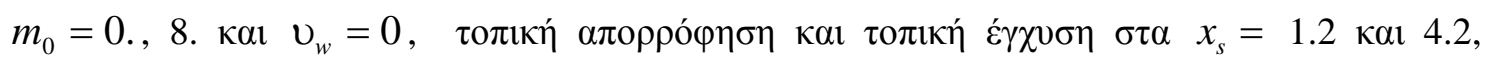

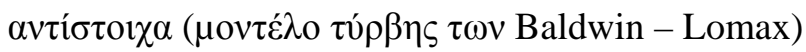

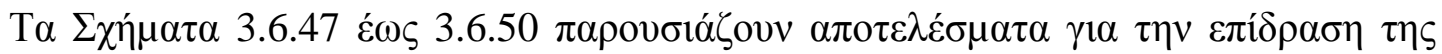

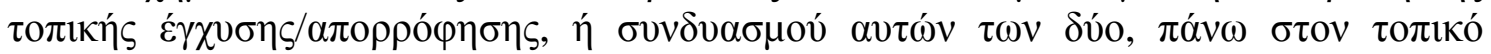

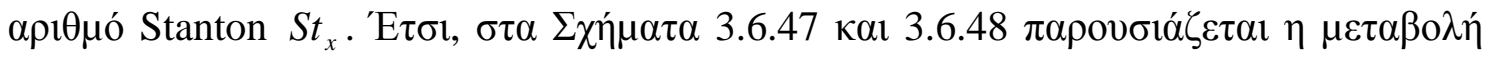

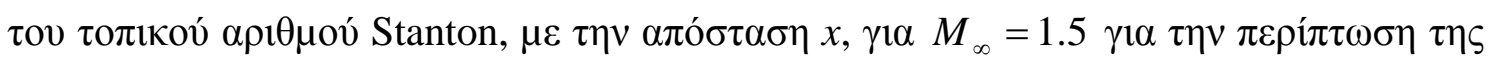

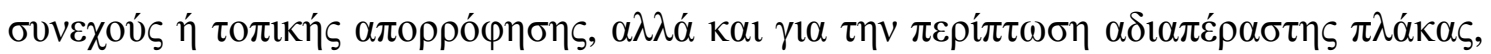

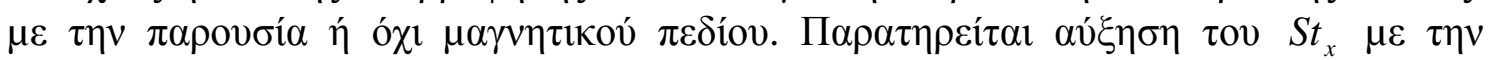

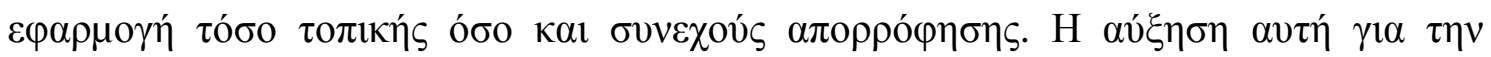

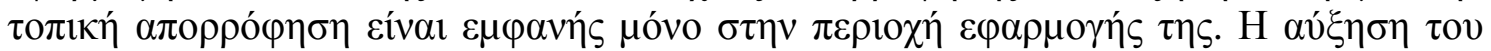

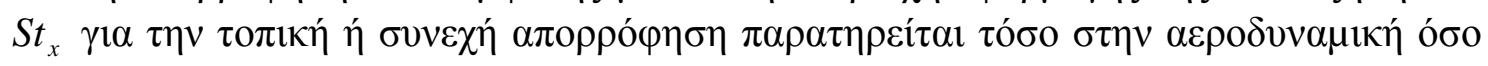

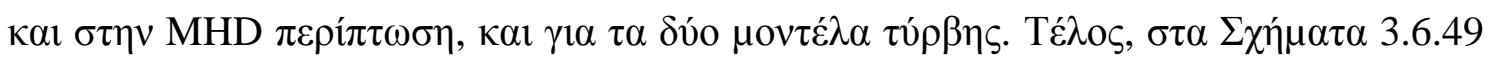

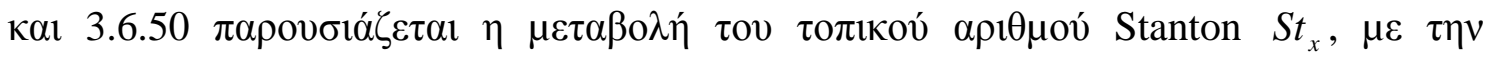

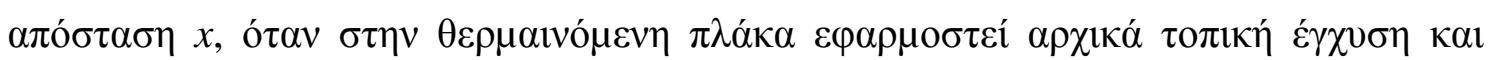

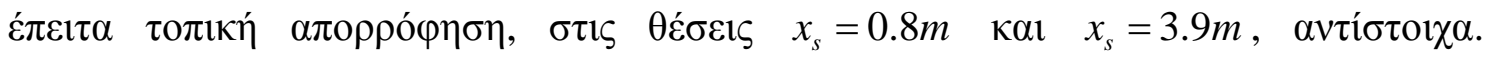

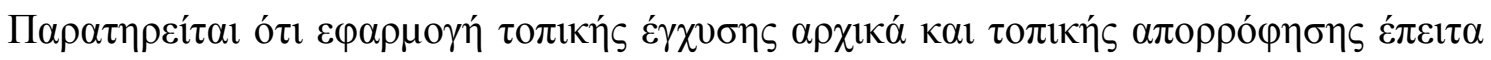

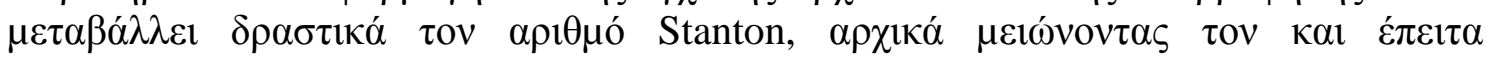

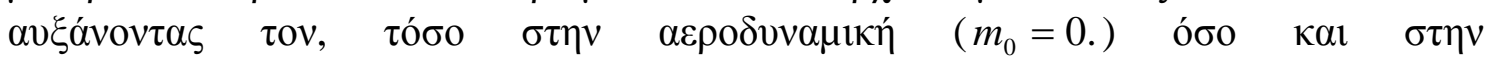

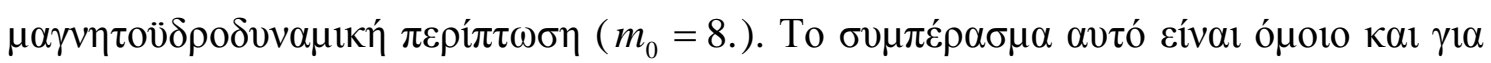

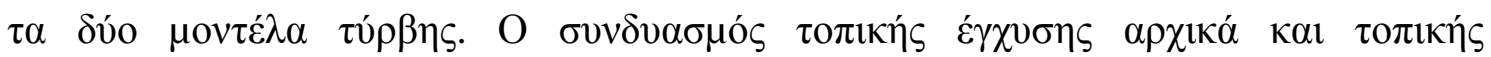

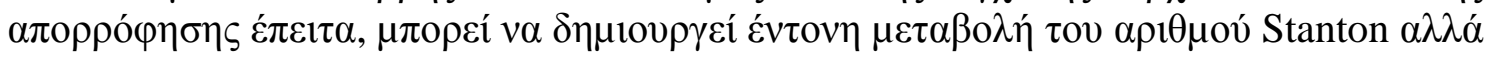

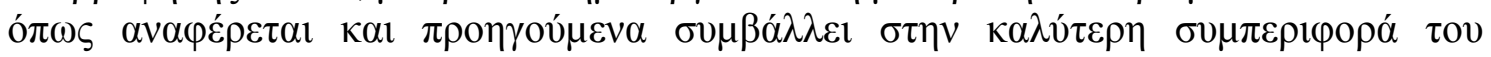

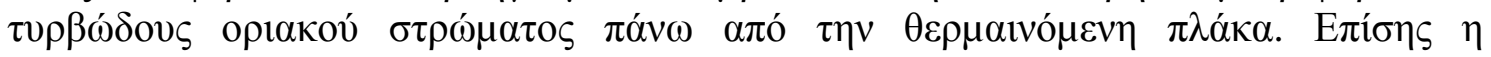




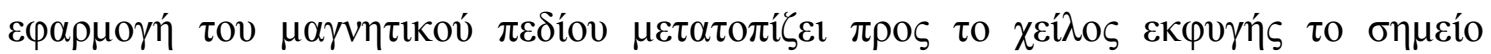

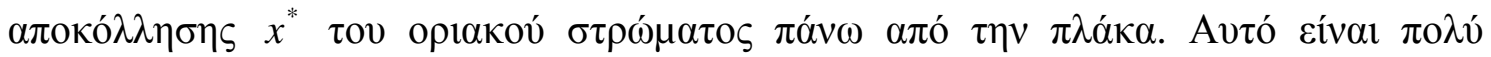

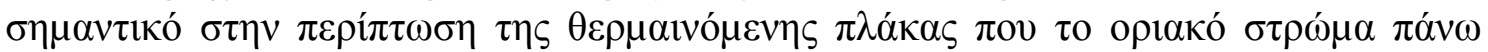

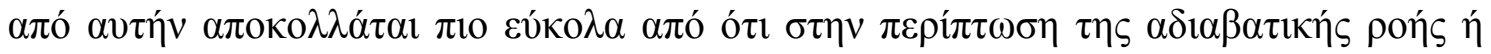
$\tau \eta \varsigma \psi v \chi o ́ \mu \varepsilon v \eta s$.

(iii) $\Psi v \chi o ́ \mu \varepsilon v \eta \pi \lambda a ́ \kappa \alpha a\left(S_{\mathrm{w}}<1\right)$

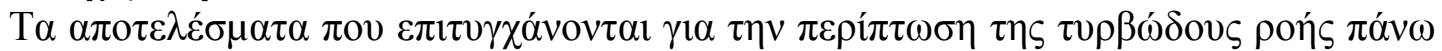

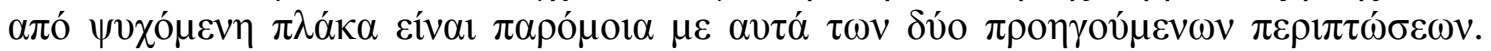

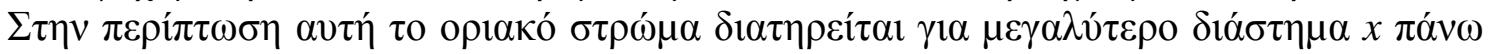

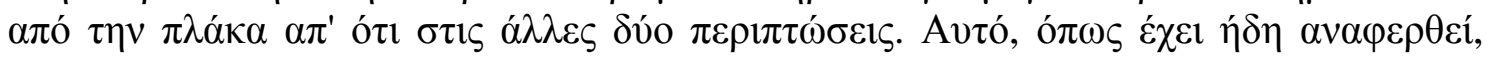

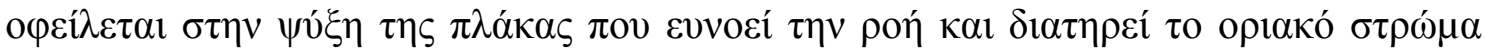

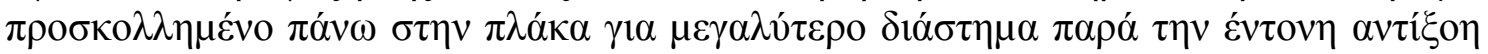

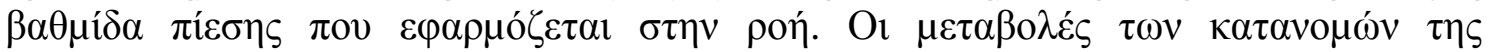

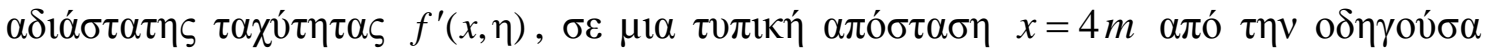

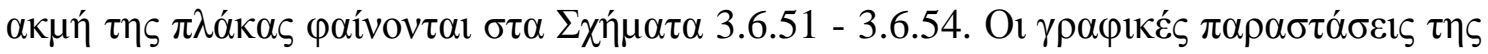

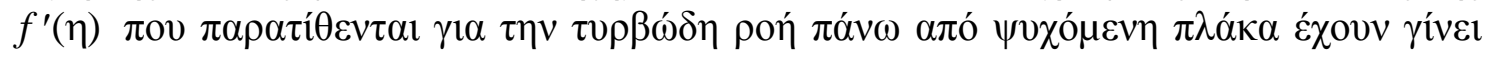

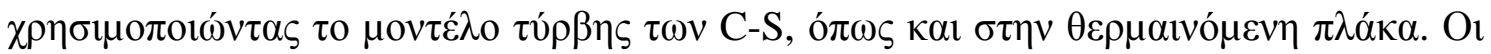

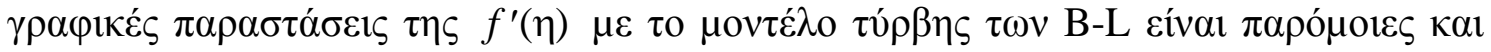

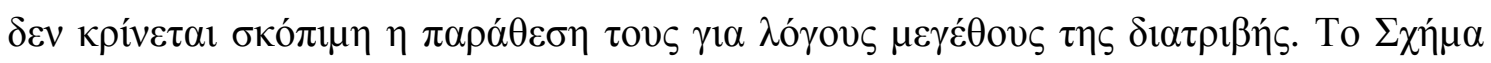

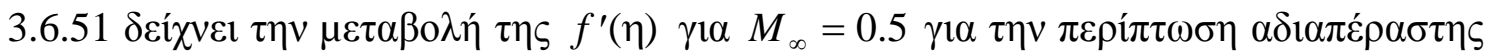

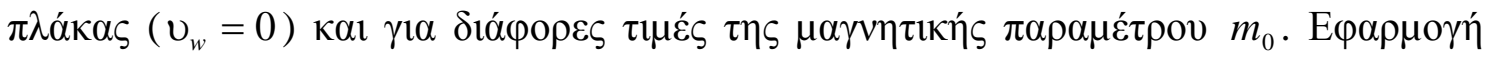

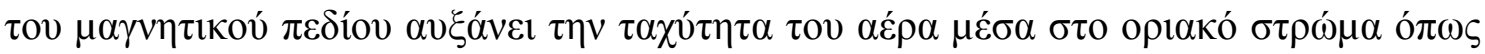

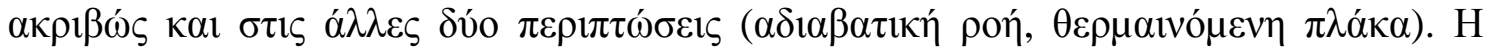

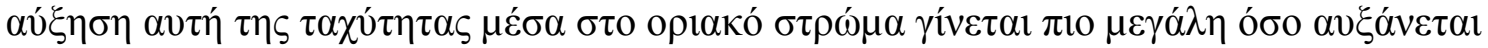

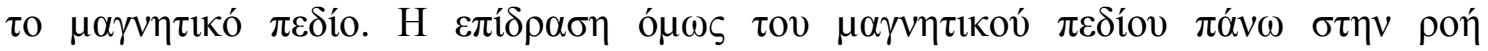

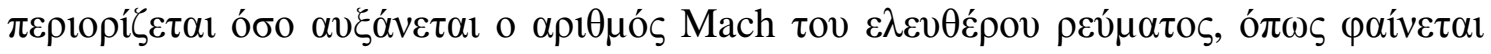

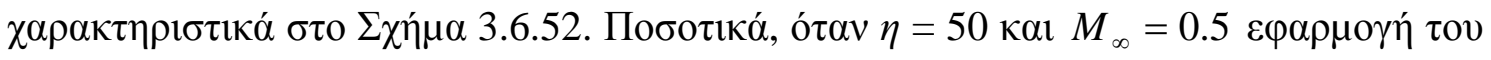

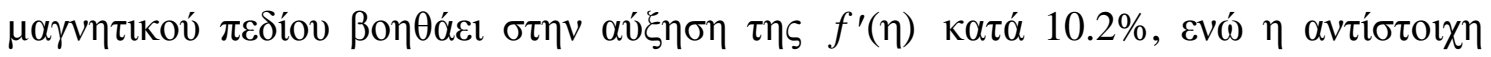

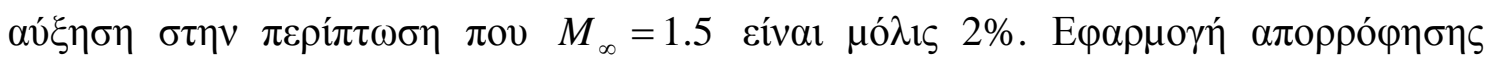

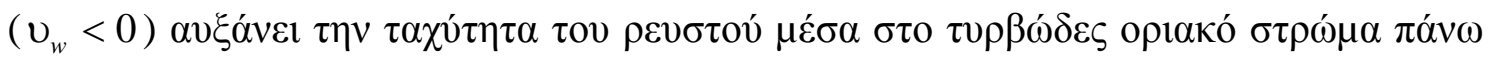

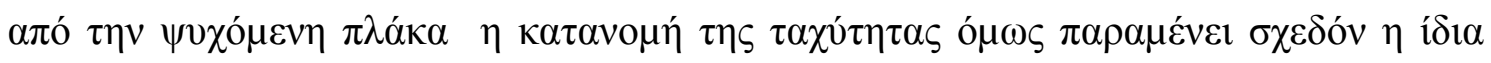

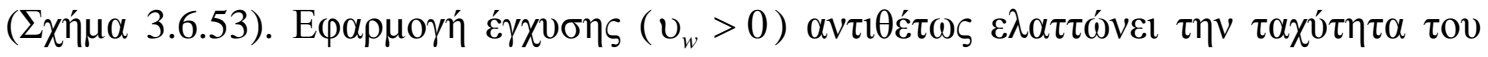

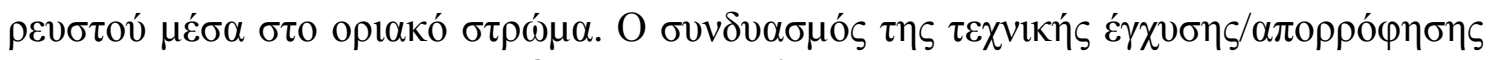

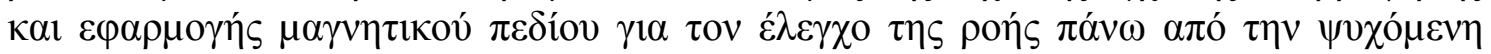

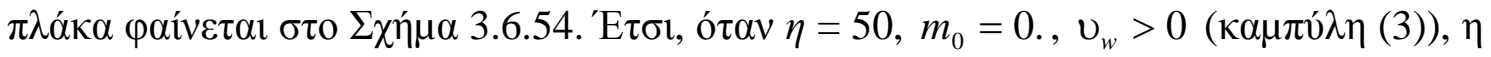

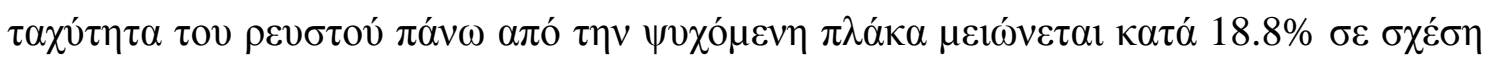

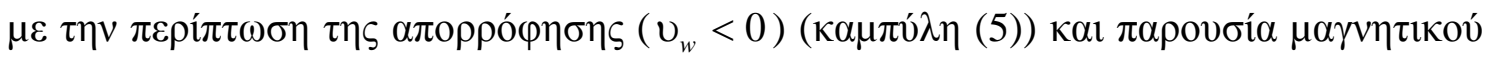
$\pi \varepsilon \delta$ íov $\left(m_{0}=8\right.$.).

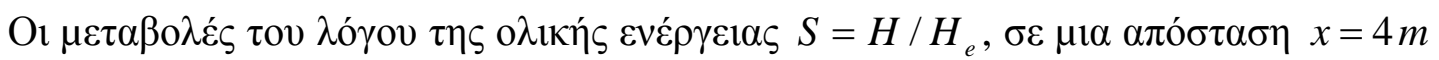

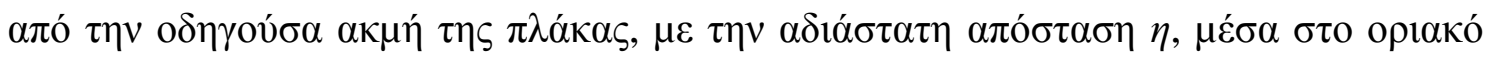

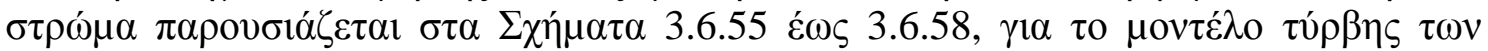

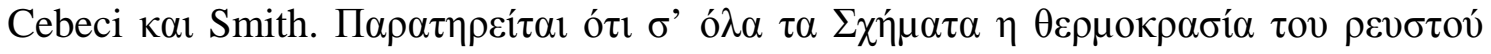

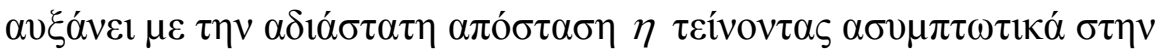




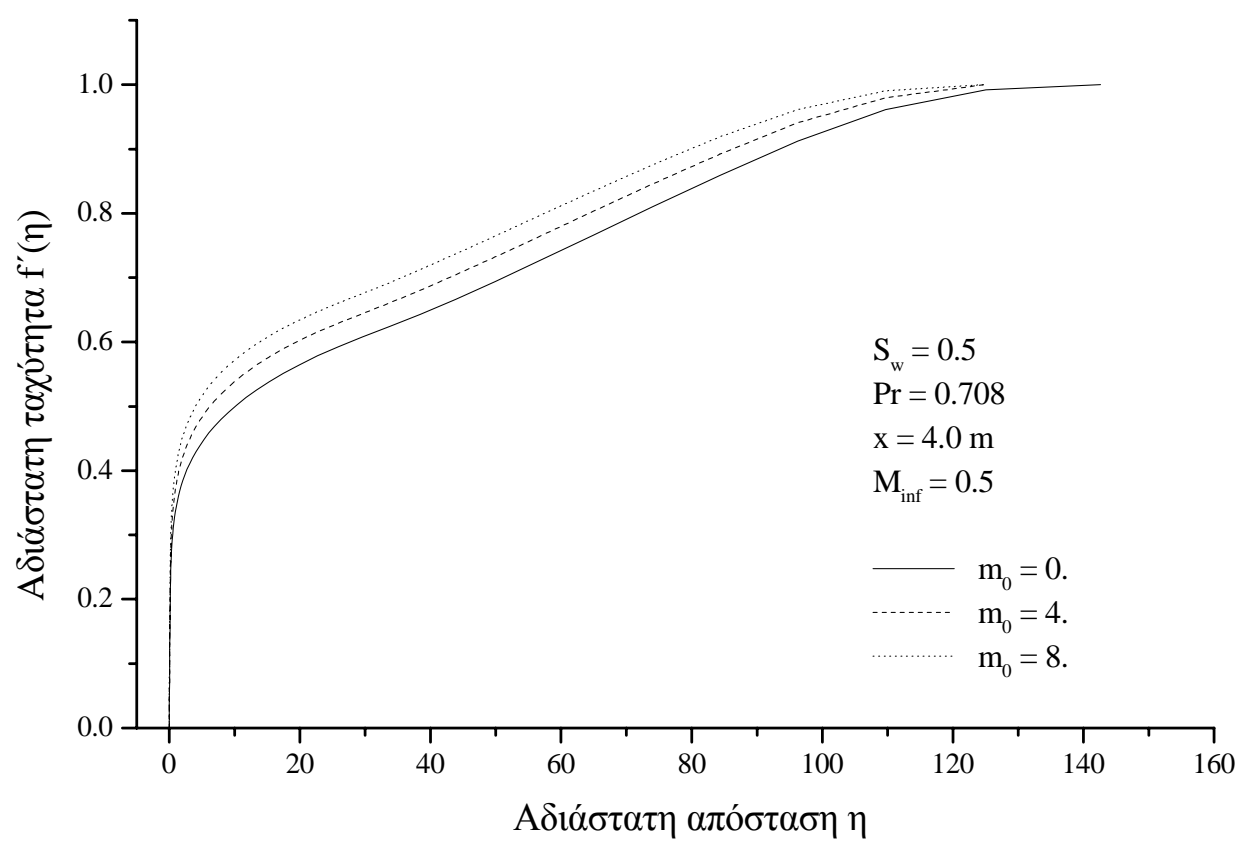

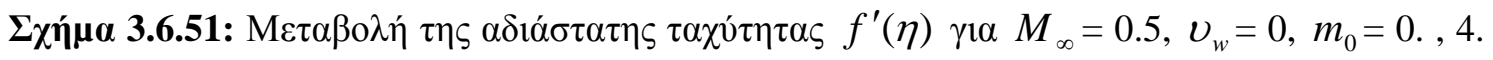

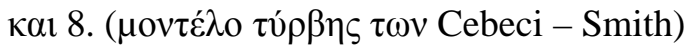

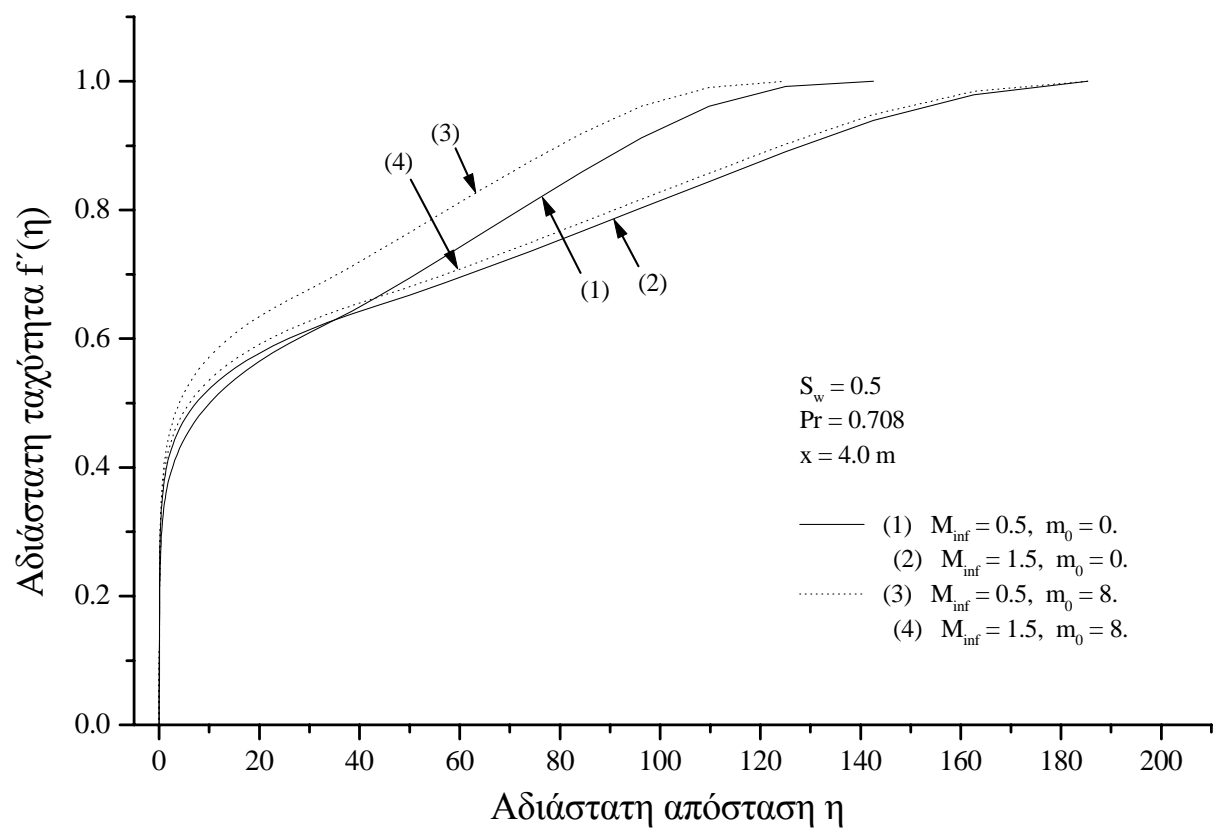

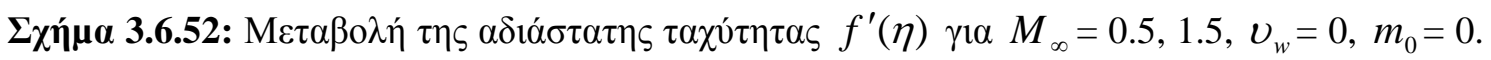

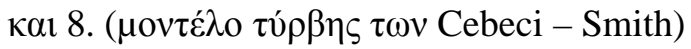




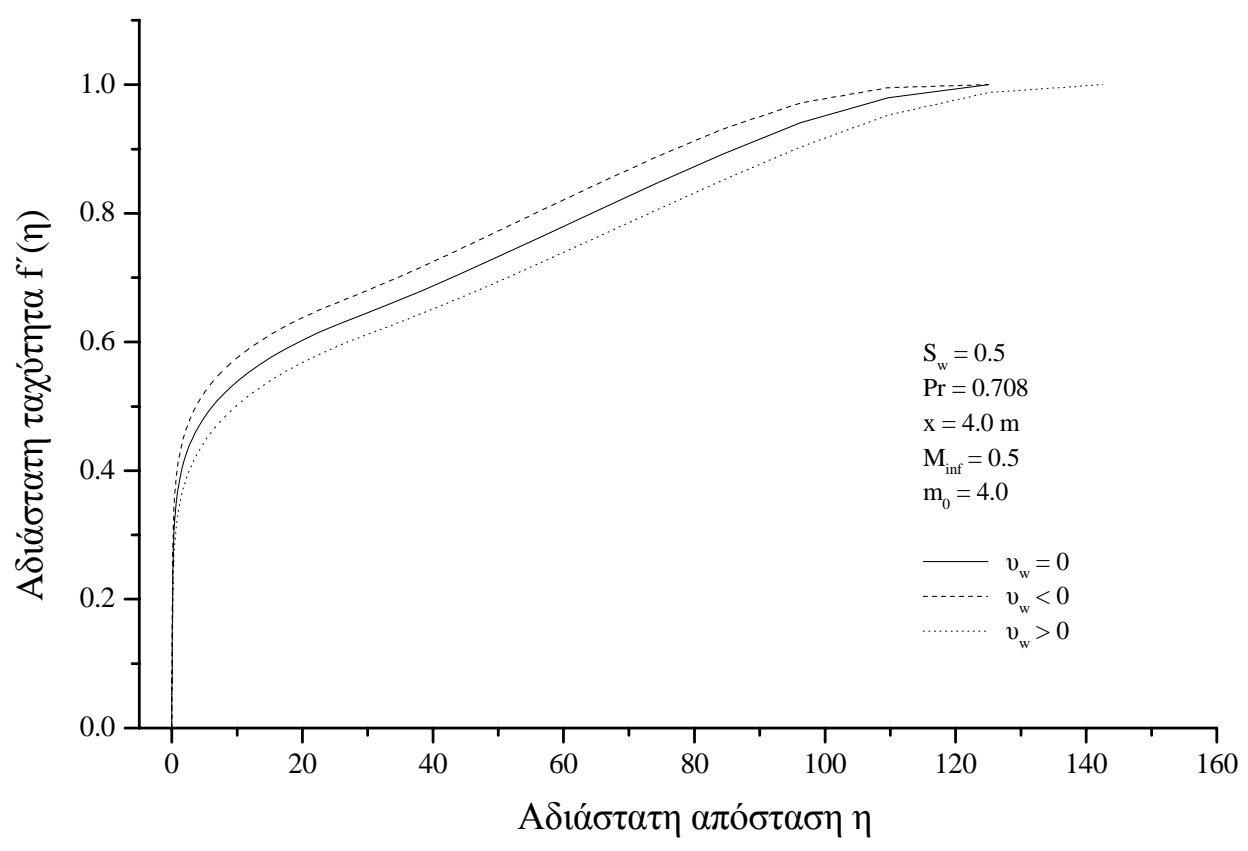

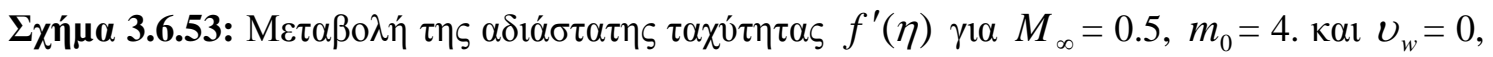

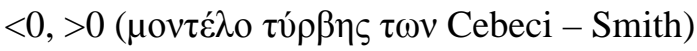

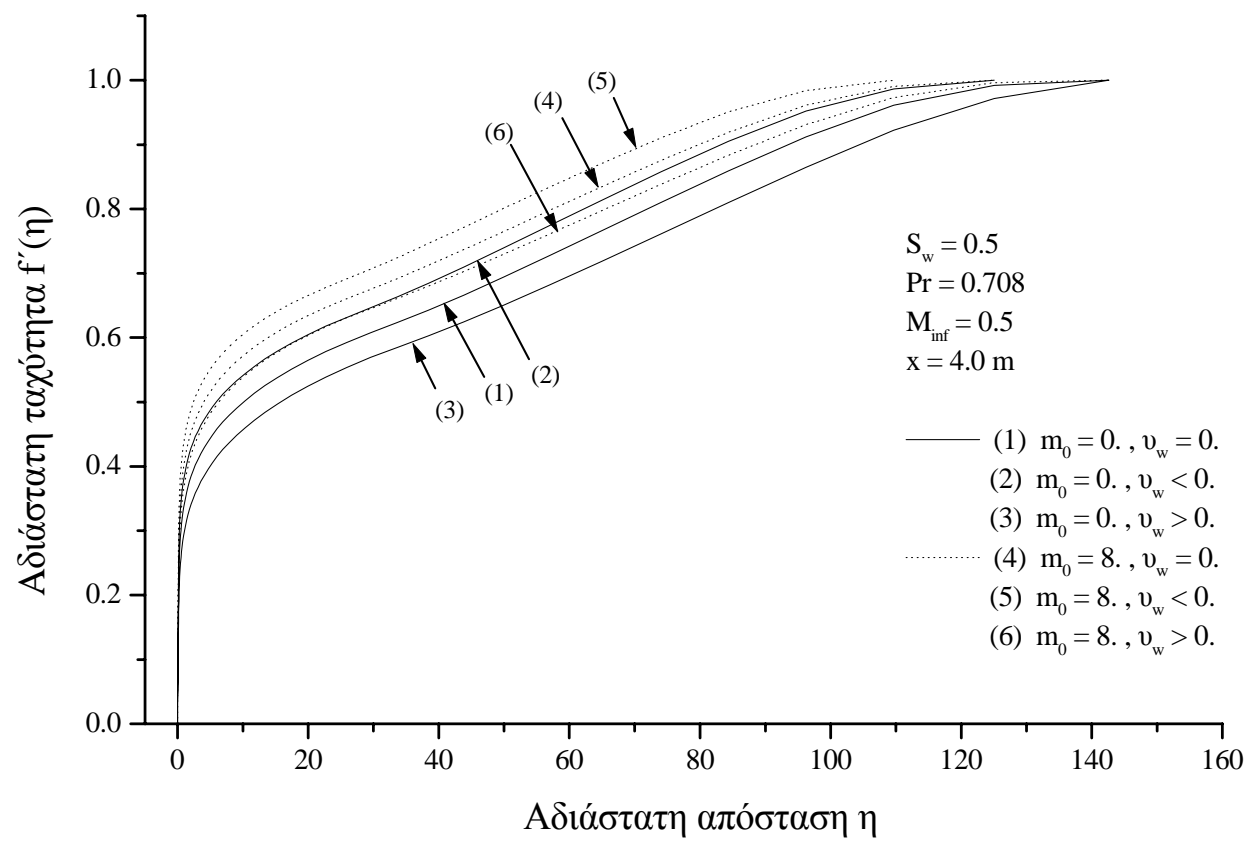

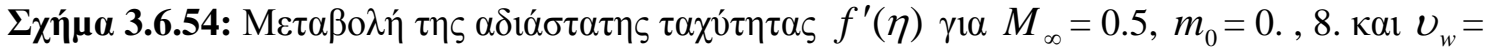

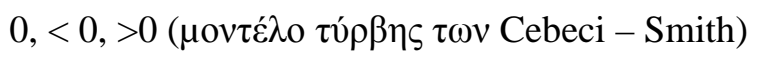




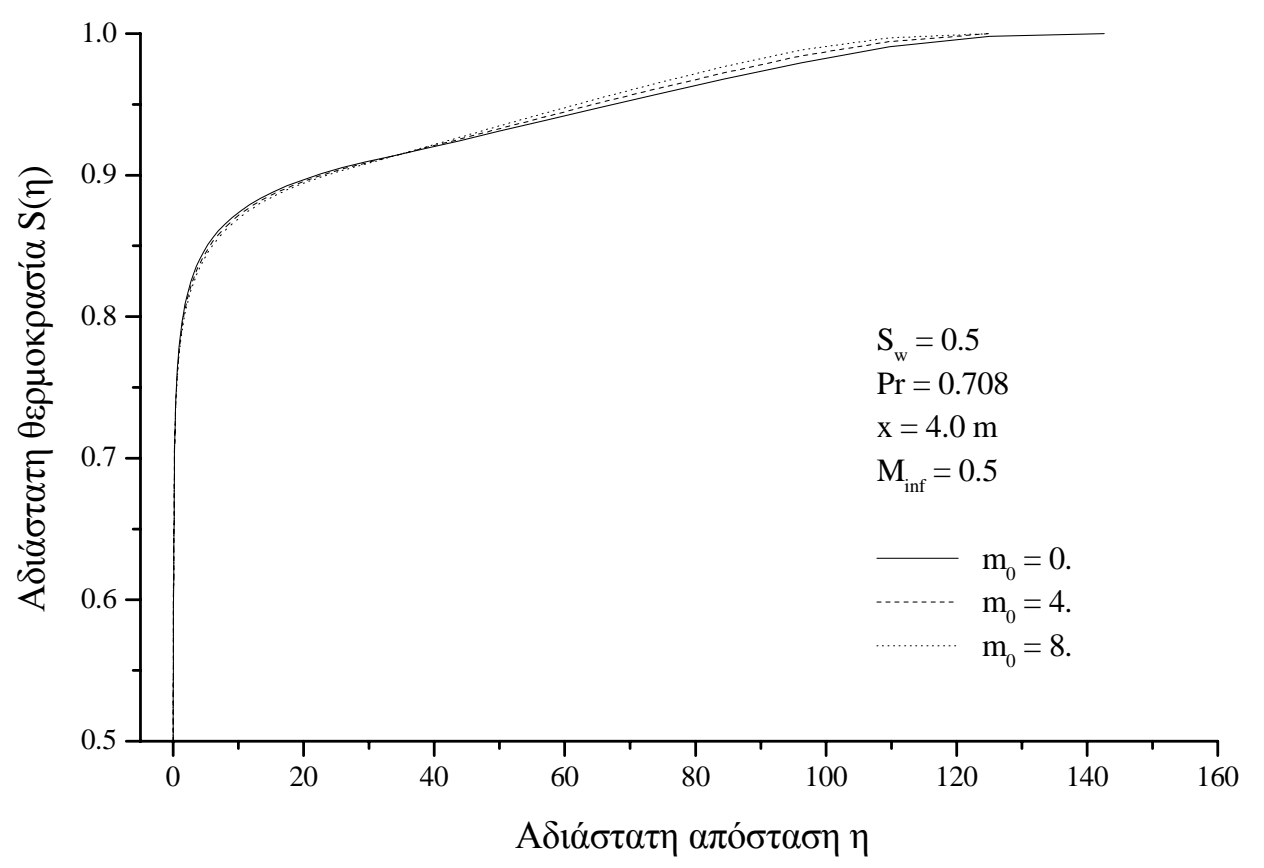

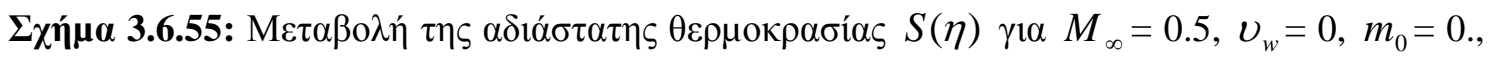

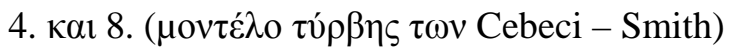

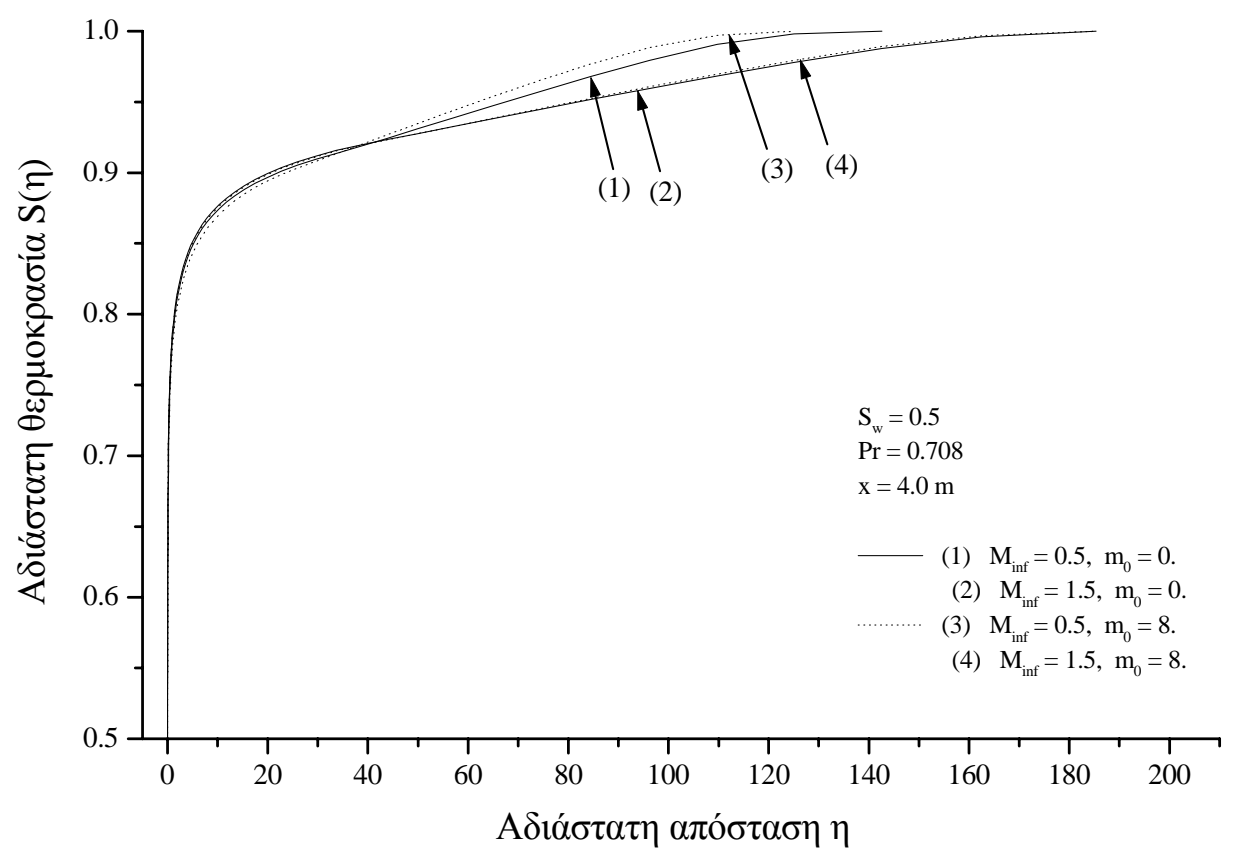

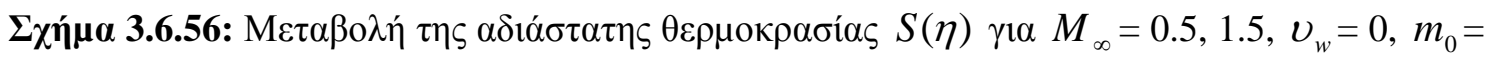

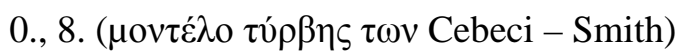




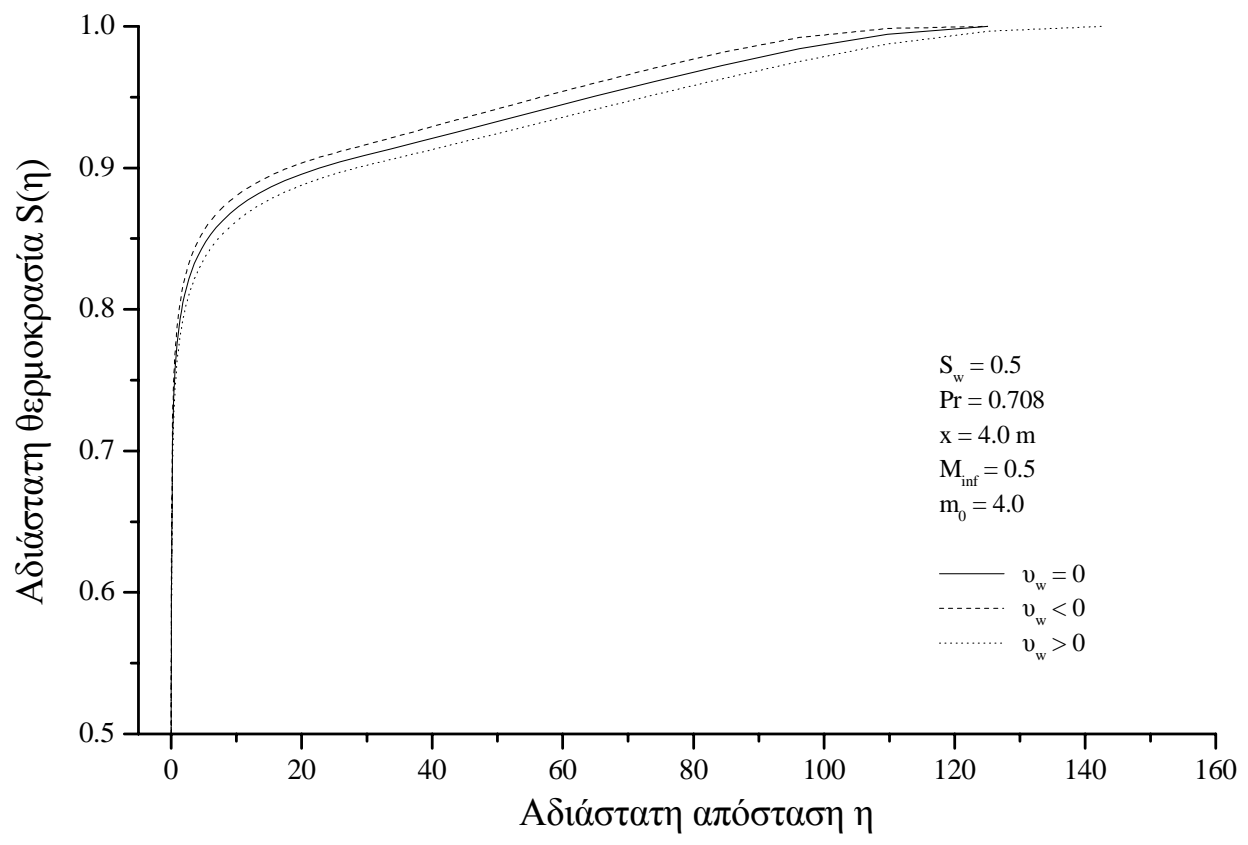

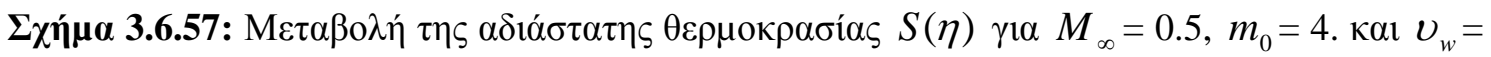

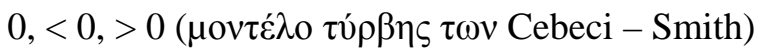

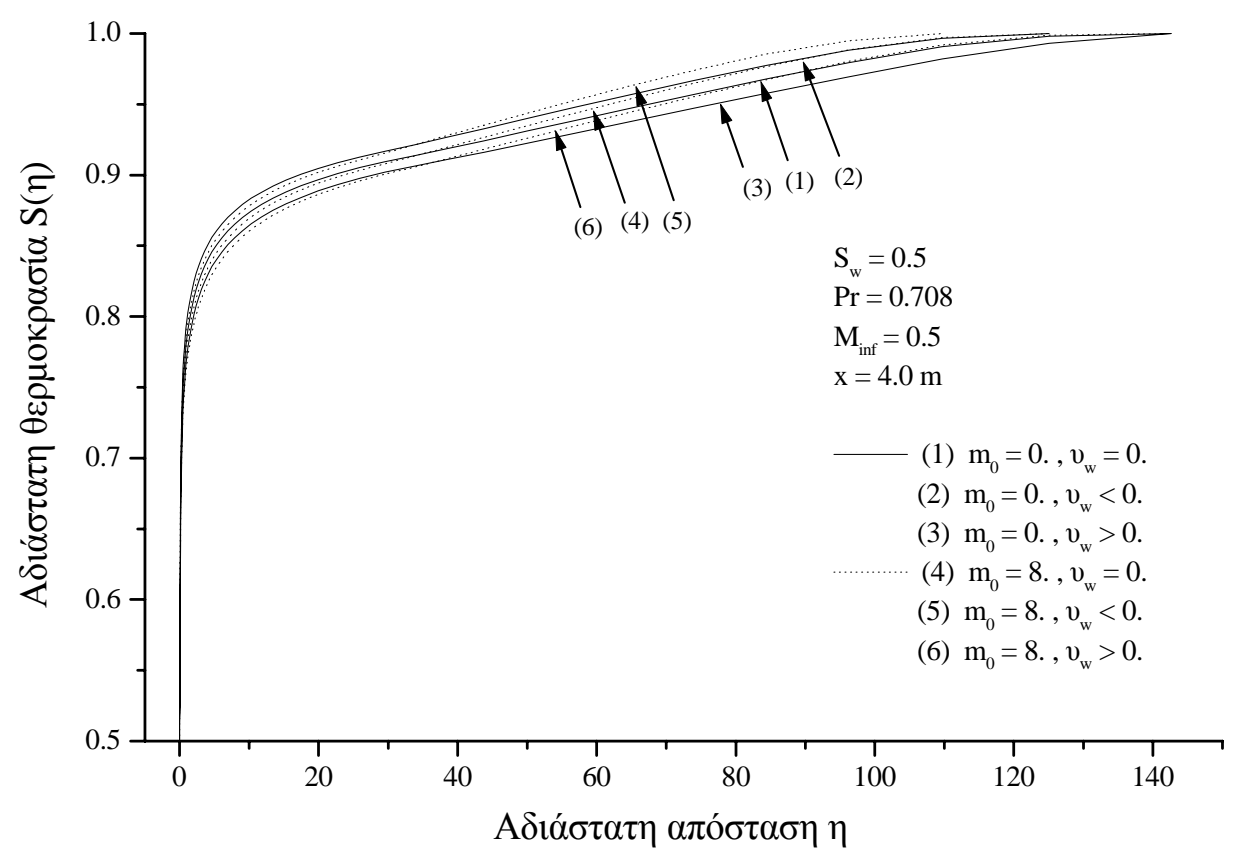

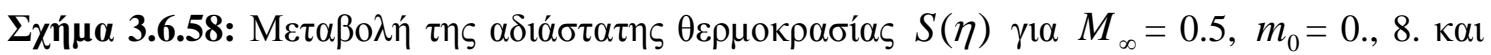

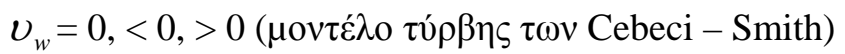




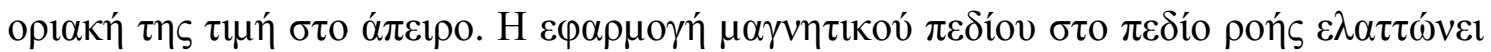

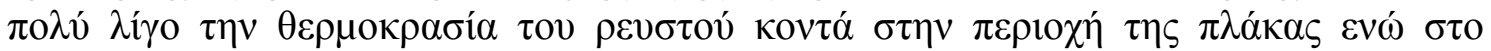

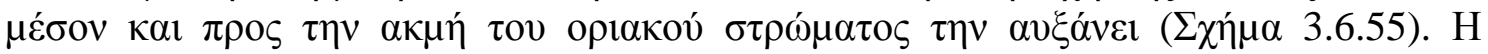

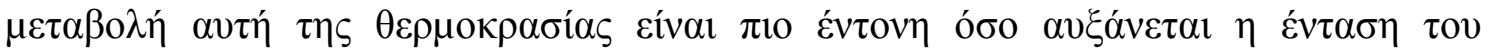

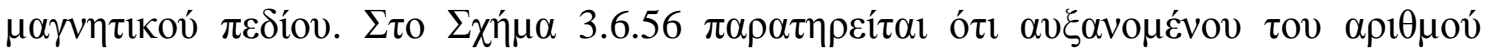

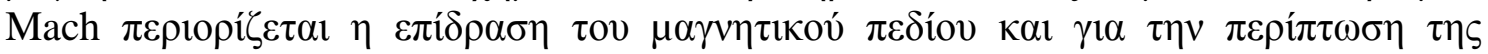

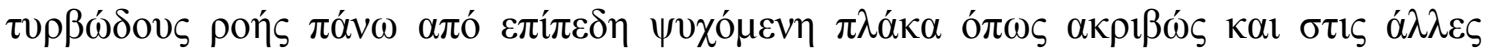

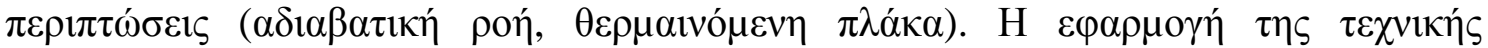

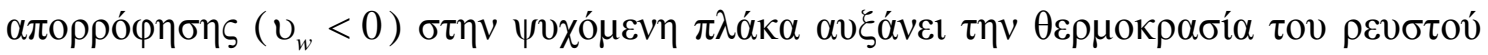

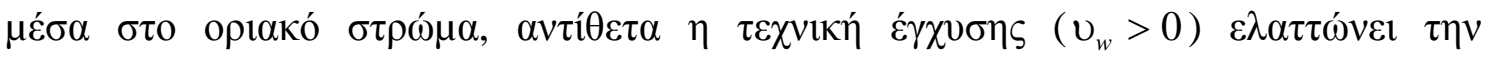

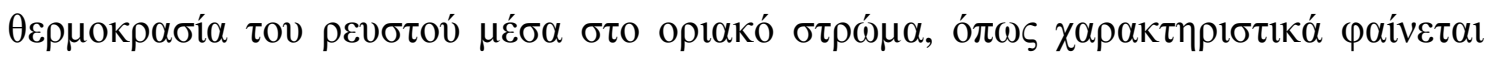

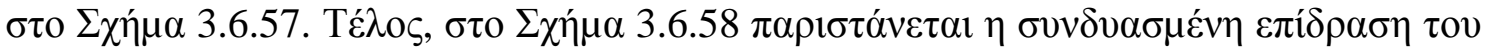

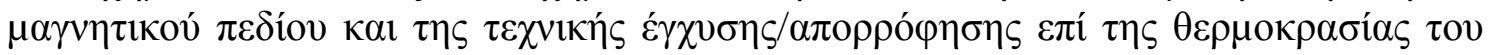


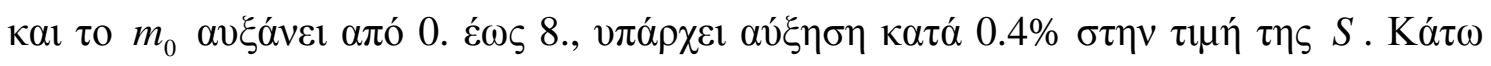

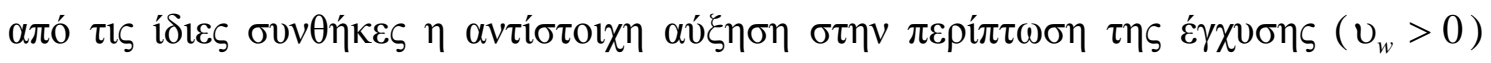

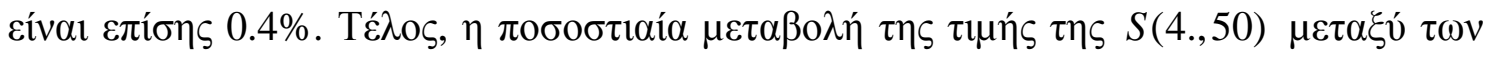

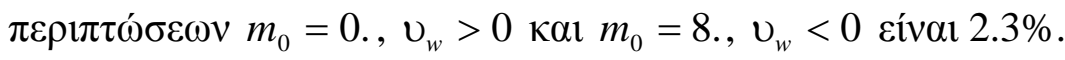

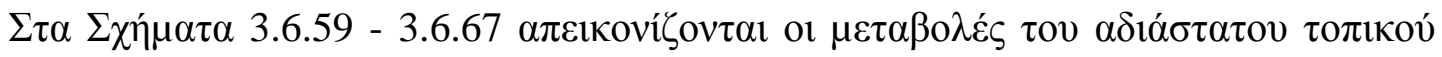

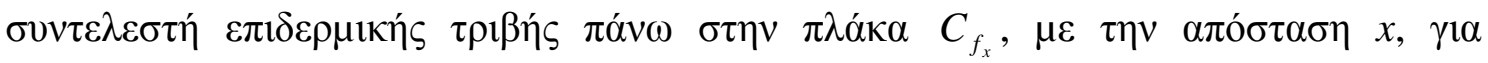
$\delta$ iá

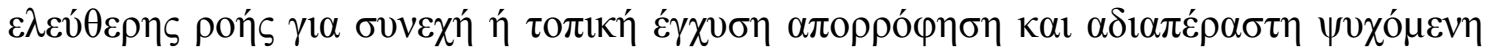

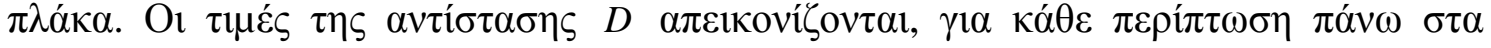

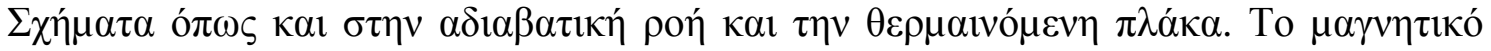

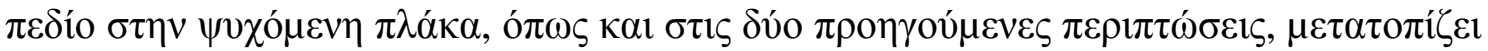

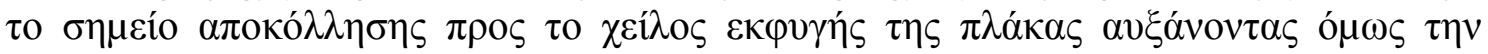

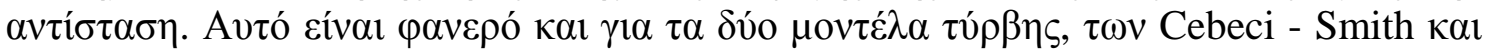

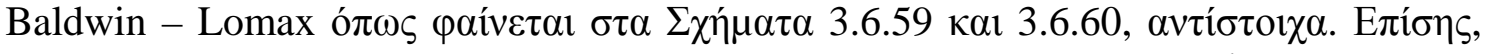

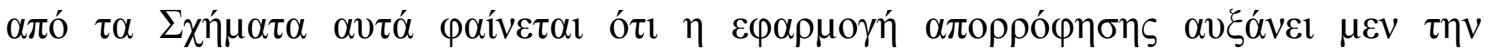

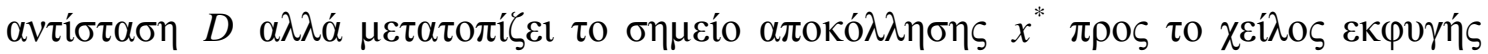

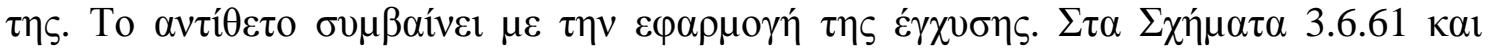

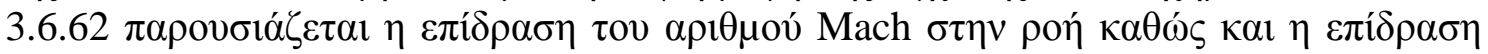

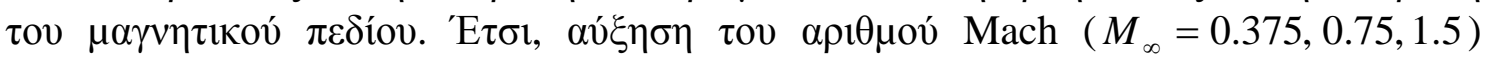

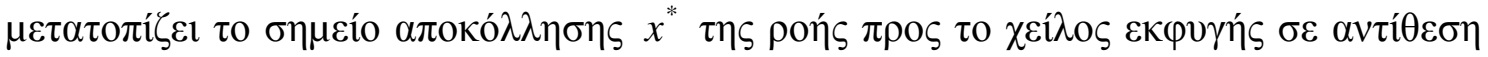

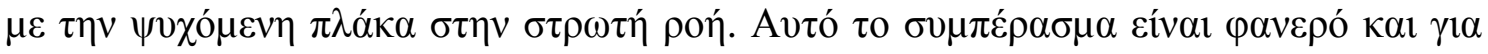

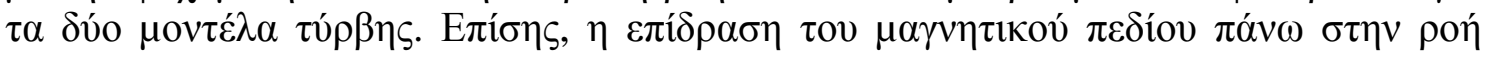

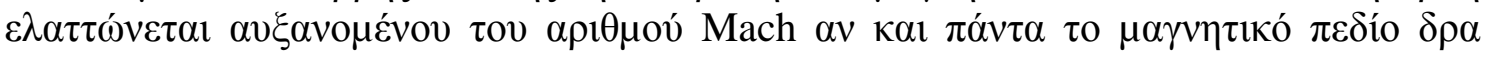

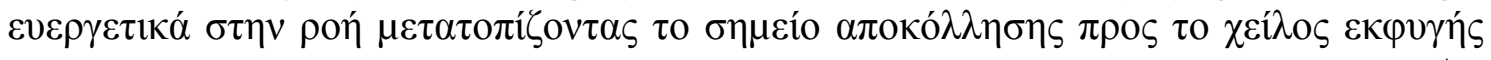

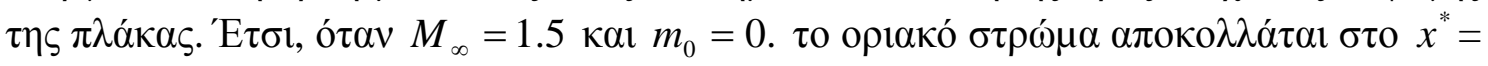

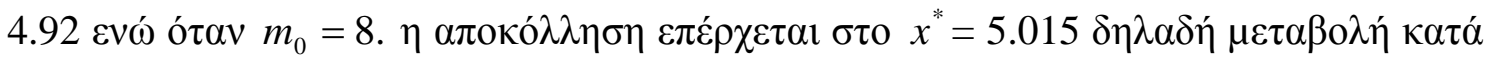

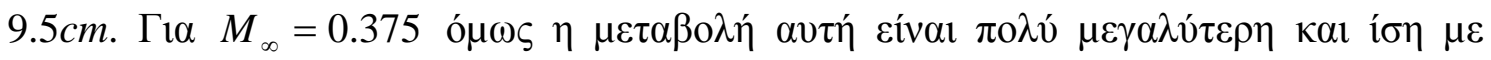

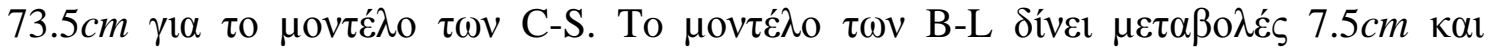
$70.5 \mathrm{~cm}, \alpha \nu \tau i ́ \sigma \tau o \imath \chi \alpha$.

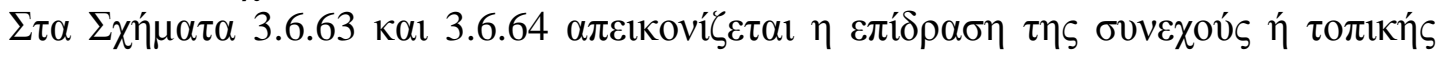

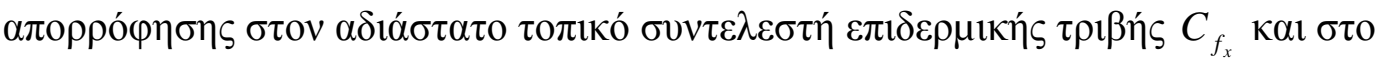




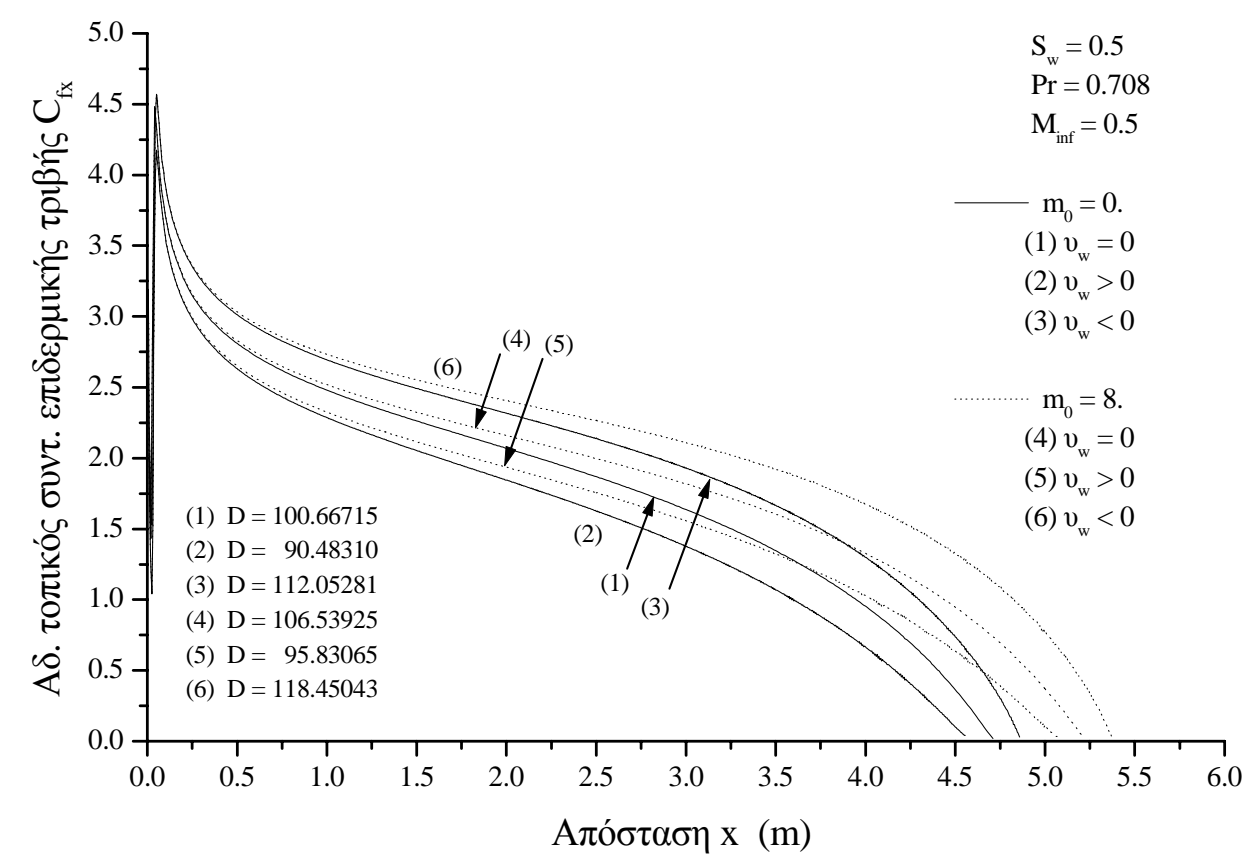

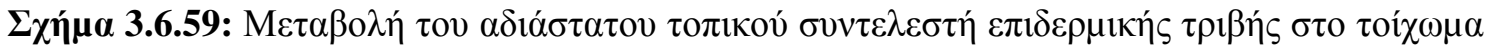

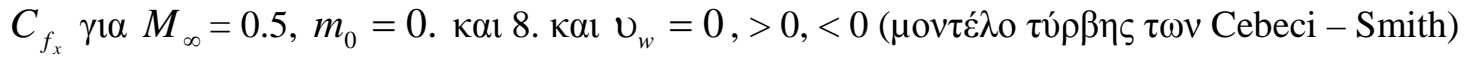

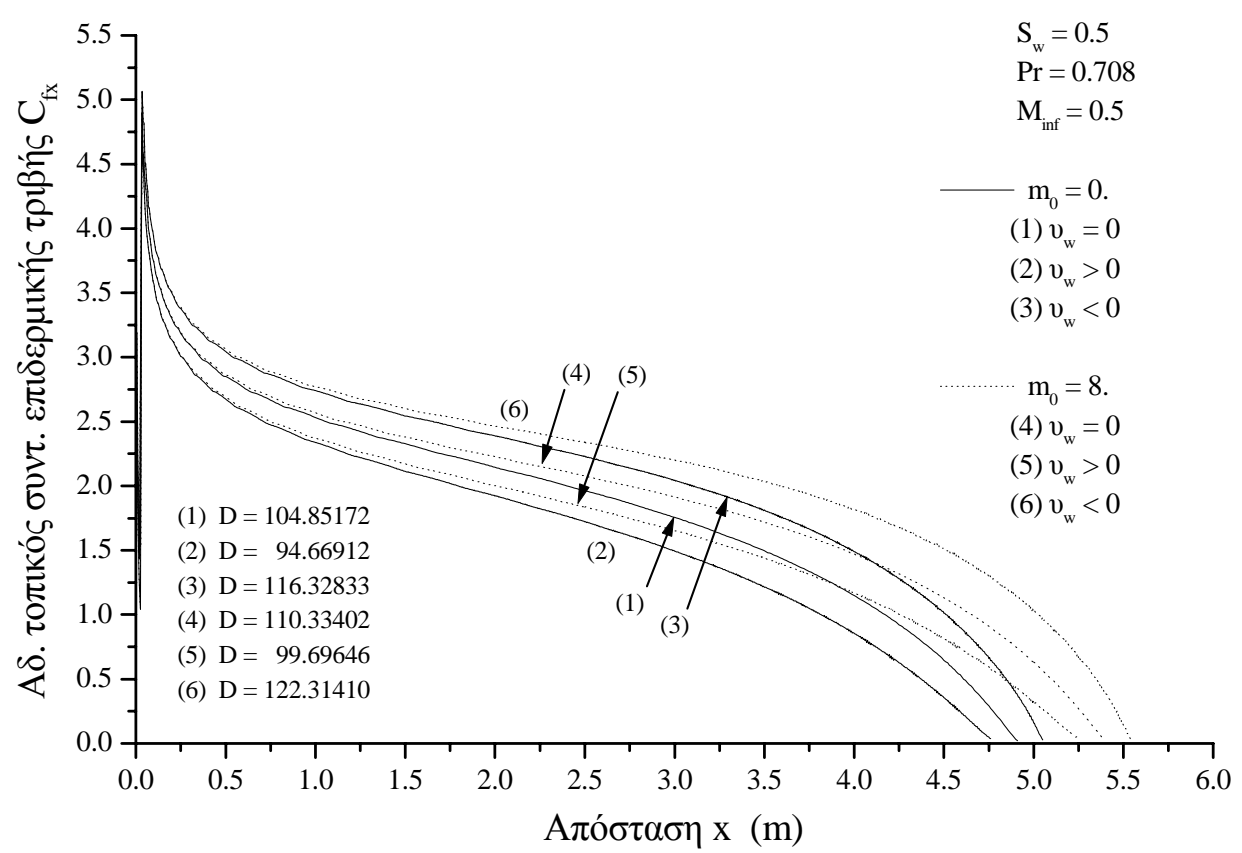

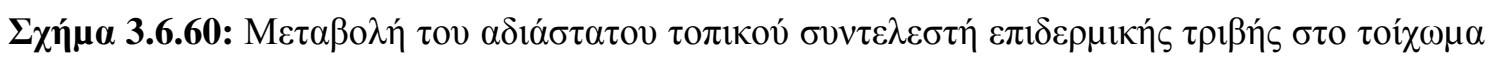

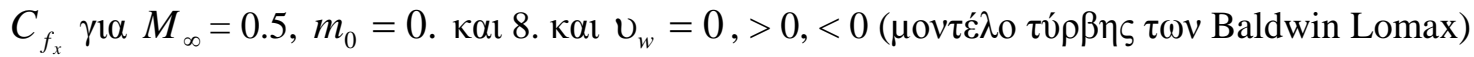




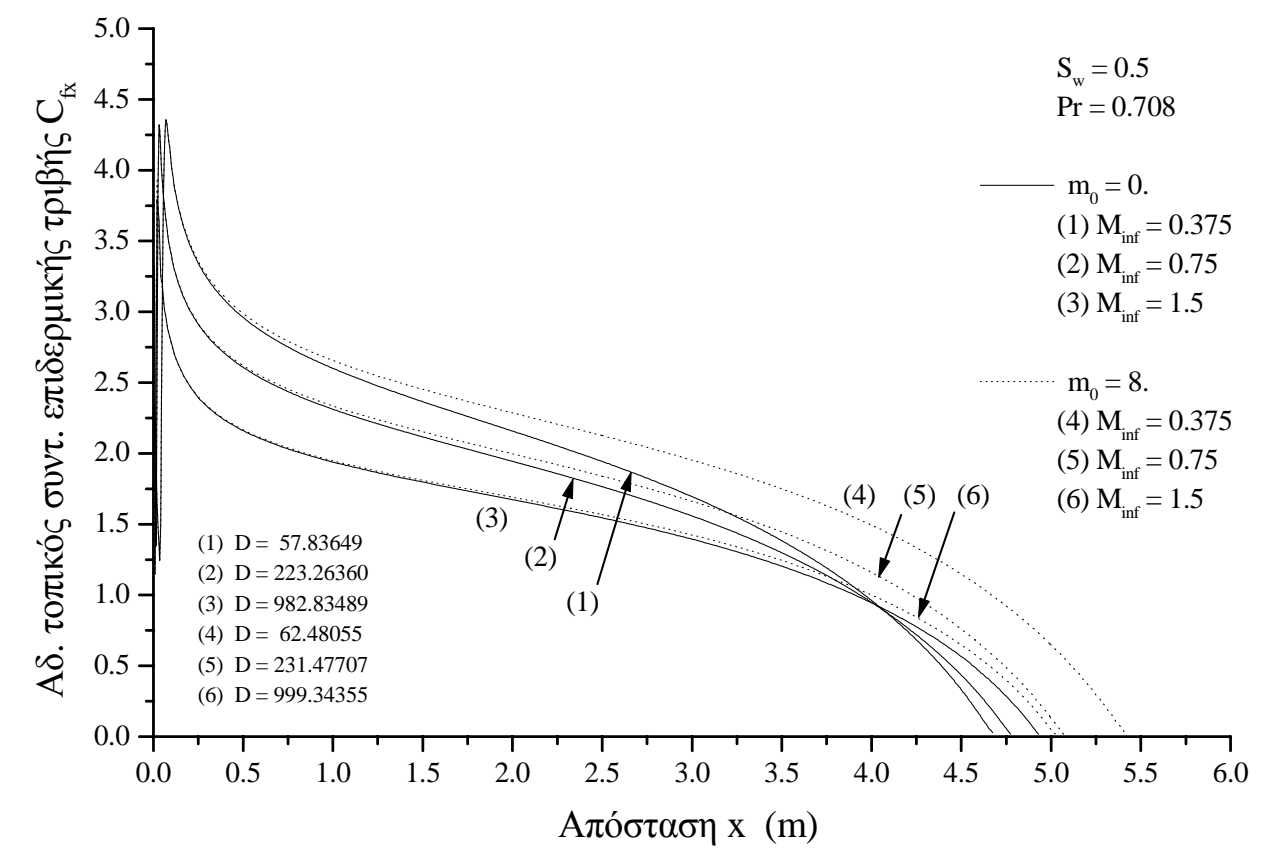

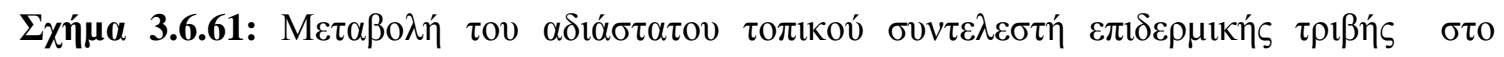

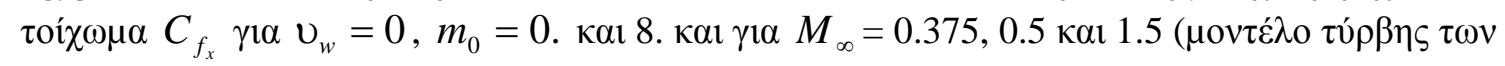
Cebeci - Smith)

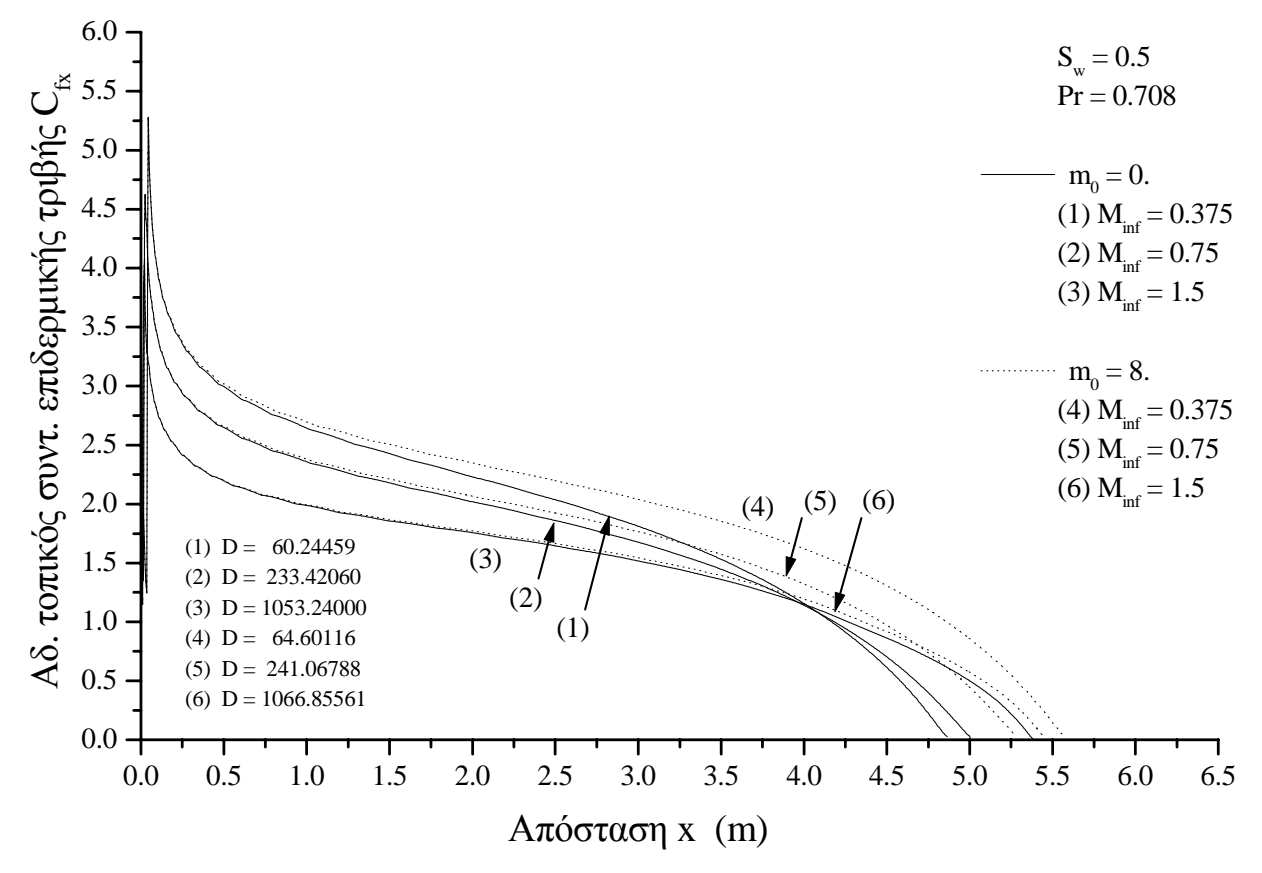

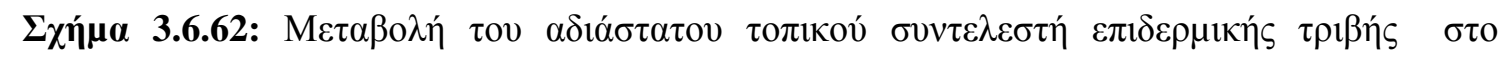

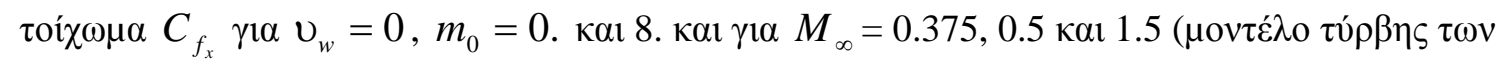
Baldwin - Lomax) 


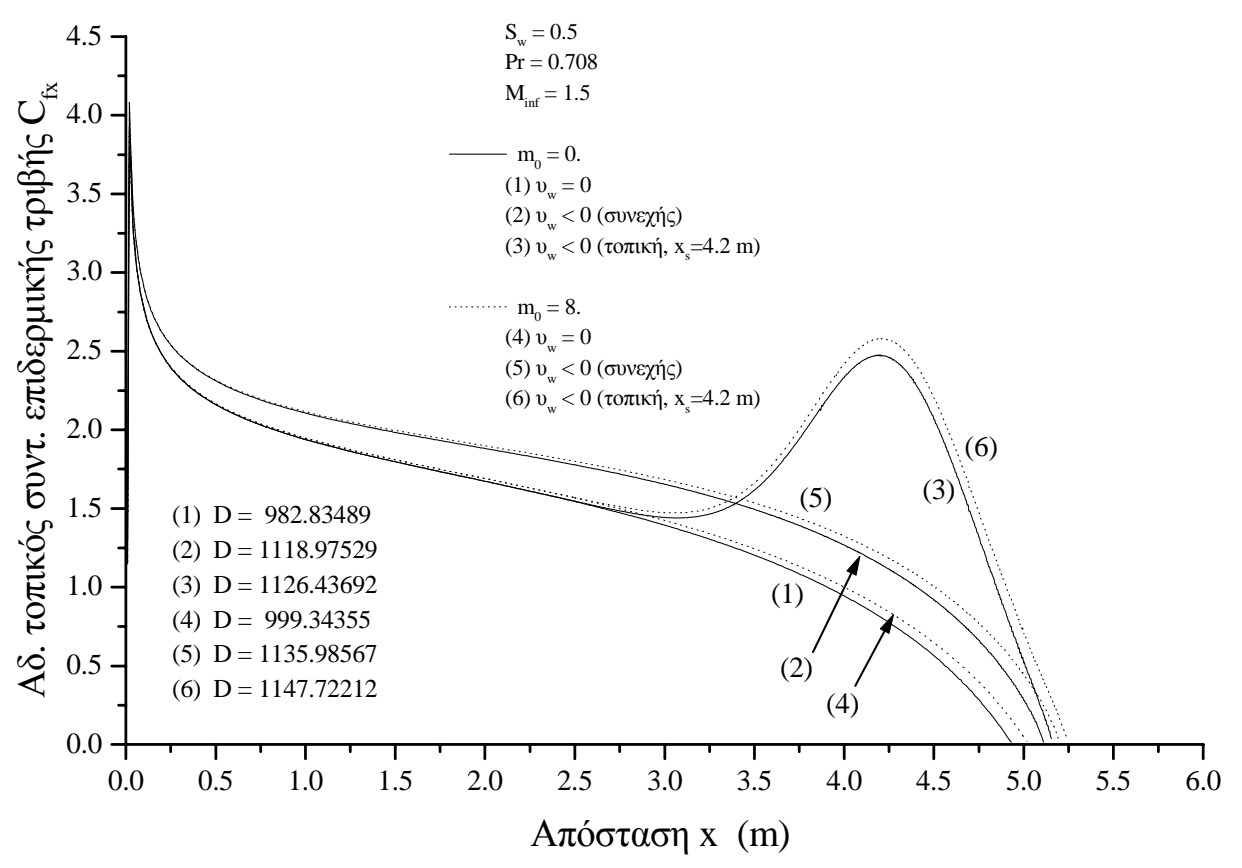

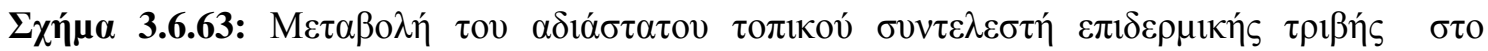

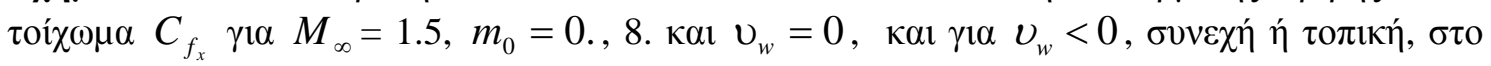
$x_{s}=4.2\left(\mu \mathrm{ov} \tau \dot{\lambda} \lambda_{0} \tau \dot{v} \rho \beta \eta \varsigma \tau \omega \nu\right.$ Cebeci - Smith)

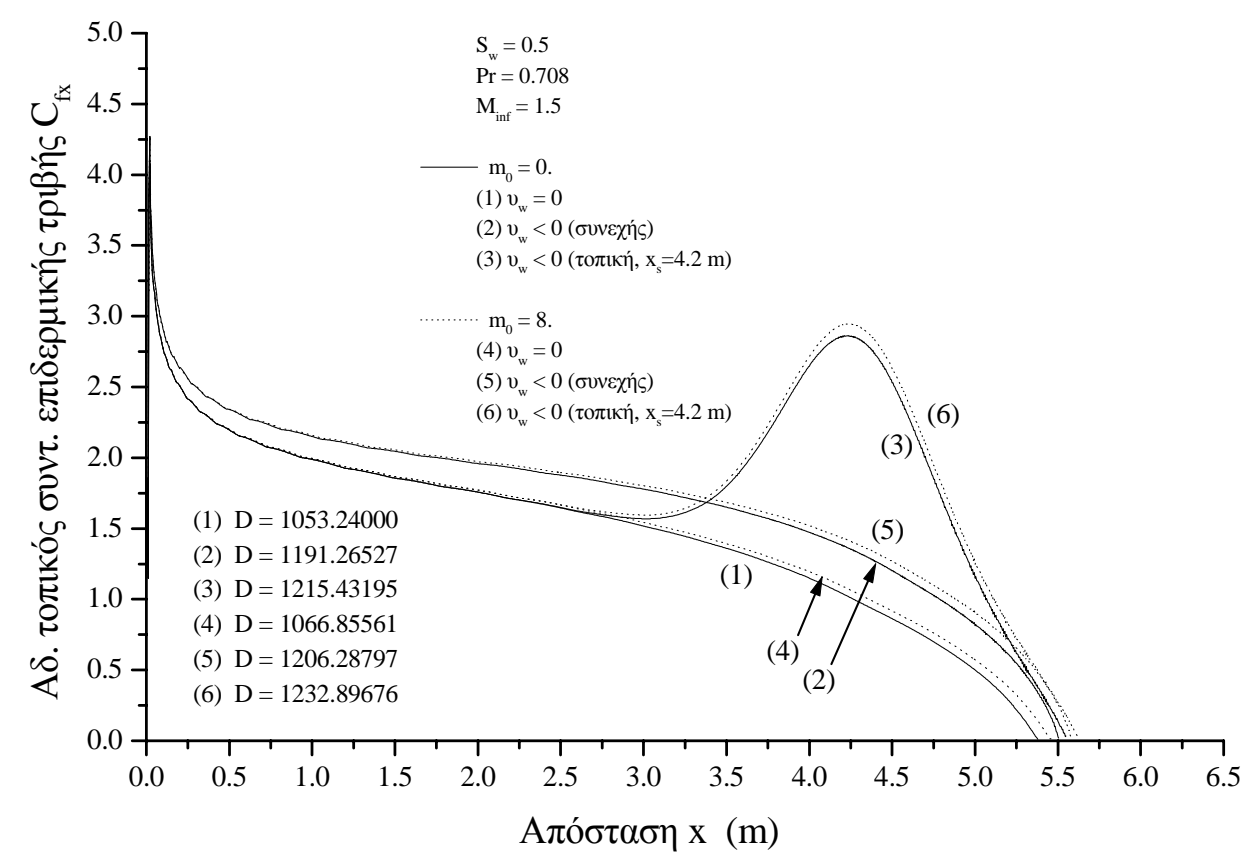

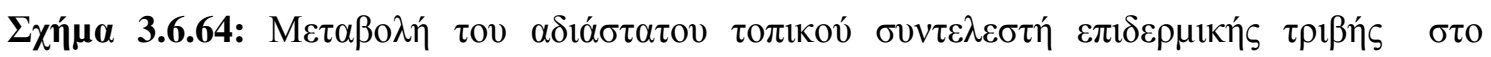

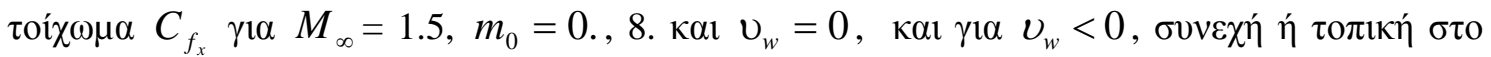

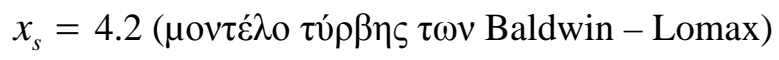




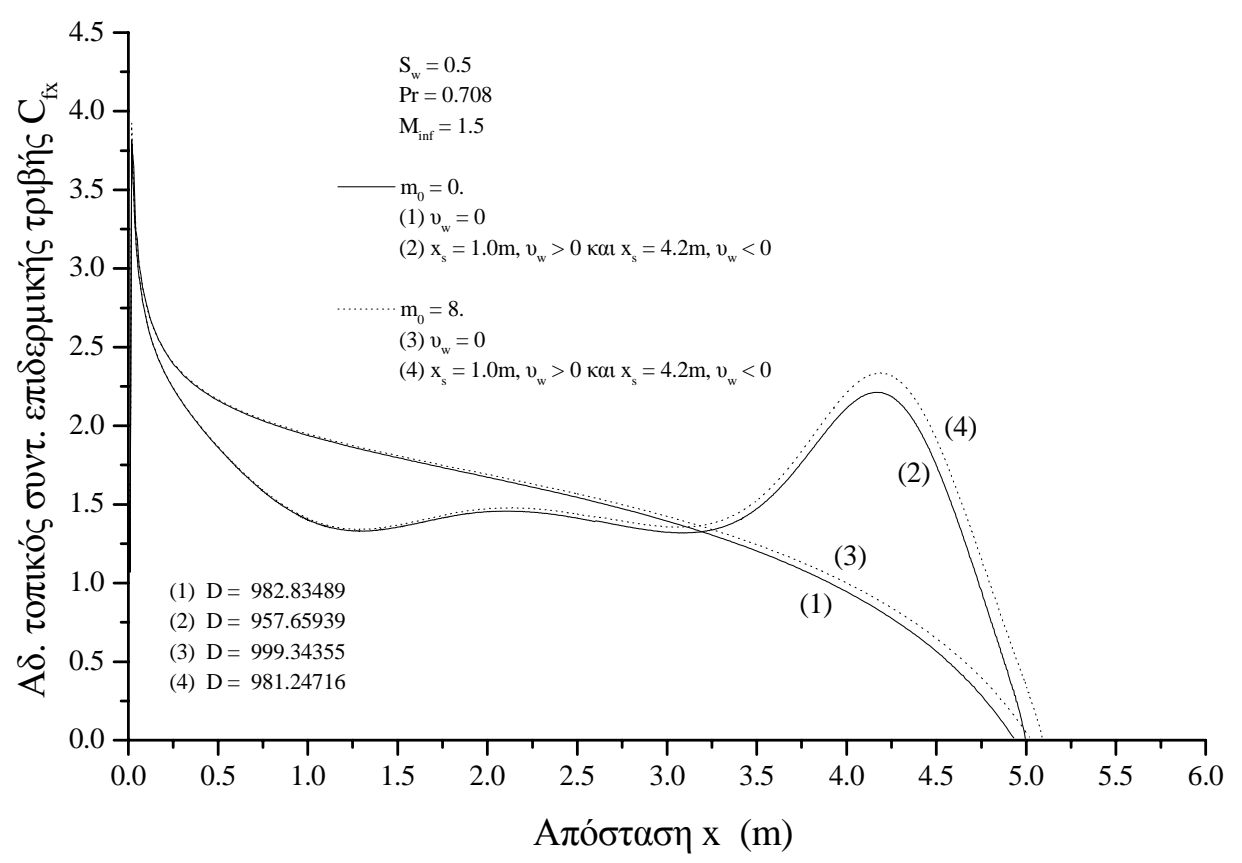

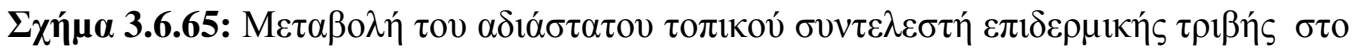

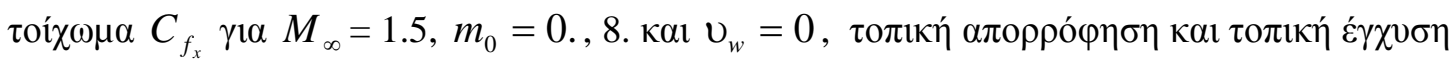

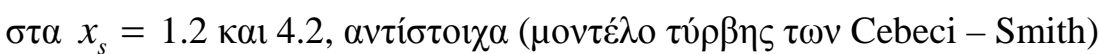

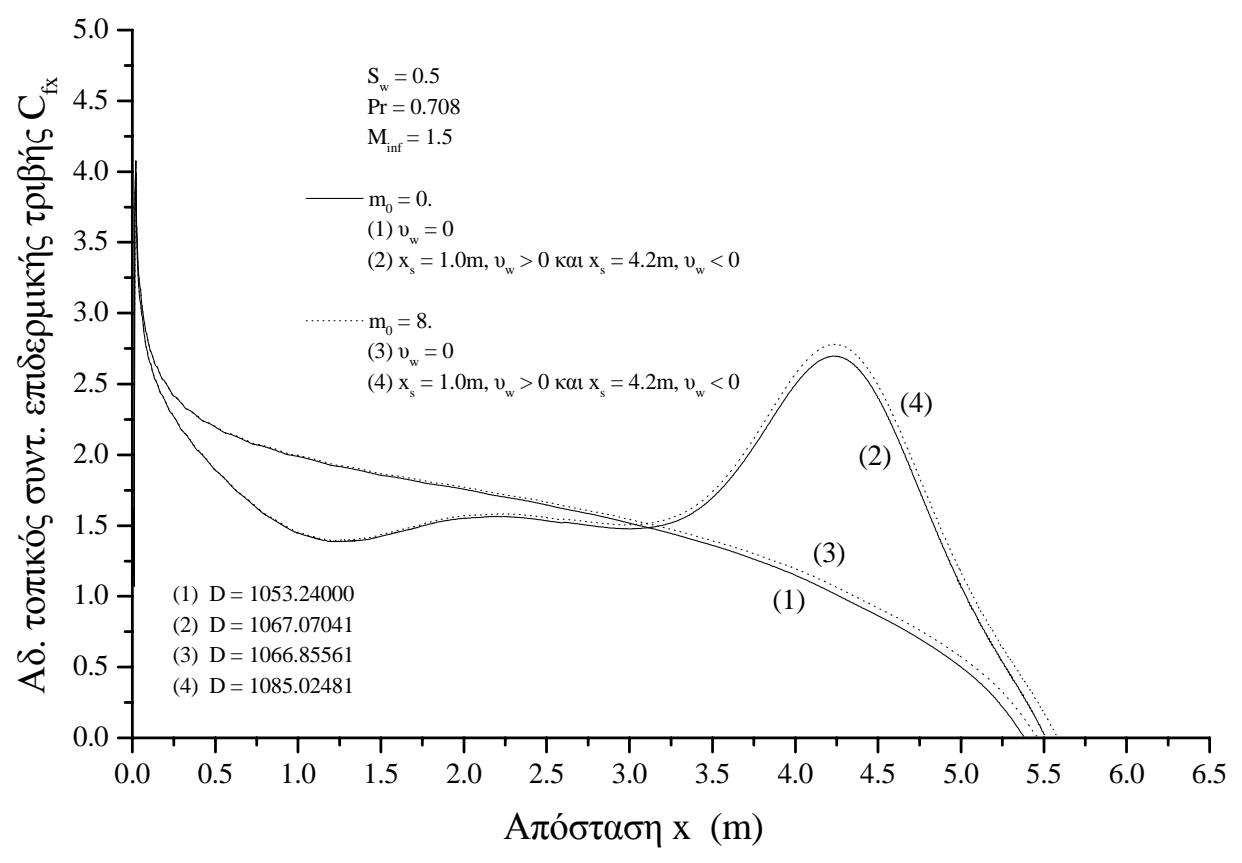

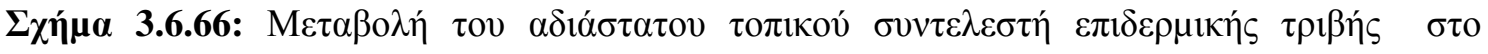

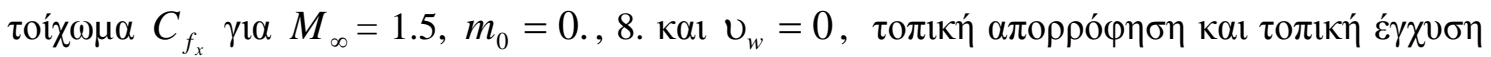

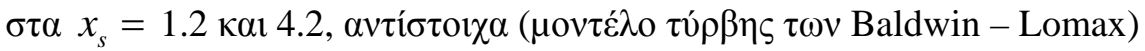




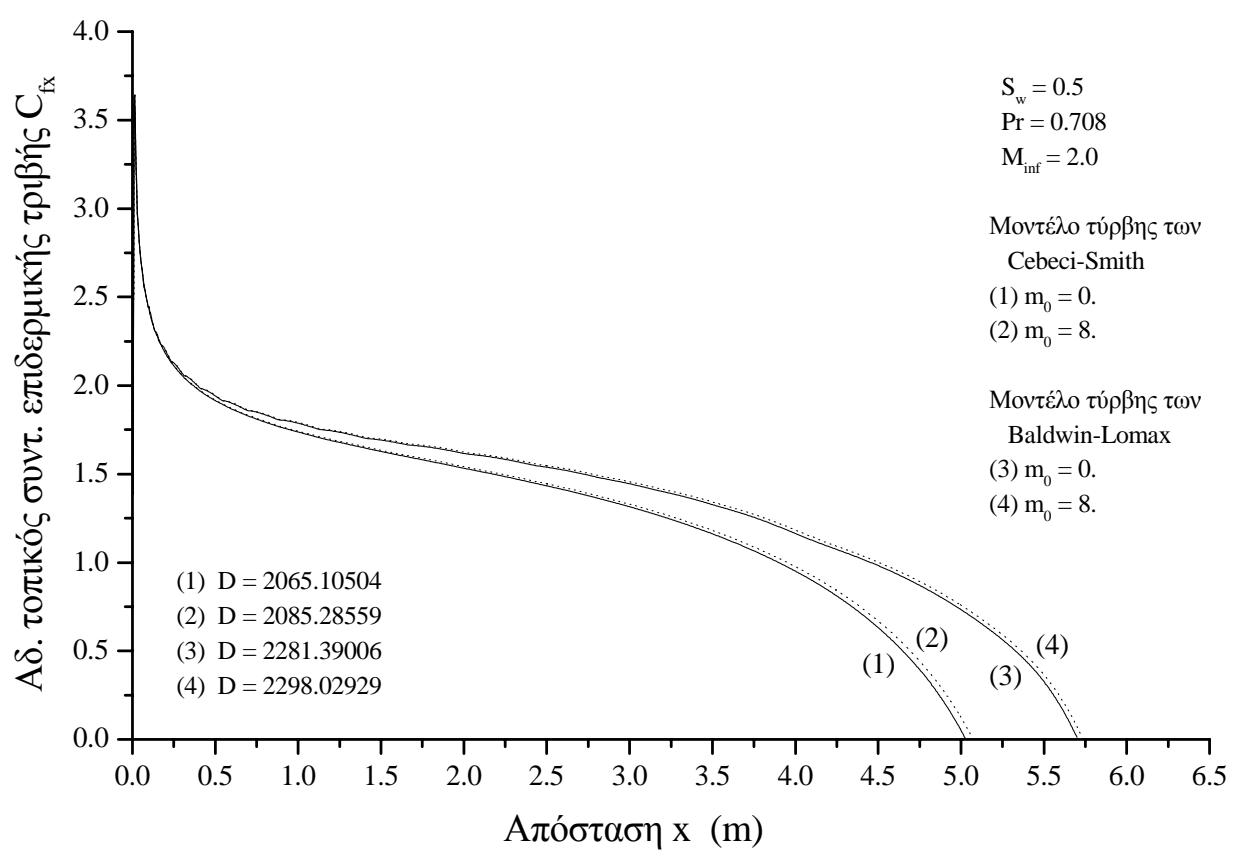

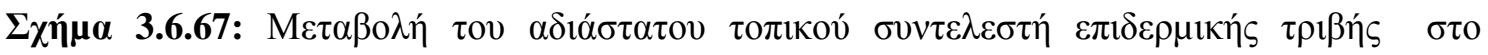

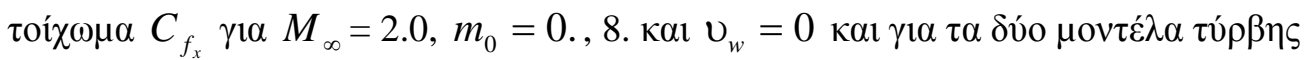

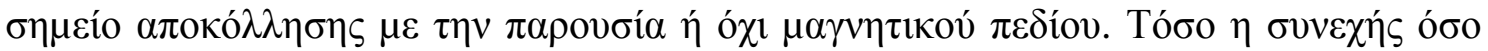

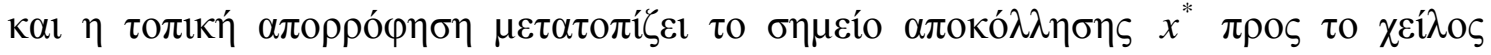

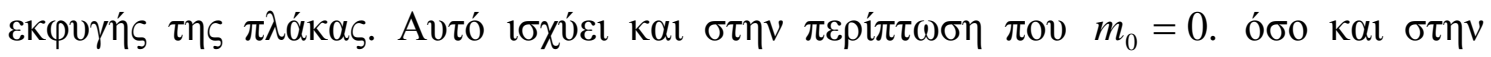

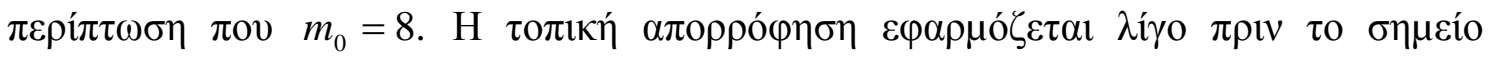

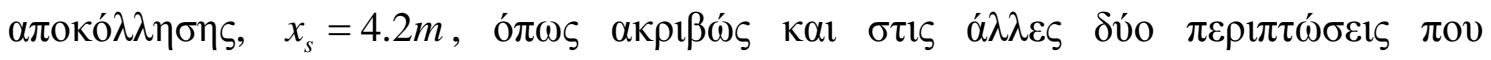

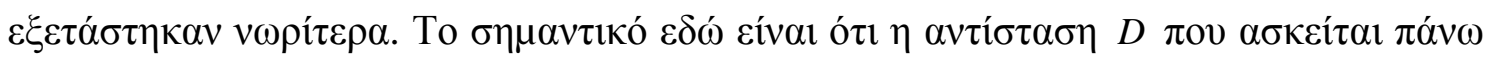

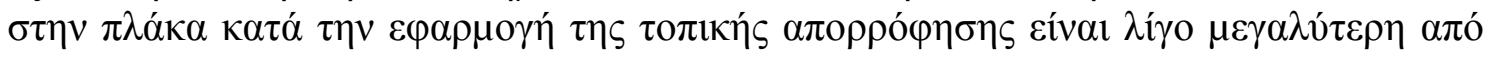

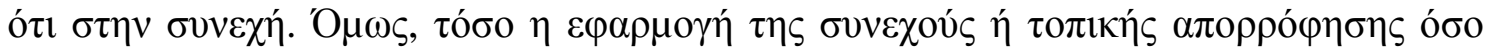

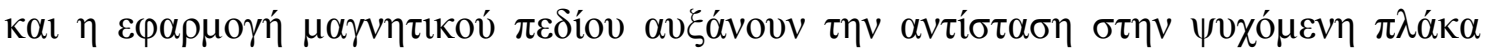

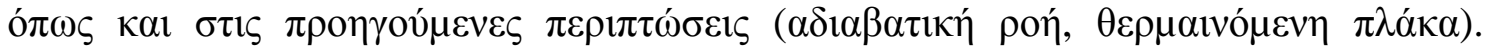

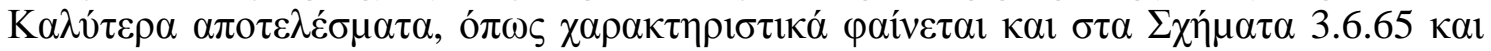

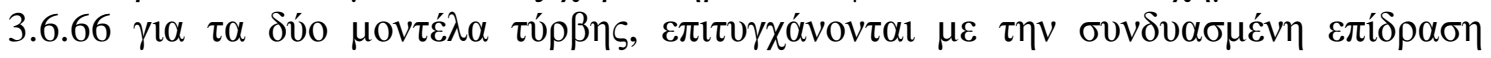

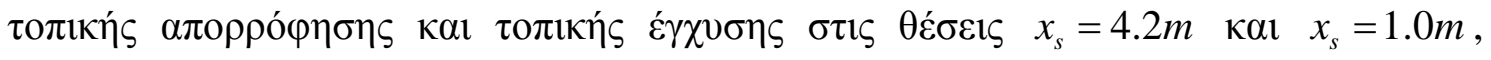

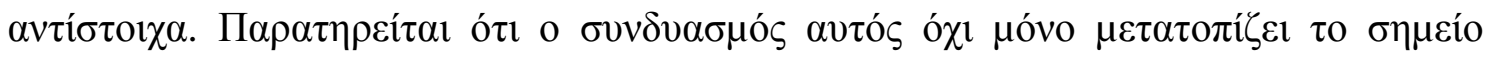

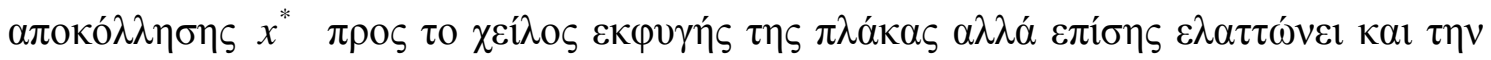

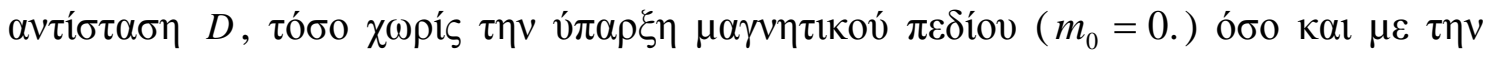

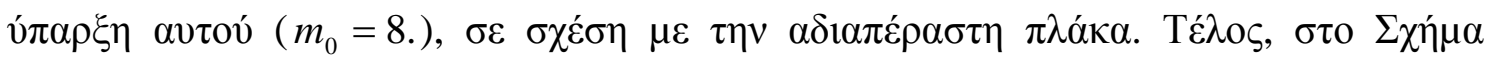

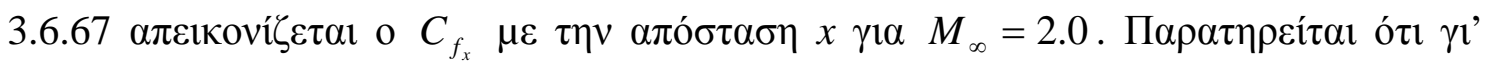

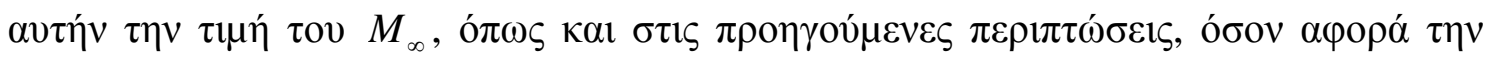

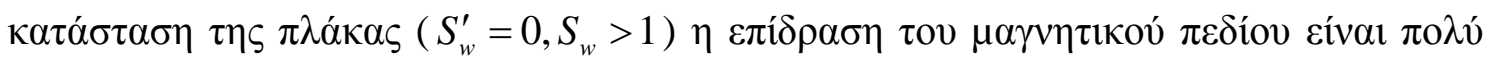

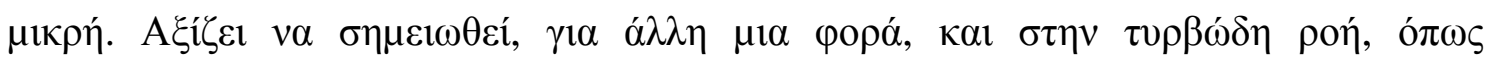

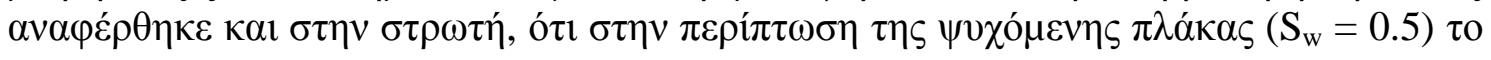




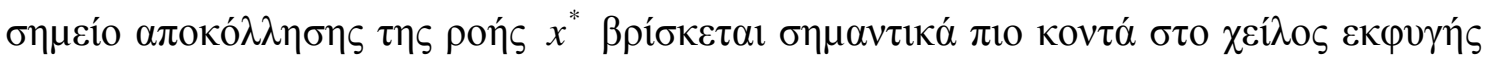

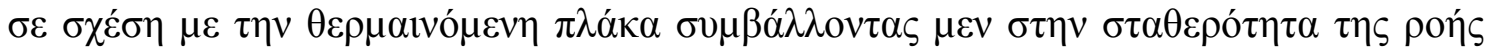

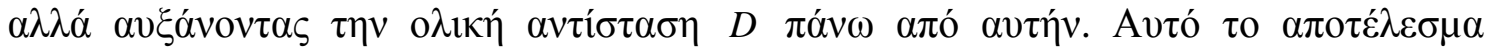

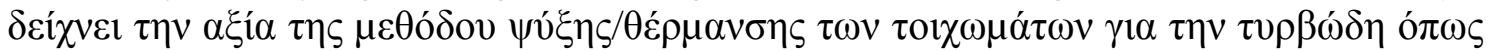

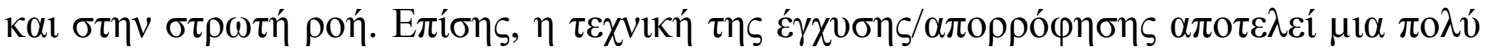

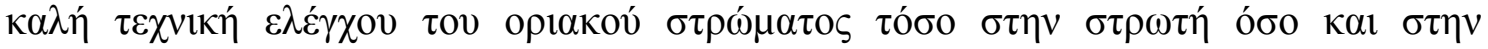

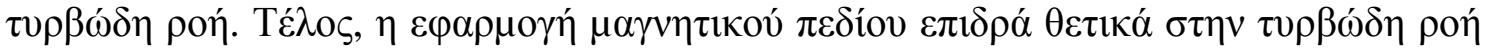

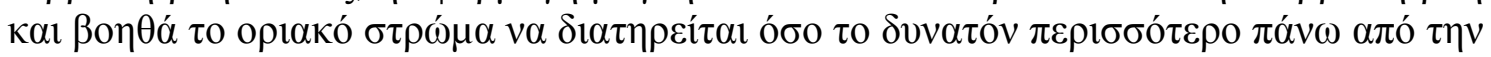
$\pi \lambda \alpha ́ \kappa \alpha$.

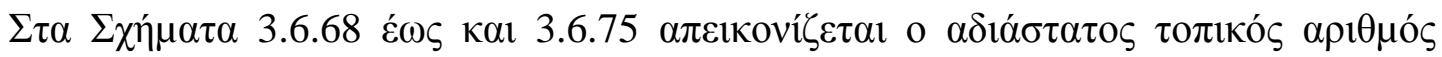

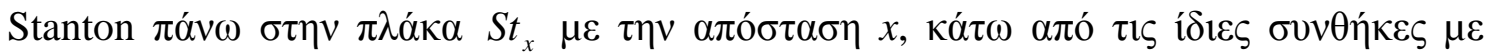

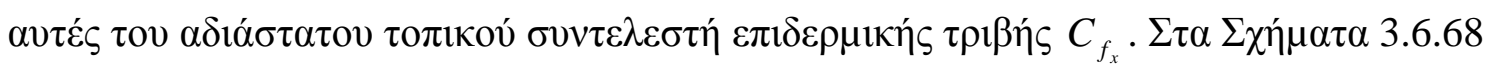

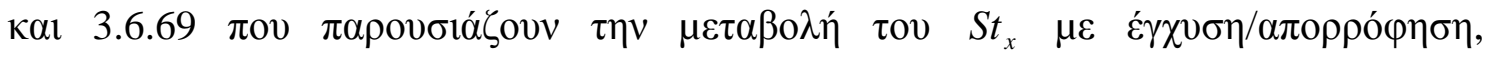

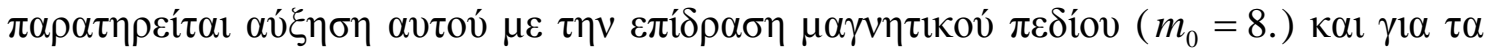

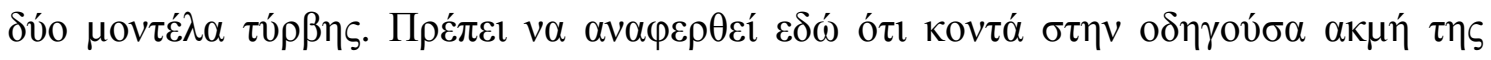

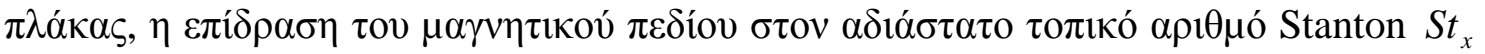

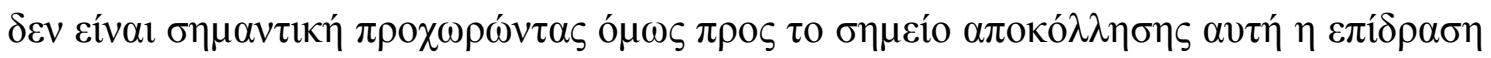

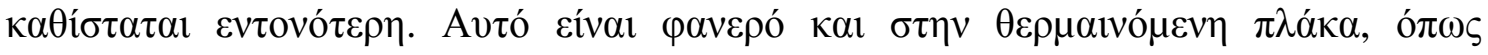

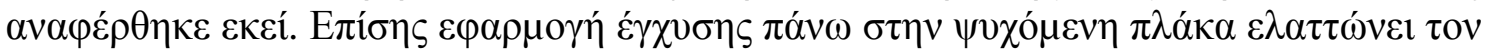

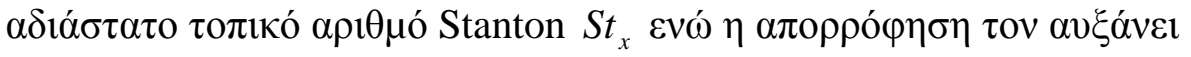

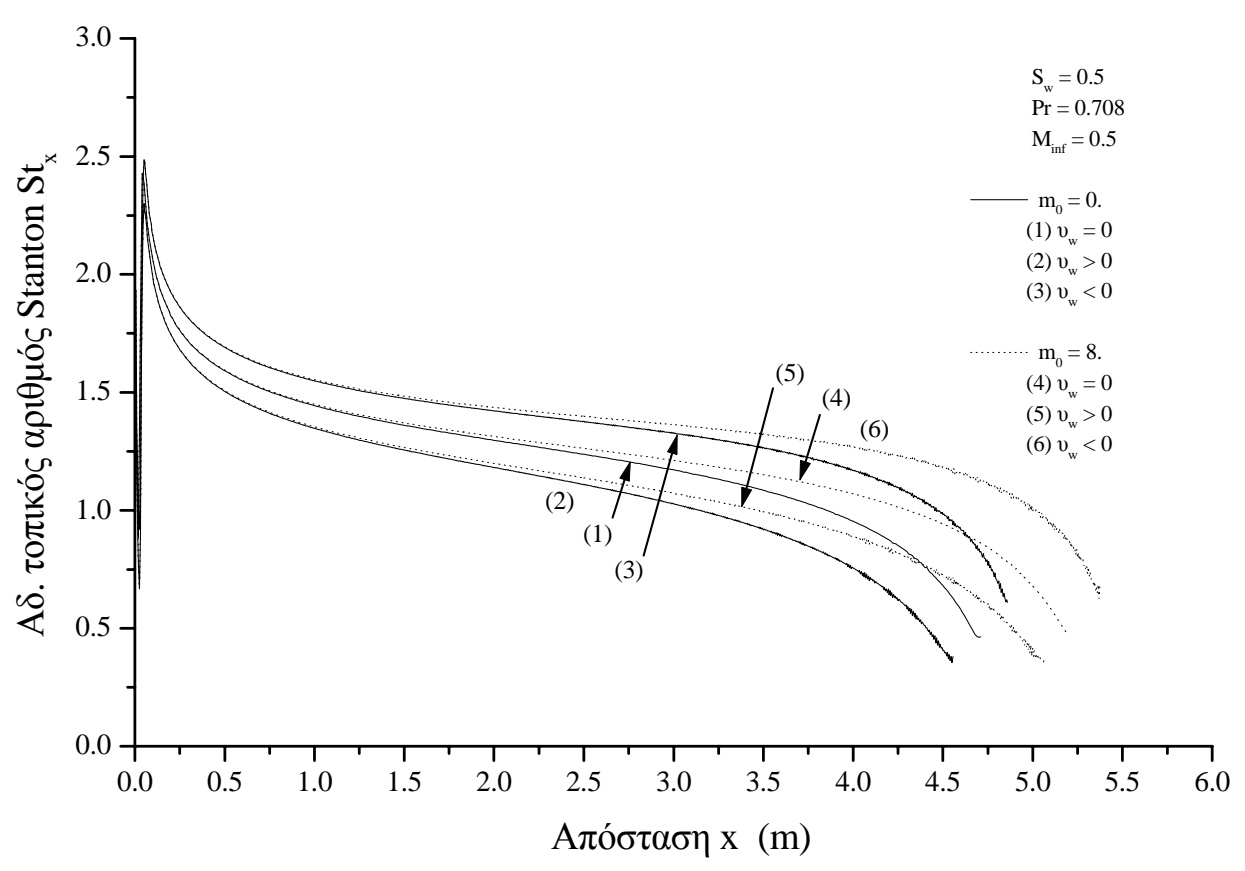

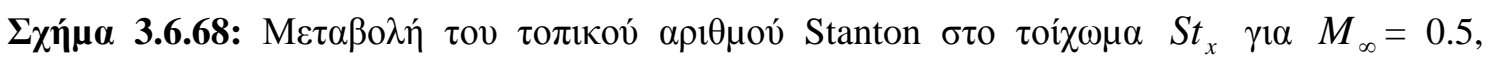

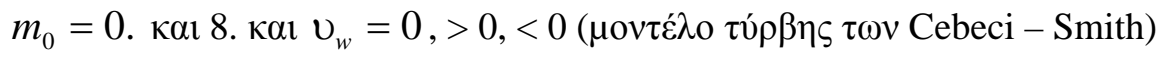




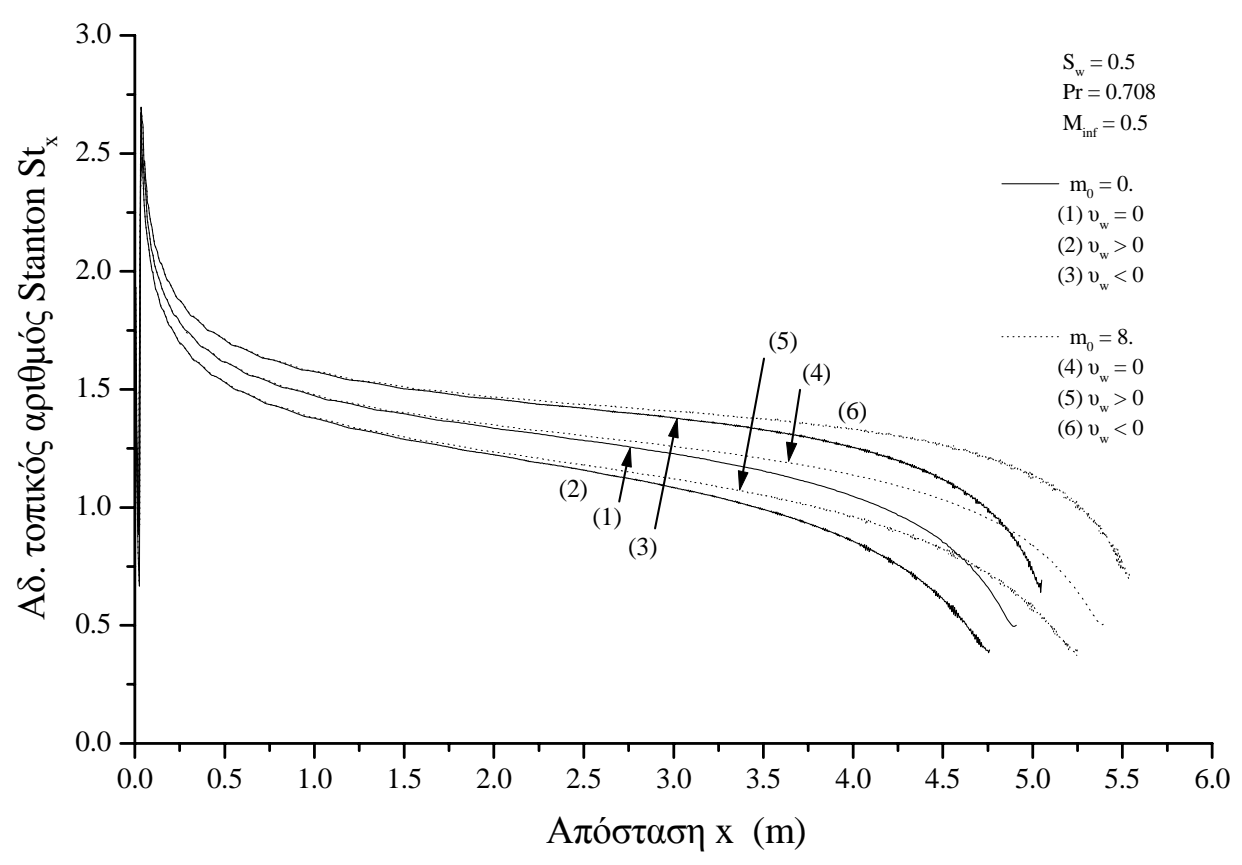

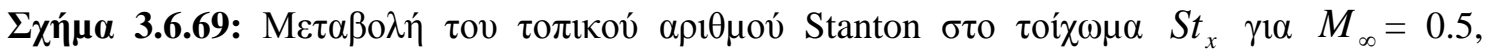

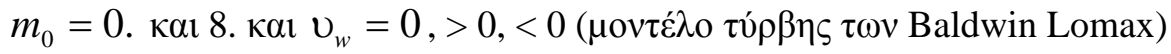

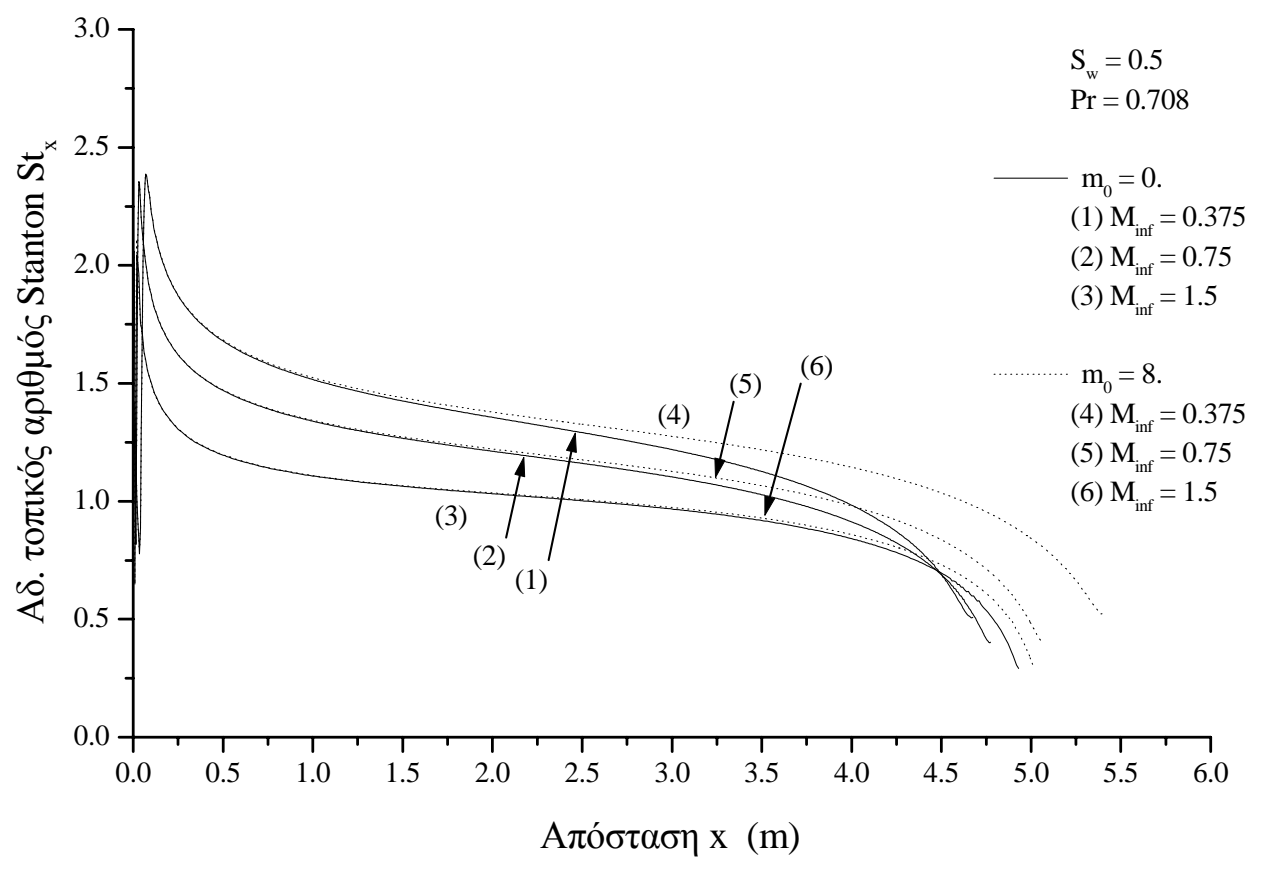

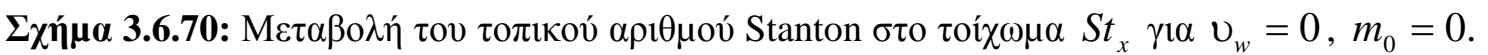

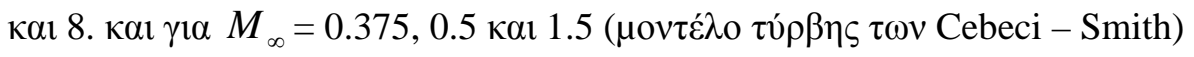




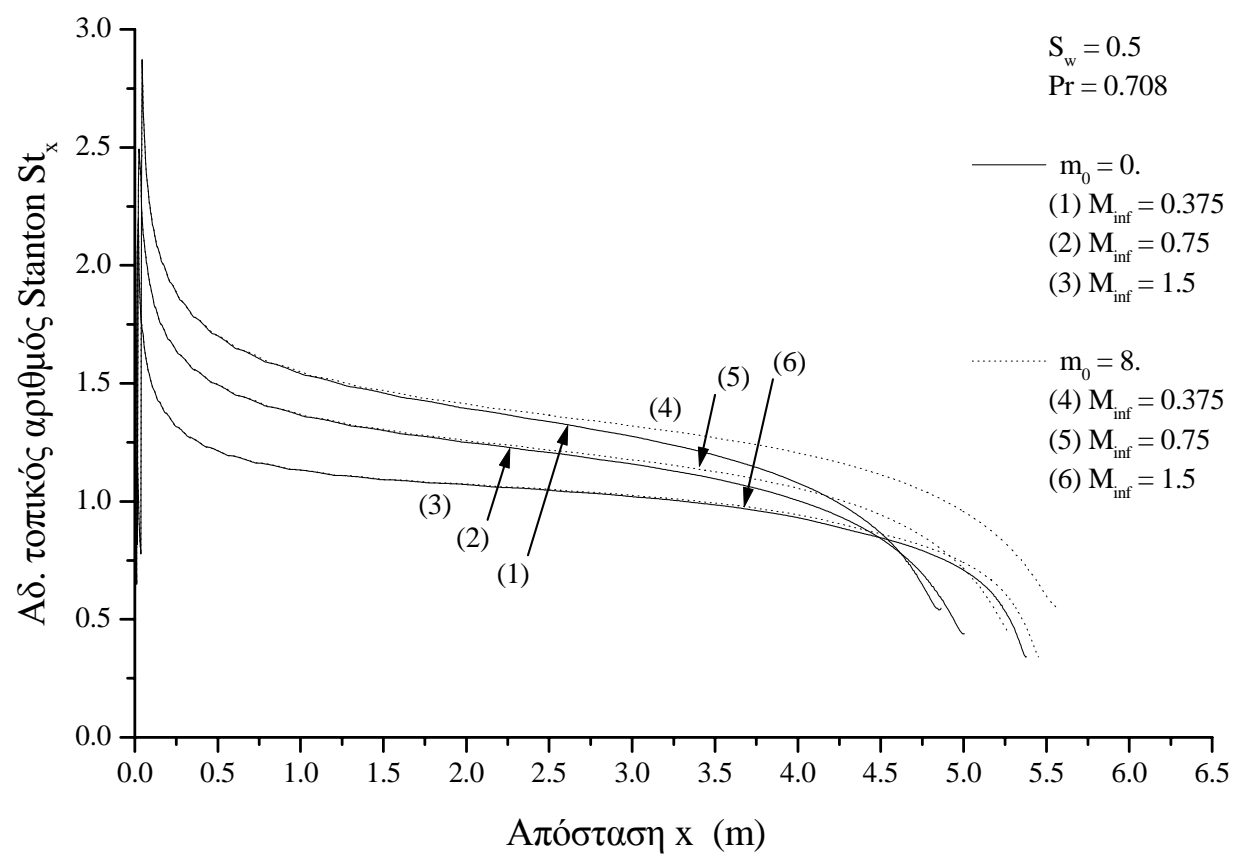

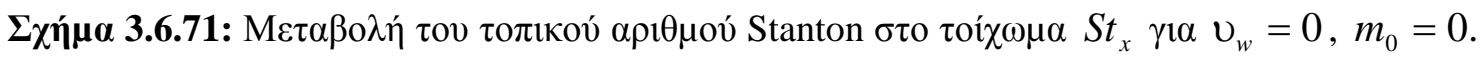

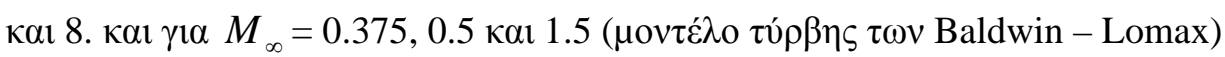

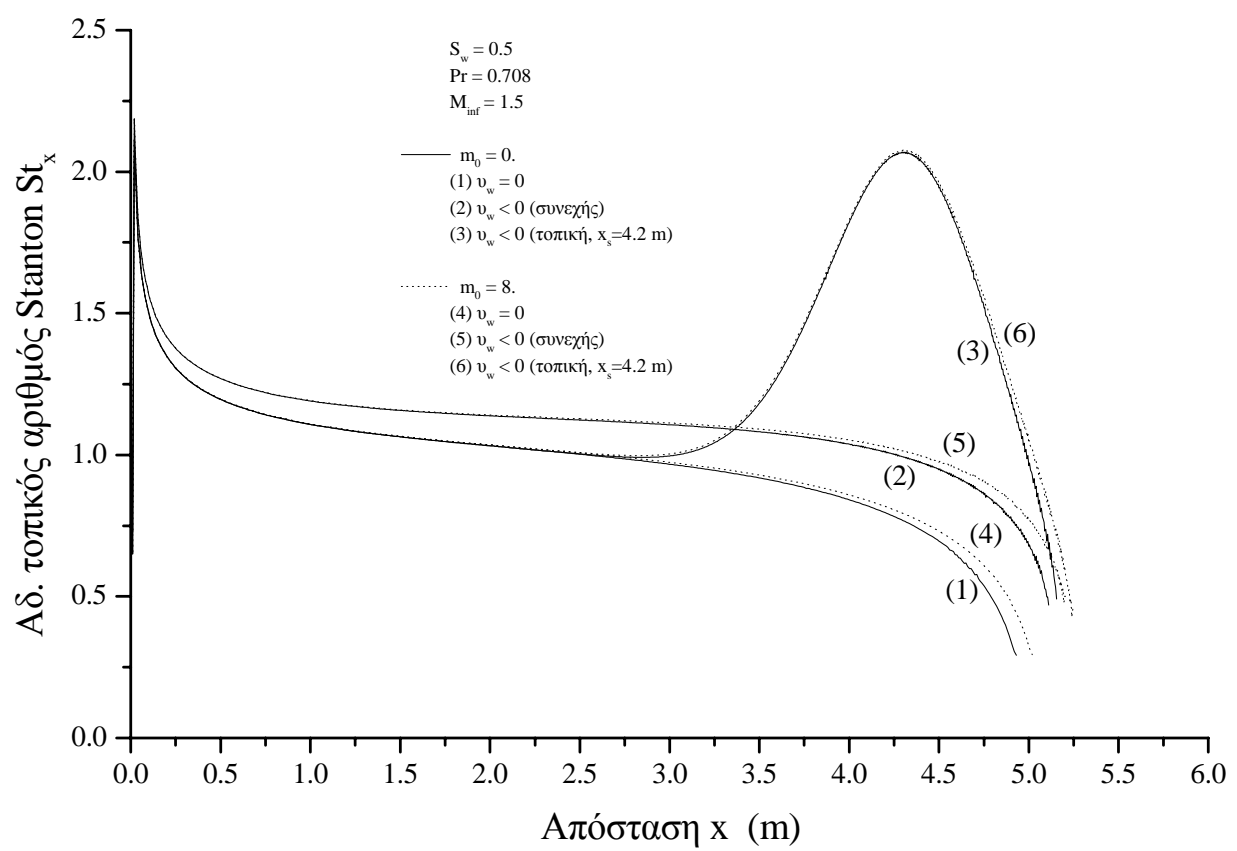

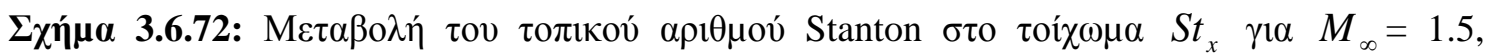

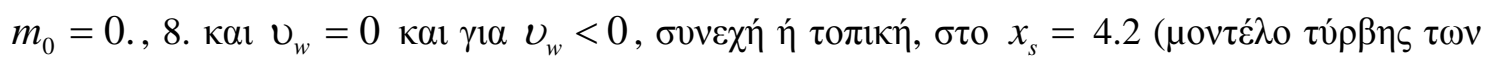
Cebeci - Smith) 


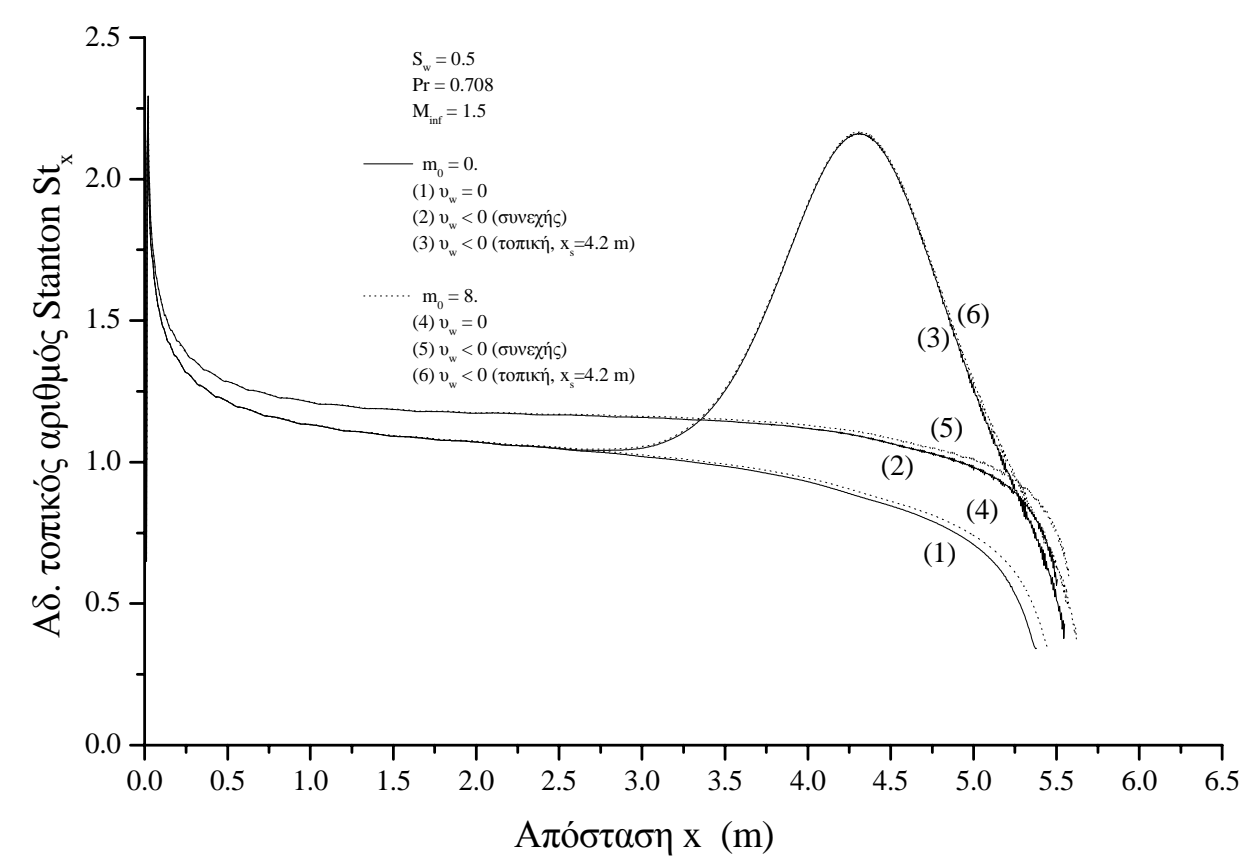

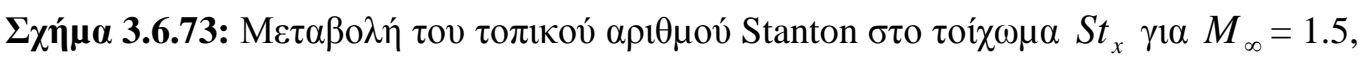

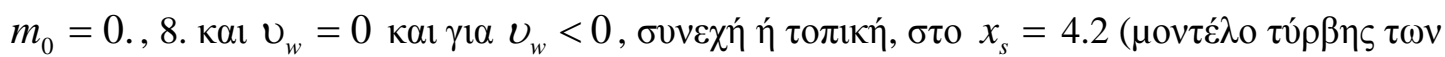
Baldwin - Lomax)

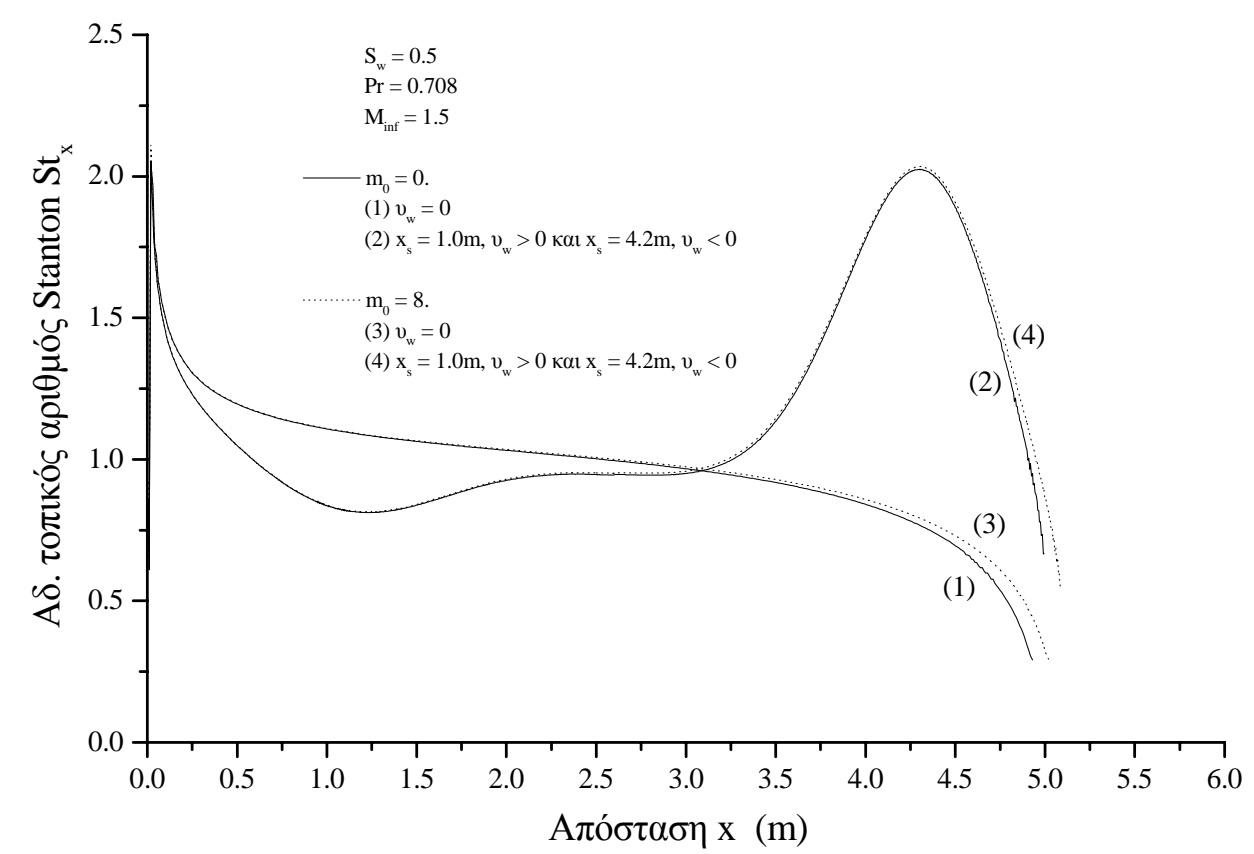

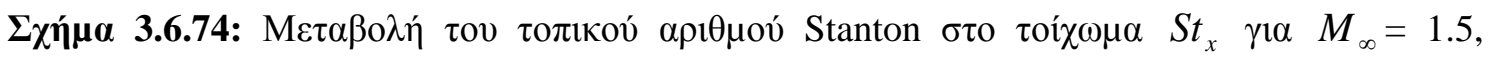

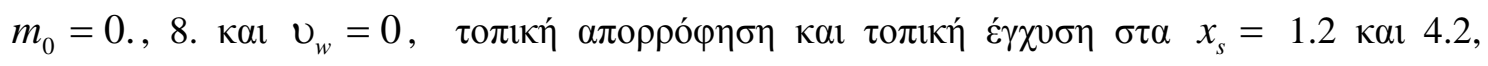

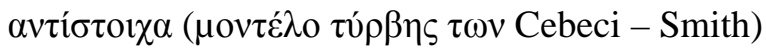




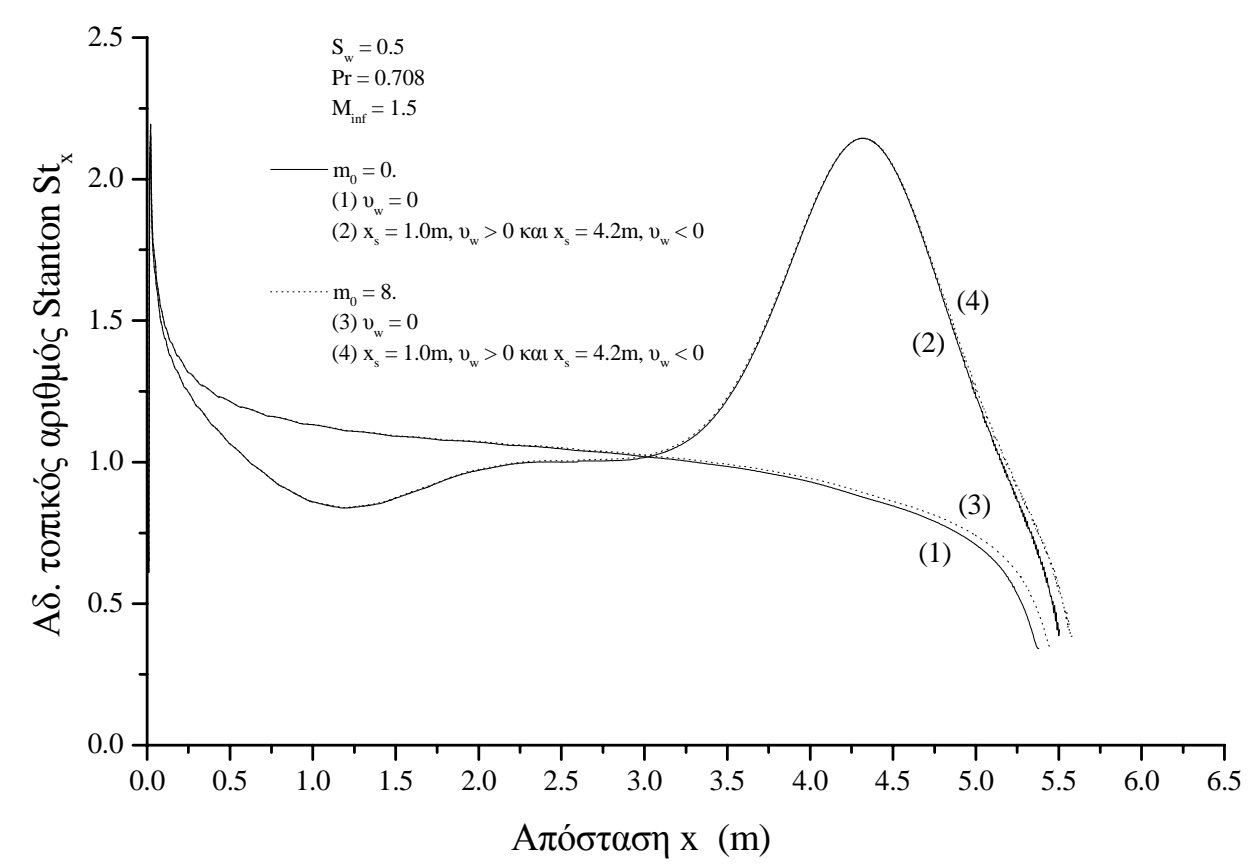

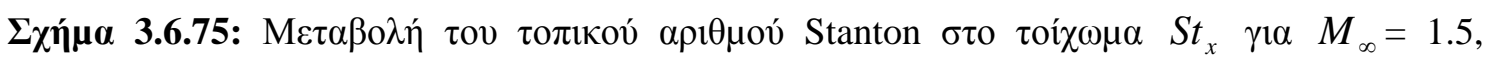

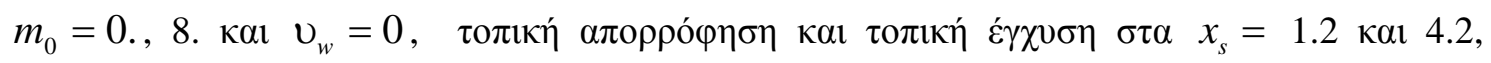

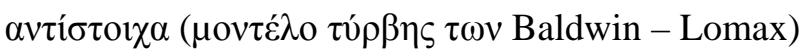

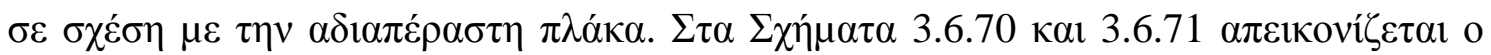

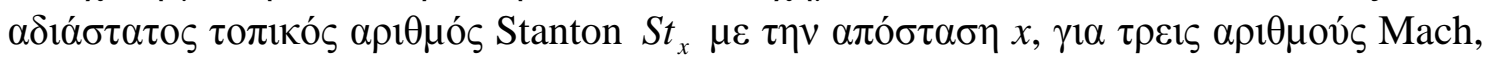

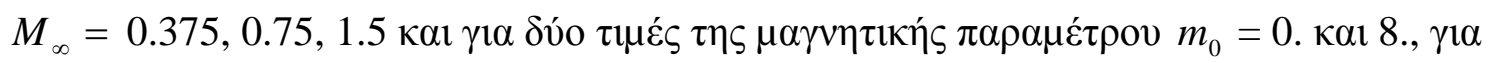

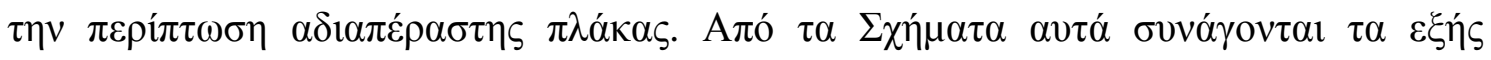

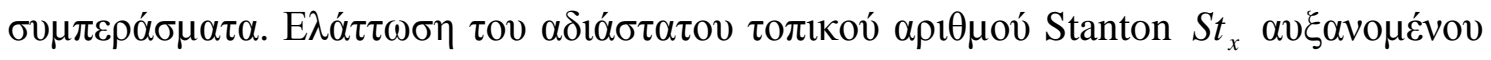

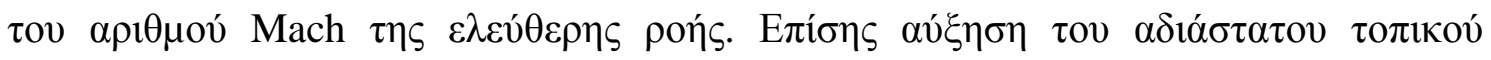

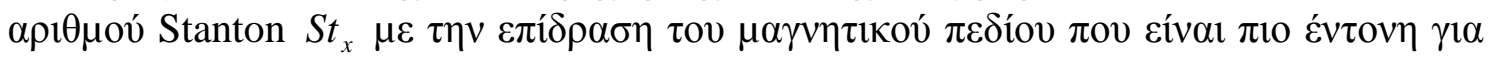
$\mu 1 \kappa \rho \varepsilon ́ \varsigma \tau$ ¿

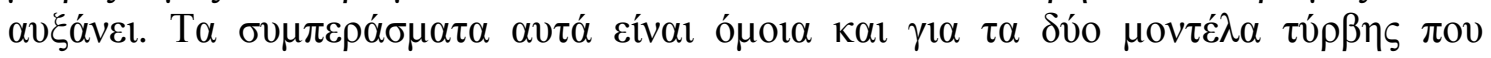

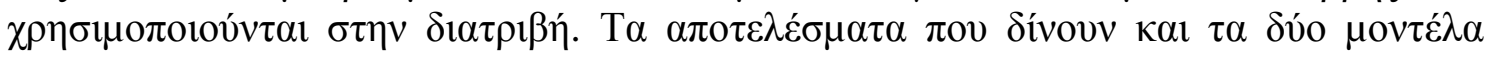

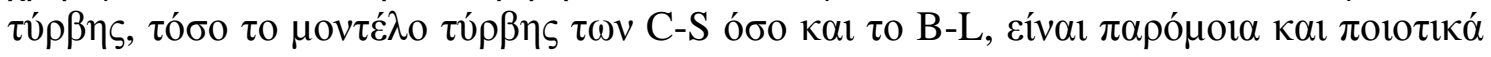

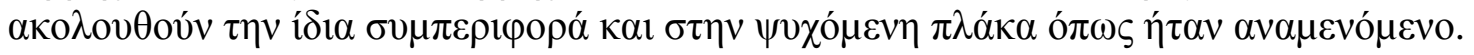

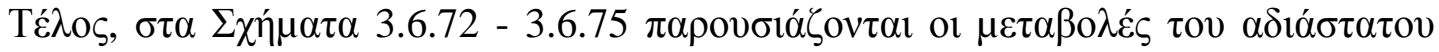

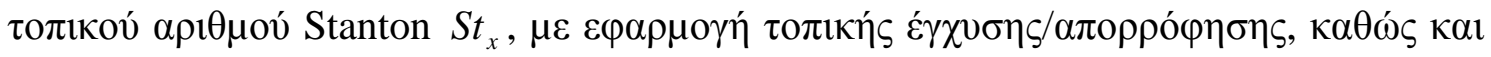

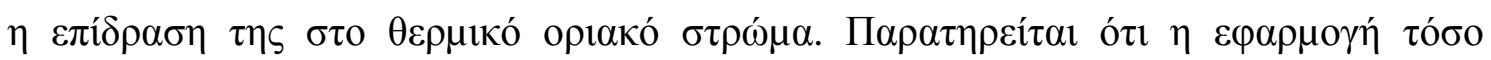

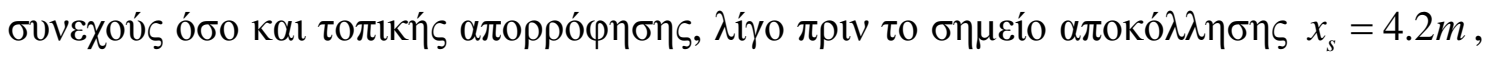

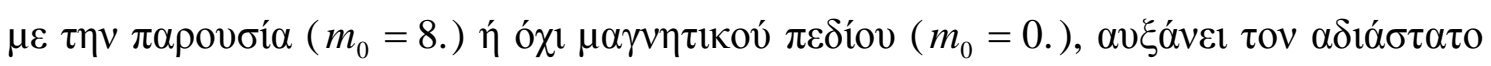

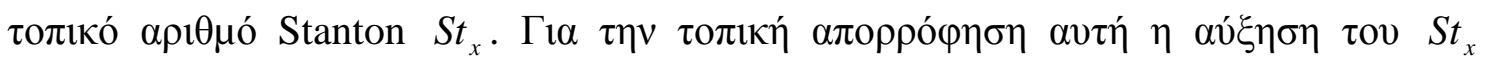

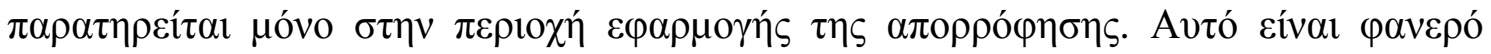

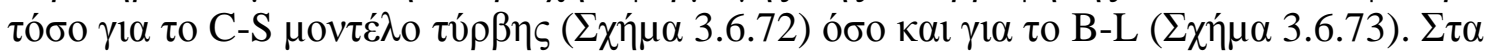

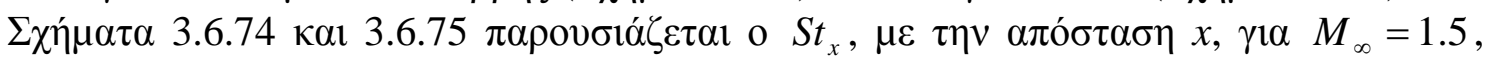




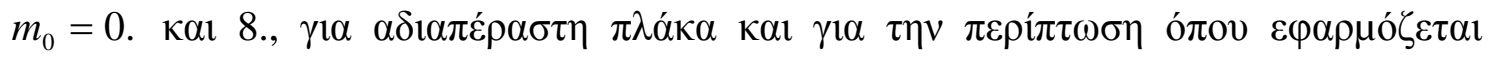

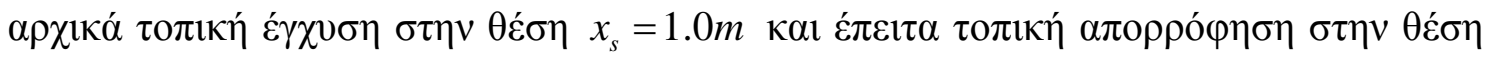

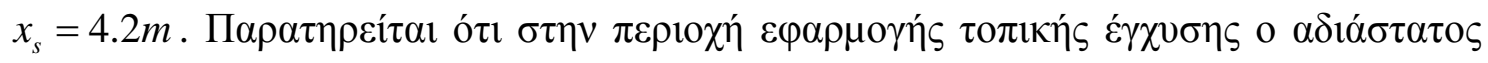

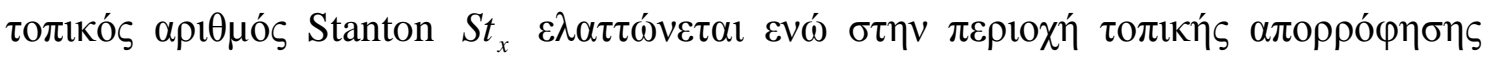

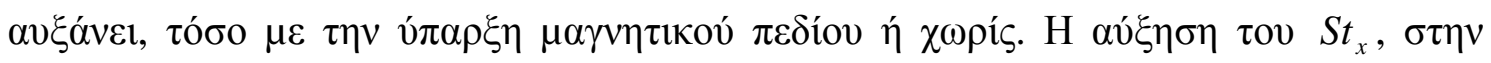

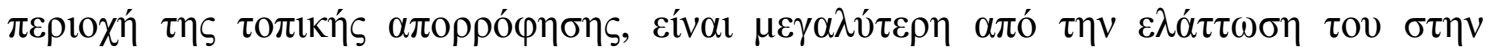

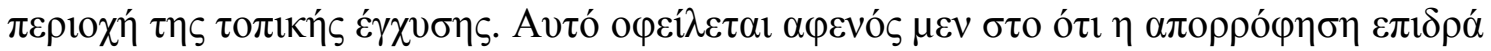

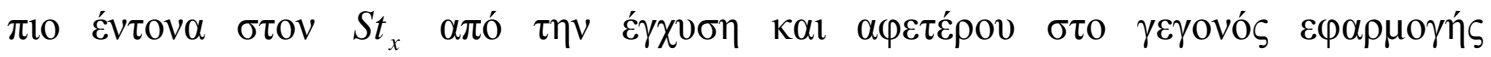

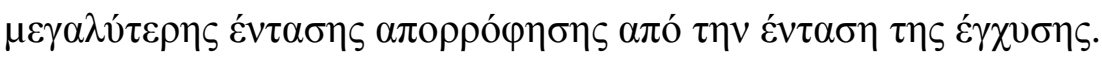

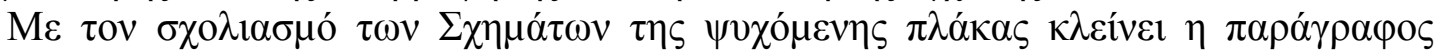

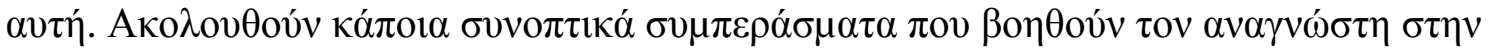

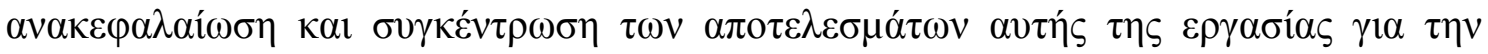

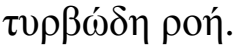

\section{$3.7 \Sigma v \mu \pi \varepsilon \rho \alpha ́ \sigma \mu \alpha \tau \alpha$}

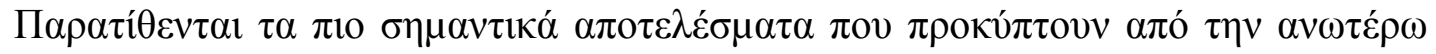

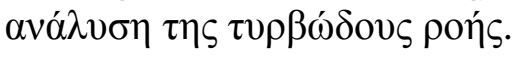

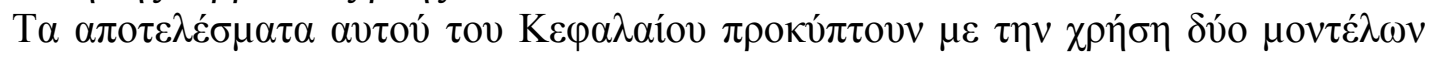

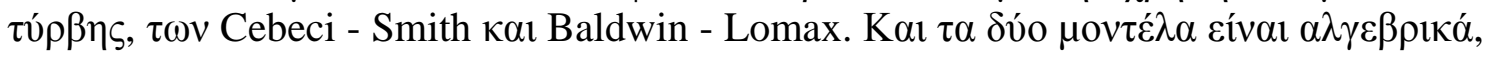

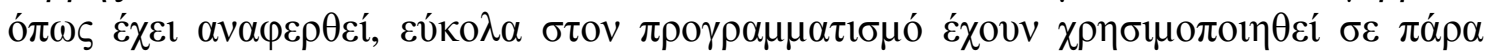

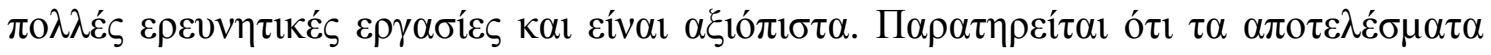

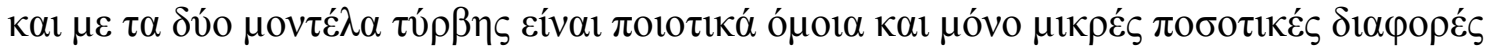

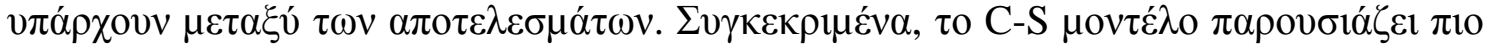

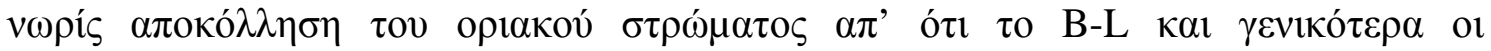

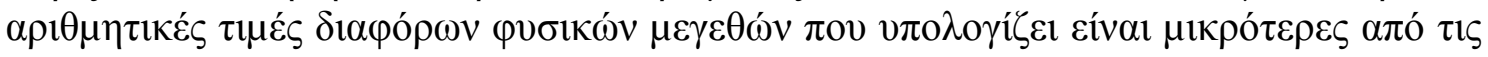

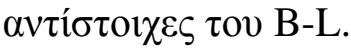

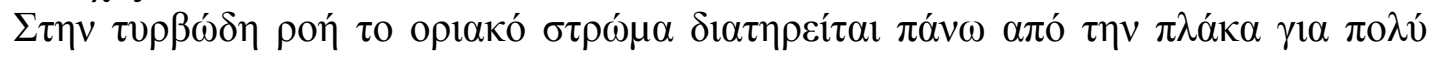

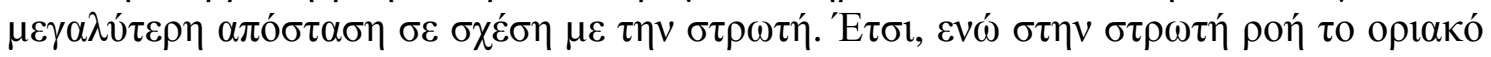

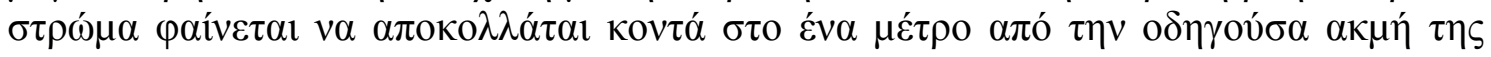

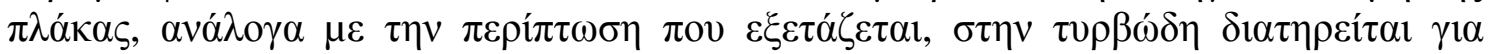

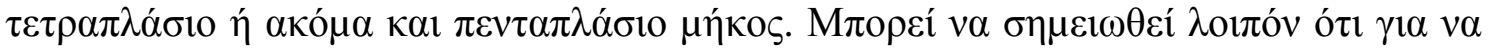

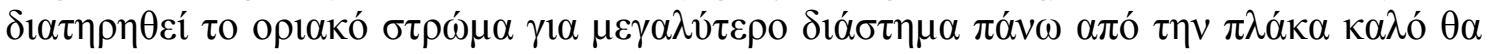

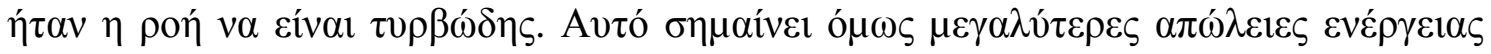

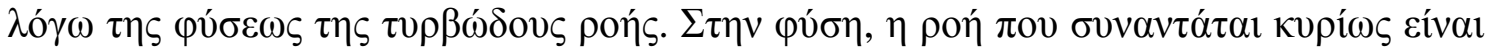

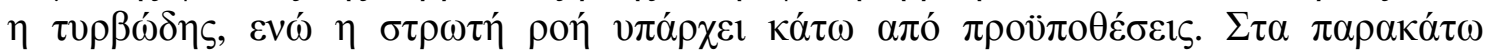

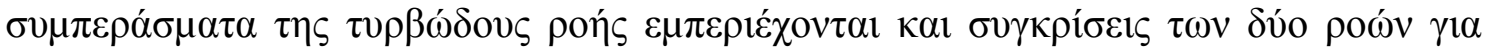

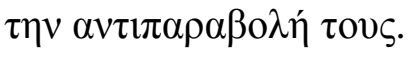

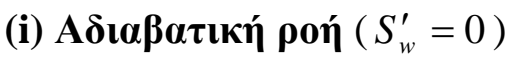

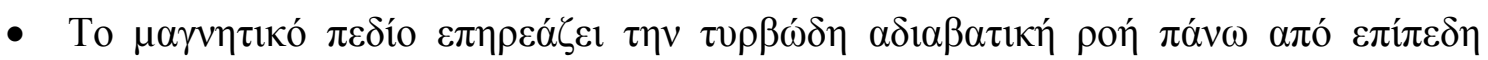

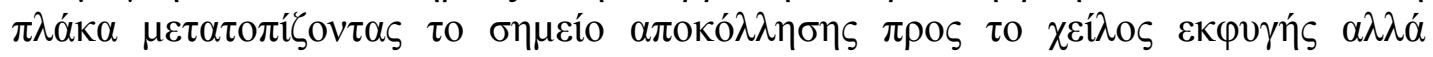

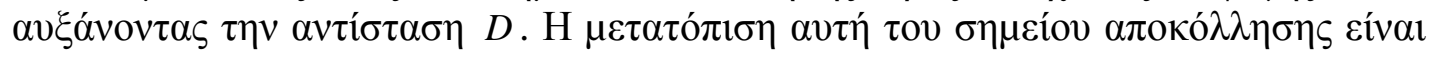

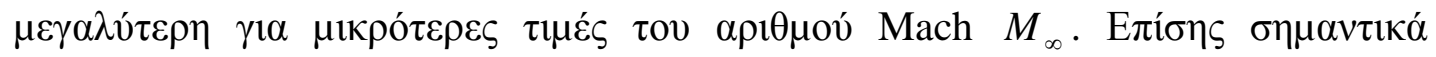

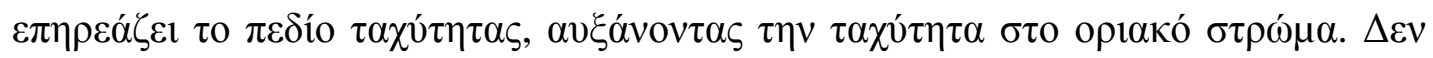

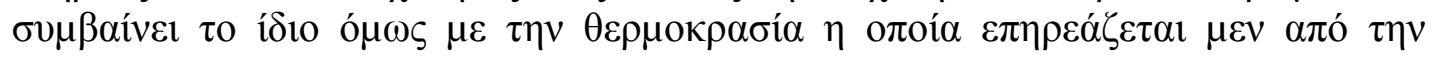




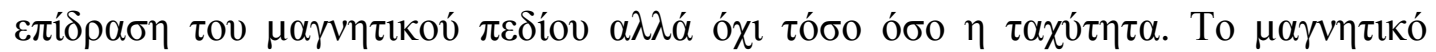

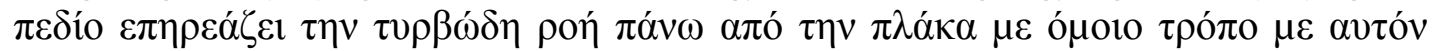

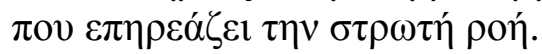

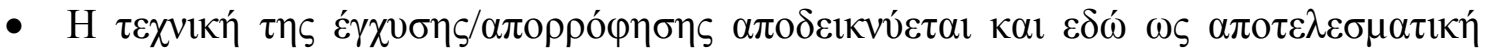

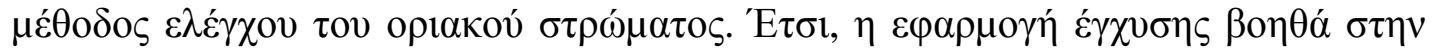

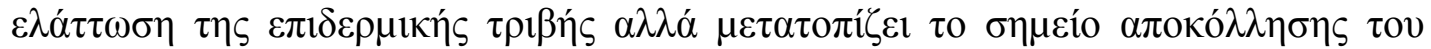

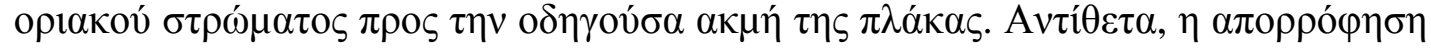

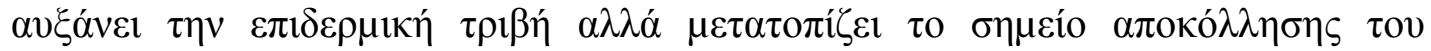

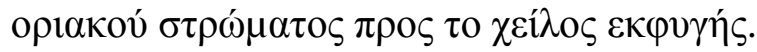

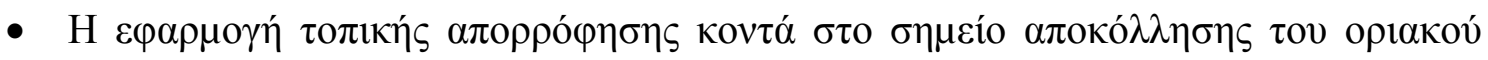

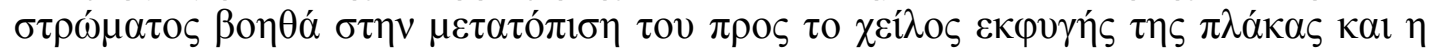

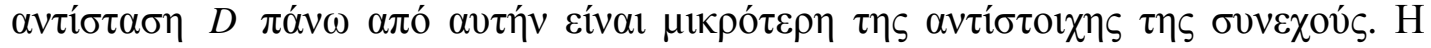

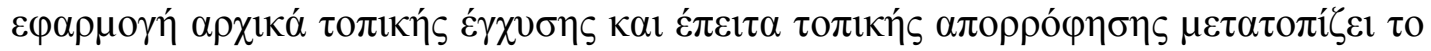

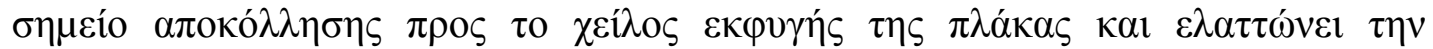

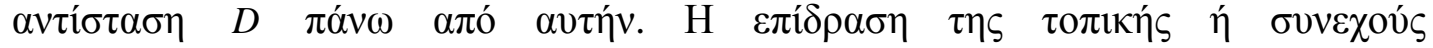

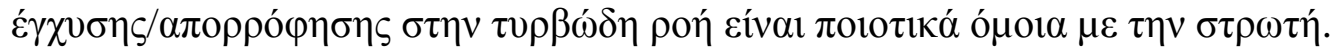

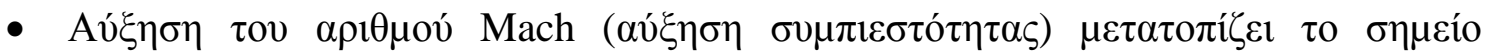

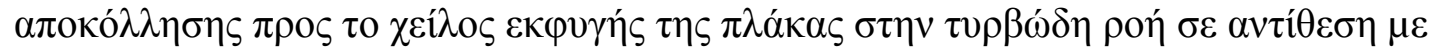
$\tau \eta \nu \sigma \tau \rho \omega \tau \eta \dot{. ~}$

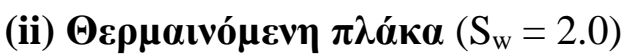

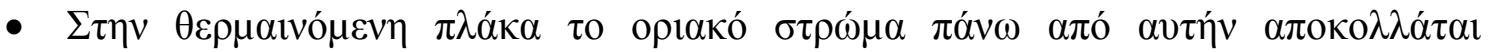

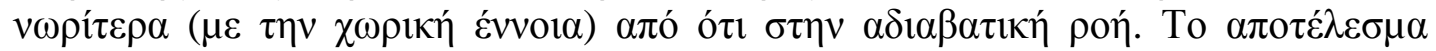
$\alpha v \tau$ cóvíva ó

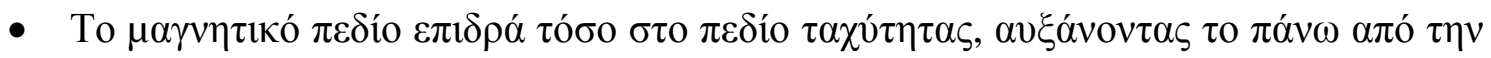

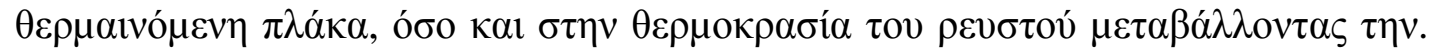

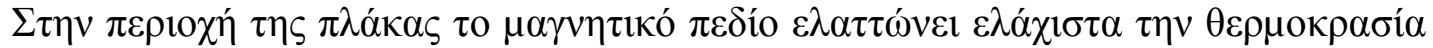

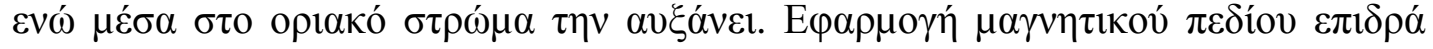

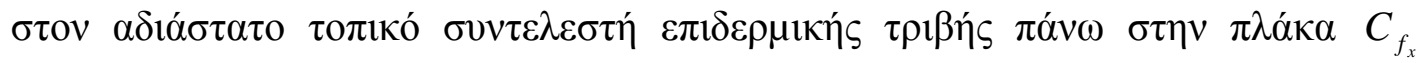

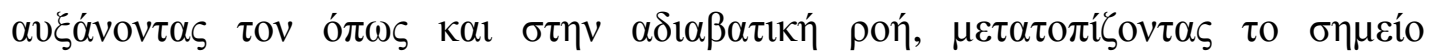

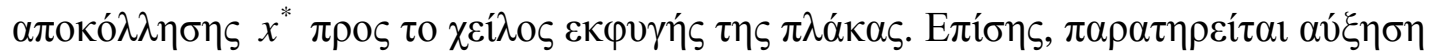

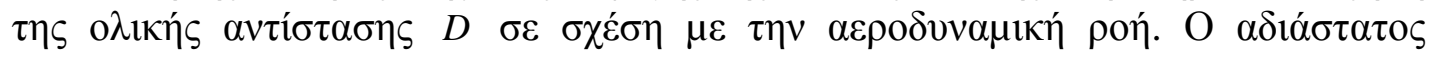

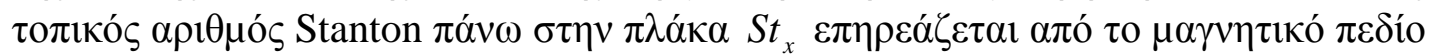

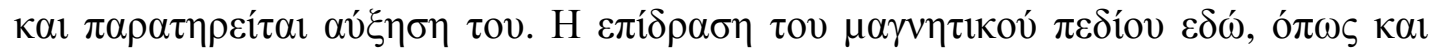

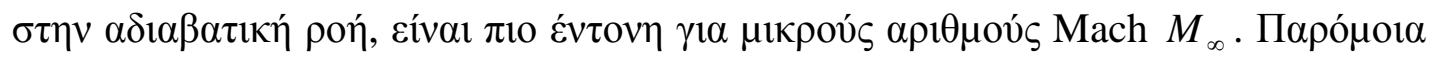

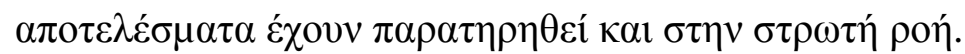

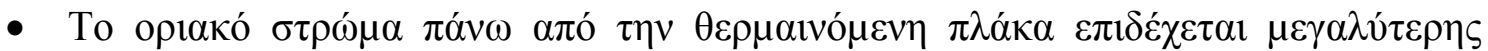

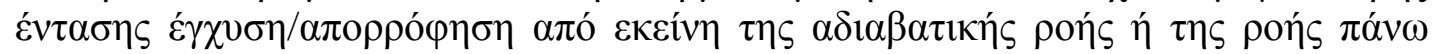

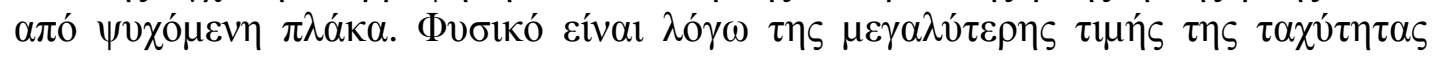

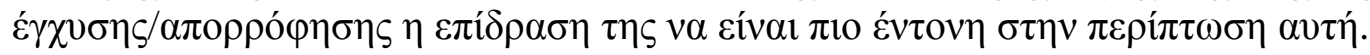

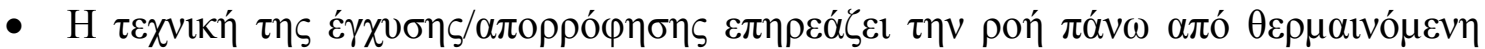

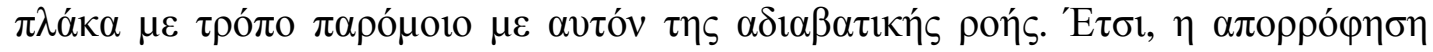




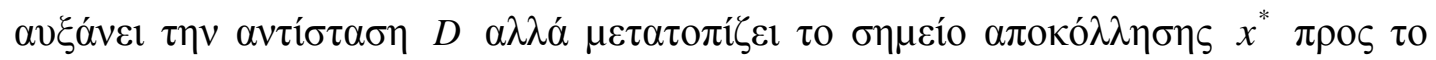

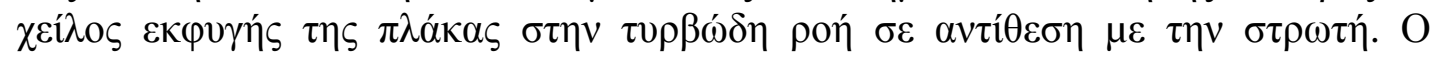

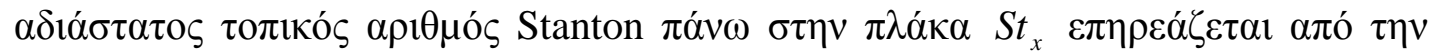

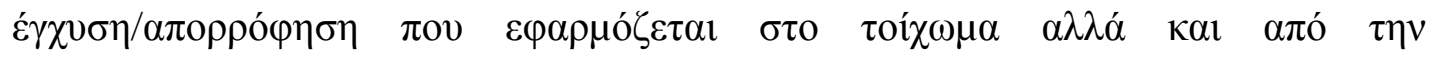
$\sigma v \mu \pi \imath \varepsilon \sigma \tau o ́ \tau \eta \tau \alpha$.

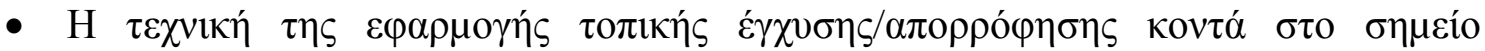

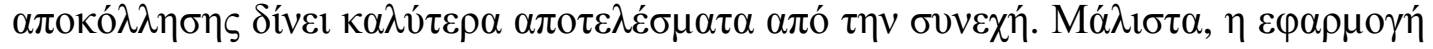

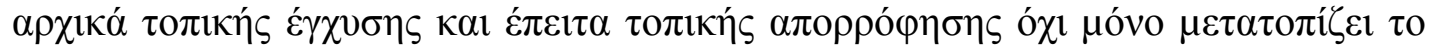

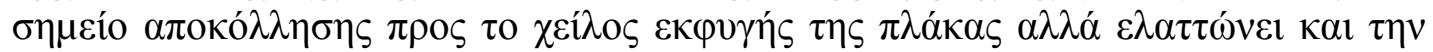

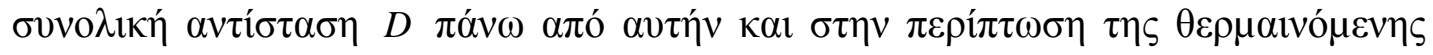

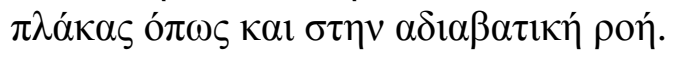

(iii) $\Psi v \chi o ́ \mu \varepsilon v \eta \pi \lambda a ́ k \alpha\left(S_{\mathrm{w}}=0.5\right)$

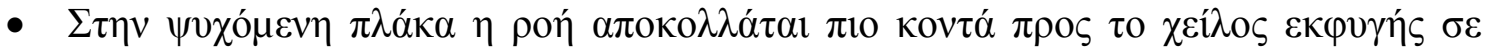

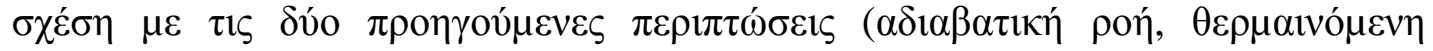

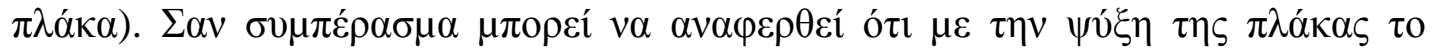

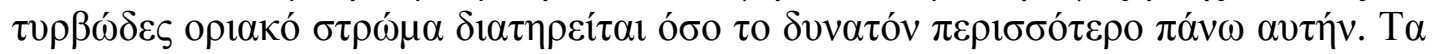

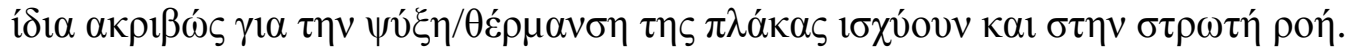

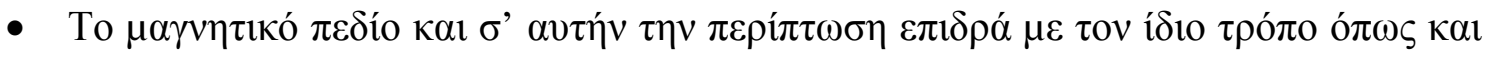

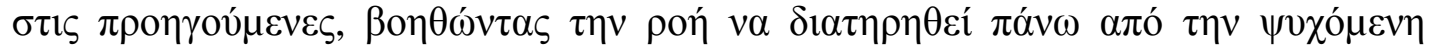

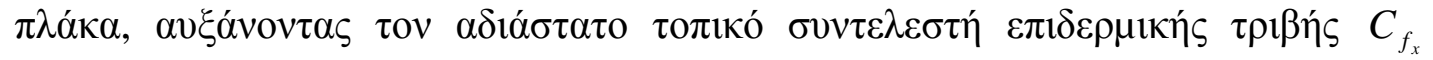

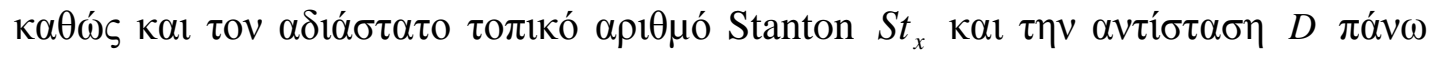
$\sigma \tau \eta \nu \pi \lambda \alpha ́ \kappa \alpha$.

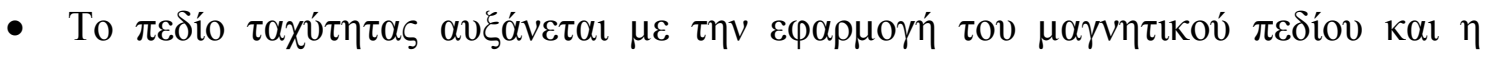

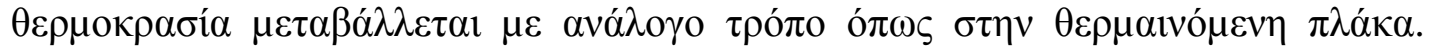

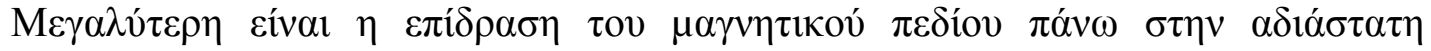

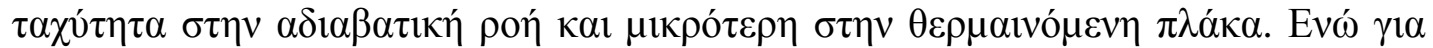

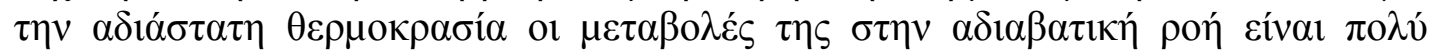

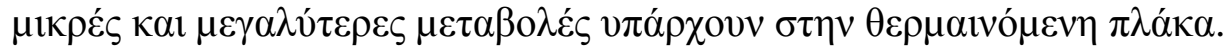

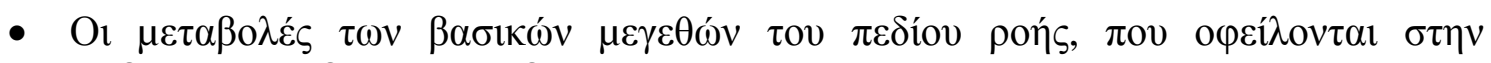

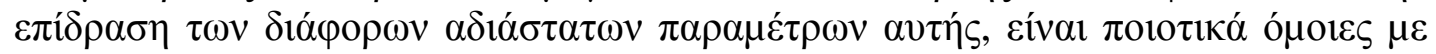
$\alpha v \tau \dot{\varepsilon} \varsigma \tau \eta \varsigma \theta \varepsilon \rho \mu \alpha 1 v o ́ \mu \varepsilon v \eta \varsigma \pi \lambda \alpha ́ \kappa \alpha \varsigma\left(S_{\mathrm{w}}=2.0\right)$.

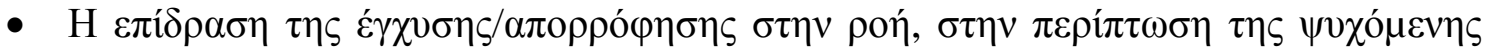

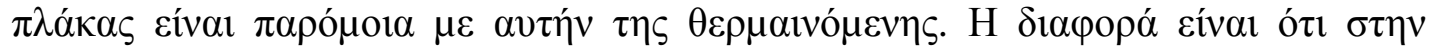

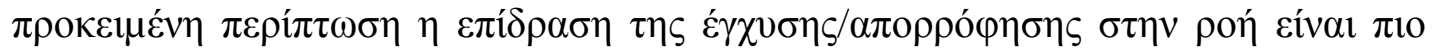

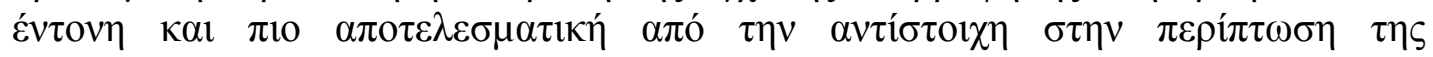
$\theta \varepsilon \rho \mu \alpha \imath{ }^{\mu} \mu \varepsilon v \eta \varsigma \pi \lambda \alpha ́ \kappa \alpha \varsigma$.

- H

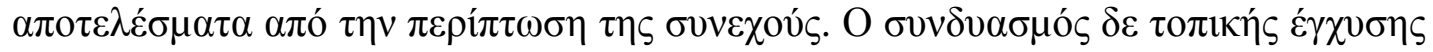

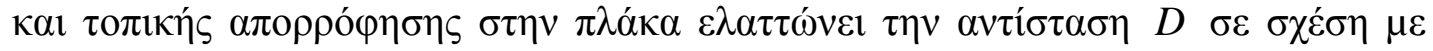

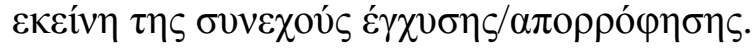




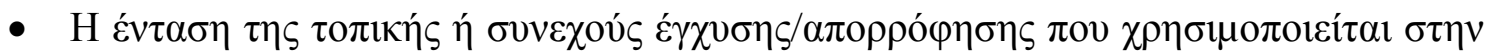

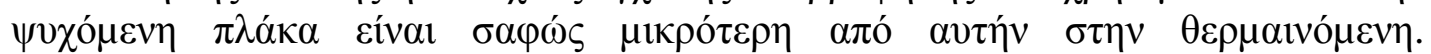

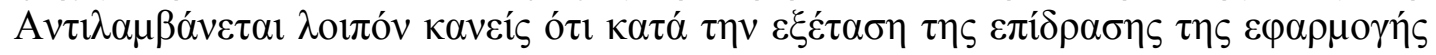

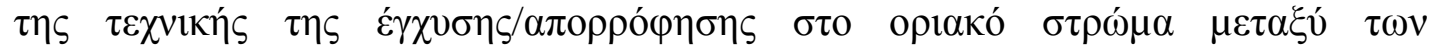

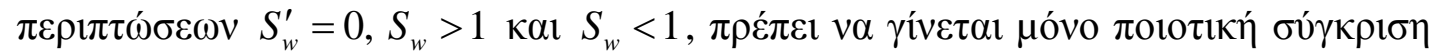

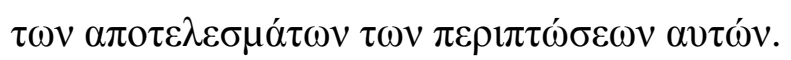




\title{
Numerical Study of Magnetohydrodynamic Compressible Boundary Layer Flow Over a Flat Plate with Adverse Pressure Gradient and Heat and Mass Transfer
}

\begin{abstract}
Summary
In this thesis the steady two-dimensional magnetohydrodynamic (MHD), compressible boundary layer flow, over a flat plate is numerically studied. The flow is subjected to an adverse pressure gradient, due to a linearly retarded velocity, that is known as Howarth's flow. The plate is electrically non-conducting and it is subjected to a suction/injection velocity, continuous or localized, normal to it. The case of an impermeable plate is also studied. The plate is parallel to the free stream of a heatconducting perfect gas (air) flowing with velocity $u_{\infty}$ along the plate. The flow field is subjected to the action of a constant magnetic field which acts normal to the plate. The fluid (air) is considered Newtonian, compressible and electrically conducting.

The fundamental equations of MHD flow are presented in Chapter I as well as the characteristic quantities of the boundary layer which are used in this study.

The laminar flow is studied in Chapter II where as the turbulent flow is studied in Chapter III. For both cases (laminar and turbulent) the partial differential equations and their boundary conditions, describing the problem under consideration, are transformed using the compressible Falkner-Skan transformation and the numerical solution of the problem is obtained by using a modification of the well known Keller's box method. The obtained numerical results for the velocity and temperature field, as well as for the associated boundary layer parameters, are shown in figures for different free-stream Mach numbers $M_{\infty}$ and for the case (i) of an adiabatic flow ( $S_{w}^{\prime}=0$ ), (ii) heating of the wall $\left(S_{w}>1\right)$ and (iii) cooling of the wall $\left(S_{w}<1\right)$, followed by an extensive discussion.

For turbulent flow, in Chapter III, the Reynolds-averaged boundary layer equations are used. Two different turbulent models, namely the model of Cebeci-Smith and Baldwin-Lomax, are used to represent eddy kinematic viscosity and eddy diffusivity of heat. These models are the most simple with acceptable generality and their accuracy has been explored for a wide range of flows for which there are experimental data. It has also been found that they give results sufficiently accurate for most engineering problems. For the turbulent Prandtl number model a modification of the extended Kays and Crawford's model is also used.

In the case of laminar flow (Chapter II) the numerical calculations showed that the application of suction moves separation point downstream, whereas injection moves the separation point towards the leading edge of the plate. The presence of the magnetic field always increases frictional drag on the wall but moves the separation point downstream for every value of free-stream Mach number. This displacement is greater for small values of $M_{\infty}$. The combined influence of the magnetic field, localized injection and localized suction moves separation point downstream reducing frictional drag. These results confirmed for the three cases (adiabatic flow, heating of the wall, cooling of the wall) of the laminar flow and for various free-stream Mach numbers.

Since most flows, which occur in practical applications, are turbulent the results in this case (Chapter III) are more important and are similar with those in laminar flow.
\end{abstract}


Precisely, application of suction moves separation point downstream but injection moves separation point towards the leading edge of the plate reducing drag. Application of localized injection and localized suction moves the separation point downstream reducing total drag. The presence of the magnetic field moves separation point downstream increasing frictional drag. The combined influence of magnetic field, localized injection and localized suction moves separation point further downstream as regards the other cases. These results confirmed for the three cases (adiabatic flow, heating of the wall, cooling of the wall) of turbulent flow, for various free-stream numbers and for two turbulent models (C-S and B-L).

It is hoped that, in the absence of detailed investigations of this problem, the obtained results, are very interesting and give a clearer insight into the mechanism of controlling a laminar or turbulent boundary layer compressible flow. 


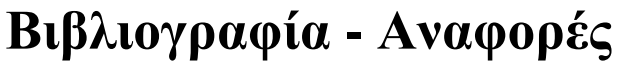

[1] M. Acharya, L.P. Singh and G.C. Dash, Heat and mass transfer over an accelerating surface with heat source in presence of suction and blowing, Int. J. Engn. Sci., Vol. 37, pp. 189-211, 1999.

[2] I.V. Adamovich, J.W. Rich, G.L. Nelson, Feasibility study of magnetohydrodynamic acceleration of unseeded and seeded airflows, AIAA Journal, Vol. 36, No. 4, pp. 590-597, 1998.

[3] R. Agarwal, Computational fluid dynamics of whole-body aircraft, Annu. Rev. Fluid Mech., Vol. 31, pp. 125-169, 1999.

[4] M.E.S. Ahmed and H.A. Attia, Magnetohydrodynamic flow and heat transfer of a non-Newtonian fluid in an eccentric annulus, Can. J. Phys., Vol. 76, pp. 391-401, 1998.

[5] P.D. Ariel, Hiemenz flow in hydromagnetics, Acta Mechanica, Vol. 103, pp. 3143, 1994.

[6] P.D. Ariel, MHD flow of a viscoelastic fluid past a stretching sheet with suction, Acta Mechanica, Vol. 105, pp. 49-56, 1994.

[7] D. Arnal, Control of laminar-turbulent transition for skin friction drag reduction, in: Control of Flow Instabilities and Unsteady Flows, CISM Course, September 18-22, 1995.

[8] D. Arnal, In Proceedings of the CISM Courses and Lectures on Control of Flow Instabilities and Unsteady Flows, Udine, Italy, 18-22 September, No. 369, 1995. Edited by G.E.A. Meier and G.H. Schnerr, Springer-Verlag, 1996.

[9] D. Arnal, Numerical and experimental studies on laminar flow control, Int. J. Num. Meth. Fluids, Vol. 30, pp. 193-204, 1999.

[10] P. Balakumar and P. Hall, Optimum suction distribution for transition control, Theoret. Comput. Fluid Dynamics, Vol. 13, pp. 1-19, 1999.

[11] B. Baldwin and T. Bath, A one-equation turbulence transport model for high Reynolds number in wall bounded flows, NASA Technical Memorandum 102847, 1978.

[12] B. Baldwin and H. Lomax, Thin-layer aproximation and algebric model for separated turbulent flows, AIAA Paper 78-205, 1978.

[13] G. Barakos and D. Drikakis, An implicit unfactored method for unsteady turbulent compressible flows with moving boundaries, Computers \& Fluids, Vol. 28, pp. 899-922, 1999. 
[14] R.F. Blackwelder, The eddy structures in bounded shear flows, in: Special Course on Skin Friction Drag Reduction (ed. J. Cousteix) AGARD Report 786, Paper 6, 1992.

[15] Z.O. Bleviss, Magnetogasdynamics of hypersonic couette flow, J. Aero/Space Sci., Vol. 25, No. 10, pp. 601-615, 1958.

[16] J.P. Boyd, Chebyshev and Fourier Spectral Methods, Dover, 2000.

[17] P. Bradshaw, Turbulence, in Science Progress, 67: 185, Oxford, 1981.

[18] P. Bradshaw (ed.), Turbulence, Topics in Applied Physics, Springer-Verlag, Berlin, Vol. 12, 1976.

[19] H. Branover (ed.), MHD - flows and turbulence, Proceedings of the Bat-Sheva international seminar, Beersheva, March 17-20, 1975.

[20] K.C. Brown and P.N. Joubert, The measurement of skin friction in turbulent boundary layer with adverse pressure gradients, J. Fluid Mech., Vol. 35, pp. 737$757,1969$.

[21] S.N. Brown and K. Stewartson, Laminar separation, Annual Review of Fluid Mech., Vol. 1, pp. 45-72, 1969.

[22] R.L. Burden, J.D. Faires, A.C. Reynolds, Numerical Analysis, Prindle, Weber \& Schmidt, Second edition, 1981.

[23] D.M. Bushnell, Longitudinal vortex control-techniques and applications, The $32^{\text {nd }}$ Lanchester Lecture, Aeronautical Quartely, 293-312, October 1992.

[24] H. Cabannes, Theoretical magnetofluid dynamics, Academic Press, New York, 1970.

[25] C. Canuto, M.Y. Hussaini, A. Quarteroni, T.A. Zang, Spectral Methods in Fluid Dynamics, Springer-Verlag, 1988.

[26] G. Casalis, M.L. Copie, Ch. Airiau, D. Arnal, Nonlinear analysis with PSE approach, IUTAM Symposium on Nonlinear Instability and Transition in ThreeDimensional Boundary Layers, Manchester, July 1995.

[27] T. Cebeci and A.M.O. Smith, Analysis of turbulent boundary layers, Academic Press, New York, 1974.

[28] T. Cebeci, A model for eddy conductivity and turbulent Prandtl number, ASME Journal of Heat Transfer, Vol. 95, pp. 227-234, 1973.

[29] T. Cebeci and P. Bradshaw, Physical and Computational Aspects of Convective Heat Transfer, Springer-Verlag, 1984. 
[30] J.M.G. Chanty, Magnetized plasma flow over an insulator at high magnetic Reynolds number, J. Thermoph. Heat Transfer, Vol. 8, No. 4, p. 795, 1994.

[31] C.J. Chen and J.S. Chiou, Laminar and turbulent heat transfer in the pipe entrance region for liquid metals, Int. J. Heat and Mass Transfer, Vol. 24, pp. 1179-1189, 1981.

[32] T.C. Chiam, A numerical solution for the laminar boundary layer flow with an exponentially decreasing velocity distribution, Acta Mechanica, Vol. 129, pp. 255261, 1998.

[33] J.S. Chiou and H.N. Kao, Heat transfer characteristics of a MHD laminar jet flowing over a convex surface, Appl. Math. Modelling, Vol. 18, No. 12, pp. 679684, 1994.

[34] P.Y. Chou, On velocity correlations and the solutions of the equations of turbulence fluctuations, Quart. Appl. Math., Vol. 3, pp. 38-564, 1945.

[35] Y.M. Chung, H.J. Sung and A.V. Boiko, Spatial simulation of the instability of channel flow with local suction/blowing, Phys. Fluids, Vol. 9, No. 11, pp. 32583266, 1997.

[36] D. Coles and A.J. Wadcock, Flying-hot-wire study of flow past an NACA 4412 airfoil at maximum lift, AIAA Journal, Vol. 17, No. 4, 1979.

[37] D.E. Coles and E.A. Hirst, Proc. AFOSF-IFP-Stanford Conf. On Computation of Turbulent Boundary Layers, Stanford, Aug. 18-25, 1968.

[38] M.A. Collins and R.L. Simpson, Flowfield prediction of separating turbulent shear layers, AIAA Journal, Vol. 16, No. 4, pp. 291-292, 1978.

[39] P. Cooper, Turbulent boundary layer on a rotating disk calculated with an effective viscosity, AIAA Journal, Vol. 9, p. 255, 1971.

[40] K.R. Cramer and S.I. Pai, Magnetofluid dynamics for engineers and applied physicists, McGraw-Hill, New York, 1973.

[41] R.M. Cummings, L.B. Schiff, J.D. Duino, Experimental investigation of tangential slot blowing on a generic chined forebody, Journal of Aircraft, Vol. 32, No. 4, pp. 812-824, 1995.

[42] R.B. Dahlburg, and J.M. Picone, Pseudospectral simulation of compressible magnetohydrodynamic turbulence, International Conference on Spectral and High Order Methods for Partial Differential Equations, North-Holland Publ. Co., Amsterdam, pp. 409-416, 1990.

[43] R.B. Dahlburg, J.P. Dahlburg and J.T. Mariska, Helical magnetohydrodynamic turbulence and the coronal heating problem, Astron. Astrophys, Vol. 198, pp. 300$310,1989$. 
[44] B.I. Davidov, On the statistical dynamics of an incompressible turbulent fluid, Dokl. Akad Nauk SSSR, Vol. 136, p. 47, 1961.

[45] J.H. Davidson, F.A. Kulacki and P.F. Dunn, Handbook of single-phase convective heat transfer, Edited by S. Kakac, R. K. Shah and W. Aung, John Wiley \& Sons, 1987.

[46] G.R. Deboy and D.E. Abbot, Examination of turbulent shear models and the prediction of compressible turbulent boundary layers by the method of weighted residuals, Tech. Rep. No FMTR-71-1, Purdue Univ. Press, Lafayette, Indiana, 1971.

[47] P. Dengel and H.H. Fernholz, An experimental investigation of an incompressible turbulent boundary layer in the vicinity of separation, J. Fluid Mech., Vol. 212, pp. 615-636, 1990.

[48] C.duP. Donaldson and H. Rosenbaum, Calculation of turbulent shear flows through closure of the Reynolds equations by invariant modeling, ARAP Inc. Rep., 127, also NASA-CR-128172, 1968.

[49] C.duP. Donaldson and R.D. Sullivan, An invariant second-order closure model of the compressible turbulent boundary layer on a flat plate, ARAP Inc. Rep., 178, 1972.

[50] C.A.J. Fletcher, Computational Techniques for Fluid Dynamics, Vol. I \& II, Springer-Verlag, 1991.

[51] D. Frei and H. Thomann, Direct measurements of skin friction in a turbulent boundary layer with a strong adverse pressure gradient, J. Fluid Mech., Vol. 101, pp. 79-95, 1980.

[52] M. Gad-el-Hak, A. Pollard, J.P. Bonnet (eds.), Flow control: Fundamentals and Practices, Springer, 1998.

[53] M. Gad-el-Hak and D.M. Bushnell, Separation control: review, J. Fluid Eng., Transactions of the ASME, Vol. 113, pp. 5-29, 1991.

[54] M. Gad-el-Hak and D.M. Bushnell, Status and outlook of flow separation control, AIAA Paper 91-0037, 1991.

[55] M. Gad-el-Hak, Flow control by suction, in: Structure of Turbulence and Drag Reduction (ed. A. Gyr) IUTAM Symposium, Zurich, Switzerland, SpringerVerlag, pp. 357-360, 1990.

[56] M. Gad-el-Hak and R.F. Blackwelder, Selective suction of controlling bursting events in a boundary layer, AIAA Journal, Vol. 27, No. 3, pp. 308-314, 1989.

[57] S. Goldstein, On laminar boundary layer flow near a point of separation, Quart. J. Mech. Appl. Math., Vol. 1, pp. 43-69, 1948. 
[58] R.S.R. Gorla, H.S. Takhar and A. Slaouti, Magnetohydrodynamic free convection boundary layer flow of a thermomicropolar fluid over a vertical plate, Int. J. Engng Sci., Vol. 36, No. 3, pp. 315-327, 1998.

[59] R.S.R. Gorla, J.K. Lee, S. Nakamura and I. Pop, Effects of transverse magnetic field on mixed convection in wall plume of power-law fluids, Int. J. Engn. Sci., Vol. 31, No. 7, pp. 1035-1045, 1993.

[60] R.J. Grade, Turbulent Flow, John Wiley \& sons, New York, 1994.

[61] F.D. Hains and Y.A. Yoler, Axisymetric magnetohydrodynamic flow, J. Aerospace Sci., pp. 143-150, February 1962.

[62] D.D. Haldavnekar, S.S. Santpur and V.M. Soundalgekar, Unsteady MHD flow and heat transfer in boundary layers in a rotating flow with variable suction, Modeling, Simulation \& Control B: Mechanical and Thermal Engineering, Materials \& Resources, Chemistry, Vol. 54, p. 1, 1994.

[63] K. Hanjalic and B.E. Launder, A Reynolds-stress model of turbulence, and its application to thin shear flows, J. Fluid Mech., Vol. 52, p. 609, 1972.

[64] F.H. Harlow and P.I. Nakayama, Turbulence transport equations, Phys. Fluids, Vol. 10, p. 2323, 1967.

[65] J.E. Harris, Numerical solution of the equation for compressible laminar, transitional, and turbulent boundary layers and comparisons with experimental data, NASA Tech. Rep. No R-368, 1971.

[66] J. Hartmann, Hg-Dynamics I, Theory of the laminar flow of an electrically conductive liquid in a homogenous magnetic field, Det. Kgl. Danske Videnskabernes Selskab, Mathematisk-fysiske Meddeleser, XV, 6, 1937.

[67] J. Hartmann and F. Lazarus, Hg-Dynamics II, Experimental investigations on the flow of mercury in a homogeneous magnetic field, Det. Kgl. Danske Videnskabernes Selskab, Mathematisk-fysiske Meddeleser, XV, 7, 1937.

[68] J.N. Hefner, D.M. Bushnell, Surface drag reduction via surface mass injection, in: Viscous drag reduction in boundary layers, Progress in Astronautics and Aeronautics 123, AIAA Washington D. C., pp. 457-476, 1990.

[69] F. Hirt and H. Thomann, Measurements of wall shear stress in turbulent boundary layers subject to strong pressure gradients, J. Fluid Mech., Vol. 171, pp. 547-562, 1986.

[70] E.J. Hopkins and M. Inouye, An evaluation of theories for predicting turbulent skin friction and heat transfer on flat plates at supersonic and hypersonic mach numbers, AIAA Journal, Vol. 9, No. 6, pp. 993-1003, June 1971. 
[71] E.J. Hopkins, E.R. Keener, T.E. Polek and H.A. Dwyer, Hypersonic turbulent skin-friction and boundary layer profiles on nonadiabatic flat plates, AIAA Journal, Vol. 10, p. 40, 1972.

[72] M.A. Hossain, I. Pop and T.Y. Na, Effect of heat transfer on compressible boundary layer flow over a circular cylinder, Acta Mechanica, Vol. 131, pp. 267272, 1998.

[73] L. Howarth, On the solution of the Laminar Boundary Layer in Compressible Fluids, Oxford, 1964.

[74] W.F. Hughes and F.J. Young, The Electromagnetodynamics of Fluids, John Wiley $\&$ Sons, Inc. New York, $1^{\text {st }}$ Ed., 1966.

[75] E.O. Hulburt, Advances in the physics of the upper air since 1950, NRL Report 4600, October 25, 1955.

[76] C.M. Hung, Computation of Navier-Stokes equations for three-dimensional flow separation, AIAA Journal, Vol. 29, No. 10, pp. 1659-1667, 1991.

[77] F. Ilinca, D. Pelletier and L. Ignat, Adaptive finite element solution of compressible turbulent flows, AIAA Journal, Vol. 36, No. 12, pp. 2187-2194, 1998.

[78] P.N. Inman and P. Bradshaw, Mixing length in low Reynolds number turbulent boundary layers, AIAA Journal, Vol. 19, No. 5, pp. 653-655, 1981.

[79] M. Jischa, H.B. Rieke, About the prediction of turbulent Prandtl and Schmidt number, Int. J. Heat Mass Transfer, Vol. 22, pp. 1547-1555, 1979.

[80] B.A. Kader and A.M. Yaglom, Similarity treatment of moving-equilibrium turbulent boundary layers in adverse pressure gradients, J. Fluid Mech., Vol. 89, pp. 305-342, 1978.

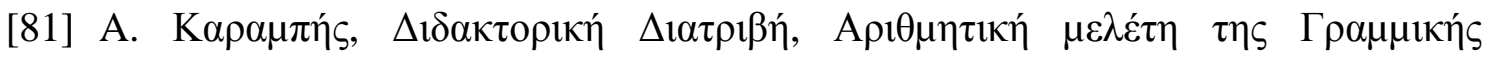

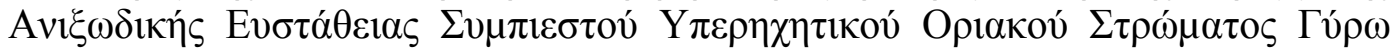

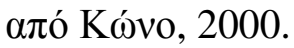

[82] N. Kafoussias, A. Karabis and M. Xenos, Numerical study of two dimensional laminar boundary layer compressible flow with pressure gradient and heat and mass transfer, Inter. J. Engn. Sci., Vol. 37, pp. 1795-1812, 1999.

[83] N.G. Kafoussias and M.A. Xenos, Numerical investigation of two-dimensional turbulent boundary-layer compressible flow with adverse pressure gradient and heat and mass transfer, Acta Mechanica, Vol. 141, pp. 201-223, 2000.

[84] N.G. Kafoussias and N.D. Nanousis, Magnetohydrodynamic laminar boundarylayer flow over a wedge with suction or injection, Can. J. Phys. Vol. 75, pp. 733$745,1997$. 


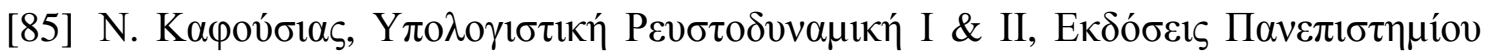

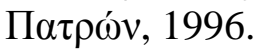

[86] A.K. Kalkan and G. Talmage, Heat transfer in liquid metals with electric currents and magnetic fields: the conduction case, I. J. Heat Mass Transfer, Vol. 37, No. 3, pp. 511-521, 1994.

[87] W.M. Kays and M.E. Crawford, Convective Heat and Mass Transfer, $3^{\text {rd }}$ edn., McGraw-Hill, New York, 1993.

[88] H.B. Keller, A new difference scheme for parabolic problems. Numerical Solutions of Partial Differential Equations, II, J. Bramble (ed.). Academic Press, New York, pp. 265- 293, 1970.

[89] J.J. Kim, Study of turbulence structure through numerical simulations : the perspective of drag and reduction, in : Special Course on Skin Friction Drag Reduction (ed. J. Cousteix) AGARD Report 786, Paper 7, 1992.

[90] K. Kinoshita, K. Tsunoda and K. Yoshikawa, Heat transfer in a disk MHD generator under the influence of a Lorentz force, Heat Transfer Japanese Research, Vol. 23, No. 3, pp. 230-, 1994.

[91] S.J. Kline, S.K. Robinson, Turbulent boundary layer structure: progress, status and challenges, in: Structure of Turbulence and Drag Reduction (ed. A. Gyr) IUTAM Symposium, Zurich, Spriger-Verlag, pp. 3-22, 1990.

[92] A.N. Kolmogorov, Equations of Turbulent Motion of an Incompressible Turbulent Fluid, Izv. Akad. Nauk SSSR Ser Phys. VI, No. 1-2, 1942.

[93] S. Kotake, K. Hijikata, Numerical Simulations of Heat Transfer and Fluid Flow on a Personal Computer, Transport Processes in Engineering, 3, Elsevier, Amsterdam, 1993.

[94] M. Kumari, MHD flows over a wedge with large blowing rates, Int. J. Engng. Sci., Vol. 36, No. 3, pp. 299-314, 1988.

[95] B.E. Launder, A. Morse, W. Rodi and D.B. Spalding, The prediction of free shear flows-a comparison of the performance of six turbulent models, Proc. Conf. Free Turbulent Shear Flows, NASA-SP-321, 1973.

[96] C.H. Lewis, E.W. Miner and E.C. Anderson, Effects of strong axial pressure gradients on turbulent boundary-layer flows, AGARD Conf. Proc. No 93, Turbulent Shear Flows, London, Proc. N72-20273, paper N72-20281, 1971.

[97] C.R. Lin and C.K. Chen, Hydromagnetic flow and heat transfer about a fluid underlying the axisymmetric spreading surface, Int. J. Engn. Sci., Vol. 31, No. 2, pp. 251-261, 1993. 
[98] I. Marusic, A.E. Perry, A wall-wake model for the turbulence structure of boundary layers, Part 2, Further experimental support, J. Fluid Mech., Vol. 298, pp. 389-407, 1995.

[99] G.E.A. Meier, In Proceedings of the CISM Courses and Lectures on Control of Flow Instabilities and Unsteady Flows, Udine, Italy, 18-22 September, No. 369, 1995. Edited by G.E.A. Meier and G.H. Schnerr, Springer-Verlag, 1996.

[100]M. Meneguzzi, A. Pouquet, P.L. Sulem (eds.), Turbulence and Nonlinear Dynamics in MHD Flows, Proceedings of the Workshop on Turbulence and Nonlinear Dynamics in MHD Flows, Cargèse, France, July 4-8, North-Holland, 1988.

[101]W.J. Minkowycz, E.M. Sparrow, Schneider, G.E., Pletcher, R. H.: Handbook of Numerical Heat Transfer, John Wiley \& Sons, Inc. 1988.

[102]R.J. Moffat and W.M. Kays, A review of turbulent boundary layer heat transfer, Research at Stanford, 1958-1983, Adv. In Heat Transfer, Vol. 16, pp. 241-365. 1984.

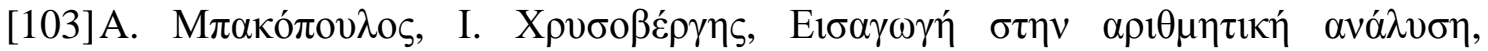

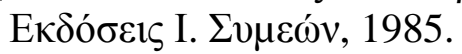

[104] V.S. Murthy and W.C. Rose, Wall shear stress measurements in a shock-wave boundary-layer interaction, AIAA Journal, Vol. 16, No. 7, pp. 667-672, 1978.

[105]R.Y. Myose, R.F. Blackwelder, Control of streamwise vortices using selective suction, AIAA Journal, Vol. 33, No. 6, pp. 1076-1080, 1995.

[106] Y. Nagano, M. Shimada, H. Yamada and M. Kuroishi, Effects of adverse pressure gradient of mean and fluctuating flow characteristics of turbulent boundary layer, National Heat Transfer Symposium of Japan, Vol. 33, No. 1, pp. 1-2, 1996.

[107]Y. Nagano, M. Tagawa and T. Tsuji, Effects of adverse pressure gradients on mean flows and turbulent statistics in a boundary layer, Eighth Symp. On Turbulent Shear Flows, Sept. 9-11, Munich, Springer, 1991.

[108]L.H. Norris, W.C. Reynolds, Turbulent channel flow with a moving wavy boundary, Rept. No. FM - 10, Stanford Univ. Mech. Eng. Dept., USA, 1975.

[109]E.N. Petropoulou and P.D. Siafarikas, Bounded solutions of a class of linear delay and advanced partial difference equations, Dynamics Systems and Applications, Vol. 10, pp. 243-260, 2001.

[110]E.N. Petropoulou and P.D. Siafarikas, Solutions of non-linear delay and advanced partial difference equations in the space $\ell_{\mathrm{N} \times \mathrm{N}}^{1}$, Computers and Mathematics with Applications, Special Issue: Advances in Difference IV, (to appear)

[111]I. Pop and T.Y. Na, A note on MHD flow over a stretching permeable surface, Mech. Res. Comm., Vol. 25, No. 3, pp. 263-269, 1998. 
[112]I. Pop, M. Kumari and G. Nath, Conjugate MHD flow past a flat plate, Acta Mechanica, Vol. 106, pp. 215-220, 1994.

[113]L. Prandtl, Uber Ein Neues Formelsystem fur die Ausgebidete Trubulenz. Nachrichten van der Akad der Wissenschaft in Gottingen, 1945.

[114]T.H. Pulliam and J. L. Steger, Impicit Finite-Difference Simulations of ThreeDimensional Compressible Flow, AIAA Journal, Vol. 18, No. 2, pp. 159-167, 1980 .

[115]D.A.S. Rees, Three dimensional free convection boundary layers in porous media induced by a heated surface with spanwise temperature variations, Transactions ASME, J. of Heat Transfer, Vol. 119, pp. 792-798, 1997.

[116]E.L. Resler and W.R. Sears, The prospects for magneto-aerodynamics, J. Aeronautical Sci., Vol. 25, No. 4, pp. 235-258, 1958.

[117] W.S. Reynolds, W.S. Saric, Experiments on the stability of the flat plate boundary layer with suction, AIAA Paper 82-1026, 1982.

[118] S.K. Robinson, Coherent motions in the turbulent boundary layer, Ann. Rev. Fluid Mech. 23, pp. 603-639, 1991.

[119]W. Rodi, Turbulence models and their applications in hydraulics, A state-of-theart review, A.A. Balkema, Rotterdam, Brookfield, 1993.

[120] V.J. Rossow, On flow of electrically conducting fluids over a flat plate in the presence of a transverse magnetic field, Report 1358, 1957.

[121] V.J. Rossow, On magneto-aerodynamic boundary layers, ZAMP, Vol. IX b, pp. 519-527, 1958.

[122] J.C. Rotta, Statistiche theorie nichthomogener turbulenz, Z. Phys., Vol. 129, pp. 547-572, 1951.

[123] S. Roy, Nonuniform slot injection (suction) into a compressible flow, Acta Mechanica, Vol. 139, pp. 43-56, 2000.

[124]H. Schlichting, Boundary-Layer Theory, Trans. by Kestin J., $7^{\text {th }}$ Ed., McGrawHill, Inc. New York, 1979.

[125] S. Schreier, Compressible Flow, Wiley, New York, 1982.

[126]J.V. Shebalin and D. Montgomery, Magnetic field amplification in mechanically driven compressible magnetohydrodynamic turbulence, AIAA Journal, Vol. 28, No. 8, pp. 1360-1368, 1990.

[127] Y. Shimomura, Large eddy simulation of magnetohydrodynamic turbulent channel flow under a uniform magnetic field, Phys. Fluids A, Vol. 3, No. 12, pp. 30983106, 1991. 
[128]R.L. Simpson, J.H. Strickland, and P.W. Berr, Features of a separating turbulent boundary layer in the vicinity of separation, J. Fluid Mech., Vol. 79, pp. 553-594, 1977.

[129]R.L. Simpson, Y.T. Chew, and B.G. Shivaprasad, The structure of a separating turbulent boundary layer. Part 1. Mean flow and Reynolds stresses, J. Fluid Mech., Vol. 113, pp. 23-51, 1981.

[130]R.L. Simpson, Y.T. Chew, and B.G. Shivaprasad, The structure of a separating turbulent boundary layer. Part 2. Higher-order turbulence results, J. Fluid Mech., Vol. 113, pp. 53-73, 1981.

[131] M. Sokolov, R.A. Antonia, Response of a turbulent boundary layer to intensive suction through a porous strip, in: Proceedings $9^{\text {th }}$ Symposium on Turbulent Shear Flows, Kyoto Japan, Paper 5-3, 1993.

[132]P.R. Spalart and J.H. Watmuff, Experimental and numerical study of a turbulent boundary layer with pressure gradients, J. Fluid Mech., Vol. 249, pp. 337-371, 1993.

[133]P.R. Spalart, G.N. Coleman, Numerical study of a separation bubble with heat transfer, Europ. J. Mech. B16, pp. 169-189, 1997.

[134]P.R. Spalart, R.D. Moser, M.M. Rogers, Spectral methods for the Navier-Stokes equations with one infinite and two periodic directions, J. Comp. Phys., Vol. 96, pp. 297-324, 1991.

[135]D.B. Spalding and W. Rodi, A two parameter model of turbulence and its application to free jets, Wärme and Stoffübertragung, No. 3, p. 85, 1970.

[136]E.T. Spyropoulos and G.A. Blaisdell, Large-eddy simulation of a spatialy evolving supersonic turbulent boundary-layer flow, AIAA Journal, Vol. 36, No. 11, pp. 1983-1990, 1998.

[137]B.S. Stratford, Flow in the laminar boundary layer near separation, ARC, RM 3002, pp. 1-27, 1957.

[138] J.L. Steger, Impicit finite difference simulation of flow about arbitrary geometries with application to airfoils, AIAA Paper 77-665, presented at AIAA $12^{\text {th }}$ Thermophysics Conference at Albuqurque, New Mex., June 27-29, 1977.

[139] Y. Sumitani, N. Kasagi, Direct Numerical Simulation of turbulent transport with uniform wall injection and suction, AIAA Journal, Vol. 33, No. 7, pp. 1220-1228, 1995.

[140]G.W. Sutton and A. Sherman, Engineering Magnetohydrodynamics, McGrawHill, Inc. New York, 1965. 
[141]S. Suwa and Y. Sasaki, On the magnetohydrodynamical incompressible radial wall jet in the presence of a transverse magnetic field, Transac. Japanese Soc. Aeron. Space Sci., Vol. 37, No. 115, p. 42, 1994.

[142]H.S. Takar and G. Nath, Self-similar solution of the unstedy flow in the stagnation point region of a rotating sphere with a magnetic field, Heat and Mass Transfer, Vol. 36, pp. 89-96, 2000.

[143]H.S. Takar, A.J. Chamkha and G. Nath, Unsteady flow and heat transfer on a semi-infinite flat plate with an aligned magnetic field, Int. J. Engng. Sci., Vol. 37, pp. 1723-1736, 1999.

[144]B.E. Thompson and J.H. Whitelaw, Characteristics of a trailing-edge flow with turbulent boundary-layer separation, J. Fluid Mech., Vol. 157, pp. 305-326, 1985.

[145]T. Watanabe and I. Pop, Thermal boundary layers in magnetohydrodynamic flow over a flat plate in the presence of a transverse magnetic field, Acta Mechanica, Vol. 105, pp. 233-238, 1994.

[146]B. Weigand, J.R. Ferguson, M.E. Crawford, An extended Kays and Crawford turbulent Prandtl number model, Int. J. Heat Mass Transfer, Vol. 40, No. 17, pp. 4191-4196, 1997.

[147]F.M. White, Viscous Fluid Flow, Second Edition, McGraw-Hill, New-York, 1991.

[148] S.P. Wilkinson, J.B. Anders, B.S. Lazos, D.M. Bushnell, Turbulent drag reduction research of NASA Langlay: progress and plans, Int. J. of Heat and Fluid Flow, Vol. 9, pp. 266-277, 1988.

[149] W.P. Wolf and S.S. Ochs, CFD calculations of S809 aerodynamic characteristics, AIAA Paper 97-0973, 1997.

[150]M. Wolfsthein, The velocity and temperature distributions of one-dimensional flow with turbulence augmentation and pressure gradient, Inter. J. Heat Mass Transfer, Vol. 12, pp. 301-318, 1969.

[151]X. Wu and K.D Squires, Large eddy simulation of an equilibrium threedimensional turbulent boundary layer, AIAA Journal, Vol. 35, No. 1, pp. 67-74, 1997.

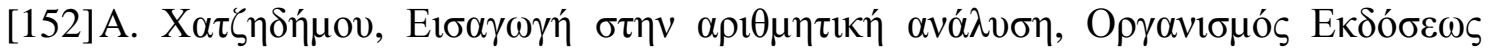

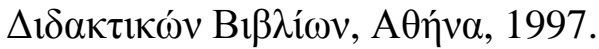

[153] M. Xenos, N. Kafoussias and G. Karahalios, Magnetohydrodynamic compressible laminar boundary layer adiabatic flow with adverse pressure gradient and continuous or localized mass transfer, Canadian Journal of Physics, Vol. 79, pp. 247-1263, 2001. 
[154]K.A. Yih, The effect of uniform suction/blowing on heat transfer of magnetohydrodynamic Hiemenz flow through porous media, Acta Mechanica, Vol. 130, pp. 147-158, 1998.

[155]A. Yoshizawa, Hydrodynamic and magnetohydrodynamic turbulent flows, Modelling and statistical theory, Kluwer Academic Publishers, Dordrecht, 1998. 